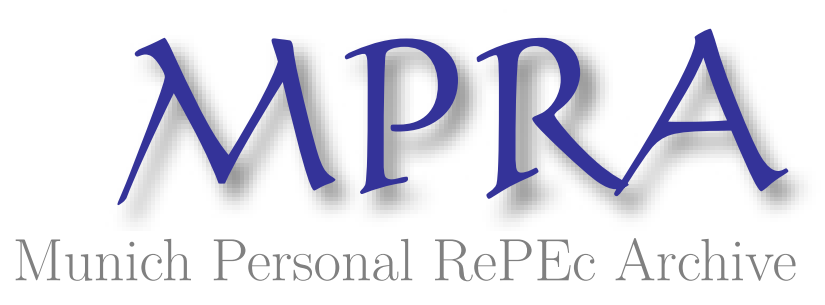

\title{
Option Pricing Under the Variance Gamma Process
}

Fiorani, Filo

April 2004

Online at https://mpra.ub.uni-muenchen.de/15395/

MPRA Paper No. 15395, posted 03 Jun 2009 00:12 UTC 


\title{
UNIVERSITÀ DEGLI STUDI DI TRIESTE
}

Sede Amministrativa del Dottorato di Ricerca

UNIVERSITÀ DEGLI STUDI DI TORINO

LIBERA UNIVERSITÀ COMMERCIALE LUIGI BOCCONI

UNIVERSITÀ DEGLI STUDI DI VENEZIA CA' FOSCARI

UNIVERSITÀ DEGLI STUDI DI UDINE

Sedi Convenzionate

XV CICLO DEL

DOTTORATO DI RICERCA IN

MATEMATICA PER LE DECISIONI ECONOMICHE

\section{OPTION PRICING UNDER THE VARIANCE GAMMA PROCESS}

\author{
DOTTORANDO \\ Dott. FILIPPO FIORANI
}

COORDINATORE DEL COLLEGIO DEI DOCENTI

CHIAR.MO PROF. MARCO ZECCHIN, UNIVERSITÀ DEGLI STUDI DI TRIESTE

FIRMA:

TUTORE

CHIAR.MA PROF.SSA ELISA LUCIANO, UNIVERSITÀ DEGLI STUDI DI TORINO FIRMA:

RELATORE

CHIAR.MA PROF.SSA ELISA LUCIANO, UNIVERSITÀ DEGLI STUDI DI TORINO FIRMA: 
To my parents and to Vittorio 


\section{Acknowledgments}

We wish to sincerely thank Professor Elisa Luciano for all the precious teachings and advices she has given us in these many years starting from our years in college and continuing with our PhD. Her approach to research has always been a model and a guide for us.

We want to thank very much Professor Marco Zecchin for the interest he has shown in our research and for his advices, his help and his availability during our PhD work.

We wish to especially thank Dr. Filipe Aires for useful comments and stimulating discussions on these topics. He has succeed in passing us his passion and enthusiasm for research and his suggestions have been of great help in giving originality to this work.

We are grateful to Professor Ali Hirsa for sharing precious ideas and for first stimulating our interest in the topic.

We want to thank Don Devendorf, Carol Chen and Richard Truong for challenging conversations, mainly on the vanilla option pricing.

We are very grateful to Aristeia Capital and especially to Satyajit Bose, Kevin Toner, Robert Lynch and Anthony Frascella for useful discussions, mainly on the implied volatility analysis.

Last, but not least, a special thank has to go to our family and to Li Zhou for their support during this work. 


\section{Contents}

1 Introduction $\quad 10$

1.1 Limits of the Black, Scholes and Merton Approach for Option Pricing . . . . . . . . . . . . . . 10

1.2 Purely Discontinuous Price Processes and Lévy Processes . . . 16

1.3 Variance Gamma Process . . . . . . . . . . . . . . . . . . . . 22

2 The Variance Gamma Model: Properties and Pricing of European Options $\quad 27$

2.1 Introduction and Brief History of the Model . . . . . . . . . . 27

2.2 Variance Gamma as Time Changed Brownian Motion . . . . . 28

2.3 Parameters of the Variance Gamma Process . . . . . . . . . . 30

2.4 Symmetric Variance Gamma . . . . . . . . . . . . . . . . . . . 33

2.5 Variance Gamma Process as Difference of Two Gamma Processes 35

2.6 Lévy Measure for the Variance Gamma Process . . . . . . . . 37

2.7 Properties of Processes Describing Stock returns . . . . . . . . 39

2.7.1 Completely Monotone Lévy Densities . . . . . . . . . . 39

2.7.2 Finite Variation Processes . . . . . . . . . . . . . 40

2.7.3 Finite Activity Processes . . . . . . . . . . . . . . . . . 41

2.7.4 Variance Gamma Properties . . . . . . . . . . . . . . 41

2.8 The Statistical and Risk Neutral Variance Gamma Stock Price

Process . . . . . . . . . . . . . . . . . . 42

2.8.1 The Statistical Variance Gamma Stock Price Process . 42

2.8.2 The Risk Neutral Variance Gamma Stock Price Process 43

2.9 European Option Price Under Variance Gamma . . . . . . . . 43

2.10 Empirical Tests of the Variance Gamma Model for European

Options . . . . . . . . . . . . . . . . . . 48

2.10.1 Skewness and Kurtosis Results . . . . . . . . . . . . . 48

2.10.2 Pricing Performance of the Variance Gamma . . . . . . 49 
2.10 .3 Conclusion. . . . . . . . . . . . . . . . . . . 50

3 Extensions of the Variance Gamma Model: CGMY Model and Stochastic Volatility Models 52

3.1 Introduction . . . . . . . . . . . . . . . . 52

3.2 Lévy Measure and Parameters for the CGMY Process . . . . . 53

3.2 .1 Definition of the Model . . . . . . . . . . . . . . . 53

3.2.2 Parameters of the CGMY Process . . . . . . . . . . . 53

3.3 Characteristic Function of the CGMY Process . . . . . . . . 55

3.4 The Statistical and Risk neutral CGMY Stock Price Process . 56

3.4.1 The Statistical CGMY Stock Price Process . . . . . . . 57

3.4.2 The Risk neutral CGMY Stock Price Process . . . . . 58

3.5 Variance and Higher Moments of the CGMY Distribution . . . 59

3.6 Decomposition of the Quadratic Variation . . . . . . . . . 60

3.7 Empirical Test of the CGMY Model for European Options . . 60

3.7.1 Skewness and Kurtosis Results . . . . . . . . . . . . . . 61

3.7.2 Diffusion Component Results . . . . . . . . . . . . 62

3.7.3 Results on the Fine Structure of Returns . . . . . . . . 62

3.8 Stochastic Volatility for Variance Gamma and CGMY Processes 63

3.8.1 Pricing of Options with Different Maturities and of Option Time Series . . . . . . . . . . . . . . . . 63

3.8.2 Stochastic Volatility Models as Time Changed Processes 66

3.8.3 Stochastic Volatility Variance Gamma and CGMY . . 67

3.8.4 Stock Price Processes for Stochastic Volatility Variance Gamma and CGMY . . . . . . . . . . . . . . 69

3.8.5 Empirical Tests of Stochastic Volatility Variance Gamma

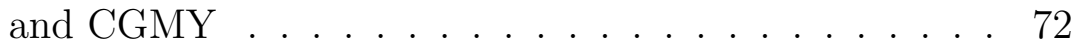

3.9 Conclusions . . . . . . . . . . . . . . . . . . . 73

4 Numerical Solution for the European and American Plain Vanilla Option Price Under the Variance Gamma Process 75

4.1 Introduction . . . . . . . . . . . . . . . 75

4.2 Plain Vanilla European Options . . . . . . . . . . . . . . 76

4.2.1 The Variance Gamma PIDE for European Vanilla Options ...................... 76

4.2.2 Numerical Solution for Vanilla European Options . . . 79

4.2.3 Solution of the Jump Integral for Vanilla European Op-

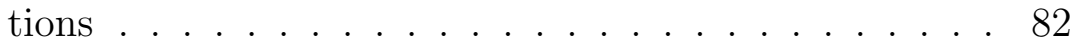


4.2.4 Difference Equation for European Vanilla Options . . . 89

4.3 Experimental Results for European Vanilla Options . . . . . . 91

4.3.1 Calibration .................... 91

4.3.2 Stability of the Scheme . . . . . . . . . . . . 92

4.3.3 Calls: Sensitivity Analysis . . . . . . . . . . . . . 110

4.3.4 Puts: Sensitivity Analysis . . . . . . . . . . . . . . . 119

4.3.5 Implied Volatility Analysis . . . . . . . . . . . . . . . 129

4.3.6 Comparison with geometric Brownian motion . . . . . 145

4.4 Plain Vanilla American Options . . . . . . . . . . . . . . . . . 149

4.4.1 The Variance Gamma PIDE for American Vanilla Options . . . . . . . . . . . . . . . . . . . 149

4.4.2 Numerical Solution for American Vanilla Options . . . 154

4.4.3 Solution of the Jump Integral for American Vanilla Options . . . . . . . . . . . . . . . . 155

4.4.4 Solution of the Integral in the Heaviside Term for American Vanilla Options . . . . . . . . . . . . . . 157

4.4.5 Difference Equation for American Vanilla Options . . . 161

4.5 Experimental Results for American Vanilla Options . . . . . . 163

4.5.1 Calls: Sensitivity Analysis . . . . . . . . . . . . . 163

4.5.2 Puts: Sensitivity Analysis . . . . . . . . . . . 167

5 Numerical Solution for the European and American Barrier Option Price Under the Variance Gamma Process $\quad 180$

5.1 Introduction to Barrier Options . . . . . . . . . . . . . . . . 180

5.2 European Barrier Options . . . . . . . . . . . . . . . . 182

5.2.1 The Variance Gamma PIDE for European Barrier Options ........................... 182

5.2.2 Numerical Solution for European Barrier Options . . . 184

5.2.3 Solution of the Jump Integral for European Barrier Options . . . . . . . . . . . . . . . . 185

5.2.4 Difference Equation for European Barrier Options . . . 191

5.3 Experimental Results for European Barrier Options . . . . . . 192

5.3.1 Up-And-Out Calls: Sensitivity Analysis . . . . . . . . . 193

5.3.2 Down-And-Out Calls: Sensitivity Analysis . . . . . . . 198

5.3.3 Up-And-Out Puts: Sensitivity Analysis . . . . . . . . . 201

5.3.4 Down-And-Out Puts: Sensitivity Analysis . . . . . . . 204

5.4 American Barrier Options . . . . . . . . . . . . . . 222

5.4 .1 Introduction . . . . . . . . . . . . . . . . 222 
5.4.2 The Variance Gamma PIDE for American Barrier Op-

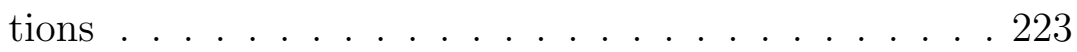

5.4.3 Numerical Solution for American Barrier Options . . . 225

5.4.4 Solution of the Jump Integral for American Barrier Options . . . . . . . . . . . . . . 226

5.4.5 Solution of the Integral in the Heaviside Term for American Barrier Options . . . . . . . . . . . . . . . . 228

5.4.6 Difference Equation for American Barrier Option . . . 231

5.5 Experimental Results for American Barrier Options . . . . . . 233

5.5.1 Up-And-Out Calls: Sensitivity Analysis . . . . . . . . . 234

5.5.2 Down-And-Out Calls: Sensitivity Analysis . . . . . . . 236

5.5.3 Up-And-Out Puts: Sensitivity Analysis . . . . . . . . . 239

5.5.4 Down-And-Out Puts: Sensitivity Analysis . . . . . . . 243

A Appendix

A.1 Numerical Solution of the Jump Integral in the Variance Gamma

PIDE for Vanilla European Options . . . . . . . . . . . . . 257

A.1.1 Solution of Integrals (A.6 and A.5) . . . . . . . . . . 258

A.1.2 Solution of Integrals (A.7 and A.4) . . . . . . . . . 260

A.1.3 Solution of Integral (A.8) in the Case of Vanilla Put

Options . . . . . . . . . . . . . . 262

A.1.4 Solution of Integral (A.3) in the Case of Vanilla Put Options . . . . . . . . . . . . . . . 263

A.1.5 Numerical Solution of the Jump Integral for European Vanilla Put Options . . . . . . . . . . . . . . . 264

A.1.6 Solution of Integral (A.8) in the Case of Vanilla Call Options . . . . . . . . . . . . 266

A.1.7 Solution of Integral (A.3) in the Case of Vanilla Call

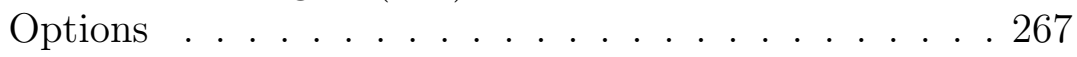

A.1.8 Numerical Solution of the Jump Integral for European Vanilla Call Options . . . . . . . . . . . . . . 268

A.2 Variance Gamma Difference Equation for European Vanilla Options . . . . . . . . . . . . . . . . 270

A.2.1 Difference Equation for Call Options . . . . . . . . . 270

A.2.2 Difference Equation for Put Options . . . . . . . . 271 
A.3 Numerical Solution of the Jump Integral in the Variance Gamma PIDE for American Vanilla Options . . . . . . . . . . . . . . . 273

A.3.1 Solution of Jump Integral for American Vanilla Call Options . . . . . . . . . . . . . . . . . 273

A.3.2 Solution of Jump Integral for American Vanilla Put Options . . . . . . . . . . . . . . . 277

A.4 Numerical Solution of the Integral in the Heaviside Term of the PIDE for American Vanilla Options . . . . . . . . . . . 280

A.4.1 Integral for American Vanilla Call Options . . . . . . . 280

A.4.2 Solution of Integral (A.34) . . . . . . . . . . . . . 281

A.4.3 Solution of Integral (A.33) . . . . . . . . . . . . 285

A.4.4 Numerical Solution of the Integral in the Heaviside

Term of the American PIDE for Vanilla Call Options . 286

A.4.5 Integral for American Vanilla Put Options . . . . . . . 286

A.4.6 Solution of Integral (A.48) . . . . . . . . . . . . 287

A.4.7 Solution of Integral (A.49) . . . . . . . . . . . . . . 289

A.4.8 Numerical Solution of the Integral in the Heaviside

Term of the American PIDE for Vanilla Put Options . 289

A.5 Variance Gamma Difference Equation for American Vanilla

Options . . . . . . . . . . . . . . . . 290

A.5.1 Difference Equation for Call Options . . . . . . . . . 290

A.5.2 Difference Equation for Put Options . . . . . . . . . 292

A.6 Numerical Solution of the Jump Integral in the Variance Gamma PIDE for European Barrier Options . . . . . . . . . . . . . . . 294

A.6.1 Solution of Integral for Up-And-Out Call Options . . . 295

A.6.2 Solution of Integral for Down-And-Out Call Options . 299

A.6.3 Solution of Integral for Up-And-Out Put Options . . . 301

A.6.4 Solution of Integral for Down-And-Out Put Options . . 303

A.7 Variance Gamma Difference Equation for European Barrier

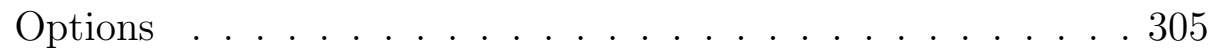

A.7.1 Difference Equation for European Up-And-Out Calls . 305

A.7.2 Difference Equation for European Down-And-Out Calls 307

A.7.3 Difference Equation for European Up-And-Out Puts . 308

A.7.4 Difference Equation for European Down-And-Out Puts 310

A.8 Numerical Solution of Jump Integral in the Variance Gamma

PIDE for American Barrier Options . . . . . . . . . . . . . . 311

A.8.1 Solution of the Jump Integral for American Up-And-

Out Call Options . . . . . . . . . . . . . . 313 
A.8.2 Solution of the Jump Integral for American Down-AndOut Call Options . . . . . . . . . . . . . . . . . 314

A.8.3 Solution of the Jump Integral for American Up-AndOut Put Options . . . . . . . . . . . . . . 317

A.8.4 Solution of the Jump Integral for American Down-AndOut Put Options . . . . . . . . . . . . . . . 321

A.9 Numerical Solution of the Integral in the Heaviside Term of the Variance Gamma PIDE for American Barrier Options . . 323 A.9.1 Numerical Solution of the Integral for American Barrier Call Options . . . . . . . . . . . . . . . . . 323

A.9.2 Numerical Solution of the Integral for American Barrier Put Options . . . . . . . . . . . . . . . . 327

A.10 Variance Gamma Difference Equation for American Barrier

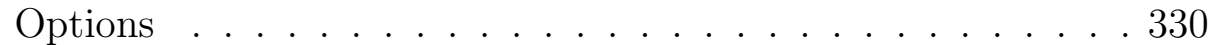
A.10.1 Difference Equation for American Up-And-Out Calls . 330 A.10.2 Difference Equation for American Down-And-Out Calls 332 A.10.3 Difference Equation for American Up-And-Out Puts . 334 A.10.4 Difference Equation for American Down-And-Out Puts 336

B Option Pricing under the Variance Gamma Process C Code338 


\section{Chapter 1}

\section{Introduction}

\subsection{Limits of the Black, Scholes and Merton Approach for Option Pricing}

Since the seminal work of Black and Scholes ${ }^{1}$ and Merton ${ }^{2}$, many authors have worked and have published papers on option pricing. The focus has gone first of all in the direction of better understanding the Black, Scholes and Merton approach with the purpose of extending the technique to related cases. In this way a number of exotic options have been priced on the basis of this theoretical framework and numerical solutions have been proposed for cases in which the Black and Scholes partial differential equation could not be solved analytically. At the same time several authors studied the limits of this classic pricing method, suggested extensions and variations of the model to overcome these limits and realized tests of the comparative pricing and hedging performance of alternative option pricing models.

It is well known in literature that most of the assumptions used by Black, Scholes and Merton are indeed not true, starting from the fact that it is not possible to realized a perfect hedging and continuing with the consideration that underlying returns are generally not normally distributed. Several authors showed on this last point that stock returns generally present kurtosis higher than the one of the normal distribution and that they often present

\footnotetext{
${ }^{1}$ Fisher Black and Myron Scholes, "The Pricing of Options and Corporate Liabilities", Journal of Political Economy, Vol. 81, No. 3, May/June 1973, pages 637-654.

${ }^{2}$ Robert C. Merton, "Theory of Rational Option Pricing", Bell Journal of Economics and Management Science, Vol. 4, 1973, pages 141-183.
} 
negative skewness. The topic was already object of study by Mandelbrot ${ }^{3}$ and by Fama ${ }^{4}$, before the Black, Scholes and Merton approach was developed. More recently Kon ${ }^{5}$, Jorion ${ }^{6}$ and Carr, Geman, Madan and Yor ${ }^{7}$, among others, have reported evidence in this direction. The consequences of these uncorrect assumptions are that the prices obtained under the Black and Scholes model are not consistent with market data as the moneyness and time to maturity vary. Jarrow and Rudd ${ }^{8}$ and Heston ${ }^{9}$ note, on this point, that the mispricing of the classic model can be explained by skewness and kurtosis in the distribution of the underlying returns. Rubinstein ${ }^{10}$ shows that the Black and Scholes model underprices put options because the risk neutral distribution of returns is possibly negatively skewed with a fatter left tail. Nandi ${ }^{11}$ moreover finds that the kurtosis higher than in the normal case causes the model to undervalue out of the money options. The impact of the non correct specification of the underlying process is reflected in the necessity of using different levels of volatilities to price options on the same asset under the Black and Scholes model. Volatility smiles have been studied in numerous papers ${ }^{12}$ and more recently authors have started to analyze the behavior

\footnotetext{
${ }^{3}$ B. Mandelbrot, "The Variation of Certain Speculative Prices", The Journal of Business, 36, 1963.

${ }^{4}$ Eugene F. Fama, "The Behavior of Stock Market Prices", The Journal of Business, 38,1965 , pages 34-105.

${ }^{5}$ T. S. Kon, "Models of Stock Returns: A Comparison", The Journal of Finance, Vol. 39, No. 1, 1984, pages 147-165.

${ }^{6}$ P. Jorion, "On Jump Processes in the Foreign Exchange and Stock Market", Review of Financial Studies, Vol. 1, No. 4, 1988.

${ }^{7}$ Peter Carr, Hélyette Geman, Dilip B. Madan and Marc Yor, "The Fine Structure of Asset Returns: An Empirical Investigation", The Journal of Business, Vol. 75, No. 2, 2002, pages 305-332.

${ }^{8}$ Robert A. Jarrow and Andrew Rudd, "Approximate Option Valuation for Arbitrary Stochastic Processes", Journal of Financial Economics, Vol. 10, No. 3, November 1982, pages 349-369.

${ }^{9}$ Steven L. Heston, "Invisible Parameters in Option Pricing", The Journal of Finance, Vol. 48, No. 3, 1993, pages 933-947.

${ }^{10}$ Mark Rubinstein, "Implied Binomial Trees", The Journal of Finance, Vol. 49, 1994, pages $771-818$.

${ }^{11}$ Saikat Nandi, "Pricing and Hedging Index Options under Stochastic Volatility: An Empirical Examination", working paper 96-9, Federal Reserve Bank of Atlanta, August 1996.

${ }^{12}$ David S. Bates, "Jumps and Stochastic Volatility : Exchange Rate Processes Implicit in Deutsche Mark Options", The Review of Financial Studies, Vol. 9, No. 1, 1996, pages 69-107; Gurdip Bakshi, Charles Cao and Zhiwu Chen, "Empirical Performance of
} 
of the whole volatility surface ${ }^{13}$, trying to understand the interaction of the impact of different moneyness levels with different maturities.

As a consequence of the limits of the Black and Scholes approach, a number of authors proposed alternative option models. None of the new approaches has however gained a general recognition as the right solution to the problems left by the classic approach. As a result, the Black and Scholes pricing model still remains the model of comparison to judge improvements. Alternative models and generalizations of the Black and Scholes model presented in literature can be divided in two main families: parametric and non parametric models. The non parametric models are based on the inference of the underlying distribution from the market data. This technique has been sometimes called expansion method, because it is based on the inference of the different terms of the expansion to reconstitute the distributions ${ }^{14}$. The parametric models on the other side assume that the equation describing the evolution of the underlying process is specified as having a particular functional form. In between the two groups are the semi-parametric models where the functional form is not specified in precise terms, for example Benhamou ${ }^{15}$ defines a semi-parametric model in which the underlying price process is modelled as a Lévy process, but no specific conditions on the underlying process are given apart from some technical conditions.

Parametric models have tried to generalize the Black and Scholes model in several ways. One approach has been to use a stochastic volatility model:

\footnotetext{
Alternative Option Pricing Models", The Journal of Finance, Vol. 52, No. 5, December 1997, pages 2003-2049; Bernard Dumas, Jeff Fleming and Robert E. Whaley, "Implied Volatility Functions: Empirical Tests", The Journal of Finance, Vol. 53, No. 6, 1998, pages 2059-2106. For a review of methodologies and empirical findings from 93 papers on volatility forecasting, with a coverage also of implied volatility, see Ser-Huang Poon and Clive W. J. Granger, "Forecasting Volatility in Financial Markets: A Review", Journal of Economic Literature, Vol. 41, No. 2, June 2003, pages 478-539.

${ }^{13}$ Thierry Ané and Chiraz Labidi, "Implied Volatility Surfaces and Market Activity over Time", Journal of Economics and Finance, Vol. 25, Nr. 3, Fall 2001, pages 259-275.

${ }^{14}$ See for example Robert A. Jarrow and Andrew Rudd, "Approximate Option Valuation for Arbitrary Stochastic Processes", Journal of Financial Economics, Vol. 10, No. 3, November 1982, pages 349-369 and M. Bouchaud, R. Cont, M. Potters, "Financial Markets as Adaptive Systems", Europhysics Letters, Vol. 41, No. 3, 1998.

${ }^{15}$ Eric Benhamou, "Option Pricing with Lévy Process", working paper, 2000.
} 
Hull and White ${ }^{16}$, Johnson and Shanno ${ }^{17}$, Scott $^{18}$, Wiggins ${ }^{19}$, Bailey and Stulz ${ }^{20}$, Melino and Turnbull ${ }^{21}$, Stein and $\mathrm{Stein}^{22}$, Amin and $\mathrm{Ng}^{23}$, Heston ${ }^{24}$, Nandi $^{25}$, Bates ${ }^{26}$, Bakshi and Chen ${ }^{27}$ and Duffie, Pan and Singleton ${ }^{28}$, for example, followed this approach. Another method has been to use a deterministic or local volatility assumption. The main idea behind this approach

\footnotetext{
${ }^{16}$ John C. Hull and A. White, "The Pricing of Options on Assets with Stochastic Volatility", The Journal of Finance, 42, 1987, pages 281-300.

${ }^{17}$ H. Johnson and D. Shanno, "Option Pricing when the Variance is Changing", Journal of Financial and Quantitative Analysis, Vol. 22, 1987, pages 143-151.

${ }^{18}$ Louis O. Scott, "Option Pricing when the Variance Changes Randomly: Theory, Estimation and an Application", Journal of Financial and Quantitative Analysis, Vol. 22, 1987, pages 419-438.

${ }^{19}$ James Wiggins, "Option Values under Stochastic Volatility", Journal of Financial Economics, Vol. 19, 1987, pages 351-372.

${ }^{20}$ Warren Bailey and René Stulz, "The Pricing of Stock Index Options in a General Equilibrium Model", Journal of Financial and Quantitative Analysis, Vol. 24, 1989, pages 1-12. The authors incorporate here also stochastic interest rates.

${ }^{21}$ Angelo Melino and Stuart M. Turnbull, "Pricing Foreign Currency Options with Stochastic Volatility", Journal of Econometrics, Vol. 45, No. 1-2, July-Aug. 1990, pages 239-265 and Angelo Melino and Stuart M. Turnbull, "Misspecification and the Pricing and Hedging of Long-Term Foreign Currency Options", Journal of International Money and Finance, Vol. 4, 1995, pages 373-393.

${ }^{22}$ Elias Stein and Jeremy Stein, "Stock Price Distribution with Stochastic Volatility: An Analysis Approach", Review of Financial Studies, Vol. 4, 1991, pages 727-752.

${ }^{23}$ Kaushik I. Amin and Victor K. Ng, "Option Valuation with Systematic Stochastic Volatility", The Journal of Finance, Vol. 48, No. 3, 1993, pages 881-910. In this case the model includes also stochastic interest rates.

${ }^{24}$ Steven L. Heston, "A Closed Form Solution for Options with Stochastic Volatility with Applications to Bond and Currency Options", Review of Financial Studies, Vol. 6, No. 2, 1993, pages 327-343.

${ }^{25}$ Saikat Nandi, "Pricing and Hedging Index Options under Stochastic Volatility: An Empirical Examination", working paper 96-9, Federal Reserve Bank of Atlanta, August 1996.

${ }^{26}$ David S. Bates, "Jumps and Stochastic Volatility : Exchange Rate Processes Implicit in Deutsche Mark Options", The Review of Financial Studies, Vol. 9, No. 1, 1996, pages 69-107 develops a model which combines stochastic volatility with a jump diffusion process.

${ }^{27}$ Gurdip Bakshi and Zhiwu Chen, "An Alternative Valuation Model for Contingent Claims", Journal of Financial Economics, Vol. 44, 1997, pages 123-165 and Gurdip Bakshi and Zhiwu Chen, "Equilibrium Valuation of Foreign Exchange Claims", The Journal of Finance, Vol. 52, 1997, pages 799-826. In these papers the authors study a model which incorporates both stochastic volatility and stochastic interest rates.

${ }^{28}$ D. Duffie, J. Pan and K. Singleton, "Transform Analysis and Asset Pricing for Affine Jump-Diffusions", Econometrica, Vol. 68, 2000, pages 1343-1376. The authors present here a model which is a combination of jump diffusion and stochastic volatility.
} 
is that option valuation using stochastic volatility generally requires a market price of risk which is clearly difficult to estimate. An exception occurs when the volatility is a deterministic function of asset price or time, in this case option valuation based on the Black and Scholes PDE remains possible, though not by means of the Black and Scholes formula itself; this is the case of deterministic volatility models. These methods attempts to decipher the cross section of option prices and deduce the future behavior of volatility as anticipated by market participants. The approach is to look for a binomial or a trinomial lattice that achieves a cross sectional fit of reported option prices. Rubinstein ${ }^{29}$ for example uses an implied volatility tree whose branches at each node are designed by choice of up-and-down increment sizes or probabilities to reflect the time variation of volatility. Similar approaches are followed by Dupire ${ }^{30}$ and by Derman and Kani ${ }^{31}$. Other modifications of the Black and Scholes model consist in the addition of stochastic interest rate ${ }^{32}$, in the constant elasticity of variance model of Cox and Ross ${ }^{33}$ and in the option pricing framework based on the GARCH process suggested by Duan ${ }^{34}$.

An important class of extensions of the classical option pricing is given by the presence of jumps in the underlying process. Discontinuities in the underlying price in the form of jumps have a long history in the financial literature. Merton considered in $1976^{35}$ the addition of a jump component to the classical geometric Brownian motion model for option pricing. Even

\footnotetext{
${ }^{29}$ Mark Rubinstein, "Implied Binomial Trees", The Journal of Finance, Vol. 49, 1994, pages $771-818$.

${ }^{30}$ Bruno Dupire, "Pricing with a Smile", Risk, 7, 1994, pages 18-20.

${ }^{31}$ Emanuel Derman and Iraj Kani, "Riding on the Smile", Risk, 7, 1994, pages 32-39 and Emanuel Derman and Iraj Kani, "Stochastic Implied Trees: Arbitrage Pricing with Stochastic Term and Strike Structure of Volatility", International Journal of Theoretical and Applied Finance, Vol. 3, 1998, pages 7-22.

${ }^{32}$ Robert C. Merton, "Theory of Rational Option Pricing", Bell Journal of Economics and Management Science, Vol. 4, 1973, pages 141-183 and Kaushik I. Amin and Robert A. Jarrow, "Pricing Options on Risky Assets in a Stochastic Interest Rate Economy", Mathematical Finance, Vol. 2, 1992, pages 217-237.

${ }^{33}$ J. C. Cox, S. A. Ross, "The Valuation of Options for Alternative Stochastic Processes", Journal of Financial Economics, 3, 1976, pages 145-166.

${ }^{34}$ Jin-Chuan Duan, "The GARCH Option Pricing Model", Mathematical Finance, Vol. 5, No. 1, January 1995, pages 13-32.

${ }^{35}$ Robert C. Merton, "Option Pricing when Underlying Stock Returns are Discontinuous", Journal of Financial Economics, Vol. 3, 1976, pages 125-144 and Robert C. Merton, "The Impact on Option Pricing of Specification Error in the Underlying Stock Price Returns", The Journal of Finance, Vol. 31, No. 2, May 1976, pages 333-350.
} 
before that time, the possibility of jumps in asset prices has been considered by Mandelbrot ${ }^{36}$ and Press ${ }^{37}$. The importance of introducing a jump component in the underlying process has been noted for example by Bakshi, Cao and Chen $^{38}$ who argue that models based on pure diffusion processes have difficulties in explaining volatility smiles in general and in particular for short dated option prices. Moreover Broadie, Chernov and Johannes ${ }^{39}$ find strong evidence in favor of both jumps in the underlying returns and in the volatility of the returns using all S\&P 500 future option transactions from 1987 to 2003. Inside the class of processes presenting jumps in the underlying process, it is possible to further distinguish between jump diffusion processes and pure jump processes. Models presenting a Poisson jump on top of the diffusion process, like the one presented by Merton in 1976, are examples of jump diffusion models. The assumption that the stock returns follow a jump diffusion process has been used to improve option pricing as well as other theories like the capital asset pricing model. Authors who studied jump diffusion processes include Cox and Ross ${ }^{40}$, Jarrow and Rosenfeld ${ }^{41}$, Ahn and

\footnotetext{
${ }^{36}$ Benoit Mandelbrot, "New Methods in Statistical Economics", The Journal of Political Economy, Vol. 71, No. 5, October 1963, pages 421-440.

${ }^{37}$ S. J. Press, "A Compound Events Model for Security Prices", The Journal of Business, 40, July 1967, pages 317-335.

${ }^{38}$ Gurdip Bakshi, Charles Cao and Zhiwu Chen, "Empirical Performance of Alternative Option Pricing Models", The Journal of Finance, Vol. 52, No. 5, December 1997, pages 2003-2049.

${ }^{39}$ Mark Broadie, Mikhail Chernov and Michael Johannes, "Model Specification and Risk Premiums: The Evidence from the Future Options", working paper, 2003.

${ }^{40}$ J. C. Cox, S. A. Ross, "The Valuation of Options for Alternative Stochastic Processes", Journal of Financial Economics, 3, 1976, pages 145-166.

${ }^{41}$ Robert A. Jarrow and Eric R. Rosenfeld, "Jump Risks and the Intertemporal Capital Asset Pricing Model, The Journal of Business, Vol. 57, No. 3, 1984, pages 337-351.
} 
Thompson ${ }^{42}$, Naik and Lee ${ }^{43}$, Aase $^{44}$, Amin $^{45}$, Bates ${ }^{46}$, Bakshi and Chen ${ }^{47}$, Scott $^{48}, \mathrm{Kou}^{49}$ and Duffie, Pan and Singleton ${ }^{50}$. Pure jump processes, on the other side, lack of the diffusion elements and hence of the continuous component. The presence of an infinite number of discontinuities in the pure jump processes makes them a quite different class of processes compared with the traditional Black, Scholes and Merton approach.

\subsection{Purely Discontinuous Price Processes and Lévy Processes}

Prices of assets and in particular of stocks are generally viewed as continuous functions of time. This is for example the case of the geometric Brownian motion used to describe the dynamic of the underlying returns. Continuity of prices has served economic theory as a powerful assumption delivering market completeness and unique pricing of contingent claim by arbitrage. Part of the financial literature has however started to question the validity of this

\footnotetext{
${ }^{42}$ Ahn Chang M. and Thompson Howard E., "Jump-Diffusion Processes and the Term Structure of Interest Rates", The Journal of Finance, Vol. 43, No. 1, 1988, pages 155-174.

${ }^{43}$ V. Naik and M. Lee, "General Equilibrium Pricing of Options on the Market Portfolio with Discontinuous Returns", Review of Financial Studies, Vol. 3, 1990, pages 493-521.

${ }^{44}$ Knut K. Aase, "A Jump Diffusion Consumption Based Capital Asset Pricing Model and the Equity Premium Puzzle", Mathematical Finance, Vol. 3, 1993, pages 65-84.

${ }^{45}$ Kaushik I. Amin, "Jump Diffusion Option Valuation in Discrete Time", The Journal of Finance, Vol. 48, No. 5, December 1993, pages 1833-1863.

${ }^{46}$ David S. Bates, "The Crash of '87: Was It Expected? The Evidence from Options Markets", The Journal of Finance, Vol. 46, No. 3, July 1991, pages 1009-1044 and David S. Bates, "Jumps and Stochastic Volatility : Exchange Rate Processes Implicit in Deutsche Mark Options", The Review of Financial Studies, Vol. 9, No. 1, 1996, pages 69-107. Bates here combines a jump diffusion process with stochastic volatility.

${ }^{47}$ Gurdip Bakshi and Zhiwu Chen, "An Alternative Valuation Model for Contingent Claims", Journal of Financial Economics, Vol. 44, 1997, pages 123-165.

${ }^{48}$ Luis O. Scott, "Pricing Stock Options in a Jump-Diffusion Model with Stochastic Volatility and Interest Rates: Applications of Fourier Inversion Methods", Mathematical Finance, Vol. 7, No. 4, October 1997, pages 413-426.

${ }^{49}$ S. G. Kou, "A Jump Diffusion Model for Option Pricing with Three Properties: Leptokurtic Feature, Volatility Smile, and Analytical Tractability", working paper, 1999.

${ }^{50}$ D. Duffie, J. Pan and K. Singleton, "Transform Analysis and Asset Pricing for Affine Jump-Diffusions", Econometrica, Vol. 68, 2000, pages 1343-1376. In this case, the model presents both a jump diffusion process and stochastic volatility.
} 
approach as an appropriate model for the stock returns and has suggested the use of pure jump models, that is purely discontinuous models. If the process followed by the underlying is purely discontinuous, options can no longer be replicated with the dynamic hedging technique suggested by Black and Scholes. Hence without dynamic hedging, options are no more redundant assets in the market and they become a completing asset useful in hedging jump risks.

As we have seen in the previous section, a number of authors have considered the case of jump diffusion models, that is models which combine the presence of a diffusion component in addition to a jump. In this case, the process accounts for high activity small price moves using an infinite variation diffusion process and for low activity large moves using an orthogonal pure jump process. On the contrary in the case of pure jump processes, price jumps are the rule and all the moves occur via jumps. In the case of processes like the variance gamma, high (or actually better, infinite) activity is accounted for by an infinite number of small jumps. Moreover the activity of various jump sizes is analytically connected by the requirement that small jumps occur at a higher rate than larger jumps. Furthermore models like variance gamma are processes of finite variation, that is the sum of absolute changes in price is finite.

Although the processes considered here are pure jump processes of finite variation, and as such very different from the process used by the traditional Black Scholes approach, it is possible to view these processes as time changed continuous processes. Already in 1973 Clark $^{51}$ studied subordinated processes, where a process is nested in another process. Clark, in particular, considered processes where prices were represented by a geometric Brownian motion and time was given by another independent Brownian motion. The economic interpretation of the time change is the passage from a calendar time to an economic activity time represented for example by the number of trades in the exchange. On this point, Ané and Geman ${ }^{52}$ show empirically that returns based on high frequency data on FTSE 100 futures, although non normal in calendar time, are very close to normality when a stochastic clock driven by the number of trades is used. Several authors, including

\footnotetext{
${ }^{51}$ P. K. Clark, "A Subordinated Stochastic Process Model with Finite Variance for Speculative Prices", Econometrica, Vol. 41, 1973, pages 135-156.

${ }^{52}$ Thierry Ané and Hélyette Geman, "Order Flow, Transaction Clock and Normality of Asset Returns", The Journal of Finance, Vol. 55, 2000, pages 2259-2284.
} 
Tauchen and Pitts ${ }^{53}$, Karpoff ${ }^{54}$ and Gallant, Rossi and Tauchen ${ }^{55}$, have considered number of trades or volume as measures of activity to be related to price changes.

Let's now consider a bit more how to relate the pure jump approach to the traditional theory developed by Black, Scholes and Merton ${ }^{56}$. The initial step of the analysis is the consideration that the model to be used has to be free of arbitrage. The theory of arbitrage is the basis of the Black, Scholes and Merton approach as well as of the martingale pricing approach first developed by Harrison and Kreps ${ }^{57}$ and by Harrison and Pliska ${ }^{58}$. Although financial markets may display arbitrage opportunities at times, it is clear that in general the model of price processes to be employed in a derivative pricing model has to be free of arbitrage.

The hypothesis of absence of arbitrage is related to the existence of an equivalent martingale measure. Without entering into the details, we can say that, depending on the context and meaning of absence of arbitrage opportunities, no arbitrage may be equivalent to the existence of an equivalent martingale measure. In discrete time and with finitely many states equivalence of the two concepts has been proved already by Harrison and Kreps. At the other extreme, there is the situation of continuous time and states given, at a minimum, by a relatively large set consisting of the paths of the stock price process. In this case, the existence of a martingale measure implies the absence of arbitrage, but the implication in the other direction is not available. Essentially, the definition of absence of arbitrage as merely asserting that one cannot combine a portfolio of existing assets to earn a non negative, non zero, cash flow is too weak to deduce the existence of a mar-

\footnotetext{
${ }^{53}$ G. Tauchen and M. Pitts, "The Price-Volume Relationship on Speculative Markets", Econometrica, Vol. 51, 1983, pages 485-505.

${ }^{54}$ Jonathan M. Karpoff, "The Relationship Between Price Changes and Trading Volume: A Survey", The Journal of Financial and Quantitative Analysis, Vol. 22, 1987, pages 109126.

${ }^{55}$ A. R. Gallant, P. E. Rossi and G. Tauchen, "Stock Prices and Volume", Review of Financial Studies, Vol. 5, 1992, pages 199-242.

${ }^{56}$ We follow here the approach presented in Dilib B.Madan, "Purely Discontinuous Asset Price Processes", working paper, 1999.

${ }^{57}$ J. Michael Harrison and David M. Kreps, "Martingales and Arbitrage in Multiperiod Securities Markets", Journal of Economic Theory, Vol. 20, 1979, pages 381-408.

${ }^{58}$ J. Michael Harrison and Stanley R. Pliska, "Martingales and Stochastic Integrals in the Theory of Continuous Trading", Stochastic Processes and their Applications, Vol. 11, 1981, pages 215-260.
} 
tingale measure. In this contest, it is necessary to strengthen the hypothesis of no arbitrage to allow the deduction of a martingale measure. The results in this direction are due to Delbaen and Schachermayer ${ }^{59}$. They employ a strong and uniform sense of no arbitrage and show that if there is no random sequence of zero cost trading strategies converging in this strong sense to a non negative, non zero cash flow with the random sequence being uniformly bounded below by a negative constant, then there exists a martingale measure and the converse holds as well. Delbaen and Schachermayer call this hypothesis "no free lunch with vanishing risk" and prove that it is equivalent to the existence of an equivalent martingale measure.

Moreover Delbaen and Schachermayer point out that if there exists a change of measure from the true statistical measure $P$ to a martingale risk neutral measure $Q$, such that under $Q$ discounted asset prices are martingales, then it must be that under $P$ the price process was a semimartingale to begin with. This is a very useful result, because it tells us that, given that we want to consider a model which is arbitrage free in the contest defined by Delbaen and Schachermayer, we can restrict our attention to the class of semimartingale processes ${ }^{60}$. We have to note, however, that the semimartingale has to be a semimartingale with non zero martingale component to assure non arbitrage.

Now that we know that the stochastic process that we need to consider is a semimartingale, we can use Monroe ${ }^{61}$ proof that every semimartingale can be written as a Brownian motion, possibly defined on some adequately extended probability space, evaluated at a random time. Hence, because the process we need to use is a time changed Brownian motion, it will be continuous only if the time change is continuous. However Revuz and Yor $^{62}$ show that the time change is an increasing process which can be continuous only if it is locally deterministic. Since this time change is viewed as measure of economic activity, for example proxied by volume or number of trades, you can expect

\footnotetext{
${ }^{59}$ Freddy Delbaen and Walter Schachermayer, "A General Version of the Fundamental Theorem of Asset Pricing", Mathematische Annalen, Vol. 300, 1994, pages 463-520.

${ }^{60}$ Without entering into the details, we can say that a semimartingale can be described by its decomposition in a martingale plus a adapted process of bounded variation. See L. C. Rogers and D. Williams, Diffusions, Markov Processes and Martingales, Vol. 2, John Wiley \& Sons, New York, 1987, page 313.

${ }^{61}$ I. Monroe, "Processes That Can Be Embedded In A Brownian Motion", The Annals of Probability, Vol. 6, 1978, pages 42-56.

${ }^{62}$ Daniel Revuz and Marc Yor, Continuous Martingales and Brownian Motion, SpringerVerlag, Berlin, 1991, page 190.
} 
some local uncertainty in the time change with the consequence that the time changed Brownian motion is going to be discontinuous. From another point of view, we can also say that the continuity would imply that the process of stock returns is locally Gaussian. Because there is large empirical evidence of the contrary, as discussed in the previous session, we can again conclude that the process cannot be continuous. Continuity may however be recovered when the time is given by the stochastic clock.

To summarize the results presented so far in this section, we can say that no arbitrage implies, via the existence of an equivalent martingale measure, that the price process is a semimartingale. Moreover we said that all semimartingales are time changed Brownian motion, and in particular time changed by a random increasing time change. The resulting process can be continuous only if the time change is locally deterministic, hypothesis this that we rejected leading us to the conclusion that the process has to be purely discontinuous.

It is clear that the class of semimartingales is very wide and hence there are a lot of stochastic processes which satisfy the non arbitrage condition. Among these process particular attention goes to the class of Lévy processes for the properties which characterize them. A general discussion on Lévy processes and their properties is outside the scope of this work ${ }^{63}$, we can however say that $X=\{X(t)\}_{t \geq 0}$ is a Lévy process if

1. $X$ has independent increments;

2. $X(0)=0 \quad$ a.s.;

3. $X$ is continuous in probability, i.e. if for $s \geq 0$

$$
X(t+s)-X(s) \stackrel{P}{\longrightarrow} 0 \quad \text { as } t \rightarrow 0 ;
$$

4. $X$ is time homogeneous, i.e. for $t \geq 0, \mathscr{L}(X(t+s)-X(s))$ does not depend on $s \geq 0$

5. $X$ is right continuous with left limits a.s.;

\footnotetext{
${ }^{63}$ See, for example, K. Sato, Lévy Processes and Infinitely Divisible Distributions, Cambridge University Press, 1999 for a more general presentation of the topic.
} 
The characteristic function of this kind of processes is completely characterized by the Lévy-Khintchine theorem ${ }^{64}$. According to this theorem, let $X(t)=\ln (S(t))$ be a Lévy process for the logarithm of the stock price with mean $\mu t$, then there exists a unique measure $\Pi$ defined in $\mathbb{R}-\{0\}$ such that

$$
\phi_{X(t)}(u) \triangleq E[\exp (i u X(t))]=\exp \left[i u \mu t+t \int_{-\infty}^{\infty}\left(e^{i u x}-1\right) \Pi(d x)\right]
$$

The measure $\Pi$ is called Lévy measure. In particular when the measure has a density $k(x)$, the characteristic function can be written as

$$
\phi_{X(t)}(u)=\exp \left[i u \mu t+t \int_{-\infty}^{\infty}\left(e^{i u x}-1\right) k(x) d x\right]
$$

and the function $k(x)$ is called Lévy density. Heuristically the function $k(x)$ specifies the arrival rate of jumps of size $x$ and the Lévy process can be thought as a compound Poisson process with a finite arrival rate if the integral of the Lévy density is finite. In general however we are interested in Lévy processes with infinite arrival rate, since this is the case of the variance gamma process. For this case, we can remember that the Lévy process may always be approximated by a compound Poisson process obtained by truncating the Lévy density in a neighborhood of zero and using an arrival rate of

$$
\lambda=\int_{|x|>\epsilon} k(x) d x
$$

and a density for the jump magnitude conditional on the arrival of

$$
g(x)=\frac{k(x) 1_{|x|>\epsilon}}{\lambda}
$$

This approximation will converge to the Lévy process as $\epsilon \rightarrow 0$.

Having a representation of the characteristic function is important because option prices are easily obtained from the characteristic function as it is described in Bakshi and Madan ${ }^{65}$ and in Carr and Madan ${ }^{66}$. Carr and

\footnotetext{
${ }^{64}$ See W. E. Feller, An Introduction to Probability Theory and its Applications, 2nd Edition, Wiley, New York, 1971 and J. Bertoin, Lévy Processes, Cambridge University Press, Cambridge, 1996.

${ }^{65}$ Gurdip Bakshi and Dilip B. Madan, "Spanning and Derivative-Security Valuation", Journal of Financial Economics, 55, 2000, pages 205-238.

${ }^{66}$ Peter Carr and Dilip B. Madan, "Option Valuation Using the Fast Fourier Transform", Journal of Computational Finance, 2, 1998, pages 61-73.
} 
Madan, in particular, show how to write analytically the Fourier transform in the log strike of an exponentially dampened call price in terms of the characteristic function of the logarithm of the stock price. The dampen call price and the call price are then obtained by Fourier inversion.

\subsection{Variance Gamma Process}

Inside the class of processes which allow to build a model free from arbitrage, which we have seen corresponds to the class of semimartingales, the attention is focused on the variance gamma process. This Lévy process can, as every semimartingale, be written as a Brownian motion evaluated at a random time. In particular the variance gamma process can be obtained by replacing the time in the Brownian motion with a gamma process. The first complete presentation of the model, in its simplified symmetric form, is due to Madan and Seneta in $1990^{67}$. In 1991 Madan and Milne ${ }^{68}$ published a paper in which they study the equilibrium option pricing for the symmetric variance gamma process in a representative agent model, under a constant relative risk aversion utility function. The resulting risk neutral process is identical with the more general non symmetric variance gamma process. This general model is described more completely in a 1998 paper by Madan, Carr and Chang $^{69}$ where also a closed form solution for European vanilla options is presented.

The variance gamma model, in its general version, has two additional parameters compared with the Black and Scholes model. These parameters allow to control the skewness and kurtosis of the process followed by the underlying returns, allowing to price options with different strikes, without need to modify implied volatility or other parameters as the moneyness changes.

The attention in this dissertation is focused on the numerical solution of the option pricing under variance gamma. As usual, it is not possible to price American options with an analytical formula and numerical procedures

\footnotetext{
${ }^{67}$ Dilib B. Madan and Eugene Seneta "The Variance Gamma (V.G.) Model for Share Market Return", The Journal of Business, vol 63. no.4, 1990, pages 511-524.

${ }^{68}$ Dibip B. Madan and Frank Milne, "Option Pricing with V.G. Martingale Components", Mathematical Finance, Vol. 1, No. 4, October 1991, pages 39-55.

${ }^{69}$ Dilip B Madan, Peter P. Carr and Eric C. Chang, "The Variance Gamma Process and Option Pricing", European Finance Review, 2, 1998, pages 79-105.
} 
need to be used instead and, in particular, our interest is in the solution of the problem via a finite difference scheme. The dynamic of the option price under variance gamma can be expressed in the form of a partial integro differential equation. The integrals, one for the European option case and two for the American option case, describe the impact on the option price of jumps in the underlying weighted by the probability of these jumps. It is important to discretize these integrals properly to obtain a stable solution of the finite difference scheme. An algorithm to price American vanilla options under variance gamma has been recently presented in literature by Hirsa and Madan $^{70}$. They present a way to discretize the integrals in the PIDE which treats the jumps in different ways depending on the size of the jumps. The result is a mixed finite difference scheme in which parts of the jumps are treated implicitly and parts explicitly. Slightly modifying this algorithm, we wrote a code in $\mathrm{C}$ language to price vanilla options and we realized numerous experiments in terms of sensitivity of the option price with respect to the parameters controlling volatility, skewness and kurtosis of the underlying process and with respect to interest rates and dividend yield. In this work, we also show how it is possible to use the prices obtained under the variance gamma process to compute corresponding implied volatility based on the Black and Scholes model. Finally we present results in terms of comparison between option prices obtained under geometric Brownian motion and under variance gamma process.

In this dissertation we also show how to price European and American barrier options under variance gamma. The approach requires the modification of the finite difference scheme used for the vanilla case to price the exotic option. Given that it is always possible to obtain knock-in value as difference between vanilla options and the corresponding knock-out prices, the attention is focused on the knock-out pricing. In designing the numerical solution, the extreme of the stock range considered in the finite difference scheme is positioned exactly at the barrier level so that we avoid the issue of oscillation in the convergence of the option price as the number of steps increases. Because the process considered is a pure jump process, we cannot automatically ignore the values of the option for stock prices outside the barrier, as we would have done for a knock-out priced under geometric Brownian motion. Brownian motion, in fact, is a continuous process and hence the stock cannot

\footnotetext{
${ }^{70}$ Ali Hirsa and Dilip B. Madan, "Pricing American Options Under Variance Gamma", Journal of Computational Finance, Vol. 7, No. 2, Winter 2003/2004, pages 63-80.
} 
reach any value outside the barrier without having reached the barrier itself first. In this continuous case, because upon reaching the barrier the option is knocked-out, we would not be interested in studying what happens outside the barrier. The variance gamma on the other side is a purely discontinuous process and as such it may jump outside the barrier without having actually touched the barrier. Clearly in the presence of a rebate, this has to be paid also for cases where the process jumps across the barrier without touching it. In this work we show how to handle this issue by properly discretizing the integrals describing the impact of underlying jumps. This approach is then implemented with code in $\mathrm{C}$ language and the program is used to generate a number of experiments of barrier option pricing. In particular, we present a sensitivity analysis of the option prices for both European and American barrier options to the parameters controlling volatility, skewness and kurtosis and to the rebate for different cases, depending on the type of option and on the relative position of the barrier with respect to the stock price.

The outline of the work is the following: the first part of the dissertation, including the second chapter, the third chapter and part of the fourth chapter, presents results already known in literature, while part of the fourth chapter and the fifth chapter contain our original contribution to the research. In particular the innovation of this work consists first of all in a detailed study of the nature of the variance gamma process. We analyze the process from a theoretical point of view and we study how to implement numerical schemes to price options under this process. We also use the numerical scheme developed by Hirsa and Madan, for vanilla options, improving it when necessary, to study the price of European and American vanilla options. Moreover our original contribution is in the generalization of the numerical solution to the pricing of European and American barrier options. Numerous experiments are conducted for the pricing of both vanilla and barrier options under variance gamma using these numerical schemes.

Going more into the detail of the contents of the chapters we can say that in the second chapter we present a general introduction to the variance gamma and to its statistical properties. In this chapter we show that the variance gamma process can be defined both as a time changed Brownian motion and as a difference of two gamma processes. The pricing of European options under variance gamma with a closed form solution and the corresponding empirical tests of the pricing performance are reported. Moreover we show some empirical tests on the presence of skewness and kurtosis in the distribution of stock returns, both from a statistical and from a risk 
neutral point of view. Results reported here are in favor of the conclusion that the variance gamma does provide an improvement with respect to the Black and Scholes model in terms of pricing option with different moneyness.

The third chapter deals with some extensions of the variance gamma. Here the CGMY model ${ }^{71}$ is presented; this model extends variance gamma by adding an additional parameter whose value defines the process as being of either finite or infinite variation and of either finite or infinite activity. The variance gamma is a particular case of this model in which the process is of infinite activity and of finite variation. Another extension to the model considered here consists in adding a stochastic volatility process to the variance gamma and to the CGMY ${ }^{72}$. Stochastic volatility is obtained here by randomly changing the time of the process and its introduction has the purpose of obtaining a model which can consistently price options with different maturities.

The fourth chapter presents the numerical solution for vanilla options under variance gamma. The finite difference scheme of Hirsa and Madan, with a little modification, is presented. Following this scheme, it is showed how to discretize integrals in the PIDE for both European and American vanilla options and how to properly write the difference equation for the different cases. In this chapter we present several numerical experiments showing sensitivity analysis of option prices and comparison with values obtainable under the correspondent geometric Brownian motion. Part of the developments presented in this chapter has been the subject of a project for the Master in Mathematics of Finance at Columbia University in New York in the Spring 2001.

The fifth chapter deals with the numerical solution of the pricing problem under the variance gamma process for European and American barrier options. Here we show how to discretize the integrals describing the impact of the stock jumps on option values and how to properly write the difference equations to be used to price the barrier options. We also present a number of numerical experiments with particular attention to sensitivity analysis of the option price.

The sixth chapter presents the conclusions of the work and the perspec-

\footnotetext{
${ }^{71}$ Peter Carr, Hélyette Geman, Dilip B. Madan and Marc Yor, "The Fine Structure of Asset Returns: An Empirical Investigation", The Journal of Business, Vol. 75, No. 2, 2002, pages 305-332.

${ }^{72}$ Peter Carr, Hélyette Geman, Dilip. B. Madan, Marc Yor, "Stochastic Volatility for Lévy Processes", Mathematical Finance, Vol. 13, No. 3, July 2003, pages 345-382.
} 
tives for future research. In the appendix we provide the detailed numerical solutions for vanilla and barrier options. In particular we show how to discretize all the integrals in the PIDEs for all the cases of European and American vanilla and barrier options. Moreover for all these cases, we show how to write the difference equations to be solved.

Finally the code in $\mathrm{C}$ language to price options under the variance gamma process is given in the appendix. This is the code used to realize all the numerical experiments reported in chapters four and five and it covers all the cases of European and American vanilla and barrier options. 


\section{Chapter 2}

\section{The Variance Gamma Model: Properties and Pricing of European Options}

\subsection{Introduction and Brief History of the Model}

The variance gamma model has been know in the financial literature for several years. The model was introduced as an extension of geometric Brownian motion to overcome some issues that the Black and Scholes model has in pricing options. The model presents two additional parameters, compared with the geometric Brownian motion, which allow to control the skewness and the kurtosis of the distribution of stock price returns.

The first complete presentation of the model is due to Madan and Seneta in $1990^{1}$. The model presented in this paper is however a symmetric variance gamma model, where there is only an additional parameter which controls the kurtosis, while the skewness is still not considered. Before 1990, some authors already presented some works related to variance gamma, among others Madan and Seneta published two articles in $1987^{2}$ where some prop-

\footnotetext{
${ }^{1}$ Dilib B. Madan and Eugene Seneta "The Variance Gamma (V.G.) Model for Share Market Return", The Journal of Business, vol 63. no.4, 1990, pages 511-524.

${ }^{2}$ Dilip B. Madan and Eugene Seneta, "Simulation of Estimates Using the Empirical Characteristic Function", International Statistical Review, 55, 1987, pages 153-161 and Dilip B. Madan and Eugene Seneta, "Chebyshev Polynomial Approximation and Characteristic Function Estimation ", Journal of the Royal Statistical Society, ser. B, 49, 1987, pages 163-169.
} 
erties of the variance gamma are discussed and empirical comparisons with other models are shown.

In 1991 Madan and Milne published a paper ${ }^{3}$, where they study equilibrium option pricing for the symmetric variance gamma process using a representative agent model under a constant relative risk aversion utility function. The resulting risk neutral process is identical to the non symmetric (or general) variance gamma process, with the drift in the time changed Brownian motion being negative for positive risk aversion. This general non symmetric process is described more completely in the 1998 paper by Madan, Carr and Chang ${ }^{4}$ where also a closed form solution for European vanilla options is presented. Through our work, we will generally talk about this general case of variance gamma, without specifying that it is the non symmetric.

\subsection{Variance Gamma as Time Changed Brow- nian Motion}

The variance gamma model is an extension of Brownian motion which can be obtained evaluating a normal process at a random time defined by a gamma process, that is replacing the time in the Brownian motion with a gamma process $^{5}$. Define a Brownian motion with drift $\vartheta$ and volatility $\sigma$,

$$
b(t ; \vartheta, \sigma)=\vartheta t+\sigma W(t)
$$

Consider a gamma process of independent gamma increments over nonoverlapping intervals of time $(t, t+h), \gamma(t ; \mu, \nu)$, where $\mu$ is the mean rate and $\nu$ is the variance rate. The increment of $\gamma$ over a time period is distributed

\footnotetext{
${ }^{3}$ Dibip B. Madan and Frank Milne, "Option Pricing with V.G. Martingale Components", Mathematical Finance, Vol. 1, No. 4, October 1991, pages 39-55.

${ }^{4}$ Dilip B Madan, Peter P. Carr and Eric C. Chang, "The Variance Gamma Process and Option Pricing", European Finance Review, 2, 1998, pages 79-105.

${ }^{5}$ For a more complete discussion on the approach and on the results of time changes for several Lévy processes, see See Hélyette Geman, Dilip B. Madan and Marc Yor, "Time Changes for Lévy Processes", Mathematical Finance, Vol. 11, No. 1, January 2001, pages 79-96. For a study of Brownian motion evaluated at a time change given by an independent and purely discontinuous subordinator and of the relationship between the quadratic variation of the process and the time change, see Hélyette Geman, Dilip B. Madan and Marc Yor, "Time Changes Hidden in Brownian Subordination", working paper, 2000 .
} 
with a gamma density function with mean $\mu h$ and variance $\nu h$. Defining the increment as $g=\gamma(t+h ; \mu, \nu)-\gamma(t ; \mu, \nu)$, with $g>0$ and denoting by $\Gamma(\cdot)$ a gamma function, we can write the density function of the increment as

$$
f_{h}(g)=\left(\frac{\mu}{\nu}\right)^{\frac{\mu^{2} h}{\nu}} \frac{g^{\frac{\mu^{2} h}{\nu}-1} \exp \left(-\frac{\mu}{\nu} g\right)}{\Gamma\left(\frac{\mu^{2} h}{\nu}\right)}
$$

The gamma density has a characteristic function $\phi_{\gamma(t)}(u)=E[\exp (i u \gamma(t ; \mu, \nu))]$ given by

$$
\phi_{\gamma(t)}(u)=\left(\frac{1}{1-i u \frac{\nu}{\mu}}\right)^{\frac{\mu^{2} t}{\nu}}
$$

Now we can define a variance gamma process, $X(t ; \sigma, \nu, \vartheta)$ as a Brownian motion where the time is stochastic and it is given by a gamma process with unit mean rate, $\gamma(t ; 1, \nu)$, in compact form we can write:

$$
X(t ; \sigma, \nu, \vartheta)=b(\gamma(t ; 1, \nu) ; \vartheta, \sigma)
$$

The economic interpretation of this is that while a calendar unit, for example a calendar year has a length which is fixed in itself, its economic value is different depending on the situations and so you can have calendar years which are more or less than an economic year. The number of economic time units in a calendar time unit in this model is random and defined by the gamma distribution. In this way we can say following Madan and Seneta that one can think about the gamma process which defines the economic time as "a formal statement of the remark, 'Didn't have much of a year this year,' by allowing for an interpretation of how much of a year one actually had" 6 . Note that this economic time measure given by the random process has to be interpreted as referred to the whole economy and not to a specific individual, because we are are going to study distribution of prices and from them option prices which reflect the market and not just a specific individual. Hence a candidate for this economic year measure could be the cumulated gross domestic product.

The density function for variance gamma process at a time $t$ can be expressed as a normal density function conditional on the realization of the

\footnotetext{
${ }^{6}$ Dilib B. Madan and Eugene Seneta "The Variance Gamma (V.G.) Model for Share Market Returns", The Journal of Business, vol 63. no.4, page 517, 1990, pages 511-524.
} 
time change given by the gamma distribution. If we integrate over the gamma distributed increments $g$, having the density function (2.2), we can obtain the unconditional density function for variance gamma $\mathrm{as}^{7}$

$$
f_{X(t)}(X)=\int_{0}^{+\infty} \frac{1}{\sigma \sqrt{2 \pi g}} \exp \left(-\frac{(X-\vartheta g)^{2}}{2 \sigma^{2} g}\right) \frac{g^{\frac{t}{\nu}-1} \exp \left(-\frac{g}{\nu}\right)}{\nu^{\frac{t}{\nu}} \Gamma\left(\frac{t}{\nu}\right)} d g
$$

In the same way, the characteristic function for the variance gamma process can be expressed conditional on the gamma time. The unconditional characteristic function, $\phi_{X(t)}(u)=E[\exp (i u X(t))]$, is

$$
\phi_{X(t)}(u)=\left(\frac{1}{1-i \vartheta \nu u+\left(\sigma^{2} \nu / 2\right) u^{2}}\right)^{\frac{t}{\nu}}
$$

\subsection{Parameters of the Variance Gamma Pro- cess}

From equation (2.4), we can see that the variance gamma distribution depends on three parameters: the volatility of the Brownian motion $\sigma$, the variance rate of the gamma time change $\nu$ and the drift of the Brownian motion $\vartheta$. Let's study the first 4 moments of the variance gamma distribution, this will allow us to give an interpretation of these parameters

Over an interval of length $t$ and conditional on the gamma time change, $g$, the variance gamma process, $X(t)$ is normally distributed with mean $\vartheta g$ and variance $\sigma \sqrt{g} z$, that is

$$
X(t)=\vartheta g+\sigma \sqrt{g} z
$$

where $z$ is a standard normal independent of the gamma distribution $g$. Let's start considering some relationship which will be useful in determining the moments. $g$, over an interval of length $\mathrm{t}$, has a mean of $t$ and a variance $\nu t$. From $V(y)=E\left[y^{2}\right]-(E[y])^{2}$, where $V(\cdot)$ is the variance, we have

$$
E\left[g^{2}\right]=\nu t+t^{2}
$$

\footnotetext{
${ }^{7}$ See Dilip B Madan, Peter P. Carr and Eric C. Chang, "The Variance Gamma Process and Option Pricing", European Finance Review, 2, 1998, pages 83.
} 
Moreover by explicit integration of the gamma density, we have

$$
E\left[g^{3}\right]=\nu^{3}\left(2+\frac{t}{\nu}\right)\left(1+\frac{t}{\nu}\right) \frac{t}{\nu}=t^{3}+3 \nu t^{2}+2 \nu^{2} t
$$

And also by integration of the gamma density, we can write

$$
E\left[g^{4}\right]=(3 \nu+t)(2 \nu+t)(\nu+t) t=6 \nu^{3} t+11 \nu^{2} t^{2}+6 \nu t^{3}+t^{4}
$$

Using these relationships it is easy to compute the moments of the variance gamma distribution. Clearly

$$
E[X(t)]=\vartheta t
$$

The variance can be easily determined by considering

$$
X(t)-E[X(t)]=\vartheta(g-t)+\sigma \sqrt{g} z
$$

Squaring and taking the expectation we get

$$
E\left[(X(t)-E[X(t)])^{2}\right]=E\left[\vartheta^{2}(g-t)^{2}+\sigma^{2} g z^{2}+2 \vartheta(g-t) \sigma \sqrt{g} z\right]
$$

Using equation (2.6), we have

$$
E\left[(X(t)-E[X(t)])^{2}\right]=\vartheta^{2}\left(\nu t+t^{2}+t^{2}-2 t^{2}\right)+\sigma^{2} t+0
$$

or, compactly,

$$
E\left[(X(t)-E[X(t)])^{2}\right]=\left(\vartheta^{2} \nu+\sigma^{2}\right) t
$$

Let's now compute the third moment of the variance gamma distribution:

$$
\begin{array}{r}
E\left[(X(t)-E[X(t)])^{3}\right]= \\
=E\left[\vartheta^{3}(g-t)^{3}+3 \vartheta^{2}(g-t)^{2} \sigma \sqrt{g} z+3 \vartheta(g-t) \sigma^{2} g z^{2}+\sigma^{3} g^{\frac{3}{2}} z^{3}\right] \\
=\vartheta^{3} E\left[g^{3}-3 g^{2} t+3 g t^{2}-t^{3}\right]+0+3 \vartheta \sigma^{2} E\left[g^{2} z^{2}-t g z^{2}\right]+0
\end{array}
$$

Remembering equation (2.6) and (2.7), we get

$$
\begin{array}{r}
E\left[(X(t)-E[X(t)])^{3}\right]= \\
=\vartheta^{3}\left(t^{3}+3 \nu t^{2}+2 \nu t-3 \nu t^{2}-3 t^{3}+3 t^{3}-t^{3}\right)+3 \vartheta \sigma\left(\nu t+t^{2}-t^{2}\right)
\end{array}
$$


that is

$$
E\left[(X(t)-E[X(t)])^{3}\right]=\left(2 \vartheta^{3} \nu^{2}+3 \sigma^{2} \vartheta \nu\right) t
$$

Finally let's compute the fourth moment.

$$
\begin{array}{r}
E\left[(X(t)-E[X(t)])^{4}\right]=E\left[\vartheta^{4}(g-t)^{4}+4 \vartheta(g-t)^{3} \sigma \sqrt{g} z+\right. \\
\left.+6 \vartheta^{2}(g-t)^{2} \sigma^{2} g z^{2}+4 \vartheta(g-t) \sigma^{3} g^{\frac{3}{2}} z^{3}+\sigma^{4} g^{2} z^{4}\right]
\end{array}
$$

Using equations (2.6), (2.7) and (2.8) and remembering that $E\left[z^{4}\right]=3$ we get

$$
\begin{array}{r}
E\left[(X(t)-E[X(t)])^{4}\right]=\vartheta^{4}\left[6 \nu^{3} t+11 \nu^{2} t^{2}+6 v t^{3}+t^{4}+\right. \\
\left.-4 t\left(t^{3}+3 \nu t^{2}+2 \nu^{2} t\right)+6\left(\nu t+t^{2}\right) t^{2}-4 t^{4}+t^{4}\right]+ \\
+0+6 \vartheta^{2} \sigma^{2}\left(t^{3}+3 \nu t^{2}+2 \nu^{2} t+t^{3}-2 \nu t^{2}-2 t^{3}\right)+0+3 \sigma^{4}\left(\nu t+t^{2}\right)
\end{array}
$$

which gives you

$$
\begin{gathered}
E\left[(X(t)-E[X(t)])^{4}\right]= \\
=\left(3 \sigma^{4} \nu+12 \sigma^{2} \vartheta^{2} \nu^{2}+6 \vartheta^{4} \nu^{3}\right) t+\left(3 \sigma^{4}+6 \sigma^{2} \vartheta^{2} \nu+3 \vartheta^{4} \nu^{2}\right) t^{2}
\end{gathered}
$$

We can now use the four moments to interpret $\vartheta$ and $\nu$ as parameters which provide a way to control skewness and kurtosis. It is clear from the exam of equations (2.9) - (2.12) that $\vartheta$ and $\nu$ are not themselves the skewness and kurtosis of the variance gamma distribution, but rather they reflect only indirectly these characteristics of the distribution. In particular, from equation (2.11) we can see that if $\vartheta=0$ than there is no skewness and that the skewness has the same sign as $\vartheta$. Therefore a negative skewness, which is generally what can be observed in the market, is the same as asking for a negative drift in the time changed Brownian motion. Finally we can observe in equation (2.10) that where there is no skewness, the volatility is expressed by $\sigma$.

As for $\nu$, we can see from equation (2.12) that if $\vartheta=0$, then dividing the fourth moment by the square of the variance, $\sigma^{2} t$, we obtain, for a single time interval,

$$
3(1+\nu)
$$

Hence we can interpret $\nu$ as a percentage excess kurtosis over 3, the kurtosis of the normal distribution. In general, when we do not consider just a single 
interval, the kurtosis divided by the square of the variance is, in the case $\vartheta=0$, equal to

$$
3\left(1+\frac{\nu}{t}\right)
$$

Hence as $t$ increases, the kurtosis converges to 3 . This property of variance gamma is consistent with empirical evidence that fat tails tend to be present in daily returns while monthly returns tend to be normally distributed.

\subsection{Symmetric Variance Gamma}

The first case of Variance Gamma presented in literature by Madan and Seneta $^{8}$ is the case where there is no skewness in the process; this process is obtained as a time changed Brownian motion without drift. In this 1990 paper also a multivariate extension of the model is obtained, considering a vector of random variables distributed, conditional on a gamma random variable, as a multivariate normal with mean vector zero and a certain variance covariance matrix.

As mentioned, before 1990 some authors already presented some works related to variance gamma. Madan and Seneta in 1987 published an article ${ }^{9}$ in which, among other things, they show that a unit period variance gamma has finite moments of all orders. In another paper in the same year, Madan and Seneta ${ }^{10}$ compare empirically the variance gamma model to the normal distribution model, the stable model of Mandelbrot ${ }^{11}$, which considers independent symmetric stable increments, and the compound event model obtained combining normally distributed jumps at Poisson jump times presented by Press ${ }^{12}$. In this paper, Madan and Seneta use a $\chi^{2}$ goodness of fit

\footnotetext{
${ }^{8}$ Dilib B. Madan and Eugene Seneta "The Variance Gamma (V.G.) Model for Share Market Return", The Journal of Business, vol 63. no.4, 1990, pages 511-524.

${ }^{9}$ Dilip B. Madan and Eugene Seneta, "Simulation of Estimates Using the Empirical Characteristic Function", International Statistical Review, 55, 1987, pages 153-161.

${ }^{10}$ Dilip B. Madan and Eugene Seneta, "Chebyshev Polynomial Approximation and Characteristic Function Estimation ", Journal of the Royal Statistical Society, ser. B, 49, 1987, pages 163-169.

${ }^{11}$ Benoit Mandelbrot, "New Methods in Statistical Economics", The Journal of Political Economy, Vol. 71, No. 5, October 1963, pages 421-440.

${ }^{12}$ S. J. Press, "A Compound Events Model for Security Prices", The Journal of Business, 40, July 1967, pages 317-335.
} 
statistics on seven class intervals for unit sample variance on 19 stocks quotes on the Sydney Stock Exchange. For 12 of the 19 stocks the minimum $\chi^{2}$ is attained by the variance gamma model. Among the remaining cases, five were best characterized by the Press compound event model and two by the stable distribution model, with none best fitted by the normal distribution.

In 1991 Madan and Milne published a paper ${ }^{13}$, where they study equilibrium option pricing for the variance gamma process using a representative agent model under a constant relative risk aversion utility function. In this paper the model considered is still the symmetric variance gamma. However the resulting risk neutral process is identical to the non symmetric (or general) variance gamma process, with the drift in the time changed Brownian motion being negative for positive risk aversion.

Following Naik and Lee ${ }^{14}$, Madan and Milne observe that it is not possible to price European options using the cost of a hedging strategy, when the underlying follows a pure jump process, because a self financing continuous trading strategy in the underlying asset and in the riskless bond that replicates the payoff does not exist. Naik and Lee obtain a necessarily incomplete market equilibrium option price by solving a one individual equilibrium model, employing a constant relative risk aversion utility function for the individual. Madan and Milne use a model similar to Naik and Lee where there is one fully equity financed firm with a unit of stock outstanding engaged in the costless production of a single perishable consumption good. An approximation to an incomplete market equilibrium price is developed when the underlying follows a variance gamma process. The approximation is realized by taking first order Taylor series approximation to the change in the jump compensator induced by the measure change. A density process is also identified by Madan and Milne as the relevant change of measure for an exact equilibrium of a Lucas $^{15}$ type economy, following the method proposed by Naik and Lee. The coefficient of the change of measure is then interpreted as coefficient of relative risk aversion scaled by the asset return standard deviation. Although the process initially considered here is the symmetric variance gamma, the resulting risk neutral stock price process is the same

\footnotetext{
${ }^{13}$ Dibip B. Madan and Frank Milne, "Option Pricing with V.G. Martingale Components", Mathematical Finance, Vol. 1, No. 4, October 1991, pages 39-55.

${ }^{14}$ V. Naik and M. Lee, "General Equilibrium Pricing of Options on the Market Portfolio with Discontinuous Returns", Review of Financial Studies, Vol. 3, 1990, pages 493-521.

${ }^{15}$ R. E. Lucas, "Asset Prices in an Exchange Economy", Econometrica, 46, 1978, pages 1429-1445.
} 
as the one which characterizes the general variance gamma process, with the negative drift in the time changed Brownian motion being the equivalent of the positive risk aversion parameter of Madan and Milne.

Madan and Milne also present a comparison of the variance gamma model with the Black and Scholes one, finding that Black and Scholes formula generally underprices options with the percent bias rising as the stock gets out of the money and as the time to maturity increase. The bias is also found to be increasing with the expected rate of return in the stock and the level of kurtosis, and decreasing with the increase in standard deviation.

\subsection{Variance Gamma Process as Difference of Two Gamma Processes}

Since the variance gamma is a process of finite variation ${ }^{16}$, it can be written as difference of two increasing processes, the first representing the increases in the process and the second representing the decreases. In particular the two increasing processes here are themselves gamma processes. We can then write

$$
X(t ; \sigma, \nu, \vartheta)=\gamma_{p}\left(t ; \mu_{p}, \nu_{p}\right)-\gamma_{n}\left(t ; \mu_{n}, \nu_{n}\right)
$$

To prove this, we can consider two gamma distribution having, as in equation (2.3), the following characteristic functions:

$$
\begin{aligned}
\phi_{\gamma_{p}(t)}(u) & =\left(\frac{1}{1-i u \frac{\nu_{p}}{\mu_{p}}}\right)^{\frac{\mu_{p}^{2} t}{\nu_{p}}} \\
\phi_{-\gamma_{n}(t)}(u) & =\left(\frac{1}{1+i u \frac{\nu_{n}}{\mu_{n}}}\right)^{\frac{\mu_{n}^{2} t}{\nu n}}
\end{aligned}
$$

If $\mu_{p}, \mu_{n}, \nu_{p}$ and $\nu_{n}$ satisfy the following equations:

$$
\frac{\mu_{p}^{2}}{\nu_{p}}=\frac{\mu_{n}^{2}}{\nu_{n}}=\frac{1}{\nu}
$$

\footnotetext{
${ }^{16}$ See more about this in section 2.7 .4 .
} 


$$
\begin{gathered}
\frac{\nu_{p} \nu_{n}}{\mu_{p} \mu_{n}}=\frac{\sigma^{2} \nu}{2} \\
\frac{\nu_{p}}{\mu_{p}}-\frac{\nu_{n}}{\mu_{n}}=\vartheta \nu
\end{gathered}
$$

the product of these characteristic functions (2.15) and (2.16) gives

$$
\left(\frac{1}{1-i u\left(\frac{\nu_{p}}{\mu_{p}}-\frac{\nu_{n}}{\mu_{n}}\right)-i^{2} u^{2} \frac{\mu_{p} \mu_{n}}{\nu_{p} \nu_{n}}}\right)^{\frac{t}{\nu}}
$$

which, remembering that $i^{2}=-1$, is the characteristic function of a variance gamma distribution as presented in equation (2.5). It follows that the variance gamma is the difference of two gamma processes which have respectively mean rates $\mu_{p}$ and $\mu_{n}$ and variance rates $\nu_{p}$ and $\nu_{n}$

Using equations (2.17) - (2.19), we can obtain explicit expressions for the mean rate and variance rate of the gamma processes representing increasing and decreasing of the variance gamma distribution. In particular from equations (2.18) and (2.19), we have

$$
\left(\frac{\nu_{p}}{\mu_{p}}\right)^{2}-\vartheta \nu\left(\frac{\nu_{p}}{\mu_{p}}\right)-\frac{\sigma^{2} \nu}{2}=0
$$

a second order equation which can be solved to obtain

$$
\frac{\nu_{p}}{\mu_{p}}=\frac{\vartheta \nu+\sqrt{\vartheta^{2} \nu^{2}+2 \sigma^{2} \nu}}{2}
$$

where we choose the positive sign of the square root because the parameter $\mu$ and $\nu$ of the gamma process, $\gamma(t ; \mu, \nu)$, are required to be positive. In the same way we can obtain

$$
\left(\frac{\nu_{n}}{\mu_{n}}\right)^{2}+\vartheta \nu\left(\frac{\nu_{n}}{\mu_{n}}\right)-\frac{\sigma^{2} \nu}{2}=0
$$

which gives

$$
\frac{\nu_{n}}{\mu_{n}}=\frac{-\vartheta \nu+\sqrt{\vartheta^{2} \nu^{2}+2 \sigma^{2} \nu}}{2}
$$


Now, from equation (2.17), we have

$$
\mu_{p}=\frac{\nu_{p}}{\mu_{p}} \cdot \frac{1}{\nu}
$$

which combined with equation (2.20) gives

$$
\begin{array}{r}
\mu_{p}=\frac{1}{2} \sqrt{\vartheta^{2}+\frac{2 \sigma^{2}}{\nu}}+\frac{\vartheta}{2} \\
\nu_{p}=\left(\frac{1}{2} \sqrt{\vartheta^{2}+\frac{2 \sigma^{2}}{\nu}}+\frac{\vartheta}{2}\right)^{2} \nu
\end{array}
$$

Finally equations (2.17) and (2.21) give

$$
\begin{array}{r}
\mu_{n}=\frac{1}{2} \sqrt{\vartheta^{2}+\frac{2 \sigma^{2}}{\nu}}-\frac{\vartheta}{2} \\
\nu_{n}=\left(\frac{1}{2} \sqrt{\vartheta^{2}+\frac{2 \sigma^{2}}{\nu}}-\frac{\vartheta}{2}\right)^{2} \nu
\end{array}
$$

\subsection{Lévy Measure for the Variance Gamma Process}

The variance gamma process is an example of Lévy process, other examples being the gamma process and the Brownian motion. We can think about Lévy process as a natural continuous time analog of a sequence of partial sums of independently and identically distributed random variables ${ }^{17}$.

\footnotetext{
${ }^{17}$ A detailed presentation of Lévy process can be found in B. Fristedt, Sample Properties of Stochastic Processes with Stationary, Independent Increments. In P. Ney and S. C. Port (editors), Advances in Probability and Related Topics, Vol. 3, New York, Marcel Dekker, 1974, pages 241-396.
} 
If we think about the variance gamma process as a time changed Brownian motion, we can write the Lévy measure as ${ }^{18}$

$$
k_{X}(x) d x=\frac{\exp \frac{\vartheta x}{\sigma^{2}}}{\nu|x|} \exp \left(-\frac{\sqrt{\frac{2}{\nu}+\frac{\vartheta^{2}}{\sigma^{2}}}}{\sigma}|x|\right) d x
$$

In the case $\vartheta=0$, the Lévy measure is symmetric about zero. We can also see from equation (2.22) that if $\vartheta<0$, negative values of $x$ receive a higher relative probability than positive ones. This means that a negative value of $\vartheta$ lead to a negative skewness. Moreover we can see that a large value of $\nu$ lower the exponential decay rate of the Lévy measure symmetrically around zero. This increases the probability of large jumps and it can therefore represent a measure of kurtosis. These observations about $\vartheta$ and $\nu$ confirm the results obtained by direct computation of the moments of the variance gamma distribution.

The Lévy measure for the variance gamma process has also a representation as difference of two gamma processes. The Lévy measure of a gamma process, $\gamma(t ; \mu, \nu)$, is

$$
k_{\gamma}(x) d x=\frac{\mu^{2} \exp \left(-\frac{\mu}{\nu} x\right)}{\nu x} d x, \text { for } x>0 \text { and } 0 \text { otherwise }
$$

hence the Lévy measure for the variance gamma process can be written as

$$
k_{X}(x) d x= \begin{cases}\frac{\mu_{n}^{2} \exp \left(-\frac{\mu_{n}}{\nu_{n}}|x|\right)}{\nu_{n}|x|} d x & \text { for } x<0 \\ \frac{\mu_{p}^{2} \exp \left(-\frac{\mu_{p}}{\nu_{p}} x\right)}{\nu_{p} x} d x & \text { for } x>0\end{cases}
$$

A third representation of Lévy measure for variance gamma process is in terms of a symmetric variance gamma process subject to a measure change induced by a constant relative risk aversion utility function. Following Madan and Milne ${ }^{19}$, the risk neutral variance gamma process for stock prices can be derived from a Lucas general Equilibrium economy where the representative

\footnotetext{
${ }^{18}$ See Dilip B Madan, Peter P. Carr and Eric C. Chang, "The Variance Gamma Process and Option Pricing", Europen Finance Review, 2, 1998, page 84.

${ }^{19}$ Dibip B. Madan and Frank Milne, "Option Pricing with V.G. Martingale Components", Mathematical Finance, Vol. 1, No. 4, October 1991, pages 39-55.
} 
agent has a constant relative risk aversion utility function, with a relative risk aversion $\zeta$ and in which the process followed by the logarithm of the stock price is a symmetric variance gamma. In this case the risk neutral Lévy measure is given by

$$
k_{X}(x) d x=\frac{\exp (-\zeta x)}{\nu|x|} \exp \left(-\frac{\sqrt{2}}{s \sqrt{\nu}}|x|\right)
$$

If we use as parameters of this equation the following

$$
\begin{array}{r}
\zeta=-\frac{\vartheta}{\sigma^{2}} \\
s=\frac{\sigma}{\sqrt{1+\left(\frac{\vartheta}{\sigma}\right)^{2} \frac{\nu}{2}}}
\end{array}
$$

we obtain again the representation in equation (2.22) and therefore the two definitions are in agreement.

\subsection{Properties of Processes Describing Stock returns}

\subsubsection{Completely Monotone Lévy Densities}

From an empirical point of view it is clear that it is reasonable to ask that, fixing the direction of the move of the stock price, large moves are less frequent that small moves, that is jumps of larger size have a lower arrival rate than smaller rates. Mathematically a structural property of the Lévy densities is that of monotonicity. This property amounts to asserting that for differentiable densities, the derivative is positive for negative jumps and negative for positive jumps. The property of monotonicity may be strengthened to complete monotonicity by requiring that derivatives of the same order have the same sign and be alternating in sign. This property links analytically the arrival rate of small and large jumps, requiring that large jumps arrive less frequently than small jumps. By Bernstein's theorem all completely monotone Lévy densities are mixtures of exponential functions and are given by 
the Laplace transform of positive measures $\rho(d a)$ on the positive half line and can be expressed $\operatorname{as}^{20}$

$$
k(y)=\int_{0}^{+\infty} e^{-a y} \rho(d a)
$$

Although this requirement of complete monotonicity seems to be quite intuitive, we note that not all the option pricing models presented in literature satisfy it. In particular the jump diffusion model based on the reflected normal distribution for the jump size is not completely monotone as easily observed by seeing that the normal density shifts from being a concave function near zero to a convex function near infinity.

Another desirable property of processes describing stock returns is that, once fixed the size of the move, down moves have an arrival rate and a risk neutral price independent and higher than those of the corresponding up moves. The independence of the down an up moves can be obtained using two non negative functions, each of them having a single argument which is a positive real. One of these functions determines the arrival rate associated with the absolute size of down move, the other determining the arrival rate of up moves. A premium for negative moves can be created by requiring that the function determining the arrival rates related with down moves has higher mean than the one determining arrival rates for up moves. Completely monotone functions satisfy these requirements of independence of up and down moves and of premium for negative moves.

\subsubsection{Finite Variation Processes}

Consider a process $x(t)$ defined on an interval [0,T]. If $P=\left\{0=t_{0}<\right.$ $\left.t_{1}<\ldots<t_{n}=T\right\}$ is a partition of $[0, T]$, write $\Delta x_{t}=x\left(t_{k}\right)-x\left(t_{k-1}\right)$, for $t=1,2, \ldots, n$. If for any path there exists a positive number $M$ such that

$$
\sum_{i=1}^{n}\left|\Delta x_{t}\right| \leq M
$$

for all partitions of $[0, T]$, then $x$ is said to be of finite, or bounded, variation on $[0, T]$. It can be proved that a function is of finite variation if and only if it can be expressed as the difference of two increasing functions.

\footnotetext{
${ }^{20}$ See Hélyette Geman, Dilip B. Madan and Marc Yor, "Time Changes for Lévy Processes", Mathematical Finance, Vol. 11, No. 1, January 2001, page 86.
} 
As Carr, Madan, Geman and Yor note ${ }^{21}$, processes of finite variations are potentially more useful than those ones of infinite variation in explaining the measure change from the statistical to the risk neutral process as they allow greater flexibility between the local characteristic of the martingale components under the two measures. In the case of infinite activity processes like the Brownian motion, the volatility, and hence the local martingale component, is invariant under an equivalent change in measure. This equivalence of measure change for infinite variation jump processes implies that the difference between the risk neutral and the statistical Lévy densities is of finite variation. This requires that the two processes have the same exponent. On the other side, if the processes are themselves of finite variation, then the difference in the Lévy densities will automatically be of finite variation and therefore no parametric restriction on the processes is required.

\subsubsection{Finite Activity Processes}

A pure jump process is defined to be of finite activity if the number of jumps in any interval of time is finite. On the other side, if the number of jump in any interval is infinite, we define the process as of infinite activity. We can think about an infinite activity process as an approximation of a highly liquid market with large activity.

\subsubsection{Variance Gamma Properties}

Let's consider again the representation of the Lévy measure for the variance gamma process as difference of two gamma processes presented in equation (2.23). We can see that the Lévy density is divided by the absolute value of the jump size, therefore the Lévy density has the behavior of $\frac{1}{|x|}$ in the neighborhood of zero and the resulting process is one of infinite activity, as the variance gamma Lévy measure integrates to infinity. Moreover, since $|x|$ is integrable with respect to the variance gamma Lévy density, the process is one of finite variation.

\footnotetext{
${ }^{21}$ Peter Carr, Hélyette Geman, Dilip B. Madan and Marc Yor, "The Fine Structure of Asset Returns: An Empirical Investigation", The Journal of Business, Vol. 75, No. 2, 2002 , page 312 .
} 


\subsection{The Statistical and Risk Neutral Vari- ance Gamma Stock Price Process}

This section defines the stochastic process followed by a stock price under the variance gamma model both from a statistical point of view and from a risk neutral point of view. This will allow an estimate of the parameters which characterize the variance gamma model, $\sigma, \vartheta$ and $\nu$. This parameter can be estimated statistically, that is using the underlying values, and risk neutrally, which refers to the parameters implied by the option prices. It is worth nothing that, in contrast with the diffusion based continuous price processes, here the statistical parameters do not have to be the same as the risk neutral ones ${ }^{22}$ The estimate of the parameters allows an analysis of the importance of being able to model the skewness and kurtosis of stock return distribution. Moreover we can analyze how much the variance gamma model add to the symmetric variance gamma and to the geometric Brownian motion in terms of description of underlying price moves and option prices.

\subsubsection{The Statistical Variance Gamma Stock Price Pro- cess}

The statistical stock price dynamic can be obtained by replacing the geometric Brownian motion with the variance gamma process in the equation used by Black and Scholes in their famous model ${ }^{23}$. We have then the following process:

$$
S(t)=S(0) \exp \left[m t+X\left(t ; \sigma_{S}, \nu_{S}, \vartheta_{S}\right)+\omega_{S} t\right]
$$

where $X(\cdot)$ is a variance gamma process, $m$ is the mean rate of return on the stock under the statistical probability measure and the subscripts " $S$ " are used to stress the fact that the parameters are the statistical ones. The values of $w$ is determined as a non arbitrage condition, by evaluating the

\footnotetext{
${ }^{22}$ See more about this in Dilib B. Madan, Peter P. Carr and Eric C. Chang, "The Variance Gamma Process and Option Pricing", European Finance Review 2, 1998, page $86-87$.

${ }^{23}$ Fisher Black and Myron Scholes, "The Pricing of Options and Corporate Liabilities", Journal of Political Economy, Vol. 81, No. 3, May/June 1973, pages 637-654.
} 
characteristic function for $X(t)$ at $u=1 / i$ so that

$$
E[S(t)]=S(0) \exp (m t) \Leftrightarrow E[\exp (X(t))]=\exp \left(-\omega_{S} t\right)
$$

and it is equal to

$$
\omega_{S}=\frac{1}{\nu_{S}} \ln \left(1-\vartheta_{S} \nu_{S}-\frac{\sigma^{2} \nu_{S}}{2}\right)
$$

\subsubsection{The Risk Neutral Variance Gamma Stock Price Process}

Under the risk neutral processes, stock prices discounted at the risk free interest rate are martingales and so the expected return on the stock under the risk neutral probability measure is the continuously compounded risk free interest rate $r$. The risk neutral process can be written as

$$
S(t)=S(0) \exp \left[r t+X\left(t ; \sigma_{R N}, \nu_{R N}, \vartheta_{R N}\right)+\omega_{R N} t\right]
$$

where the subscripts " $R N$ " indicates that these are risk free parameters and, using the same condition as before applied to the risk neutral case, $\omega_{R N}$ is given by

$$
\omega_{R N}=\frac{1}{\nu_{R N}} \ln \left(1-\vartheta_{R N} \nu_{R N}-\frac{\sigma^{2} \nu_{R N}}{2}\right)
$$

\subsection{European Option Price Under Variance Gamma}

We present in this section an analytical formula which allows to price options under the variance gamma model. As in the case of geometric Brownian motion, it is not possible to obtain close form solution for American options when the stock returns are explained by the variance gamma model and 
it is necessary instead to use numerical solution for them. Chapter 5 will deal with this issue providing an algorithm which allows to solve numerically the partial differential equation describing the dynamic of the option price. This numerical approach will be used to price both European and American options.

The first step required to obtain an analytical pricing formula is to write the density function of stock return expressed as logarithm of the stock price relative

$$
z=\ln \left[\frac{S(t)}{S(0)}\right]
$$

We denote this density function as $h(z)$. Because the variance gamma process is a time changed Brownian motion as defined in equation (2.4), we can say that over an interval of length $t, h(z)$ is a normal density function, conditional on the realization of the gamma time change. Let's compute this conditional probability. In the computation of the density function $h(z)$, we omit the subscripts " $S$ " to the parameters to reduce the notation, but it is understood that these are the parameters we are using. Considering the combination of equations (2.1), (2.4) and (2.26), we can see that, conditional on the gamma random variable $g, z$ is normally distributed with mean

$$
m t+\frac{t}{\nu} \ln \left(1-\vartheta \nu-\frac{\sigma^{2} \nu}{2}\right)+\vartheta g
$$

and variance $\sigma^{2} g$. Hence the conditional density function is

$$
h(z \mid g)=\frac{1}{\sigma \sqrt{2 \pi g}} \exp \left\{-\frac{\left[z-m t-\frac{t}{\nu} \ln \left(1-\vartheta \nu-\frac{\sigma^{2} \nu}{2}\right)-\vartheta g\right]^{2}}{2 \sigma^{2} g}\right\}
$$

The unconditional density can be obtained as conditional density given $g$ times the marginal density of $g$, with the random variable $g$ integrated out. The gamma density function (2.2) with a unit mean rate and a time interval of length $t$ is

$$
f_{t}(g)=\frac{g^{\frac{t}{\nu}-1} \exp \left(-\frac{g}{\nu}\right)}{\nu^{\frac{t}{\nu}} \Gamma\left(\frac{t}{\nu}\right)}
$$


Hence combining (2.28) and (2.29), we have

$$
\begin{gathered}
h(z)=\int_{0}^{+\infty} \frac{1}{\sigma \sqrt{2 \pi g}} \exp \left\{-\frac{\left[z-m t-\frac{t}{\nu} \ln \left(1-\vartheta \nu-\frac{\sigma^{2} \nu}{2}\right)-\vartheta g\right]^{2}}{2 \sigma^{2} g}\right\} . \\
\cdot \frac{g^{\frac{t}{\nu}-1} \exp \left(-\frac{g}{\nu}\right)}{\nu^{\frac{t}{\nu}} \Gamma\left(\frac{t}{\nu}\right)} d g
\end{gathered}
$$

Using the results from Gradshetyn and Ryzhik ${ }^{24}$ this integral can be solved as

$$
h(z)=\frac{2 \exp \left(\frac{\vartheta x}{\sigma^{2}}\right)}{\nu^{\frac{t}{\nu}} \sqrt{2 \pi} \sigma \Gamma\left(\frac{t}{\nu}\right)}\left(\frac{x^{2}}{\frac{2 \sigma^{2}}{\nu}+\vartheta^{2}}\right)^{\frac{t}{2 \nu}-\frac{1}{4}} \cdot K_{\frac{t}{\nu}-\frac{1}{2}}\left[\frac{1}{\sigma^{2}} \sqrt{x^{2}\left(\frac{2 \sigma^{2}}{\nu}+\vartheta^{2}\right)}\right]
$$

where $K$ is a modified Bessel function of second kind and

$$
x=z-m t-\frac{t}{\nu} \ln \left(1-\vartheta \nu-\frac{\sigma^{2} \nu}{2}\right)
$$

The price of a European call options $C(S(0), K, t)$, where $K$ is the strike and $t$ is the maturity, can be written with the familiar expression

$$
C(S(0), K, t)=e^{-r t} E[\max (S(t)-K, 0)]
$$

where the expectation is taken under the risk neutral process defined in equation (2.27). To obtain the close price we can proceed in the same way we did for the density function $h(z)$ : we first obtain the call value conditional on the time change given by the gamma distribution $g$ and then we integrate out the gamma random variable. Conditional on $g, X(t)$ is normally distributed, hence the conditional option price can be written $\mathrm{as}^{25}$

$$
\begin{aligned}
C(g)=S(0) & \left(1-\frac{\nu(\zeta s+s)^{2}}{2}\right)^{\frac{t}{\nu}} \exp \left(\frac{(\zeta s+s)^{2} g}{2}\right) \cdot N\left[\frac{d}{\sqrt{g}}+(\zeta s+s) \sqrt{g}\right]+ \\
& -K e^{-r t}\left[1-\frac{\nu(\zeta s)^{2}}{2}\right]^{\frac{t}{\nu}} \exp \left[\frac{(\zeta s)^{2} g}{2}\right] \cdot N\left(\frac{d}{\sqrt{g}}+\zeta s \sqrt{g}\right)
\end{aligned}
$$

\footnotetext{
${ }^{24}$ I. S. Gradshetyn and I. M. Ryzhik, Tables of Integrals, Series and Products, Academic Press, New York, 1980, 3.471.9.

${ }^{25}$ Dibip B. Madan and Frank Milne, "Option Pricing with V.G. Martingale Components", Mathematical Finance, Vol. 1, No. 4, October 1991, page 49, with $\alpha=\zeta s$.
} 
where $N(\cdot)$ is the cumulative distribution function of a standard normal random variable, with $\zeta$ and $s$ given by equations (2.24) and (2.25) and where we define

$$
d=\frac{1}{s}\left\{\ln \left[\frac{S(0)}{K}\right]+r t+\frac{t}{\nu} \ln \left[\frac{1-c_{1}}{1-c_{2}}\right]\right\}
$$

with

$$
\begin{array}{r}
c_{1}=\frac{\nu(\zeta s+s)^{2}}{2} \\
c_{2}=\frac{\nu(\zeta s)^{2}}{2}
\end{array}
$$

The unconditional call price $C(S(0), K, t)$ can be obtained integrating over $g$ the conditional price $C(g)$ times the marginal density of $g$ in equation (2.29), that is

$$
C(S(0), K, t)=\int_{0}^{+\infty} c(g) \frac{g^{\frac{t}{\nu}-1} \exp \left(-\frac{g}{\nu}\right)}{\nu^{\frac{t}{\nu}} \Gamma\left(\frac{t}{\nu}\right)} d g
$$

Madan and Milne provide a numerical solution of the integral, while an analytical solution, in terms of the Bessel functions and of the degenerate hypergeometric functions of two variables, can be found in Madan, Carr and Chang $^{26}$ and is equal to

$$
\begin{aligned}
C(S(0), K, t)= & S(0) \Psi\left[d \sqrt{\frac{1-c_{1}}{\nu}},(\zeta s+s) \sqrt{\frac{\nu}{1-c_{1}}}, \frac{t}{\nu}\right]+ \\
& -K e^{-r t} \Psi\left[d \sqrt{\frac{1-c_{2}}{\nu}}, \zeta s^{2} \sqrt{\frac{\nu}{1-c_{2}}}, \frac{t}{\nu}\right]
\end{aligned}
$$

In which the generic function $\Psi(a, b, \gamma)$ is the solution of the following integral

$$
\int_{0}^{+\infty} N\left(\frac{a}{\sqrt{u}}+b \sqrt{u}\right) \frac{u^{\gamma-1} e^{-u}}{\Gamma(\gamma)} d u
$$

${ }^{26}$ Dilib B. Madan, Peter P. Carr and Eric C. Chang, "The Variance Gamma Process and Option Pricing", European Finance Review 2, 1998, pages 99-102. 
and is given by

$$
\begin{aligned}
& \Psi(a, b, \gamma)=\frac{c^{\gamma+\frac{1}{2}} \exp [\operatorname{sign}(a) c](1+u)^{\gamma}}{\sqrt{2 \pi} \Gamma(\gamma) \gamma} \\
& \cdot K_{\gamma+\frac{1}{2}}(c) \Phi\left[\gamma, 1-\gamma, 1+\gamma ; \frac{1+u}{2},-\operatorname{sign}(a) c(1+u)\right]+ \\
& -\operatorname{sign}(a) \frac{c^{\gamma+\frac{1}{2}} \exp [\operatorname{sign}(a) c](1+u)^{1+\gamma}}{\sqrt{2 \pi} \Gamma(\gamma)(1+\gamma)} . \\
& \cdot K_{\gamma-\frac{1}{2}}(c) \Phi\left[1+\gamma, 1-\gamma, 2+\gamma ; \frac{1+u}{2},-\operatorname{sign}(a) c(1+u)\right]+ \\
& +\operatorname{sign}(a) \frac{c^{\gamma+\frac{1}{2}} \exp [\operatorname{sign}(a) c](1+u)^{\gamma}}{\sqrt{2 \pi} \Gamma(\gamma) \gamma} . \\
& \cdot K_{\gamma-\frac{1}{2}}(c) \Phi\left[\gamma, 1-\gamma, 1+\gamma ; \frac{1+u}{2},-\operatorname{sign}(a) c(1+u)\right]
\end{aligned}
$$

where we have defined the following variables

$$
\begin{gathered}
c=|a| \sqrt{2+b^{2}} \\
u=\frac{b}{\sqrt{2+b^{2}}}
\end{gathered}
$$

and where $K_{\alpha}$ is a modified Bessel function of the second kind of order $\alpha$ and $\Phi(\cdot)$ is the degenerate hypergeometric function of two variables, which has the following integral representation ${ }^{27}$

$$
\Phi(\alpha, \beta, \gamma ; x, y)=\frac{\Gamma(\gamma)}{\Gamma(\alpha) \Gamma(\gamma-\alpha)} \cdot \int_{0}^{1} u^{\alpha-1}(1-u)^{\gamma-\alpha-1}(1-u x)^{-\beta} e^{u y} d u
$$

The call price in equation (2.31) has a form similar to the Black Scholes formula having the stock price times a probability minus the present value of the strike price times a second probability element. It is possible to prove that the second probability element is the risk neutral probability that $S(t)>K$. The first probability element on the other side is the probability that $S(t)>K$

\footnotetext{
${ }^{27}$ Pierre Humbert, "The confluent hypergeometric function of two variables", Proceedings of the Royal Society of Edinburgh, 1920, pages 73-85.
} 
obtained on normalizing the stock price with the risk neutral density of the stock price. As usual put prices can be determined by using put-call parity.

\subsection{Empirical Tests of the Variance Gamma Model for European Options}

This section describes some results presented by Madan, Carr and Chang ${ }^{28}$. The authors compare the variance gamma process with the symmetric variance gamma and with the geometric Brownian motion using options on the Standard \& Porr's 500. Options from January 1992 to September 1994 are considered and several options prices on or around the close for each day are used to ensure liquidity and avoid issues of non synchronous trading. Up to fours strike for each of four maturities were used.

The test is realized both from a statistical point of view, using underlying prices to determine the values of the statistical parameters, and from a risk neutral point of view, by determining the risk neutral parameters implied by option prices. Moreover the pricing errors are studied to determine if the models are biased.

\subsubsection{Skewness and Kurtosis Results}

To compute statistical parameters for the variance gamma, maximum likelihood estimation with the density function presented in equation (2.30) is used. The estimation of the symmetric variance gamma is realized in the same way, but constraining the $\vartheta=0$, while in the case of the Black Scholes model, the underlying process is the lognormal.

Statistical mean returns and volatilities estimated by the three models are very similar. As for the kurtosis, both symmetric and general variance gamma model, have an estimated statistical $\nu$ equal to 0.002 . Remembering from equation (2.13) that, when $\vartheta=0, \nu$ provides a measure of percentage excess kurtosis over 3 for a unit time period, here one year, measuring the time in years. Therefore the corresponding daily kurtosis is given by $3 *(1+$

\footnotetext{
${ }^{28}$ Madan Dilib B., Carr Peter P. and Chang Eric C., "The Variance Gamma Process and Option Pricing", European Finance Review 2, 1998, pages 79-105.
} 
$0.002 * 365)=5.19$, where we converted years in days using calendar days instead of trading days. Finally we can say that statistical skewness for the variance gamma is insignificant.

Risk neutral parameters are obtained on a weekly basis, again using maximum likelihood. The average estimates of $\nu$ for symmetric and general variance gamma are much higher than the statistical counterparts, moreover a negative skew is showed in the case of (general) variance gamma.

\subsubsection{Pricing Performance of the Variance Gamma}

We saw that from a statistical point of view, kurtosis is an important factor in describing the dynamics of stock returns, while, from a risk neutral point of view, both skewness and kurtosis appear to be relevant. Madan, Carr and Chang try to give a more definitive opinion on the performance of variance gamma, compared with its symmetrical counterpart and with geometric Brownian motion by studying the pricing error of the models. In particular a regression on the pricing errors from each model is realized to determined the presence of biases. Factors used are maturity, the moneyness, the square of the moneyness and the level of interest rate. Moneyness enters twice in the regression to allow volatility smiles where both out of the money puts and calls exhibit higher implied volatilities.

All four regressors together with the constant are significant in the case of the Black and Scholes model at a $5 \%$ level. Consistently with expectation, the coefficient of the degree of moneyness is negative, while the one for the square of moneyness is positive. Positive are also the coefficient for the maturity and the interest rate. Adjusted $R^{2}$ for this model is high at $16 \%$ and the F statistics takes to a conclusion that we must reject the hypothesis of orthogonality of errors to the regressors. The symmetric variance gamma performs only slightly better, with all the variables, but the interest rates significant at a $5 \%$ level. Adjusted $R^{2}$ is high at $17 \%$ and $\mathrm{F}$ again requires to reject the hypothesis of orthogonality of the explanatory variables. The symmetric variance gamma seems to have over adjusted the smile issue presented by Black and Scholes and now the smile is inverted with a positive coefficient for moneyness and a negative coefficient of the square of the moneyness.

The variance gamma model, on the contrary, produced a market improvement over the other two models, based on this data. Moneyness, square of moneyness and interest rate, together with the constant are now insignificant 
even at a $1 \%$ level. Adjusted $R^{2}$ is is $0.1 \%$ and the $\mathrm{F}$ statistic is not significant. The only problems which is not solved by the variance gamma model is the maturity bias: the model presents a negative coefficient for maturity which is significant at a $1 \%$ level.

Another empirical test on the pricing performances of the variance gamma model which is worth mentioning is the one realized by Lam, Chang and $\mathrm{Lee}^{29}$. In this paper the authors test both the symmetric and the general variance gamma on the pricing of the Hang Seng Index call options, which are European style options. The test of the model when the options fair values are computed using the closed form solution is better carried on European options like these ones rather than on American options, like the one used by Madan, Carr and Chang, because in this way no additional error related to the conversion of American to European prices is added. The test is realized on intraday prices over a three year time frame and the data are closely examined and matched so that they are as synchronous as possible. The presence of this large database allows for the use of robust statistical tests, with respect to outliers, without loosing too much test power. The conclusion found by Lam, Chang and Lee is that the variance gamma option pricing model performs someway better than the Black and Scholes model. Under the historical approach, the variance gamma can quite well correct some of the systematic biases of the Black and Scholes model. However, under the implied approach, the variance gamma continues to exhibit predictable biases.

\subsubsection{Conclusion}

Based on the empirical tests realized on the model, especially on those ones presented by Madan, Carr and Chang, the variance gamma model presents a material improvement over the geometric Brownian motion and the symmetric variance gamma model. The variance gamma is no more moneyness biased, however it still presents problems related with the estimation of options with different maturities. As we will see in section (3.8), this can be corrected using a further extension of the model which presents stochastic volatility. The stochastic volatility model is however beyond the scope of this

\footnotetext{
${ }^{29}$ K. Lam, E. Chang and M. C. Lee, "An Empirical Test of the Variance Gamma Option Pricing Model", Pacific-Basin Finance Journal, Vol. 10, No. 3, 2002, pages 267-285.
} 
work and the attention, when presenting the numerical procedure for pricing European and American options in chapters 5, will be focus on the variance gamma model and not on the stochastic volatility extension. 


\section{Chapter 3}

\section{Extensions of the Variance Gamma Model: CGMY Model and Stochastic Volatility Models}

\subsection{Introduction}

As described in the previous chapter, stochastic processes describing the dynamic of stock prices can exhibit infinite of finite variation and infinite of finite variation. The variance gamma, in particular, is a process of infinite activity and of finite variation. Carr, Geman, Madan and Yor $^{1}$ presented an extension of the variance gamma which allows for both infinite and finite activity and for both infinite and finite variation. The name of the model, CGMY, is derived from the initials of the authors.

\footnotetext{
${ }^{1}$ The authors presented a series of articles on the subject, the most complete introduction to the model is probably in Peter Carr, Hélyette Geman, Dilip B. Madan and Marc Yor, "The Fine Structure of Asset Returns: An Empirical Investigation", The Journal of Business, Vol. 75, No. 2, 2002, pages 305-332.
} 


\subsection{Lévy Measure and Parameters for the CGMY Process}

\subsubsection{Definition of the Model}

We define CGMY process, denoted $X_{C G M Y}(t ; C, G, M, Y)$, as the infinitely divisible process of independent increments having Lévy density given by

$$
k_{C G M Y}(x)= \begin{cases}C \frac{\exp (-G|x|)}{|x|^{1+Y}} & \text { for } x<0 \\ C \frac{\exp (-M|x|)}{|x|^{1+Y}} & \text { for } x>0\end{cases}
$$

where $C>0, G \geq 0, M \geq 0$ and $Y<2$. The condition on $Y$ comes from the requirement that Lévy densities integrate $x^{2}$ in the neighborhood of 0 . It is easy to see that by choosing

$$
\begin{array}{r}
Y=0 \\
C=\frac{1}{\nu}=\frac{\mu_{p}^{2}}{\nu_{p}}=\frac{\mu_{n}^{2}}{\nu_{n}} \\
G=\frac{\mu_{n}}{\nu_{n}} \\
M=\frac{\mu_{p}}{\nu_{p}}
\end{array}
$$

we obtain the Lévy density corresponding to the measure presented in equation (2.23). Hence the variance gamma process can be seen as a particular case of the CGMY process.

\subsubsection{Parameters of the CGMY Process}

The parameters $C, G, M$ and $Y$, named after the authors of the model, describe the behavior of the stochastic process. The parameter $C$ can be interpreted as a measure of the overall level of activity. In particular, keeping the other parameters constant and integrating over all moves exceeding a small level, we can calibrate the overall level of activity with $C$. We can see the parameter $C$ as providing control over the kurtosis. Think in particular to the case where $G=M$, here the Lévy measure is symmetric and $C$ can be 
seen as a measure of kurtosis in a similar way used to obtain equation (2.13). Remember on this point that, when the parameters are chosen to obtain the variance gamma, as a special case, $C$ is equal to $1 / \nu$.

The parameters $G$ and $M$ control the rate of exponential decay on the right and on the left of the Lévy density. When they are not equal this translates into a process characterized by skewness. In particular if $G<M$, the left tail of the $X$ distribution is heavier than the right one, which is consistent with generally accepted negative skewness. We can think about $G$ and $M$ as parameters implied from the risk neutral distribution and as parameters in a statistical distribution. From the statistical point of view, the difference between $G$ and $M$ specifies the relative frequency of drops versus rises, while their sum measures how often large moves occur compared with small ones. On the other side, if we think about the parameter as implied by a risk neutral distribution, their difference is a measure of the price of a fall with respect to the price of a rise, while their sum gives the price of a large move compared with that one of a small one.

The parameter $Y$ which is really the new parameter compared with the variance gamma process and as such is the one which allows to have a process which can be of finite or infinite variation and of finite and infinite activity, moreover it also the parameter which allows to determine if the process have a completely monotone Lévy density.

First of all we can prove that the CGMY process has completely monotone Lévy density for $Y>-1$. If $Y<-1$, then $1+Y<0$ and the Lévy density

$$
x^{-(1+Y)} \exp (-\beta x) \text { for } \beta=G, M
$$

increases near zero and then declines to zero as $x$ tends to infinity. Hence the density is not monotone. On the other side, when $Y>-1$, the Lévy density can be written as $^{2}$

$$
x^{-(1+Y)} \exp (-\beta x)=\int_{\beta}^{+\infty} \frac{(\alpha-\beta)^{Y}}{\Gamma(1+Y)} e^{-\alpha x} d a
$$

hence we have complete monotonicity with weighting function

$$
1_{\alpha>\beta} \frac{(\alpha-\beta)^{Y}}{\Gamma(1+Y)}
$$

\footnotetext{
${ }^{2}$ See Peter Carr, Hélyette Geman, Dilip B. Madan and Marc Yor, "The Fine Structure of Asset Returns: An Empirical Investigation", The Journal of Business, Vol. 75, No. 2, 2002 , page 314 .
} 
Secondly we can see that CGMY is of infinite activity for $Y>0$. Note that for negative values of $Y$, the Lévy measure integrates to a finite values in the neighborhood of zero and so we have a process of finite activity; while if $Y>0$, the Lévy measure integrates to infinity near zero and the opposite is true.

Finally we can see that if $Y<1$, it follows that $|x| k_{C G M Y}(x)$, where $k_{C G M Y}(x)$ is given by equation (3.1), has a finite integral near zero and therefore the CGMY is process is of finite variation. On the contrary if $Y>1,|x| k_{C G M Y}(x)$ has an infinite integral near zero and the process is one of infinite variation.

\subsection{Characteristic Function of the CGMY Pro- cess}

We can use the Khintchine theorem to have

$$
\begin{aligned}
& \phi_{C G M Y}(u, t)=E\left[\exp \left(i u X_{C G M Y}(t)\right)\right]= \\
& =\exp \left[t \int_{-\infty}^{+\infty}\left(e^{i u x}-1\right) k_{C G M Y}(x) d x\right]
\end{aligned}
$$

Let's focus on the integral. Remembering equation (3.1), we can write the integral as

$$
\begin{array}{r}
\int_{-\infty}^{+\infty}\left(e^{i u x}-1\right) k_{C G M Y}(x) d x= \\
=\int_{-\infty}^{0}\left(e^{i u x}-1\right) C \frac{\exp (G x)}{(-x)^{1+Y}} d x+\int_{0}^{+\infty}\left(e^{i u x}-1\right) C \frac{\exp (-M x)}{x^{1+Y}} d x
\end{array}
$$

The first integral can be rewritten as difference of two integrals

$$
\begin{array}{r}
\int_{-\infty}^{0}\left(e^{i u x}-1\right) C \frac{\exp (G x)}{(-x)^{1+Y}} d x= \\
=\int_{-\infty}^{0} C \frac{\exp (i u x+G x)}{(-x)^{1+Y}} d x-\int_{-\infty}^{0} C \frac{\exp (G x)}{(-x)^{1+Y}} d x
\end{array}
$$

If we do a change of variable $w=-(i u+G) x$ in the first integral in the right hand side of equation (3.7) and a change of variable $w=-G x$ in the second 
integral, we get

$$
\int_{0}^{+\infty}(G+i u)^{Y} w^{-1-Y} C e^{-w} d w-\int_{0}^{+\infty} C G^{Y} w^{-1-Y} e^{-w} d w
$$

that is

$$
C \Gamma(-Y)\left[(G+i u)^{Y}-G^{Y}\right]
$$

In the same way the second integral in equation(3.6) can be divide in two pieces and with changes of variables $w=x(M-i u)$ and $w=M x$ we can have

$$
\begin{array}{r}
\int_{0}^{+\infty}\left(e^{i u x}-1\right) C \frac{\exp (-M x)}{x^{1+Y}} d x= \\
=\int_{0}^{+\infty} C(M-i u)^{Y} w^{-1-Y} e^{-W} d w-\int_{0}^{+\infty} C M^{Y} w^{-1-Y} e^{-w} d w
\end{array}
$$

which can besolved as

$$
C \Gamma(-Y)\left[(M-i u)^{Y}-M^{Y}\right]
$$

Now substituting equations (3.8) and (3.9) in equation (3.5) we get

$$
\begin{array}{r}
\phi_{C G M Y}(u, t ; C, G, M Y)= \\
=\exp \left\{t C \Gamma(-Y)\left[(M-i u)^{Y}-M^{Y}+(G+i u)^{Y}-G^{Y}\right]\right\}
\end{array}
$$

which is exactly the characteristic function of CGMY that we were looking for.

\subsection{The Statistical and Risk neutral CGMY Stock Price Process}

We would like to define here the stochastic process followed by a stock assuming that the martingale component of the movement in the logarithm of prices is described by a CGMY process. We do this both in terms of statistical process and in terms of risk neutral process. We can in this way 
estimate the parameters $C, G, M$ and $Y$ from a statistical and from a risk neutral point of view. Estimation from a statistical point of view is realized by direct observation of the underlying security, while the estimation in the risk neutral case, is obtained using option price. Using these parameters we can then determine skewness and kurtosis under the statistical and risk neutral densities. Moreover we want to assess the importance of the diffusion and of the jump component in the process. We measure the relative importance of the two based on the proportion of total quadratic variation contributed by each of them. To assess this we will define a parameter $\eta$ which controls the diffusion component in an extension of the CGMY process which include also a diffusion component. Finally we can study if the stock price processes are processes of finite or infinite activity and of finite or infinite variation and if they are completely monotone. This analysis allows us to judge how much the CGMY process add to the variance gamma in terms of explanation of underlying price moves and of option prices.

\subsubsection{The Statistical CGMY Stock Price Process}

Assuming that the martingale component of the movement of the logarithm of prices is given by a CGMY process, we have

$$
S(t)=S(0) \exp \left[(\mu+\omega) t+X_{C G M Y}(t ; C, G, M, Y)\right]
$$

$\mu$ here is the mean return on the stock and $\omega$ is given by

$$
e^{-\omega t}=\phi_{C G M Y}(-i, t ; C, G, M, Y)
$$

To test the relevance of an additional diffusion component in this model, Carr, Madan, Geman and Yor ${ }^{3}$ define a model called "extended CGMY", denoted $C G M Y_{e}$ which includes an orthogonal diffusion component. They consider

$$
X_{C G M Y_{e}}(t ; C, G, M, Y, \eta)=X_{C G M Y}(t ; C, G, M, Y)+\eta W(t)
$$

\footnotetext{
${ }^{3}$ Peter Carr, Hélyette Geman, Dilip B. Madan and Marc Yor, "The Fine Structure of Asset Returns: An Empirical Investigation", The Journal of Business, Vol. 75, No. 2, 2002, page 316 .
} 
where $W(t)$ is a standard Brownian motion independent of $X_{C G M Y}(t ; C, G, M, Y)$. This extended process, gives a stock prices process

$$
S(t)=S(0) \exp \left[\left(\mu+\omega-\frac{\eta^{2}}{2}\right) t+X_{C G M Y_{e}}(t ; C, G, M, Y, \eta)\right]
$$

Particularly useful in the test of the model is the characteristic function for the logarithm of the stock price at time $t$, which is given by

$$
\begin{aligned}
\phi_{\ln (S)}(u, t)= & \exp \left\{i u\left[\ln (S(0))+\left(\mu+\omega-\frac{\eta^{2}}{2}\right) t\right]\right\} \\
& \times \phi_{C G M Y}(u ; C, G, M, Y) \exp \left(-\frac{\eta^{2} u^{2}}{2}\right)
\end{aligned}
$$

\subsubsection{The Risk neutral CGMY Stock Price Process}

In this section we want to define a risk neutral process for stock prices obtained assuming that stock follows the diffusion extended CGMY model. Note that the parameters here can be different from the one obtained for the statistical stock price process. In fact the risk neutral parameters are obtained using option prices while the statistical ones are obtained from direct observation of the underlying. We denote $\tilde{C}, \tilde{G}, \tilde{M}, \tilde{Y}$ and $\tilde{\eta}$ the risk neutral parameters to distinguish them from their statistical counterparts. The risk neutral stock price process is here given by

$$
S(t)=S(0) \exp \left[\left(r+\tilde{\omega}-\frac{\tilde{\eta}^{2}}{2}\right) t+X_{C G M Y_{e}}(t ; \tilde{C}, \tilde{G}, \tilde{M}, \tilde{Y}, \tilde{\eta})\right]
$$

where $r$, is the mean risk neutral return given by the continuously compounded interest rate and $\tilde{\omega}$ is obtained by solving

$$
e^{-\tilde{\omega} t}=\phi_{C G M Y}(-i, t ; \tilde{C}, \tilde{G}, \tilde{M}, \tilde{Y})
$$

Moreover, similarly to the statistical case, the characteristic function for the logarithm of the stock price at time $t$ is given by

$$
\begin{aligned}
\tilde{\phi}_{\ln (S)}(u, t)= & \exp \left\{i u\left[\ln (S(0))+\left(r+\tilde{\omega}-\frac{\tilde{\eta}^{2}}{2}\right) t\right]\right\} \\
& \times \phi_{C G M Y}(u ; \tilde{C}, \tilde{G}, \tilde{M}, \tilde{Y}) \exp \left(-\frac{\tilde{\eta}^{2} u^{2}}{2}\right)
\end{aligned}
$$




\subsection{Variance and Higher Moments of the CGMY Distribution}

Moments of a distribution can be obtained differentiating the characteristic function. In particular, given a Lévy density $k(x)$ and a diffusion coefficient $\eta$, it is possible to prove that for the random variable $X$ which is the level of the Lévy process at time 1 , we have

$$
\begin{array}{r}
E\left\{[X-E(X)]^{2}\right\}=\eta^{2}+\int_{-\infty}^{+\infty} x^{2} k(x) d x \\
E\left\{[X-E(X)]^{3}\right\}=\eta^{2}+\int_{-\infty}^{+\infty} x^{3} k(x) d x \\
E\left\{[X-E(X)]^{4}\right\}= \\
E\left\{[X-E(X)]^{4}\right\}=3\left\{E\left[(X-E(X))^{2}\right]\right\}^{2}
\end{array}
$$

For the extended CGMY, Carr, Geman, Madan and Yor ${ }^{4}$ show that

$$
E\left\{\left[X_{C G M Y_{e}}-E\left(X_{C G M Y_{e}}\right)\right]^{2}\right\}=\eta^{2}+C \Gamma(2-Y)\left(\frac{1}{M^{2-Y}}+\frac{1}{G^{2-Y}}\right)
$$

Moreover, using the notation $V(\cdot)$ for the variance, we have

$$
\begin{gathered}
E\left\{\left[X_{C G M Y_{e}}-E\left(X_{C G M Y_{e}}\right)\right]^{3}\right\}=\frac{C \Gamma(3-Y)\left(\frac{1}{M^{3-Y}}+\frac{1}{G^{3-Y}}\right)}{\left[V\left(X_{C G M Y_{e}}\right)\right]^{\frac{3}{2}}} \\
E\left\{\left[X_{C G M Y_{e}}-E\left(X_{C G M Y_{e}}\right)\right]^{4}\right\}=3+\frac{C \Gamma(4-Y)\left(\frac{1}{M^{4-Y}}+\frac{1}{G^{4-Y}}\right)}{\left[V\left(X_{C G M Y_{e}}\right)\right]^{2}}
\end{gathered}
$$

\footnotetext{
${ }^{4}$ See Peter Carr, Hélyette Geman, Dilip B. Madan and Marc Yor, "The Fine Structure of Asset Returns: An Empirical Investigation", The Journal of Business, Vol. 75, No. 2, 2002 , page 318 .
} 


\subsection{Decomposition of the Quadratic Varia- tion}

Let's consider the statistical stock price process. The total quadratic variation over an interval $(0, t)$ of the diffusion component in the extended CGMY model having characteristic function (3.9) is deterministic and equal to $\eta^{2} t$. For the jump diffusion, on the other side, Carr, Madan, Geman and Yor show that the total quadratic variation is random and that its expectation is given by

$$
\begin{array}{r}
t \int_{0}^{+\infty} x^{2} C \frac{\exp (-M x)}{x^{1+Y}} d x+t \int_{0}^{+\infty} x^{2} C \frac{\exp (-G x)}{x^{1+Y}} d x= \\
t C \Gamma(2-Y)\left(\frac{1}{M^{2-Y}}+\frac{1}{G^{2-Y}}\right)
\end{array}
$$

We will use this decomposition of the quadratic variation in section (3.7.2) to discuss the contribute brought to the CGMY model by the addition of a diffusion term.

\subsection{Empirical Test of the CGMY Model for European Options}

In this section we would like to describe some results presented by Carr, Geman, Madan and Yor ${ }^{5}$. The authors consider the issue from two perspectives: one based on the statistical processes meaning on the underlying assets, the other related to the risk neutral processes where option prices are used to estimate the various parameters. Their work provide estimate for both $C, G, M, Y, \eta$ and for $\tilde{C}, \tilde{G}, \tilde{M}, \tilde{Y}, \tilde{\eta}$ as defined in sections (3.4.1) and (3.4.2). As for the statistical parameters, they are estimated using time series data on 13 stocks and 8 market indexes for five years from 1994 to 1998. The risk neutral parameters are estimated on a set of days from closing prices for options and underlying, following the approach used by Bakshi, Cao and

\footnotetext{
${ }^{5}$ The authors presented a series of articles on the subject, the most complete introduction to the model is probably in Peter Carr, Hélyette Geman, Dilip B. Madan and Marc Yor, "The Fine Structure of Asset Returns: An Empirical Investigation", The Journal of Business, Vol. 75, No. 2, 2002, pages 305-332.
} 
Chen ${ }^{6}$. In particular they used short dated (between one and two months) options on 4 stocks and one index for five mid month Wednesdays. To adjust for the fact that the options on the market are American, Carr, Madan, Geman and Yor first determine an implied volatility for the American options using a finite difference scheme and then use this volatility to determine the European option price.

To estimate the statistical price processes, the authors consider for each asset, a daily time series of log price relatives and then use the mean-adjusted return to compute the parameters. The fast Fourier transform is used to invert the characteristic function once for each of the parameters and the probability density level is obtained at a specified set of values for returns. With the density evaluated at these determined points, it is possible to bin the return series by counting the number of observations at each specified return point. Parameters are hence obtained by maximum likelihood estimation of these binned data.

Risk neutral estimates of the parameters are obtained using non linear least squares minimization of pricing errors from out of the money option prices. Model option prices are computed using Carr and Madan approach and inverting the fast Fourier transform in log strike of the call prices reduced by an exponential factor ${ }^{7}$.

\subsubsection{Skewness and Kurtosis Results}

From the statistical point of view, results on the skewness are mixed. Out of 20 estimation, skewness is negatively significant in five cases. Using equation (3.11), Carr, Geman, Madan adn Yor find a negative skew for three processes and a positive one for ten cases, the remaining seven being the skewness equal to zero. As for the statistical kurtosis, it is generally above 3 .

The risk neutral process, on the contrary, is definitely skewed in every case, but with a level of skewness which changes over time. As for the kurtosis implied by option prices, it is materially higher than the corresponding

\footnotetext{
${ }^{6}$ Gurdip Bakshi, Charles Cao and Zhiwu Chen, "Empirical Performance of Alternative Option Pricing Models", The Journal of Finance, Vol. 52, No. 5, December 1997, pages 2003-2049.

${ }^{7}$ Peter Carr and Dilip B. Madan, "Option Valuation Using the Fast Fourier Transform", Journal of Computational Finance, 2, 1998, pages 61-73.
} 
statistical one.

\subsubsection{Diffusion Component Results}

The importance of a diffusion element introduced in the extended CGMY model is considered here, by using the decomposition of quadratic variation presented in section (3.6). For each and every index considered, the diffusion component is absent; while for stocks it is positive but insignificant in seven cases and absent in the remaining five cases.

An economic interpretation of this result could be that the diffusion component risk is a diversifiable one, while the systematic risk is represented by the pure jump process which is reflected in the indexes. This idea suggests that the diffusion component should have a small importance in the risk neutral process since it can be diversified away. To test this hypothesis, the authors consider a sub sample of the data used for the rest of the risk neutral analysis. For each stock, out of five available days, they pick the best three in terms of fit of average pricing error and they compute the proportion of the total quadratic variation which is attributable to the diffusion term. The result is that the proportion of total quadratic variation of the risk neutral process explained by the diffusion component is zero for all the considered case.

\subsubsection{Results on the Fine Structure of Returns}

From the statistical point of view, for only three stocks the jump component process is one of finite activity. However in none of these three cases it is possible to reject the null hypothesis of a variance gamma distribution. For all the other cases, the statistical processes are of infinite activity. As for the statistical finite or infinite variation, only three cases are infinite variation process, all the others being finite variation.

From the risk neutral point of view, essentially all the processes are of infinite activity and of finite variation.

Finally all the cases, both statistically and risk neutrally are consistent with a Lévy density characterized by complete monotonicity. 


\subsection{Stochastic Volatility for Variance Gamma and CGMY Processes}

\subsubsection{Pricing of Options with Different Maturities and of Option Time Series}

Variance Gamma and CGMY allow to model skewness and kurtosis of the distribution of logarithm of stocks and the option pricing models based on them allow to explain the volatility smile. They therefore represent a big improvement from the empirical point of view with respect to several other models starting from the Black and Scholes one ${ }^{8}$. One problem which however remains unsolved is the fit of option prices across maturities. Models like variance gamma and CGMY generally provide a good fit if calibrated based on a specific maturity, but calibrations obtained from short maturity options do not work fine for long maturity options and vice versa. Note on this point that Carr, Madan, Geman and Yor test the empirical performance of the CGMY model based on short dated (one to two months) options and the results presented in section (3.7) are all based on this short maturity. Konikov and Madan ${ }^{9}$ argue that the reason of these poor empirical performances across maturity is related with the fact that these homogeneous Lévy processes impose strict condition on the term structure of the risk neutral variance, skewness and kurtosis. In fact these models requires that the variance is constant over the term structure, the skewness is inversely proportional to the square root of the term and kurtosis is inversely proportional to the term. However real data show that variance, skewness and kurtosis often increase as the time to maturity increase. This increase makes sense from an economically intuitive point of view because as the term increases the uncertainty of the investor increases and so the return distribution should spread out as the time to maturity increase. On the other side, risk premia generally display mean reversion and this impacts the skewness and kurtosis of the risk neutral process. It is therefore desirable to incorporate a richer behavior of these parameters across maturities than the one implied by homogeneous Lévy processes.

\footnotetext{
${ }^{8}$ Fisher Black and Myron Scholes, "The Pricing of Options and Corporate Liabilities", Journal of Political Economy, Vol. 81, No. 3, May/June 1973, pages 637-654.

${ }^{9}$ Mikhail Konikov and Dilip B. Madan, "Stochastic Volatility via Markov Chain", working paper, Robert H. Smith School of Business, University of Mariland, 2001.
} 
Moreover several authors including Clark $^{10}$, Engle ${ }^{11}$, Nelson ${ }^{12}$, Ander$\operatorname{sen}^{13}$, Bates ${ }^{14}$ and Duan ${ }^{15}$ observed that volatility estimates from the time series are usually clustered. This property is known as volatility persistence and it is inconsistent with homogeneous Lévy processes not allowing therefore a consistent option pricing across maturities.

The problem of valuing options which models which are consistent across maturities becomes of particular interest when considering instruments with significantly longer maturities than the options generally traded on the market. Assets like convertibles have often fairly long maturities. Portfolio managers often have an average life of convertible in the 3-5 year range, even considering corrections for the fact that most of the bonds are callable and puttable. This portfolios of long dated convertibles are often hedged with instruments which have a sufficient liquidity in the market, for example options on the Standard \& Poor's 500. The liquid options on this asset do not generally have a maturity much longer than six months. The manager therefore finds herself in the position of making decisions in terms of the terms structure of volatility which allows her to price both the convertible long maturity portfolio and the short maturity hedge in a meaningful way. Given the absence of liquid long dated options trading on exchanges, the financial literature has focus its attention on option pricing models effective for short term options. It is clear that a single model which allows to price instruments with both long and short maturities in a way consistent with the market prices, without having to be recalibrated for each maturity (or set of similar maturities), would be of practical interest.

Furthermore variance gamma cannot properly address the pricing of op-

\footnotetext{
${ }^{10}$ P. K. Clark, "A Subordinated Stochastic Process Model with Finite Variance for Speculative Prices", Econometrica, Vol. 41, 1973, pages 135-156.

${ }^{11}$ R. F. Engle, "Autoregressive Conditional Heteroskedasticity with Estimates of the Variance of United Kingdom Inflation", Econometrica, Vol. 61, 1982, pages 929-952.

${ }^{12}$ D. Nelson, "Conditional Heteroskedasticity in Asset Returns: A New Approach", Econometrica, Vol. 59, 1991, pages 347-370.

${ }^{13}$ Torben G. Andersen, "Stochastic Autoregressive Volatility: A Framework for Volatility Modelling", Mathematical Finance, Vol. 4, 1994, pages 75-102.

${ }^{14}$ David S. Bates, "The Crash of '87: Was It Expected? The Evidence from Options Markets", The Journal of Finance, Vol. 46, No. 3, July 1991, pages 1009-1044 and David S. Bates, "Jumps and Stochastic Volatility : Exchange Rate Processes Implicit in Deutsche Mark Options", The Review of Financial Studies, Vol. 9, No. 1, 1996, pages 69-107.

${ }^{15}$ Jin-Chuan Duan, "The GARCH Option Pricing Model", Mathematical Finance, Vol. 5, No. 1, January 1995, pages 13-32.
} 
tion time series. The problem concerns the status of the estimated parameters in the model across time. If they are constant then the model can be used for out of sample forecast of option prices consequent a movement of calendar time. The issue is that parameters estimated at a point of time from data on market prices synthesize important dimensions of information content in option prices, like the risk premium charged for a large move relative to a small move regardless of the direction and the premium charged for a down move relative to a comparable up move. These market premia may vary across time with the size and level of market interest in insuring against various moves. As a consequence, we are going to see a time variation in the parameters which makes the out sample use of the model problematic.

An approach which can be used to deal with these problems consists in employing a stochastic volatility process, as it is going to be described in the remaining part of this chapter. An alternative method is the one suggested by Konikov and Madan ${ }^{16}$ : they employ a Markov chain that jumps at infinitesimal time intervals between two homogeneous parametric specifications that calibrate to different levels of volatility, skewness and kurtosis. In particular they use a variance gamma process as homogeneous process creating a two state variance gamma Markov model. Konikov and Madan claims that the Markov chain methodology is an attractive and tractable way to incorporate the phenomenon of volatility clustering. Albanese, Jaimungal and Rubisov ${ }^{17}$ suggest an approach similar to the one proposed by Konikov and Madan, the difference is that the parameter switching occurs at finite time intervals, which in applications have a typical duration of about 3-5 weeks. In this setting, it is possible to obtain a closed form pricing formula for European options. However the resulting expression would have a complex combinatorial structure whose numerical valuation would not practically viable. To overcome this problem, the authors introduce a resummation scheme that reduces the algorithmic complexity by exploiting systematic sign cancellations between terms in the combinatorial expression and reduce the computational complexity. The algorithm is based on the model of lines introduced to streamline the pricing problem for the ordinary variance gamma model ${ }^{18}$.

\footnotetext{
${ }^{16}$ Mikhail Konikov and Dilip B. Madan, "Option Pricing Using Variance Gamma Markov Chains", Review of Derivatives Research, Vol. 5, 2002, pages 81-115.

${ }^{17}$ Claudio Albanese, Sebastian Jaimungal and Dimitri H. Rubisov, "A Two-State Jump Model", Quantitative Finance, Vol. 3, 2003, pages 145-154.

${ }^{18}$ Claudio Albanese, Sebastian Jaimungal and Dimitri H. Rubisov, "Jumping in Line", Risk, February 2001, pages 65-68.
} 
The model contains seven parameters that allow the adjustment of several moments of the return distributions.

\subsubsection{Stochastic Volatility Models as Time Changed Processes}

Carr, Madan, Geman and Yor ${ }^{19}$ present extensions of the variance gamma model and of the CGMY models which incorporate stochastic and mean reverting volatilities. The intuition behind these stochastic volatility models is related to the scaling property of Brownian motion, which says that changes in time are related to changes in scale; so it is possible to randomly change the volatility by randomly change the time. The rate of time change has to be mean reverting to allow the random time changes to persist, moreover the instantaneous rate of time change should be positive otherwise the time would be decreasing. The author in particular generate these new processes which have the desired volatility properties by subordinating the variance gamma and the CGMY to the time integral of a Cox, Ingersoll and Ross ${ }^{20}$ square root process. Define the instantaneous rate of time change, $y(t)$, as the solution of the differential equation

$$
d y=k(\eta-y) d t+\lambda \sqrt{y} d W
$$

where $W(t)$ is a standard Brownian motion independent of all the other processes encountered so far; $\eta$ is the long run rate of time change; $k$ is the rate of mean reversion and $\lambda$ control the volatility of the time change. The randomness of the process induces stochastic volatility, while the mean reversion in this process creates the volatility clustering desired. We can see that here changes in volatility are independent of asset returns. This is not consistent with empirical evidence showing a correlation, which is often negative in the case of stocks, between returns and their volatilities. Carr and $\mathrm{Wu}^{21}$ extend this approach by allowing that changes in volatility are

\footnotetext{
${ }^{19}$ Peter Carr, Hélyette Geman, Dilip. B. Madan, Marc Yor, "Stochastic Volatility for Lévy Processes", Mathematical Finance, Vol. 13, No. 3, July 2003, pages 345-382.

${ }^{20}$ J. Cox, J Ingersoll and S. Ross, "A Theory of the Term Structure of Interest Rates", Econometrica, 41, 1985, pages 135-156.

${ }^{21}$ Peter Carr and Liuren Wu, "Time-Changed Lévy Processes and Option Pricing", forthcoming in Journal of Financial Economics.
} 
independent of asset returns. In particular Carr and Wu work with Lévy processes in general, and employ a measure change in the complex plane to obtain the characteristic function of the time changed Lévy process and then they use fast Fourier transform to price contingent claims.

Going back to the the approach presented by Carr, Geman, Madan and Yor, using the process $y(t)$, from equation (3.12), the new clock is given by

$$
Y(t)=\int_{0}^{t} y(u) d u
$$

Cox, Ingersoll and Ross show that the characteristic function of $Y(t)$ can be written as

$$
E[\exp (i u Y(t))]=\phi(u, t, y(0) ; k, n, \lambda)=A(t, u) \exp (B(t, u) y(0))
$$

where we have used the following notation:

$$
\begin{array}{r}
A(t, u)=\frac{\exp \left(\frac{k^{2} \eta t}{\lambda^{2}}\right)}{\left(\cosh \left(\frac{\gamma t}{2}\right)+\frac{k}{\gamma} \sinh \left(\frac{\gamma t}{2}\right)\right)^{\frac{2 k \eta}{\lambda^{2}}}} \\
B(t, u)=\frac{2 i u}{k+\gamma \operatorname{coth}\left(\frac{\gamma t}{2}\right)} \\
\gamma=\sqrt{k^{2}-2 \lambda^{2} i u}
\end{array}
$$

\subsubsection{Stochastic Volatility Variance Gamma and CGMY}

To obtain the extensions of varance gamma and CGMY having stochastic volatility, we take the process and we subordinate them to the process $Y(t)$ through a time change. Carr, Geman, Madan and Yor defines the new processes as VGSV (Variance Gamma Stochastic Volatility) and CGMYSV (CGMY Stochastic Volatility). It is possible to obtain characteristic functions of these processes. In particular if $X(t)$ is a Lévy process, it has a characteristic function in the form of

$$
E[\exp (i u X(t))]=\exp \left(t \psi_{X}(u)\right)
$$


Assume that $X(t)$ is a process of finite variation, like the variance gamma process, and assume that the Lévy density exists and is denoted by $k(x)$, then log characteristic function at unit time $\psi_{X}(u)$ id given by

$$
\psi_{X}(u)=\int_{-\infty}^{+\infty}\left(e^{i u x}-1\right) k(x) d x
$$

Now we can define the class of stochastic volatility Lévy processes as

$$
Z(t)=X(Y(t))
$$

where $Y(\cdot)$ is independent of $Z(\cdot)$. $Z(\cdot)$ can be obtained by the Bochner's procedure of subordinating $X(\cdot)$ to $Y(\cdot)$. The characteristic function for these processes can be obtained as

$$
E[\exp (i u Z(t))]=E\left[\exp \left(Y(t) \psi_{X}(u)\right)\right]=\phi\left(-i \psi_{X}(u), t, y(0) ; k, n, \lambda\right)
$$

Carr, Madan, Geman and Yor provide also the specific characteristic function of the VGSV and CGMYSV processes. Following their results, in the case of the variance gamma we have,

$$
Z_{V G}(t)=X_{V G}(Y(t) ; \sigma, \nu, \vartheta)
$$

and the characteristic function is given by

$$
E\left[\exp \left(i u Z_{V G}(t)\right)\right]=\phi\left(-i \psi_{V G}(u ; 1, G, M), t, C ; k, \eta, \lambda\right)
$$

where the parameters $C, G$ and $M$ are the one given by equations (3.2),(3.3) and (3.4) which allow to see the variance gamma as a parametric case of CGMY. The stochastic volatility version of the CGMY process, the CGMYSV is given by

$$
Z_{C G M Y}(t)=X_{C G M Y}\left(Y(t) ; C_{p}, G, M, Y_{p}, Y_{n}, \zeta\right)
$$

where the notation $C_{p}, Y_{n}$ and $Y_{n}$ refers to the fact that the parameters $C$ and $Y$ can have different values for $x>0$ and for $x<0$ in the CGMY Lévy density (3.1), $p$ being the case $x>0$. The characteristic function for the CGMYSV process is given by

$$
E\left[\exp \left(i u Z_{C G M Y}(t)\right)\right]=\phi\left(-i \psi_{C G M Y}\left(1, G, M, Y_{p}, Y_{n}, \zeta\right), t, C ; k, \eta, \lambda\right)
$$




\subsubsection{Stock Price Processes for Stochastic Volatility Variance Gamma and CGMY}

From the stochastic volatility processes we now want to construct the appropriate stock price processes. Carr, Geman, Madan and Yor use two different approaches which differs in terms of the filtration in which the martingale condition is based on. The first approach prohibits arbitrage based only on the stock price, by assuming that investors can only condition trades on the level of the stock price. The second approach instead assumes that trades can be conditioned also on the level of the Lévy process and the time as defined after the time change. This second approach therefore is arbitrage free not just from the point of view of stock based arbitrage, but also from that one based on the level of the Lévy process and the new clock. This second approach though more appealing theoretically has the drawback that the variables which determine the stock price process are less easily observable than the stock price itself.

To implement the first approach, Carr, Madan, Geman and Yor determine the risk neutral distribution for the stock price at each future time as a the exponential of the VGSV process and of the GCMYSV process, normalized to reflect the initial term structure of forward prices. Note that here the exponential is just the ordinary exponential and not a stochastic exponential as it will be in the second approach. By doing this the model is spot-forward arbitrage free; furthermore arbitrage of calendar spreads of option is also not allowed since it is based only on the stock price. Variance gamma and CGMY models modifications realized with this approach are defined VGSA and CGMYSA, where the letters "SA" remembers that they are free only from stock price based arbitrage. To write the formal expressions of the characteristic functions, let's define $S(t)$ the stock price at time $t, r$ the constant continuously compounded interest rate and $q$ the constant continuously compounded dividend yield. Consider the class of stochastic volatility Lévy processes, $Z(t)$ from equation (3.13), then we have and we can define the stock price at time $t$ as

$$
S(t)=S(0) \frac{\exp [(r-q) t+Z(t)}{E[\exp (Z(t))]}
$$

Noting that

$$
E[\exp (Z(t))]=\phi\left(-i \psi_{X}(-i), t, y(0) ; k, \eta, \lambda\right)
$$


Carr, Madan, Geman and Yor show that the characteristic function for the logarithm of the stock price at time $t$ for the generic stochastic volatility Lévy process is given by

$$
\begin{aligned}
& E[\exp (i u \ln (S(t)))]= \\
& =\exp \{i u[\ln (S(0))+(r-q) t]\} \times \frac{\phi\left(-i \psi_{X}(u), t, y(0) ; k, \eta, \lambda\right)}{\phi\left(-i \psi_{X}(-i), t, y(0) ; k, \eta, \lambda\right)^{i u}}
\end{aligned}
$$

In the particular case of the VGSA model, the characteristic function for the logarithm of the stock price at time $t$ is given by

$$
\exp \{i u[\ln (S(0))+(r-q) t]\} \times \frac{\phi\left(-i \psi_{V G}(u ; 1, G, M), t, C ; k, \eta, \lambda\right)}{\phi\left(-i \psi_{V G}(-i ; 1, G, M), t, C ; k, \eta, \lambda\right)^{i u}}
$$

while for the CGMYSA model, the characteristic function for the logarithm of the stock price at time $t$ can be written as

$$
\begin{array}{r}
\exp \{i u[\ln (S(0))+(r-q) t]\} \\
\times \frac{\phi\left(-i \psi_{C G M Y}\left(u ; 1, G, M, Y_{p}, Y_{n}, \zeta\right), t, C ; k, \eta, \lambda\right)}{\phi\left(-i \psi_{C G M Y}\left(-i ; 1, G, M, Y_{p}, Y_{n}, \zeta\right), t, C ; k, \eta, \lambda\right)^{i u}}
\end{array}
$$

The second approach is realized by compensating the pure jump processes VGSV and CGMYSV to form martingales. These martingales are then exponentiated to yield martingale candidates for forward prices. The authors name VGSAM and CGMYSAM the models created with this approach, where the letter "M" stays for martingale. Without entering in the detail of the derivation of the results ${ }^{22}$, we can say that for generic stochastic volatility Lévy process, the stock price can be define by

$$
S(t)=S(0) \exp [(r-q) t] \exp \left\{X[Y(t)]-Y(t) \psi_{X}(-i)\right\}
$$

where it can proved that

$$
\exp \left\{X[Y(t)]-Y(t) \psi_{X}(-i)\right\}
$$

\footnotetext{
${ }^{22}$ For more details see Peter Carr, Hélyette Geman, Dilip B. Madan and Marc Yor, "Stochastic Volatility for Lévy Processes", Mathematical Finance, Vol. 13, No. 3, July 2003, page 359-360.
} 
is a martingale. In this case the characteristic function for the logarithm of the stock price at time $t$ is given by

$$
\begin{array}{r}
E[\exp (i u \ln (S(t)))]= \\
=\exp \{i u[\ln (S(0))+(r-q) t]\} \times \phi\left(-i \psi_{X}(u)-u \psi_{X}(-i), t, y(0) ; k, \eta, \lambda\right)
\end{array}
$$

Moreover for the VGSAM, the characteristic function for the logarithm of the stock price at time $t$ is given by

$$
\begin{array}{r}
\exp \{i u[\ln (S(0))+(r-q) t]\} \\
\times \phi\left[-i \psi_{V G}(u, 1, G, M)-u \psi_{V G}(-i, 1, G, M), t, C ; k, \eta, \lambda\right]
\end{array}
$$

while for the CGMYSAM the characteristic function for the logarithm of the stock price at time $t$ is

$$
\begin{array}{r}
\exp \{i u[\ln (S(0))+(r-q) t]\} \times \phi\left[-i \psi_{C G M Y}\left(u, 1, G, M, Y_{p}, Y_{n}, \zeta\right)+\right. \\
\left.-u \psi_{C G M Y}\left(-i, 1, G, M, Y_{p}, Y_{n}, \zeta\right), t, C ; k, \eta, \lambda\right]
\end{array}
$$

The stochastic processes obtained using this second approach are appealing from a theoretical but not from practical point of view because they are based on information which is not available in the market. VGSAM and CGMYSAM models are in fact martingales with respect to the enlarged filtration, which includes information from the driving Lévy process and knowledge about the subordinator given by the time integrated Cox, Ingersoll and Ross process. If these two processes cannot be separately ascertained from a time series of prices, then serious problems in terms of relevance of the associated martingale condition. Geman, Madan and Yor ${ }^{23}$ provides conditions under which the two processes can be determined from a time series of underlying prices. However even if the two processes can be determined from a time series, most likely the rich dynamics of the option price cannot be adequately captured by a martingale which reflects movements only in two processes. Hence this martingale condition based on a filtration which cannot be observed is not really interesting from a practical perspective.

The attention is therefore focused on the models obtained with the first approach: VGSA and CGMYSA, which provide a better empirical performance than the martingale ones. VGSA and CGMYSA require only the ability to observe stock prices and so they generate risk neutral price processes

\footnotetext{
${ }^{23}$ Hélyette Geman, Dilip B. Madan and Marc Yor, "Stochastic Volatility, Jumps and Hidden Time Changes", forthcoming in Finance and Stochastic, 2001.
} 
whose expectation is consistent with the initial term structure of forward prices, but they do not require that the forward prices are martingales with respect to the filtration generated by the Lévy process and the subordinator.

\subsubsection{Empirical Tests of Stochastic Volatility Variance Gamma and CGMY}

The authors test VGSA and CGMYSA. The test include also the martingale corresponding processes, CGSAM and GCGMYSAM and stochastic volatility models based on the normal inverse Gaussian process ${ }^{24}$, which we are not going to analyze here. The test is realized on closing prices of out of the money options on S\&P 500 and on 20 stocks for maturities between a month and a year for each second Wednesday of the month for the year 2000. Given that it is possible to obtain characteristic functions of these processes we can price European options under these models using the fast Fourier transform method proposed by Carr and Madan ${ }^{25}$.

Parameters in the model are computed by minimizing the root mean square error between the market option prices and the model option prices. The root mean square error is taken over all strikes and maturities. Average absolute error as a percentage of the mean price is also computed as a measure of overall quality of the fit. First of all, the authors show that the models based on the first approach (VGSA and CGMYSA) always outperform VGSAM and CGMYSAM, which we can therefore ignore.

Both VGSA and CGMYSA risk neutral parameters obtained using the S\&P 500 options and the single stock options are consistent with a marked negative skew which confirms results from the CGMY risk neutral test in section (3.7.1). In the case of test based on S\&P 500 data, VGSA and CGMYSA show a comparable rate of mean reversion in volatility. The absolute pricing errors are larger for shorter maturities and for out of the money options. This is confirmed by a regression of the absolute errors on the moneyness, the square of moneyness and maturity. Carr, Geman, Madan and Yor $^{26}$ show that all the three coefficient in this regression are statistically

\footnotetext{
${ }^{24}$ The normal inverse Gaussian process is obtained by subordinating an arithmetic Brownian motion to an inverse Gaussian process.

${ }^{25}$ Peter Carr and Dilip B. Madan, "Option Valuation using Fast Fourier Transform", Journal of Computational Finance, 2, 1998, pages 61-73.

${ }^{26}$ Peter Carr, Hélyette Geman, Dilip B. Madan and Marc Yor, "Stochastic Volatility for
} 
significant. However the reason of larger pricing error for shorter maturity options and for put of the money options could be simply related to the fact that the authors try to minimize the absolute error rather then the relative error. In the case of S\&P 500, the best performing model appears to be the CGMYSA, which has the lowest pricing error and whose parameters are more stable over time, however when we go to the single stocks, VGSA (and the same is true also the stochastic volatility normal inverse Gaussian process) performance well. The authors think that this may be a consequence of the fact that fewer options are available for calibration, with the result that the lower dimensional models are sufficient to capture their variation.

\subsection{Conclusions}

The CGMY model is definitely an attractive one, for its flexibility in modelling processes of both finite and infinite activity and of both finite and infinite variation. Moreover it allows to work with Lévy density not completely monotone. Clearly, being the variance gamma obtainable as a particular parametric case of the CGMY, from a theoretical point of view the CGMY is more desirable. However as shown in section (3.7) and more in particular in section (3.7.3), the additional properties that the CGMY exhibits do not seem to make noticeable improvement over the variance gamma, at least in the examples analyzed in the current literature. Price processes seem to be completely monotone and also from an intuitive point of view, it is more interesting to think about a stochastic process which exhibits large jumps less often than small jumps. Hence the additional CGMY feature represented by non monotonicity does not appear of much practical use. As for finite and infinite variation and finite and infinite activity, Carr, Geman, Madan and Yor observe that the large majority of the statistical processes are consistent with finite variation and infinite activity. Moreover where the processes are of finite activity, the variance gamma process null hypothesis cannot be rejected. Furthermore, substantially all the risk neutral processes are consistent with the variance gamma model. This is reasonable because the pricing process focuses on large moves while small moves do not get too much attention, while the infinite variation comes from a high degree of activity near zero which does not seem to be justified. For all these reasons, it appears

Lévy Processes", Mathematical Finance, Vol. 13, No. 3, July 2003, table 9.1, page 372. 
that the variance gamma model allows to obtain results comparable to the CGMY in terms of quality of modelling of the price processes at a lower cost represented by an easier model having one less variable. It would be interesting to investigate which stocks may benefit from the additional properties of the CGMY.

On the other side the stochastic volatility extension for both the variance gamma and the CGMY model, in the form of VGSA and CGMYSA, seems very promising. Of particular appeal from both a theoretical and a practical point of view is the fact that the models fit well options of different maturities and of different strikes. This result is generally not reached by option pricing model as reflected by the practice of comparing the models partitioning the data by term and moneyness in order to get adequate pricing quality ${ }^{27}$. The fact that the Variance Gamma can be extended to include a stochastic volatility is very positive, because it shows the quality of the model and opens perspective of future research. Moreover the fact that the stochastic volatility is obtained as a time changed process with the same approach used to define variance gamma as a time changed Brownian motion, shows that the subordination technique can be applied in a consistent and successful way to obtained the desired results. However we believe that a good strategy to treat these models is to investigate deeply the variance gamma first before working on further extensions, because there are still areas of interest inside the variance gamma model which can be better understood and whose study may facilitate the analysis of any further extension. For these reasons, we decide to concentrate our research effort on the variance gamma process in this work, leaving the stochastic volatility extensions for future research.

\footnotetext{
${ }^{27}$ This is for example the approach followed by Gurdip Bakshi, Charles Cao and Zhiwu Chen, "Empirical Performance of Alternative Option Pricing Models", The Journal of Finance, Vol. 52, No. 5, December 1997, pages 2003-2049.
} 


\section{Chapter 4}

\section{Numerical Solution for the European and American Plain Vanilla Option Price Under the Variance Gamma Process}

\subsection{Introduction}

As we have seen, an analytical solution is known for the pricing of European options, when the underlying follows a variance gamma process. However in the case of American options, we cannot solve the problem in an analytical way and we have therefore to use numerical methods. Numerical solution of the option price under variance gamma via Monte Carlo has been presented by Ribeiro and Webber ${ }^{1}$. They use a gamma bridge in conjunction with a stratified sampling to price both vanilla and some exotic options, including barrier options. Këllezi and Webber ${ }^{2}$ present a lattice method to solve option pricing under a Lévy process and have variance gamma as an example. In particular, they relate the transition density function of a Lévy process to its representation as time changed Brownian motion and to its time-copula,

\footnotetext{
${ }^{1}$ Nick Webber and Claudia Ribeiro, "Valuing Path-Dependent Options in the VarianceGamma Model by Monte Carlo with a Gamma Bridge", Journal of Computational Finance, Vol. 7, No. 2, Winter 2003/2004, pages 81-100.

${ }^{2}$ Evis Këllezi and Nick Webber, "Numerical Methods for Lévy Processes: Lattice Methods and the Density, the Subordinator and the Time Copula", working paper, 2003.
} 
leading to an alternative derivation of the lattice.A finite difference method which can be applied to solve only the case of European options when the underlying asset is driven by a Lévy process, and hence also by a variance gamma process as a particular case, has also been recently presented by Cont and Voltchkova ${ }^{3}$.

The scheme presented here is instead a slight modification of the numerical solution of the differential equation in terms of finite difference scheme as presented by Hirsa and Madan ${ }^{4}$ for American vanilla options.

Part of the developments presented in this chapter has been the subject of a project for the Master in Mathematics of Finance at Columbia University in New York in the Spring 2001.

\subsection{Plain Vanilla European Options}

\subsubsection{The Variance Gamma PIDE for European Vanilla Options}

We showed in section 2.8 that the dynamic of the stock price described by a variance gamma process is given by

$$
S(t)=S(0) \exp [r t+X(t ; \sigma, \nu, \vartheta)+\omega t]
$$

where $X(t ; \sigma, \nu, \vartheta)$ is a variance gamma process and

$$
\omega=\frac{1}{\nu} \ln \left(1-\vartheta \nu-\frac{\sigma^{2} \nu}{2}\right)
$$

and where we dropped the subscripts " $R N$ " because it is clear we are referring to the risk neutral process. In that section we were not considering the presence of dividends. We can easily extend this expression to the case of a stock paying a dividend with a dividend yield of $q$ by writing

$$
S(t)=S(0) \exp [(r-q+\omega) t+X(t ; \sigma, \nu, \vartheta)]
$$

\footnotetext{
${ }^{3}$ Rama Cont and Ekaterina Voltchkova, "A Finite Difference Scheme for Option Pricing in Jump Diffusion and Exponential Lévy Models", working paper, 2003.

${ }^{4}$ Ali Hirsa and Dilip B. Madan, "Pricing American Options Under Variance Gamma", Journal of Computational Finance, Vol. 7, No. 2, Winter 2003/2004, pages 63-80.
} 
Let's define the value of an generical option, either put or call, with $V(\cdot)$. In the case of an option priced under a variance gamma model, the value of the option depends on the usual parameters which determine the value of an option when the underlying follows a geometric Brownian motion: underlying price, strike price, time to maturity, volatility, interest rate, dividend rate. Moreover the option value depends on the two additional parameters skewness and kurtosis when the returns of the underlying follow a variance gamma process, hence we can write the option value as $V(S, K, t, \sigma, r, q, \vartheta, \nu)$. In solving the differential equation which describes the option price dynamic, however, all the parameters but underlying price and time will be kept fix and in general we will not explicit all the parameters to avoid redundancy in the notation. We will use instead the notation $V(S, t)$ or sometimes simply the notation $V$.

It can be proved ${ }^{5}$ that the dynamic of the option price when the underlying returns are described by a variance gamma process is given by

$$
\begin{array}{r}
\frac{\partial V(S, t)}{\partial t}+(r-q) S \frac{\partial V(S, t)}{\partial S}+ \\
+\int_{-\infty}^{+\infty}\left[V\left(S \cdot e^{y}, t\right)-V(S, t)-\frac{\partial V(S, t)}{\partial S} S\left(e^{y}-1\right)\right] k(y) d y=r V(S, t)
\end{array}
$$

$k(y) d y$ is the Lévy measure for the variance gamma process as we described in section 2.6 and in particular we will refer here to the representation of the Lévy measure as difference of two gamma processes, that is

$$
k_{X}(y) d y= \begin{cases}\frac{\mu_{n}^{2} \exp \left(-\frac{\mu_{n}}{\nu_{n}}|y|\right)}{\nu_{n}|y|} d y & \text { for } y<0 \\ \frac{\mu_{p}^{2} \exp \left(-\frac{\mu_{p}}{\nu_{p}} y\right)}{\nu_{p} y} d y & \text { for } y>0\end{cases}
$$

Let's focus the attention on the term

$$
\int_{-\infty}^{+\infty}\left(e^{y}-1\right) k(y) d y
$$

in equation (4.1). From the definition of Lévy measure, we have

$$
k(y)=\lim _{t \rightarrow 0} \frac{P\left(y_{t} \in d y \mid y_{0}=0\right)}{t}
$$

\footnotetext{
${ }^{5}$ Ali Hirsa and Dilip B. Madan, "Pricing American Options Under Variance Gamma", Journal of Computational Finance, Vol. 7, No. 2, Winter 2003/2004, pages 63-80.
} 
and therefore

$$
\int_{-\infty}^{+\infty}\left(e^{y}-1\right) k(y) d y=\frac{1}{t} \int_{-\infty}^{+\infty}\left(e^{y}-1\right) P\left(y_{t} \in d y \mid y_{0}=0\right) d y
$$

moreover we saw in section 2.8 that

$$
\int_{-\infty}^{+\infty} e^{y} \cdot P\left(y_{t} \in d y \mid y_{0}=0\right) d y=E\left(e^{X_{t}}\right)=e^{-\omega t}
$$

and of course

$$
\int_{-\infty}^{+\infty} P\left(y_{t} \in d y \mid y_{0}=0\right) d y=1
$$

Hence, substituting these results in equation 4.2 , we get

$$
\int_{-\infty}^{+\infty}\left(e^{y}-1\right) k(y) d y=\frac{e^{-\omega t}-1}{t}
$$

and taking the limit as $\mathrm{t}$ approaches zero we have

$$
\int_{-\infty}^{+\infty}\left(e^{y}-1\right) k(y) d y=-\omega
$$

We can now substitute equation (4.3) in equation (4.1) which therefore becomes

$$
\begin{array}{r}
\frac{\partial V(S, t)}{\partial t}+(r-q+\omega) S \frac{\partial V(S, t)}{\partial S}+ \\
+\int_{-\infty}^{+\infty}\left[V\left(S \cdot e^{y}, t\right)-V(S, t)\right] k(y) d y=r V(S, t)
\end{array}
$$

This PIDE, where the letter "I" refers to the presence of an integral in the partial differential equation, describe the movement in an option price under the variance gamma. It is interesting to compare this equation with the equivalent one which characterizes the dynamics of option prices if the underlying follows a geometric Brownian motion, the so famous Black and Scholes PDE

$$
\frac{\partial V(S, t)}{\partial t}+(r-q) S \frac{\partial V(S, t)}{\partial S}+\frac{\sigma^{2} S^{2}}{2} \cdot \frac{\partial^{2} V(S, t)}{\partial S^{2}}=r V(S, t)
$$


We can see that the two equations are someway similar, however the Black and Scholes PDE does not have the $\omega$ term and the diffusion term of the geometric Brownian motion is replaced with the integral expression.

This integral is really the key to understand the dynamics of the options under a variance gamma process. The size of the jump in the stock price is expressed in terms $e^{y}$ and it is reflected in the difference of the option after and before the jump itself. The integral taken on the real axis allows to consider all the possible jump sizes $y$ and the Lévy density defines the probability of the different ranges of jump size.

The PIDE (4.4) clearly holds for both calls and put, the final and boundary conditions defining the difference in the two cases. In the case of a call options the final condition is

$$
V(S, T)=\max (S-K, 0)
$$

where $T$ is the maturity of the option. Moreover we have the boundary conditions for call options

$$
\begin{array}{rr}
V(0, t)=0 & \forall t \\
V(+\infty, t)=S & \forall t
\end{array}
$$

while for put options the final condition is

$$
V(S, T)=\max (K-S, 0)
$$

and we have the boundary conditions

$$
\begin{array}{cc}
V(0, t)=K & \forall t \\
V(+\infty, t)=0 & \forall t
\end{array}
$$

\subsubsection{Numerical Solution for Vanilla European Options}

We want to solve the PIDE (4.4) using a finite difference numerical method. In order to properly implement a working solution, it is necessary to properly discretize the continuous-time partial integro differential equation and write it down as a linear system. We then had to enforce boundary conditions and finally solve the system iteratively. The solution is realized using an implicit scheme. The key to solve numerically the PIDE is in the way the linear system has to written and in particular in the way in which the jump 
integral has to be rewritten to allow a correct and computationally efficient numerical solution.

First of all let's define the space in which we build the grid for the numerical solution as

$$
[0, T] \times\left[S_{\min }, S_{\max }\right]
$$

where the minimum and maximum values at which $S$ is considered have to be respectively small and big enough such that further reducing the minimum value or increasing the maximum value does not improve the result accuracy more than a chosen threshold.

Let's now transform the problem in logarithm terms with the following change of variable:

$$
x \triangleq \ln (S)
$$

and considering the new function of $x$

$$
W(x, t) \triangleq V(S, t)
$$

From these relationship we have

$$
\begin{array}{r}
W(x+y, t)=V\left(S e^{y}, t\right) \\
\frac{\partial W(x, t)}{\partial x}=S \cdot \frac{\partial V(S, t)}{\partial S} \\
\frac{\partial W(x, t)}{\partial t}=\frac{\partial V(S, t)}{\partial t}
\end{array}
$$

Now substituting these values in equation (4.4) we get

$$
\begin{array}{r}
\frac{\partial W(x, t)}{\partial t}+(r-q+\omega) \cdot \frac{\partial W(x, t)}{\partial x}+ \\
+\int_{-\infty}^{+\infty}[W(x+y, t)-W(x, t)] k(y) d y=r W(x, t)
\end{array}
$$

In the same way we can modify final and boundary conditions. In the case of a call option, we have the following final condition

$$
W(x, T)=\max \left(e^{x}-K, 0\right)
$$


together with these boundary conditions

$$
\begin{array}{rr}
W(-\infty, t)=0 & \forall t \\
W(+\infty, t)=e^{x} & \forall t
\end{array}
$$

While for the put option we have final condition

$$
W(x, T)=\max \left(K-e^{x}, 0\right)
$$

and boundary conditions

$$
\begin{array}{ll}
W(-\infty, t)=K & \forall t \\
W(+\infty, t)=0 & \forall t
\end{array}
$$

Finally we can modify the domain of analysis (4.5) to adapt it to the new variables by writing

$$
[0, T] \times\left[\ln \left(S_{\min }\right), \ln \left(S_{\max }\right)\right]
$$

or

$$
[0, T] \times\left[x_{\min }, x_{\max }\right]
$$

let's now discretize this system using $N+1$ mesh point in the $x$-direction and $M+1$ mesh points in the $t$-direction. The size of each of the $N$ space intervals is given by

$$
\Delta x=\frac{x_{\max }-x_{\min }}{N}
$$

and the size of each of the $M$ time interval is

$$
\Delta t=\frac{T-0}{M}
$$

The notation $W\left(x_{i}, t_{j}\right)$ refers to the value of $W(\cdot)$ at the node $(i, j)$. We use the following approximation of the partial derivatives

$$
\begin{gathered}
\frac{\partial W(x, t)}{\partial t} \simeq \frac{W\left(x_{i}, t_{j+1}\right)-W\left(x_{i}, t_{j}\right)}{\Delta t} \\
\frac{\partial W(x, t)}{\partial x} \simeq \frac{W\left(x_{i+1}, t_{j}\right)-W\left(x_{i-1}, t_{j}\right)}{2 \Delta x}
\end{gathered}
$$


Substituting these values in our PIDE (4.6) we get

$$
\begin{array}{r}
\frac{W\left(x_{i}, t_{j+1}\right)-W\left(x_{i}, t_{j}\right)}{\Delta t}+(r-q+\omega) \cdot \frac{W\left(x_{i+1}, t_{j}\right)-W\left(x_{i-1}, t_{j}\right)}{2 \Delta x}+ \\
\quad+\int_{-\infty}^{+\infty}\left[W\left(x_{i}+y, t_{j+1}\right)-W\left(x_{i}, t_{j+1}\right)\right] k(y) d y=r W\left(x_{i}, t_{j}\right)
\end{array}
$$

Now multiplying this last equation by $\Delta t$ and defining

$$
h \triangleq \frac{(r-q+\omega) \Delta t}{2 \Delta x}
$$

we have

$$
\begin{array}{r}
h W\left(x_{i-1}, t_{j}\right)+(1+r \Delta t) W\left(x_{i}, t_{j}\right)-h W\left(x_{i+1}, t_{j}\right)= \\
=W\left(x_{i}, t_{j+1}\right)+\Delta t \int_{-\infty}^{+\infty}\left[W\left(x_{i}+y, t_{j+1}\right)-W\left(x_{i}, t_{j+1}\right)\right] k(y) d y
\end{array}
$$

To implement a numerical solution of this PIDE we need first of all to solve numerically the integral.

\subsubsection{Solution of the Jump Integral for Vanilla Euro- pean Options}

A detailed solution of the integral in equation 4.7 is given in the Appendix following the approach presented by Hirsa and Madan ${ }^{6}$. The basic idea is to break the integral as sum of integral depending on the size of the jumps. We distinguish three cases, each of them has to be considered twice, being possible to have positive or negative jump values. In particular we can rewrite

\footnotetext{
${ }^{6}$ Ali Hirsa and Dilip B. Madan, "Pricing American Options Under Variance Gamma", Journal of Computational Finance, Vol. 7, No. 2, Winter 2003/2004, pages 63-80.
} 
the integral in the following way:

$$
\begin{aligned}
& \int_{-\infty}^{+\infty}\left[W\left(x_{i}+y, t_{j+1}\right)-W\left(x_{i}, t_{j+1}\right)\right] k(y) d y= \\
= & \int_{-\infty}^{x_{0}-x_{i}}\left[W\left(x_{i}+y, t_{j+1}\right)-W\left(x_{i}, t_{j+1}\right)\right] k(y) d y+ \\
+ & \int_{x_{0}-x_{i}}^{-\Delta x}\left[W\left(x_{i}+y, t_{j+1}\right)-W\left(x_{i}, t_{j+1}\right)\right] k(y) d y+ \\
& +\int_{-\Delta x}^{0}\left[W\left(x_{i}+y, t_{j+1}\right)-W\left(x_{i}, t_{j+1}\right)\right] k(y) d y+ \\
& +\int_{0}^{\Delta x}\left[W\left(x_{i}+y, t_{j+1}\right)-W\left(x_{i}, t_{j+1}\right)\right] k(y) d y+ \\
+ & \int_{\Delta x}^{x_{N}-x_{i}}\left[W\left(x_{i}+y, t_{j+1}\right)-W\left(x_{i}, t_{j+1}\right)\right] k(y) d y+ \\
& +\int_{x_{N}-x_{i}}^{+\infty}\left[W\left(x_{i}+y, t_{j+1}\right)-W\left(x_{i}, t_{j+1}\right)\right] k(y) d y
\end{aligned}
$$

The first couple of cases is that one of jumps of small size, where we define small a jump $y$ which in absolute value is smaller than one space interval $\Delta x$, that is

$$
y \in[-\Delta x, 0] \text { or } y \in[0, \Delta x]
$$

The difficulty here emerges from the fact that as the variance gamma Lévy process tends to infinity as the jump size approaches zero from both above and below. Clearly being a numerical solution of a PDE, we are interested only in the values of the function at every node, but not at its values in between nodes. The discretization of the jump integral in this case is realized with a linear interpolation of option values: the difference between the option value for stock values after and before the jump is approximated by the move in the option value between the 2 consecutive nodes multiplied by the proportion between the size of the jump and the size of the space interval. This implies the assumption that the option price changes linearly between two consecutive space nodes. In mathematical terms we have that, for example, in the case of a positive jump of size smaller than $\Delta x$

$$
W\left(x_{i}+y, t_{j+1}\right)-W\left(x_{i}, t_{j+1}\right) \simeq\left[W\left(x_{i+1}, t_{j+1}\right)-W\left(x_{i}, t_{j+1}\right)\right] \cdot \frac{y}{\Delta x}
$$


Being a first order approximation, clearly the error is an $O\left(y^{2}\right)$. Note that by doing this, we were able to have a jump term $y$ in the numerator. This can be simplified with the one in the denominator of the Lévy measure, thus solving the issue of a diverging measure.

If we wanted to apply the same kind of approach to a CGMY model, we would have an extra level of difficulty here because of the form of the Lévy measure for CGMY. Remember from equation (3.1) that at the denominator of the Lévy measure we have $|x|^{1+Y}$ with $Y<2$. Hence for cases $Y>1$ it is necessary to be particularly careful in the way small jumps which approach zero are handled.

The second case of jump is characterized by jumps which in absolute values are bigger than a single space interval. These jumps however cannot be so big that the logarithm of the underlying value finishes out from the considered range of values $\left[x_{\min }, x_{\max }\right]$. For example, let's say that you are at the generic point $x_{i}$, and call $x_{0}$ and $x_{N}$ the first and the last point on the grid, then the jump size has to be

$$
y \in\left[x_{0}-x_{i},-\Delta x\right] \text { or } y \in\left[\Delta x, x_{N}-x_{i}\right]
$$

In this case, the jump will take the value at some point in between two generic nodes. We can rewrite the integral as sum of integrals. Each of these integrals represents a jump which takes you to a different space interval. Say, for example, that you are in $x_{i}$ and that there is a positive jump of size $y$. Let the jump have a size such that you jump a number of steps between $k$ and $k+1$, that is

$$
y \in[k \Delta x,(k+1) \Delta x]
$$

then the stock value will be in the region $\left[x_{i+k}, x_{i+k+1}\right]$. It is possible to use linear interpolation again to define the difference in the option value between the value after the jump in position $x_{i}+y$ and the last node available provided by the grid that is $x_{i+k}$. This approximation defines this difference as the difference in option values in the two closest nodes multiplied by the proportion between the amount by which the jump exceed the last available node of the grid and the size of a space step. In mathematical terms

$$
\begin{array}{r}
W\left(x_{i}+y, t_{j+1}\right)-W\left(x_{i+k}, t_{j+1}\right) \simeq \\
\simeq \frac{W\left(x_{i+k+1}, t_{j+1}\right)-W\left(x_{i+k}, t_{j+1}\right)}{\Delta x} \cdot(y-k \Delta x)
\end{array}
$$


The final case is represented by jumps which are so big that the underlying value finishes outside the considered grid. To value the integral in this case we need to explicit the value of the option after the considered boundary of the grid, for this reason it is necessary to consider put and call options as two separate cases. Let's start with call options and assume a positive jump. Assuming that the stock is at the point $x_{i}$ a positive jump will take the underlying value outside the considered range if

$$
y \in\left[x_{N}-x_{i},+\infty\right]
$$

In this case the value of the option is given by the discounted value of the payoff where we stock is discounted at the dividend yield and the strike is discounted at the risk free interest rate. In mathematical form we can write that

$$
W\left(x_{i}+y, t_{j+1}\right) \simeq e^{x_{i}+y} e^{-q\left(T-t_{j+1}\right)}-K e^{-r\left(T-t_{j+1}\right)}
$$

Note that we need to choose $x_{N}$ big enough so that this is true for every $j$, that is for every time to maturity in the considered time range. Now we can consider the situation where the jump is negative and negative and big, still with a call option. If the jump has a size

$$
y \in\left[-\infty, x_{0}-x_{i}\right]
$$

the underlying value becomes smaller than minimum value considered and we impose that the call option in this case has value zero. The boundary condition in this case is

$$
W\left(x_{i}+y, t_{j+1}\right) \simeq 0
$$

Again we will choose $x_{0}$ small enough so that the option value can be approximated to zero, using the desired level of precision, for every $j$.

Symmetrical values can be obtained in the case of a put option. Here if the jump is huge and positive $y>\left(x_{N}-x_{i}\right)$, the stock price passes a boundary at which the value is imposed to be zero so here

$$
W\left(x_{i}+y, t_{j+1}\right) \simeq 0
$$

Finally when the jump is negative and huge $y<\left(x_{0}-x_{i}\right)$, we can say that the put option value is given by the discounted value of the payoff where 
the stock price is discounted at the dividend yield and the strike price is discounted at the risk free interest rate:

$$
W\left(x_{i}+y, t_{j+1}\right) \simeq K e^{-r\left(T-t_{j+1}\right)}-e^{x_{i}+y} e^{-q\left(T-t_{j+1}\right)}
$$

Using this technique it is possible to write the integral in equation (4.7) for call options in the following way:

$$
\begin{gathered}
\int_{-\infty}^{+\infty}\left[W\left(x_{i}+y, t_{j+1}\right)-W\left(x_{i}, t_{j+1}\right)\right] k(y) d y= \\
=\frac{\left[W\left(x_{i+1}, t_{j+1}\right)-W\left(x_{i}, t_{j+1}\right)\right]\left(1-e^{-\frac{\mu_{p}}{\nu_{p}} \Delta x}\right)}{\nu \Delta x \frac{\mu_{p}}{\nu_{p}}}+ \\
+\frac{\left[W\left(x_{i-1}, t_{j+1}\right)-W\left(x_{i}, t_{j+1}\right)\right]\left(1-e^{-\frac{\mu_{n}}{\nu_{n}} \Delta x}\right)}{\nu \Delta x \frac{\mu_{n}}{\nu_{n}}}+
\end{gathered}
$$

$+\sum_{k=1}^{N-i-1} \frac{1}{\nu \Delta x}\left[W\left(x_{i+k+1}, t_{j+1}\right)-W\left(x_{i+k}, t_{j+1}\right)\right] \frac{\nu_{p}}{\mu_{p}}\left[e^{-\frac{\mu_{p}}{\nu_{p}} k \Delta x}-e^{-\frac{\mu_{p}}{\nu_{p}}(k+1) \Delta x}\right]+$

$+\sum_{k=1}^{N-i-1} \frac{1}{\nu}\left\{W\left(x_{i+k}, t_{j+1}\right)-W\left(x_{i}, t_{j+1}\right)-k\left[W\left(x_{i+k+1}, t_{j+1}\right)-W\left(x_{i+k}, t_{j+1}\right)\right]\right\} \cdot$

$$
\begin{gathered}
\cdot\left\{\operatorname{expint}\left(\frac{\mu_{p}}{\nu_{p}} \cdot k \Delta x\right)-\text { expint }\left[\frac{\mu_{p}}{\nu_{p}} \cdot(k+1) \Delta x\right]\right\}+ \\
+\sum_{k=1}^{i-1} \frac{1}{\nu \Delta x}\left[W\left(x_{i-k-1}, t_{j+1}\right)-W\left(x_{i-k}, t_{j+1}\right)\right] \frac{\nu_{n}}{\mu_{n}}\left[e^{-\frac{\mu_{n}}{\nu_{n}} k \Delta x}-e^{-\frac{\mu_{n}}{\nu_{n}}(k+1) \Delta x}\right]+ \\
+\sum_{k=1}^{i-1} \frac{1}{\nu}\left\{W\left(x_{i-k}, t_{j+1}\right)-W\left(x_{i}, t_{j+1}\right)-k\left[W\left(x_{i-k-1}, t_{j+1}\right)-W\left(x_{i-k}, t_{j+1}\right)\right]\right\} .
\end{gathered}
$$




$$
\begin{gathered}
\cdot\left\{\operatorname{expint}\left(\frac{\mu_{n}}{\nu_{n}} k \Delta x\right)-\text { expint }\left[\frac{\mu_{n}}{\nu_{n}}(k+1) \Delta x\right]\right\}+ \\
+\frac{1}{\nu}\left\{e^{x_{i}} e^{-q\left(T-t_{j+1}\right)} \text { expint }\left[\left(\frac{\mu_{p}}{\nu_{p}}-1\right)(N-i) \Delta x\right]+\right. \\
\left.-\left[K e^{-r\left(T-t_{j+1}\right)}+W\left(x_{i}, t_{j+1}\right)\right] \operatorname{expint}\left[\frac{\mu_{p}}{\nu_{p}}(N-i) \Delta x\right]\right\}+ \\
-\frac{1}{\nu} W\left(x_{i}, t_{j+1}\right) \text { expint }\left[\left(x_{i}-x_{0}\right) \frac{\mu_{n}}{\nu_{n}}\right]
\end{gathered}
$$

On the other side, if the option is a put then the integral can be rewritten as

$$
\begin{gathered}
\int_{-\infty}^{+\infty}\left[W\left(x_{i}+y, t_{j+1}\right)-W\left(x_{i}, t_{j+1}\right)\right] k(y) d y= \\
=\frac{\left[W\left(x_{i+1}, t_{j+1}\right)-W\left(x_{i}, t_{j+1}\right)\right]\left(1-e^{-\frac{\mu_{p}}{\nu_{p}} \Delta x}\right)}{\nu \Delta x \frac{\mu_{p}}{\nu_{p}}}+ \\
+\frac{\left[W\left(x_{i-1}, t_{j+1}\right)-W\left(x_{i}, t_{j+1}\right)\right]\left(1-e^{-\frac{\mu_{n}}{\nu_{n}} \Delta x}\right)}{\nu \Delta x_{n} \frac{\mu_{n}}{\nu_{n}}}+ \\
+\sum_{k=1}^{N-i-1} \frac{1}{\nu \Delta x}\left[W\left(x_{i+k+1}, t_{j+1}\right)-W\left(x_{i+k}, t_{j+1}\right)\right] \frac{\nu_{p}}{\mu_{p}}\left[e^{-\frac{\mu_{p}}{\nu_{p}} k \Delta x}-e^{-\frac{\mu_{p}}{\nu_{p}}(k+1) \Delta x}\right]+ \\
\left.+\sum_{k=1}^{N-i-1} \frac{1}{\nu}\left\{W\left(x_{i+k}, t_{j+1}\right)-W\left(x_{i}, t_{j+1}\right)-k\left[W\left(x_{i+k+1}, t_{j+1}\right)-W\left(x_{i+k}, t_{j+1}\right)\right]\right]\right\} . \\
\cdot\left\{\operatorname{expint}\left(\frac{\mu_{p}}{\nu_{p}} k \Delta x\right)-\operatorname{expint}\left[\frac{\mu_{p}}{\nu_{p}}(k+1) \Delta x\right]\right\}+
\end{gathered}
$$




$$
\begin{gathered}
+\sum_{k=1}^{i-1} \frac{1}{\nu \Delta x}\left[W\left(x_{i-k-1}, t_{j+1}\right)-W\left(x_{i-k}, t_{j+1}\right)\right] \frac{\nu_{n}}{\mu_{n}}\left[e^{-\frac{\mu_{n}}{\nu_{n}} k \Delta x}-e^{-\frac{\mu_{n}}{\nu_{n}}(k+1) \Delta x}\right]+ \\
+\sum_{k=1}^{i-1} \frac{1}{\nu}\left\{W\left(x_{i-k}, t_{j+1}\right)-W\left(x_{i}, t_{j+1}\right)-k\left[W\left(x_{i-k-1}, t_{j+1}\right)-W\left(x_{i-k}, t_{j+1}\right)\right]\right\} \\
\cdot\left\{\operatorname{expint}\left(\frac{\mu_{n}}{\nu_{n}} k \Delta x\right)-\operatorname{expint}\left[\frac{\mu_{n}}{\nu_{n}}(k+1) \Delta x\right]\right\} \\
+\frac{1}{\nu}\left\{\left[K e^{-r\left(T-t_{j+1}\right)}-W\left(x_{i}, t_{j+1}\right)\right] \operatorname{expint}\left[\left(x_{i}-x_{0}\right) \frac{\mu_{n}}{\nu_{n}}\right]+\right. \\
\left.-e^{-q\left(T-t_{j+1}\right)} e^{x_{i}} \operatorname{expint}\left[\left(x_{i}-x_{0}\right)\left(\frac{\mu_{n}}{\nu_{n}}+1\right)\right]\right\}
\end{gathered}
$$

In the implementation of the scheme, however, the small jumps will be implemented implicitly. That is, we are going to use expression

$$
\begin{aligned}
& \frac{\left[W\left(x_{i+1}, t_{j}\right)-W\left(x_{i}, t_{j}\right)\right]\left(1-e^{-\frac{\mu_{p}}{\nu_{p}} \Delta x}\right)}{\nu \Delta x \frac{\mu_{p}}{\nu_{p}}}+ \\
& +\frac{\left[W\left(x_{i-1}, t_{j}\right)-W\left(x_{i}, t_{j}\right)\right]\left(1-e^{-\frac{\mu_{n}}{\nu_{n}} \Delta x}\right)}{\nu \Delta x \frac{\mu_{n}}{\nu_{n}}}
\end{aligned}
$$

in substitution to the first two lines of the solutions (4.8) and (4.9) and we are going to move this term to the left hand side of the PIDE (4.7). This approach improves the stability of the system as noted by Hirsa and Madan ${ }^{7}$. In reality the approach presented by Hirsa and Madan is slightly different

\footnotetext{
${ }^{7}$ Ali Hirsa and Dilip B. Madan, "Pricing American Options Under Variance Gamma", Journal of Computational Finance, Vol. 7, No. 2, Winter 2003/2004, pages 63-80.
} 
because they treat in an implicit way also the terms containing $W\left(x_{i}, t_{j+1}\right)$ for large jumps. This corresponds to use

$$
\begin{aligned}
& -\frac{1}{\nu} \cdot W\left(x_{i}, t_{j}\right) \cdot \text { expint }\left[(N-i) \frac{\mu_{p}}{\nu_{p}} \Delta x\right]+ \\
& -\frac{1}{\nu} \cdot W\left(x_{i}, t_{j}\right) \cdot \operatorname{expint}\left[\left(x_{i}-x_{0}\right) \frac{\mu_{n}}{\nu_{n}}\right]
\end{aligned}
$$

rather then the corresponding $W\left(x_{i}, t_{j+1}\right)$ terms. We decided not to move on the left hand side of the PIDE this term because, based on our numerical experiments, it did not provide an improvement from a stability point of view.

\subsubsection{Difference Equation for European Vanilla Op- tions}

Let's consider a European call option, in this case the difference equation to be solved can be written by substituting equation (4.8), as corrected by expression (4.10) in the initial PIDE (4.7). This gives the following difference equation:

$$
\begin{aligned}
& W\left(x_{i-1}, t_{j}\right)\left\{h-\frac{\Delta t \cdot\left(1-e^{-\frac{\mu_{n}}{\nu_{n}} \Delta x}\right)}{\nu \Delta x \frac{\mu_{n}}{\nu_{n}}}\right\}+ \\
& +W\left(x_{i}, t_{j}\right)\left\{(1+r \Delta t)+\frac{\Delta t \cdot\left(1-e^{-\frac{\mu_{p}}{\nu_{p}} \Delta x}\right)}{\nu \Delta x \frac{\mu_{p}}{\nu_{p}}}+\frac{\Delta t \cdot\left(1-e^{-\frac{\mu_{n}}{\nu_{n}} \Delta x}\right)}{\nu \Delta x \frac{\mu_{n}}{\nu_{n}}}\right\}+ \\
& -W\left(x_{i+1}, t_{j}\right)\left\{h+\frac{\Delta t \cdot\left(1-e^{-\frac{\mu_{p}}{\nu_{p}} \Delta x}\right)}{\nu \Delta x \frac{\mu_{p}}{\nu_{p}}}\right\}=W\left(x_{i}, t_{j+1}\right)+
\end{aligned}
$$




$$
\begin{aligned}
& +\Delta t \cdot\left\{\sum_{k=1}^{N-i-1} \frac{1}{\nu \Delta x}\left[W\left(x_{i+k+1}, t_{j+1}\right)-W\left(x_{i+k}, t_{j+1}\right)\right] .\right. \\
& \cdot \frac{\nu_{p}}{\mu_{p}}\left[e^{-\frac{\mu_{p}}{\nu_{p}} k \Delta x}-e^{-\frac{\mu_{p}}{\nu_{p}}(k+1) \Delta x}\right]+ \\
& +\sum_{k=1}^{N-i-1} \frac{1}{\nu}\left\{W\left(x_{i+k}, t_{j+1}\right)-W\left(x_{i}, t_{j+1}\right)+\right. \\
& \left.-k\left[W\left(x_{i+k+1}, t_{j+1}\right)-W\left(x_{i+k}, t_{j+1}\right)\right]\right\} . \\
& \cdot\left\{\operatorname{expint}\left(\frac{\mu_{p}}{\nu_{p}} \cdot k \Delta x\right)-\operatorname{expint}\left[\frac{\mu_{p}}{\nu_{p}} \cdot(k+1) \Delta x\right]\right\}+ \\
& +\sum_{k=1}^{i-1} \frac{1}{\nu \Delta x}\left[W\left(x_{i-k-1}, t_{j+1}\right)-W\left(x_{i-k}, t_{j+1}\right)\right] \text {. } \\
& \cdot \frac{\nu_{n}}{\mu_{n}}\left[e^{-\frac{\mu_{n}}{\nu_{n}} k \Delta x}-e^{-\frac{\mu_{n}}{\nu_{n}}(k+1) \Delta x}\right]+ \\
& +\sum_{k=1}^{i-1} \frac{1}{\nu}\left\{W\left(x_{i-k}, t_{j+1}\right)-W\left(x_{i}, t_{j+1}\right)+\right. \\
& \left.-k\left[W\left(x_{i-k-1}, t_{j+1}\right)-W\left(x_{i-k}, t_{j+1}\right)\right]\right\} . \\
& \cdot\left\{\operatorname{expint}\left(\frac{\mu_{n}}{\nu_{n}} k \Delta x\right)-\operatorname{expint}\left[\frac{\mu_{n}}{\nu_{n}}(k+1) \Delta x\right]\right\}+ \\
& +\frac{1}{\nu}\left\{e^{x_{i}} e^{-q\left(T-t_{j+1}\right)} \operatorname{expint}\left[\left(\frac{\mu_{p}}{\nu_{p}}-1\right)(N-i) \Delta x\right]+\right. \\
& \left.-\left[K e^{-r\left(T-t_{j+1}\right)}+W\left(x_{i}, t_{j+1}\right)\right] \operatorname{expint}\left[\frac{\mu_{p}}{\nu_{p}}(N-i) \Delta x\right]\right\}+ \\
& \left.-\frac{1}{\nu} W\left(x_{i}, t_{j+1}\right) \operatorname{expint}\left[\left(x_{i}-x_{0}\right) \frac{\mu_{n}}{\nu_{n}}\right]\right\}
\end{aligned}
$$

The left hand side of this equation can be written in matrix form as a tridiagonal matrix and hence the numerical solution of the equation can be realized using the Gauss's elimination method without partial pivoting; this technique 
is sometimes known also with the name of Thomas algorithm ${ }^{8}$. By using Gauss's elimination method the solution of the problem can be obtained in a much faster way compared with inverting the matrix. In the appendix B we provide $\mathrm{C}$ code to obtain option prices by solving this difference equation.

The difference equation for the European vanilla put is available in appendix A.2.2 and it can obtain in a similar way as we obtained the call option equation by substituting equation (4.9) as modified by expression (4.10) in equation (4.7).

\subsection{Experimental Results for European Vanilla Options}

\subsubsection{Calibration}

The calibration of the parameters of the variance gamma, involves the determination of the values of the three parameters which cannot be directly observed in the market, that is $\sigma, \vartheta$ and $\nu$, which as we know control the standard deviation, the skewness and the kurtosis of the process describing the underlying return. Madan, Carr and Chang ${ }^{9}$ present a minimization criterion which can be followed to obtain the desired values. In particular, the problem to be solved is the following one:

$$
\min _{\sigma, \vartheta, \nu} \sqrt{\frac{1}{M} \sum_{i=1}^{M}\left[\ln \left(\omega_{i}\right)-\ln \left(\hat{\omega}_{i}\right)\right]^{2}}
$$

where $\omega_{i}$ is the observed price on the $i^{\text {th }}$ option while $\hat{\omega}_{i}$ is the corresponding model price. Madan, Carr and Chang use 143 weeks and 4 maturities on the S\&P 500 from 1992 to 1994 and then they average the results obtaining the following means

$$
\sigma=0.1213 ; \vartheta=-0.1436 ; \nu=0.1686 ; \sigma \text { for } \mathrm{BS}=0.1236
$$

\footnotetext{
${ }^{8}$ See more on this in John C. Strikwerda, Finite Difference Schemes and Partial Differential Equations, Wadsworth Inc., Belmont, California, 1989, pages 78-81 and G. D. Smith, "Numerical Solution of Partial Differential Equations: Finite Difference Methods", $3^{\text {rd }}$ ed., Oxford University Press, 1998, pages 24-28.

${ }^{9}$ Dilip B Madan, Peter P. Carr and Eric C. Chang, "The Variance Gamma Process and Option Pricing", European Finance Review, 2, 1998, page 91.
} 
and the following standard deviations

$$
\sigma=0.0192 ; \vartheta=0.0552 ; \nu=0.0812 ; \sigma \text { for } \mathrm{BS}=0.0165
$$

Moreover they present the following minimum values:

$$
\sigma=0.08 ; \vartheta=-0.2744 ; \nu=0.0541 ; \sigma \text { for } \mathrm{BS}=0.087
$$

and maximum values

$$
\sigma=0.1737 ; \vartheta=0.0492 ; \nu=0.6790 ; \sigma \text { for } \mathrm{BS}=0.171
$$

The issue of this approach is that it is generally not possible to fit different maturities with the same set of parameters. It is therefore necessary to calibrate the parameters separately for different maturities. This is the approach followed by Hirsa and Madan ${ }^{10}$. They use the close form solution presented in equation (2.31) as model price in the minimization problem (4.13), because the numerical solution of the PIDE is computationally expensive. Using European call and put prices for the S\&P 500 on June $30^{\text {th }}$ 1999, Hirsa and Madan present the following results:

$$
\begin{aligned}
& T=0.13972, S=1369.41: \sigma \rightarrow 0.178753, \vartheta \rightarrow-0.30649, \nu \rightarrow 0.13317 \\
& T=0.21643, S=1369.41: \sigma \rightarrow 0.185002, \vartheta \rightarrow-0.28837, \nu \rightarrow 0.22460 \\
& T=0.46575, S=1369.41: \sigma \rightarrow 0.190714, \vartheta \rightarrow-0.28113, \nu \rightarrow 0.49083 \\
& T=0.56164, S=1369.41: \sigma \rightarrow 0.207220, \vartheta \rightarrow-0.22898, \nu \rightarrow 0.50215
\end{aligned}
$$

As we can see, the values of the parameters do not look very stable, in particular the value of $\nu$ pass from 0.17 to 0.5 with the change in the time to maturity of the options.

\subsubsection{Stability of the Scheme}

A discussion of the stability of the finite difference scheme presented in this work to price options under variance gamma is beyond the scope of this work. However we want to give some flavor of the issue. The stability of the system is clearly going to depend on the parameters entering in the variance gamma

\footnotetext{
${ }^{10}$ Ali Hirsa and Dilip B. Madan, "Pricing American Options Under Variance Gamma", Journal of Computational Finance, Vol. 7, No. 2, Winter 2003/2004, pages 63-80.
} 
difference equation, starting from $\sigma, \vartheta$ and $\nu$ and on the size of the time and space intervals. In particular $\Delta t$ and $\Delta x$ enter in the difference equation (4.12) and in the corresponding put equation a number of times; in the case of $\Delta x$ often in exponential form, hence it is not trivial to define a stability condition in an analytic way using for example Von Neumann analysis.

Hirsa and Madan ${ }^{11}$, referring in particular to the American put case, present some results for a ratio $\frac{N}{M}=2$. We remember that $N$ defines the number of space intervals and $M$ indicates the number of time intervals. Hirsa and Madan find stable results for the experiments presented and they say that "computations of other ratios give similar results and lead to the conjecture that the scheme is unconditionally stable". They however add that "this conjecture is solely based on our computational experience".

Given the complexity of the stability analysis for this problem, it seems that the issue cannot be addressed simply in terms of proper ratios of $N$ and $M$, since this would imply some linear relationship between $\Delta t$ and $\Delta x$. Anyway for general reference we will report the values of $\frac{\Delta t}{\Delta x}$ through the numerical experiments presented in this work.

Dealing with the issue from just a numerical point of view, we can say that the system, though generally performing well, has however some issues. In particular we found some stability problems as the variance gamma process converges to the geometric Brownian motion case. We know that the variance gamma is a generalization of the Black and Scholes model and that this model can be obtained as a special case when $\vartheta=0$ and $\nu=0$. As these two parameters get close to zero, however, the system tends to blow up. In particular we realize some experiments where $\vartheta=0$ and $\nu$ is progressively reduced and we see how the stability is affected as the $\nu$ approaches zero. In doing this analysis we compare three different finite different schemes:

1. The initial scheme presented in section 4.2 .3 where the whole jump integral is treated explicitly meaning that also the terms corresponding to small jumps are evaluated at time $t_{j+1}$. This scheme can be obtained for the European vanilla call case by simply substituting the discretized integral obtained in equation (4.8) in the initial PIDE (4.7). In the same way, the scheme for put options can be written by substituting the discretized integral (4.9) in the PIDE (4.7). We will refer to this scheme simply as "Scheme 1".

\footnotetext{
${ }^{11}$ Ali Hirsa and Dilip B. Madan, "Pricing American Options Under Variance Gamma", Journal of Computational Finance, Vol. 7, No. 2, Winter 2003/2004, page 77.
} 
2. The scheme that we indeed follow in this dissertation which modifies the previous one by treating implicitly the small jumps by replacing parts of the discretized integrals (4.8) and (4.9) with expression (4.10). In this case the difference equation is given by equation (4.12) for the call option case. We will refer to this scheme simply as "Scheme 2".

3. The scheme used by Hirsa and Madan, which further modify the initial scheme by treating implicitly two more elements given by expression (4.11). This expression together with expression (4.10) is used to replace part of the discretized integrals (4.8) and (4.9) before substituting them in the the PIDE (4.7). We will refer to this scheme simply as "Scheme 3".

We see that while in Scheme 1 the whole jump integral is treated explicitly, moving to Scheme 2 and to Scheme 3, progressively more parts of the integral are treated implicitly. In this contest we say that part of the integral is treated implicitly if the time at which it is considered is $t_{j}$ and therefore can be taken to the left hand side of the difference equation which needs to be solved. The reason behind the different schemes is that there is a singularity in the Lévy measure at 0 and the implicit treatment of the integrands near this singularity improves the stability of the scheme. Clearly it is possible to treat further parts of the integral implicitly and in particular the whole jump integral could be treated implicitly. However if we use a fully implicit method, we would not be able to use the Gauss's elimination method to solve the system and hence we would have to numerically invert the matrix to solve the linear system and this would be much more expensive in terms of computation time. We note that none of the schemes considered suggests a different approach for the integral in the heaviside function, which we will see appear in the American option case. Hence the observations that we make here in terms of scheme comparison are valid also for the American option case.

The experiments realized to study the stability of the system as the stochastic process becomes more and more similar to the geometric Brownian motion, use a European vanilla call option. And in particular the following parameters are used:

- $T=1 \quad$ Time to maturity in years;

- $K=\$ 100$ Strike price; 
- $r=0.03$ Interest rate;

- $q=0.01$ Dividend yield;

- $\sigma=0.2$

- $\vartheta=0.0$

- $x_{\min }=1.60944$ Min $x$ value considered, corresponding to $S=\$ 5$;

- $x_{\max }=5.85793$ Max $x$ value considered, corresponding to $S=\$ 350$;

Let's first of all see a case where we do not have any problem.

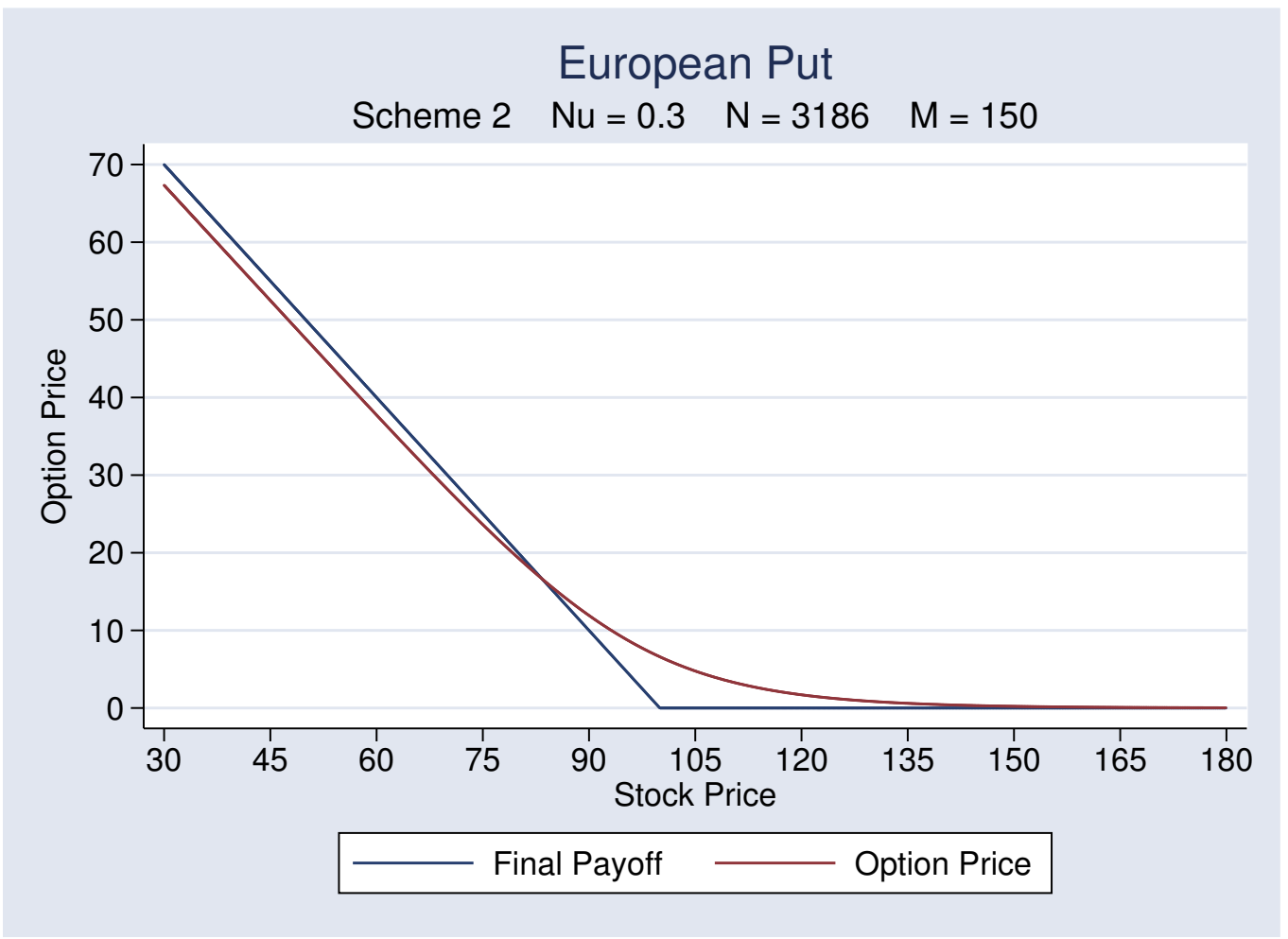

Figure 4.1: European Put Stability Analysis 
Figure 4.1 shows the option price computed using Scheme 2 when we have the following parameters in addition to the previous one:

- $\nu=0.3$

- $N=3186$ Number of space intervals;

- $M=150$ Number of time intervals;

- $\Delta x \simeq 0.0013$ Size of a space interval;

- $\Delta t \simeq 0.0067 \quad$ Size of a time interval;

- $\frac{\Delta t}{\Delta x} \simeq 5$;

We can see that the option graph is stable and smooth. Starting from these initial parameters, we study the stability of the three schemes. In particular, we want to compare four cases of $\nu$ : $0.3,0.1,0.01$ and 0.001 and three proportions of $\frac{\Delta t}{\Delta x}: 1,5$ and 10 . These proportions are obtained in different cases, one where the number of time intervals $M$ is equal to 30 and one where $M$ is equal to 150 . The stability of the system is problematic especially at the level of the discontinuity point at the strike price level $\$ 100$, so we are going to report results for this area, knowing that if the scheme is stable in this area is going to be stable in the whole range. We show in particular option values corresponding to the stock prices just above and below the strike. Because the number of space intervals is not constant the stock prices which are the closest to $\$ 100$ are going to be change, hence we show also also the stock level to which the option price corresponds. 
We first present the results for a case in which we are far from normality. Here $\nu=0.3$, a value which corresponds to a kurtosis of 3.9. This is the level of kurtosis used in figure 4.1

\begin{tabular}{|c|c|c|c|c|c|c|}
\hline \multicolumn{7}{|c|}{ Stability Analysis } \\
\hline$N$ & 127 & 127 & 637 & 637 & 1275 & 1275 \\
\hline$M$ & 30 & 30 & 30 & 30 & 30 & 30 \\
\hline$\Delta x$ & 0.0335 & 0.0335 & 0.0067 & 0.0067 & 0.0033 & 0.0033 \\
\hline$\Delta t$ & 0.0333 & 0.0333 & 0.0333 & 0.0333 & 0.0333 & 0.0333 \\
\hline$\Delta t / \Delta x$ & 1 & 1 & 5 & 5 & 10 & 10 \\
\hline Stock Price & 98.17 & 101.51 & 99.89 & 100.56 & 99.99 & 100.32 \\
\hline Scheme 1 & 7.7626 & 6.3630 & 6.6959 & 6.4191 & 6.6398 & 6.5011 \\
\hline Scheme 2 & 7.7509 & 6.3507 & 6.6953 & 6.4185 & 6.6396 & 6.5009 \\
\hline Scheme 3 & 7.7509 & 6.3507 & 6.6953 & 6.4185 & 6.6396 & 6.5009 \\
\hline \hline$N$ & 637 & 637 & 3186 & 3186 & 6372 & 6372 \\
\hline$M$ & 150 & 150 & 150 & 150 & 150 & 150 \\
\hline$\Delta x$ & 0.0067 & 0.0067 & 0.0013 & 0.0013 & 0.0007 & 0.0007 \\
\hline$\Delta t$ & 0.0067 & 0.0067 & 0.0067 & 0.0067 & 0.0067 & 0.0067 \\
\hline$\Delta t / \Delta x$ & 1 & 1 & 5 & 5 & 10 & 10 \\
\hline Stock Price & 99.89 & 100.56 & 99.93 & 100.06 & 100.00 & 100.06 \\
\hline Scheme 1 & 6.6683 & 6.3913 & 6.6322 & 6.5763 & 6.6034 & 6.5755 \\
\hline Scheme 2 & 6.6681 & 6.3912 & 6.6322 & 6.5763 & 6.6034 & 6.5755 \\
\hline Scheme 3 & 6.6681 & 6.3912 & 6.6322 & 6.5763 & 6.6034 & 6.5755 \\
\hline
\end{tabular}

The table shows in the top part results when there are 30 time intervals corresponding to $\Delta t=0.0333$. Keeping this number constant, we see the impact of changing the number of stock price intervals in the logarithmic space. We present the results for three different $N: 127,637$ and 1,275. This three numbers of stock intervals are chosen in such a way that the proportion of $\frac{\Delta t}{\Delta x}$ is about equal to 1,5 and 10 . In the bottom part of the table, the time intervals used are 150 corresponding to $\Delta t=0.0067$. The corresponding numbers of space intervals are chosen so that $\frac{\Delta t}{\Delta x}$ is again equal to 1,5 and 10. We said that the conditions to be given to $\Delta t$ and $\Delta x$ to assure stability and convergence of the scheme are more complex than just the proportion between the two. In the absence of a more precise definition, we study the 
combined approach of changing this proportion and changing the absolute size of the intervals.

For each of this $N$ and $M$ combination we report the two stock prices which are the closest to the strike price $\$ 100$. For each of these stock prices we present the corresponding option price as obtained with the 3 schemes considered. Results for Scheme 2 are obtained using the program in C language presented in appendix B. The results for the other two schemes are obtained using proper modifications of this code. We can see that the results are always stable for all the cases showed in this table. Clearly the option prices may be quite different in the different cases because as $N$ changes, the stock prices closest to the strike price move. We note however that for the same stock price, the option prices generated by the different schemes are quite close with each other. If we exclude the case $N=127$, we see that the option prices are less than 1 cent far from each other. Scheme 2 and 3 seems to be exactly the same. The difference between them is at most of order $10^{-8}$. 
We study now the case of $\nu=0.1$ which corresponds to a kurtosis of 3.3. The following table shows the results:

\begin{tabular}{|c|c|c|c|c|c|c|}
\hline \multicolumn{7}{|c|}{ Stability Analysis } \\
\hline$N$ & 127 & 127 & 637 & 637 & 1275 & 1275 \\
\hline$M$ & 30 & 30 & 30 & 30 & 30 & 30 \\
\hline$\Delta x$ & 0.0335 & 0.0335 & 0.0067 & 0.0067 & 0.0033 & 0.0033 \\
\hline$\Delta t$ & 0.0333 & 0.0333 & 0.0333 & 0.0333 & 0.0333 & 0.0333 \\
\hline$\Delta t / \Delta x$ & 1 & 1 & 5 & 5 & 10 & 10 \\
\hline Stock Price & 98.17 & 101.51 & 99.89 & 100.56 & 99.99 & 100.32 \\
\hline Scheme 1 & 8.3298 & 6.9513 & -7.540 & 19.912 & $-139,350$ & 128,025 \\
\hline Scheme 2 & 8.3062 & 6.9268 & 6.9024 & 6.6268 & 6.8266 & 6.6884 \\
\hline Scheme 3 & 8.3062 & 6.9268 & 6.9024 & 6.6268 & 6.8266 & 6.6884 \\
\hline \hline$N$ & 637 & 637 & 3186 & 3186 & 6372 & 6372 \\
\hline$M$ & 150 & 150 & 150 & 150 & 150 & 150 \\
\hline$\Delta x$ & 0.0067 & 0.0067 & 0.0013 & 0.0013 & 0.0007 & 0.0007 \\
\hline$\Delta t$ & 0.0067 & 0.0067 & 0.0067 & 0.0067 & 0.0067 & 0.0067 \\
\hline$\Delta t / \Delta x$ & 1 & 1 & 5 & 5 & 10 & 10 \\
\hline Stock Price & 99.89 & 100.56 & 99.93 & 100.06 & 100.00 & 100.06 \\
\hline Scheme 1 & 6.8777 & 6.6019 & 6.8138 & 6.7581 & 6.7839 & 6.7561 \\
\hline Scheme 2 & 6.8774 & 6.6016 & 6.8138 & 6.7581 & 6.7839 & 6.7561 \\
\hline Scheme 3 & 6.8774 & 6.6016 & 6.8138 & 6.7581 & 6.7839 & 6.7561 \\
\hline
\end{tabular}

The table is organized like the previous one with results for $M=30$ in the top and for $M=150$ in the bottom. We see that the Scheme 1 is starting to blow up for the cases $M=30$ with $\frac{\Delta t}{\Delta x}$ equal to 5 and 10. For these same cases we see that Scheme 2 and 3 produce good results. Hence we can say that they provide an improvement with respect to Scheme 1. We see that Scheme 1 is fine not only for the case $\frac{\Delta t}{\Delta x}=1$, but also when it is equal 5 and 10 if $M$ is equal 150. When $M=150$ option prices generated by the three schemes are very close with each other. This confirms our arguing that just talking about ratios of $N$ and $M$ is not enough to capture the more complex relationships exhibited by the difference equation. Finally we also remember that the option prices for the same stock levels are different with respect to the results in the previous table because the level of kurtosis is different. Let's now move more in the direction of the Black and Scholes model by 
further reducing $\nu$. In particular next table shows the results for $\nu=0.01$ which corresponds to a kurtosis of 3.03.

\begin{tabular}{|c|c|c|c|c|c|c|}
\hline \multicolumn{7}{|c|}{ Stability Analysis } \\
\hline$N$ & 127 & 127 & 637 & 637 & 1275 & 1275 \\
\hline$M$ & 30 & 30 & 30 & 30 & 30 & 30 \\
\hline$\Delta x$ & 0.0335 & 0.0335 & 0.0067 & 0.0067 & 0.0033 & 0.0033 \\
\hline$\Delta t$ & 0.0333 & 0.0333 & 0.0333 & 0.0333 & 0.0333 & 0.0333 \\
\hline$\Delta t / \Delta x$ & 1 & 1 & 5 & 5 & 10 & 10 \\
\hline Stock Price & 98.17 & 101.51 & 99.89 & 100.56 & 99.99 & 100.32 \\
\hline Scheme 1 & $10^{17}$ & $-10^{17}$ & $-10^{32}$ & $10^{32}$ & $-10^{36}$ & $10^{36}$ \\
\hline Scheme 2 & 10.8272 & 9.5370 & 6.8954 & 6.7021 & $-10^{11}$ & $-10^{11}$ \\
\hline Scheme 3 & 10.8272 & 9.5370 & 6.8954 & 6.7021 & $-10^{11}$ & $-10^{11}$ \\
\hline \hline$N$ & 637 & 637 & 3186 & 3186 & 6372 & 6372 \\
\hline$M$ & 150 & 150 & 150 & 150 & 150 & 150 \\
\hline$\Delta x$ & 0.0067 & 0.0067 & 0.0013 & 0.0013 & 0.0007 & 0.0007 \\
\hline$\Delta t$ & 0.0067 & 0.0067 & 0.0067 & 0.0067 & 0.0067 & 0.0067 \\
\hline$\Delta t / \Delta x$ & 1 & 1 & 5 & 5 & 10 & 10 \\
\hline Stock Price & 99.89 & 100.56 & 99.93 & 100.06 & 100.00 & 100.06 \\
\hline Scheme 1 & $-10^{42}$ & $10^{42}$ & $10^{88}$ & $-10^{88}$ & $-10^{102}$ & $10^{102}$ \\
\hline Scheme 2 & 7.2529 & 6.9795 & $-10^{9}$ & $-10^{9}$ & $-10^{38}$ & $-10^{38}$ \\
\hline Scheme 3 & 7.2529 & 6.9795 & $-10^{9}$ & $-10^{9}$ & $-10^{38}$ & $-10^{38}$ \\
\hline
\end{tabular}

The table shows the same cases in terms of time and space interval sizes. We see that now Scheme 1 is never stable and progressively blows up as $\frac{\Delta t}{\Delta x}$ increases. Scheme 2 and 3 on the other side are fine if $\frac{\Delta t}{\Delta x}=1$ or if $\frac{\Delta t}{\Delta x}=5$ and $M=30$. We see that the results produced using Scheme 2 and 3 are very close with each other both in the case in which the scheme is stable and in the case in which it is not stable. This suggests the idea that from a stability point of view Scheme 2 and 3 are on the same quality level. Finally we note that results for $N=127$ and $M=30$ are getting a worse approximation of the solution. Also in the cases $\nu=0.3$ and $\nu=0.1$ results generated with less intervals were less precise, here however the poor quality of the approximation is clearer. 
We study now a final case, in which the process considered is very close to a geometric Brownian motion. The following table shows results when $\nu=0.001$, corresponding to a kurtosis of 3.003 .

\begin{tabular}{|c|c|c|c|c|c|c|}
\hline \multicolumn{7}{|c|}{ Stability Analysis } \\
\hline$N$ & 127 & 127 & 637 & 637 & 1275 & 1275 \\
\hline$M$ & 30 & 30 & 30 & 30 & 30 & 30 \\
\hline$\Delta x$ & 0.0335 & 0.0335 & 0.0067 & 0.0067 & 0.0033 & 0.0033 \\
\hline$\Delta t$ & 0.0333 & 0.0333 & 0.0333 & 0.0333 & 0.0333 & 0.0333 \\
\hline$\Delta t / \Delta x$ & 1 & 1 & 5 & 5 & 10 & 10 \\
\hline Stock Price & 98.17 & 101.51 & 99.89 & 100.56 & 99.99 & 100.32 \\
\hline Scheme 1 & $10^{34}$ & $-10^{34}$ & $-10^{54}$ & $10^{54}$ & $-10^{60}$ & $10^{60}$ \\
\hline Scheme 2 & 16.1281 & 15.0215 & 8.7880 & 8.5243 & -602.17 & -526.97 \\
\hline Scheme 3 & 16.1281 & 15.0215 & 8.7880 & 8.5243 & -602.17 & -526.97 \\
\hline \hline$N$ & 637 & 637 & 3186 & 3186 & 6372 & 6372 \\
\hline$M$ & 150 & 150 & 150 & 150 & 150 & 150 \\
\hline$\Delta x$ & 0.0067 & 0.0067 & 0.0013 & 0.0013 & 0.0007 & 0.0007 \\
\hline$\Delta t$ & 0.0067 & 0.0067 & 0.0067 & 0.0067 & 0.0067 & 0.0067 \\
\hline$\Delta t / \Delta x$ & 1 & 1 & 5 & 5 & 10 & 10 \\
\hline Stock Price & 99.89 & 100.56 & 99.93 & 100.06 & 100.00 & 100.06 \\
\hline Scheme 1 & $-10^{172}$ & $10^{172}$ & $10^{227}$ & $-10^{227}$ & $-10^{243}$ & $10^{243}$ \\
\hline Scheme 2 & 8.7999 & 8.5363 & $-10^{70}$ & $-10^{70}$ & $-10^{119}$ & $-10^{119}$ \\
\hline Scheme 3 & 8.7999 & 8.5363 & $-10^{70}$ & $-10^{70}$ & $-10^{119}$ & $-10^{119}$ \\
\hline
\end{tabular}

We see that again Scheme 1 is never stable. Scheme 2 and 3 on the other side present stable results for $\frac{\Delta t}{\Delta x}=1$ and for $\frac{\Delta t}{\Delta x}=5$ when $M=30$. We find confirmation here of the fact that Scheme 2 and 3 provides an improvement over Scheme 1. We note that when we use only 127 space intervals and 30 time intervals, the option price is quite off, being over $\$ 15$ when around at the money while, when more points are used, the price is close to $\$ 8.6$. We see that also in this case Scheme 2 and 3 produce virtually the same option prices for both the stable and unstable cases. This confirm our conjecture that Scheme 3 does not provide a material improvement over Scheme 2. 
The issue described so far concerned the stability of the alternative schemes when parameters are "small" and the variance gamma model converges to the Black and Scholes model. Another problems which this scheme exhibits is the one we sometimes have when the parameters are too "large". Although in this case, we do not have stability issues, we may have in some cases finite oscillation in correspondence with discontinuity points. It is a case of non dissipation where small, high frequency oscillations are propagated without being dumped ${ }^{12}$. Some authors ${ }^{13}$ define these non dissipative schemes $A_{0}$-stable schemes, in contraposition to dissipative schemes which are defined $L_{0}$-stable schemes. Clearly $L_{0}$-stable schemes are preferable to $A_{0}$-stable ones because possible unwanted oscillation in the numerical solution are rapidly dampened and this eliminates the necessity of adding additional constraints on the time step. Moreover with $A_{0}$-stable methods it is common for unwanted finite oscillations to increase in magnitude when any attempt is made to improve accuracy by decreasing the space intervals.

To consider this problem, let's consider here some experiments on European vanilla put options, in particular we want to use the following parameters:

- $T=1$ Time to maturity in years;

- $K=\$ 100$ Strike price;

- $r=0.03$ Interest rate;

- $q=0.01 \quad$ Dividend yield;

- $\sigma=1.3$

- $\vartheta=-0.4$

- $\nu=0.7$

- $x_{\min }=1.60944$ Min $x$ value considered, corresponding to $S=\$ 5$;

\footnotetext{
${ }^{12}$ We do not enter here in the details of the problem; see more on this in John C. Strikwerda, Finite Difference Schemes and Partial Differential Equations, Wadsworth Inc., Belmont, California, 1989, page 100. A well known example of non dissipative scheme is the Crank Nicolson scheme.

${ }^{13}$ G. D. Smith, "Numerical Solution of Partial Differential Equations: Finite Difference Methods", $3^{\text {rd }}$ ed., Oxford University Press, 1998, page 119.
} 
- $x_{\text {max }}=5.85793$ Max $x$ value considered, corresponding to $S=\$ 350$;

These values of $\sigma, \vartheta$ and $\nu$ correspond to a volatility of $134 \%$, a kurtosis of 5.4 and a skewness of $-0.61^{14}$. Using these parameters we run a series of experiments to see the impact of changing the number of time and space steps. For each case we report the results obtained using each of the three schemes presented above.

Let's start considering the following table:

\begin{tabular}{|c|c|c|c|c|c|c|}
\hline \multicolumn{7}{|c|}{ Dissipation Analysis } \\
\hline$N$ & 127 & 127 & 637 & 637 & 1275 & 1275 \\
\hline$M$ & 30 & 30 & 30 & 30 & 30 & 30 \\
\hline$\Delta x$ & 0.0335 & 0.0335 & 0.0067 & 0.0067 & 0.0033 & 0.0033 \\
\hline$\Delta t$ & 0.0333 & 0.0333 & 0.0333 & 0.0333 & 0.0333 & 0.0333 \\
\hline$\Delta t / \Delta x$ & 1 & 1 & 5 & 5 & 10 & 10 \\
\hline Stock Price & 327.3 & 338.5 & 345.4 & 347.7 & 347.7 & 348.8 \\
\hline Scheme 1 & 11.71 & 17.12 & 4.95 & 19.69 & 3.01 & 20.35 \\
\hline Scheme 2 & 11.77 & 17.05 & 4.98 & 19.66 & 3.03 & 20.33 \\
\hline Scheme 3 & 11.54 & 16.54 & 4.89 & 19.03 & 2.98 & 19.68 \\
\hline \hline$N$ & 637 & 637 & 3186 & 3186 & 6372 & 6372 \\
\hline$M$ & 150 & 150 & 150 & 150 & 150 & 150 \\
\hline$\Delta x$ & 0.0067 & 0.0067 & 0.0013 & 0.0013 & 0.0007 & 0.0007 \\
\hline$\Delta t$ & 0.0067 & 0.0067 & 0.0067 & 0.0067 & 0.0067 & 0.0067 \\
\hline$\Delta t / \Delta x$ & 1 & 1 & 5 & 5 & 10 & 10 \\
\hline Stock Price & 345.4 & 347.7 & 349.1 & 349.5 & 349.5 & 349.8 \\
\hline Scheme 1 & 4.96 & 19.28 & 1.47 & 20.43 & 0.83 & 20.61 \\
\hline Scheme 2 & 4.97 & 19.27 & 1.47 & 20.43 & 0.83 & 20.61 \\
\hline Scheme 3 & 4.95 & 19.15 & 1.47 & 20.29 & 0.83 & 20.48 \\
\hline
\end{tabular}

This table reports the option prices correspondent to the the last two points on the grid before the end of the space range. The boundary conditions impose that the put option deep out of the money at the extreme of the grid is worth zero. As the stock price goes down, the option price is supposed to

\footnotetext{
${ }^{14}$ See section 4.3 .3 for a reminder of how to convert $\sigma, \vartheta$ and $\nu$ in volatility, skewness and kurtosis.
} 
increase monotonically. However when the parameters are as large as above we may have finite oscillations. In the table we observe an oscillation when the point corresponding to the lower stock price has a lower option value than the one corresponding to the high stock price. These oscillations fade as we move to lower stock prices so the case reported here corresponds to the largest oscillation. We show in this table values obtained with $N=127,637$ and 1275 and $M=30$ in the top part. They correspond to $\frac{\Delta t}{\Delta x}=1,5$ and 10. In the bottom part the same $\frac{\Delta t}{\Delta x}$ proportions have been obtained with $N=637,3186$ and 6372 and $M=150$

We can see that in all the cases presented here we observe oscillations, in fact the left option of each pair is worth less than the right one. To compare between results generated with different grids, we should keep in mind that the stock levels at which option prices are computed change and so the size of the oscillation is affected by this. In particular as the oscillations fade getting far from the discontinuity point, we are going to have larger oscillations when the grid is very fine in the stock price, because the points at which the oscillation is measured are closer to the discontinuity point. If we compare among schemes for a specific stock price, we can see that the results are overall similar. Although Scheme 2 has in general a smaller oscillation size than Scheme 1 and Scheme 3 has a smaller oscillation size than Scheme 2 , none of them really outperforms the other. In particular there are no cases in this table where a scheme does not present oscillations. 
Let's consider now the following series of cases, presenting a lower $\frac{\Delta t}{\Delta x}$, run on the same option.

\begin{tabular}{|c|c|c|c|c|c|c|}
\hline \multicolumn{7}{|c|}{ Dissipation Analysis } \\
\hline$N$ & 127 & 127 & 127 & 127 & 127 & 127 \\
\hline$M$ & 150 & 150 & 300 & 300 & 1500 & 1500 \\
\hline$\Delta x$ & 0.0335 & 0.0335 & 0.0335 & 0.0335 & 0.0335 & 0.0335 \\
\hline$\Delta t$ & 0.0067 & 0.0067 & 0.0033 & 0.0033 & 0.0007 & 0.0007 \\
\hline$\Delta t / \Delta x$ & 0.20 & 0.20 & 0.10 & 0.10 & 0.02 & 0.02 \\
\hline Stock Price & 327.3 & 338.5 & 327.3 & 338.5 & 327.3 & 338.5 \\
\hline Scheme 1 & 11.63 & 16.72 & 11.62 & 16.68 & 11.61 & 16.64 \\
\hline Scheme 2 & 11.64 & 16.71 & 11.63 & 16.67 & 11.62 & 16.64 \\
\hline Scheme 3 & 11.60 & 16.61 & 11.61 & 16.62 & 11.61 & 16.62 \\
\hline \hline$N$ & 127 & 127 & 127 & 127 & 127 & 127 \\
\hline$M$ & 3,000 & 3,000 & 15,000 & 15,000 & 30,000 & 30,000 \\
\hline$\Delta x$ & 0.0335 & 0.0335 & 0.0335 & 0.0335 & 0.0335 & 0.0335 \\
\hline$\Delta t$ & 0.0003 & 0.0003 & 0.0001 & 0.0001 & 0.00003 & 0.00003 \\
\hline$\Delta t / \Delta x$ & 0.010 & 0.010 & 0.002 & 0.002 & 0.001 & 0.001 \\
\hline Stock Price & 327.3 & 338.5 & 327.3 & 338.5 & 327.3 & 338.5 \\
\hline Scheme 1 & 11.61 & 16.63 & 11.61 & 16.63 & 11.61 & 16.63 \\
\hline Scheme 2 & 11.61 & 16.63 & 11.61 & 16.63 & 11.61 & 16.63 \\
\hline Scheme 3 & 11.61 & 16.63 & 11.61 & 16.63 & 11.61 & 16.63 \\
\hline
\end{tabular}

In this table we leave the number of space steps constant and we progressively increase the number of time steps from 150 to 30,000. The following proportion $\frac{\Delta t}{\Delta x}$ are considered: 0.2, 0.1, 0.02, 0.01, 0.002 and 0.001. Because in these experiments we keep $N$ constant the stock price levels at which we measure the oscillations are kept constant, hence it is going to be easier to compare the different cases. We can see that increasing the number of time steps does not provide any meaningful improvement. Moreover we can see that the advantage presented by Scheme 3 and Scheme 2 over Scheme 1 disappears here as the proportion $\frac{\Delta t}{\Delta x}$ is reduced.

Since it seems it is hard to improve the scheme by increasing $M$ when $N$ is fixed we try to go in the reverse direction of reducing $N$. The following table shows results of experiments realized with this approach. 


\begin{tabular}{|c|c|c|c|c|c|c|}
\hline \multicolumn{7}{|c|}{ Dissipation Analysis } \\
\hline$N$ & 63 & 63 & 63 & 63 & 63 & 63 \\
\hline$M$ & 1500 & 1500 & 1000 & 1000 & 500 & 500 \\
\hline$\Delta x$ & 0.0674 & 0.0674 & 0.0674 & 0.0674 & 0.0674 & 0.0674 \\
\hline$\Delta t$ & 0.0007 & 0.0007 & 0.0010 & 0.0010 & 0.0020 & 0.0020 \\
\hline$\Delta t / \Delta x$ & 0.010 & 0.010 & 0.015 & 0.015 & 0.030 & 0.030 \\
\hline Stock Price & 305.8 & 327.2 & 305.8 & 327.2 & 305.8 & 327.2 \\
\hline Scheme 1 & 14.77 & 15.91 & 14.77 & 15.92 & 14.78 & 15.93 \\
\hline Scheme 2 & 14.77 & 15.91 & 14.77 & 15.92 & 14.78 & 15.93 \\
\hline Scheme 3 & 14.77 & 15.90 & 14.77 & 15.90 & 14.76 & 15.90 \\
\hline \hline$N$ & 50 & 50 & 50 & 50 & 50 & 50 \\
\hline$M$ & 1500 & 1500 & 1000 & 1000 & 500 & 500 \\
\hline$\Delta x$ & 0.0850 & 0.0850 & 0.0850 & 0.0850 & 0.0850 & 0.0850 \\
\hline$\Delta t$ & 0.0007 & 0.0007 & 0.0010 & 0.0010 & 0.0020 & 0.0020 \\
\hline$\Delta t / \Delta x$ & 0.008 & 0.008 & 0.012 & 0.012 & 0.024 & 0.024 \\
\hline Stock Price & 295.3 & 321.5 & 295.3 & 321.5 & 295.3 & 321.5 \\
\hline Scheme 1 & 15.93 & 15.90 & 15.93 & 15.91 & 15.93 & 15.92 \\
\hline Scheme 2 & 15.93 & 15.90 & 15.93 & 15.91 & 15.94 & 15.92 \\
\hline Scheme 3 & 15.92 & 15.89 & 15.92 & 15.89 & 15.92 & 15.89 \\
\hline
\end{tabular}

In the top part of the table we use 63 stock price intervals and we match them with 1,500,1,000 and 500 time steps, corresponding to 0.01, 0.015 and $0.03 \frac{\Delta t}{\Delta x}$ proportions. Being the grid built with larger space in between stock price points, we are now more far from the discontinuity point and hence the size of the oscillation is reduced. However we see that the oscillations still exist as we can see from the put price going down from about $\$ 15.92$ to about $\$ 14.77$ when the stock price is reduced from $\$ 327.2$ to $\$ 305.8$. We note also in this case that changing the number of time steps does not produce any meaningful effect. Moreover we see that the three schemes produce virtually the same results.

The bottom part of the table correspond to the case where we have only 50 stock price intervals. We try again 1,500, 1,000 and 500 time intervals here. This is finally a case where there are no more oscillations, we see however that to reach this result we had to use only 50 space intervals with the results that, because stock is expressed in logarithmic terms in the grid, the first two point on the left of the highest margin of the stock range are very 
far from each other: $\$ 321.5$ and $\$ 295.3$. We see again here that changing the number of time steps does not impact much the results and that the three schemes are equivalent.

Overall we can conclude that for the non dissipation problem the three schemes are equivalent. Moreover we can conclude that the oscillations depend more on the proximity to the end of the range than to the proportion of $\frac{\Delta t}{\Delta x}$. The following graph shows the results as generated by using Scheme 2 in the case $N=6,372$ and $M=150$ which corresponds to a proportion of 10 between time and space interval sizes.

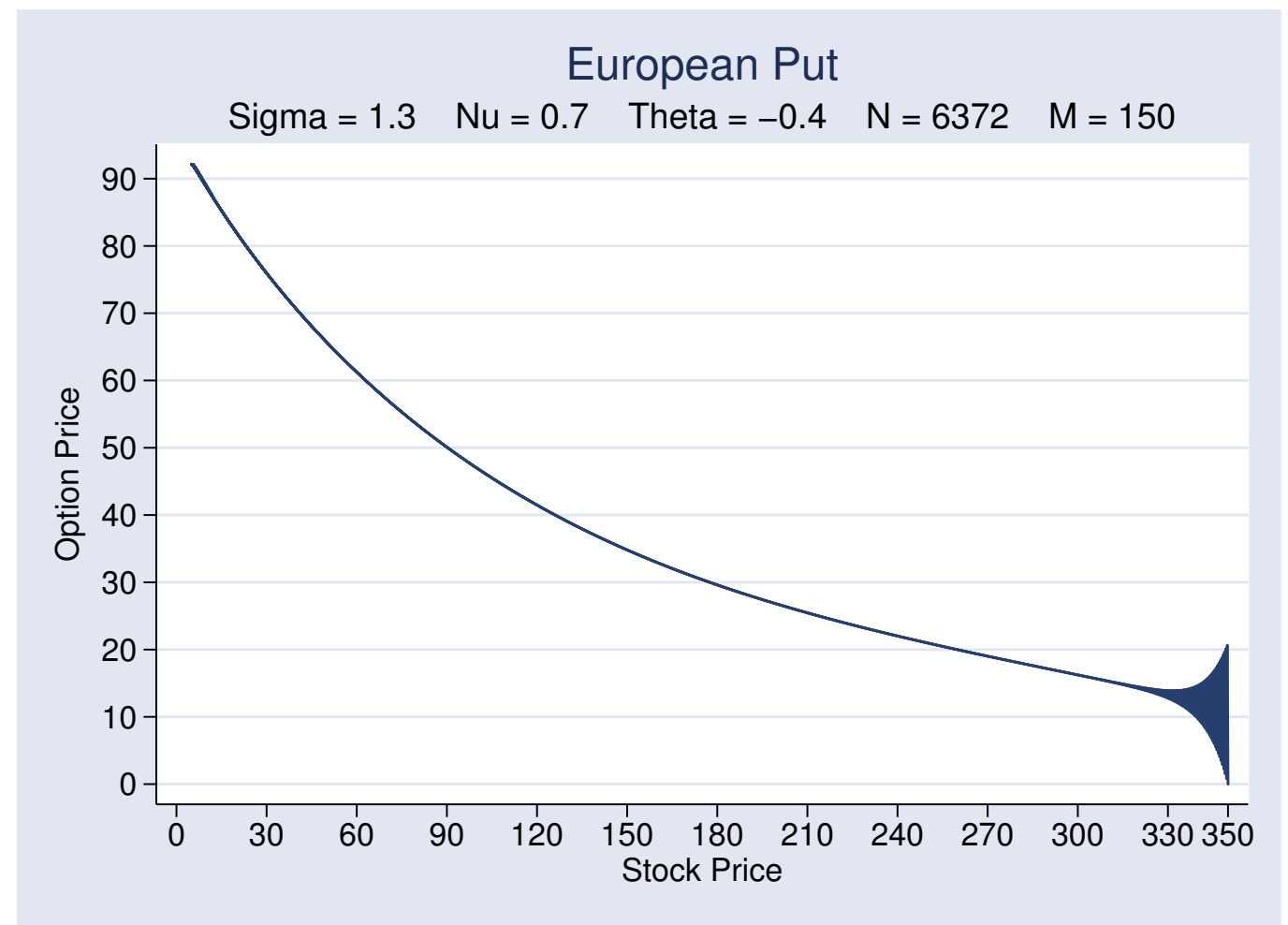

Figure 4.2: European Put Dissipation Analysis

This figure helps to understand some of the issues encountered here. We can see that given the large volatility and kurtosis, combined with the maturity 
of 1 year, the stock has still a non negligible value near the end of the grid. Hence when the boundary condition requiring the option to have a zero value at $\$ 350$ is applied, the discontinuity is pretty large and the scheme is unable to provide smooth option prices in the neighborhood of the discontinuity point.

We can tentatively conclude, solely on the basis of our computational experience, that overall this finite difference scheme seems to perform well. Although we cannot claim unconditional stability for the cases in which the process is very close to the Black and Scholes process, we had unconditional stability for all the other cases studied. Pricing options as the variance gamma converges to the geometric Brownian motion case is still possible and we could find a stable solution for the case $\sigma=0.2, \vartheta=0, \nu=0.001$ and $\frac{\Delta t}{\Delta x}=1$, which is close enough to the Black and Scholes model corresponding to a kurtosis of 3.003 and no skewness. As for the non dissipation issue, we can say that it is most of the time not a major problem, because it often corresponds to large values of the parameters which are not extremely common: in the example presented volatility was $134 \%$ and it was coupled with a kurtosis of 5.4. Although these are possible values, they are definitely very unusual especially if we consider that they are implied parameters for a 1 year option. Furthermore we can see that, when we are far from the discontinuity point, the price is good: in figure 4.2 we can see that for a $\$ 100$ strike option, it is possible to obtain prices not affected by oscillations until about $\$ 310$ underlying price. We can also here push further out the margin of the grid, at the cost of a longer computational time for the same interval size, to move the oscillation area in a region in which we are not interested.

Coming now to the comparison between the different schemes, we can say that Scheme 2 and 3 provide a material improvement over Scheme 1, as we have seen in the case of the convergence to the Black and Scholes case. Having to choose between Scheme 2 and 3, we noted that scheme three does not provide any material further improvement over Scheme 2. In particular we could not find any case where the stability or the oscillation issue was solved by using Scheme 3 rather than 2. On the other side we prefer Scheme 2 over Scheme 3 because we believe it is more consistent in terms of theoretical foundations. In fact Scheme 2 treats implicitly the small jumps of size smaller than a space interval; this is important because we know that the Lévy measure has a singularity point for jumps of size zero. In addition to this, Scheme 3 treats implicitly other two elements corresponding to parts of 
the integrals representing very large jumps. This does not seem to provide stability improvement as showed by the numerical experiments. Moreover this seems to be a bit arbitrary: we agree that having the whole integral treated fully implicitly would be too expensive, but there are other terms containing $W\left(x_{i-1}, t_{j+1}\right), W\left(x_{i}, t_{j+1}\right)$ and $W\left(x_{i+1}, t_{j+1}\right)$ in the jumps of size larger than a single space intervals but still not so big to take the stock outside the range. These elements could be moved to the left hand side of the equations without impairing the possibility of solving the system with the Gauss's elimination technique. Hence we believe that, while choosing to treat only the small jumps implicitly is justified, it is not justified to discriminate among the other terms and we therefore prefer to stick with Scheme 2. Therefore in the remaining part of this work we will always use Scheme 2 or modifications based on this approach to price American options and barrier options. All the numerical experiments presented in the remaining part of this dissertation will be realized using Scheme 2. Appendix B provides code in $\mathrm{C}$ language to price options under variance gamma utilizing Scheme 2. 


\subsubsection{Calls: Sensitivity Analysis}

In this section we want to study the sensitivity of vanilla European call prices to the parameters affecting volatility, skewness and kurtosis of the distribution of the underlying returns. We remember from section 2.3 that the three parameters which enter in the solutions we have seen above, that is $\sigma, \nu$ and $\vartheta$, are not themselves directly volatility, skewness and kurtosis. In particular we have seen that the second, third and fourth moment can be written in the following way:

$$
\begin{gathered}
E\left[(X(t)-E[X(t)])^{2}\right]=\left(\vartheta^{2} \nu+\sigma^{2}\right) t \\
E\left[(X(t)-E[X(t)])^{3}\right]=\left(2 \vartheta^{3} \nu^{2}+3 \sigma^{2} \vartheta \nu\right) t \\
E\left[(X(t)-E[X(t)])^{4}\right]= \\
=\left(3 \sigma^{4} \nu+12 \sigma^{2} \vartheta^{2} \nu^{2}+6 \vartheta^{4} \nu^{3}\right) t+\left(3 \sigma^{4}+6 \sigma^{2} \vartheta^{2} \nu+3 \vartheta^{4} \nu^{2}\right) t^{2}
\end{gathered}
$$

Although we generally think of $\sigma$ as the parameter which impacts volatility the most, and although we consider $\nu$ as the main driver of kurtosis and $\vartheta$ as the main variable affecting skewness, we can see that each of the three parameters enters in the definition of each of the moments. Hence it is not possible in general to separate the impact of a parameter on a moment without affecting the other moments too. An exception is the case of $\vartheta=0$ that is the case of a symmetric distribution. In this case the volatility is controlled solely by $\sigma$; the kurtosis, as measured by the proportion between the fourth moment and square of the second moment, is controlled only by $\nu$ and the skewness is controlled only by $\vartheta$ in the sense that if $\vartheta=0$ the third moment is equal to zero regardless of the values of the other two parameters. To facilitate the understanding of the graphs that we will present below, we therefore report together $\sigma$ and the value of the standard deviation. Moreover we will write $\vartheta$ together with the proportion obtained by dividing the third moment by the cube of the square root of the second moment. Finally we will show $\nu$ together with the proportion between the fourth moment and the square of the second moment.

The numerical experiments presented here can be obtained using the code in $\mathrm{C}$ language available in appendix B. The vanilla call case studied here can is realized by choosing the following variable values:

- callput $=1$ 
- euroamerican $=0$

- barrier_switch $=0$

in the $\mathrm{C}$ code.

\section{Sensitivity with Respect to $\sigma$}

Let's start considering the sensitivity of the vanilla European call option price with respect to $\sigma$. This experiment is realized using the following parameters:

- $T=1 \quad$ Time to maturity in years;

- $K=\$ 100$ Strike price;

- $r=0.03 \quad$ Interest rate;

- $q=0.01$ Dividend yield;

- $\vartheta=-0.1$

- $\nu=0.2$

- $N=1700$ Number of space intervals;

- $M=400 \quad$ Number of time intervals;

- $x_{\min }=1.60944$ Min $x$ value considered, corresponding to $S=\$ 5$;

- $x_{\max }=5.85793$ Max $x$ value considered, corresponding to $S=\$ 350$;

- $\Delta x \simeq 0.0025 \quad$ Size of a space interval;

- $\Delta t=0.0025 \quad$ Size of a time interval;

- $\frac{\Delta t}{\Delta x} \simeq 1$

In this contest we vary $\sigma$ from $10 \%$ to $70 \%$, while keeping $\nu, \vartheta$ and the other variables constant. At the various level of $\sigma$ we have the following levels of variance, skewness and kurtosis: 


\begin{tabular}{|c|c|c|c|c|c|}
\hline$\sigma$ & 0.1 & 0.2 & 0.3 & 0.5 & 0.7 \\
\hline$\sqrt{E\left[(X(t)-E[X(t)])^{2}\right]}$ & 0.110 & 0.205 & 0.303 & 0.502 & 0.701 \\
\hline$\frac{E\left[(X(t)-E[X(t)])^{3}\right]}{\sqrt{\left\{E\left[(X(t)-E[X(t)])^{2}\right]\right\}^{3}}}$ & -0.517 & -0.288 & -0.196 & -0.119 & -0.085 \\
\hline$\frac{E\left[(X(t)-E[X(t)])^{4}\right]}{\left\{E\left[(X(t)-E[X(t)])^{2}\right]\right\}^{2}}$ & 3.783 & 3.656 & 3.626 & 3.609 & 3.605 \\
\hline
\end{tabular}

We can see that as $\sigma$ increases clearly volatility increases. Moreover we can note that, on the other side, the absolute value of skewness and kurtosis decreases as $\sigma$ becomes larger. The graph shows that as $\sigma$ increases, the option value increases.

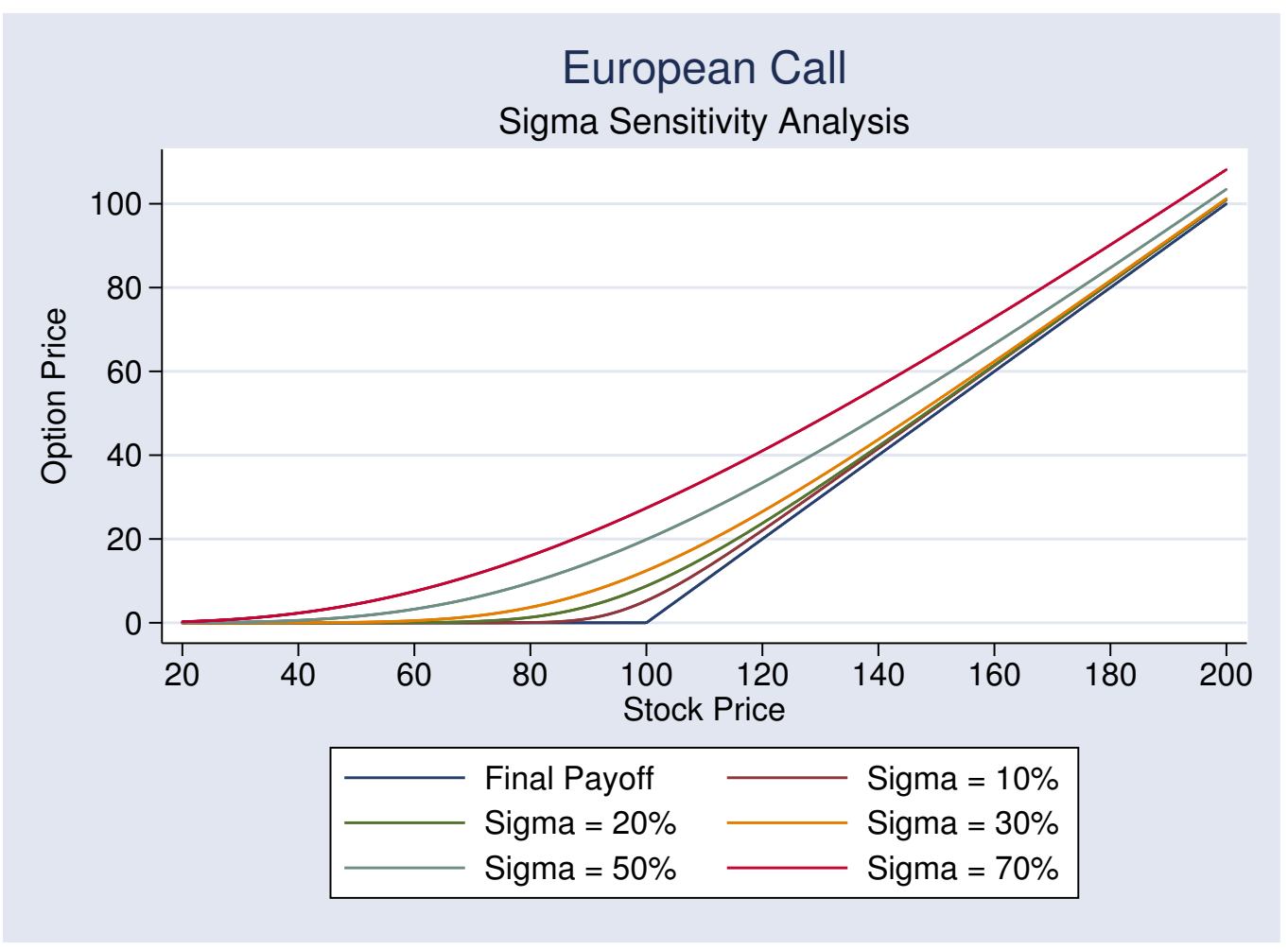

Figure 4.3: European Call: $\sigma$ Sensitivity Analysis 


\section{Sensitivity with Respect to $\nu$}

Let's now consider the sensitivity of the vanilla European call price with respect to $\nu$. The parameters used in this experiment are the following:

- $T=1 \quad$ Time to maturity in years;

- $K=\$ 100$ Strike price;

- $r=0.03$ Interest rate;

- $q=0.01 \quad$ Dividend yield;

- $\sigma=0.2$

- $\vartheta=0$;

- $N=1700$ Number of space intervals;

- $M=400$ Number of time intervals;

- $x_{\min }=1.60944$ Min $x$ value considered, corresponding to $S=\$ 5$;

- $x_{\max }=5.85793$ Max $x$ value considered, corresponding to $S=\$ 350$;

- $\Delta x \simeq 0.0025 \quad$ Size of a space interval;

- $\Delta t=0.0025 \quad$ Size of a time interval;

- $\frac{\Delta t}{\Delta x} \simeq 1$;

Starting from these values we modify $\nu$ so that it takes values $0.01,0.5$ and 2.0. We can see that here $\vartheta=0$, hence the change in $\nu$ will affect only the kurtosis of the distribution. In fact equation (4.14) for the second moment becomes simply $\sigma^{2} t$. Moreover the skewness remains fixed at zero as $\nu$ changes. The impact of $\nu$ changes in this experiment is summarized in this table:

\begin{tabular}{|c|c|c|c|}
\hline$\nu$ & 0.01 & 0.5 & 2 \\
\hline$\sqrt{E\left[(X(t)-E[X(t)])^{2}\right]}$ & 0.2 & 0.2 & 0.2 \\
\hline$\frac{E\left[(X(t)-E[X(t)])^{3}\right]}{\sqrt{\left\{E\left[(X(t)-E[X(t)])^{2}\right]\right\}^{3}}}$ & 0 & 0 & 0 \\
\hline$\frac{E\left[(X(t)-E[X(t)])^{4}\right]}{\left\{E\left[(X(t)-E[X(t)])^{2}\right]\right\}^{2}}$ & 3.03 & 4.5 & 9 \\
\hline
\end{tabular}


We can note that the first case considered in the experiment with $\vartheta=0$ and $\nu=0.01$ is very close to the geometric Brownian motion case, where both $\vartheta$ and $\nu$ are equal to zero. Moreover we can see that when $\vartheta=0, \nu$ can be interpreted as a percentage increase in kurtosis above the normal level, in fact here, for one time interval, as it is the case here, we have

$$
\frac{E\left[(X(t)-E[X(t)])^{4}\right]}{\left\{E\left[(X(t)-E[X(t)])^{2}\right]\right\}^{2}}=3(1+\nu)
$$

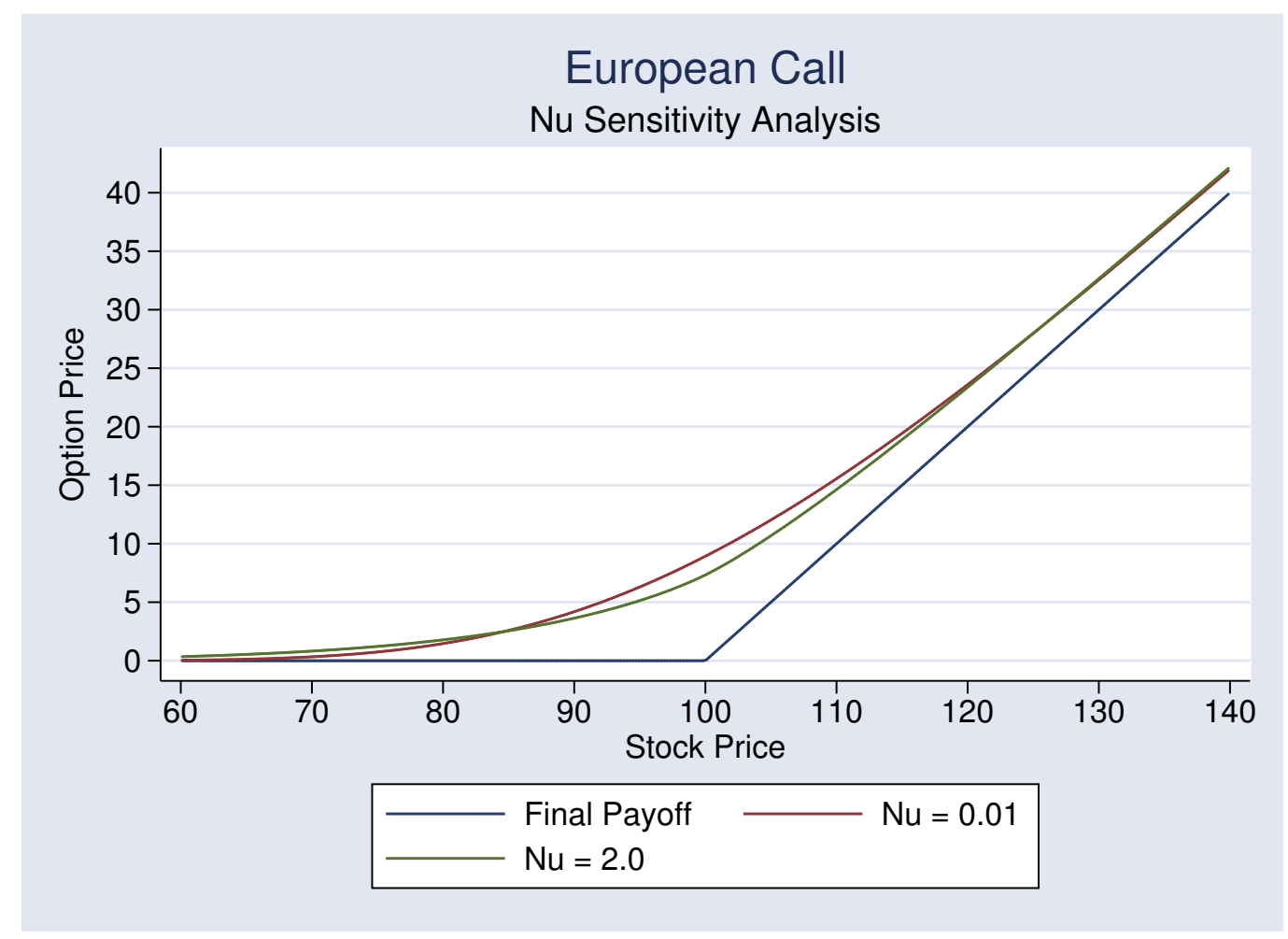

Figure 4.4: European Call: $\nu$ Sensitivity Analysis

In this first graph, we compare just the cases of very small kurtosis (a standardized value of 3.03) and a case of large kurtosis (a standardized value of 9). We can see the that, depending on the region considered, it is different 
which option is worth more. In particular we can see that there is an area around at the money where the option with lower $\nu$ is worth more, while there are two areas on the sides where the option whose underlying has higher $\nu$ is worth more.

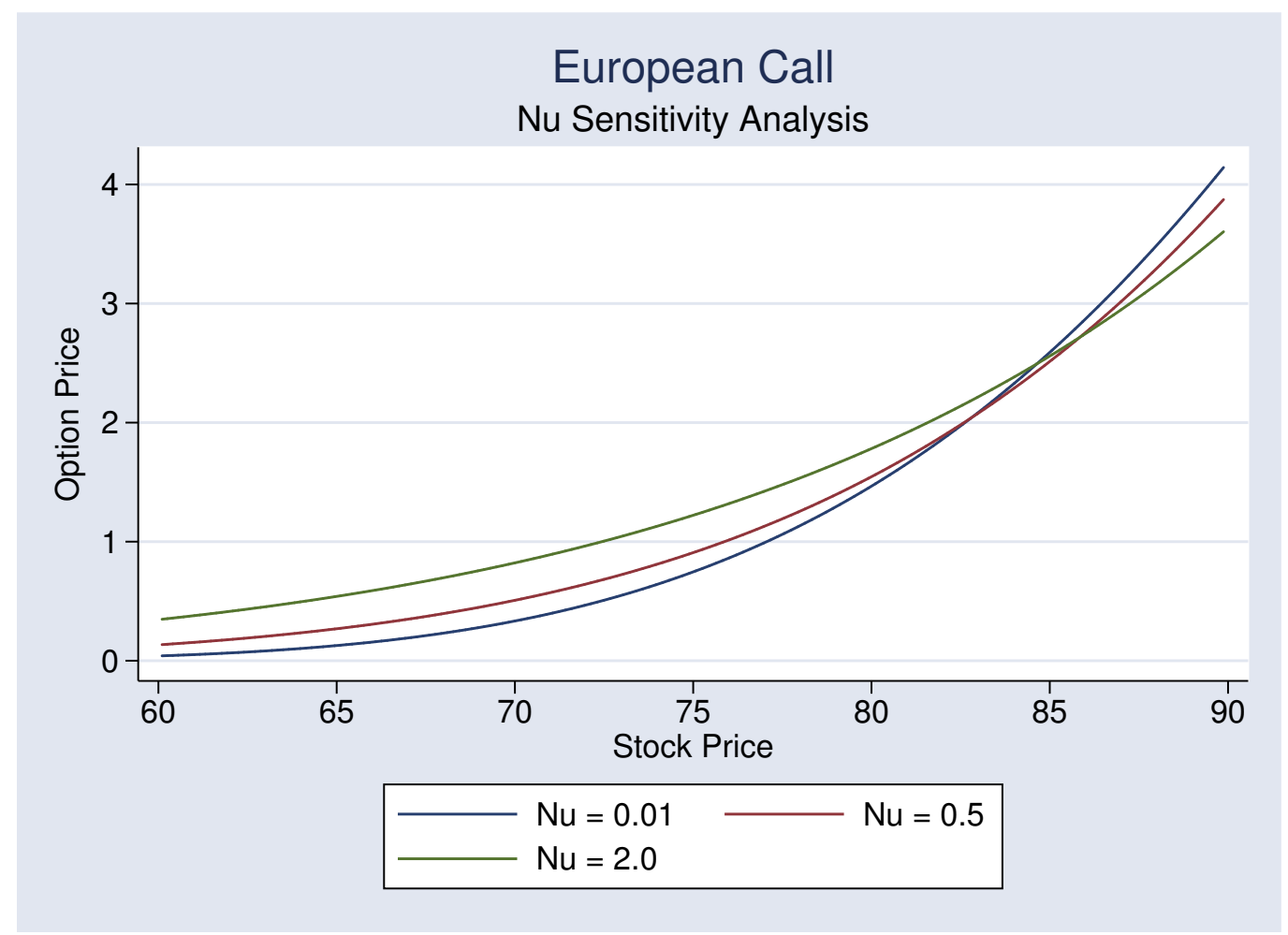

Figure 4.5: European Call: $\nu$ Sensitivity Analysis, Detail

Above we present a zoom for the out of the money area, where we also include a third case with $\nu=0.5$. We can better note in this graph the fact that the the higher kurtosis does not produce a larger option value everywhere. The reason is the fact that being the probability of all possible moves fixed at one, if we increase the probability of larger jumps, we reduce the probability of smaller moves and so the option near the money may be negatively affected by the increased kurtosis. Note that in this case, we assume $\vartheta=0$ and so 
we do not have interaction of volatility and skewness. If $\vartheta$ had been different from zero an increase in $\nu$ would have produced also an increase in volatility and skewness and so the analysis would have been more complex. In figure 4.5 we can see that in the area where the stock is more out of the money, a higher kurtosis increases the option price, because it more likely that the stock will jump to an in the money position.

The last case we show here is a zoom in the area where the option is around at the money. In this case, having a large kurtosis reduces the value of the option.

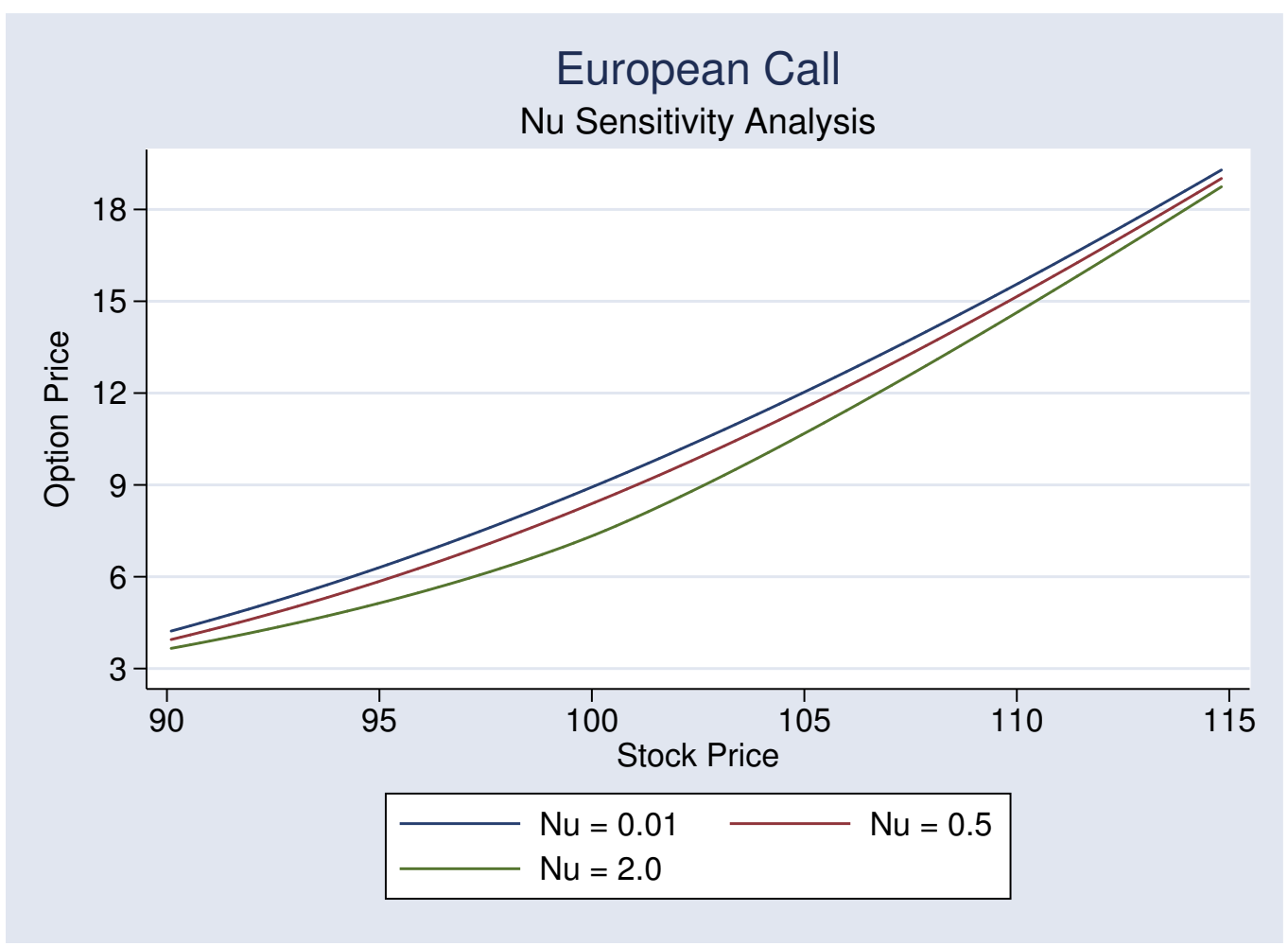

Figure 4.6: European Call: $\nu$ Sensitivity Analysis, Detail 


\section{Sensitivity with Respect to $\vartheta$}

We show now some analysis of the sensitivity of a vanilla European call option with respect to $\vartheta$. The parameters used in this case are the following:

- $T=1 \quad$ Time to maturity in years;

- $K=\$ 100$ Strike price;

- $r=0.03 \quad$ Interest rate;

- $q=0.01 \quad$ Dividend yield;

- $\sigma=0.2$;

- $\nu=0.2$;

- $N=1700$ Number of space intervals;

- $M=400$ Number of time intervals;

- $x_{\min }=1.60944$ Min $x$ value considered, corresponding to $S=\$ 5$;

- $x_{\max }=5.85793$ Max $x$ value considered, corresponding to $S=\$ 350$;

- $\Delta x \simeq 0.0025$ Size of a space interval;

- $\Delta t=0.0025 \quad$ Size of a time interval;

- $\frac{\Delta t}{\Delta x} \simeq 1$

Keeping these values constant, we study the impact of moving $\vartheta$ from +0.2 to -1.0 . The change of $\vartheta$ produces the following impact on volatility, skewness and kurtosis:

\begin{tabular}{|c|c|c|c|c|c|c|}
\hline$\vartheta$ & +0.2 & 0.0 & -0.2 & -0.5 & -0.7 & -1.0 \\
\hline$\sqrt{E\left[(X(t)-E[X(t)])^{2}\right]}$ & 0.22 & 0.20 & 0.22 & 0.30 & 0.37 & 0.49 \\
\hline$\frac{E\left[(X(t)-E[X(t)])^{3}\right]}{\sqrt{\left\{E\left[(X(t)-E[X(t)])^{2}\right]\right\}^{3}}}$ & +0.52 & 0.00 & -0.52 & -0.81 & -0.86 & -0.88 \\
\hline$\frac{E\left[(X(t)-E[X(t)])^{4}\right]}{\left\{E\left[(X(t)-E[X(t)])^{2}\right]\right\}^{2}}$ & 3.78 & 3.60 & 3.78 & 4.08 & 4.15 & 4.18 \\
\hline
\end{tabular}


In the first graph we concentrate on cases where there is no skewness or the skewness in negative. Negative skewness or at most no skewness is the case most in agreement with market data and it is the case which makes more sense also from a theoretical point of view. From the table we can see that, if we start from $\vartheta=0$ and we move to progressively larger negative values of course the negative skewness will increase. Moreover and more interestingly we can see that volatility and skewness will increase too. This explain the fact that the graph shows an increasing option value as the $\vartheta$ become negative larger in absolute value.

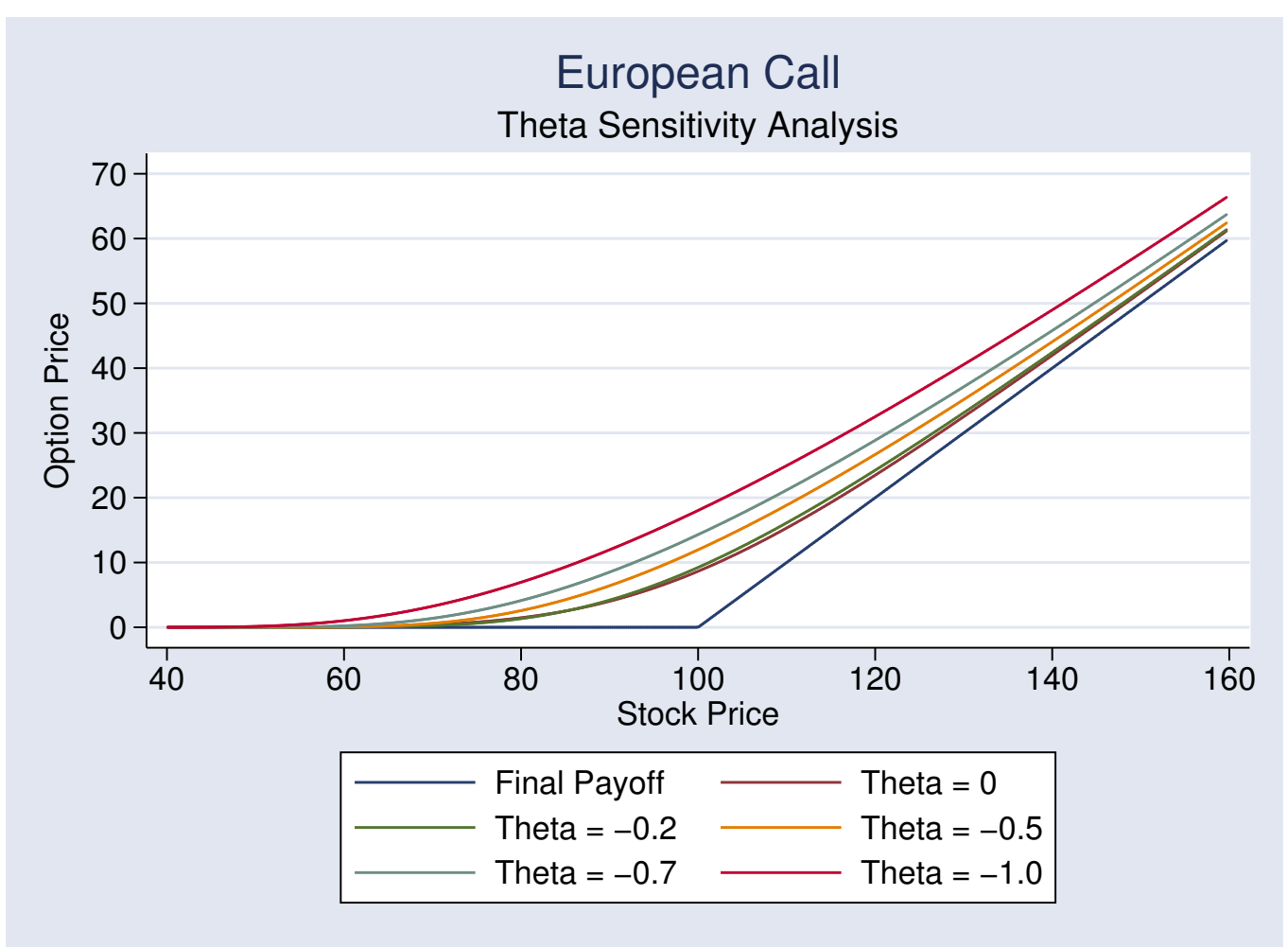

Figure 4.7: European Call: $\vartheta$ Sensitivity Analysis 
In this second graph we compare the case of $\vartheta=-0.2$ with the case $\vartheta=$ +0.2 . From table above, we can see that in these two scenarios volatility and kurtosis are unchanged, because $\vartheta$ enters in equations (4.14) and (4.16) only in the form of square of fourth power. Hence in this experiment all the difference is in the skewness itself. In the case of positive skewness, the option has a higher value when it is out of the money since it is more likely that there will be larger jumps taking it in the money.

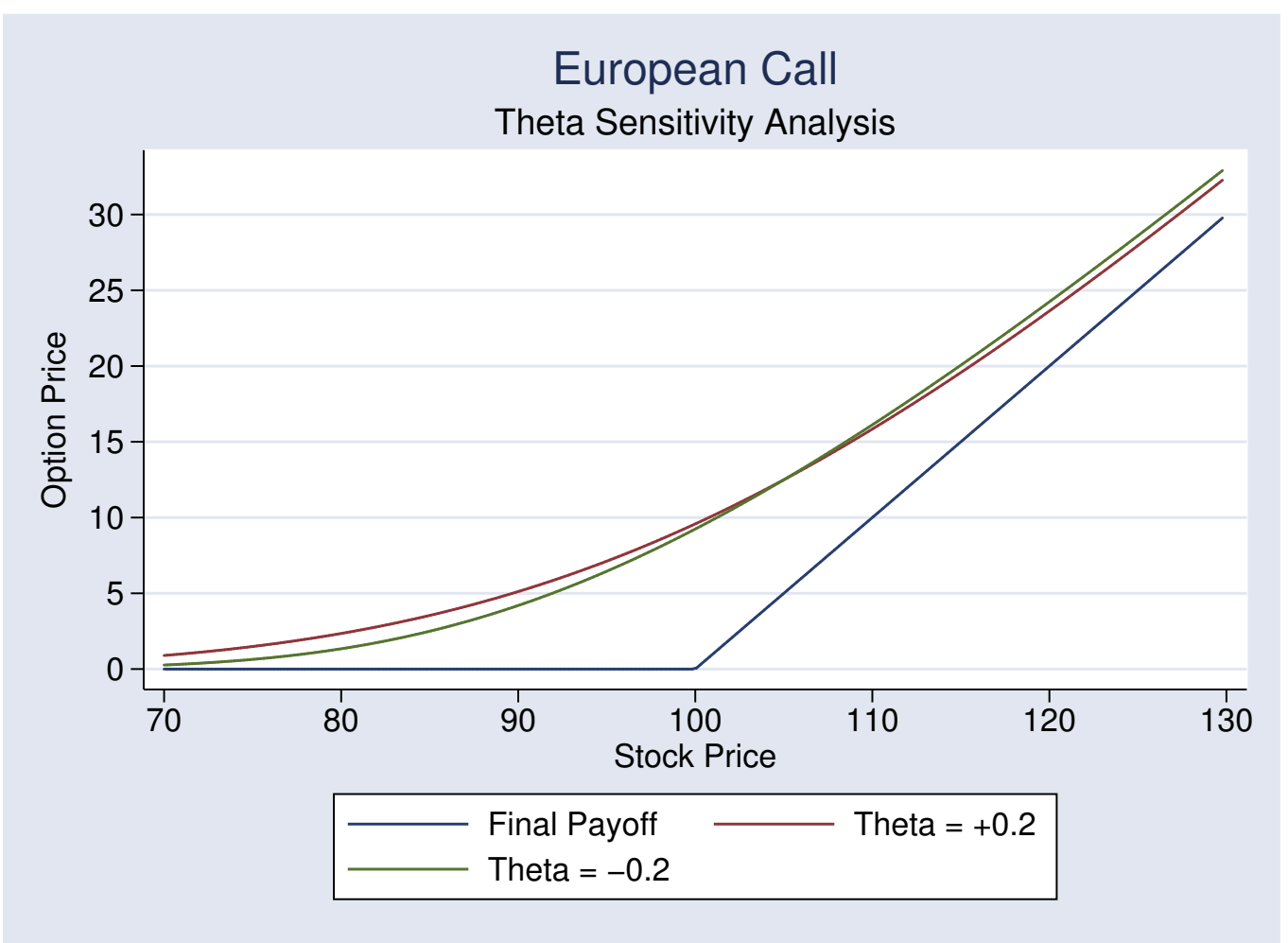

Figure 4.8: European Call: $\vartheta$ Sensitivity Analysis, Detail

\subsubsection{Puts: Sensitivity Analysis}

We consider here some experiments on the sensitivity of vanilla European put prices as computed under variance gamma as $\sigma, \nu$ and $\vartheta$ changes. 
The numerical experiments presented here can be obtained using the code in $\mathrm{C}$ language available in appendix B. The vanilla put case studied here can is realized by choosing the following variable values:

- callput $=0$

- euroamerican $=0$

- barrier_switch $=0$

in the $\mathrm{C}$ code.

\section{Sensitivity with Respect to $\sigma$}

Let's consider here the sensitivity of vanilla European put option prices to $\sigma$. The parameters used in this case are the following:

- $T=1 \quad$ Time to maturity in years;

- $K=\$ 100$ Strike price;

- $r=0.03$ Interest rate;

- $q=0.01 \quad$ Dividend yield;

$\bullet \vartheta=-0.1$

- $\nu=0.2$;

- $N=1700$ Number of space intervals;

- $M=400$ Number of time intervals;

- $x_{\text {min }}=1.60944$ Min $x$ value considered, corresponding to $S=\$ 5$;

- $x_{\max }=5.85793$ Max $x$ value considered, corresponding to $S=\$ 350$;

- $\Delta x \simeq 0.0025 \quad$ Size of a space interval;

- $\Delta t=0.0025 \quad$ Size of a time interval;

- $\frac{\Delta t}{\Delta x} \simeq 1$

Keeping $\vartheta, \nu$ and the other parameters constant, we move $\sigma$ from $10 \%$ to 90\%. Volatility, skewness and kurtosis are affected in the following way: 


\begin{tabular}{|c|c|c|c|c|c|c|c|}
\hline$\sigma$ & 0.1 & 0.2 & 0.3 & 0.4 & 0.5 & 0.7 & 0.9 \\
\hline$\sqrt{E\left[(X(t)-E[X(t)])^{2}\right]}$ & 0.110 & 0.205 & 0.303 & 0.402 & 0.502 & 0.701 & 0.901 \\
\hline$\frac{E\left[(X(t)-E[X(t)])^{3}\right]}{\sqrt{\left\{E\left[(X(t)-E[X(t)])^{2}\right]\right\}^{3}}}$ & -0.517 & -0.288 & -0.196 & -0.148 & -0.119 & -0.085 & -0.067 \\
\hline$\frac{E\left[(X(t)-E[X(t)])^{4}\right]}{\left\{E\left[(X(t)-E[X(t)])^{2}\right]\right\}^{2}}$ & 3.783 & 3.656 & 3.626 & 3.615 & 3.609 & 3.605 & 3.603 \\
\hline
\end{tabular}

We can see that when $\sigma$ increases, volatility increases and skewness and kurtosis are reduced. The graph below shows that the option value increases as $\sigma$ increases.

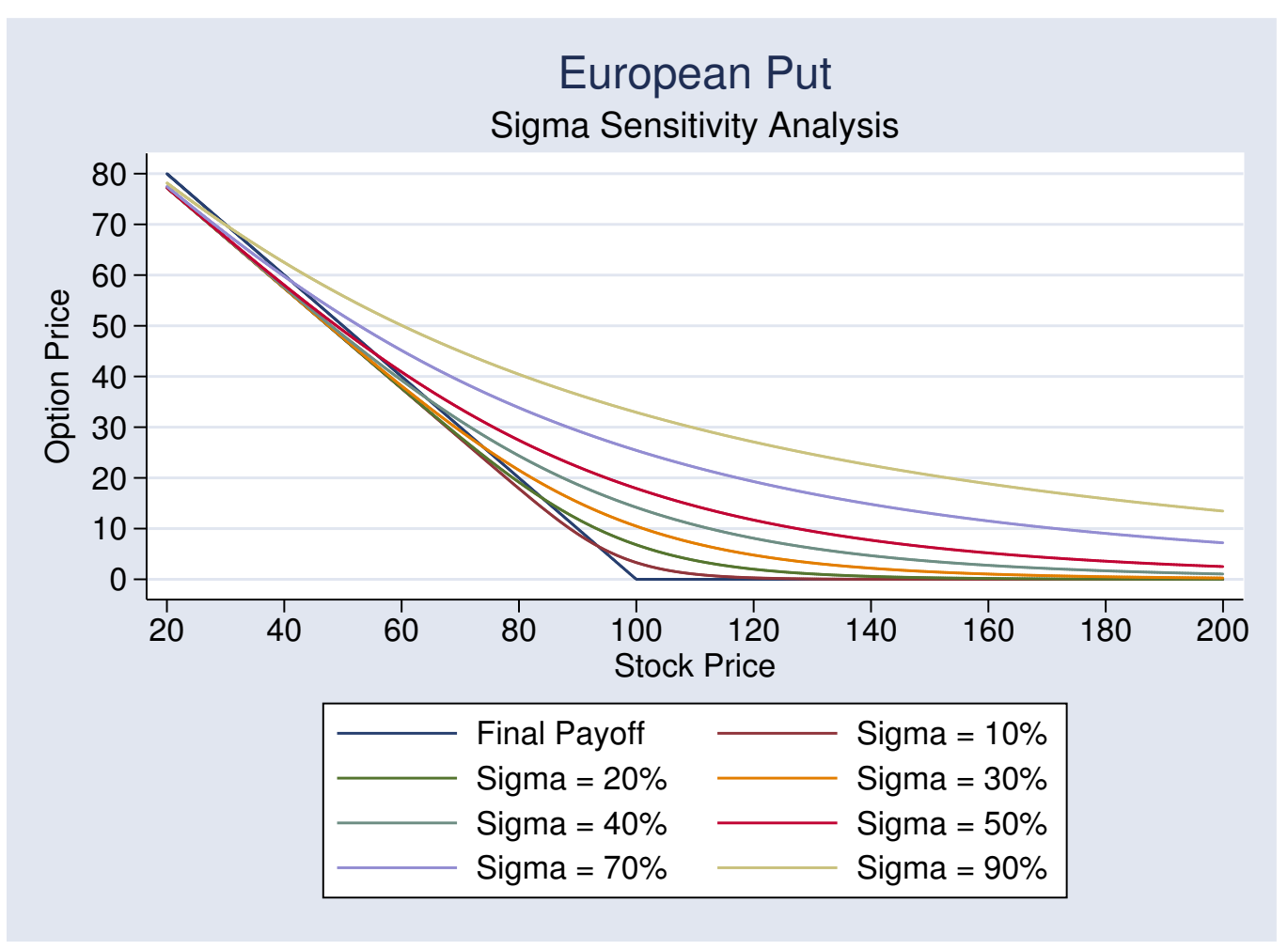

Figure 4.9: European Put: $\sigma$ Sensitivity Analysis 


\section{Sensitivity with Respect to $\nu$}

We consider here the sensitivity of a vanilla European put option with respect to $\nu$. The parameters used for these experiments are the following:

- $T=1 \quad$ Time to maturity in years;

- $K=\$ 100$ Strike price;

- $r=0.03 \quad$ Interest rate;

- $q=0.01 \quad$ Dividend yield;

- $\sigma=0.2$;

- $\vartheta=0$;

- $N=1700$ Number of space intervals;

- $M=400$ Number of time intervals;

- $x_{\min }=1.60944$ Min $x$ value considered, corresponding to $S=\$ 5$;

- $x_{\max }=5.85793$ Max $x$ value considered, corresponding to $S=\$ 350$;

- $\Delta x \simeq 0.0025 \quad$ Size of a space interval;

- $\Delta t=0.0025 \quad$ Size of a time interval;

- $\frac{\Delta t}{\Delta x} \simeq 1$;

Using these values, we modify $\nu$ from 0.01 to 2.0 . We note that here, as we did for the call case, we constrained $\vartheta=0$. By doing this, volatility and and skewness are not affected by $\nu$ and the impact of changing $\nu$ is reflected only on the kurtosis. In particular we have the following values as $\nu$ changes: 


\begin{tabular}{|c|c|c|c|c|}
\hline$\nu$ & 0.01 & 0.5 & 1 & 2 \\
\hline$\sqrt{E\left[(X(t)-E[X(t)])^{2}\right]}$ & 0.2 & 0.2 & 0.2 & 0.2 \\
\hline$\frac{E\left[(X(t)-E[X(t)])^{3}\right]}{\sqrt{\left\{E\left[(X(t)-E[X(t)])^{2}\right]\right\}^{3}}}$ & 0 & 0 & 0 & 0 \\
\hline$\frac{E\left[(X(t)-E[X(t)])^{4}\right]}{\left\{E\left[(X(t)-E[X(t)])^{2}\right]\right\}^{2}}$ & 3.03 & 4.5 & 6 & 9 \\
\hline
\end{tabular}

In the first graph we compare a case of low kurtosis with a case of higher kurtosis. We remember that when $\vartheta=0$ and $\nu=0.01$ we are quite close to the geometric Brownian motion case.

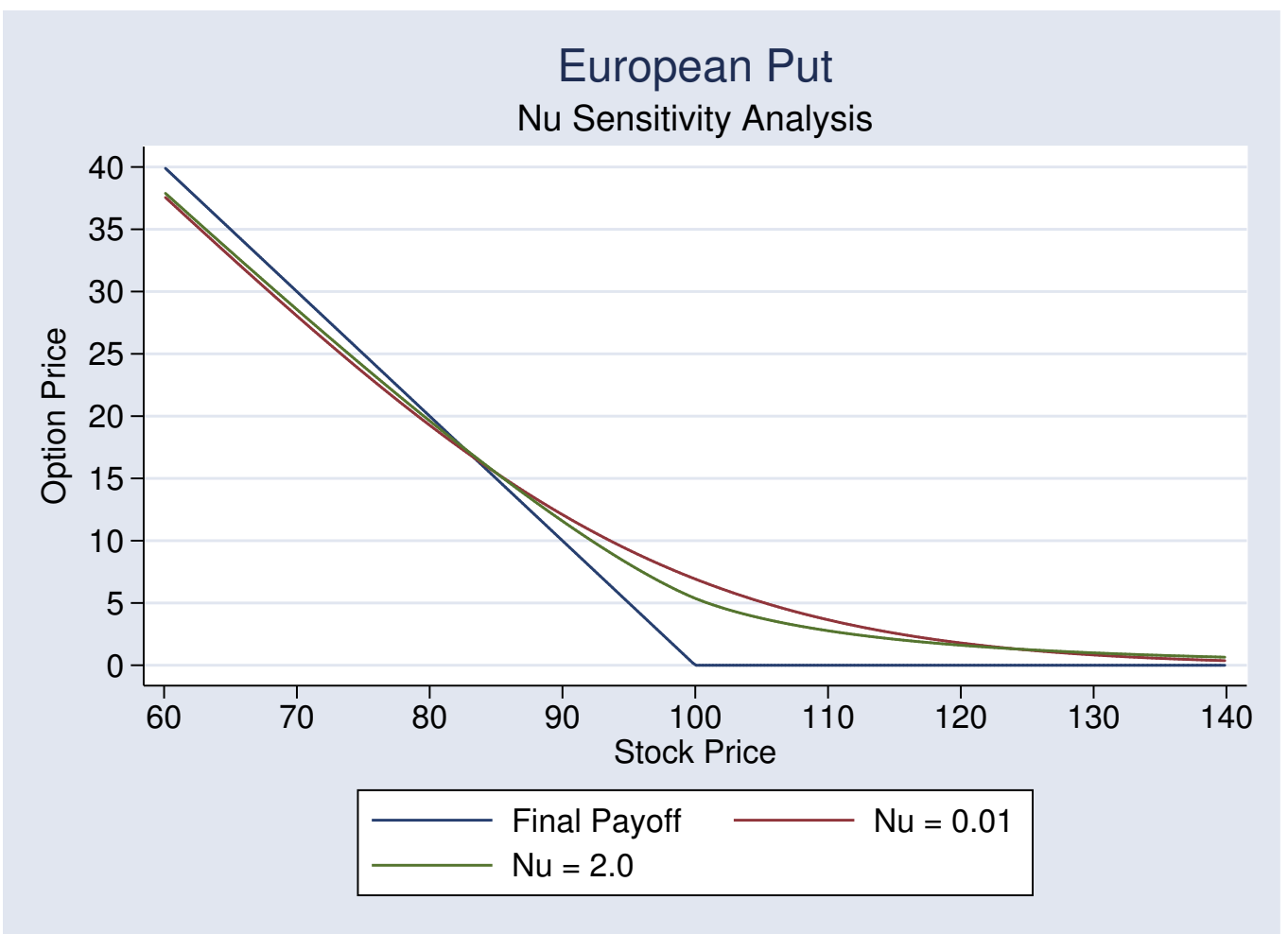

Figure 4.10: European Put: $\nu$ Sensitivity Analysis

We can see from this graph that we have a region around at the money where 
the option whose underlying has lower kurtosis is worth more, while on the two sides the option on the stock with higher $\nu$ is worth more.

In this second case we consider a zoom in the area near the money. We can see that in this area a large kurtosis reduces the value of the option.

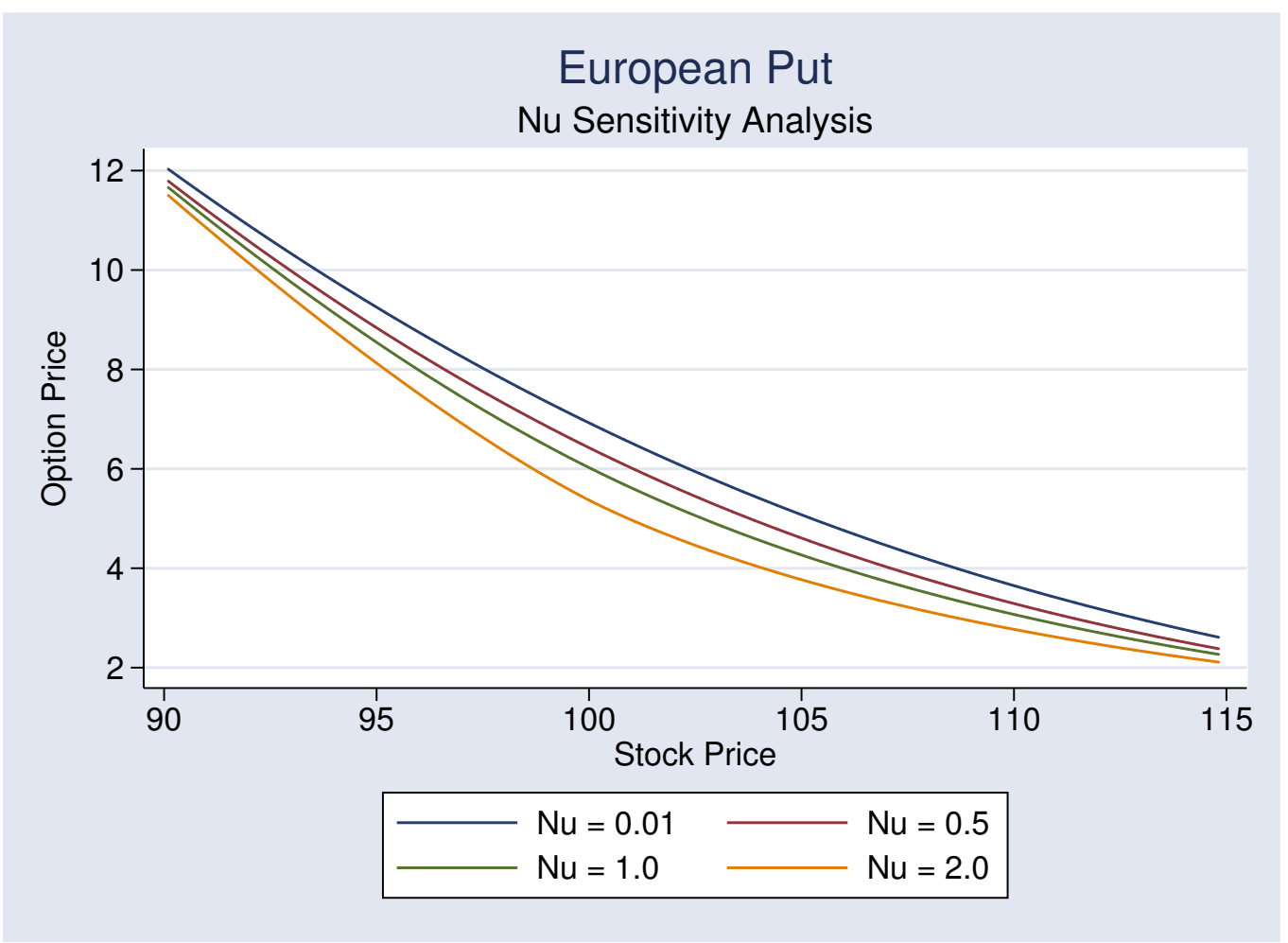

Figure 4.11: European Put: $\nu$ Sensitivity Analysis, Detail 
In this last case, we consider an in the money area. We compare here a level of kurtosis close to the normal one with a larger kurtosis case and we see that the option having large kurtosis is worth more as the stock moves far from the money.

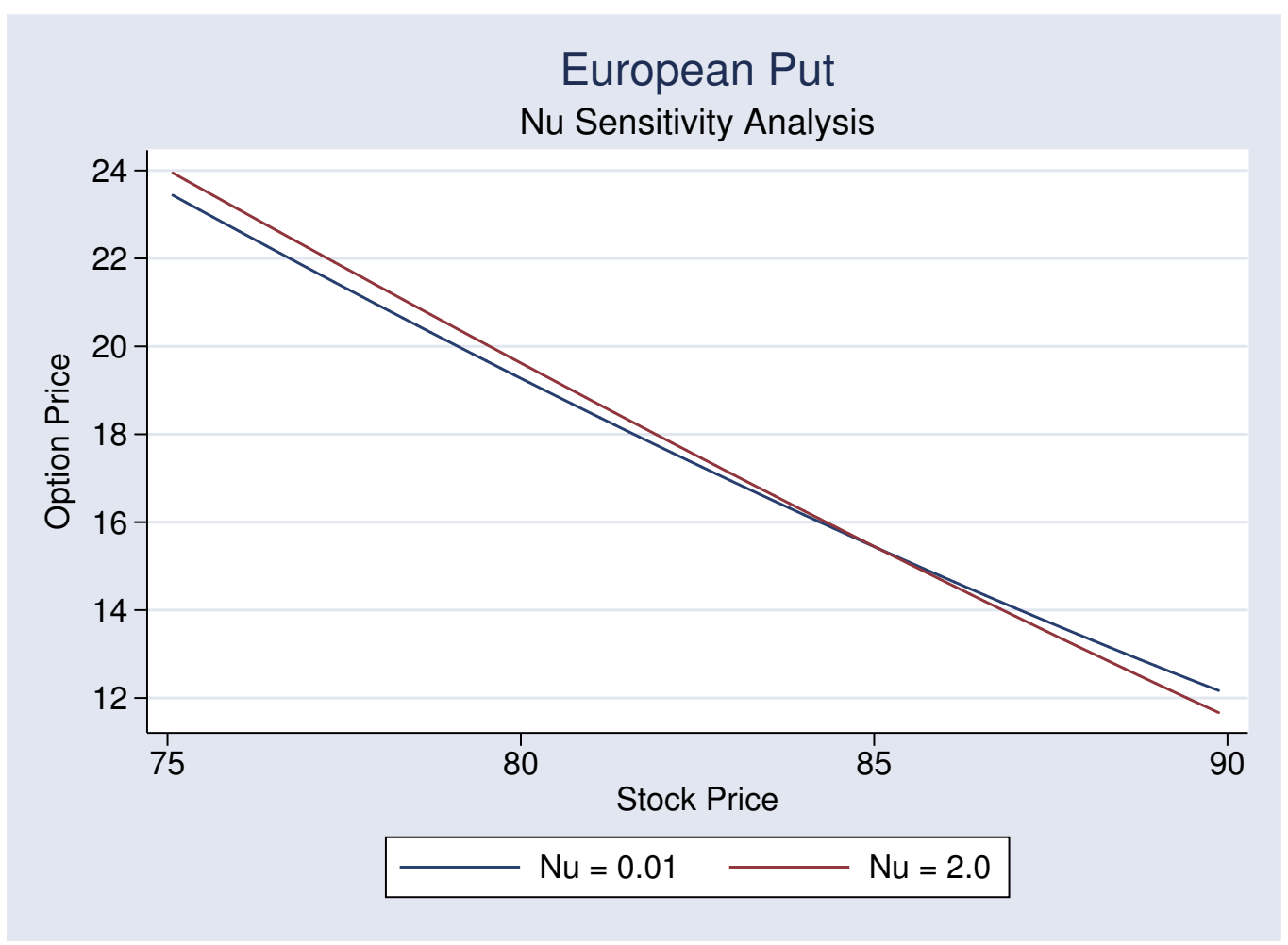

Figure 4.12: European Put: $\nu$ Sensitivity Analysis, Detail 


\section{Sensitivity with Respect to $\vartheta$}

We consider now the sensitivity of European vanilla put options with respect to $\vartheta$. In particular let's consider the following parameters:

- $T=1 \quad$ Time to maturity in years;

- $K=\$ 100$ Strike price;

- $r=0.03 \quad$ Interest rate;

- $q=0.01 \quad$ Dividend yield;

- $\sigma=0.2$;

- $\nu=0.2$;

- $N=1700$ Number of space intervals;

- $M=400$ Number of time intervals;

- $x_{\min }=1.60944$ Min $x$ value considered, corresponding to $S=\$ 5$;

- $x_{\max }=5.85793$ Max $x$ value considered, corresponding to $S=\$ 350$;

- $\Delta x \simeq 0.0025 \quad$ Size of a space interval;

- $\Delta t=0.0025 \quad$ Size of a time interval;

- $\frac{\Delta t}{\Delta x} \simeq 1$;

We consider here the impact of moving $\vartheta$ from +0.2 to -1.0 . The following table shows the impact on volatility, skewness and kurtosis of a move in $\vartheta$ as the other variables are unchanged

\begin{tabular}{|c|c|c|c|c|c|c|}
\hline$\vartheta$ & +0.2 & 0.0 & -0.2 & -0.5 & -0.7 & -1.0 \\
\hline$\sqrt{E\left[(X(t)-E[X(t)])^{2}\right]}$ & 0.22 & 0.20 & 0.22 & 0.30 & 0.37 & 0.49 \\
\hline$\frac{E\left[(X(t)-E[X(t)])^{3}\right]}{\sqrt{\left\{E\left[(X(t)-E[X(t)])^{2}\right]\right\}^{3}}}$ & +0.52 & 0.00 & -0.52 & -0.81 & -0.86 & -0.88 \\
\hline$\frac{E\left[(X(t)-E[X(t)])^{4}\right]}{\left\{E\left[(X(t)-E[X(t)])^{2}\right]\right\}^{2}}$ & 3.78 & 3.60 & 3.78 & 4.08 & 4.15 & 4.18 \\
\hline
\end{tabular}


In this first graph we consider only the case of a non skewed or negative skewed distribution. This case is consistent with market data and with the economic interpretation of skewness in stock price processes. From the table above, we see that as $\vartheta$ becomes increasingly negative, the negative skewness increases. Moreover we can see that volatility and kurtosis increases as $\vartheta$ goes from 0 to -1.0. As a result of this when the put price increases as $\vartheta$ moves from zero to larger negative values.

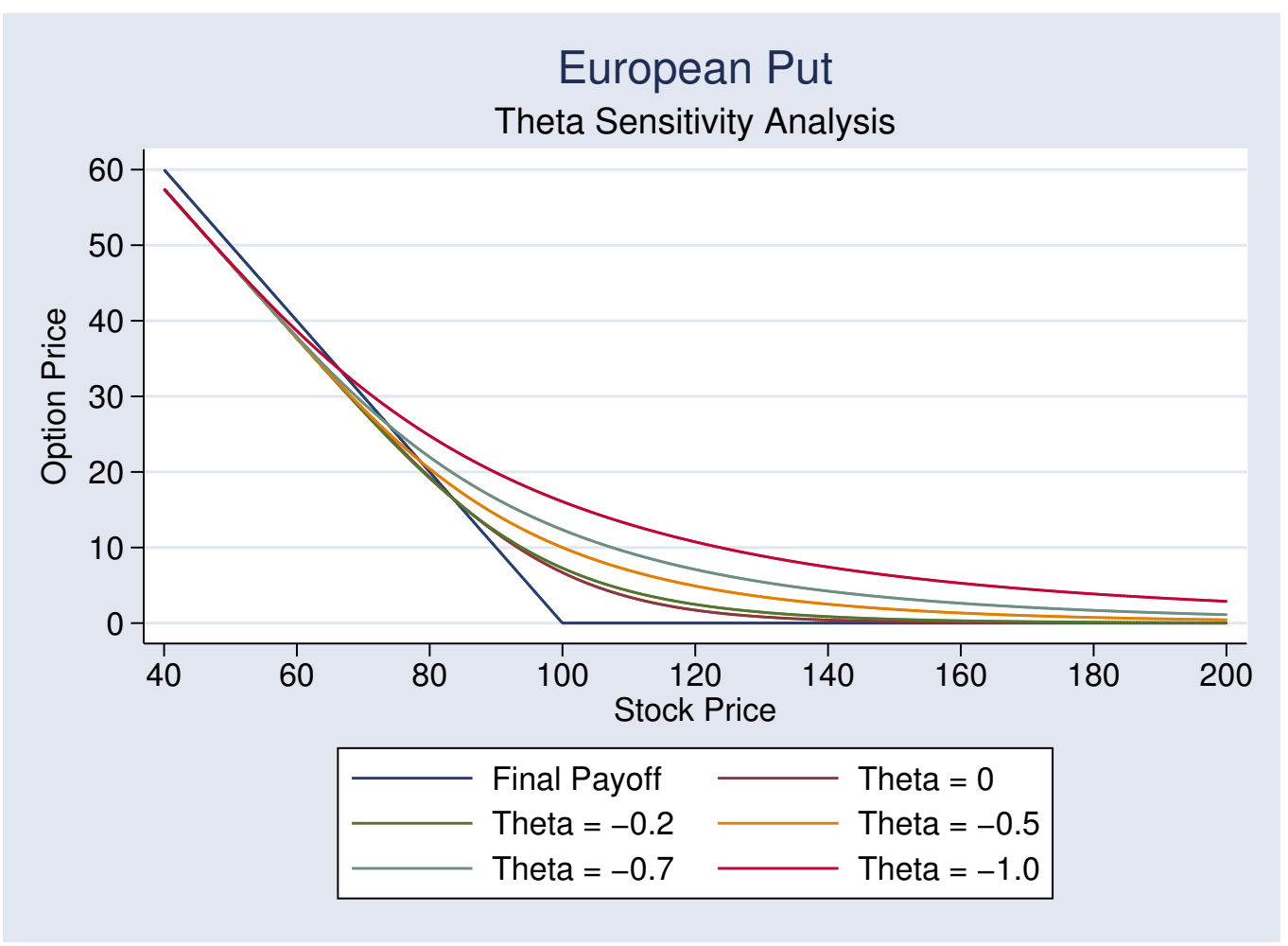

Figure 4.13: European Put: $\vartheta$ Sensitivity Analysis 
In this second graph we compare the case of $\vartheta=-0.2$ with the case of $\vartheta=+0.2$. Because $\vartheta$ impacts the second and fourth moments only in the form of its square and its fourth power, the fact that we change the sign of the variable does not produce any impact on volatility and kurtosis. In this way we isolated the skewness effect on the put price. We can see that when the option is out of the money, the option with negative skew is worth more because it is more likely that it will jump in the money.

This is consistent with what we found for the call case: in that situation the out of the money option was worth more if the skewness was positive since a positive jump was required to bring the option in the money. In the put case, on the other side, we need a negative jump to move in the money, that is why the out of the money option with negative skewness is the one having the highest value.

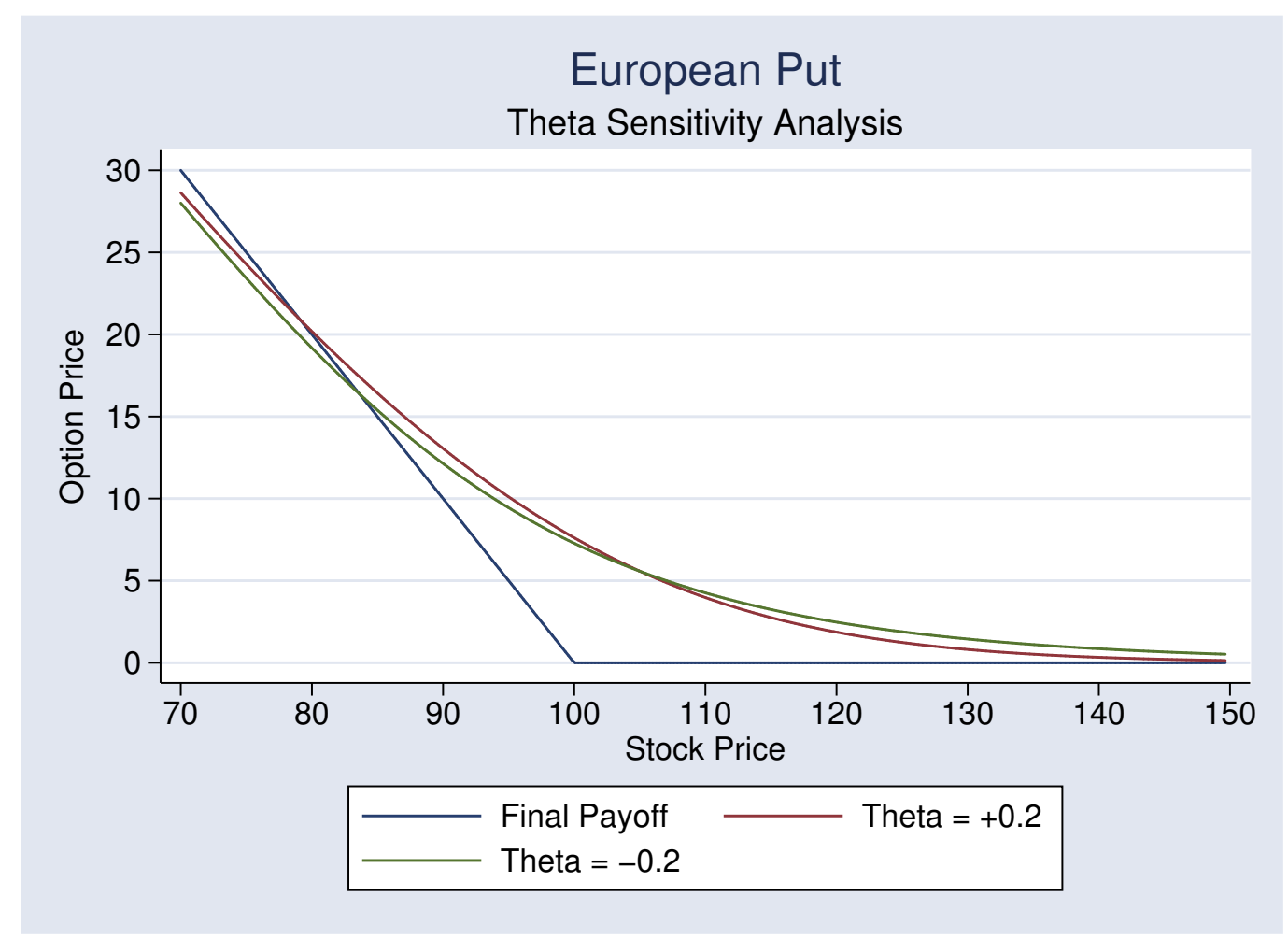

Figure 4.14: European Put: $\vartheta$ Sensitivity Analysis 


\subsubsection{Implied Volatility Analysis}

In this section we want to study the behavior of the implied volatilities corresponding to the vanilla European option prices obtained under the variance gamma process. The approach is to first price options under variance gamma and then use the obtained prices to compute correspondent implied volatilities by inverting Black and Scholes pricing formula.

\section{Experiment 1}

For this experiment, we use the following parameters:

- $T=1 \quad$ Time to maturity in years;

- $K=\$ 100$ Strike price;

- $r=0.066$ Interest rate;

- $q=0.012$ Dividend yield;

- $\sigma=0.3$

$\bullet \vartheta=-0.3$;

- $\nu=0.25$;

- $N=250$ Number of space intervals;

- $M=300$ Number of time intervals;

- $x_{\min }=2.30259$ Min $x$ value considered, corresponding to $S=\$ 10$;

- $x_{\max }=5.56068$ Max $x$ value considered, corresponding to $S=\$ 260$;

- $\Delta x=0.01303 \quad$ Size of a space interval;

- $\Delta t=0.00333 \quad$ Size of a time interval;

- $\frac{\Delta t}{\Delta x}=3.9097$; 
The vanilla European call option value given by these parameters is given by the following graph.

\section{Call Option}

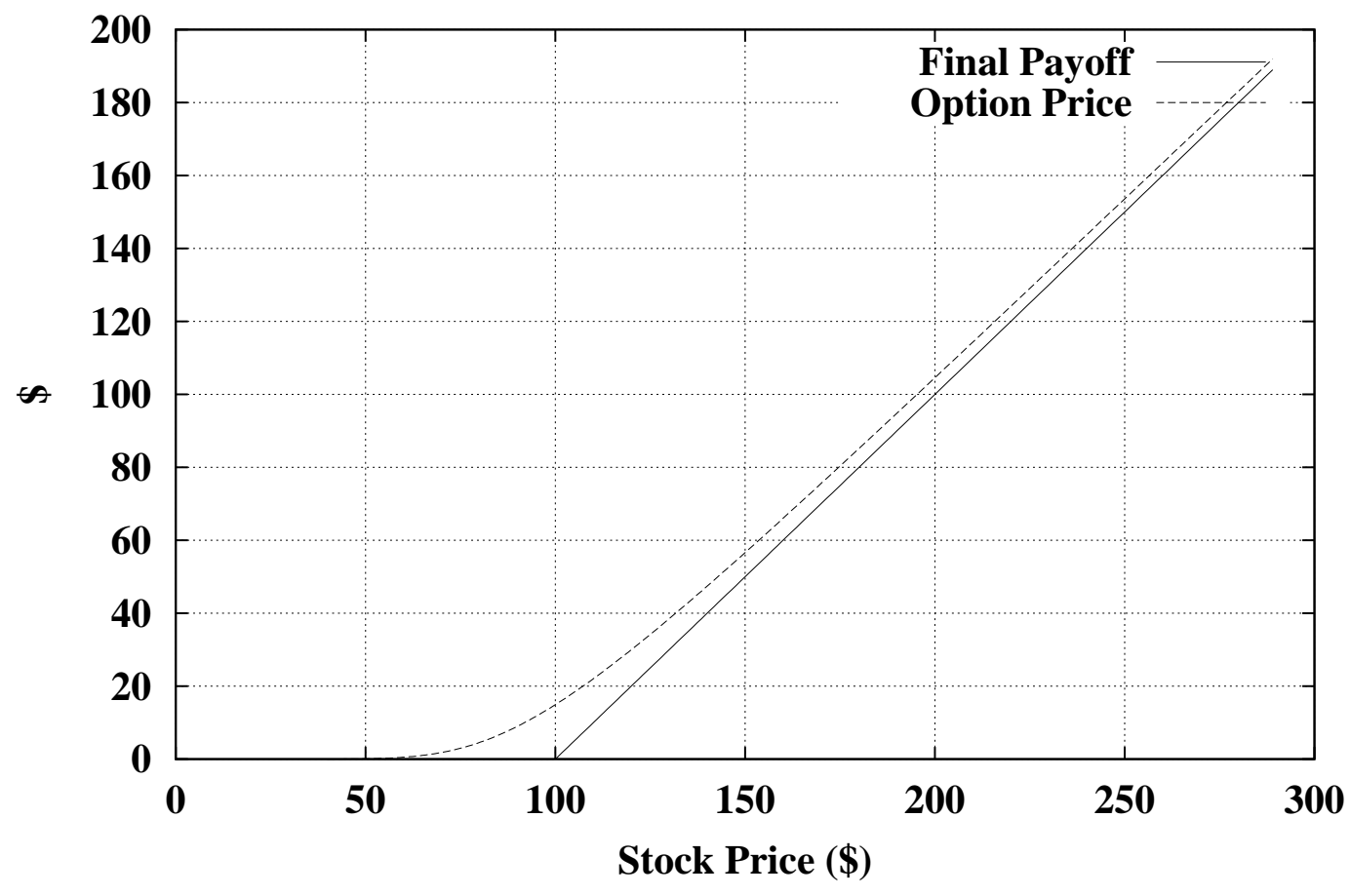

Figure 4.15: European call option under VG process 
The vanilla European put option value given by same parameters is given by the following graph.

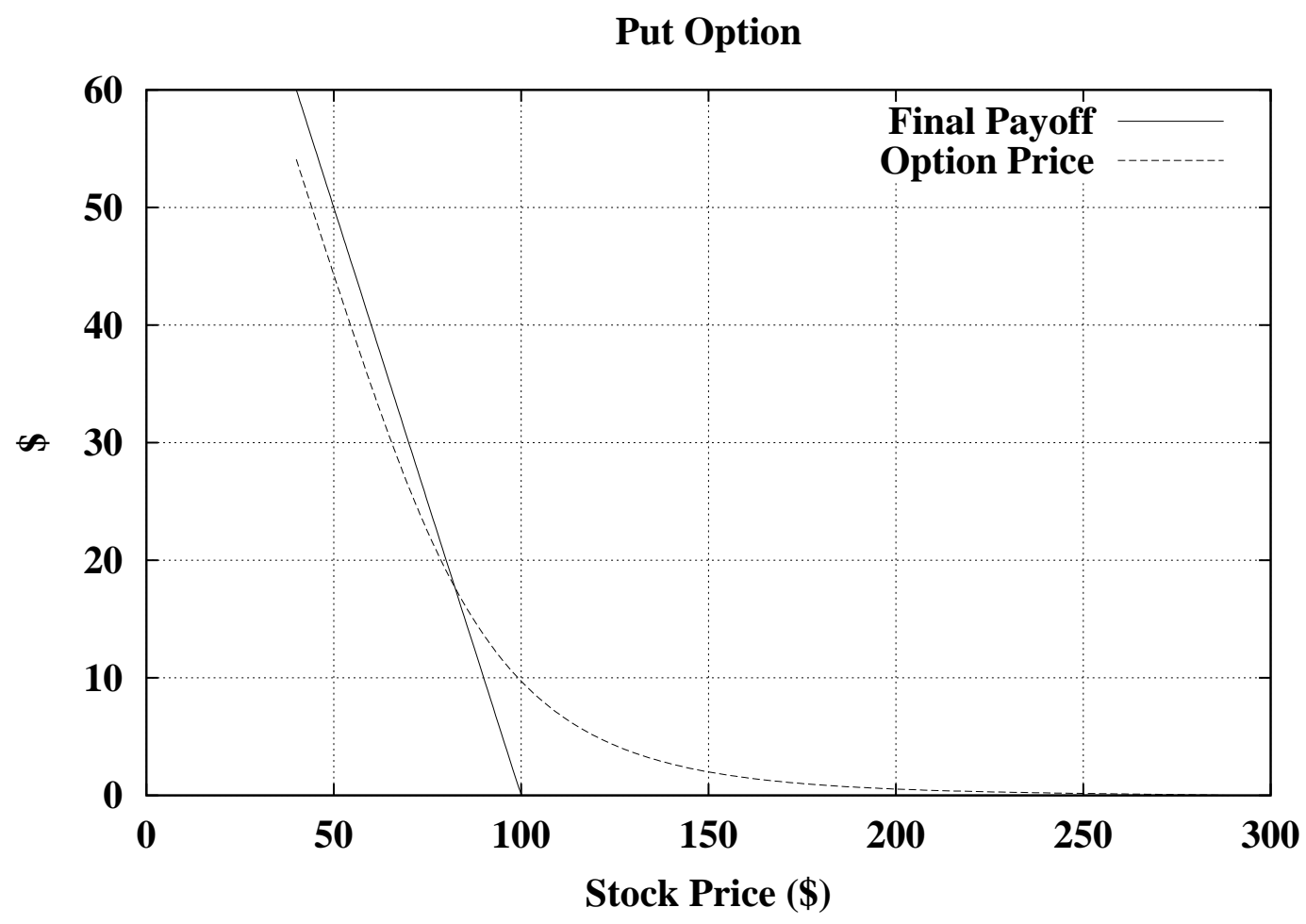

Figure 4.16: European put option under VG process 
Let's consider now the corresponding implied volatilities. We plug the option values obtained under variance gamma in the Black and Scholes formula to obtain the corresponding implied volatilities. This graph shows the implied volatility for the vanilla European call option case.

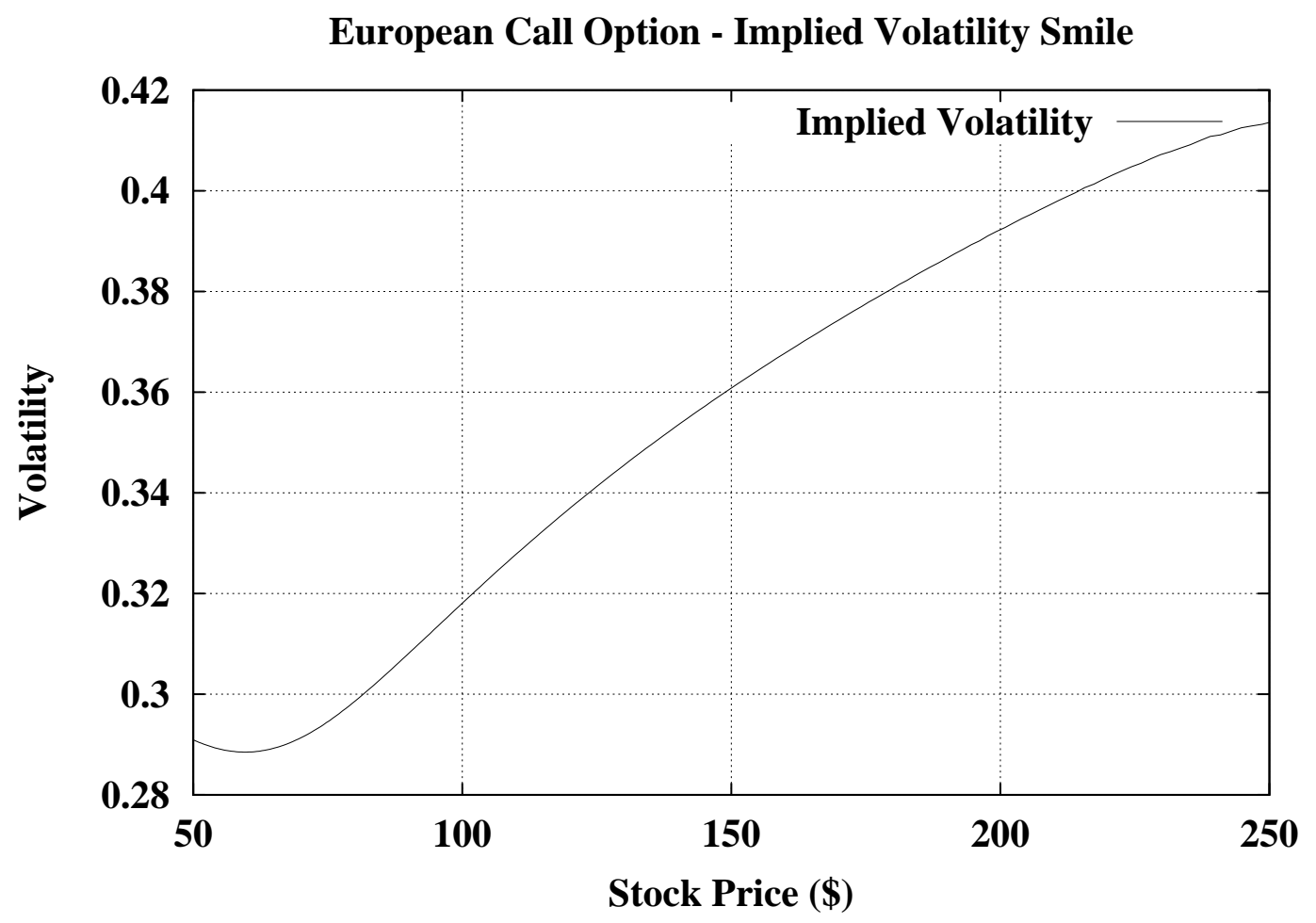

Figure 4.17: Implied volatility smile for European call option under VG process 
This graphs shows the implied volatility for the put option computed in figure 4.16. If we compare the implied volatility in this graph with the one for the call showed in figure 4.17, we can see that the implied volatilities are very close with each other, especially when the option is at the money or close to at the money.

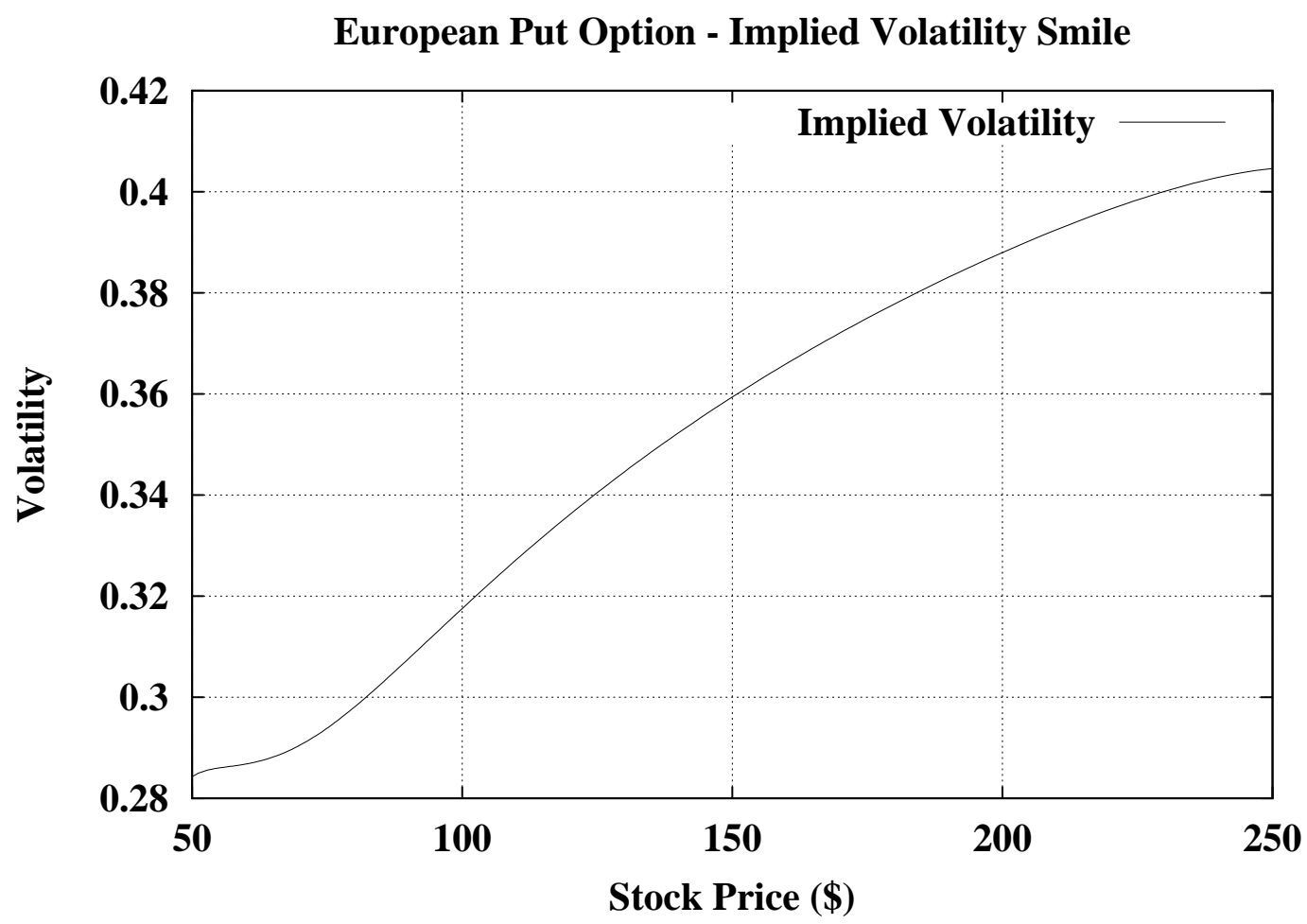

Figure 4.18: Implied volatility smile for European put option under VG process 


\section{Experiment 2}

For this experiment, we use the following parameters:

- $T=1 \quad$ Time to maturity in years;

- $K=\$ 100 \quad$ Strike price;

- $r=0.0514$ Interest rate;

- $q=0.014$ Dividend yield;

- $\sigma=0.20722$;

- $\vartheta=-0.22898$

- $\nu=0.50215$;

- $N=250$ Number of space intervals;

- $M=300$ Number of time intervals;

- $x_{\min }=2.30259$ Min $x$ value considered, corresponding to $S=\$ 10$;

- $x_{\max }=5.56068$ Max $x$ value considered, corresponding to $S=\$ 260$;

- $\Delta x=0.01303 \quad$ Size of a space interval;

- $\Delta t=0.00333 \quad$ Size of a time interval;

- $\frac{\Delta t}{\Delta x}=3.9097$; 
Using these parameters we can graph the values of a vanilla European vanilla call option in the following way:

\section{European Call Option}

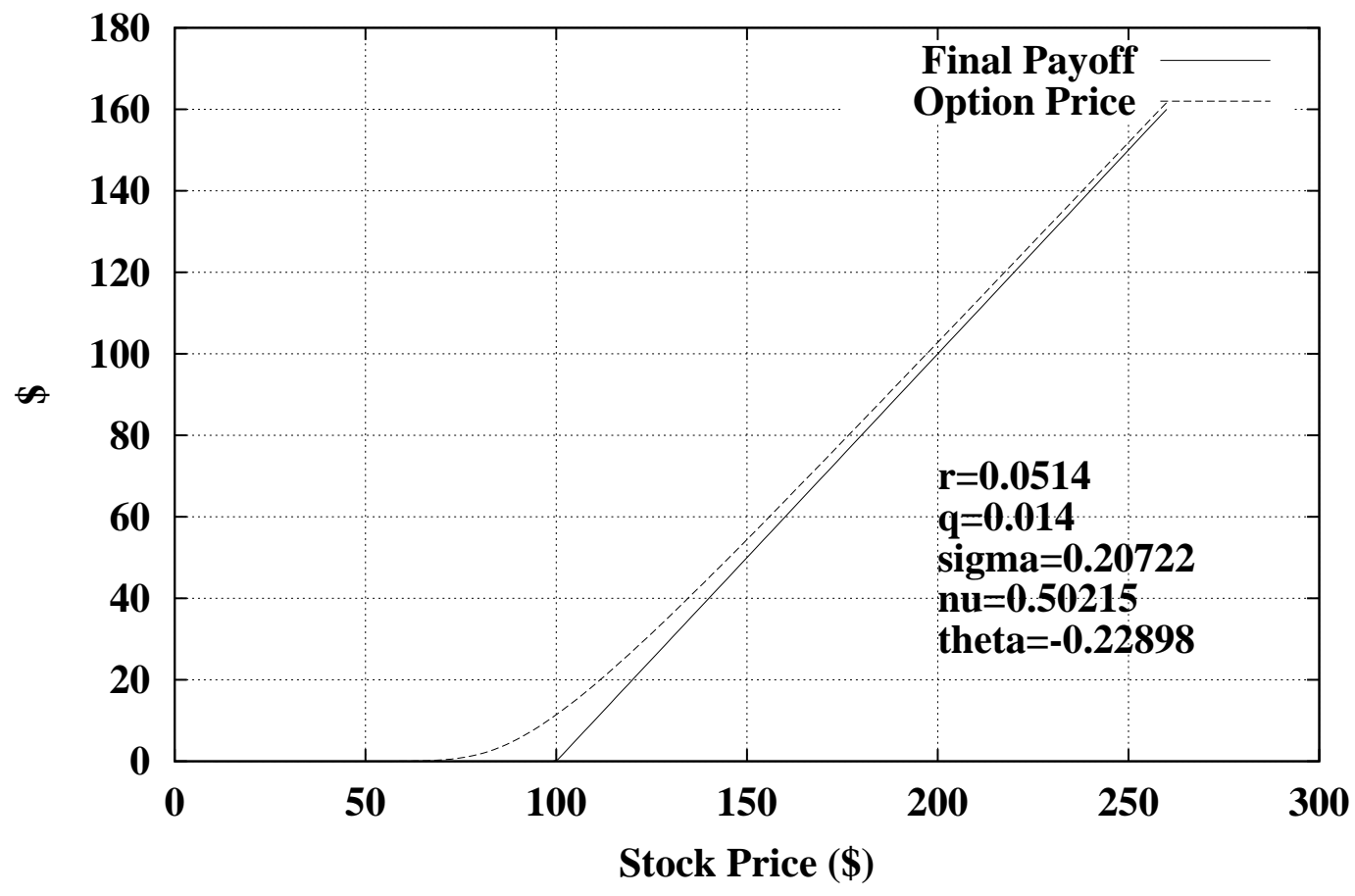

Figure 4.19: European call option under VG process 
In the same we can plot the graph of the corresponding European vanilla put option.

\section{European Put Option}

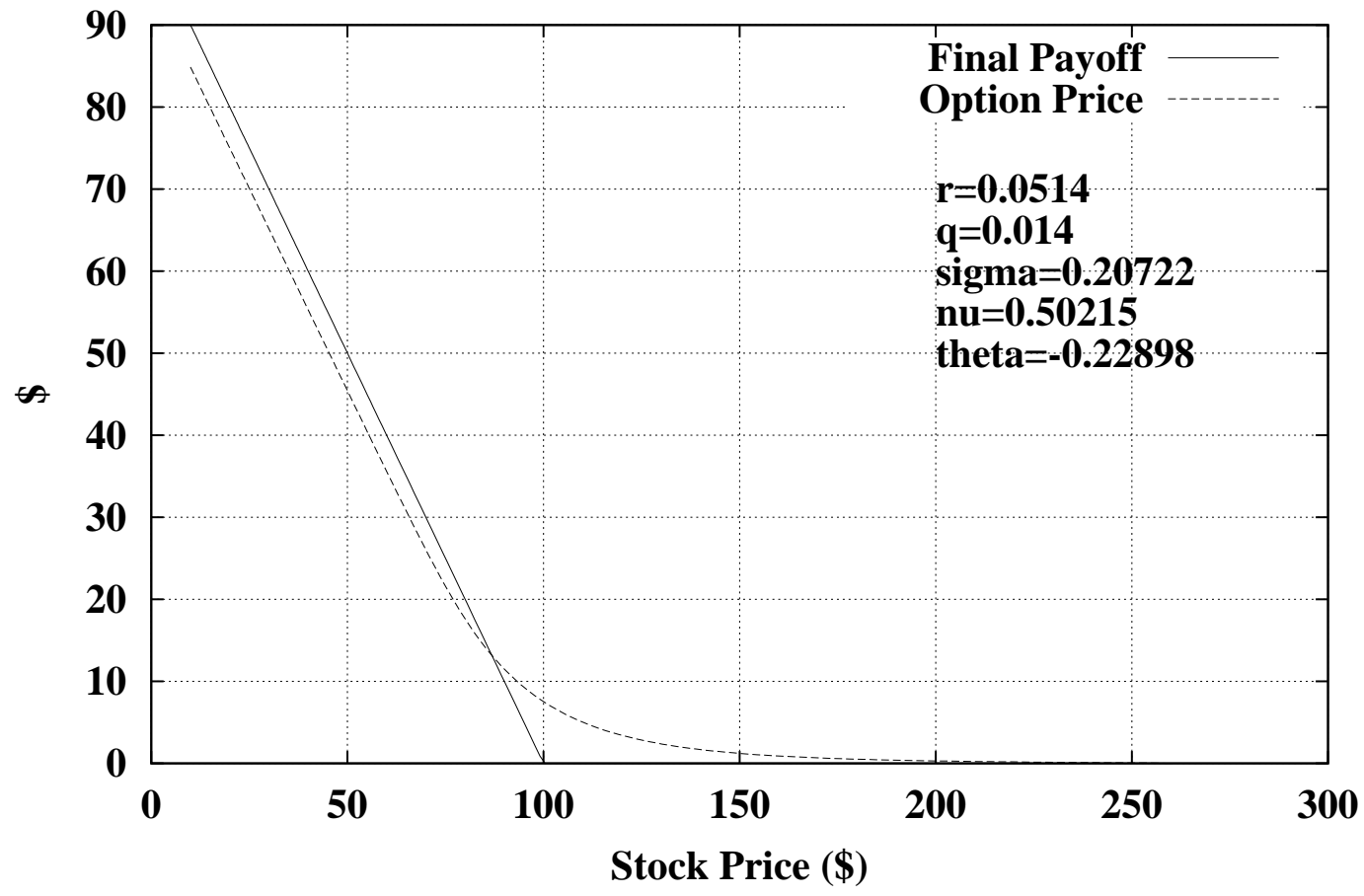

Figure 4.20: European put option under VG process 
We invert the Black and Scholes formula to compute the implied volatility for the vanilla call option.

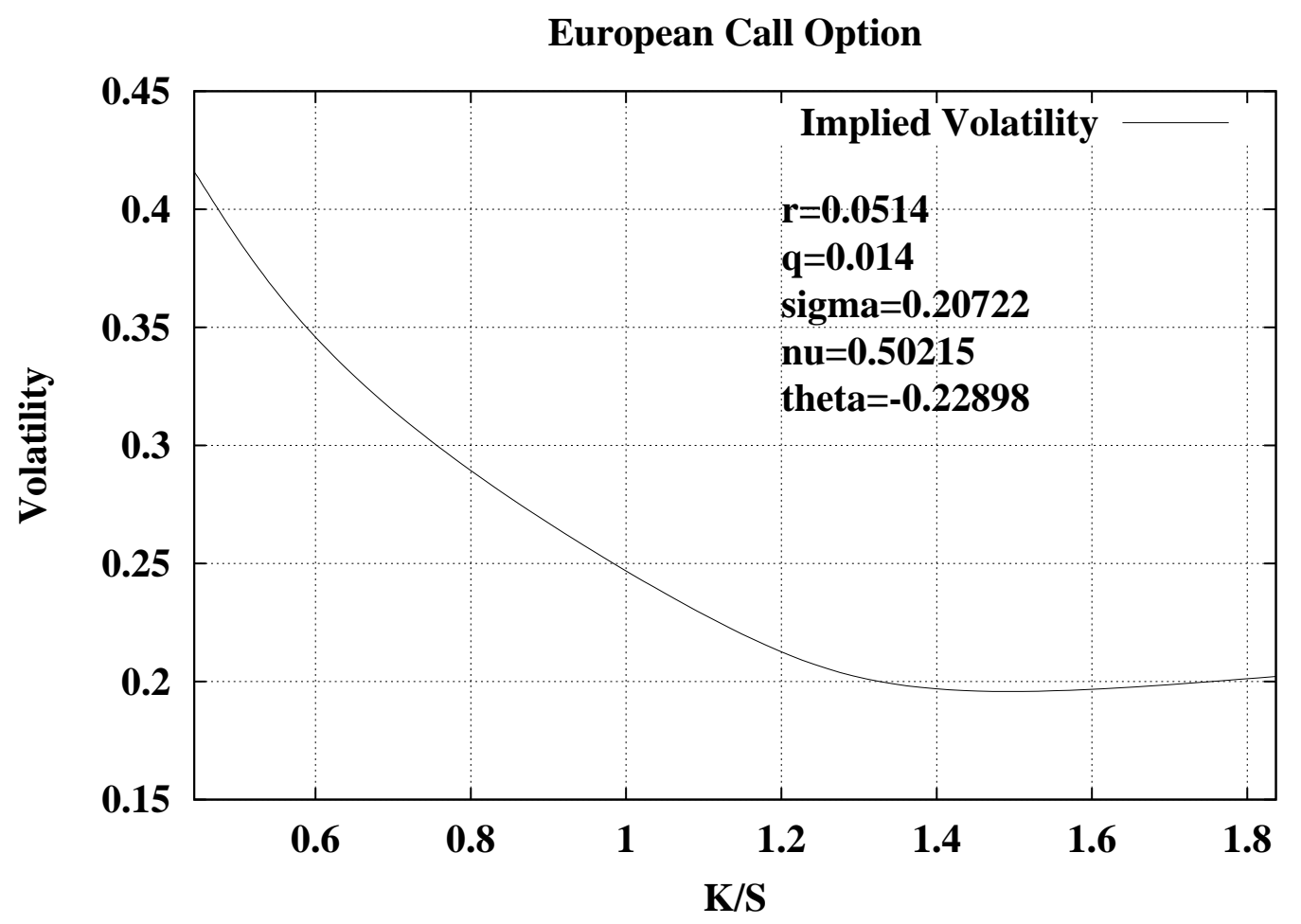

Figure 4.21: Implied volatility for European call option under VG process 
Here we have the implied volatility for the European put option case. If we compare this graph with figure 4.21, we can see that the difference between call and put implied volatility is larger here than in the previous experiment.

\section{European Put Option}

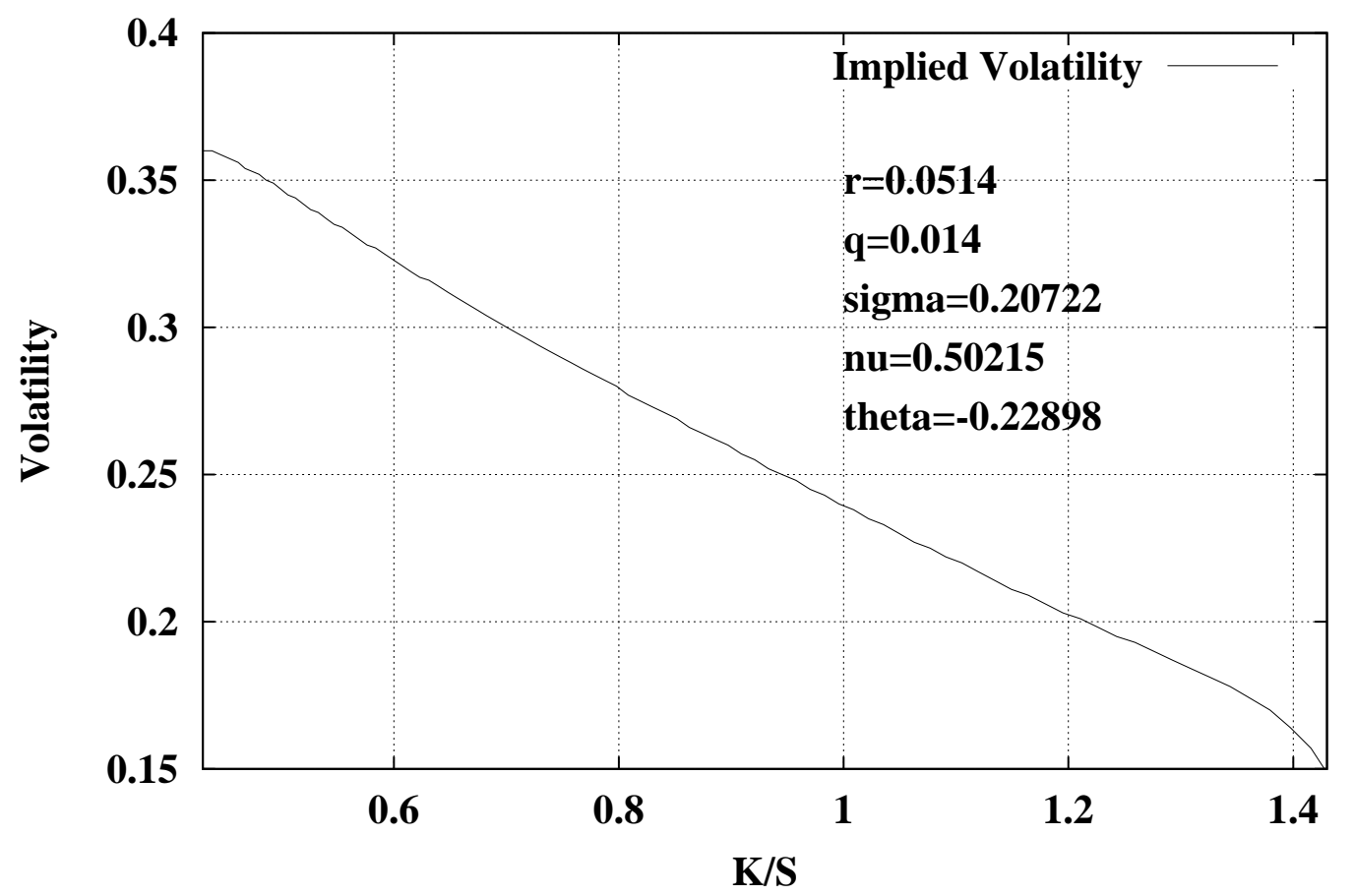

Figure 4.22: Implied volatility for European put option under VG process 


\section{Experiment 3}

For this experiment, we use the following parameters:

- $T=1$ Time to maturity in years;

- $K=\$ 100 \quad$ Strike price;

- $r=0.0514$ Interest rate;

- $q=0.014$ Dividend yield;

- $\sigma=0.178753$;

- $\vartheta=-0.30649$;

- $\nu=0.13317$

- $N=250$ Number of space intervals;

- $M=300$ Number of time intervals;

- $x_{\min }=2.30259$ Min $x$ value considered, corresponding to $S=\$ 10$;

- $x_{\max }=5.56068$ Max $x$ value considered, corresponding to $S=\$ 260$;

- $\Delta x=0.01303 \quad$ Size of a space interval;

- $\Delta t=0.00333 \quad$ Size of a time interval;

- $\frac{\Delta t}{\Delta x}=3.9097$; 
The graph below shows European put prices computed under the variance gamma process using the parameters above.

\section{European Put Option}

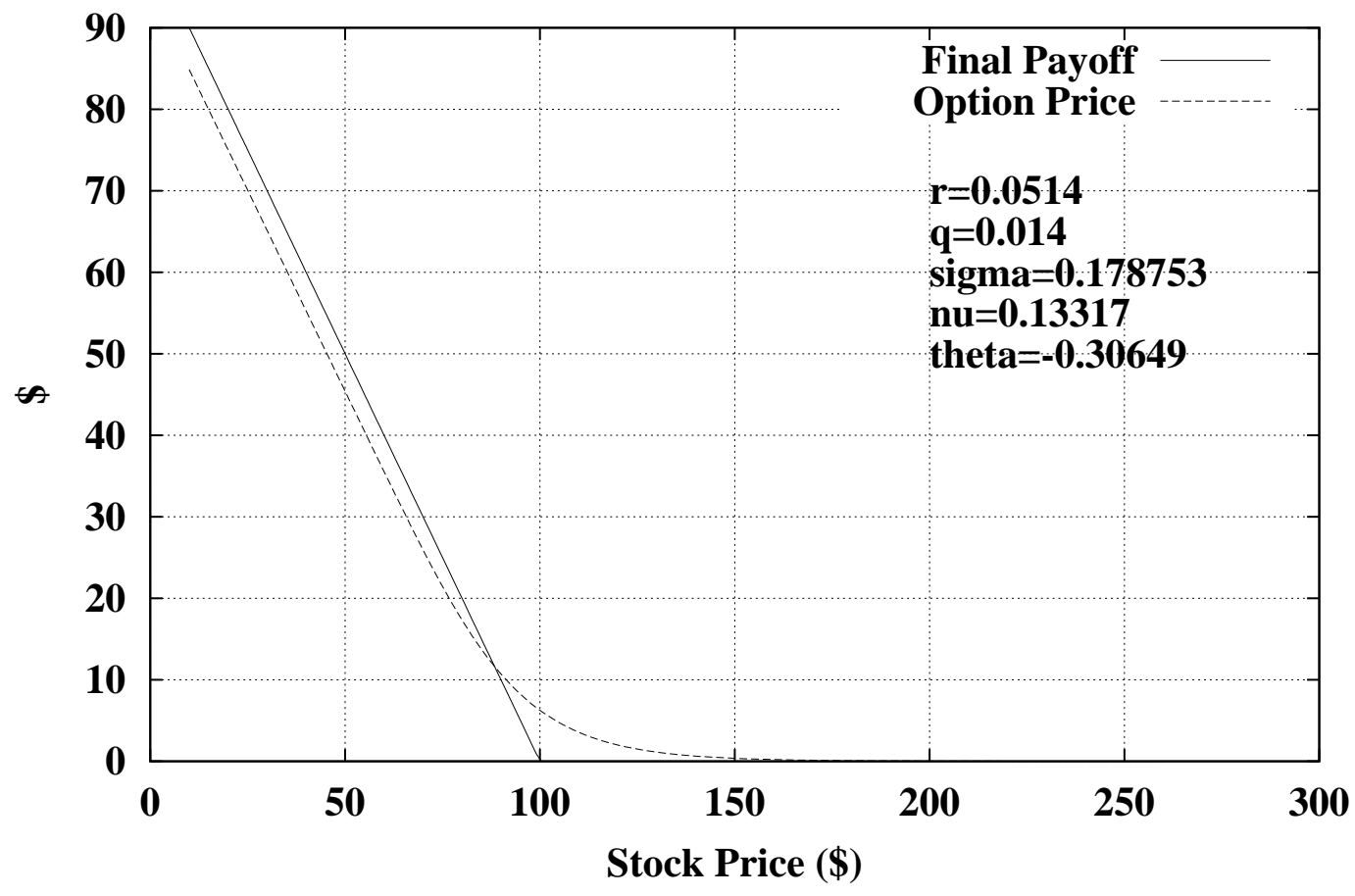

Figure 4.23: European put option under VG process 
This is the graph of the corresponding implied volatility computed by inverting the Black and Scholes formulas.

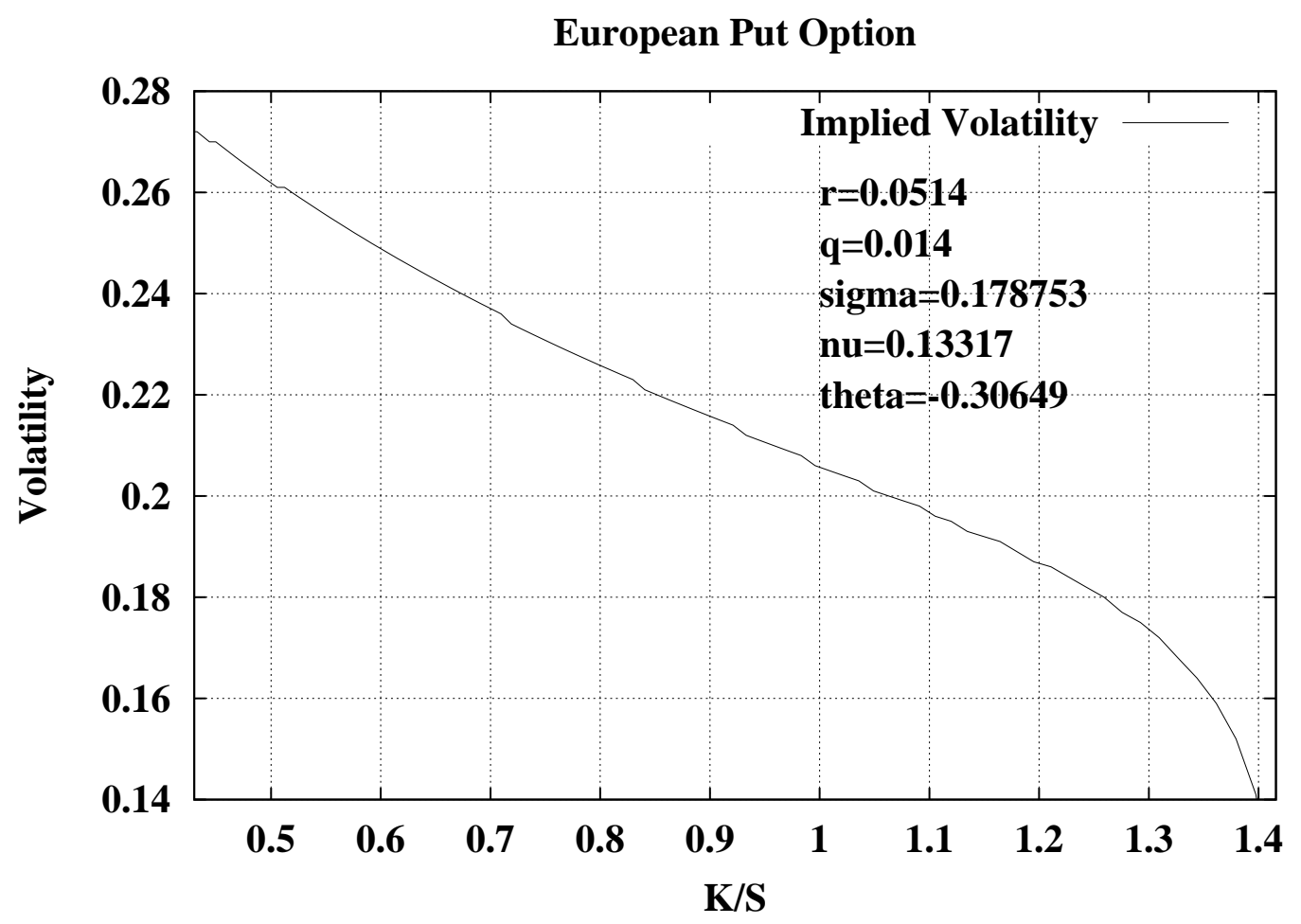

Figure 4.24: Implied volatility for European put option under VG process 


\section{Experiment 4}

For this experiment, we use the following parameters:

- $T=1 \quad$ Time to maturity in years;

- $K=\$ 100 \quad$ Strike price;

- $r=0.0514$ Interest rate;

- $q=0.014$ Dividend yield;

- $\sigma=0.178753$;

- $\vartheta=0.3$

- $\nu=0.13317$

- $N=250$ Number of space intervals;

- $M=300$ Number of time intervals;

- $x_{\min }=2.30259$ Min $x$ value considered, corresponding to $S=\$ 10$;

- $x_{\max }=5.56068$ Max $x$ value considered, corresponding to $S=\$ 260$;

- $\Delta x=0.01303 \quad$ Size of a space interval;

- $\Delta t=0.00333 \quad$ Size of a time interval;

- $\frac{\Delta t}{\Delta x}=3.9097$;

In this experiment we used the same values as in experiment 3, the only difference is that here the use a positive skewness, which is something which generally does not agree with market data. 
This is the graph of the put price computed using the parameters defined above.

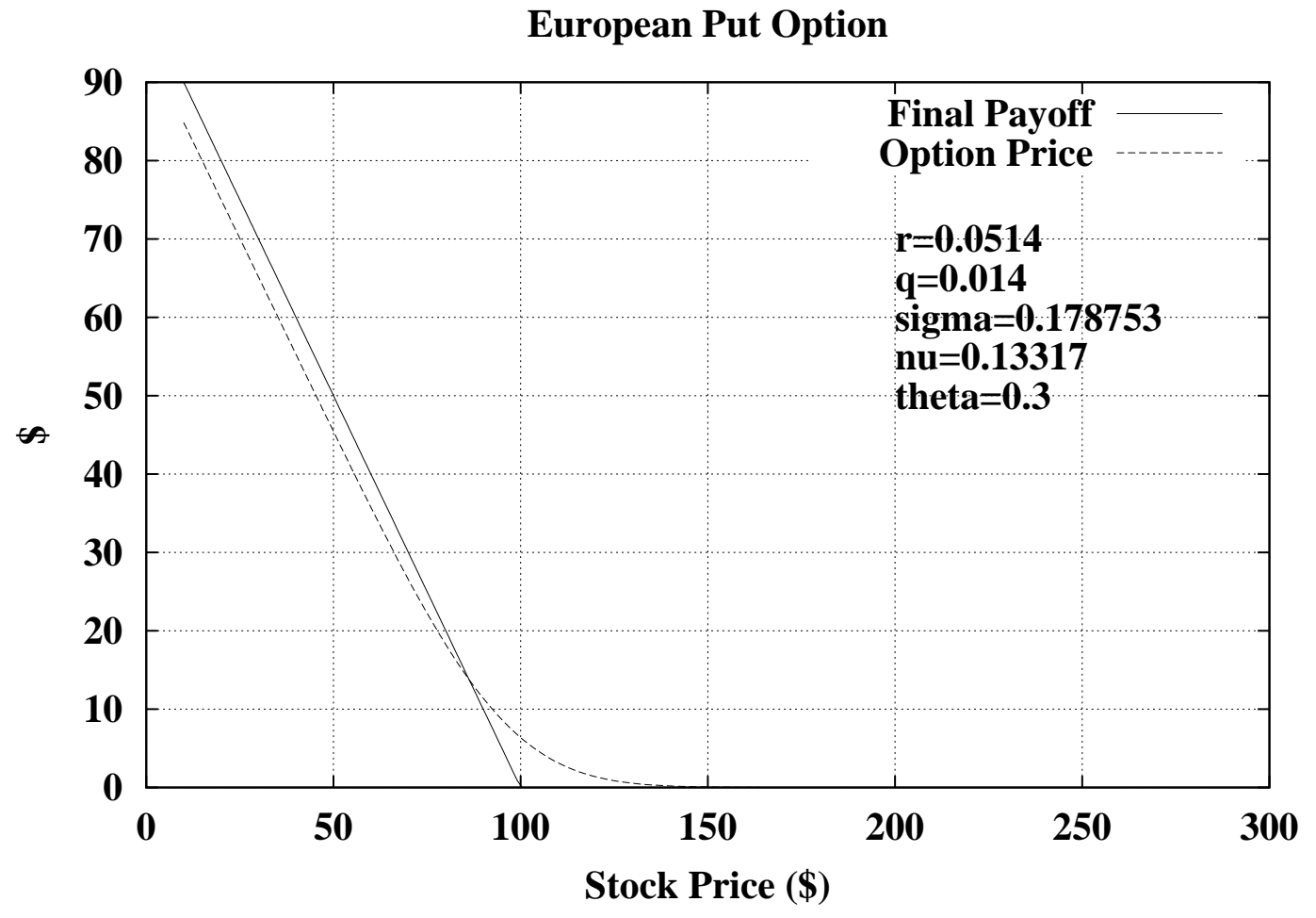

Figure 4.25: European put option under VG process 
This is the correspondent implied volatility. For this case having positive $\vartheta$, we see that the implied volatility has a unusual shape. We note anyway that the values are in a limited range from $18 \%$ to $22.5 \%$.

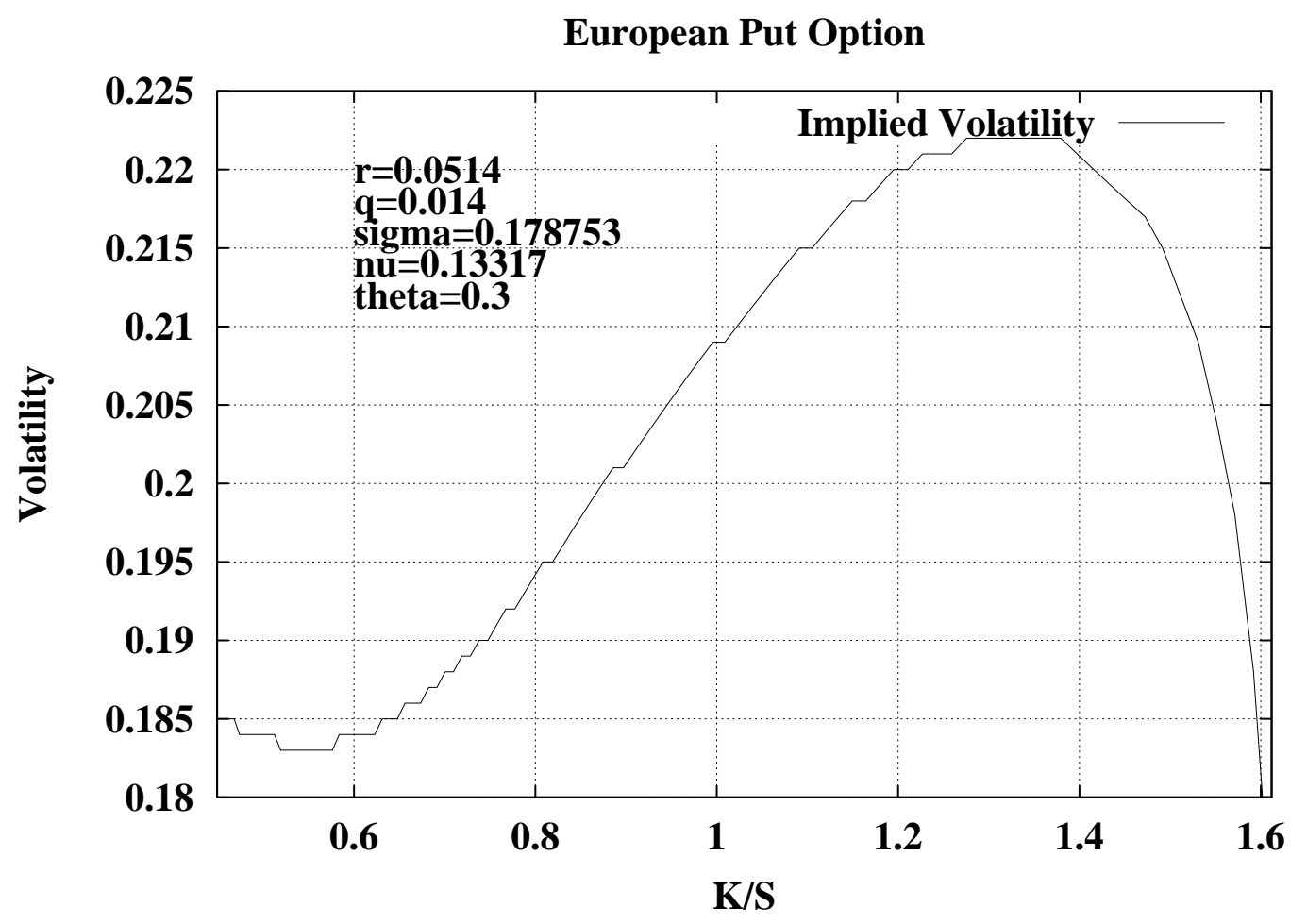

Figure 4.26: Implied volatility for European put option under VG process 


\subsubsection{Comparison with geometric Brownian motion}

In this experiment, we compare the option prices obtained under the geometric Brownian motion with those ones obtained under the variance gamma process. We have used the following parameters: For this experiment, we use the following parameters:

- $T=1 \quad$ Time to maturity in years;

- $K=\$ 100$ Strike price;

- $r=0.0514$ Interest rate;

- $q=0.014$ Dividend yield;

- $\sigma=0.1213$ for variance gamma process;

- $\sigma=0.1236$ for geometric Brownian motion;

- $\vartheta=-0.1436$

- $\nu=0.1686$;

- $N=250$ Number of space intervals;

- $M=300$ Number of time intervals;

- $x_{\min }=2.30259$ Min $x$ value considered, corresponding to $S=\$ 10$;

- $x_{\max }=5.56068$ Max $x$ value considered, corresponding to $S=\$ 260$;

- $\Delta x=0.01303 \quad$ Size of a space interval;

- $\Delta t=0.00333 \quad$ Size of a time interval;

- $\frac{\Delta t}{\Delta x}=3.9097$;

In realizing the comparison between geometric Brownian motion and variance gamma, it is important to use the right parameters. In particular it is necessary to have a different $\sigma$ for the two cases. The parameters used here are the implied ones as computed for each of the two models on the same S\&P 500 option prices by Madan, Carr and Chang ${ }^{15}$.

\footnotetext{
${ }^{15}$ Dilip B Madan, Peter P. Carr and Eric C. Chang, "The Variance Gamma Process and Option Pricing", European Finance Review, 2, 1998, page 92.
} 
The graph shows theoretical prices obtained under the two models.

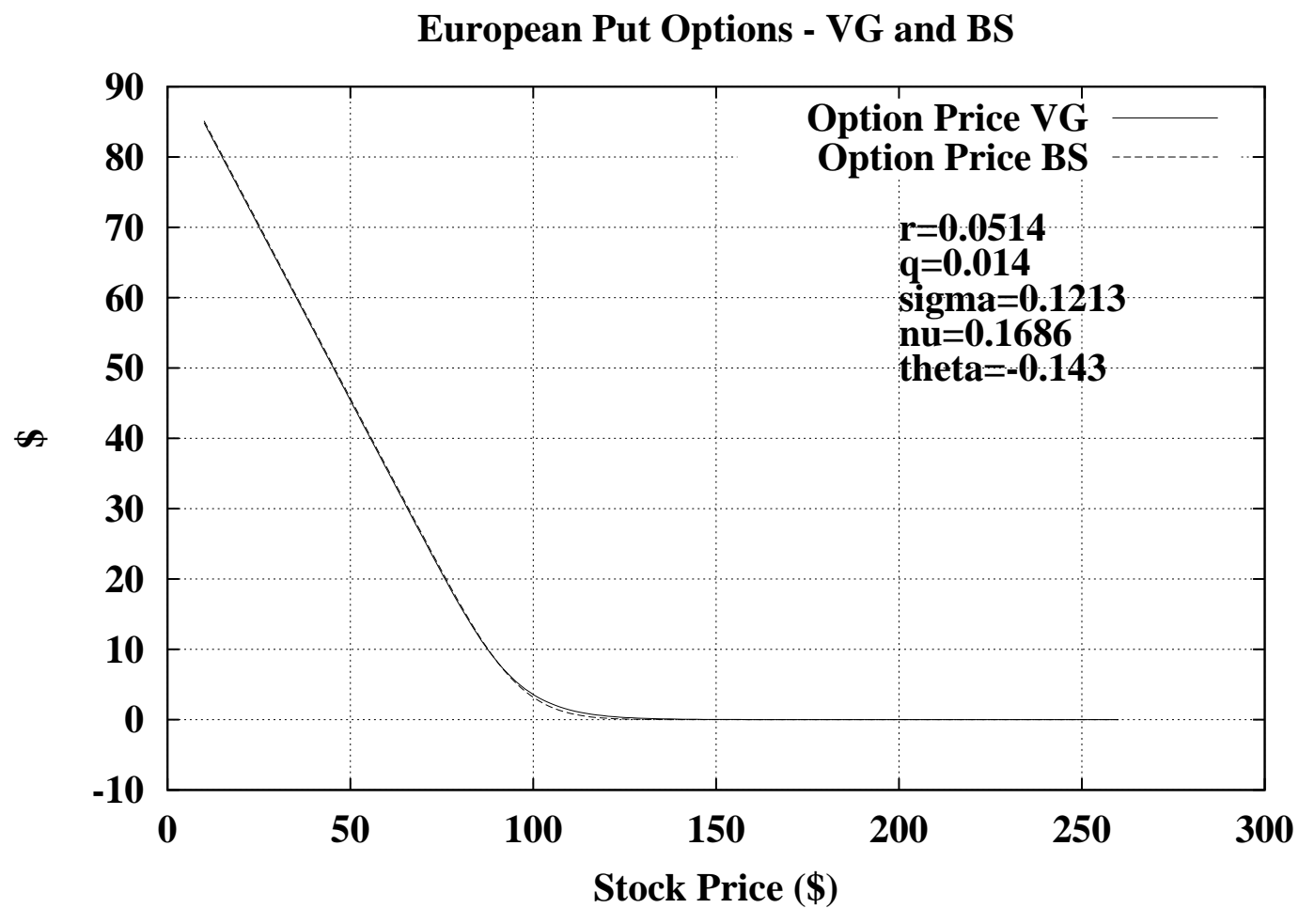

Figure 4.27: European put option under VG process compared to Geometric Brownian motion 
In the previous graph, the difference between the two graphs seems quite small. However if we zoom we can see that the difference is not negligible.

\section{European Put Options - VG and BS}

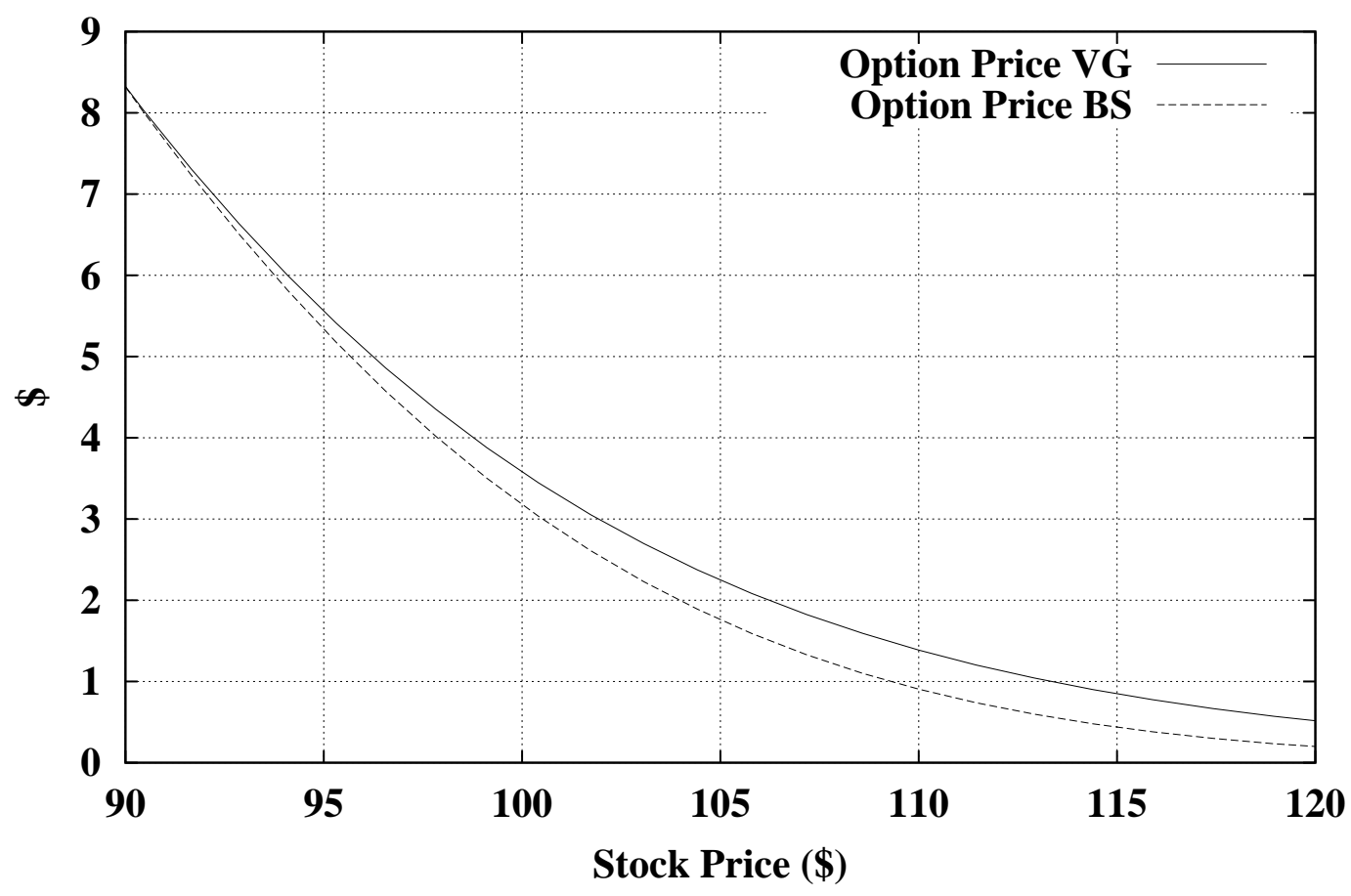

Figure 4.28: European put option under VG process compared to Geometric Brownian motion (same as previous graph, but for a different scale) 
This graph shows the implied volatility computed using the option prices obtained with the variance gamma process and the geometric Brownian motion. In particular the line denoted "Implied Volatility VG" corresponds to values obtained by inverting the Black and Scholes formula for option prices calculated under variance gamma. The line denoted "Implied Volatility BS" represents the implied volatility when the prices are computed using the Black and Scholes formula. Clearly because the Black and Scholes model assumes a constant volatility the implied volatility is just the original volatility used as original input.

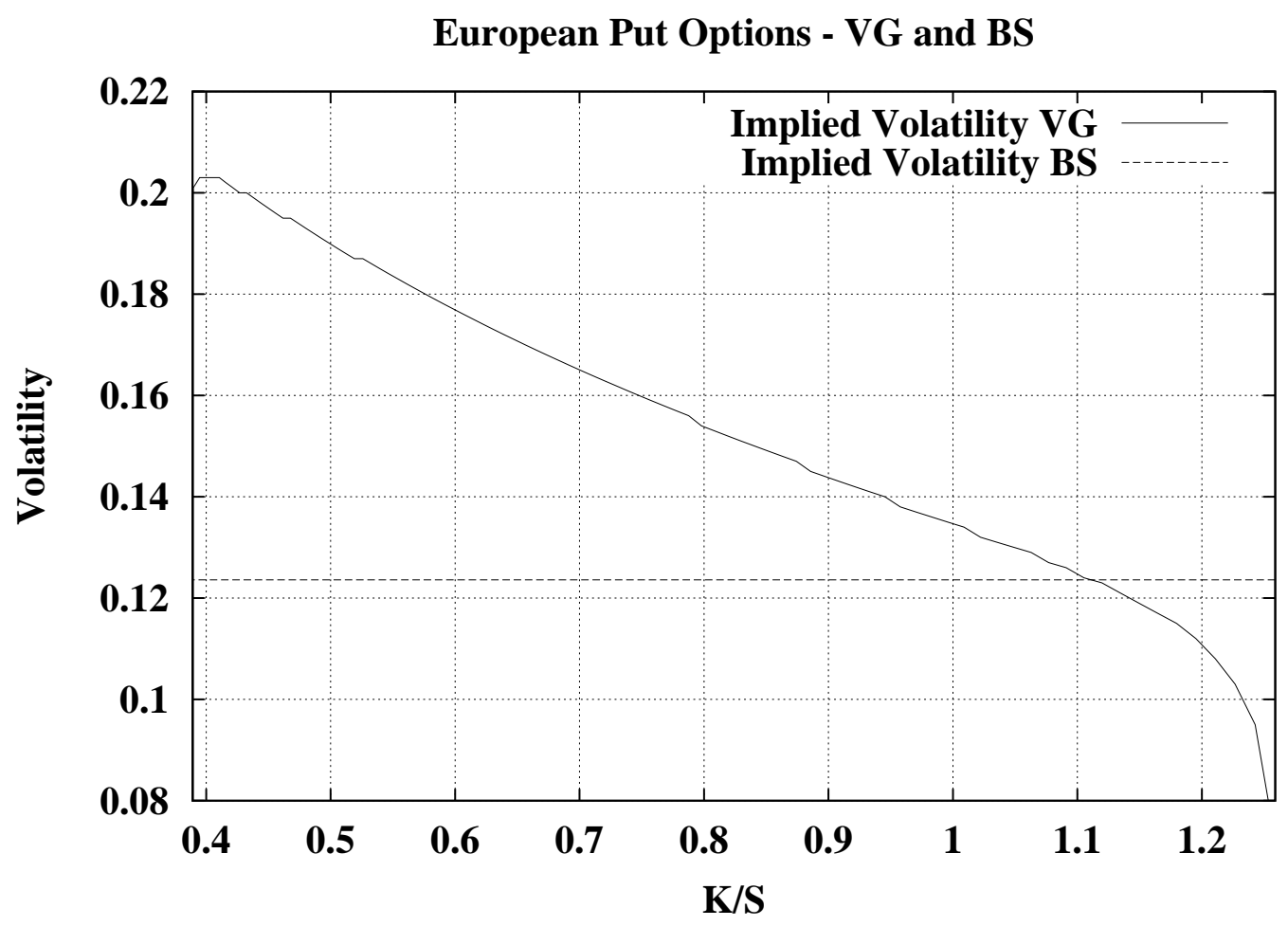

Figure 4.29: Implied volatility for European put option under VG process 


\subsection{Plain Vanilla American Options}

\subsubsection{The Variance Gamma PIDE for American Vanilla Options}

American options allow early exercise at any time, therefore the value of an American call option $V(S, t)$ can be written as

$$
V(S, t)=\sup _{t \leq \tau \leq T} e^{-r \tau} E\left[(S(\tau)-K)^{+}\right]
$$

It can be proved ${ }^{16}$ that for each $t$ there exists a stock price $S^{*}(t)$ such that if $S(t) \geq S^{*}(t)$ the value of the American call option is the payoff which can be obtained by immediately exercising the option, that is $[S(t)-K]$, while if $S(t)<S^{*}(t)$ the call value exceeds this payoff. The curve $S^{*}(t)$ is called exercise boundary as it defines over time the level at which after which it is best to exercise the option. On the exercise boundary the value of the non exercised option is the same as the value of the exercised option. The region

$$
\left\{(S, t) \mid S<S^{*}(t)\right\}
$$

is defined the continuation region of an American call option. Its complement is called exercise region. Symmetric results apply to the American put option: its value can be expressed as

$$
P(S, t)=\sup _{t \leq \tau \leq T} e^{-r \tau} E\left[(K-S(\tau))^{+}\right]
$$

and for each $t$ there exists a stock price $S^{*}(t)$ such that if $S(t) \leq S^{*}(t)$, the value of the American put option is the payoff $[K-S(t)]$, while if $S(t)>$ $S^{*}(t)$ the put value exceeds this payoff. The continuation region for the American put option is given by

$$
\left\{(S, t) \mid S>S^{*}(t)\right\}
$$

the exercise region being its complement.

It can be proved ${ }^{17}$ that the discounted price of the option is a martingale in the continuation region and hence the PIDE (4.6) holds. However in the

\footnotetext{
${ }^{16}$ Ioannis Karatzas and Steven E. Shreve, Brownian Motion and Stochastic Calculus, Springer Verlag, second edition, 1991.

${ }^{17}$ Ioannis Karatzas and Steven E. Shreve, Methods of Mathematical Finance, Application of Mathematics, Vol. 39, Springer, New York and Heidelberg, 1998.
} 
exercise region the PIDE does not hold anymore. Being the exercise and the continuation region being switched for calls and puts with respect to the exercise boundary it is a good idea to treat the two cases distinctly.

\section{The Variance Gamma PIDE for American Vanilla Call Options}

We know that the PIDE (4.6) does not apply to the case where the option is exercised. Let's define the exercise region for call options in terms of our variable $x \triangleq \ln (S)$ as the area where $x>x(\tau)$. Hence $x(\tau)$ is the exercise barrier expressed in terms of $\ln (S)$, as a function of time, in the same as $S^{*}(t)$ defined the barrier before. Let's write an equivalent expression for the PIDE in the exercise region; in particular we want to write the value of the infinitesimal generator for the Markov process $x$ correspondent to the PIDE we wrote. Let's define the operator $\mathscr{L}(f)$ applied to the generical function $f(x, t)$ as the infinitesimal generator

$$
\begin{gathered}
\mathscr{L}(f) \triangleq \frac{\partial f(x, t)}{\partial t}+(r-q+\omega) \cdot \frac{\partial f(x, t)}{\partial x}+ \\
+\int_{-\infty}^{+\infty}[f(x+y, t)-f(x, t)] k(y) d y-r f(x, t)
\end{gathered}
$$

When we are in the exercise region, the option is worth its payoff that is

$$
W(x, t)=e^{x}-K
$$

and hence we have

$$
\begin{gathered}
\frac{\partial W(x, t)}{\partial t}=0 \\
\frac{\partial W(x, t)}{\partial x}=e^{x}
\end{gathered}
$$

We can now substitute this values in the operator $\mathscr{L}(f)$ to obtain its value in the exercise region. We get

$$
\begin{array}{r}
\mathscr{L}(W)=(r-q+\omega) e^{x}+ \\
+\int_{-\infty}^{+\infty}\left[W(x+y, t)-\left(e^{x}-K\right)\right] k(y) d y-r\left(e^{x}-K\right)
\end{array}
$$


Because values of the underlying over $x(\tau)$ correspond to the exercise region, we have that after a jump of size $y$, we are in the exercise region if $x+y>x(\tau)$, that is if the size of the jump is bigger than $x(\tau)-x$. In this area

$$
W(x+y, t)=e^{x+y}-K
$$

We can therefore divide the integral in equation (4.18) in two pieces dividing them at the level $x(\tau)-x$. Moreover remembering that

$$
\int_{-\infty}^{+\infty}\left(e^{y}-1\right) k(y) d y=-\omega
$$

and dividing also this integral in two pieces we can rewrite equation (4.18) as

$$
\begin{array}{r}
\mathscr{L}(W)=(r-q) e^{x}-r\left(e^{x}-K\right)+ \\
+\int_{-\infty}^{x(\tau)-x}\left[W(x+y, t)-\left(e^{x}-K\right)+\left(1-e^{y}\right) e^{x}\right] k(y) d y+ \\
+\int_{x(\tau)-x}^{+\infty}\left[e^{x+y}-K-\left(e^{x}-K\right)+\left(1-e^{y}\right) e^{x}\right] k(y) d y
\end{array}
$$

The second integral is equal to zero and we remain with

$$
\mathscr{L}(W)=r K-q e^{x}+\int_{-\infty}^{x(\tau)-x}\left[W(x+y, t)+K-e^{x+y}\right] k(y) d y
$$

We found that for American call options, the PIDE (4.6) holds in the continuation region therefore $\mathscr{L}(W)=0$ for $x<x(\tau)$; moreover for $x>x(\tau)$ $\mathscr{L}(W)$ is given by equation (4.20). Therefore we can incorporate both the behavior in the continuation and in the exercise region by writing

$$
\begin{array}{r}
\frac{\partial W(x, t)}{\partial t}+(r-q+\omega) \cdot \frac{\partial W(x, t)}{\partial x}+ \\
+\int_{-\infty}^{+\infty}[W(x+y, t)-W(x, t)] k(y) d y-r W(x, t)+ \\
-1_{x>x(\tau)}\left\{r K-q e^{x}+\int_{-\infty}^{x(\tau)-x}\left[W(x+y, t)+K-e^{x+y}\right] k(y) d y\right\}=0
\end{array}
$$

where the indicator function is defined as

$$
1_{A}\left\{\begin{array}{l}
1 \text { if } A \\
0 \text { if not } A
\end{array}\right.
$$


It is interesting to compare the European and American PIDE: we can see that the difference is given by the part multiplied by the indicator function. To obtain the European PIDE it is necessary to extract from the American option the value of early exercise which is expressed by the dividend yield minus the interest on the strike times the time the stock spends in the exercise region ${ }^{18}$. In the case of a pure jump process, this amount has to be further modified by adding the expected shortfall the strategy may experience because of the jumping back of the process in the continuation region after having reached the exercised region ${ }^{19}$. This last correction is realized with the integral in the last term.

This PIDE has to be solved by imposing the following final condition:

$$
W(x, T)=\max \left(e^{x}-K, 0\right)
$$

Moreover, being the option American we need to impose an early exercise condition:

$$
W(x, t) \geqslant \max \left(e^{x}-K, 0\right) \forall t<T
$$

Finally we have the usual boundary conditions for call options

$$
\begin{array}{rr}
W(-\infty, t)=0 & \forall t \\
W(+\infty, t)=e^{x} & \forall t
\end{array}
$$

\section{The Variance Gamma PIDE for American Vanilla Put Options}

The approached used for call options can be replicated for put options. We define here the exercise region as the area where $x<x(\tau)$. In this region the following equations are true

$$
\begin{array}{r}
W(x, t)=K-e^{x} \\
\frac{\partial W(x, t)}{\partial t}=0 \\
\frac{\partial W(x, t)}{\partial x}=-e^{x}
\end{array}
$$

\footnotetext{
${ }^{18}$ See on this Peter Carr, R. A. Jarrow and R. Myneni, "Alternative Characterization of American Put Options", Mathematical Finance, 2, 1992, pages 87-106.

${ }^{19}$ See more on this in C. R. Gukhal, "Analytical Valuation of American Options on Jump-Diffusion Processes", Mathematical Finance, 11, 2001, pages 97-115.
} 
Therefore the operator $\mathscr{L}(f)$ as defined in equation (4.17), in the exercise region, is equal to

$$
\begin{array}{r}
\mathscr{L}(W)=-(r-q+\omega) e^{x}+ \\
+\int_{-\infty}^{+\infty}\left[W(x+y, t)-\left(K-e^{x}\right)\right] k(y) d y-r\left(K-e^{x}\right)
\end{array}
$$

Using again equation (4.19) and remembering that this time the process jumps in the exercise region if $y<x(\tau)-x$, we can write

$$
\begin{array}{r}
\mathscr{L}(W)=(q-r) e^{x}-r\left(K-e^{x}\right)+ \\
+\int_{-\infty}^{x(\tau)-x}\left[K-e^{x+y}-\left(K-e^{x}\right)+\left(e^{y}-1\right) e^{x}\right] k(y) d y+ \\
+\int_{x(\tau)-x}^{+\infty}\left[W(x+y, t)-\left(K-e^{x}\right)+\left(e^{y}-1\right) e^{x}\right] k(y) d y
\end{array}
$$

that is equal to

$$
\mathscr{L}(W)=q e^{x}-r K+\int_{x(\tau)-x}^{+\infty}\left[W(x+y, t)-K+e^{x+y}\right] k(y) d y
$$

For American put option we can say that in the continuation region $\mathscr{L}(W)=$ 0 and in the exercise region $\mathscr{L}(W)$ is given by equation (4.22). Everything can be written in the same equation as

$$
\begin{array}{r}
\frac{\partial W(x, t)}{\partial t}+(r-q+\omega) \cdot \frac{\partial W(x, t)}{\partial x}+ \\
+\int_{-\infty}^{+\infty}[W(x+y, t)-W(x, t)] k(y) d y-r W(x, t)+ \\
-1_{x<x(\tau)}\left\{q e^{x}-r K+\int_{x(\tau)-x}^{+\infty}\left[W(x+y, t)-K+e^{x+y}\right] k(y) d y\right\}=0
\end{array}
$$

The difference between European and American PIDE for put option can be interpreted in the same way as we did for the call options as subtraction of the early exercise value from the American option. However here the European case is obtained by subtracting the interest on the strike minus the dividend yield for the time spent in the exercise region and adding the expected shortfall the strategy would have if the stock jumps back in the continuation region after having reached the exercise region. 
The PIDE for American put options will be solved imposing the following final condition:

$$
W\left(e^{x}, T\right)=\max \left(K-e^{x}, 0\right)
$$

Moreover since early exercise is allowed for American option, we need to impose

$$
W\left(e^{x}, t\right) \geqslant \max \left(K-e^{x}, 0\right) \forall t<T
$$

And finally we have boundary conditions for put options

$$
\begin{array}{ll}
W(-\infty, t)=K & \forall t \\
W(+\infty, t)=0 & \forall t
\end{array}
$$

\subsubsection{Numerical Solution for American Vanilla Options}

A finite difference numerical method is employed to solve equations (4.21) and (4.23) in a similar to the European case. It is necessary to discretize the continuous time PIDE to write down as linear system. As before the most significant part of the process is in the way the integrals are written to allow the numerical computation. Let's start defining the range of values we are going to consider in our computation as

$$
[0, T] \times\left[x_{\min }, x_{\max }\right]
$$

in the same way as we did for the European case, we can discretize the system using let's now discretize this system using $N+1$ mesh point in the $x$-direction and $M+1$ mesh points in the $t$-direction, with the size of space and time intervals given respectively by

$$
\begin{array}{r}
\Delta x=\frac{x_{\max }-x_{\min }}{N} \\
\Delta t=\frac{T-0}{M}
\end{array}
$$

The notation $W\left(x_{i}, t_{j}\right)$ refers to the value of $W(\cdot)$ at the node $(i, j)$ and we use the following approximation of the partial derivatives

$$
\begin{gathered}
\frac{\partial W(x, t)}{\partial t} \simeq \frac{W\left(x_{i}, t_{j+1}\right)-W\left(x_{i}, t_{j}\right)}{\Delta t} \\
\frac{\partial W(x, t)}{\partial x} \simeq \frac{W\left(x_{i+1}, t_{j}\right)-W\left(x_{i-1}, t_{j}\right)}{2 \Delta x}
\end{gathered}
$$


Finally we define

$$
h \triangleq \frac{(r-q+\omega) \Delta t}{2 \Delta x}
$$

Using these transformations and multiplying all the terms in the equation by $\Delta t$, we can write the partial integro differential equation for American call options (4.21) as

$$
\begin{aligned}
& h W\left(x_{i-1}, t_{j}\right)+(1+r \Delta t) W\left(x_{i}, t_{j}\right)-h W\left(x_{i+1}, t_{j}\right)=W\left(x_{i}, t_{j+1}\right)+ \\
& +\Delta t \int_{-\infty}^{+\infty}\left[W\left(x_{i}+y, t_{j+1}\right)-W\left(x_{i}, t_{j+1}\right)\right] k(y) d y-1_{x_{i}>x\left(\tau_{j+1}\right)} \cdot \Delta t . \\
& \cdot\left\{r K-q e^{x_{i}}+\int_{-\infty}^{x\left(\tau_{j+1}\right)-x_{i}}\left[W\left(x_{i}+y, t_{j+1}\right)+K-e^{x_{i}+y}\right] k(y) d y\right\}
\end{aligned}
$$

Similarly equation (4.23) for American put options can be written as

$$
\begin{aligned}
& h W\left(x_{i-1}, t_{j}\right)+(1+r \Delta t) W\left(x_{i}, t_{j}\right)-h W\left(x_{i+1}, t_{j}\right)=W\left(x_{i}, t_{j+1}\right)+ \\
& +\Delta t \int_{-\infty}^{+\infty}\left[W\left(x_{i}+y, t_{j+1}\right)-W\left(x_{i}, t_{j+1}\right)\right] k(y) d y-1_{x_{i}<x\left(\tau_{j+1}\right)} \cdot \Delta t . \\
& \cdot\left\{q e^{x_{i}}-r K+\int_{x\left(\tau_{j+1}\right)-x_{i}}^{+\infty}\left[W\left(x_{i}+y, t_{j+1}\right)-K+e^{x_{i}+y}\right] k(y) d y\right\}
\end{aligned}
$$

Note that all terms in the integrals present the time valued at the step $j+1$. To implement a numerical solution of these equations, it is necessary to write the integrals in a proper way.

\subsubsection{Solution of the Jump Integral for American Vanilla Options}

We define "jump integral" the first integral in (4.24) and (4.25). This integral is the same as the one we found in the European option PIDE. The second integral in both equations will be called "integral in the heaviside term", in this way we can easily refer to the part of the partial integro differential 
equation we are interested into. We want here to rewrite the jump integral in a proper way to be implemented numerically, in a similar way as what we did for the European options. A detailed solution of the integral can be found in appendix A.3. The solution is going to be very similar to the European case, however we need to keep into account the early exercise option. In particular, if the option is deep in the money and the proportion between risk free interest rate and dividend yield is such that exercise is profitable, we can impose that the option will be early exercised at the boundary level.

In the case of European put option, when the jump is negative and large, we imposed that the option value was the discounted value of the payoff. For American put option we can instead impose that the option value is the payoff, whenever the early exercise is profitable. Mathematically, we can write that if

$$
y \in\left[-\infty, x_{0}-x_{i}\right]
$$

the value of the put option can be written as

$$
W\left(x_{i}+y, t_{j+1}\right) \simeq K-e^{x_{i}+y}
$$

when early exercise is profitable. All the other integrals for the put option would be unaffected. Equation (4.26) does not always hold. It does however for $r>q$, which is generally the case we can see in financial markets. In general, we can write that for put options for $y<x_{0}-x_{i}$

$$
W\left(x_{i}+y, t_{j+1}\right) \simeq \max \left[K e^{-r\left(T-t_{j+1}\right)}-e^{x_{i}+y} e^{-q\left(T-t_{j+1}\right)}, K-e^{x_{i}+y}\right]
$$

In the case of a call option, if the jump is positive and large the American option would be exercised provided that is profitable to do so. We can write that for

$$
y \in\left[x_{N}-x_{i},+\infty\right]
$$

the option value can be approximated by

$$
W\left(x_{i}+y, t_{j+1}\right) \simeq \max \left[e^{x_{i}+y} e^{-q\left(T-t_{j+1}\right)}-K e^{-r\left(T-t_{j+1}\right)}, e^{x_{i}+y}-K\right]
$$

Note that in this case, when $r>q$ and $q>0$, as the stock value increases there is going to be a point where it starts to be profitable to early exercise. This point can also be outside the grid range $\left[x_{0}, x_{N}\right]$. In this case for values 
of $y$ relatively close to $x_{N}-x_{i}$, the maximum in equation (4.27) is the discounted value, while as $y$ increases further, the solution becomes the actual payoff.

All the other integrals for the call option remains the same as for the European case.

\subsubsection{Solution of the Integral in the Heaviside Term for American Vanilla Options}

The exercise region is for small stock values for American put options and is for large stock values for American call options, therefore the heaviside term which corrects the European PIDE to obtain the American equivalent is different for calls and put.

\section{Integral for Call Options}

Let's start considering the solution of the heaviside term for call options in equation (4.24) and in particular the integral consider inside this term. A detailed solution of the integral is provided in the appendix A.4.1- A.4.4 following the approach which Hirsa and Madan apply to put options ${ }^{20}$. Similarly to the European option case, the technique requires to break the integral in pieces depending on the size of the jump.

First of all, we can see that the integral is multiplied by the indicator function $1_{x_{i}>x\left(\tau_{j+1}\right)}$, therefore it is taken over a strictly negative range. This is not a useless observation, because it means that we do not have to worry about the divergent behavior of the Lévy density in the neighborhood of zero. Moreover we can note that, since we are working on a discrete space, $x\left(\tau_{j+1}\right)$ has to be on a node of the grid and $x_{i}-x\left(\tau_{j+1}\right)$ as to be at least as big as $\Delta x$, that is one space step in the grid. Hence we can define

$$
x\left(\tau_{j+1}\right) \triangleq l \Delta x=x_{l}
$$

for some integer $l$ between 0 and $N$ whose value determines the position of the exercise boundary in the grid. In reality $l$ must be larger than zero, since

\footnotetext{
${ }^{20}$ Ali Hirsa and Dilip B. Madan, "Pricing American Options Under Variance Gamma", Journal of Computational Finance, Vol. 7, No. 2, Winter 2003/2004, pages 63-80.
} 
a minimum condition for early exercise is having a positive intrinsic value, which is achieved for $e^{x_{i}} \geq K$. If the option is not exercised for any point in the grid at a certain time step, $l$ is equal $N$.

Using this notation, we can split the integral in two pieces in the following way.

$$
\begin{aligned}
& \int_{-\infty}^{x\left(\tau_{j+1}\right)-x_{i}}\left[W\left(x_{i}+y, t_{j+1}\right)+K-e^{x_{i}+y}\right] k(y) d y= \\
& =\int_{-\infty}^{x_{0}-x_{i}}\left[W\left(x_{i}+y, t_{j+1}\right)+K-e^{x_{i}+y}\right] k(y) d y+ \\
& \quad+\int_{x_{0}-x_{i}}^{(l-i) \Delta x}\left[W\left(x_{i}+y, t_{j+1}\right)+K-e^{x_{i}+y}\right] k(y) d y
\end{aligned}
$$

The last integral, (4.29), relates to negative jumps which are at least as big as a space interval but such that the underlying does not finish outside the range of values considered. That is, assuming to start from the generic point $x_{i}$,

$$
y \in\left[x_{0}-x_{i},(l-i) \Delta x\right]
$$

We can rewrite the integral as sum of integrals each of them corresponding to a jump which takes the stock to a subsequent space interval. Redefine $y \triangleq-y$ and say that the jump has a size

$$
y \in[k \Delta x,(k+1) \Delta x]
$$

for $k=(i-l),(i-l+1), \ldots,(i-1)$. This jump will take the stock value between two consecutive nodes and it is possible to approximate the difference in the value of the option after the jump and the value of the option at the last node passed by the stock with the jump, as a linear interpolation of the option values in the two nodes which are the closest to the real position. In mathematical terms, we can write

$$
\begin{gathered}
W\left(x_{i}-y, t_{j+1}\right)-W\left(x_{i-k}, t_{j+1}\right) \simeq \\
\simeq \frac{W\left(x_{i-k-1}, t_{j+1}\right)-W\left(x_{i-k}, t_{j+1}\right)}{\Delta x} \cdot(y-k \Delta x)
\end{gathered}
$$

Integral (4.28), on the other side, define the case where the negative jump is so big that the underlying finishing outside the range of values considered. 
Because we are considering a call option here, we impose that values on the boundary $x_{0}$ or outside that boundary are zero. Hence we can write

$$
W\left(x_{i}+y, t_{j+1}\right) \simeq 0 \quad \text { for } \quad y \in\left[-\infty, x_{0}-x_{i}\right]
$$

With this technique, it is possible to rewrite the integral in the following way:

$$
\begin{gathered}
\int_{-\infty}^{x\left(\tau_{j+1}\right)-x_{i}}\left[W\left(x_{i}+y, t_{j+1}\right)+K-e^{x_{i}+y}\right] k(y) d y= \\
=\sum_{k=i-l}^{i-1}\left\{\frac{W\left(x_{i-k-1}, t_{j+1}\right)-W\left(x_{i-k}, t_{j+1}\right)}{\nu \frac{\mu_{n}}{\nu_{n}} \Delta x} \cdot\left[e^{-\frac{\mu_{n}}{\nu_{n}} k \Delta x}-e^{-\frac{\mu_{n}}{\nu_{n}}(k+1) \Delta x}\right]+\right. \\
+\frac{1}{\nu}\left[W\left(x_{i-k}, t_{j+1}\right)-k\left(W\left(x_{i-k-1}, t_{j+1}\right)-W\left(x_{i-k}, t_{j+1}\right)\right)\right] \cdot \\
\left.\cdot\left[\operatorname{expint}\left(\frac{\mu_{n}}{\nu_{n}} k \Delta x\right)-\text { expint }\left(\frac{\mu_{n}}{\nu_{n}}(k+1) \Delta x\right)\right]\right\}+ \\
+\frac{K}{\nu} \cdot \text { expint }\left[\frac{\mu_{n}}{\nu_{n}}(i-l) \Delta x\right]-\frac{e^{x_{i}}}{\nu} \cdot \operatorname{expint}\left[\left(1+\frac{\mu_{n}}{\nu_{n}}\right)(i-l) \Delta x\right]
\end{gathered}
$$

\section{Integral for Put Options}

We consider now the integral in the heaviside term for put options in equation (4.25). A detailed solution of the integral is provided in appendices A.4.5 A.4.8, following the approach of Hirsa and Madan ${ }^{21}$. This time the integral is multiplied by $1_{x_{i}<x\left(\tau_{j+1}\right)}$ and therefore it is taken over a strictly positive range, allowing us to ignore issues related to divergence of the Lévy measure as $x \rightarrow 0$. Given that we are in a discrete environment $x\left(\tau_{j+1}\right)-x_{i}$ has to be at least as big as $\Delta x$. We define

$$
x\left(\tau_{j+1}\right) \triangleq l \Delta x=x_{l}
$$

for some integer $l$ between 0 and $N$ whose value determines the position of the exercise boundary in the grid. In reality $l$ has to be less than $N$

\footnotetext{
${ }^{21}$ Ali Hirsa and Dilip B. Madan, "Pricing American Options Under Variance Gamma", Journal of Computational Finance, Vol. 7, No. 2, Winter 2003/2004, pages 63-80.
} 
because necessary condition for the early exercise of the put option is that the underlying price is lower than the strike price. If the option is not exercised for any grid point at a certain time step, then $l=0$.

The integral is divided into two pieces depending on the size of the jump by writing

$$
\begin{gathered}
\int_{x\left(\tau_{j+1}\right)-x_{i}}^{+\infty}\left[W\left(x_{i}+y, t_{j+1}\right)-K+e^{x_{i}+y}\right] k(y) d y= \\
\int_{(l-i) \Delta x}^{(N-i) \Delta x}\left[W\left(x_{i}+y, t_{j+1}\right)-K+e^{x_{i}+y}\right] k(y) d y+ \\
\int_{(N-i) \Delta x}^{+\infty}\left[W\left(x_{i}+y, t_{j+1}\right)-K+e^{x_{i}+y}\right] k(y) d y
\end{gathered}
$$

Integral (4.31) refers to positive jumps which takes the underlying back to the continuation region, but whose size is such that the underlying value is still inside the range of values considered. To discretize this integral it is necessary to make use of a linear interpolation for which given a jump of size $y \in[k \Delta x,(k+1) \Delta x]$, we can write

$$
\begin{array}{r}
W\left(x_{i}+y, t_{j+1}\right)-W\left(x_{i+k}, t_{j+1}\right) \simeq \\
\simeq \frac{W\left(x_{i+k+1}, t_{j+1}\right)-W\left(x_{i+k}, t_{j+1}\right)}{\Delta x} \cdot(y-k \Delta x)
\end{array}
$$

Integral (4.32) refers to jumps which are so large that the underlying finishes outside the range of values considered. Given that we are dealing with a put option, its value is imposed to be equal to zero once the right boundary at $x_{N}$ is reached. Hence we say

$$
W\left(x_{i}+y, t_{j+1}\right) \simeq 0 \quad \text { for } \quad y \in\left[x_{N}-x_{i}, \infty\right]
$$


This approach allows to reach the following result for American put options:

$$
\begin{gathered}
\int_{x\left(\tau_{j+1}\right)-x_{i}}^{+\infty}\left[W\left(x_{i}+y, t_{j+1}\right)-K+e^{x_{i}+y}\right] k(y) d y= \\
=\sum_{k=l-i}^{N-i-1}\left\{\frac{W\left(x_{i+k+1}, t_{j+1}\right)-W\left(x_{i+k}, t_{j+1}\right)}{\nu \frac{\mu_{p}}{\nu_{p}} \Delta x} \cdot\left[e^{-\frac{\mu_{p}}{\nu_{p}} k \Delta x}-e^{-\frac{\mu_{p}}{\nu_{p}}(k+1) \Delta x}\right]+\right. \\
+\frac{1}{\nu}\left[W\left(x_{i+k}, t_{j+1}\right)-k\left(W\left(x_{i+k+1}, t_{j+1}\right)-W\left(x_{i+k}, t_{j+1}\right)\right)\right] \cdot \\
\left.\cdot\left[\operatorname{expint}\left(\frac{\mu_{p}}{\nu_{p}} k \Delta x\right)-\operatorname{expint}\left(\frac{\mu_{p}}{\nu_{p}}(k+1) \Delta x\right)\right]\right\}+ \\
-\frac{K}{\nu} \cdot \text { expint }\left[\frac{\mu_{p}}{\nu_{p}}(l-i) \Delta x\right]+\frac{e^{x_{i}}}{\nu} \cdot \operatorname{expint}\left[\left(\frac{\mu_{p}}{\nu_{p}}-1\right)(l-i) \Delta x\right]
\end{gathered}
$$

\subsubsection{Difference Equation for American Vanilla Op- tions}

Let's consider an American vanilla call options. To write the difference equation to be used to price the option under variance gamma, we can substitute the results for the integral in the heaviside function obtained in equation (4.30) and the solution of the jump integral (A.29) as modified by (A.30) into the PIDE (4.24) to obtain:

$$
\begin{gathered}
W\left(x_{i-1}, t_{j}\right)\left\{h-\frac{\Delta t \cdot\left(1-e^{-\frac{\mu_{n}}{\nu_{n}} \Delta x}\right)}{\nu \Delta x \frac{\mu_{n}}{\nu_{n}}}\right\}+ \\
+W\left(x_{i}, t_{j}\right)\left\{(1+r \Delta t)+\frac{\Delta t \cdot\left(1-e^{-\frac{\mu_{p}}{\nu_{p}} \Delta x}\right)}{\nu \Delta x \frac{\mu_{p}}{\nu_{p}}}+\frac{\Delta t \cdot\left(1-e^{-\frac{\mu_{n}}{\nu_{n}} \Delta x}\right)}{\nu \Delta x \frac{\mu_{n}}{\nu_{n}}}\right\}+ \\
-W\left(x_{i+1}, t_{j}\right)\left\{h+\frac{\Delta t \cdot\left(1-e^{-\frac{\mu_{p}}{\nu_{p}} \Delta x}\right)}{\nu \Delta x \frac{\mu_{p}}{\nu_{p}}}\right\}=W\left(x_{i}, t_{j+1}\right)+ \\
+\Delta t \cdot\left\{\sum_{k=1}^{N-i-1} \frac{1}{\nu \Delta x}\left[W\left(x_{i+k+1}, t_{j+1}\right)-W\left(x_{i+k}, t_{j+1}\right)\right] \cdot\right.
\end{gathered}
$$




$$
\begin{aligned}
& \cdot \frac{\nu_{p}}{\mu_{p}}\left[e^{-\frac{\mu_{p}}{\nu_{p}} k \Delta x}-e^{-\frac{\mu_{p}}{\nu_{p}}(k+1) \Delta x}\right]+ \\
& +\sum_{k=1}^{N-i-1} \frac{1}{\nu}\left\{W\left(x_{i+k}, t_{j+1}\right)-W\left(x_{i}, t_{j+1}\right)-k\left[W\left(x_{i+k+1}, t_{j+1}\right)-W\left(x_{i+k}, t_{j+1}\right)\right]\right\} . \\
& \cdot\left\{\operatorname{expint}\left(\frac{\mu_{p}}{\nu_{p}} k \Delta x\right)-\operatorname{expint}\left[\frac{\mu_{p}}{\nu_{p}}(k+1) \Delta x\right]\right\}+ \\
& +\sum_{k=1}^{i-1}\left\{\frac{1}{\nu \Delta x}\left[W\left(x_{i-k-1}, t_{j+1}\right)-W\left(x_{i-k}, t_{j+1}\right)\right] \frac{\nu_{n}}{\mu_{n}}\left[e^{-\frac{\mu_{n}}{\nu_{n}} k \Delta x}-e^{-\frac{\mu_{n}}{\nu_{n}}(k+1) \Delta x}\right]\right\}+ \\
& +\sum_{k=1}^{i-1} \frac{1}{\nu}\left\{W\left(x_{i-k}, t_{j+1}\right)-W\left(x_{i}, t_{j+1}\right)-k\left[W\left(x_{i-k-1}, t_{j+1}\right)-W\left(x_{i-k}, t_{j+1}\right)\right]\right\} . \\
& \cdot\left\{\operatorname{expint}\left(\frac{\mu_{n}}{\nu_{n}} k \Delta x\right)-\operatorname{expint}\left[\frac{\mu_{n}}{\nu_{n}}(k+1) \Delta x\right]\right\}+ \\
& +\frac{1}{\nu}\left\{e^{x_{i}} \operatorname{expint}\left[\left(\frac{\mu_{p}}{\nu_{p}}-1\right)(N-i) \Delta x\right]+\right. \\
& \left.-\left[K+W\left(x_{i}, t_{j+1}\right)\right] \operatorname{expint}\left[\frac{\mu_{p}}{\nu_{p}}(N-i) \Delta x\right]\right\}+ \\
& \left.-\frac{1}{\nu} W\left(x_{i}, t_{j+1}\right) \operatorname{expint}\left(\frac{\mu_{n}}{\nu_{n}} i \Delta x\right)\right\}+ \\
& -1_{x_{i}>x\left(\tau_{j+1}\right)} \cdot \Delta t \cdot\left\{r K-q e^{x_{i}}+\right. \\
& \sum_{k=i-l}^{i-1}\left\{\frac{W\left(x_{i-k-1}, t_{j+1}\right)-W\left(x_{i-k}, t_{j+1}\right)}{\nu \frac{\mu_{n}}{\nu_{n}} \Delta x} \cdot\left[e^{-\frac{\mu_{n}}{\nu_{n}} k \Delta x}-e^{-\frac{\mu_{n}}{\nu_{n}}(k+1) \Delta x}\right]+\right. \\
& +\frac{1}{\nu}\left[W\left(x_{i-k}, t_{j+1}\right)-k\left(W\left(x_{i-k-1}, t_{j+1}\right)-W\left(x_{i-k}, t_{j+1}\right)\right)\right] . \\
& \left.\cdot\left[\operatorname{expint}\left(\frac{\mu_{n}}{\nu_{n}} k \Delta x\right)-\operatorname{expint}\left(\frac{\mu_{n}}{\nu_{n}}(k+1) \Delta x\right)\right]\right\}+ \\
& \left.+\frac{K}{\nu} \cdot \operatorname{expint}\left[\frac{\mu_{n}}{\nu_{n}}(i-l) \Delta x\right]-\frac{e^{x_{i}}}{\nu} \cdot \operatorname{expint}\left[\left(1+\frac{\mu_{n}}{\nu_{n}}\right)(i-l) \Delta x\right]\right\}
\end{aligned}
$$


The left hand side of the equation can be written as a tridiagonal matrix and hence the system can be solved using a Gauss's elimination method. In appendix $\mathrm{B}$ we provide $\mathrm{C}$ code to solve this difference equation numerically.

The American vanilla put case be obtained in the same way by substituting equation (4.33) and equation (A.31) as adjusted by (A.32) into the PIDE (4.25). The difference equation for put options can be found in appendix A.5.2.

\subsection{Experimental Results for American Vanilla Options}

\subsubsection{Calls: Sensitivity Analysis}

In this section we want to study the sensitivity of vanilla American call prices. American vanilla call prices are generally not too far from the corresponding European call options, provided that the dividends are not too high. We study here a sensitivity analysis with respect to the dividend yield.

The numerical experiments presented here can be obtained using the code in $\mathrm{C}$ language available in appendix B. The vanilla American call case studied here can be realized by choosing the following variable values:

- callput $=1$

- euroamerican $=1$

- barrier_switch $=0$

in the $\mathrm{C}$ code.

\section{Sensitivity with Respect to the Dividend Yield}

We consider an American call option and we use the following parameters:

- $T=1 \quad$ Time to maturity in years;

- $K=\$ 100$ Strike price;

- $r=0.03 \quad$ Interest rate;

- $\sigma=0.2$ 
- $\vartheta=-0.1$;

- $\nu=0.2$

- $N=4500$ Number of space intervals;

- $M=260$ Number of time intervals;

- $x_{\min }=1.60944$ Min $x$ value considered, corresponding to $S=\$ 5$;

- $x_{\max }=5.85793$ Max $x$ value considered, corresponding to $S=\$ 350$;

- $\Delta x=0.000944$ Size of a space interval;

- $\Delta t=0.003846 \quad$ Size of a time interval;

- $\frac{\Delta t}{\Delta x} \simeq 4$

Since in this experiment we move only the dividend yield; volatility, skewness and kurtosis do not change. In particular we have the following values

\begin{tabular}{|c|c|}
\hline$\sqrt{E\left[(X(t)-E[X(t)])^{2}\right]}$ & 0.205 \\
\hline$\frac{E\left[(X(t)-E[X(t)])^{3}\right]}{\sqrt{\left\{E\left[(X(t)-E[X(t)])^{2}\right]\right\}^{3}}}$ & -0.288 \\
\hline$\frac{E\left[(X(t)-E[X(t)])^{4}\right]}{\left\{E\left[(X(t)-E[X(t)])^{2}\right]\right\}^{2}}$ & 3.656 \\
\hline
\end{tabular}


In the first graph we consider we have the case of a call option with an underlying which has a $1 \%$ dividend yield.

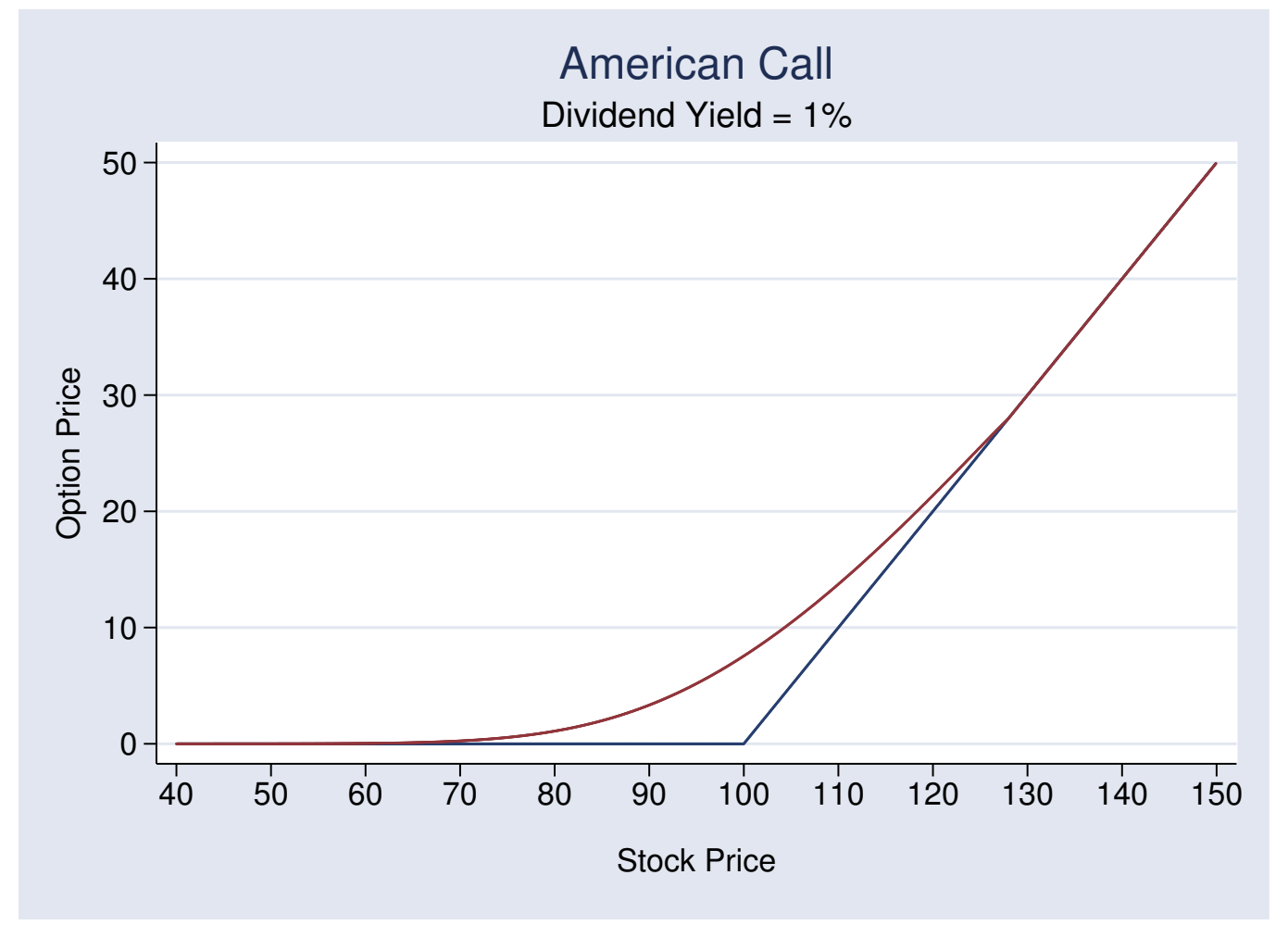

Figure 4.30: American Call: Starting Level for Dividend Yield Sensitivity Analysis 
In this graph we see the impact of raising the dividend yield from $0 \%$ to $3 \%$. As expect, when the dividend yield increases, the option prices is reduced.

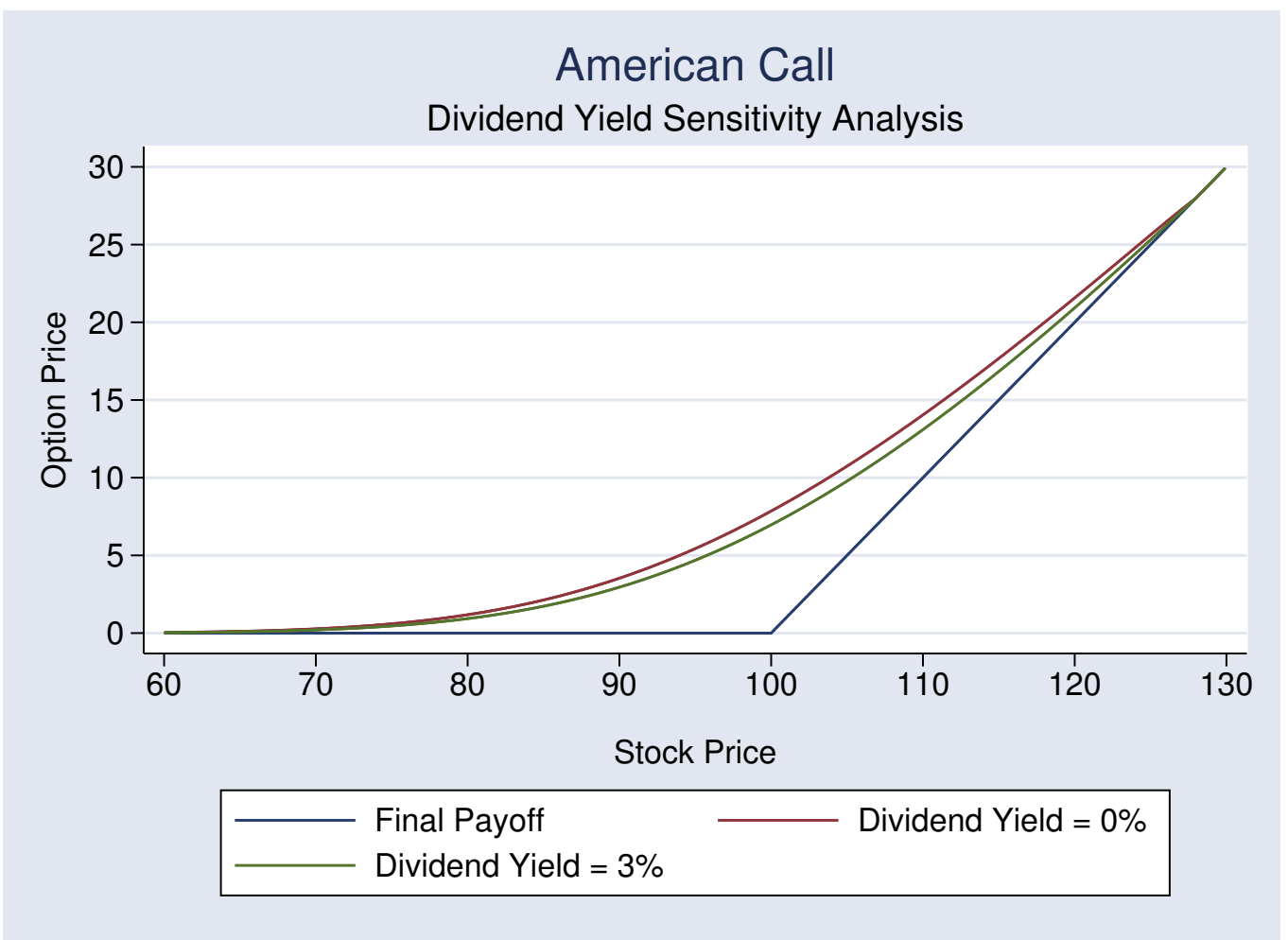

Figure 4.31: American Call: Dividend Yield Sensitivity Analysis 


\subsubsection{Puts: Sensitivity Analysis}

In this section we want to study the sensitivity of vanilla American put prices to the risk free interest rate and to the parameters affecting volatility, skewness and kurtosis of the distribution of the underlying returns. We remember from section 2.3 that the three parameters which enter in the solutions we have seen above, that is $\sigma, \nu$ and $\vartheta$, are not themselves directly volatility, skewness and kurtosis. In particular we have seen that the second, third and fourth moment can be written in the following way:

$$
\begin{gathered}
E\left[(X(t)-E[X(t)])^{2}\right]=\left(\vartheta^{2} \nu+\sigma^{2}\right) t \\
E\left[(X(t)-E[X(t)])^{3}\right]=\left(2 \vartheta^{3} \nu^{2}+3 \sigma^{2} \vartheta \nu\right) t \\
E\left[(X(t)-E[X(t)])^{4}\right]= \\
=\left(3 \sigma^{4} \nu+12 \sigma^{2} \vartheta^{2} \nu^{2}+6 \vartheta^{4} \nu^{3}\right) t+\left(3 \sigma^{4}+6 \sigma^{2} \vartheta^{2} \nu+3 \vartheta^{4} \nu^{2}\right) t^{2}
\end{gathered}
$$

The numerical experiments presented here can be obtained using the code in $\mathrm{C}$ language available in appendix B. The vanilla call case studied here is realized choosing the following variable values:

- callput $=0$

- euroamerican $=0$

- barrier_switch $=0$

in the $\mathrm{C}$ code. 


\section{Sensitivity with Respect to the Interest Rate}

As first experiment, we consider the sensitivity of a vanilla American put price with respect to the risk free interest rate. The parameters used in this experiment are the following:

- $T=1 \quad$ Time to maturity in years;

- $K=\$ 100$ Strike price;

- $q=0.01 \quad$ Dividend yield;

- $\sigma=0.2$;

- $\vartheta=-0.1$;

- $\nu=0.2$

- $N=4500$ Number of space intervals;

- $M=260$ Number of time intervals;

- $x_{\min }=1.60944$ Min $x$ value considered, corresponding to $S=\$ 5$;

- $x_{\max }=5.85793$ Max $x$ value considered, corresponding to $S=\$ 350$;

- $\Delta x=0.000944$ Size of a space interval;

- $\Delta t=0.003846 \quad$ Size of a time interval;

- $\frac{\Delta t}{\Delta x} \simeq 4$

The change in interest rate does not affect the volatility, skewness and kurtosis of the distribution. In particular we have the following constant values:

\begin{tabular}{|c|c|}
\hline$\sqrt{E\left[(X(t)-E[X(t)])^{2}\right]}$ & 0.205 \\
\hline$\frac{E\left[(X(t)-E[X(t)])^{3}\right]}{\sqrt{\left\{E\left[(X(t)-E[X(t)])^{2}\right]\right\}^{3}}}$ & -0.288 \\
\hline$\frac{E\left[(X(t)-E[X(t)])^{4}\right]}{\left\{E\left[(X(t)-E[X(t)])^{2}\right]\right\}^{2}}$ & 3.656 \\
\hline
\end{tabular}


Let's start consider the American put price in a scenario of $1 \%$ risk free interest rate.

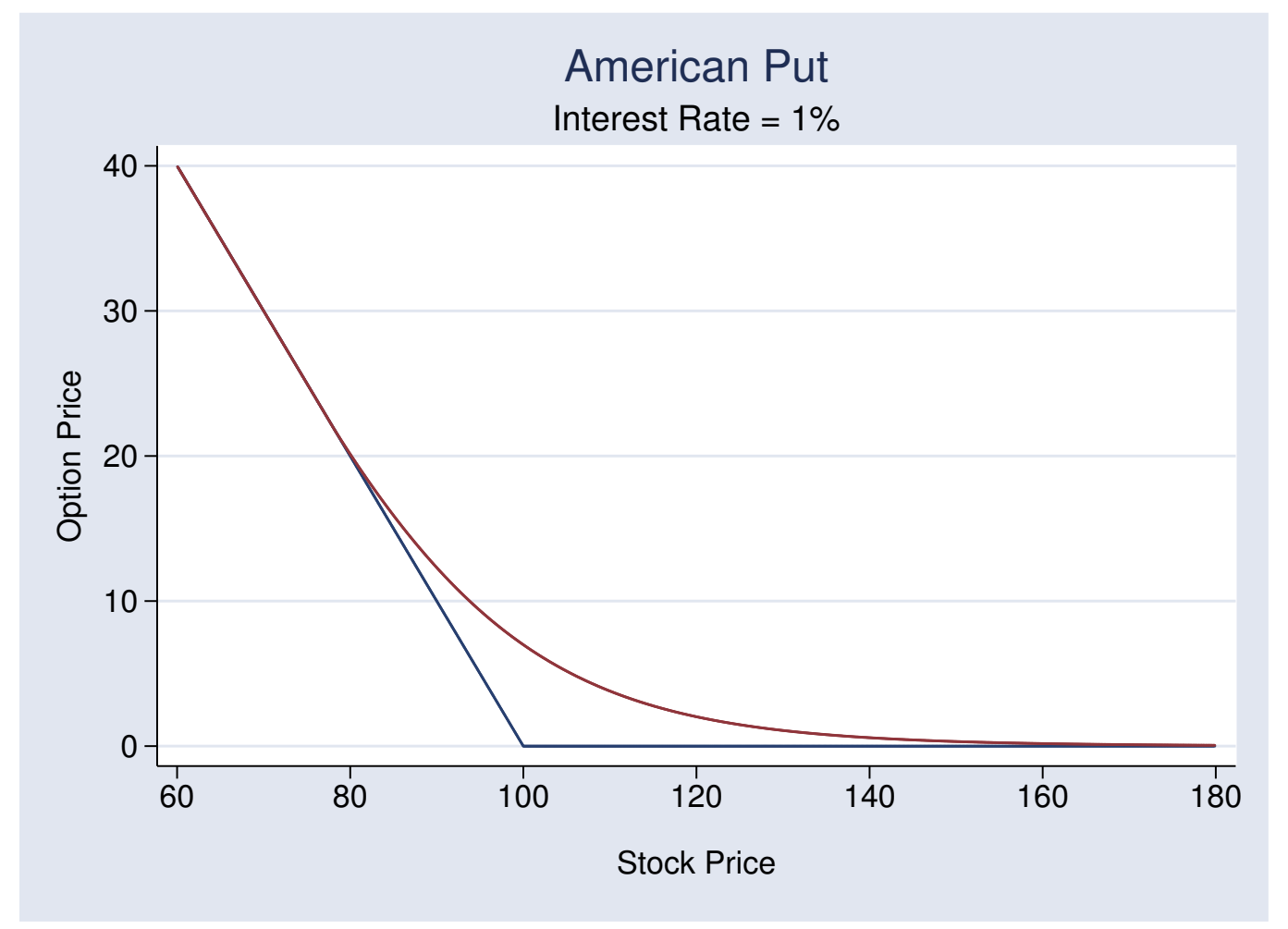

Figure 4.32: American Put: Starting Level for Interest Rate Sensitivity Analysis 
We now consider the impact on the option prices of moving the interest rate from $1 \%$ to $7 \%$. As expected, we can see that as the interest rate increases, the put price decreases.

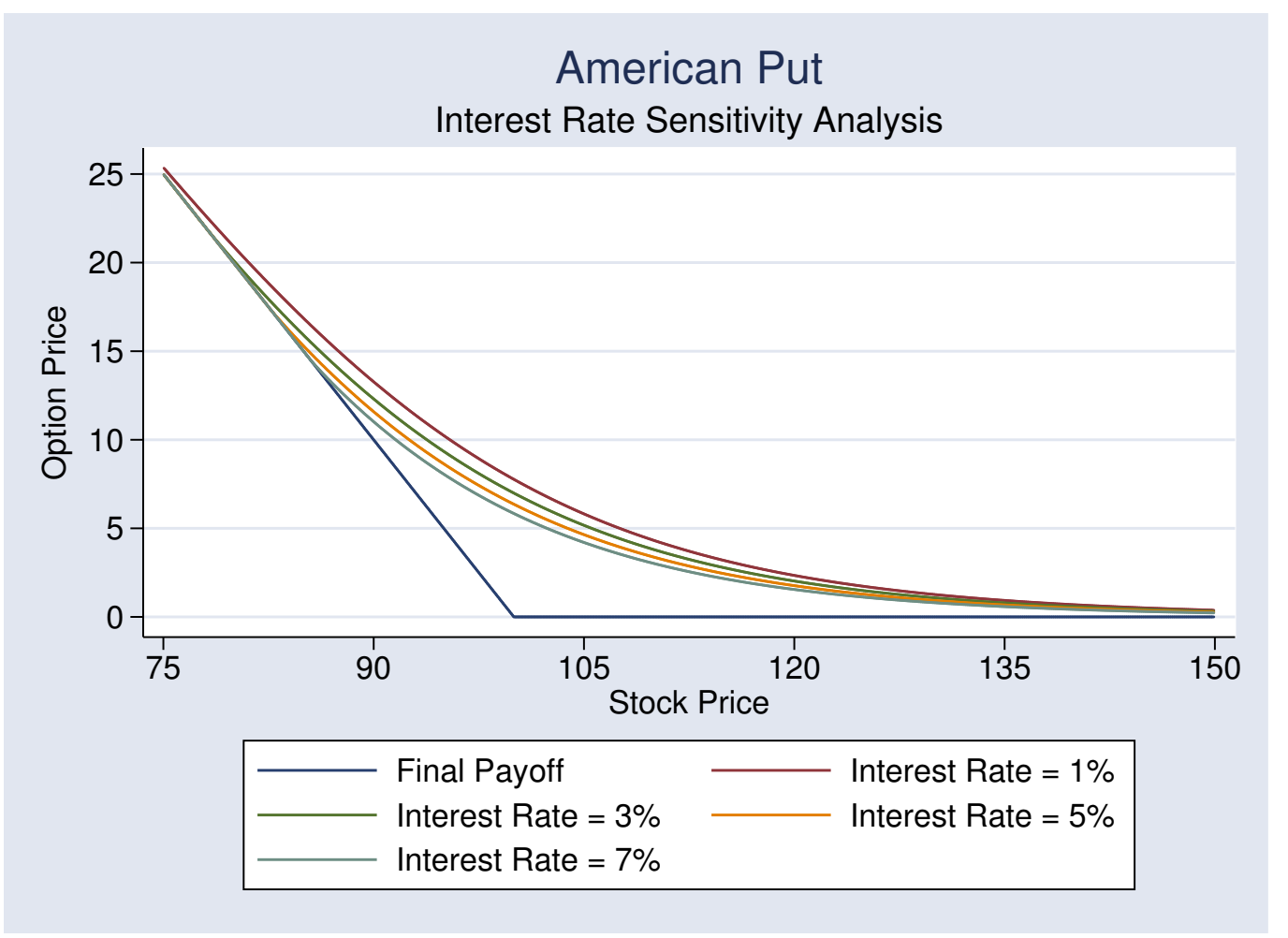

Figure 4.33: American Put: Interest Rate Sensitivity Analysis 


\section{Sensitivity with Respect to $\sigma$}

We study now the sensitivity of vanilla American options with respect to $\sigma$. The parameters used in this experiment are the following:

- $T=1 \quad$ Time to maturity in years;

- $K=\$ 100$ Strike price;

- $r=0.03$ Interest rate;

- $q=0.01$ Dividend yield;

- $\vartheta=-0.1$;

- $\nu=0.2$;

- $N=4000$ Number of space intervals;

- $M=235$ Number of time intervals;

- $x_{\min }=1.60944$ Min $x$ value considered, corresponding to $S=\$ 5$;

- $x_{\max }=5.85793$ Max $x$ value considered, corresponding to $S=\$ 350$;

- $\Delta x=0.00106 \quad$ Size of a space interval;

- $\Delta t=0.00426 \quad$ Size of a time interval;

- $\frac{\Delta t}{\Delta x} \simeq 4$

Using these parameters, we move $\sigma$ from $10 \%$ to $50 \%$. As $\sigma$ values are modified, volatility, skewness and kurtosis change. In particular we have the following values:

\begin{tabular}{|c|c|c|c|c|c|c|}
\hline$\sigma$ & 0.1 & 0.15 & 0.2 & 0.3 & 0.4 & 0.5 \\
\hline$\sqrt{E\left[(X(t)-E[X(t)])^{2}\right]}$ & 0.110 & 0.157 & 0.205 & 0.303 & 0.402 & 0.502 \\
\hline$\frac{E\left[(X(t)-E[X(t)])^{3}\right]}{\sqrt{\left\{E\left[(X(t)-E[X(t)])^{2}\right]\right\}^{3}}}$ & -0.517 & -0.373 & -0.288 & -0.196 & -0.148 & -0.119 \\
\hline$\frac{E\left[(X(t)-E[X(t)])^{4}\right]}{\left\{E\left[(X(t)-E[X(t)])^{2}\right]\right\}^{2}}$ & 3.783 & 3.694 & 3.656 & 3.626 & 3.615 & 3.609 \\
\hline
\end{tabular}


Of course when $\sigma$ increases, volatility increases. We see moreover that as $\sigma$ becomes larger, skewness and kurtosis are reduced. The graph shows that option prices increase as $\sigma$ increases.

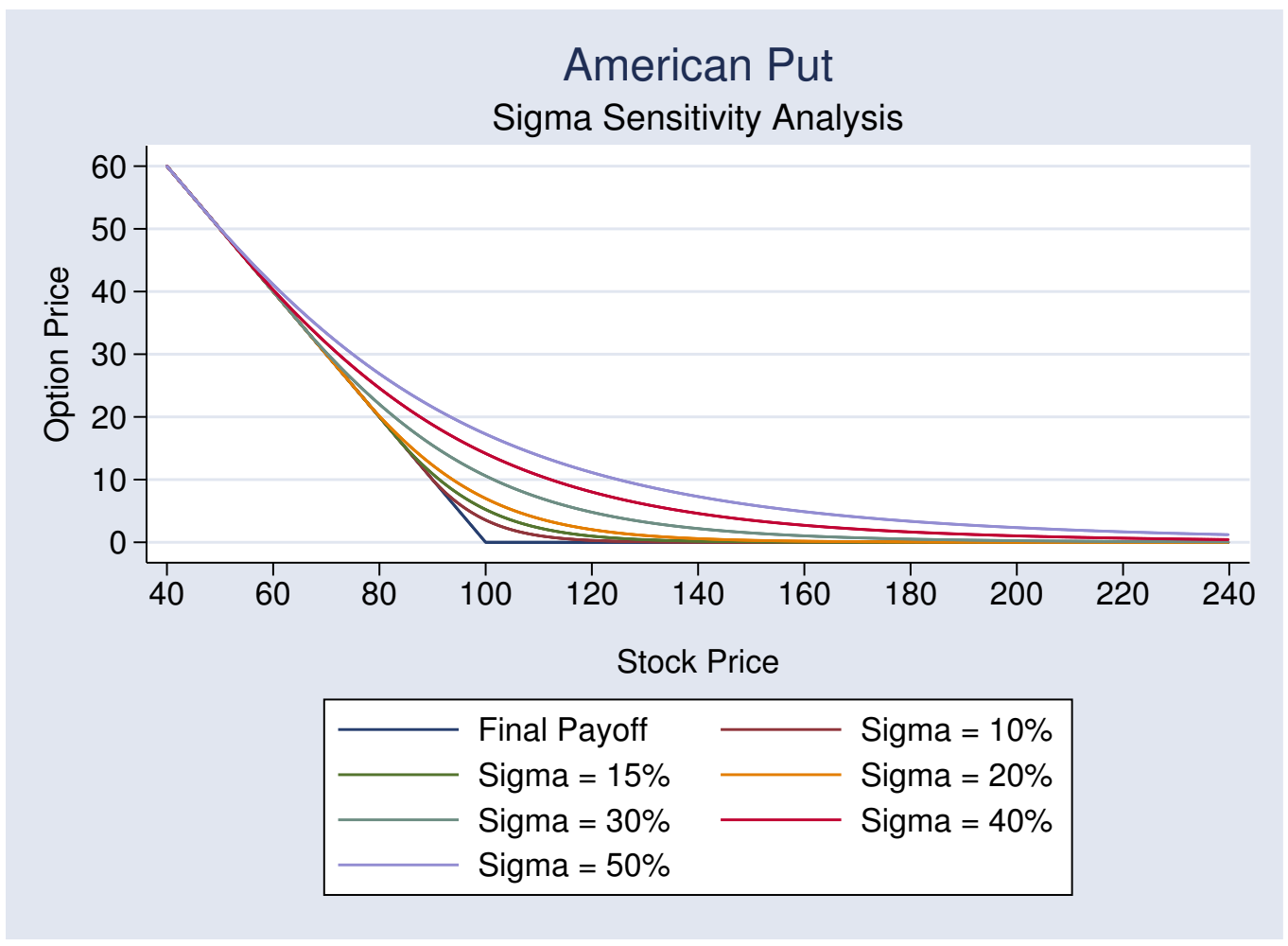

Figure 4.34: American Put: $\sigma$ Sensitivity Analysis 


\section{Sensitivity with Respect to $\nu$}

We study now the sensitivity of vanilla American options with respect to $\nu$. The parameters used in this experiment are the following:

- $T=1 \quad$ Time to maturity in years;

- $K=\$ 100$ Strike price;

- $r=0.03 \quad$ Interest rate;

- $q=0.01 \quad$ Dividend yield;

- $\sigma=0.2$

- $\vartheta=-0.1$;

- $N=4000$ Number of space intervals;

- $M=235 \quad$ Number of time intervals;

- $x_{\min }=1.60944$ Min $x$ value considered, corresponding to $S=\$ 5$;

- $x_{\max }=5.85793$ Max $x$ value considered, corresponding to $S=\$ 350$;

- $\Delta x=0.00106 \quad$ Size of a space interval;

- $\Delta t=0.00426 \quad$ Size of a time interval;

- $\frac{\Delta t}{\Delta x} \simeq 4$;

While keeping fixed $\sigma, \vartheta$ and the other parameters, we move $\nu$ from 0.1 to 1.0 and we study the impact of this on the option price. Volatility, skewness and kurtosis are affected by the move in $\nu$ in the following way:

\begin{tabular}{|c|c|c|c|c|}
\hline$\nu$ & 0.1 & 0.3 & 0.7 & 1.0 \\
\hline$\sqrt{E\left[(X(t)-E[X(t)])^{2}\right]}$ & 0.202 & 0.207 & 0.217 & 0.224 \\
\hline$\frac{E\left[(X(t)-E[X(t)])^{3}\right]}{\sqrt{\left\{E\left[(X(t)-E[X(t)])^{2}\right]\right\}^{3}}}$ & -0.147 & -0.424 & -0.921 & -1.252 \\
\hline$\frac{E\left[(X(t)-E[X(t)])^{4}\right]}{\left\{E\left[(X(t)-E[X(t)])^{2}\right]\right\}^{2}}$ & 3.314 & 4.021 & 5.679 & 7.080 \\
\hline
\end{tabular}


We see that in this case we use a value of $\vartheta \neq 0$. When we studied the sensitivity of European vanilla option prices to $\nu$, we assumed the presence of symmetric distribution of stock returns. Here we add a level of complexity because if the distribution is skewed, a move in $\nu$ will impact also volatility and skewness. In particular as we can see from the table above, as $\nu$ increases of course the kurtosis increases. Moreover we observe that the volatility and the negative skewness increase as well.

The figure compares two hypothesis: $\nu=0.1$ and $\nu=1.0$. We see that the option is worth more in the case of large $\nu$ when it is more out of the money, while the low $\nu$ case is worth more for in the money options. Note that as $\nu$ increase volatility increases too, so the option with higher $\nu$ is proportionally worth more, for the same level of skewness and kurtosis.

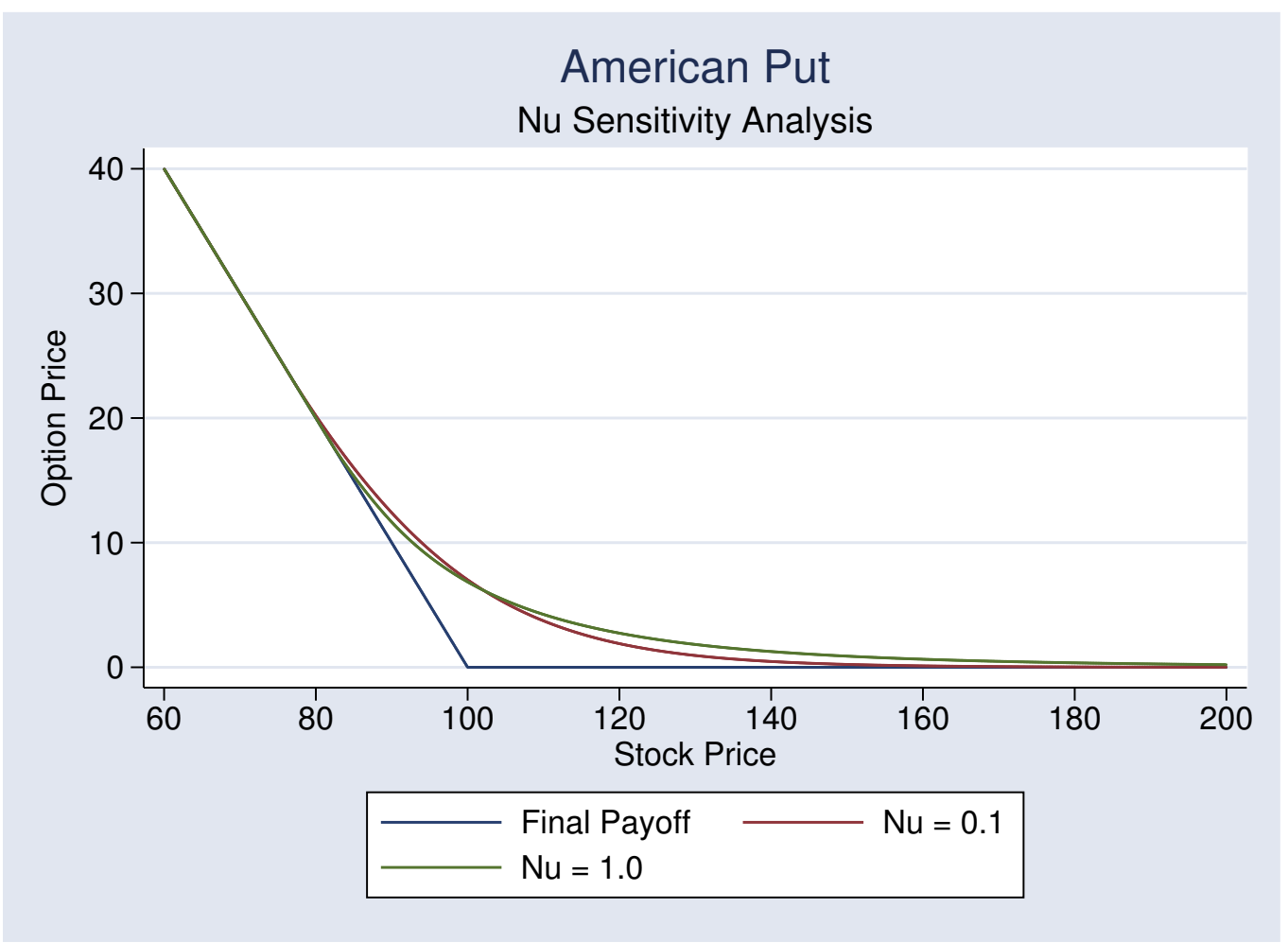

Figure 4.35: American Put: $\nu$ Sensitivity Analysis 
In this second graph, we present a zoom of the previous graph. We see that for the in the money area the option whose underlying has a lower $\nu$ is worth less. Because the put option is American at some point the two value will converge, because both option will reach the early exercise level.

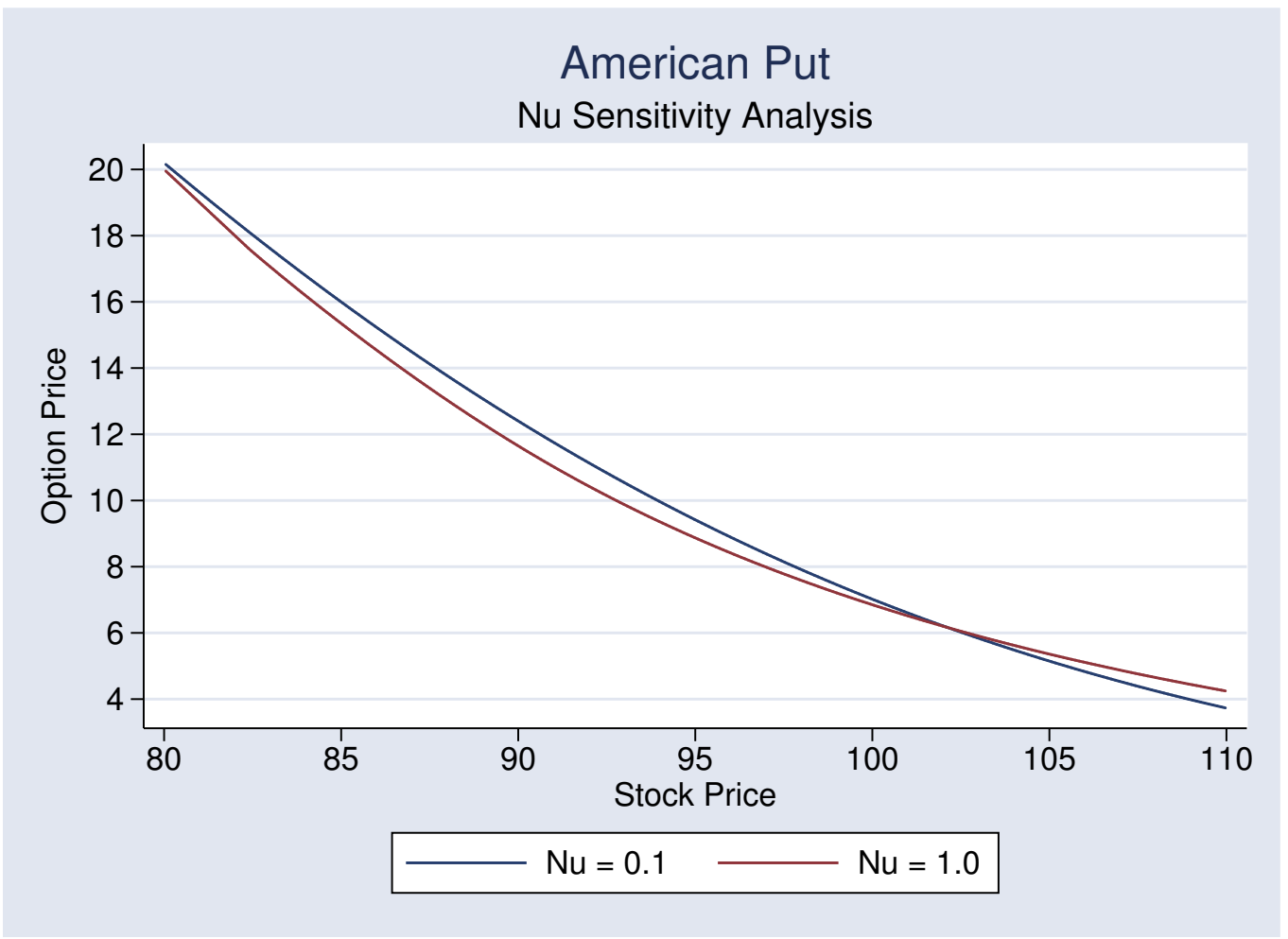

Figure 4.36: American Put: $\nu$ Sensitivity Analysis, Detail 
In this graph we study the out of the money region. Here higher values of $\nu$ produce higher option value. We see that the combination of a larger volatility, negative skewness and kurtosis can produce a material difference in the option price. For example when the stock is around $\$ 130$ the option having $\nu=0.1$ is worth 94 cents while the option with $\nu=1.0$ has a price of $\$ 1.83$.

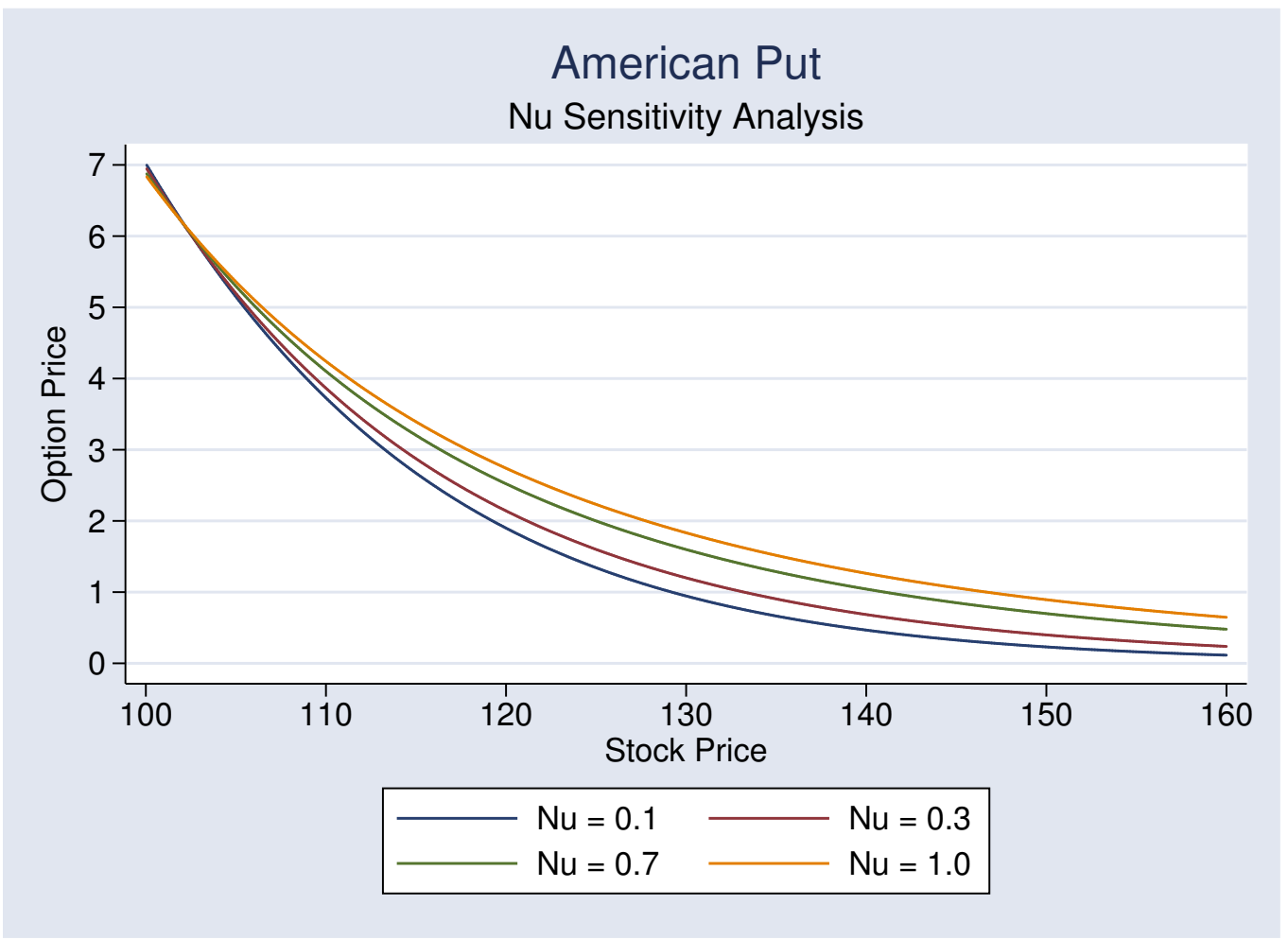

Figure 4.37: American Put: $\nu$ Sensitivity Analysis, Detail 


\section{Sensitivity with Respect to $\vartheta$}

Let's consider now the sensitivity of a vanilla American put option with respect to $\vartheta$. The experiments will use the following parameters:

- $T=1 \quad$ Time to maturity in years;

- $K=\$ 100$ Strike price;

- $r=0.03 \quad$ Interest rate;

- $q=0.01 \quad$ Dividend yield;

- $\sigma=0.2$

- $\nu=0.2$;

- $N=4000$ Number of space intervals;

- $M=235 \quad$ Number of time intervals;

- $x_{\text {min }}=1.60944$ Min $x$ value considered, corresponding to $S=\$ 5$;

- $x_{\max }=5.85793$ Max $x$ value considered, corresponding to $S=\$ 350$;

- $\Delta x=0.00106 \quad$ Size of a space interval;

- $\Delta t=0.00426 \quad$ Size of a time interval;

- $\frac{\Delta t}{\Delta x} \simeq 4$

Keeping these values fixed, we move $\vartheta$ from 0 to -0.5 . As $\vartheta$ changes, volatility, skewness and kurtosis are affected in the following way:

\begin{tabular}{|c|c|c|c|c|c|}
\hline$\vartheta$ & 0.0 & -0.2 & -0.3 & -0.4 & -0.5 \\
\hline$\sqrt{E\left[(X(t)-E[X(t)])^{2}\right]}$ & 0.20 & 0.22 & 0.24 & 0.27 & 0.30 \\
\hline$\frac{E\left[(X(t)-E[X(t)])^{3}\right]}{\sqrt{\left\{E\left[(X(t)-E[X(t)])^{2}\right]\right\}^{3}}}$ & 0.00 & -0.52 & -0.67 & -0.76 & -0.81 \\
\hline$\frac{E\left[(X(t)-E[X(t)])^{4}\right]}{\left\{E\left[(X(t)-E[X(t)])^{2}\right]\right\}^{2}}$ & 3.60 & 3.78 & 3.91 & 4.01 & 4.08 \\
\hline
\end{tabular}


An increase in the absolute value of negative $\vartheta$ of course produces an increase the negative skewness. Moreover it produces an increase in both volatility and kurtosis. For this reason, we see in this graph that as $\vartheta$ becomes more negative the option price increase.

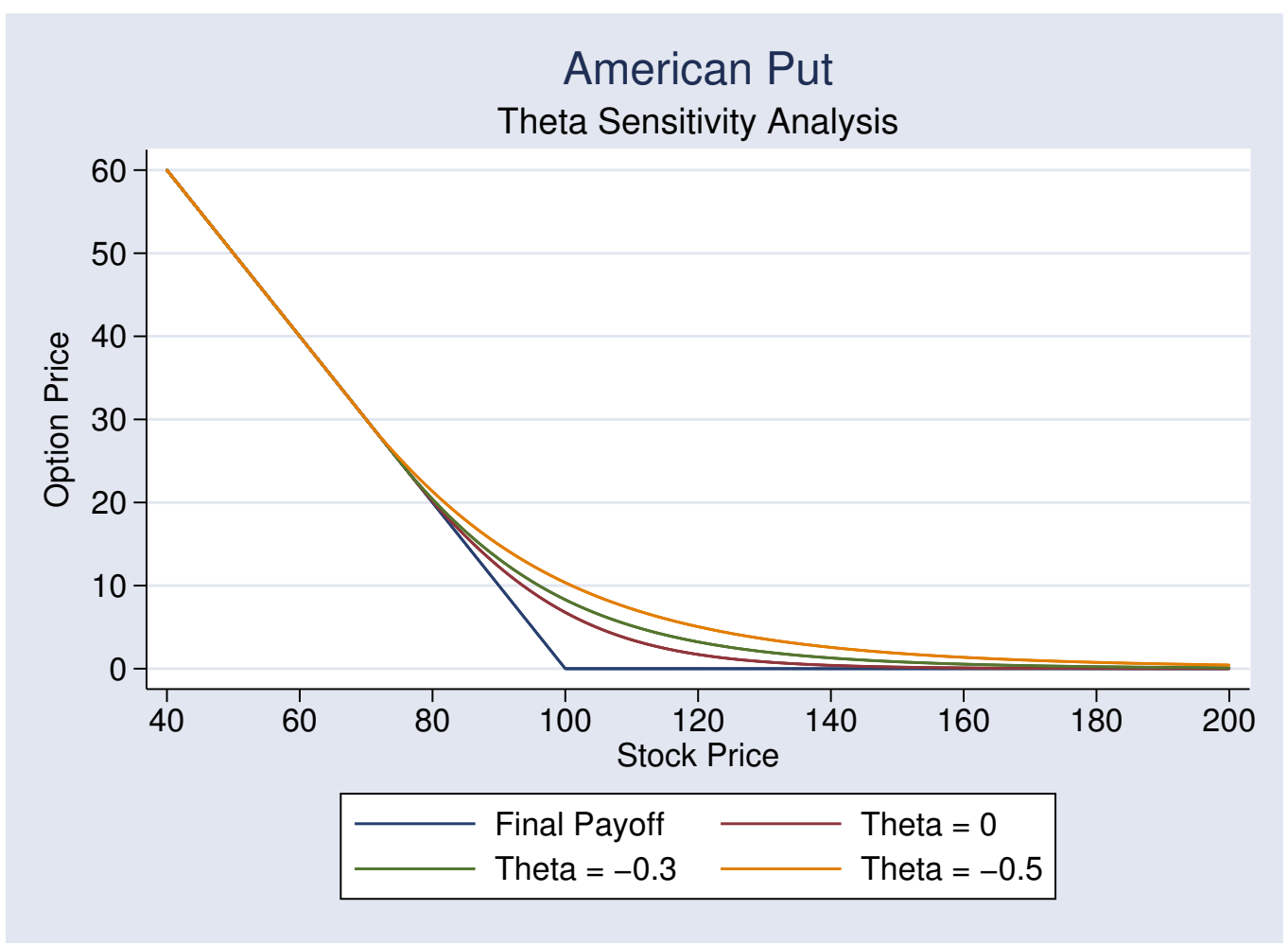

Figure 4.38: American Put: $\vartheta$ Sensitivity Analysis 
In this graph we have a zoom of the area considered in the previous figure. We consider here 5 different levels of $\vartheta$ and note that as $\vartheta$ increases in absolute value, the fair value increase too.

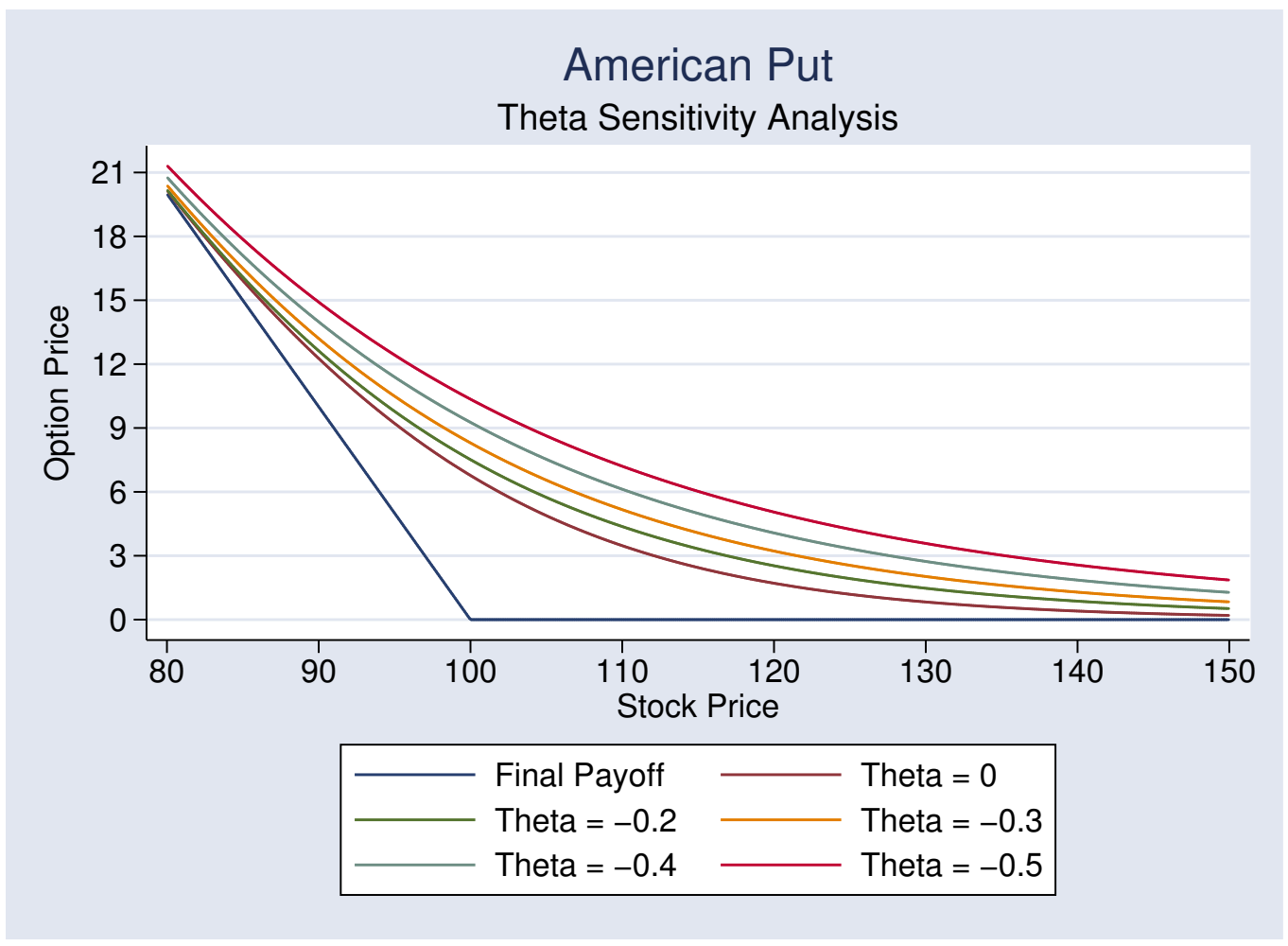

Figure 4.39: American Put: $\vartheta$ Sensitivity Analysis, Detail 


\section{Chapter 5}

\section{Numerical Solution for the European and American Barrier Option Price Under the Variance Gamma Process}

\subsection{Introduction to Barrier Options}

Barrier options are options whose value depends upon the underlying reaching a particular level defined as barrier ${ }^{1}$. There are two main group of barrier options: in-barriers, also known as knock-in options, and out-barriers, or knock-out options. Knock-ins are options which expires worthless if the underlying has not reached the barrier during the life of the of the option. They become plain vanilla options as soon as the underlying reaches the barrier. Knock-out options, on the other side, have a final payoff at expiration which is the same as plain vanilla options if the barrier has not been reached during the life of the derivative. They expires worthless as soon as the barrier is reached. What we described so far is the case where there is no rebate. It is also possible to have a rebate: in this case the knock-out option pays a fixed rebate as soon as the barrier is passed, while the knock-in option pays the

\footnotetext{
${ }^{1}$ For an introduction to barrier options, to their valuation in terms of closed form solution, and to their static and dynamic replication see Filippo Fiorani, Le Barrier Option nel Mercato Finanziario: Replica Statica e Dinamica, Tesi discussa alla Facoltà di Economia e Commercio, Università degli Studi di Torino, academic year 1998-1999.
} 
rebate at expiration if the barrier has not been reached.

The barrier can be at a higher or at a lower stock level compared with the value of the underlying at the moment of issue. We use sometimes the expression "straight barrier" to indicate the fact that the barrier is reached when the option is out of the money. Straight barriers are up-and-out put, down-and-out call, down-and-in call e up-and-in put. We use the expression "reverse barrier" to define the situation where the barrier is reached when the option is in the money. Reverse barriers are up-and-out call, down-andout put, down-and-in put e up-and-in call. The straight barriers are more similar to the plain vanilla options, while the reverse barrier have a behavior which can be quite different from their regular counterparts. The reason is that reverse barrier options when are activate or deactivated by reaching the barrier, have a high intrinsic value ${ }^{2}$ and hence their value may change dramatically as it approaches the barrier. Because of this, reverse barrier options are more difficult to be valued and hedged.

Apart from the typologies described so far, it possible to distinguish several more classes of barrier options, depending, for example, on the barrier being monitored continuously or at discrete times, on the presence of multiple barriers (both above and below the stock price), on the option being activated or deactivated instantaneously or gradually as it reaches further barrier levels and so on. We will focus our attention here only on the case where there is only one barrier, the stock price is monitored continuously to determine if the barrier has been reached and the options is activated or deactivated completely and instantaneously upon reaching of the barrier.

The pricing of barrier options under variance gamma via numerical solution of a finite different scheme is our innovation. Hirsa presented some ideas behind the pricing of European up-and-out call options with this approach at a seminar in Columbia University in the Spring 2001. The actual solution of the problem and all the remaining European barrier option cases as well as the whole American barrier option pricing are our own original contribution to the research.

\footnotetext{
${ }^{2}$ With intrinsic value, we mean the value the option has if exercised right away.
} 


\subsection{European Barrier Options}

\subsubsection{The Variance Gamma PIDE for European Bar- rier Options}

Similarly to the plain vanilla option case, it possible to obtain a closed form solution for the price of European barrier options, however it is not necessary to solve numerically American barrier option. We want to present here a technique which follows the same approach used for the plain vanilla case and that as such can be applied to both European and American barrier option.

It is known that when the underlying follows a geometric Brownian motion, barrier options follows the same dynamics as plain vanilla options, that is barrier option have to satisfy the Black and Scholes PDE. However this options will have to satisfy different boundary conditions. The same is true when the underlying follows a variance gamma process. We can therefore say that a European barrier option follows the PIDE (4.6) which describe the dynamic of European vanilla options under the variance gamma, that is

$$
\begin{array}{r}
\frac{\partial W(x, t)}{\partial t}+(r-q+\omega) \cdot \frac{\partial W(x, t)}{\partial x}+ \\
+\int_{-\infty}^{+\infty}[W(x+y, t)-W(x, t)] k(y) d y=r W(x, t)
\end{array}
$$

where as before $x \triangleq \ln (S)$. The boundary and final conditions will depend on the kind of barrier option we are considering. Before going to solve numerically our problem let's remember the following relationship:

$$
\text { Vanilla Option }=\text { Knock-in }+ \text { Knock-out }
$$

Because we are already able to price vanilla options under the variance gamma, we do not need to be able to price both in-barriers and out-barriers since one of the two cases can be obtained by difference. Pricing of knock-in is generally considerably more difficult than the pricing of knock-out, therefore we decide to find a solution to the problem of pricing the out-barrier numerically and to obtain the correspondent knock-in just by difference.

Let's define now the final and boundary conditions required to solve this PIDE in the different cases of knock-out options. Let's start with up-andout-call options. Here the final condition, provided that the stock has not 
reached the barrier is that the option at expiration is equal to its payoff. That is

$$
W(x, T)=\max \left(e^{x}-K, 0\right) \quad \text { if }\left\{e^{x}<B \quad \forall t\right\}
$$

where we denoted with $B>0$ the barrier level. Clearly if the stock reaches the barrier at any time before expiration, the final condition is no more relevant since the option cease to exist. Up-and-out call boundary conditions are

$$
\begin{array}{rr}
W(-\infty, t)=0 & \forall t \\
W(\ln (B), t)=R & \forall t
\end{array}
$$

with $R \geqslant 0$ denoting the rebate value. We can see that only the last boundary condition has changed with respect to the plain vanilla case: the convergence of the option value with to the stock value as the underlying tends to infinity is now replaced with the option paying the rebate value (which could also be equal to zero) as soon as the stock reaches the barrier. For down-and-out call the final condition is

$$
W(x, T)=\max \left(e^{x}-K, 0\right) \quad \text { if }\left\{e^{x}>B \quad \forall t\right\}
$$

while the boundary conditions become

$$
\begin{array}{rr}
W(\ln (B), t)=R & \forall t \\
W(+\infty, t)=e^{x} & \forall t
\end{array}
$$

As for up-and-out put, the final condition is

$$
W\left(e^{x}, T\right)=\max \left(K-e^{x}, 0\right) \quad \text { if }\left\{e^{x}<B \quad \forall t\right\}
$$

and the boundary conditions are

$$
\begin{array}{cc}
W(-\infty, t)=K & \forall t \\
W(\ln (B), t)=R & \forall t
\end{array}
$$

Finally, for down-and-out put the final condition is

$$
W\left(e^{x}, T\right)=\max \left(K-e^{x}, 0\right) \text { if }\left\{e^{x}>B \quad \forall t\right\}
$$

and the boundary conditions are

$$
\begin{array}{r}
W(\ln (B), t)=R \quad \forall t \\
W(+\infty, t)=0 \quad \forall t
\end{array}
$$




\subsubsection{Numerical Solution for European Barrier Op- tions}

The PIDE describing the dynamics of European option prices, can be solved with a finite difference approach quite similar to the one presented for plain vanilla options. Let's start defining the range of value inside which we will build the grid. This time it is necessary to distinguish between typology of options. Given that we are going to work on knock-out options, the options disappear when they reach the barrier level, it is therefore a good choice to fix the limit of the grid at the barrier level. In this way we never face the issue of considering jumps of the stock from positions where it does not exist anymore. This approach has also the advantage that we will be sure to have a node exactly at the barrier level. Remember in fact that when pricing a barrier option with the binomial method you have to be particularly careful about how to position the nodes with respect to the barrier to avoid problems created by the oscillation around the barrier of the nodes as the number of steps is increased ${ }^{3}$.

We can say that for the cases where the barrier is for higher value of the underlying (up-and-out calls and puts) the range of values considered is

$$
[0, T] \times\left[x_{\min }, \ln (B)\right]
$$

while when the barrier is below the stock level (down-and-out calls and puts) the range of value is given by

$$
[0, T] \times\left[\ln (B), x_{\max }\right]
$$

where $x_{\min }$ and $x_{\max }$ are such that the value of the option on the boundary is correct up to the level of precision required. We can now realize the discretization using $N+1$ points in the $x$-direction and $M+1$ points in the $t$-direction. The size of space steps is therefore equal to

$$
\Delta x=\frac{\ln (B)-x_{\min }}{N}
$$

for up and out options, while it is given by

$$
\Delta x=\frac{x_{\max }-\ln (B)}{N}
$$

\footnotetext{
${ }^{3}$ See more on this in Phelim P. Boyle and Sok Hoon Lau, "Bumping Up Against the Barrier with the Binomial Method", The Journal of Derivatives, Summer 1994, pages 6-14.
} 
for down and out options. The size of a time step is the same for both types of options and it is equal to

$$
\Delta t=\frac{T}{M}
$$

The notation $W\left(x_{i}, t_{j}\right)$ refers to the value of $W(\cdot)$ at the node $(i, j)$ and, as for the plain vanilla case, the partial derivatives can be approximated in the following way

$$
\begin{gathered}
\frac{\partial W(x, t)}{\partial t} \simeq \frac{W\left(x_{i}, t_{j+1}\right)-W\left(x_{i}, t_{j}\right)}{\Delta t} \\
\frac{\partial W(x, t)}{\partial x} \simeq \frac{W\left(x_{i+1}, t_{j}\right)-W\left(x_{i-1}, t_{j}\right)}{2 \Delta x}
\end{gathered}
$$

and by substitution the PIDE (5.1) can be approximated as follow:

$$
\begin{array}{r}
h W\left(x_{i-1}, t_{j}\right)+(1+r \Delta t) W\left(x_{i}, t_{j}\right)-h W\left(x_{i+1}, t_{j}\right)= \\
=W\left(x_{i}, t_{j+1}\right)+\Delta t \int_{-\infty}^{+\infty}\left[W\left(x_{i}+y, t_{j+1}\right)-W\left(x_{i}, t_{j+1}\right)\right] k(y) d y
\end{array}
$$

Note that this is exactly the same as PIDE (4.7) which described the plain vanilla case.

\subsubsection{Solution of the Jump Integral for European Bar- rier Options}

As for the plain vanilla options, the most interesting part of the solution of the system is in the way the integral is treated. A detailed solution of the integral is presented in the appendix A.6. Some ideas behind the solution the integral for European up-and-out calls have been presented by Ali Hirsa at the Mathematics of Finance Practitioner Seminar in Spring 2001, everything else is our own original development.

We can separate the integral as sum of integral in the same way we did before, dividing three main cases: jumps smaller than a space step, jumps bigger than a step, but still inside the range, jump so big that the stock finishes outside the range considered. For each of these three cases, we have 
two subcases given by positive and negative jumps. The integral can be written as

$$
\begin{aligned}
& \int_{-\infty}^{+\infty}\left[W\left(x_{i}+y, t_{j+1}\right)-W\left(x_{i}, t_{j+1}\right)\right] k(y) d y= \\
= & \int_{-\infty}^{x_{0}-x_{i}}\left[W\left(x_{i}+y, t_{j+1}\right)-W\left(x_{i}, t_{j+1}\right)\right] k(y) d y+ \\
+ & \int_{x_{0}-x_{i}}^{-\Delta x}\left[W\left(x_{i}+y, t_{j+1}\right)-W\left(x_{i}, t_{j+1}\right)\right] k(y) d y+ \\
& +\int_{-\Delta x}^{0}\left[W\left(x_{i}+y, t_{j+1}\right)-W\left(x_{i}, t_{j+1}\right)\right] k(y) d y+ \\
& +\int_{0}^{\Delta x}\left[W\left(x_{i}+y, t_{j+1}\right)-W\left(x_{i}, t_{j+1}\right)\right] k(y) d y+ \\
+ & \int_{\Delta x}^{x_{N}-x_{i}}\left[W\left(x_{i}+y, t_{j+1}\right)-W\left(x_{i}, t_{j+1}\right)\right] k(y) d y+ \\
& +\int_{x_{N}-x_{i}}^{+\infty}\left[W\left(x_{i}+y, t_{j+1}\right)-W\left(x_{i}, t_{j+1}\right)\right] k(y) d y
\end{aligned}
$$

Note that here $x_{N}=\ln (B)$ for up-and-out options and $x_{0}=\ln (B)$ for downand-out options. The prices imposed at the barrier level $\ln (B)$ is going to be different than the price imposed at the boundary level for regular options. However, inside the barrier, the dynamics of the option value is unchanged compared with the plain vanilla option case. Hence the four integrals which define jumps after which the stock price remains inside the range considered, integrals (5.5) to (5.8), can be rewritten in the exact same way we did for plain vanilla options. The only difference is that for up barrier, $W\left(x_{N}, \cdot\right)=R$ and for down barrier $W\left(x_{0}, \cdot\right)=R$, since these would represent first passages of the barrier, where $R \geq 0$ denotes the rebate. Remember that we do not need to distinguish between puts and calls to deal with these four integrals and therefore they will be written in the same way for all the cases of knockout options that we are going to consider here.

\section{Solution for Up-And-Out Call Options}

Let's now focus our attention on large jumps which take the stock outside the considered range, in this case the behavior is different depending on the fact 
that the option is a put or a call and on the position of the barrier $B$ with respect to the underlying. We start considering up-and-out call options. In this situation, if the jump is large and positive, the stock passes the barrier and therefore explodes. The option holder would get the rebate, if any, at the very moment the stock reaches the barrier and after that her option will have no more value. Therefore, provided that the stock price has always been lower than the barrier, if

$$
y \in\left[x_{N}-x_{i},+\infty\right]
$$

where $x_{N}=\ln (B)$, we can say that the up-and-out call value is

$$
W\left(x_{i}+y, t_{j+1}\right)=R
$$

Instead of $R$, we could have written $W\left(x_{N}, t_{j+1}\right)$ since the boundary condition (5.2) requires that at the barrier level the option is worth the rebate, if this is the first time that the stock reaches the barrier and the upper limit of the grid is fixed at the barrier level.

Note that, starting from a situation in which the barrier has never been reached, we get the rebate both if we jump exactly at the barrier level and if we cross the barrier jumping further. The remark is necessary because the variance gamma is not a continuous process and so it does not necessarily reach the barrier level before reaching a stock level higher than the barrier and, clearly, it would not make any sense to receive the rebate payment only for jumps exactly at the barrier level. On the other side, the rebate has to be paid only once and if the stock goes back for a second time to the barrier level, nothing happens since the option does not exist anymore. Given that we initially imposed that the grid boundary is given by the barrier, we do not have to be worried to check for second or further barrier passages, because we do not study the stock behavior outside the grid and so we would never have a case of a stock passing the barrier level more than once. We can say that here the boundary is absorbent meaning that the process does not come back once the boundary is reached. We can also say that the three values at time $t$ which are solved in each equation of the linear system, $W\left(x_{i-1}, t_{j}\right), W\left(x_{i}, t_{j}\right)$ and $W\left(x_{i+1}, t_{j}\right)$, are a function of option values at time $t+1$ corresponding to stocks inside the grid. These stocks are allowed to jump through the barrier, as in the case of the jump described by integral (5.9). However time $t$ option prices are not affected by options corresponding to stocks which are already outside the barrier and are jumping starting from a position outside the grid. 
This condition is assured by imposing that the grid boundary is given by the barrier itself.

Moving now to the case in which the jump is big and negative the option value can be approximated to zero because it is a call option, formally if

$$
y \in\left[-\infty, x_{0}-x_{i}\right]
$$

we have the same approximation we used for plain vanilla call options, that is

$$
W\left(x_{i}+y, t_{j+1}\right) \simeq 0
$$

Using these approximations for the large positive and negative jumps together with the result we had for the plain vanilla options for smaller jumps, leads to the following way to rewrite the integral in the case of up-and-out calls:

$$
\begin{gathered}
\int_{-\infty}^{+\infty}\left[W\left(x_{i}+y, t_{j+1}\right)-W\left(x_{i}, t_{j+1}\right)\right] k(y) d y= \\
=\frac{\left[W\left(x_{i+1}, t_{j+1}\right)-W\left(x_{i}, t_{j+1}\right)\right]\left(1-e^{-\frac{\mu_{p}}{\nu_{p}} \Delta x}\right)}{\nu \Delta x \frac{\mu_{p}}{\nu_{p}}}+ \\
+\frac{\left[W\left(x_{i-1}, t_{j+1}\right)-W\left(x_{i}, t_{j+1}\right)\right]\left(1-e^{-\frac{\mu_{n}}{\nu_{n}} \Delta x}\right)}{\nu \Delta x \frac{\mu_{n}}{\nu_{n}}}+ \\
+\sum_{k=1}^{N-i-1} \frac{1}{\nu \Delta x}\left[W\left(x_{i+k+1}, t_{j+1}\right)-W\left(x_{i+k}, t_{j+1}\right)\right] \frac{\nu_{p}}{\mu_{p}}\left[e^{-\frac{\mu_{p}}{\nu_{p}} k \Delta x}-e^{-\frac{\mu_{p}}{\nu_{p}}(k+1) \Delta x}\right]+ \\
+\sum_{k=1}^{N-i-1} \frac{1}{\nu}\left\{W\left(x_{i+k}, t_{j+1}\right)-W\left(x_{i}, t_{j+1}\right)-k\left[W\left(x_{i+k+1}, t_{j+1}\right)-W\left(x_{i+k}, t_{j+1}\right)\right]\right\} \\
+\left\{e x p i n t\left(\frac{\mu_{p}}{\nu_{p}} k \Delta x\right)-e x p i n t\left[\frac{\mu_{p}}{\nu_{p}}(k+1) \Delta x\right]\right\}+ \\
+\sum_{k=1}^{i-1} \frac{1}{\nu \Delta x}\left[W\left(x_{i-k-1}, t_{j+1}\right)-W\left(x_{i-k}, t_{j+1}\right)\right] \frac{\nu_{n}}{\mu_{n}}\left[e^{-\frac{\mu_{n}}{\nu_{n}} k \Delta x}-e^{-\frac{\mu_{n}}{\nu_{n}}(k+1) \Delta x}\right]+ \\
+\sum_{k=1}^{i-1} \frac{1}{\nu}\left\{W\left(x_{i-k}, t_{j+1}\right)-W\left(x_{i}, t_{j+1}\right)-k\left[W\left(x_{i-k-1}, t_{j+1}\right)-W\left(x_{i-k}, t_{j+1}\right)\right]\right\}
\end{gathered}
$$




$$
\begin{gathered}
\cdot\left\{\operatorname{expint}\left(\frac{\mu_{n}}{\nu_{n}} k \Delta x\right)-\text { expint }\left[\frac{\mu_{n}}{\nu_{n}}(k+1) \Delta x\right]\right\}+ \\
+\frac{1}{\nu}\left[R-W\left(x_{i}, t_{j+1}\right)\right] \cdot \text { expint }\left[\frac{\mu_{p}}{\nu_{p}}(N-i) \Delta x\right]+ \\
-\frac{1}{\nu} W\left(x_{i}, t_{j+1}\right) \cdot \operatorname{expint}\left(\frac{\mu_{n}}{\nu_{n}} i \Delta x\right)
\end{gathered}
$$

As usual in the implementation of the scheme the small jumps will be implemented implicitly, to improve the stability of the system. That is, we are going to use expression

$$
\begin{aligned}
& \frac{\left[W\left(x_{i+1}, t_{j}\right)-W\left(x_{i}, t_{j}\right)\right]\left(1-e^{-\frac{\mu_{p}}{\nu_{p}} \Delta x}\right)}{\nu \Delta x \frac{\mu_{p}}{\nu_{p}}}+ \\
& +\frac{\left[W\left(x_{i-1}, t_{j}\right)-W\left(x_{i}, t_{j}\right)\right]\left(1-e^{-\frac{\mu_{n}}{\nu_{n}} \Delta x}\right)}{\nu \Delta x \frac{\mu_{n}}{\nu_{n}}}
\end{aligned}
$$

instead of the first two lines of the right hand side part of equation (5.10).

\section{Solution for Down-And-Out Call Options}

Let's now consider down-and-out call options. If the jump is positive and so large to finish outside the boundary, we are sure the option will be exercised and the stock won't reach the down barrier, in fact the upper boundary can be chosen to be big enough so that the option values is equal to the discounted value of the payoff, where the stock is discounted at the dividend yield and the strike is discounted at the risk free interest rate, for every value of $t$. In mathematical terms we can write that if

$$
y \in\left[x_{N}-x_{i},+\infty\right]
$$

we can approximate

$$
W\left(x_{i}+y, t_{j+1}\right) \simeq e^{x_{i}+y} e^{-q\left(T-t_{j+1}\right)}-K e^{-r\left(T-t_{j+1}\right)}
$$

Remember that this is the same expression we used for regular call options. On the other side if the jump is large and negative and the stock reaches the down barrier, the rebate is paid and the option becomes worthless. So if

$$
y \in\left[-\infty, x_{0}-x_{i}\right]
$$


where $x_{0}=\ln (B)$, we can write

$$
W\left(x_{i}+y, t_{j+1}\right)=R
$$

We note that also here the rebate is paid both for jumps at the barrier or for jumps which takes the stock outside the barrier, provided that this is the first time that the barrier is passed. The solution for down-and-out calls is provided by equation (A.70) which is derived in appendix A.6.2.

\section{Solution for Up-And-Out Put Options}

We move now to consider up-and-out put options. Similarly to the up-andout call options, if the stock has a positive jump and passes the barrier, the option explodes and the rebate is paid. So for $y>\left(x_{N}-x_{i}\right)$, where $x_{N}=\ln (B)$, the up-and-out put value is given by by

$$
W\left(x_{i}+y, t_{j+1}\right)=R
$$

When the jump is negative and the stock is outside the considered range, that is when $y<\left(x_{0}-x_{i}\right)$, the up-and-out put is worth the discounted payoff where the stock is discounted at the dividend yield and the strike is discounted at the risk free interest rate. This is the same value we found for regular put option and it is given by

$$
W\left(x_{i}+y, t_{j+1}\right) \simeq K e^{-r\left(T-t_{j+1}\right)}-e^{x_{i}+y} e^{-q\left(T-t_{j+1}\right)}
$$

The correct way to rewrite the PIDE for up-and-out put is derived in appendix A.6.3 and is given by equation (A.74).

\section{Solution for Down-And-Out Put Options}

Finally we have the case of down-and-out puts, if the jump is positive and larger than $\left(x_{N}-x_{i}\right)$, the stock is so out of the money that we can impose its value to be equal to zero. On the other side if the jump is negative and the underlying passes the barrier, the option explodes and the rebate is paid. Hence for large positive jumps the down-and-out put value can be approximated in the following way.

$$
W\left(x_{i}+y, t_{j+1}\right) \simeq 0
$$


while for large negative jumps, it can be written as

$$
W\left(x_{i}+y, t_{j+1}\right)=R
$$

Solution is derived in appendix A.6.4 and is given by equation (A.78).

\subsubsection{Difference Equation for European Barrier Op- tions}

Let's consider an up-and-out call option. We can replace the discretization of the jump integral provided by equation (5.10) as adjusted by (5.11) in the PIDE (5.3) to obtain the following difference equation:

$$
\begin{aligned}
& W\left(x_{i-1}, t_{j}\right)\left\{h-\frac{\Delta t \cdot\left(1-e^{-\frac{\mu_{n}}{\nu_{n}} \Delta x}\right)}{\nu \Delta x \frac{\mu_{n}}{\nu_{n}}}\right\}+ \\
& +W\left(x_{i}, t_{j}\right)\left\{(1+r \Delta t)+\frac{\Delta t \cdot\left(1-e^{-\frac{\mu_{p}}{\nu_{p}} \Delta x}\right)}{\nu \Delta x \frac{\mu_{p}}{\nu_{p}}}+\frac{\Delta t \cdot\left(1-e^{-\frac{\mu_{n}}{\nu_{n}} \Delta x}\right)}{\nu \Delta x \frac{\mu_{n}}{\nu_{n}}}\right\}+ \\
& -W\left(x_{i+1}, t_{j}\right)\left\{h+\frac{\Delta t \cdot\left(1-e^{-\frac{\mu_{p}}{\nu_{p}} \Delta x}\right)}{\nu \Delta x \frac{\mu_{p}}{\nu_{p}}}\right\}=W\left(x_{i}, t_{j+1}\right)+ \\
& +\Delta t \cdot\left\{\sum_{k=1}^{N-i-1} \frac{1}{\nu \Delta x}\left[W\left(x_{i+k+1}, t_{j+1}\right)-W\left(x_{i+k}, t_{j+1}\right)\right] \cdot\right. \\
& \cdot \frac{\nu_{p}}{\mu_{p}}\left[e^{-\frac{\mu_{p}}{\nu_{p}} k \Delta x}-e^{-\frac{\mu_{p}}{\nu_{p}}(k+1) \Delta x}\right]+ \\
& +\sum_{k=1}^{N-i-1} \frac{1}{\nu}\left\{W\left(x_{i+k}, t_{j+1}\right)-W\left(x_{i}, t_{j+1}\right)-k\left[W\left(x_{i+k+1}, t_{j+1}\right)-W\left(x_{i+k}, t_{j+1}\right)\right]\right\} \cdot \\
& \cdot\left\{\operatorname{expint}\left(\frac{\mu_{p}}{\nu_{p}} k \Delta x\right)-\operatorname{expint}\left[\frac{\mu_{p}}{\nu_{p}}(k+1) \Delta x\right]\right\}+ \\
& +\sum_{k=1}^{i-1} \frac{1}{\nu \Delta x}\left[W\left(x_{i-k-1}, t_{j+1}\right)-W\left(x_{i-k}, t_{j+1}\right)\right] \frac{\nu_{n}}{\mu_{n}}\left[e^{-\frac{\mu_{n}}{\nu_{n}} k \Delta x}-e^{-\frac{\mu_{n}}{\nu_{n}}(k+1) \Delta x}\right]+ \\
& +\sum_{k=1}^{i-1} \frac{1}{\nu}\left\{W\left(x_{i-k}, t_{j+1}\right)-W\left(x_{i}, t_{j+1}\right)-k\left[W\left(x_{i-k-1}, t_{j+1}\right)-W\left(x_{i-k}, t_{j+1}\right)\right]\right\} \text {. }
\end{aligned}
$$




$$
\begin{gathered}
\cdot\left\{\operatorname{expint}\left(\frac{\mu_{n}}{\nu_{n}} k \Delta x\right)-\text { expint }\left[\frac{\mu_{n}}{\nu_{n}}(k+1) \Delta x\right]\right\}+ \\
+\frac{1}{\nu}\left[R-W\left(x_{i}, t_{j+1}\right)\right] \cdot \operatorname{expint}\left[\frac{\mu_{p}}{\nu_{p}}(N-i) \Delta x\right]+ \\
\left.\quad-\frac{1}{\nu} W\left(x_{i}, t_{j+1}\right) \cdot \operatorname{expint}\left(\frac{\mu_{n}}{\nu_{n}} i \Delta x\right)\right\}
\end{gathered}
$$

As usual the left hand side can be written as a tridiagonal matrix and hence the Gauss's elimination method can be employed to obtain a fast solution of the system. Appendix B provides $\mathrm{C}$ code to numerically solve the difference equation.

In a similar way we can obtain difference equations for down-and-out call option, up-and-out put options and down-and-out put options. These difference equations are obtained respectively in appendix A.7.2, A.7.3 and A.7.4.

\subsection{Experimental Results for European Bar- rier Options}

In this section, we realize a number of experiments of European barrier option pricing. In particular, we want to study the sensitivity of barrier option prices to the rebate and to the parameters controlling volatility, skewness and kurtosis. We know that $\sigma, \vartheta$ and $n u$ do not represent themselves volatility, skewness and kurtosis, but are linked to them by the following relationships:

$$
\begin{gathered}
E\left[(X(t)-E[X(t)])^{2}\right]=\left(\vartheta^{2} \nu+\sigma^{2}\right) t \\
E\left[(X(t)-E[X(t)])^{3}\right]=\left(2 \vartheta^{3} \nu^{2}+3 \sigma^{2} \vartheta \nu\right) t \\
E\left[(X(t)-E[X(t)])^{4}\right]= \\
=\left(3 \sigma^{4} \nu+12 \sigma^{2} \vartheta^{2} \nu^{2}+6 \vartheta^{4} \nu^{3}\right) t+\left(3 \sigma^{4}+6 \sigma^{2} \vartheta^{2} \nu+3 \vartheta^{4} \nu^{2}\right) t^{2}
\end{gathered}
$$




\subsubsection{Up-And-Out Calls: Sensitivity Analysis}

Let's study here the sensitivity analysis of up-and-out call options. We will focus our attention here on the sensitivity to the rebate and to $\sigma$. The numerical experiments presented here can be obtained using the code in $\mathrm{C}$ language available in appendix B. The European up-and-out call case studied here is realized by choosing the following variable values:

- callput $=1$

- euroamerican $=0$

- barrier_switch $=1$

- up_and_out $=1$

in the $\mathrm{C}$ code.

\section{Sensitivity with Respect to the Rebate}

We consider here the sensitivity of an European up-and-out call option with respect to the rebate. The parameters used in this experiment are:

- $T=0.5$ Time to maturity in years;

- $K=\$ 100$ Strike price;

- barrier $=4.86753$, corresponding to $S=\$ 130$;

- $r=0.03$ Interest rate;

- $q=0.01 \quad$ Dividend yield;

- $\sigma=0.2$;

- $\vartheta=-0.1$

- $\nu=0.2$

- $N=5100$ Number of space intervals;

- $M=300$ Number of time intervals;

- $x_{\min }=1.60944$ Min $x$ value considered, corresponding to $S=\$ 5$; 
- $x_{\max }=4.86753$ Max $x$ value considered, corresponding to $S=\$ 130$;

- $\Delta x=0.000639$ Size of a space interval;

- $\Delta t=0.001667$ Size of a time interval;

- $\frac{\Delta t}{\Delta x}=2.609$;

We see that this option is knocked out when the intrinsic value is $\$ 30$, hence we consider the impact of changing the rebate from 0to30. Because we do not modify $\sigma, \vartheta$ and $\nu$; volatility, skewness and kurtosis are constant in this experiment. In particular we have the following annualized values:

\begin{tabular}{|c|c|}
\hline$\sqrt{E\left[(X(t)-E[X(t)])^{2}\right]}$ & 0.205 \\
\hline$\frac{E\left[(X(t)-E[X(t)])^{3}\right]}{\sqrt{\left\{E\left[(X(t)-E[X(t)])^{2}\right]\right\}^{3}}}$ & -0.288 \\
\hline$\frac{E\left[(X(t)-E[X(t)])^{4}\right]}{\left\{E\left[(X(t)-E[X(t)])^{2}\right]\right\}^{2}}$ & 3.656 \\
\hline
\end{tabular}


This graph shows the impact of increasing the rebate. Clearly the higher the rebate, the higher the option value. We note that for low rebate values the option value is not monotonic with respect to the stock price. In this case, in fact, the option price may first increase and then go down again as the barrier is approaching. On the other side if the rebate is high enough, the option will have a monotonic increase as the stock price increases.

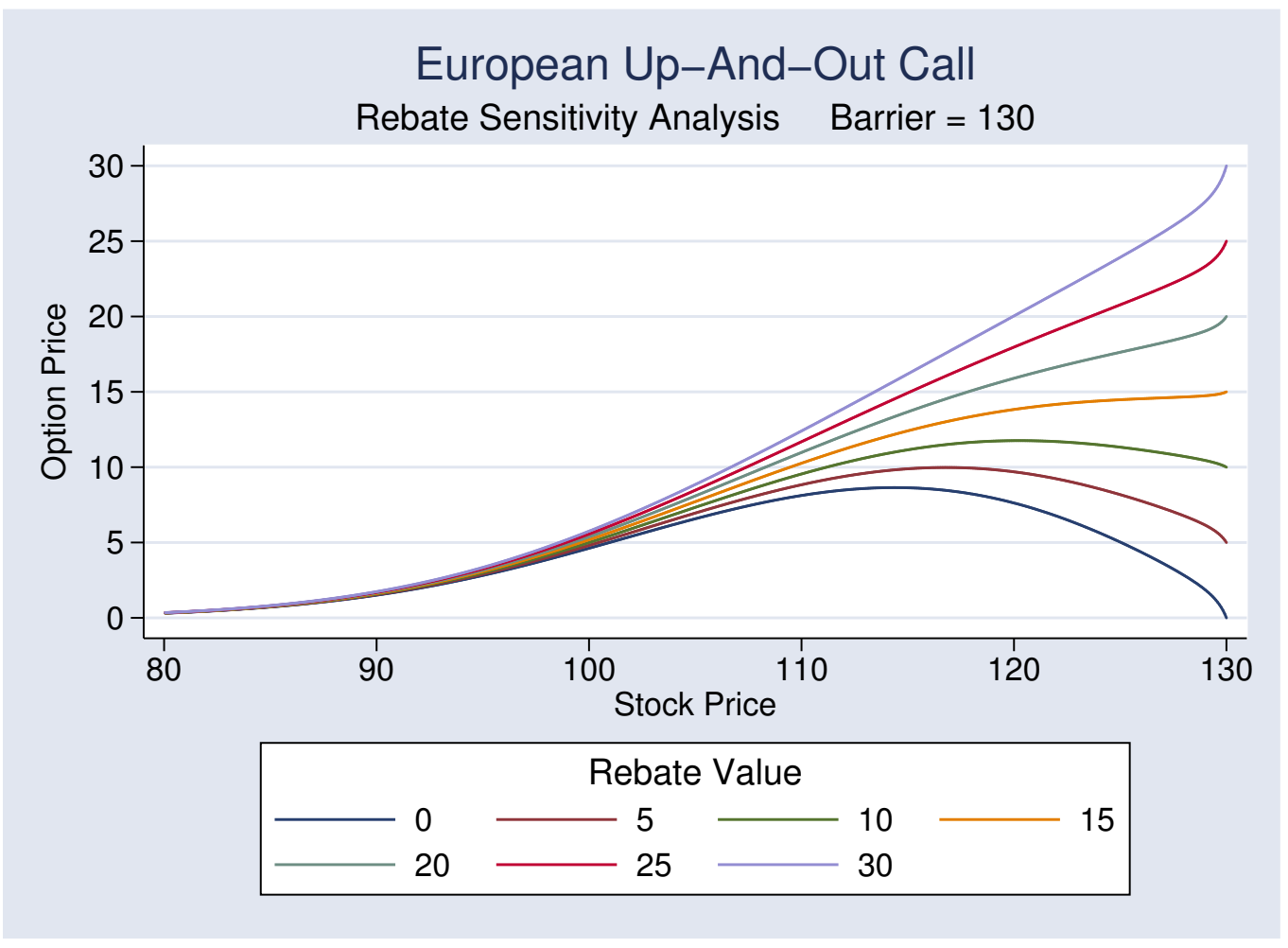

Figure 5.1: European Up-And-Out Call: Rebate Sensitivity Analysis 


\section{Sensitivity with Respect to $\sigma$}

We consider here the sensitivity of an European up-and-out call option with respect to $\sigma$. The parameters used in this experiment are:

- $T=0.5$ Time to maturity in years;

- $K=\$ 100$ Strike price;

- $r=0.03 \quad$ Interest rate;

- $q=0.01 \quad$ Dividend yield;

- $\vartheta=-0.1$

- $\nu=0.2$;

- $N=5100$ Number of space intervals;

- $M=300$ Number of time intervals;

- $x_{\min }=1.60944$ Min $x$ value considered, corresponding to $S=\$ 5$;

- $x_{\max }=4.86753$ Max $x$ value considered, corresponding to $S=\$ 130$;

- $\Delta x=0.000639$ Size of a space interval;

- $\Delta t=0.001667 \quad$ Size of a time interval;

- $\frac{\Delta t}{\Delta x}=2.609$;

Using these parameters we move $\sigma$ from $10 \%$ to $50 \%$. The change in $\sigma$ produces an impact on volatility, skewness and kurtosis of the process describing the underlying returns. In particular we have the following values:

\begin{tabular}{|c|c|c|c|c|}
\hline$\sigma$ & 0.1 & 0.2 & 0.3 & 0.5 \\
\hline$\sqrt{E\left[(X(t)-E[X(t)])^{2}\right]}$ & 0.110 & 0.205 & 0.303 & 0.502 \\
\hline$\frac{E\left[(X(t)-E[X(t)])^{3}\right]}{\sqrt{\left\{E\left[(X(t)-E[X(t)])^{2}\right]\right\}^{3}}}$ & -0.517 & -0.288 & -0.196 & -0.119 \\
\hline$\frac{E\left[(X(t)-E[X(t)])^{4}\right]}{\left\{E\left[(X(t)-E[X(t)])^{2}\right]\right\}^{2}}$ & 3.783 & 3.656 & 3.626 & 3.609 \\
\hline
\end{tabular}


We can see that as $\sigma$ increases, volatility increases. Moreover as $\sigma$ becomes larger, skewness and kurtosis are reduced.

We realize this sensitivity analysis experiments in two hypotheses. The first is the case where the option pays no rebate if it is knocked out. The following graph shows this case of no rebate.

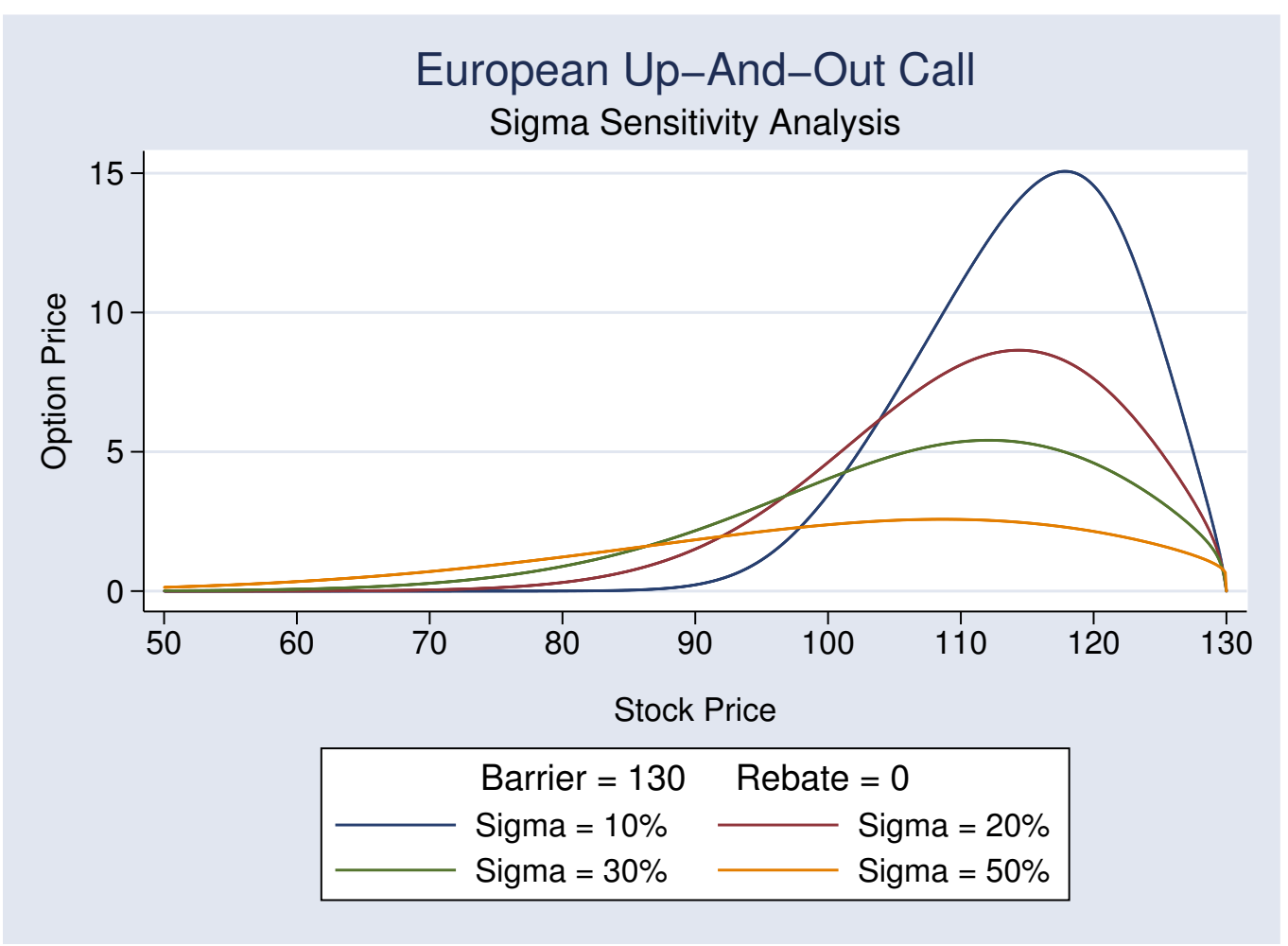

Figure 5.2: European Up-And-Out Call: $\sigma$ Sensitivity Analysis with Rebate $=0$ 
In this second graph we consider the situation of a rebate equal to the intrinsic value of the option when it reaches the barrier.

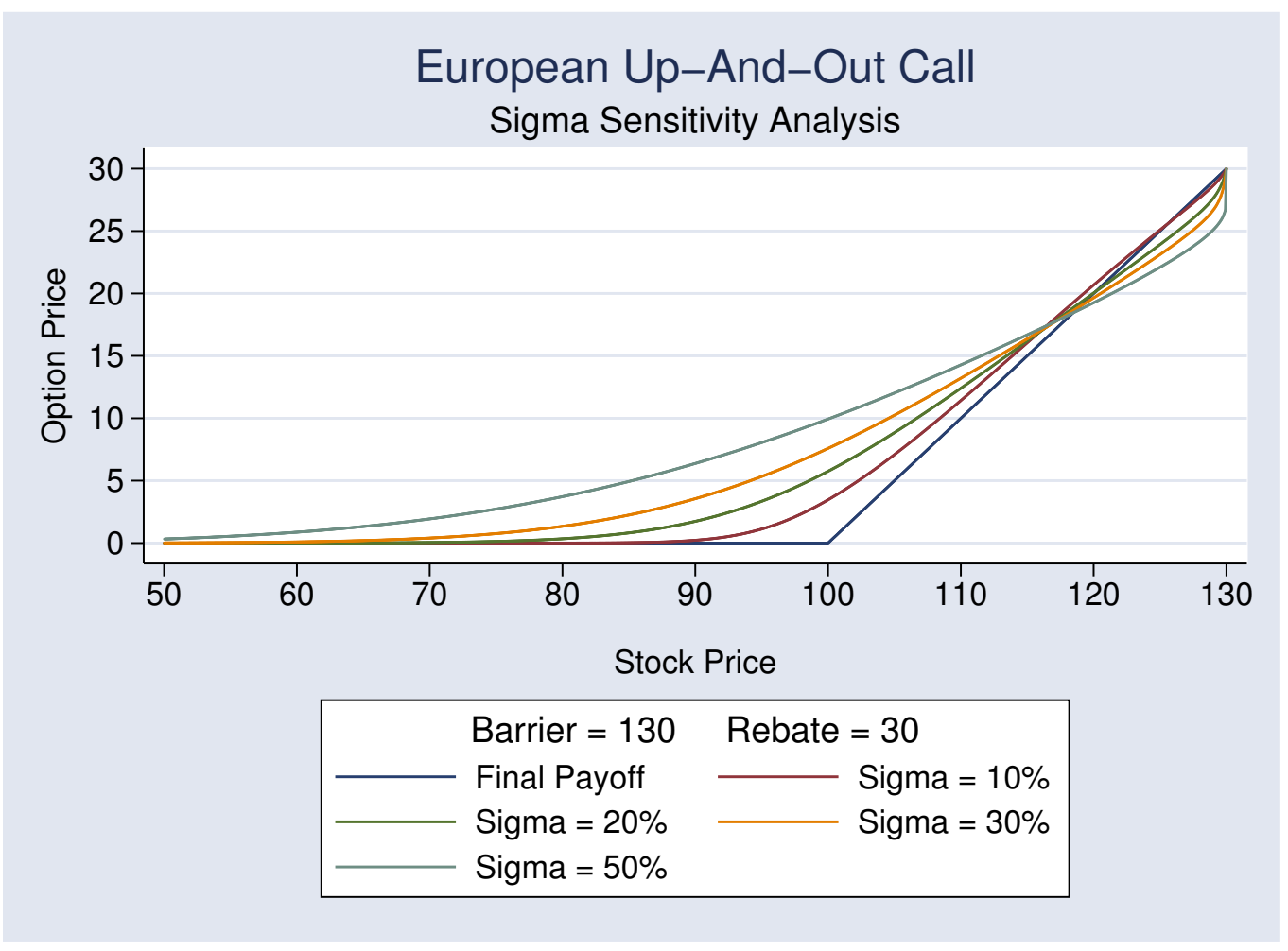

Figure 5.3: European Up-And-Out Call: $\sigma$ Sensitivity Analysis with Rebate $=30$

\subsubsection{Down-And-Out Calls: Sensitivity Analysis}

Let's study here the sensitivity analysis of down-and-out call options. We are going to study in particular the sensitivity with respect to the rebate. The numerical experiments presented here can be obtained using the code in $\mathrm{C}$ language available in appendix B. The European down-and-out call case studied here is realized by choosing the following variable values:

- callput $=1$ 
- euroamerican $=0$

- barrier_switch $=1$

- up_and_out $=0$

in the $\mathrm{C}$ code.

\section{Sensitivity with Respect to the Rebate}

We consider here the sensitivity of an European down-and-out call option with respect to the rebate. The parameters used in this experiment are:

- $T=0.5$ Time to maturity in years;

- $K=\$ 100$ Strike price;

- barrier $=4.2485$, corresponding to $S=\$ 70$;

- $r=0.03$ Interest rate;

- $q=0.01 \quad$ Dividend yield;

- $\sigma=0.2$

- $\vartheta=-0.1$;

- $\nu=0.2$;

- $N=5100$ Number of space intervals;

- $M=300 \quad$ Number of time intervals;

- $x_{\min }=4.2485$ Min $x$ value considered, corresponding to $S=\$ 70$;

- $x_{\max }=5.85793$ Max $x$ value considered, corresponding to $S=\$ 350$;

- $\Delta x=0.000316$ Size of a space interval;

- $\Delta t=0.001667$ Size of a time interval;

- $\frac{\Delta t}{\Delta x}=5.281$;

$\sigma, \vartheta$ and $\nu$ are unchanged in this experiment, hence volatility, skewness and kurtosis are fixed. More in detail, we have the following values: 


\begin{tabular}{|c|c|}
\hline$\sqrt{E\left[(X(t)-E[X(t)])^{2}\right]}$ & 0.205 \\
\hline$\frac{E\left[(X(t)-E[X(t)])^{3}\right]}{\sqrt{\left\{E\left[(X(t)-E[X(t)])^{2}\right]\right\}^{3}}}$ & -0.288 \\
\hline$\frac{E\left[(X(t)-E[X(t)])^{4}\right]}{\left\{E\left[(X(t)-E[X(t)])^{2}\right]\right\}^{2}}$ & 3.656 \\
\hline
\end{tabular}

In a down-and-out call option, the barrier is reached when the option is out of the money. Here the barrier level is $\$ 30$ out of the money and we want to compare the option prices in the case that no rebate or a $\$ 5$ rebate is paid upon reaching the barrier. The graph shows the results of the experiment.

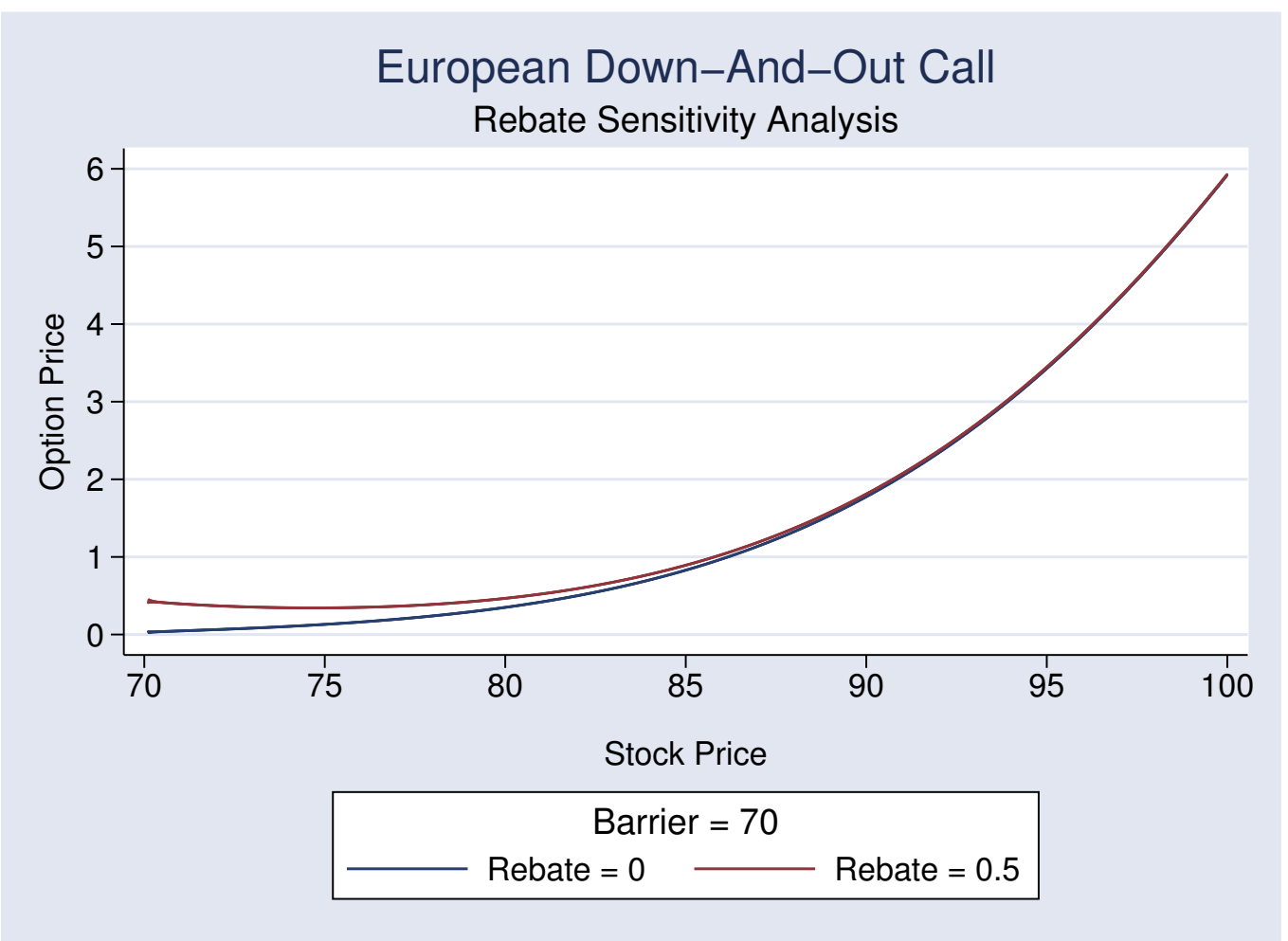

Figure 5.4: European Down-And-Out Call: Rebate Sensitivity Analysis 


\subsubsection{Up-And-Out Puts: Sensitivity Analysis}

Let's study here the sensitivity analysis of an up-and-out put option. We are going to study in particular the sensitivity with respect to the rebate. The numerical experiments presented here can be obtained using the code in $\mathrm{C}$ language available in appendix B. The European up-and-out put case studied here is realized by choosing the following variable values:

- callput $=0$

- euroamerican $=0$

- barrier_switch $=1$

- up_and_out $=1$

in the $\mathrm{C}$ code.

\section{Sensitivity with Respect to the Rebate}

We consider here the sensitivity of an European up-and-out put option with respect to the rebate. The parameters used in this experiment are:

- $T=1 \quad$ Time to maturity in years;

- $K=\$ 100$ Strike price;

- barrier $=4.86753$, corresponding to $S=\$ 130$;

- $r=0.03$ Interest rate;

- $q=0.01 \quad$ Dividend yield;

- $\sigma=0.2$

- $\vartheta=-0.1$

- $\nu=0.2$

- $N=5100$ Number of space intervals;

- $M=300$ Number of time intervals;

- $x_{\min }=1.60944 \quad$ Min $x$ value considered, corresponding to $S=\$ 5$; 
- $x_{\max }=4.86753$ Max $x$ value considered, corresponding to $S=\$ 130$;

- $\Delta x=0.000639$ Size of a space interval;

- $\Delta t=0.003333$ Size of a time interval;

- $\frac{\Delta t}{\Delta x}=5.218$

In this experiment, the parameters affecting volatility, skewness and kurtosis are unchanged. We have the following values:

\begin{tabular}{|c|c|}
\hline$\sqrt{E\left[(X(t)-E[X(t)])^{2}\right]}$ & 0.205 \\
\hline$\frac{E\left[(X(t)-E[X(t)])^{3}\right]}{\sqrt{\left\{E\left[(X(t)-E[X(t)])^{2}\right]\right\}^{3}}}$ & -0.288 \\
\hline$\frac{E\left[(X(t)-E[X(t)])^{4}\right]}{\left\{E\left[(X(t)-E[X(t)])^{2}\right]\right\}^{2}}$ & 3.656 \\
\hline
\end{tabular}


In an up-and-out put, the barrier is reached when the option is out of the money. Here the barrier is positioned $30 \%$ out of the money. In the following graph we consider four different levels of rebate for this case: $\$ 0, \$ 0.5, \$ 1$ and $\$ 1.5$. We can see that, as expected, the higher the rebate, the higher the option price

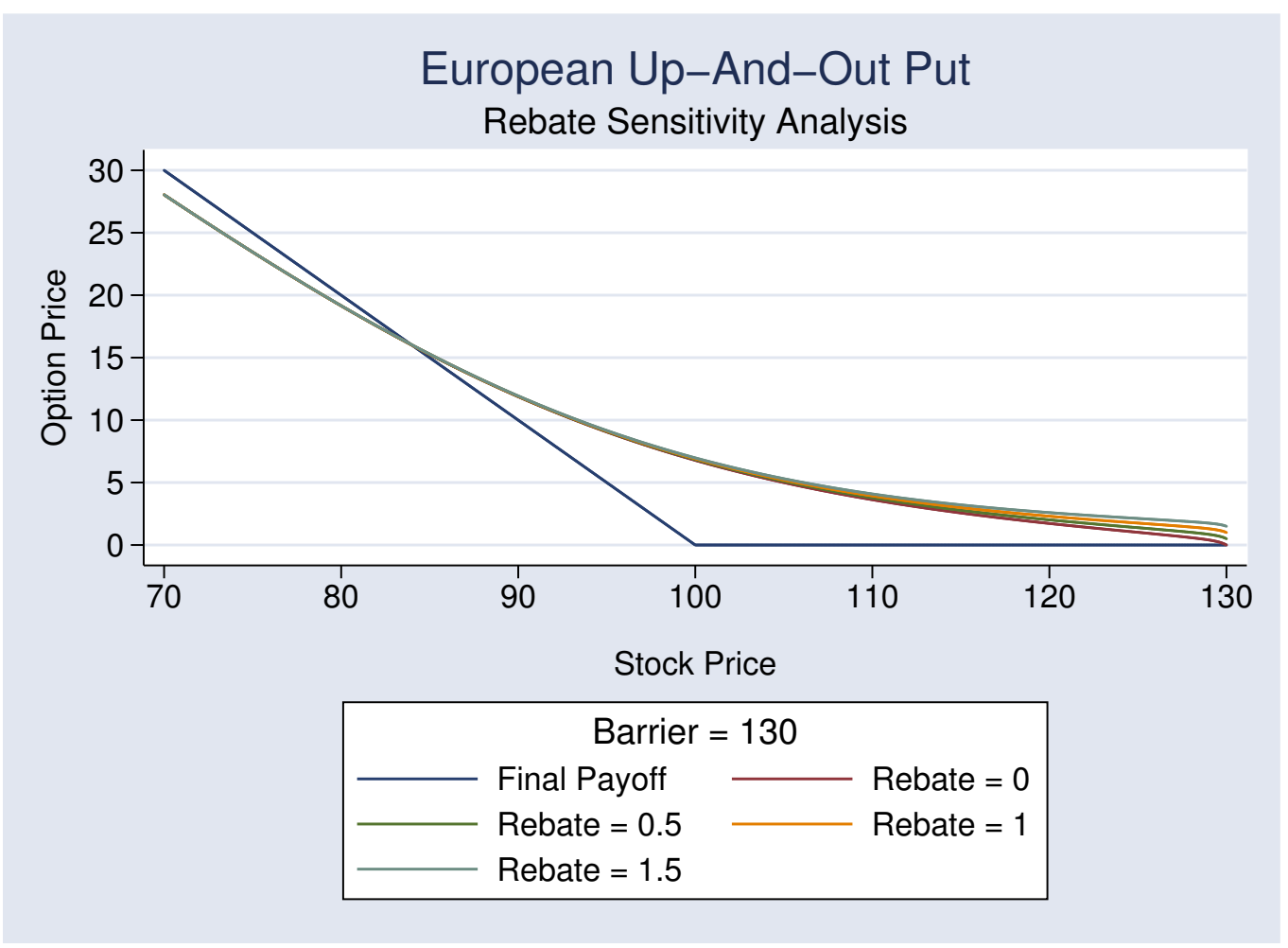

Figure 5.5: European Up-And-Out Put: Rebate Sensitivity Analysis 
The following graph is a zoom in the area near the barrier. The parameters are the same used in the previous figure.

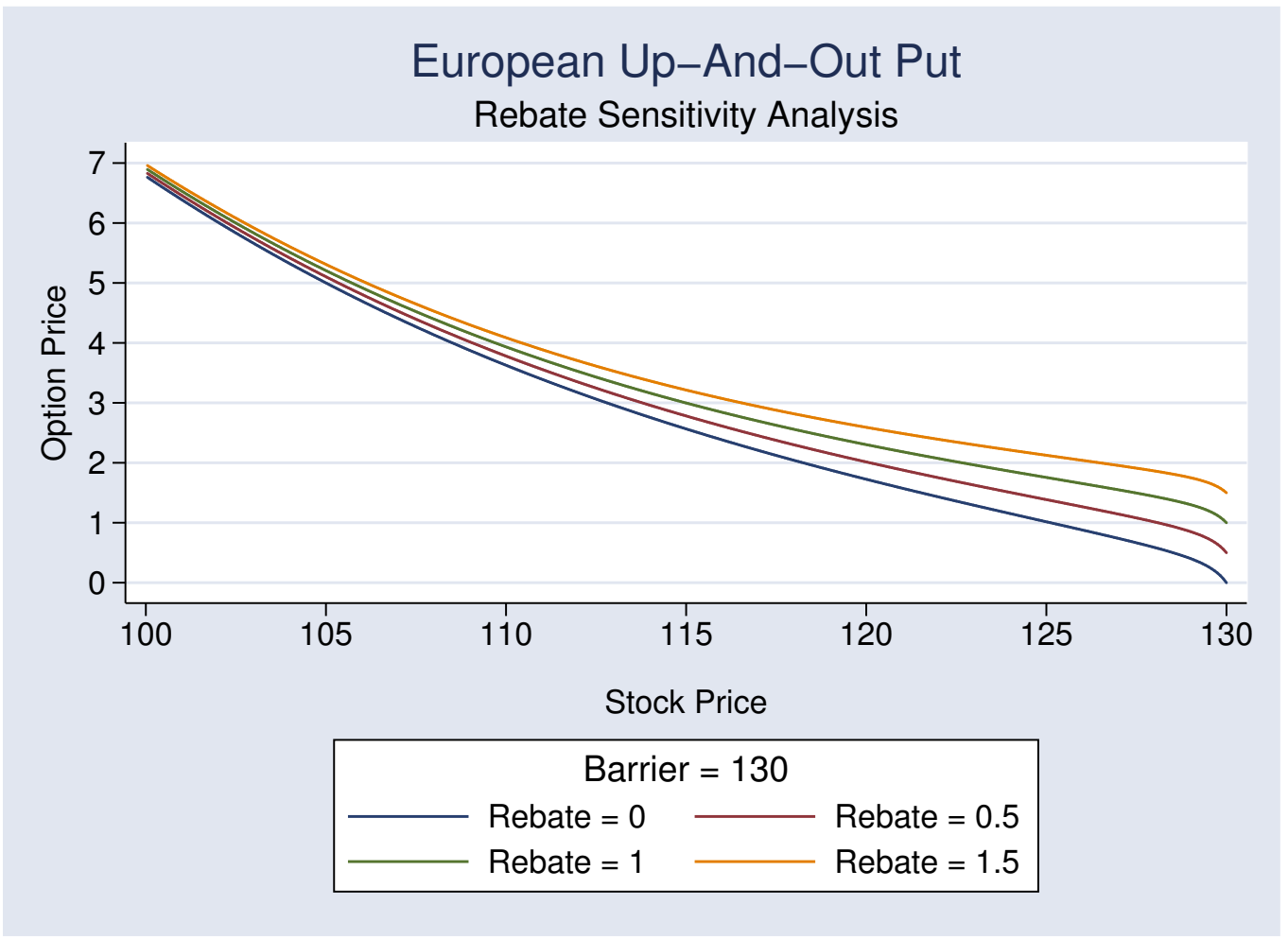

Figure 5.6: European Up-And-Out Put: Rebate Sensitivity Analysis, Detail

\subsubsection{Down-And-Out Puts: Sensitivity Analysis}

We consider now some experiments on down-and-out put options. In particular we want to study sensitivity analysis with respect to the rebate and with respect to $\nu$ and $\vartheta$. The numerical experiments presented here can be obtained using the code in $\mathrm{C}$ language available in appendix B. The European down-and-out put case studied here is realized by choosing the following variable values:

- callput $=0$ 
- euroamerican $=0$

- barrier_switch $=1$

- up_and_out $=0$

in the $\mathrm{C}$ code.

\section{Sensitivity with Respect to the Rebate}

We consider here the sensitivity of an European up-and-out put option with respect to the rebate. The parameters used in this experiment are:

- $T=1 \quad$ Time to maturity in years;

- $K=\$ 100$ Strike price;

- barrier $=4.09434$, corresponding to $S=\$ 60$;

- $r=0.03 \quad$ Interest rate;

- $q=0.01 \quad$ Dividend yield;

- $\sigma=0.2$;

$\bullet \vartheta=-0.1$

- $\nu=0.2$

- $N=5100$ Number of space intervals;

- $M=300$ Number of time intervals;

- $x_{\min }=4.09434$ Min $x$ value considered, corresponding to $S=\$ 60$;

- $x_{\max }=5.85793$ Max $x$ value considered, corresponding to $S=\$ 350$;

- $\Delta x=0.000346$ Size of a space interval;

- $\Delta t=0.003333$ Size of a time interval;

- $\frac{\Delta t}{\Delta x}=9.639$;

In this first experiment on up-and-out put options, we keep the $\sigma, \vartheta$ and $\nu$ unchanged. So the we have the following values in terms of volatility, skewness and kurtosis: 


\begin{tabular}{|c|c|}
\hline$\sqrt{E\left[(X(t)-E[X(t)])^{2}\right]}$ & 0.205 \\
\hline$\frac{E\left[(X(t)-E[X(t)])^{3}\right]}{\sqrt{\left\{E\left[(X(t)-E[X(t)])^{2}\right]\right\}^{3}}}$ & -0.288 \\
\hline$\frac{E\left[(X(t)-E[X(t)])^{4}\right]}{\left\{E\left[(X(t)-E[X(t)])^{2}\right]\right\}^{2}}$ & 3.656 \\
\hline
\end{tabular}

Here the option reaches the barrier when it is $\$ 40$ in the money. The following graph shows the option prices corresponding to a rebate of $\$ 20$ and $\$ 30$.

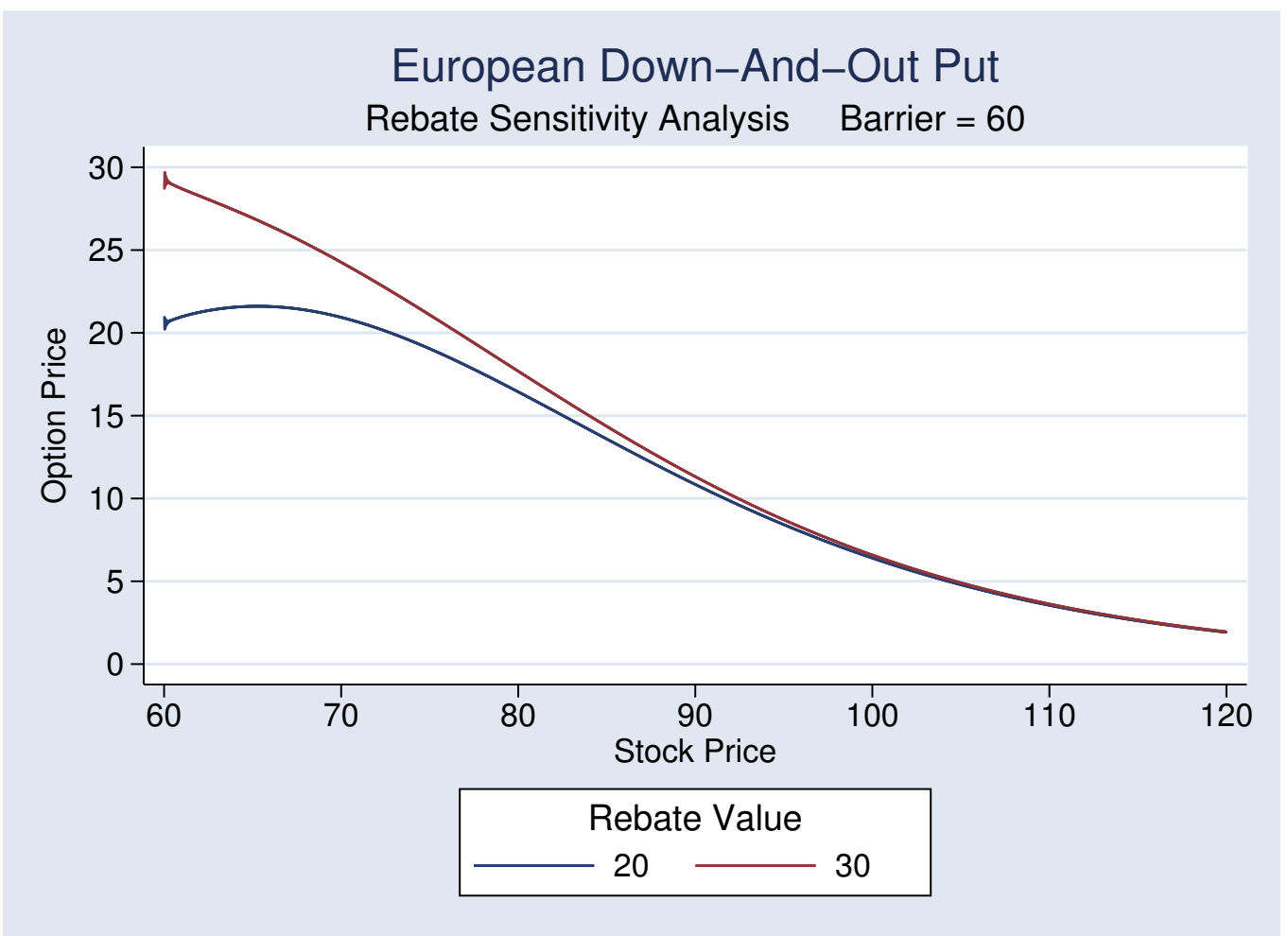

Figure 5.7: European Down-And-Out Put: Rebate Sensitivity 


\section{Sensitivity with Respect to $\nu$ with Rebate $=\mathbf{0}$}

We study here the sensitivity of an European up-and-out put option with respect to $\nu$. We assume here to receive no rebate if the option is knocked out. Later we will see the same experiment with an option paying a $\$ 40$ rebate if the barrier is reached. The parameters used in this experiment are:

- $T=1 \quad$ Time to maturity in years;

- $K=\$ 100$ Strike price;

- barrier $=4.09434$, corresponding to $S=\$ 60$;

- rebate $=0$;

- $r=0.03$ Interest rate;

- $q=0.01 \quad$ Dividend yield;

- $\sigma=0.2$;

- $\vartheta=-0.1$

- $N=5100$ Number of space intervals;

- $M=300 \quad$ Number of time intervals;

- $x_{\min }=4.09434$ Min $x$ value considered, corresponding to $S=\$ 60$;

- $x_{\max }=5.85793$ Max $x$ value considered, corresponding to $S=\$ 350$;

- $\Delta x=0.000346$ Size of a space interval;

- $\Delta t=0.003333$ Size of a time interval;

- $\frac{\Delta t}{\Delta x}=9.639$;

Using these values, we study the impact of moving $\nu$ from 0.1 to 0.7 . Because $\vartheta \neq 0$, if we change $\nu$, also volatility and skewness will be affected. In particular we are going to have the following values: 


\begin{tabular}{|c|c|c|c|c|c|}
\hline$\nu$ & 0.1 & 0.2 & 0.3 & 0.5 & 0.7 \\
\hline$\sqrt{E\left[(X(t)-E[X(t)])^{2}\right]}$ & 0.202 & 0.205 & 0.207 & 0.212 & 0.217 \\
\hline$\frac{E\left[(X(t)-E[X(t)])^{3}\right]}{\sqrt{\left\{E\left[(X(t)-E[X(t)])^{2}\right]\right\}^{3}}}$ & -0.147 & -0.288 & -0.424 & -0.681 & -0.921 \\
\hline$\frac{E\left[(X(t)-E[X(t)])^{4}\right]}{\left\{E\left[(X(t)-E[X(t)])^{2}\right]\right\}^{2}}$ & 3.314 & 3.656 & 4.021 & 4.815 & 5.679 \\
\hline
\end{tabular}

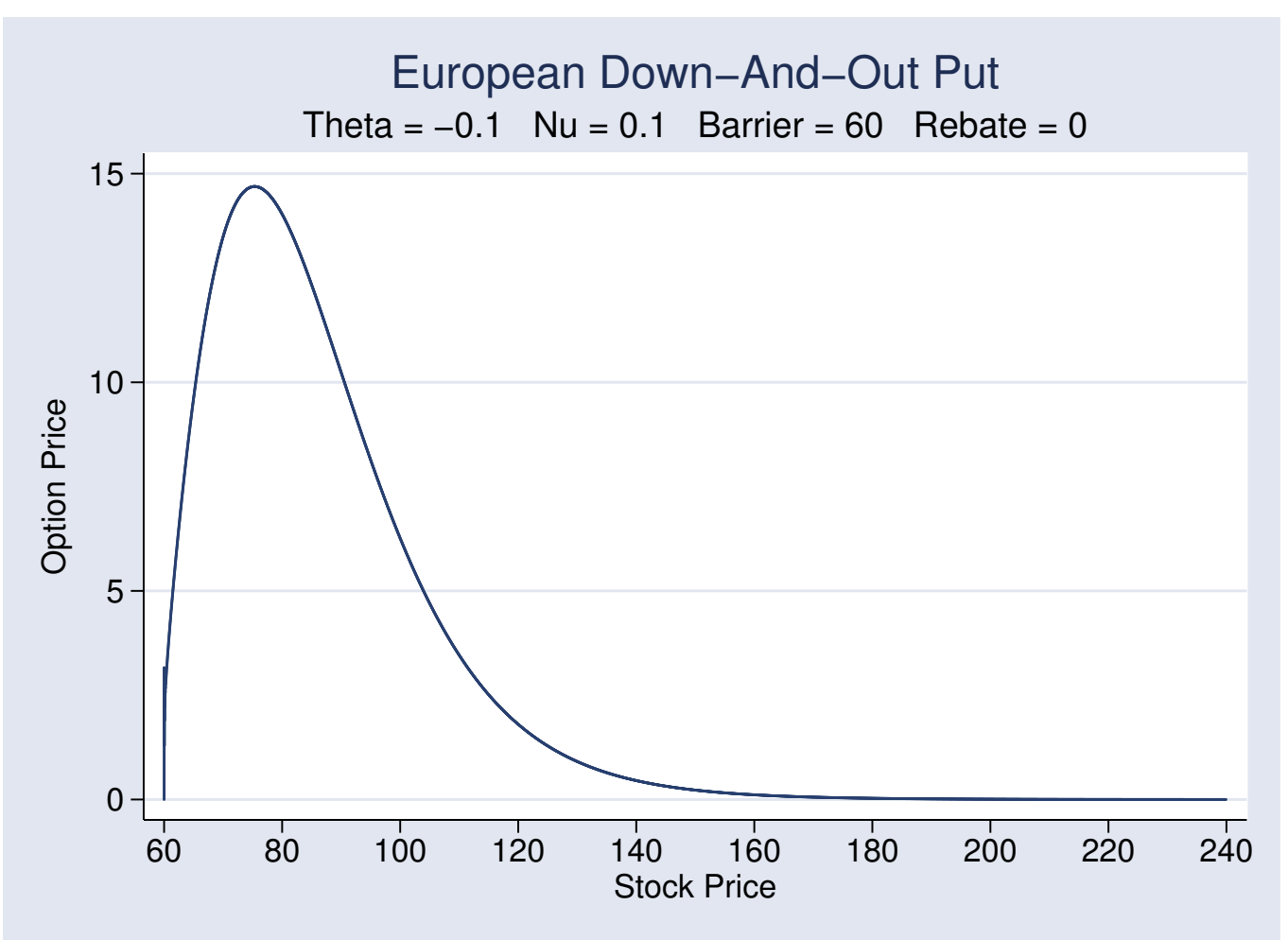

Figure 5.8: European Down-And-Out Put: Starting Level for $\nu$ Sensitivity Analysis, with Rebate $=0$

Clearly an increase in $\nu$ produces an increase in kurtosis. Moreover we can see from the table that as $\nu$ becomes larger, volatility and skewness increase. 
Figure 5.8 presents a starting scenario, where $\nu=0.1$. We can see that, because there is no rebate, the option price first increases as the stock price goes down, and later decreases as the stock price keeps on going down and approaches the rebate.

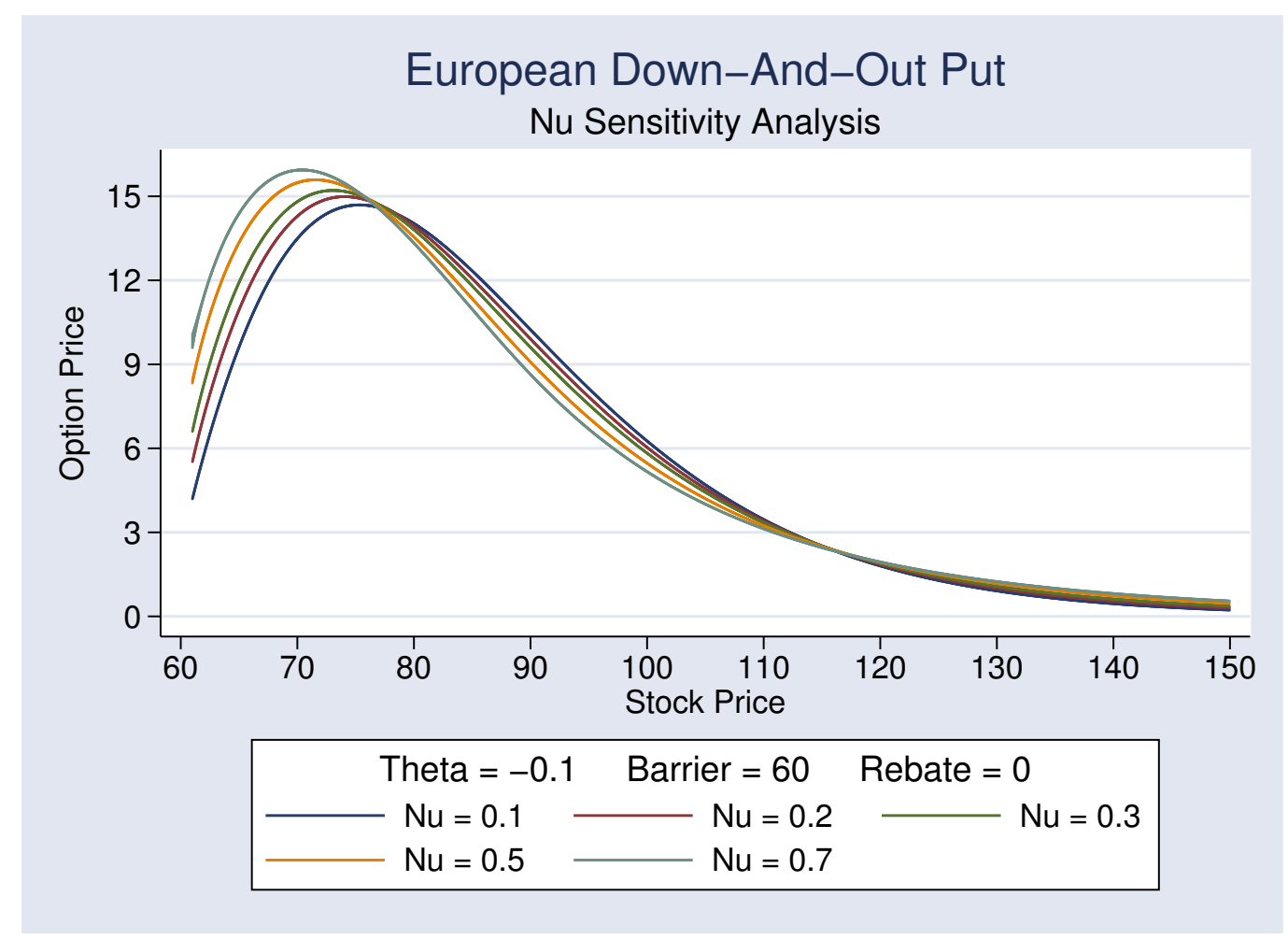

Figure 5.9: European Down-And-Out Put: $\nu$ Sensitivity Analysis with Rebate $=0$

In figure 5.9 we consider 5 different levels of $\nu$. We can see that the impact on the option price is mixed depending on how much the option is in the money. There is a central area where the stock is relatively close to at the money where the option whose underlying has the lowest $\nu$ has the highest value. On the contrary, on the two sides, where the option is more far from being at the money, the options are worth more as $\nu$ increases. 
The following graph is a zoom of the previous one showing the in the money area. We can see how $\nu$ has different impacts on the option prices.

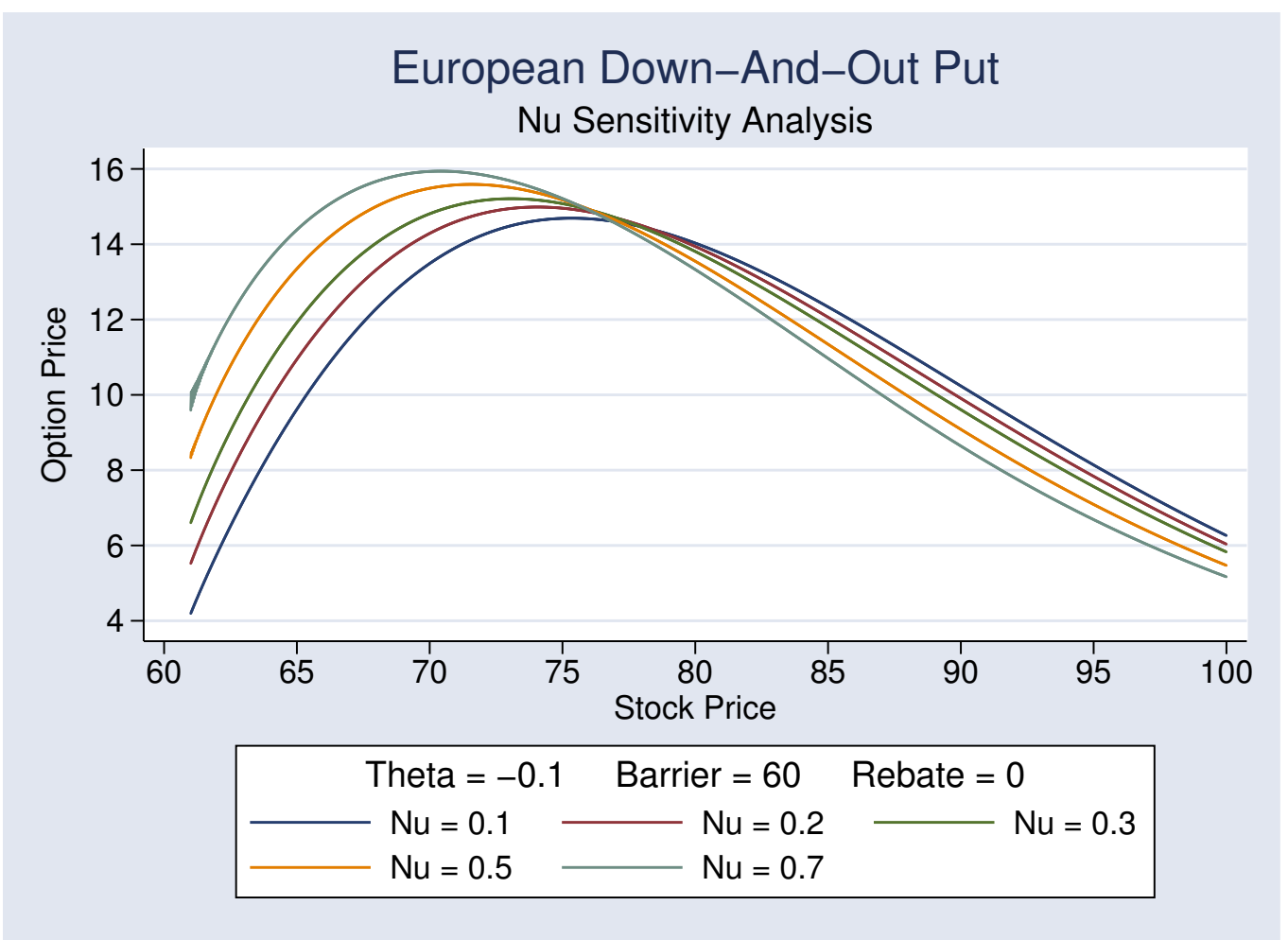

Figure 5.10: European Down-And-Out Put: $\nu$ Sensitivity Analysis, Detail 
This graph shows a detail of the in out of the money area. Again we can note that the increase in the level of $\nu$ produces a mixed effect depending on the position of the moneyness.

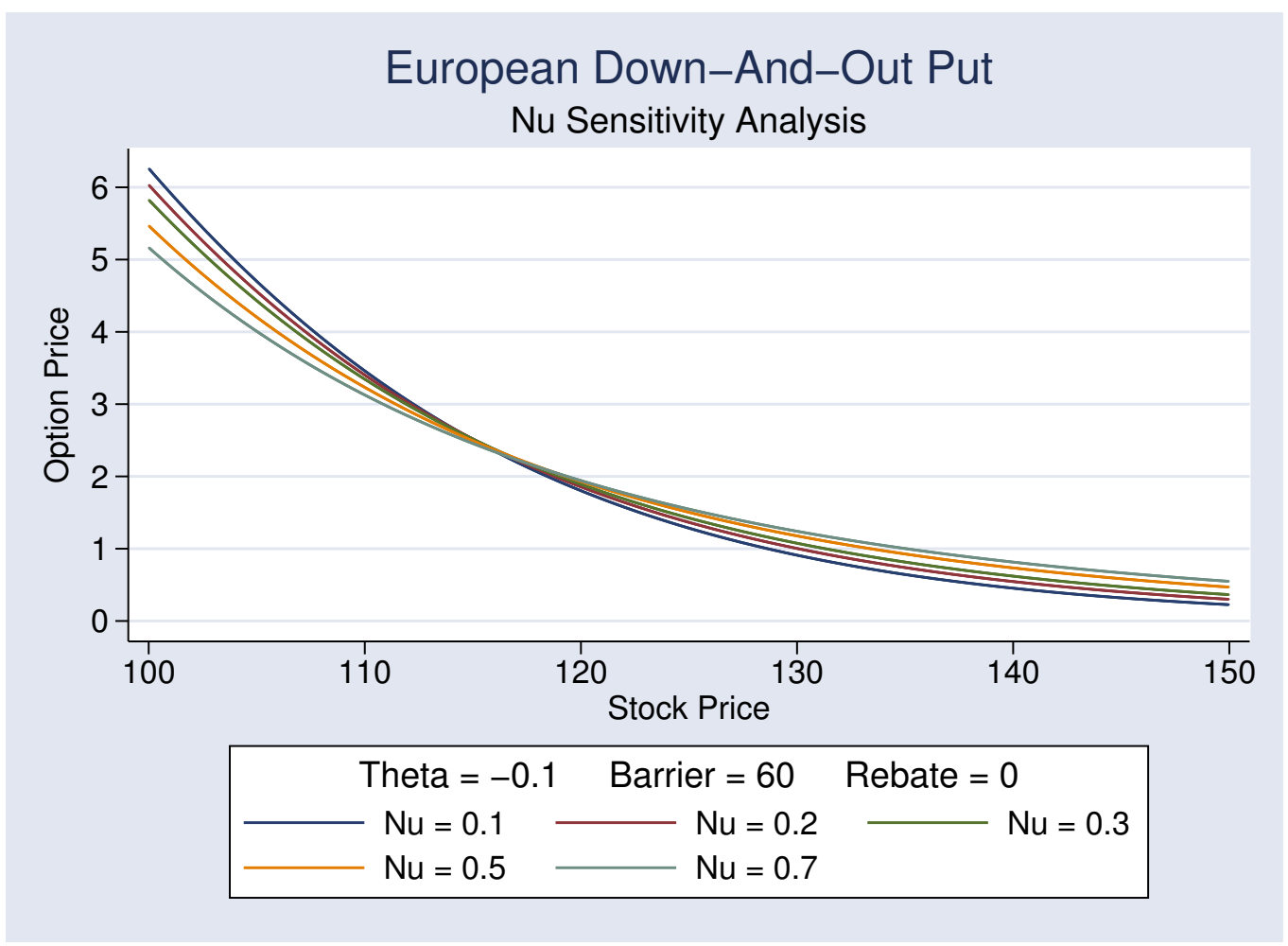

Figure 5.11: European Down-And-Out Put: $\nu$ Sensitivity Analysis, Detail 


\section{Sensitivity with Respect to $\vartheta$ with Rebate $=0$}

We consider now the sensitivity of an European up-and-out put option with respect to $\vartheta$. We assume here to receive no rebate if the option is knocked out. Later we will see the same experiment with an option paying a $\$ 40$ rebate if the barrier is reached. The parameters used in this experiment are:

- $T=1 \quad$ Time to maturity in years;

- $K=\$ 100$ Strike price;

- barrier $=4.09434$, corresponding to $S=\$ 60$;

- rebate $=0$;

- $r=0.03$ Interest rate;

- $q=0.01 \quad$ Dividend yield;

- $\sigma=0.2$;

- $\nu=0.2$;

- $N=5100$ Number of space intervals;

- $M=300 \quad$ Number of time intervals;

- $x_{\min }=4.09434$ Min $x$ value considered, corresponding to $S=\$ 60$;

- $x_{\max }=5.85793$ Max $x$ value considered, corresponding to $S=\$ 350$;

- $\Delta x=0.000346$ Size of a space interval;

- $\Delta t=0.003333$ Size of a time interval;

- $\frac{\Delta t}{\Delta x}=9.639$;

Using these parameters we study the impact of moving $\vartheta$ from 0 to -0.5 . The impact on volatility, skewness and kurtosis of changing $\vartheta$ is summarized in this table. 


\begin{tabular}{|c|c|c|c|c|}
\hline$\vartheta$ & 0 & -0.1 & -0.3 & -0.5 \\
\hline$\sqrt{E\left[(X(t)-E[X(t)])^{2}\right]}$ & 0.200 & 0.205 & 0.241 & 0.300 \\
\hline$\frac{E\left[(X(t)-E[X(t)])^{3}\right]}{\sqrt{\left\{E\left[(X(t)-E[X(t)])^{2}\right]\right\}^{3}}}$ & 0.000 & -0.288 & -0.670 & -0.815 \\
\hline$\frac{E\left[(X(t)-E[X(t)])^{4}\right]}{\left\{E\left[(X(t)-E[X(t)])^{2}\right]\right\}^{2}}$ & 3.600 & 3.656 & 3.915 & 4.081 \\
\hline
\end{tabular}

We can see that as $\vartheta$ becomes increasingly negative, the negative skewness increases. Moreover volatility and kurtosis increases as $\vartheta$ increases.

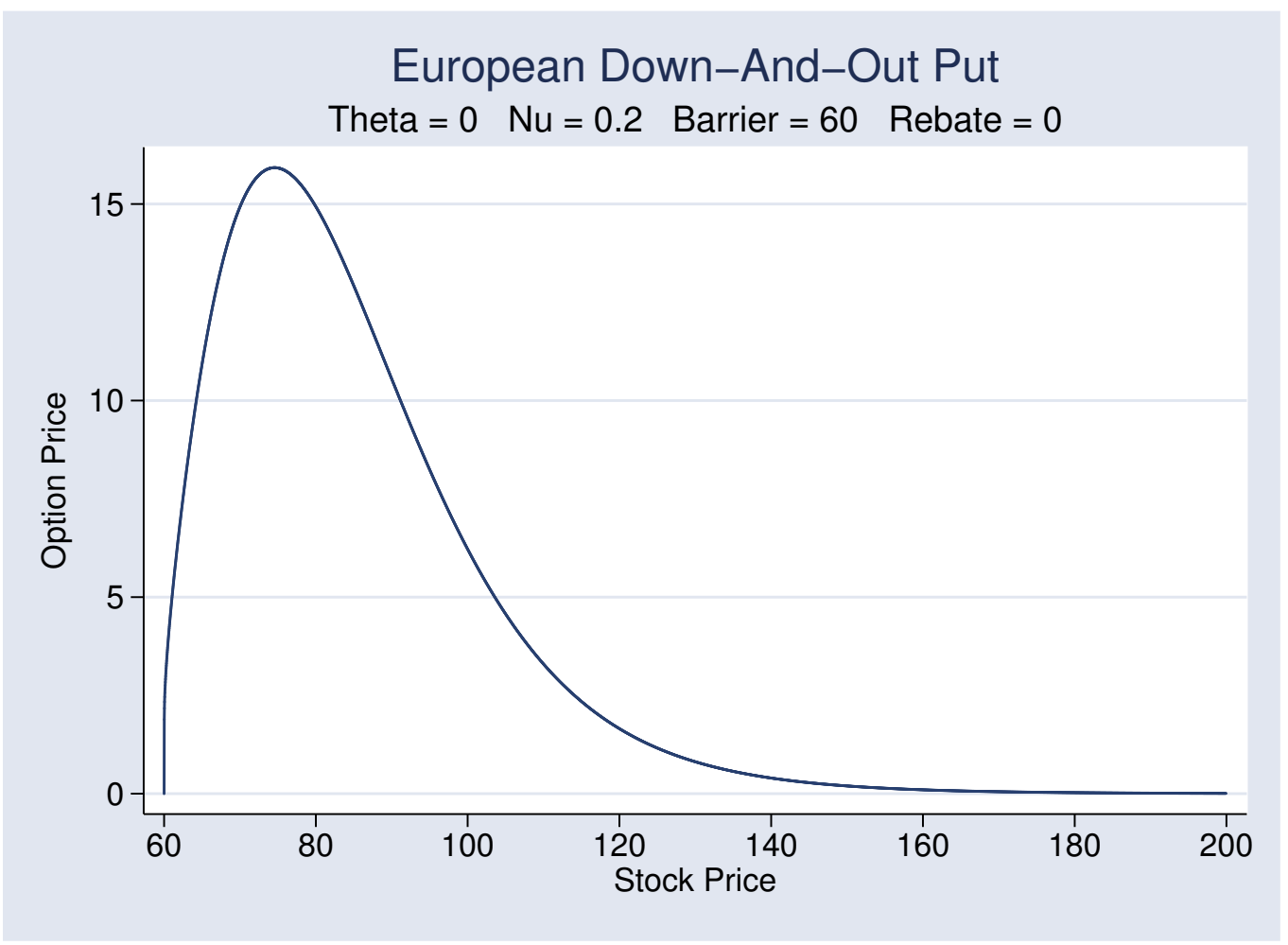

Figure 5.12: European Down-And-Out Put: Starting Level for $\vartheta$ Sensitivity Analysis, with Rebate $=0$ 
Figure 5.12 shows the starting point of the analysis with a symmetric underlying process.

The following graph shows four different levels of $\vartheta$. We see that for values far from the barrier and out of the money, an increase in $\vartheta$ produces an increase in the option price. On the other side as the stock gets closer to the barrier if $\vartheta$ increases, the option price decreases.

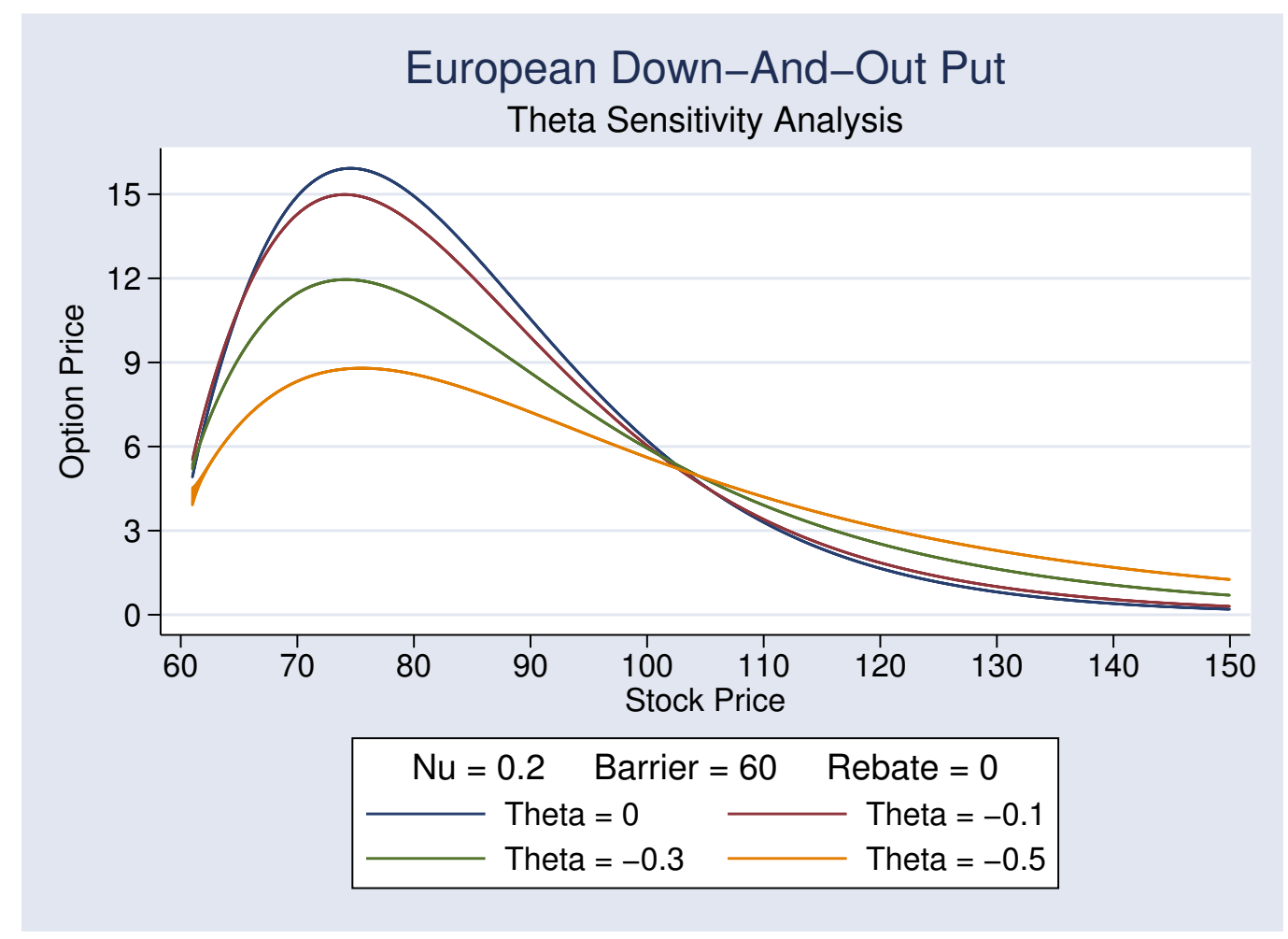

Figure 5.13: European Down-And-Out Put: $\vartheta$ Sensitivity Analysis, with Rebate $=0$ 


\section{Sensitivity with Respect to $\nu$ with Rebate $=40$}

We consider now the sensitivity of an European up-and-out put option with respect to $\vartheta$. The difference with the case presented above is that now we assume the presence of a $\$ 40$ rebate. The parameters used in this experiment are:

- $T=1 \quad$ Time to maturity in years;

- $K=\$ 100$ Strike price;

- barrier $=4.09434$, corresponding to $S=\$ 60$;

- rebate $=40$;

- $r=0.03$ Interest rate;

- $q=0.01$ Dividend yield;

- $\sigma=0.2$;

- $\vartheta=-0.1$

- $N=5100$ Number of space intervals;

- $M=300 \quad$ Number of time intervals;

- $x_{\min }=4.09434$ Min $x$ value considered, corresponding to $S=\$ 60$;

- $x_{\max }=5.85793$ Max $x$ value considered, corresponding to $S=\$ 350$;

- $\Delta x=0.000346 \quad$ Size of a space interval;

- $\Delta t=0.003333$ Size of a time interval;

- $\frac{\Delta t}{\Delta x}=9.639$;

Using these parameters we study the impact of changing $\nu$ from 0.1 to 0.7 . Volatility, skewness and kurtosis are affected by the movement in $\nu$ in the following way: 


\begin{tabular}{|c|c|c|c|c|c|}
\hline$\nu$ & 0.1 & 0.2 & 0.3 & 0.5 & 0.7 \\
\hline$\sqrt{E\left[(X(t)-E[X(t)])^{2}\right]}$ & 0.202 & 0.205 & 0.207 & 0.212 & 0.217 \\
\hline$\frac{E\left[(X(t)-E[X(t)])^{3}\right]}{\sqrt{\left\{E\left[(X(t)-E[X(t)])^{2}\right]\right\}^{3}}}$ & -0.147 & -0.288 & -0.424 & -0.681 & -0.921 \\
\hline$\frac{E\left[(X(t)-E[X(t)])^{4}\right]}{\left\{E\left[(X(t)-E[X(t)])^{2}\right]\right\}^{2}}$ & 3.314 & 3.656 & 4.021 & 4.815 & 5.679 \\
\hline
\end{tabular}

We can see that as $\nu$ increases, kurtosis, volatility and skewness increase. In the following graph we show an initial situation where $\nu=0.1$.

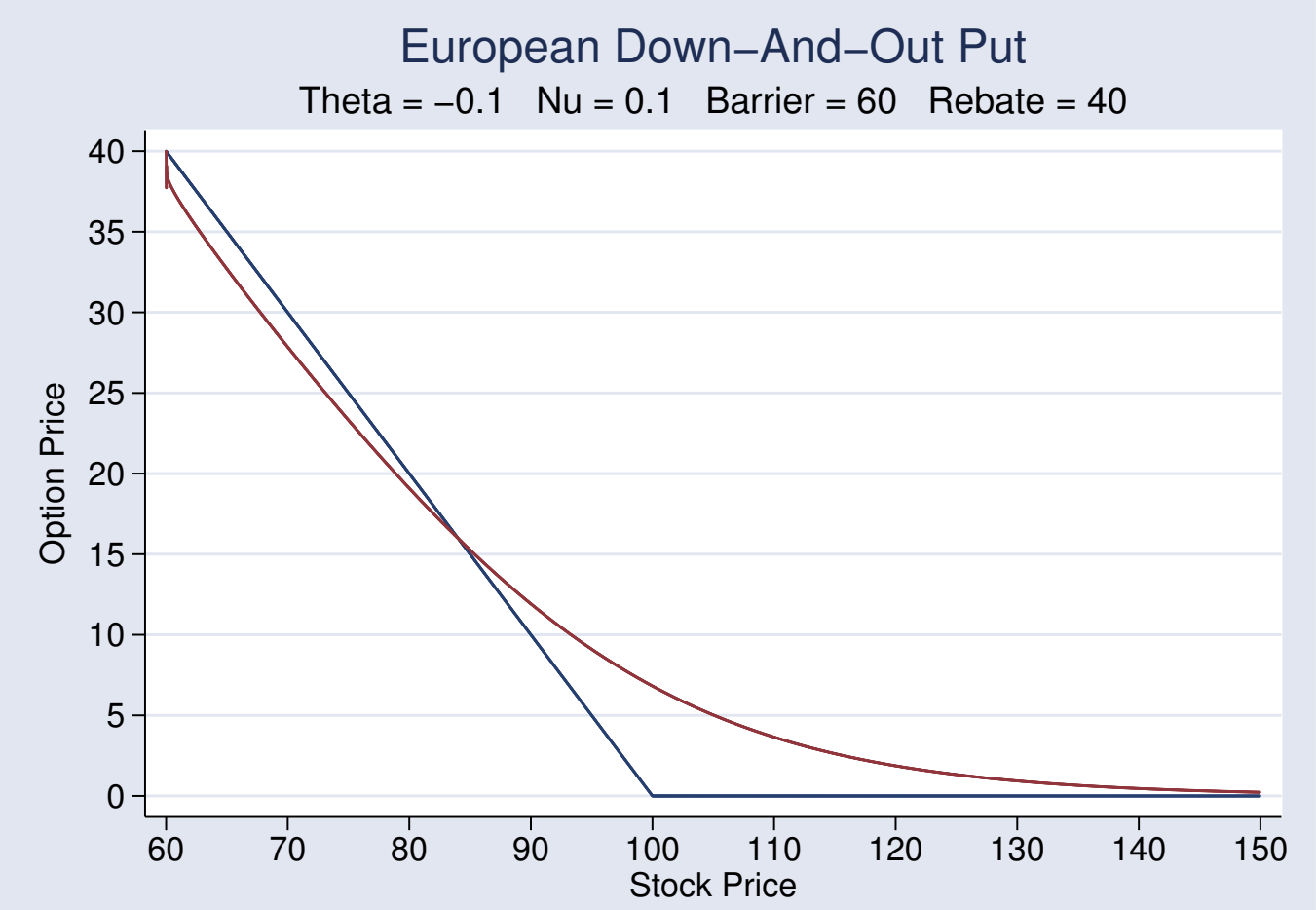

Figure 5.14: European Down-And-Out Put: Starting Level for $\nu$ Sensitivity Analysis, with Rebate $=40$ 
The following graph shows the option price in five cases of $\nu$ values. We can see that as the option becomes in the money and gets closer to the barrier, the value is higher for low levels of $\nu$, because the increased volatility and kurtosis makes more likely the fact that the option is knocked out.

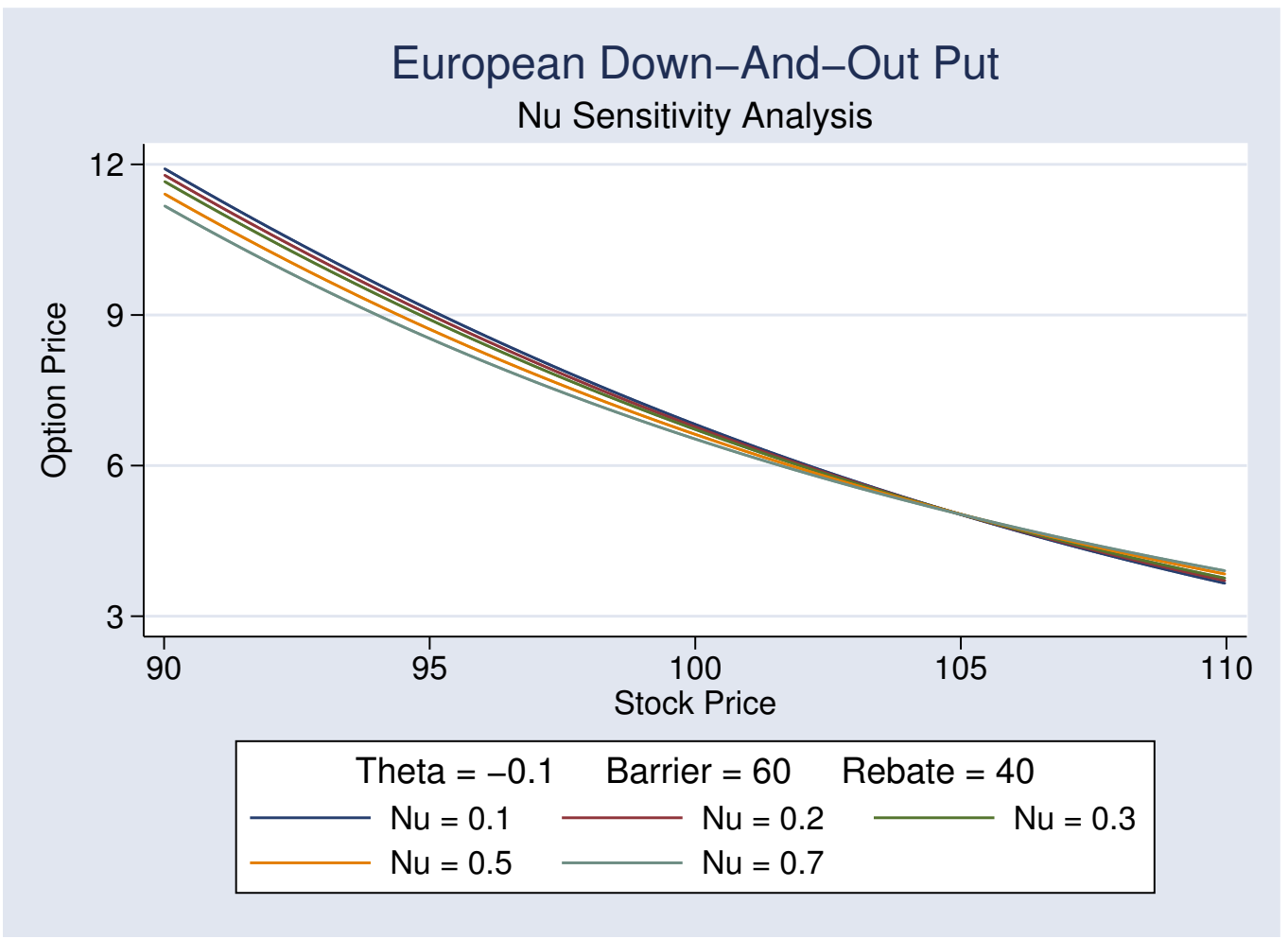

Figure 5.15: European Down-And-Out Put: $\nu$ Sensitivity Analysis, Detail 
The following graph shows a comparison between five levels of $\nu$ with special attention to the out of the money area. If the barrier option is deep out of the money, increasing volatility and kurtosis increases the option value. Hence in this case the barrier price is higher when $\nu$ is higher.

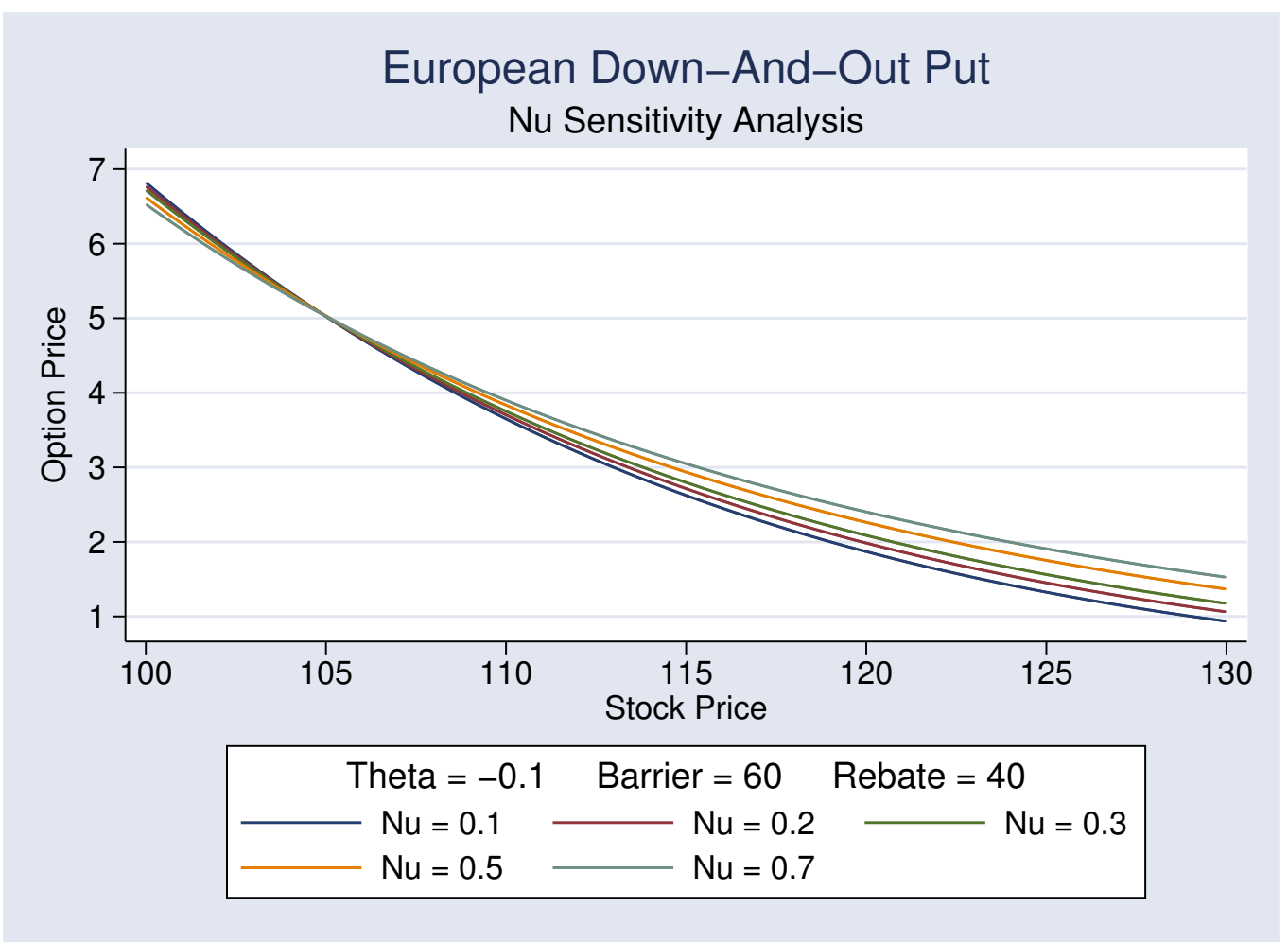

Figure 5.16: European Down-And-Out Put: $\nu$ Sensitivity Analysis, Detail 
Sensitivity with Respect to $\vartheta$ with Rebate $=40$

We consider now the sensitivity of an European up-and-out put option with respect to $\vartheta$. Here we assume that the rebate to be paid if the option is knocked out is $\$ 40$. The parameters used in this experiment are:

- $T=1 \quad$ Time to maturity in years;

- $K=\$ 100$ Strike price;

- barrier $=4.09434$, corresponding to $S=\$ 60$;

- rebate $=40$

- $r=0.03 \quad$ Interest rate;

- $q=0.01 \quad$ Dividend yield;

- $\sigma=0.2$;

- $\nu=0.2$;

- $N=5100$ Number of space intervals;

- $M=300$ Number of time intervals;

- $x_{\min }=4.09434$ Min $x$ value considered, corresponding to $S=\$ 60$;

- $x_{\max }=5.85793$ Max $x$ value considered, corresponding to $S=\$ 350$;

- $\Delta x=0.000346$ Size of a space interval;

- $\Delta t=0.003333$ Size of a time interval;

- $\frac{\Delta t}{\Delta x}=9.639$;

Using these parameters we study the impact of moving $\vartheta$ from 0 to -0.5 . The impact on volatility, skewness and kurtosis of changing $\vartheta$ is summarized in this table. 


\begin{tabular}{|c|c|c|c|c|}
\hline$\vartheta$ & 0 & -0.1 & -0.3 & -0.5 \\
\hline$\sqrt{E\left[(X(t)-E[X(t)])^{2}\right]}$ & 0.200 & 0.205 & 0.241 & 0.300 \\
\hline$\frac{E\left[(X(t)-E[X(t)])^{3}\right]}{\sqrt{\left\{E\left[(X(t)-E[X(t)])^{2}\right]\right\}^{3}}}$ & 0.000 & -0.288 & -0.670 & -0.815 \\
\hline$\frac{E\left[(X(t)-E[X(t)])^{4}\right]}{\left\{E\left[(X(t)-E[X(t)])^{2}\right]\right\}^{2}}$ & 3.600 & 3.656 & 3.915 & 4.081 \\
\hline
\end{tabular}

We see that if $\vartheta$ becomes more negative, skewness, volatility and kurtosis increase.

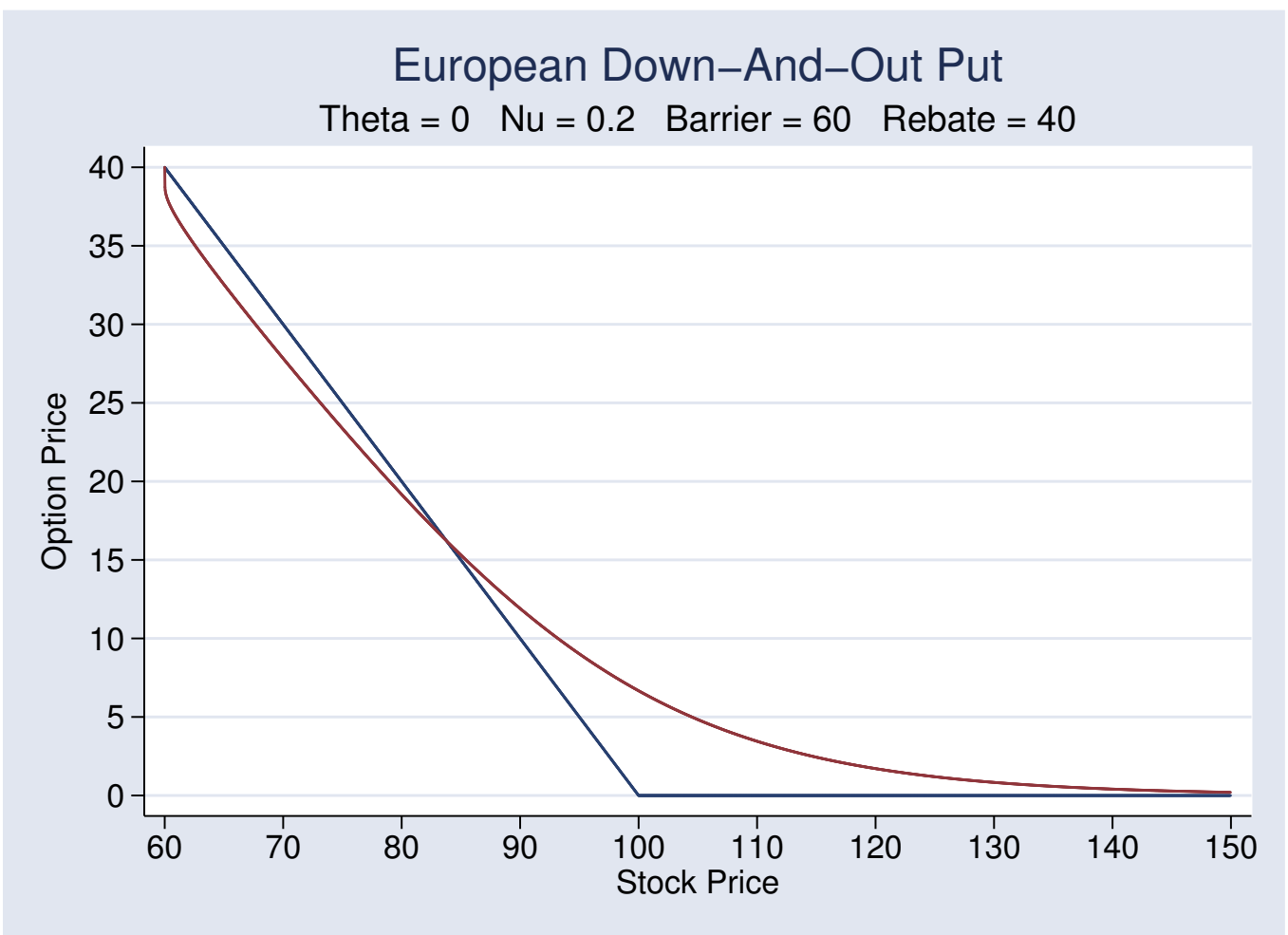

Figure 5.17: European Down-And-Out Put: Starting Level for $\vartheta$ Sensitivity Analysis, with Rebate $=40$ 
Figure 5.17 shows an initial situation where the process followed by the underlying returns is symmetric.

Figure 5.18 compares four different levels of $\vartheta$ when the stock is far from the barrier level. We can see that as $\vartheta$ increases, the option value increases.

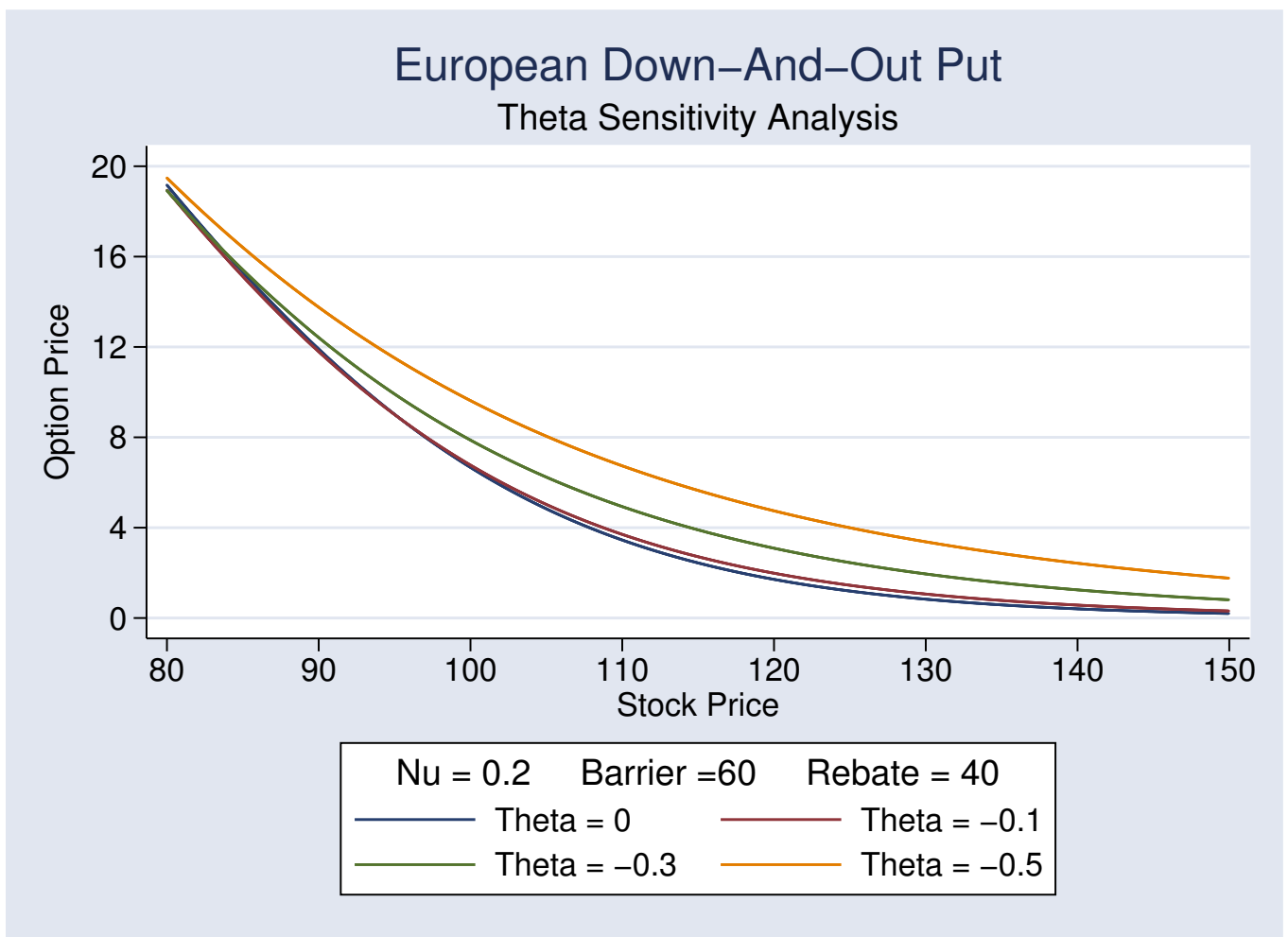

Figure 5.18: European Down-And-Out Put: $\vartheta$ Sensitivity Analysis, with Rebate $=40$ 


\subsection{American Barrier Options}

\subsubsection{Introduction}

We define as American Barrier Options, those barrier options which can be exercised at any time before expiration, in the same way as we used the term "American" for plain vanilla options. We note however that some authors used the term with a different meaning. For example Berger ${ }^{4}$ refers the early exercise options to the knock-out case only. The reason could be that it does not make sense to exercise an in-barrier before it gets knocked in, especially if there is no rebate. However this distinction does not seem to be correct because after having reached the barrier, the in-barrier could be exercised. Other authors ${ }^{5}$ use the distinction between European and American barrier options not on the basis of the early exercise, but depending on how often it is tested if the barrier has been reached. So if the option is such that as soon as the barrier is reached the in or out event is realized, they speak about American barrier option; while if only some specified times (for example daily close) are relevant to activate or deactivate the option, they speak about European barrier options. As said, in this work American option will always refers to the early exercise, using instead the expression "discretely monitored barrier options" or "local barrier options" to define an options where only if the price passes the barrier at certain relevant times the option is knocked in or out.

American barrier options are quite popular among practitioner, for example a number of convertible bonds presents contingent conversion which is defined in terms of an up-and-in call and basically all these convertible bonds can be exercised at any time before maturity.

The variance gamma PIDE describing the dynamics of these options is the same as the one we derived for American vanilla options. The difference is going to emerge from the boundary conditions which will be imposed in the same way as we did for European barrier options. As we will have to distinguish between call and put cases and between the fact if the barrier

\footnotetext{
${ }^{4}$ Eric Berger, "Barrier Options", in Israel Nelken, edit., The Handbook of Exotic Options: Instruments, Analysis and Applications, Richard D. Irwin, New York, 1996, page 215 .

${ }^{5}$ See for example Robert R. Trippi and Don M. Chance, "Quick Valuation of the 'Bermuda' Capped Option", Journal of Portfolio Management, Vol. 20, N. 1, 1993, pages 93-99.
} 
level is higher or lower the current stock price. In the same way we did for the European case, we will focus our attention on the knock-out case which is materially easier to be solved numerically, obtaining the corresponding knock-in with the formula

Vanilla Option $=$ Knock-in + Knock-out

\subsubsection{The Variance Gamma PIDE for American Bar- rier Options}

\section{The Variance Gamma PIDE for American Barrier Call Options}

We know from the vanilla case that the American call options has to follow the following partial integro differential equation.

$$
\begin{array}{r}
\frac{\partial W(x, t)}{\partial t}+(r-q+\omega) \cdot \frac{\partial W(x, t)}{\partial x}+ \\
+\int_{-\infty}^{+\infty}[W(x+y, t)-W(x, t)] k(y) d y-r W(x, t)+ \\
-1_{x>x(\tau)}\left\{r K-q e^{x}+\int_{-\infty}^{x(\tau)-x}\left[W(x+y, t)+K-e^{x+y}\right] k(y) d y\right\}=0
\end{array}
$$

Let's define now the final and boundary conditions required to solve this PIDE in the different cases of an up-and-out-call options. Here the final condition, provided that the stock has not reached the barrier is that the option at expiration is equal to its payoff. That is

$$
W(x, T)=\max \left(e^{x}-K, 0\right) \quad \text { if }\left\{e^{x}<B \quad \forall t\right\}
$$

where, as usual, $B>0$ is the barrier level. Moreover at any time before expiration, the value of the option has to be at least as big as its exercise value, provided that the underlying has not reached the barrier, that is

$$
W(x, t) \geqslant \max \left(e^{x}-K, 0\right) \forall t<T \quad \text { if }\left\{e^{x}<B \quad \forall t\right\}
$$

Finally we have the following conditions

$$
\begin{array}{rr}
W(-\infty, t)=0 & \forall t \\
W(\ln (B), t)=R & \forall t
\end{array}
$$


where $R \geqslant 0$ is the value of the rebate. If we consider a down-and-out call options, on the other side, the final condition and the early exercise condition are

$$
\begin{aligned}
W(x, T)=\max \left(e^{x}-K, 0\right) & \text { if }\left\{e^{x}>B \quad \forall t\right\} \\
W(x, t) \geqslant \max \left(e^{x}-K, 0\right) \forall t<T & \text { if }\left\{e^{x}>B \quad \forall t\right\}
\end{aligned}
$$

while the boundary conditions become

$$
\begin{array}{rr}
W(\ln (B), t)=R & \forall t \\
W(+\infty, t)=e^{x} & \forall t
\end{array}
$$

\section{The Variance Gamma PIDE for American Barrier Put Options}

The equation we need to solve for American barrier puts is the same as the correspondent vanilla one:

$$
\begin{array}{r}
\frac{\partial W(x, t)}{\partial t}+(r-q+\omega) \cdot \frac{\partial W(x, t)}{\partial x}+ \\
+\int_{-\infty}^{+\infty}[W(x+y, t)-W(x, t)] k(y) d y-r W(x, t)+ \\
-1_{x<x(\tau)}\left\{q e^{x}-r K+\int_{x(\tau)-x}^{+\infty}\left[W(x+y, t)-K+e^{x+y}\right] k(y) d y\right\}=0
\end{array}
$$

Up-and-out puts have the following final condition

$$
W\left(e^{x}, T\right)=\max \left(K-e^{x}, 0\right) \text { if }\left\{e^{x}<B \quad \forall t\right\}
$$

moreover they satisfy the early exercise condition

$$
W\left(e^{x}, t\right) \geqslant \max \left(K-e^{x}, 0\right) \forall t<T \quad \text { if }\left\{e^{x}<B \quad \forall t\right\}
$$

Boundary conditions for up-and-out put options are

$$
\begin{array}{cc}
W(-\infty, t)=K & \forall t \\
W(\ln (B), t)=R & \forall t
\end{array}
$$

For down-and-out put options, final and boundary conditions are

$$
\begin{array}{r}
W\left(e^{x}, T\right)=\max \left(K-e^{x}, 0\right) \quad \text { if }\left\{e^{x}>B \quad \forall t\right\} \\
W\left(e^{x}, t\right) \geqslant \max \left(K-e^{x}, 0\right) \forall t<T \quad \text { if }\left\{e^{x}>B \quad \forall t\right\}
\end{array}
$$


and the boundary conditions are

$$
\begin{array}{r}
W(\ln (B), t)=R \quad \forall t \\
W(+\infty, t)=0 \quad \forall t
\end{array}
$$

\subsubsection{Numerical Solution for American Barrier Op- tions}

As we did for the previous cases, we want now to rewrite the PIDEs in such a way that can be solved using a finite difference approach. First of all we need to define the interval inside which we are going to solve the equations. We make sure that the barrier level represents the limit of the range. In particular we choose

$$
[0, T] \times\left[x_{\text {min }}, \ln (B)\right]
$$

for the up-and out call and put options, while we consider

$$
[0, T] \times\left[\ln (B), x_{\max }\right]
$$

for down-and-out call and put options. We divide the stock price range in $N$ intervals of equal size. The size of each space is

$$
\Delta x=\frac{\ln (B)-x_{m i n}}{N}
$$

for up-and-out options while it is

$$
\Delta x=\frac{x_{\max }-\ln (B)}{N}
$$

for down-and-out options. The time space is divided in $M$ intervals of size

$$
\Delta t=\frac{T}{M}
$$

The notation $W\left(x_{i}, t_{j}\right)$ refers to the value of $W(\cdot)$ at the node $(i, j)$ and using this notation, we can write the PIDE for American call barrier options (5.15) 


$$
\begin{aligned}
& h W\left(x_{i-1}, t_{j}\right)+(1+r \Delta t) W\left(x_{i}, t_{j}\right)-h W\left(x_{i+1}, t_{j}\right)=W\left(x_{i}, t_{j+1}\right)+ \\
& +\Delta t \int_{-\infty}^{+\infty}\left[W\left(x_{i}+y, t_{j+1}\right)-W\left(x_{i}, t_{j+1}\right)\right] k(y) d y-1_{x_{i}>x\left(\tau_{j+1}\right)} \cdot \Delta t . \\
& \cdot\left\{r K-q e^{x_{i}}+\int_{-\infty}^{x\left(\tau_{j+1}\right)-x_{i}}\left[W\left(x_{i}+y, t_{j+1}\right)+K-e^{x_{i}+y}\right] k(y) d y\right\}
\end{aligned}
$$

We can see that this is the same PIDE as (4.24) that is the equation describing the dynamics of the American vanilla call option.

Similarly equation (5.16) can be written in its discrete form as

$$
\begin{aligned}
& h W\left(x_{i-1}, t_{j}\right)+(1+r \Delta t) W\left(x_{i}, t_{j}\right)-h W\left(x_{i+1}, t_{j}\right)=W\left(x_{i}, t_{j+1}\right)+ \\
& +\Delta t \int_{-\infty}^{+\infty}\left[W\left(x_{i}+y, t_{j+1}\right)-W\left(x_{i}, t_{j+1}\right)\right] k(y) d y-1_{x_{i}<x\left(\tau_{j+1}\right)} \cdot \Delta t . \\
& \cdot\left\{q e^{x_{i}}-r K+\int_{x\left(\tau_{j+1}\right)-x_{i}}^{+\infty}\left[W\left(x_{i}+y, t_{j+1}\right)-K+e^{x_{i}+y}\right] k(y) d y\right\}
\end{aligned}
$$

which is the same as equation (4.25), the PIDE for American vanilla put options.

Equations (5.17) and (5.18) presents two integral, the first of them is the same for calls and puts and it is the same as the one we had in the European barrier option, while the second defines the case of the stock jumping back to the continuation region after having reached the exercise region. Let's examine how to rewrite these two integrals to allow a numerical implementation.

\subsubsection{Solution of the Jump Integral for American Bar- rier Options}

We call "jump integrals" the first integrals in equations (5.17) and (5.18) to distinguish them from the second integrals. These other integrals will be referred to simply as integrals in the heaviside term. A detailed solution of the jump integral for American barrier options is available in appendix A.8. 
This integral can be solved in a similar way we did for the European barrier case. The only difference is for those integrals which describe the situations where the stock has jumped outside the range and it is so in the money and far from the barrier that we can impose it will be exercised for sure. In this case, if the proportion between interest rate and dividend yield is such that it is profitable to exercise the option, the option will be exercised right away. Specifically when we consider down-and-out call options, if the jump is large and positive such that

$$
y \in\left[x_{N}-x_{i},+\infty\right]
$$

we can approximate

$$
W\left(x_{i}+y, t_{j+1}\right) \simeq \max \left[e^{x_{i}+y} e^{-q\left(T-t_{j+1}\right)}-K e^{-r\left(T-t_{j+1}\right)}, e^{x_{i}+y}-K\right]
$$

If the dividend yield is zero, early exercise of the call is never a profitable option.

If $r>q$ and $q>0$, as the stock price increases, there is going to be a point at which it starts to be profitable to early exercise. It is possible that this point is outside the considered grid. In this case, the maximum in (5.19) is given by the discounted payoff for values of the jump which takes the stock relatively close to $x_{N}$, while for larger jumps the maximum is given by the non discounted payoff.

The correspondent case for put options is given by the up-and-out put. Here if the jump is large and negative such that $y<\left(x_{0}-x_{i}\right)$, the value of the option is set equal to

$$
W\left(x_{i}+y, t_{j+1}\right) \simeq \max \left[K e^{-r\left(T-t_{j+1}\right)}-e^{x_{i}+y} e^{-q\left(T-t_{j+1}\right)}, K-e^{x_{i}+y}\right]
$$

For up-and-out puts, it is possible that the exercise boundary is outside the grid at a level corresponding to a smaller value than the lower limit of the grid. This is however unlikely, because it would require $r<q$.

All the other integrals which constitutes the jump integral for American barrier options are valued in the same way we did for European barrier options. 


\subsubsection{Solution of the Integral in the Heaviside Term for American Barrier Options}

Because continuation and exercise regions are switched for calls and put options, calls and puts have two different heaviside term and we can therefore examine them one at a time. A detailed solution of these integrals is available in appendix A.9.

\section{Integral for American Barrier Call Options}

The integral we are interested into is

$$
\int_{-\infty}^{x\left(\tau_{j+1}\right)-x_{i}}\left[W\left(x_{i}+y, t_{j+1}\right)+K-e^{x_{i}+y}\right] k(y) d y
$$

from equation (5.17). Because the integral is multiplied by the indicator function $1_{x_{i}>x\left(\tau_{j+1}\right)}$, it is taken over a strictly negative range. We can split this integral in two pieces based upon the size of the jump in the following way

$$
\begin{aligned}
& \int_{-\infty}^{x_{0}-x_{i}}\left[W\left(x_{i}+y, t_{j+1}\right)+K-e^{x_{i}+y}\right] k(y) d y+ \\
& +\int_{x_{0}-x_{i}}^{(l-i) \Delta x}\left[W\left(x_{i}+y, t_{j+1}\right)+K-e^{x_{i}+y}\right] k(y) d y
\end{aligned}
$$

where we have used the usual notation

$$
x\left(\tau_{j+1}\right) \triangleq l \Delta x=x_{l}
$$

for some integer $l$ between 0 and $N$ whose value determines the position of the exercise boundary in the grid. In reality $l$ has to be larger than zero, since a necessary condition for early exercise is that the option is in the money. Moreover note that if the option is not exercised for any point in the grid at a certain time step $t_{j+1}$, then $l=N$ for that time step.

If the jump is relatively small and the stock value remains inside the range, the problem can be approached in the same way we did for American vanilla options. Hence integral (5.22) can be valued as we did for the vanilla case. The only difference is that if the option considered is a down and out 
call option, the value of the option when the stock is at the node $x_{0} \triangleq \ln (B)$ is the rebate received upon reaching the barrier.

Let's examine now integral (5.21) and let's start talking about an up-andout call. Here we have a negative jump which takes the stock outside the considered range. Because it is a call option, we can say that for minimal stock prices, the option price is near zero that is

$$
W\left(x_{i}-y, t_{j+1}\right) \simeq 0 \quad \text { for } \quad y \in\left[x_{i}-x_{0},+\infty\right]
$$

where we used the usual change of variable $y \triangleq-y$. If instead we are considering a down-and-out call option, we have that the lower boundary is given by the barrier, that is $x_{0}=\ln (B)$. If the stock has a negative jump and passes the boundary, the option is knocked out and has no value. At the time of the first passage of the barrier the rebate is paid. Because here, similarly to the case we saw for jump integrals, the passage of the barrier is going to be a first passage, we can impose that the rebate is paid every time the stock jumps through the barrier. Hence we can impose that the option in this case is worth its rebate and we can write

$$
W\left(x_{i}-y, t_{j+1}\right)=R \quad \text { for } \quad y \in\left[x_{i}-\ln (B),+\infty\right]
$$

Putting these together we can value the integral in the heaviside term in the case of a down-and-out call option as

$$
\begin{array}{r}
\int_{-\infty}^{x\left(\tau_{j+1}\right)-x_{i}}\left[W\left(x_{i}+y, t_{j+1}\right)+K-e^{x_{i}+y}\right] k(y) d y= \\
=\sum_{k=i-l}^{i-1}\left\{\frac{W\left(x_{i-k-1}, t_{j+1}\right)-W\left(x_{i-k}, t_{j+1}\right)}{\nu \frac{\mu_{n}}{\nu_{n}} \Delta x} \cdot\left[e^{-\frac{\mu_{n}}{\nu_{n}} k \Delta x}-e^{-\frac{\mu_{n}}{\nu_{n}}(k+1) \Delta x}\right]+\right. \\
+\frac{1}{\nu}\left\{W\left(x_{i-k}, t_{j+1}\right)-k\left[W\left(x_{i-k-1}, t_{j+1}\right)-W\left(x_{i-k}, t_{j+1}\right)\right]\right\} \\
+\frac{K}{\nu} \cdot \operatorname{expint}\left[\frac{\mu_{n}}{\nu_{n}}(i-l) \Delta x\right]-\frac{e^{x_{i}}}{\nu} \cdot \operatorname{expint}\left[\left(1+\frac{\mu_{n}}{\nu_{n}} k \Delta x\right)(i-l) \Delta x\right]+ \\
+\frac{R}{\nu} \operatorname{expint}\left(\frac{\mu_{n}}{\nu_{n}} i \Delta x\right)
\end{array}
$$


The solution of the integral in the heaviside function for American up-andout call options is given in appendix A.9.1, equation (A.110).

\section{Integral for American Barrier Put Options}

The integral for put options from equation (5.18) is

$$
\int_{x\left(\tau_{j+1}\right)-x_{i}}^{+\infty}\left[W\left(x_{i}+y, t_{j+1}\right)-K+e^{x_{i}+y}\right] k(y) d y
$$

The integral is multiplied buy $1_{x_{i}<x\left(\tau_{j+1}\right)}$, hence it is taken over a strictly positive interval and it can be divided into

$$
\begin{gathered}
\int_{(l-i) \Delta x}^{(N-i) \Delta x}\left[W\left(x_{i}+y, t_{j+1}\right)-K+e^{x_{i}+y}\right] k(y) d y+ \\
\int_{(N-i) \Delta x}^{+\infty}\left[W\left(x_{i}+y, t_{j+1}\right)-K+e^{x_{i}+y}\right] k(y) d y
\end{gathered}
$$

where

$$
x\left(\tau_{j+1}\right) \triangleq l \Delta x=x_{l}
$$

for some integer $l$ between 0 and $N$ whose value determines the position of the exercise boundary in the grid. In reality $l$ has to be smaller than $N$ because it has at least to be such that $e^{x_{i}} \leq K$. Note also that if the option is not exercised for any grid point at a certain time step, we fix $l$ corresponding to the lower boundary of the grid $x_{0}$.

Integral (5.25) can be solved in the same way we did for American vanilla options; we just need to remember to impose the rebate as boundary condition at $x_{N} \triangleq \ln (B)$ for up-and-out put options.

Integral (5.26), on the other side, describes the case where the jump is outside the range of values considered. If we are considering an up-andout put option, this means that the stock has reached the barrier and this determines the payment of the rebate and the deactivation of the stock. Hence we can write

$$
W\left(x_{i}+y, t_{j+1}\right)=R \quad \text { for } \quad y \in\left[\ln (B)-x_{i},+\infty\right]
$$


where as usual we chose the range limit value $x_{N}$ to be equal to $\ln (B)$. In this up-and-out put case integral (5.24) can be solved as

$$
\begin{array}{r}
\int_{x\left(\tau_{j+1}\right)-x_{i}}^{+\infty}\left[W\left(x_{i}+y, t_{j+1}\right)-K+e^{x_{i}+y}\right] k(y) d y= \\
=\sum_{k=l-i}^{N-i-1}\left\{\frac{W\left(x_{i+k+1}, t_{j+1}\right)-W\left(x_{i+k}, t_{j+1}\right)}{\nu \frac{\mu_{p}}{\nu_{p}} \Delta x} \cdot\left[e^{-\frac{\mu_{p}}{\nu_{p}} k \Delta x}-e^{-\frac{\mu_{p}}{\nu_{p}}(k+1) \Delta x}\right]+\right. \\
+\frac{1}{\nu}\left\{W\left(x_{i+k}, t_{j+1}\right)-k\left[W\left(x_{i+k+1}, t_{j+1}\right)-W\left(x_{i+k}, t_{j+1}\right)\right]\right\} \\
\left.\cdot\left[\operatorname{expint}\left(\frac{\mu_{p}}{\nu_{p}} k \Delta x\right)-\operatorname{expint}\left(\frac{\mu_{p}}{\nu_{p}}(k+1) \Delta x\right)\right]\right\}+ \\
-\frac{K}{\nu} \cdot \text { expint }\left[\frac{\mu_{p}}{\nu_{p}}(l-i) \Delta x\right]+\frac{e^{x_{i}}}{\nu} \cdot \operatorname{expint}\left[\left(\frac{\mu_{p}}{\nu_{p}}-1\right)(l-i) \Delta x\right] \\
+\frac{R}{\nu} \text { expint }\left[\frac{\mu_{p}}{\nu_{p}}(N-i) \Delta x\right]
\end{array}
$$

On the other side, if the option considered is a down-and-out put, when we have a positive jump which takes the stock outside the range of considered values, we can approximate the value of the option with zero

$$
W\left(x_{i}+y, t_{j+1}\right) \simeq 0 \quad \text { for } \quad y \in\left[x_{N}-x_{i},+\infty\right]
$$

The solution of the integral in the heaviside function for down-and-out put options is given by equation (A.119), in appendix A.9.2.

\subsubsection{Difference Equation for American Barrier Op- tion}

Let's consider an American up-and-out call option. To obtain the difference equation under variance gamma, we replace the integral in the heaviside term in the PIDE (5.17) with the discretization in equation (A.110) and the jump integral in the PIDE with the approximation in equation (A.91) as modified 
by expression (A.92). We obtain in this way the following difference equation.

$$
\begin{aligned}
& W\left(x_{i-1}, t_{j}\right)\left\{h-\frac{\Delta t \cdot\left(1-e^{-\frac{\mu_{n}}{\nu_{n}} \Delta x}\right)}{\nu \Delta x \frac{\mu_{n}}{\nu_{n}}}\right\}+ \\
& +W\left(x_{i}, t_{j}\right)\left\{(1+r \Delta t)+\frac{\Delta t \cdot\left(1-e^{-\frac{\mu_{p}}{\nu_{p}} \Delta x}\right)}{\nu \Delta x \frac{\mu_{p}}{\nu_{p}}}+\frac{\Delta t \cdot\left(1-e^{-\frac{\mu_{n}}{\nu_{n}} \Delta x}\right)}{\nu \Delta x \frac{\mu_{n}}{\nu_{n}}}\right\}+ \\
& -W\left(x_{i+1}, t_{j}\right)\left\{h+\frac{\Delta t \cdot\left(1-e^{-\frac{\mu_{p}}{\nu_{p}} \Delta x}\right)}{\nu \Delta x \frac{\mu_{p}}{\nu_{p}}}\right\}=W\left(x_{i}, t_{j+1}\right)+ \\
& +\Delta t \cdot\left\{\sum_{k=1}^{N-i-1} \frac{1}{\nu \Delta x}\left[W\left(x_{i+k+1}, t_{j+1}\right)-W\left(x_{i+k}, t_{j+1}\right)\right] .\right. \\
& \cdot \frac{\nu_{p}}{\mu_{p}}\left[e^{-\frac{\mu_{p}}{\nu_{p}} k \Delta x}-e^{-\frac{\mu_{p}}{\nu_{p}}(k+1) \Delta x}\right]+ \\
& +\sum_{k=1}^{N-i-1} \frac{1}{\nu}\left[W\left(x_{i+k}, t_{j+1}\right)-W\left(x_{i}, t_{j+1}\right)-k\left[W\left(x_{i+k+1}, t_{j+1}\right)-W\left(x_{i+k}, t_{j+1}\right)\right]\right] . \\
& \cdot\left\{\operatorname{expint}\left(\frac{\mu_{p}}{\nu_{p}} k \Delta x\right)-\operatorname{expint}\left[\frac{\mu_{p}}{\nu_{p}}(k+1) \Delta x\right]\right\}+ \\
& +\sum_{k=1}^{i-1} \frac{1}{\nu \Delta x}\left[W\left(x_{i-k-1}, t_{j+1}\right)-W\left(x_{i-k}, t_{j+1}\right)\right] \frac{\nu_{n}}{\mu_{n}}\left[e^{-\frac{\mu_{n}}{\nu_{n}} k \Delta x}-e^{-\frac{\mu_{n}}{\nu_{n}}(k+1) \Delta x}\right]+ \\
& +\sum_{k=1}^{i-1} \frac{1}{\nu}\left\{W\left(x_{i-k}, t_{j+1}\right)-W\left(x_{i}, t_{j+1}\right)-k\left[W\left(x_{i-k-1}, t_{j+1}\right)-W\left(x_{i-k}, t_{j+1}\right)\right]\right\} . \\
& \cdot\left\{\operatorname{expint}\left(\frac{\mu_{n}}{\nu_{n}} k \Delta x\right)-\operatorname{expint}\left[\frac{\mu_{n}}{\nu_{n}}(k+1) \Delta x\right]\right\}+ \\
& +\frac{1}{\nu}\left[R-W\left(x_{i}, t_{j+1}\right)\right] \cdot \operatorname{expint}\left[\frac{\mu_{p}}{\nu_{p}}(N-i) \Delta x\right]+ \\
& \left.-\frac{1}{\nu} W\left(x_{i}, t_{j+1}\right) \cdot \operatorname{expint}\left(\frac{\mu_{n}}{\nu_{n}} i \Delta x\right)\right\}+
\end{aligned}
$$




$$
\begin{gathered}
-1_{x_{i}>x\left(\tau_{j+1}\right)} \cdot \Delta t \cdot\left\{r K-q e^{x_{i}}+\right. \\
+\sum_{k=i-l}^{i-1}\left\{\frac{W\left(x_{i-k-1}, t_{j+1}\right)-W\left(x_{i-k}, t_{j+1}\right)}{\nu \frac{\mu_{n}}{\nu_{n}} \Delta x} \cdot\left[e^{-\frac{\mu_{n}}{\nu_{n}} k \Delta x}-e^{-\frac{\mu_{n}}{\nu_{n}}(k+1) \Delta x}\right]+\right. \\
+\frac{1}{\nu}\left\{W\left(x_{i-k}, t_{j+1}\right)-k\left[W\left(x_{i-k-1}, t_{j+1}\right)-W\left(x_{i-k}, t_{j+1}\right)\right]\right\} \\
\left.\cdot\left[\operatorname{expint}\left(\frac{\mu_{n}}{\nu_{n}} k \Delta x\right)-\operatorname{expint}\left(\frac{\mu_{n}}{\nu_{n}}(k+1) \Delta x\right)\right]\right\}+ \\
+\frac{K}{\nu} \cdot \operatorname{expint}\left[\frac{\mu_{n}}{\nu_{n}}(i-l) \Delta x\right]+ \\
\left.-\frac{e^{x_{i}}}{\nu} \cdot \operatorname{expint}\left[\left(1+\frac{\mu_{n}}{\nu_{n}}\right)(i-l) \Delta x\right]\right\}
\end{gathered}
$$

We can see that the left hand side of the difference equation can be written in the form of a tridiagonal matrix. Hence we can easily solve the system using the Gauss's elimination method. In Appendix B we present a C code which can be used to solve numerically the difference equation.

The difference equation to solve American down-and-out calls, up-andout puts and down-and-out puts under variance gamma, can be obtain in a similar way. Results are available respectively in the appendix A.10.2, A.10.3 and A.10.4.

\subsection{Experimental Results for American Bar- rier Options}

In this section, we realize a number of experiments of American barrier option pricing. In particular, we want to study the sensitivity of barrier option prices to the rebate and to the parameters controlling volatility, skewness and kurtosis. We know that $\sigma, \vartheta$ and $n u$ do not represent themselves volatility, 
skewness and kurtosis, but are linked to them by the following relationships:

$$
\begin{gathered}
E\left[(X(t)-E[X(t)])^{2}\right]=\left(\vartheta^{2} \nu+\sigma^{2}\right) t \\
E\left[(X(t)-E[X(t)])^{3}\right]=\left(2 \vartheta^{3} \nu^{2}+3 \sigma^{2} \vartheta \nu\right) t \\
E\left[(X(t)-E[X(t)])^{4}\right]= \\
=\left(3 \sigma^{4} \nu+12 \sigma^{2} \vartheta^{2} \nu^{2}+6 \vartheta^{4} \nu^{3}\right) t+\left(3 \sigma^{4}+6 \sigma^{2} \vartheta^{2} \nu+3 \vartheta^{4} \nu^{2}\right) t^{2}
\end{gathered}
$$

\subsubsection{Up-And-Out Calls: Sensitivity Analysis}

Let's study here the sensitivity analysis of American up-and-out call options. We consider here in particular the sensitivity with respect to $\sigma$. We note that being American option it is not worth to study the sensitivity to the rebate in this case, unless the rebate has a value larger than the intrinsic value of the option upon reaching the barrier. In fact it is always possible to early exercised the American option to cash the intrinsic value.

The numerical experiments presented here can be obtained using the code in $\mathrm{C}$ language available in appendix B. The European up-and-out call case studied here is realized by choosing the following variable values:

- callput $=1$

- euroamerican $=1$

- barrier_switch $=1$

- up_and_out $=1$

in the $\mathrm{C}$ code.

\section{Sensitivity with Respect to $\sigma$}

We consider here the sensitivity of an European up-and-out call option with respect to $\sigma$. The parameters used in this experiment are:

- $T=0.5$ Time to maturity in years; 
- $K=\$ 100$ Strike price;

- $r=0.03$ Interest rate;

- $q=0.01$ Dividend yield;

- $\vartheta=-0.1$;

- $\nu=0.2$

- $N=5100$ Number of space intervals;

- $M=300$ Number of time intervals;

- $x_{\text {min }}=1.60944$ Min $x$ value considered, corresponding to $S=\$ 5$;

- $x_{\max }=4.86753$ Max $x$ value considered, corresponding to $S=\$ 130$;

- $\Delta x=0.000639$ Size of a space interval;

- $\Delta t=0.001667 \quad$ Size of a time interval;

- $\frac{\Delta t}{\Delta x}=2.609$

Using these parameters we move $\sigma$ from $10 \%$ to $50 \%$. The change in $\sigma$ produces an impact on volatility, skewness and kurtosis of the process describing the underlying returns. In particular we have the following values:

\begin{tabular}{|c|c|c|c|c|}
\hline$\sigma$ & 0.1 & 0.2 & 0.3 & 0.5 \\
\hline$\sqrt{E\left[(X(t)-E[X(t)])^{2}\right]}$ & 0.110 & 0.205 & 0.303 & 0.502 \\
\hline$\frac{E\left[(X(t)-E[X(t)])^{3}\right]}{\sqrt{\left\{E\left[(X(t)-E[X(t)])^{2}\right]\right\}^{3}}}$ & -0.517 & -0.288 & -0.196 & -0.119 \\
\hline$\frac{E\left[(X(t)-E[X(t)])^{4}\right]}{\left\{E\left[(X(t)-E[X(t)])^{2}\right]\right\}^{2}}$ & 3.783 & 3.656 & 3.626 & 3.609 \\
\hline
\end{tabular}

We see that when $\sigma$ increases, volatility increases. On the other side, larger values of $\sigma$ determine a reduction in kurtosis and skewness. 
Figure 5.19 shows the impact of changing $\sigma$, we can see that higher values of $\sigma$ determine larger option values.

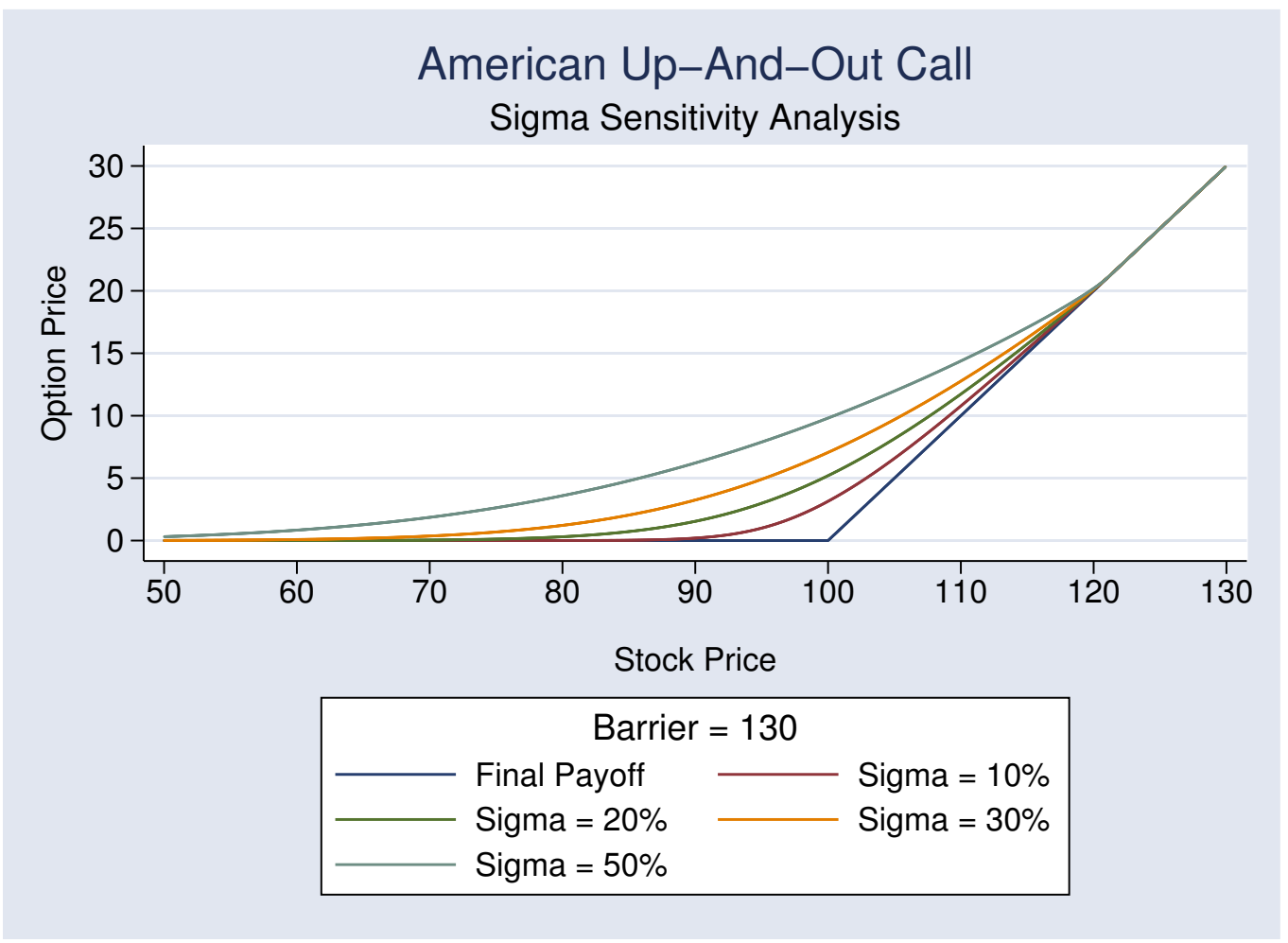

Figure 5.19: American Up-And-Out Call: $\sigma$ Sensitivity Analysis

\subsubsection{Down-And-Out Calls: Sensitivity Analysis}

We consider now the sensitivity of American down-and-out call options. We consider here in particular the sensitivity with respect to the rebate. We observe that because in this case the barrier is reached when the option is out of the money, the fact that the option is American does not make the analysis trivial as in the cases where the barrier is reached when the option is in the money. 
The numerical experiments presented here can be obtained using the code in $\mathrm{C}$ language available in appendix B. The European down-and-out call case studied here is realized by choosing the following variable values:

- callput $=1$

- euroamerican $=1$

- barrier_switch $=1$

- up_and_out $=0$

in the $\mathrm{C}$ code.

\section{Sensitivity with Respect to the Rebate}

We consider here the sensitivity of an American down-and-out call option with respect to the rebate. The parameters used in this experiment are:

- $T=0.5$ Time to maturity in years;

- $K=\$ 100$ Strike price;

- barrier $=4.2485$, corresponding to $S=\$ 70$;

- $r=0.03$ Interest rate;

- $q=0.01 \quad$ Dividend yield;

- $\sigma=0.2$

- $\vartheta=-0.1$;

- $\nu=0.2$

- $N=5100$ Number of space intervals;

- $M=300$ Number of time intervals;

- $x_{\min }=4.2485 \quad$ Min $x$ value considered, corresponding to $S=\$ 70$;

- $x_{\max }=5.85793$ Max $x$ value considered, corresponding to $S=\$ 350$;

- $\Delta x=0.000316$ Size of a space interval; 
- $\Delta t=0.001667$ Size of a time interval;

- $\frac{\Delta t}{\Delta x}=5.281$;

In this experiment we move only the rebate which is paid when the stock reaches the barrier positioned $\$ 30$ out of the money. In doing this, we keep $\sigma, \vartheta$ and $\nu$ unchanged in this experiment, hence volatility, skewness and kurtosis are fixed. More in detail, we have the following values:

\begin{tabular}{|c|c|}
\hline$\sqrt{E\left[(X(t)-E[X(t)])^{2}\right]}$ & 0.205 \\
\hline$\frac{E\left[(X(t)-E[X(t)])^{3}\right]}{\sqrt{\left\{E\left[(X(t)-E[X(t)])^{2}\right]\right\}^{3}}}$ & -0.288 \\
\hline$\frac{E\left[(X(t)-E[X(t)])^{4}\right]}{\left\{E\left[(X(t)-E[X(t)])^{2}\right]\right\}^{2}}$ & 3.656 \\
\hline
\end{tabular}


The following graph shows the option value for a zero rebate and a $\$ 0.5$ rebate. Clearly the option paying a rebate is worth more than the one without rebate, with the difference being more evident as the barrier level approaches.

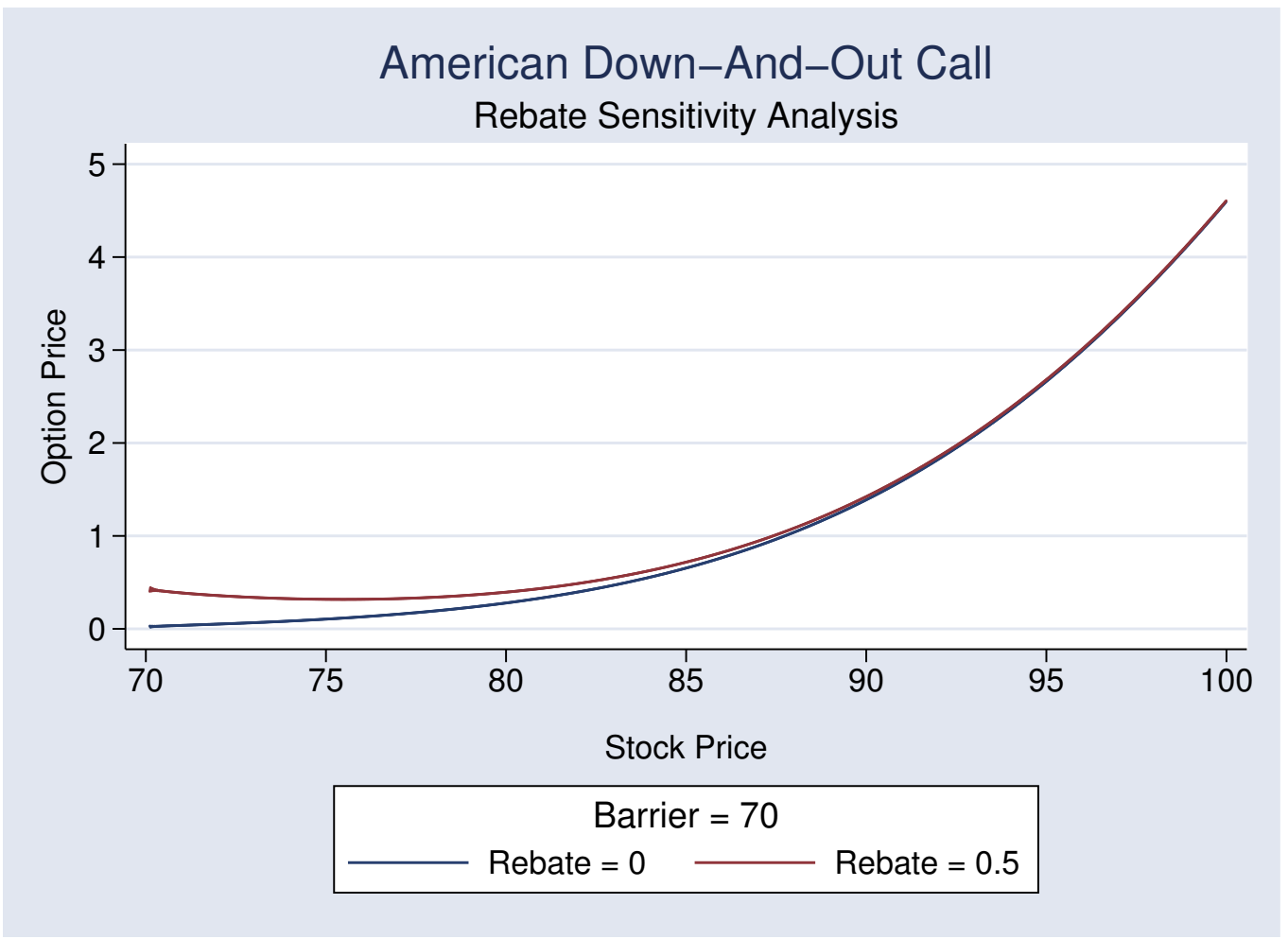

Figure 5.20: American Down-And-Out Call: Rebate Sensitivity Analysis

\subsubsection{Up-And-Out Puts: Sensitivity Analysis}

We consider now the sensitivity of American up-and-out put options. We consider here in particular the sensitivity with respect to the rebate.

The numerical experiments presented here can be obtained using the code in $\mathrm{C}$ language available in appendix B. The European up-and-out put case studied here is realized by choosing the following variable values:

- callput $=0$ 
- euroamerican $=1$

- barrier_switch $=1$

- up_and_out $=1$

in the $\mathrm{C}$ code.

\section{Sensitivity with Respect to the Rebate}

We consider here the sensitivity of an American up-and-out put option with respect to the rebate. The parameters used in this experiment are:

- $T=1 \quad$ Time to maturity in years;

- $K=\$ 100$ Strike price;

- barrier $=4.86753$, corresponding to $S=\$ 130$;

- $r=0.03$ Interest rate;

- $q=0.01 \quad$ Dividend yield;

- $\sigma=0.2$

- $\vartheta=-0.1$;

- $\nu=0.2$;

- $N=5100$ Number of space intervals;

- $M=300 \quad$ Number of time intervals;

- $x_{\text {min }}=1.60944 \quad$ Min $x$ value considered, corresponding to $S=\$ 5$;

- $x_{\max }=4.86753$ Max $x$ value considered, corresponding to $S=\$ 130$;

- $\Delta x=0.000639$ Size of a space interval;

- $\Delta t=0.003333$ Size of a time interval;

- $\frac{\Delta t}{\Delta x}=5.218$

In this experiment, the parameters affecting volatility, skewness and kurtosis are unchanged. We have the following values: 


\begin{tabular}{|c|c|}
\hline$\sqrt{E\left[(X(t)-E[X(t)])^{2}\right]}$ & 0.205 \\
\hline$\frac{E\left[(X(t)-E[X(t)])^{3}\right]}{\sqrt{\left\{E\left[(X(t)-E[X(t)])^{2}\right]\right\}^{3}}}$ & -0.288 \\
\hline$\frac{E\left[(X(t)-E[X(t)])^{4}\right]}{\left\{E\left[(X(t)-E[X(t)])^{2}\right]\right\}^{2}}$ & 3.656 \\
\hline
\end{tabular}

The graph present an up-and-out put where the barrier is positioned $30 \%$ out of the money. We compare the option value for four different level of rebate paid upon reaching the barrier: $0,0.5,1$ and 1.5.

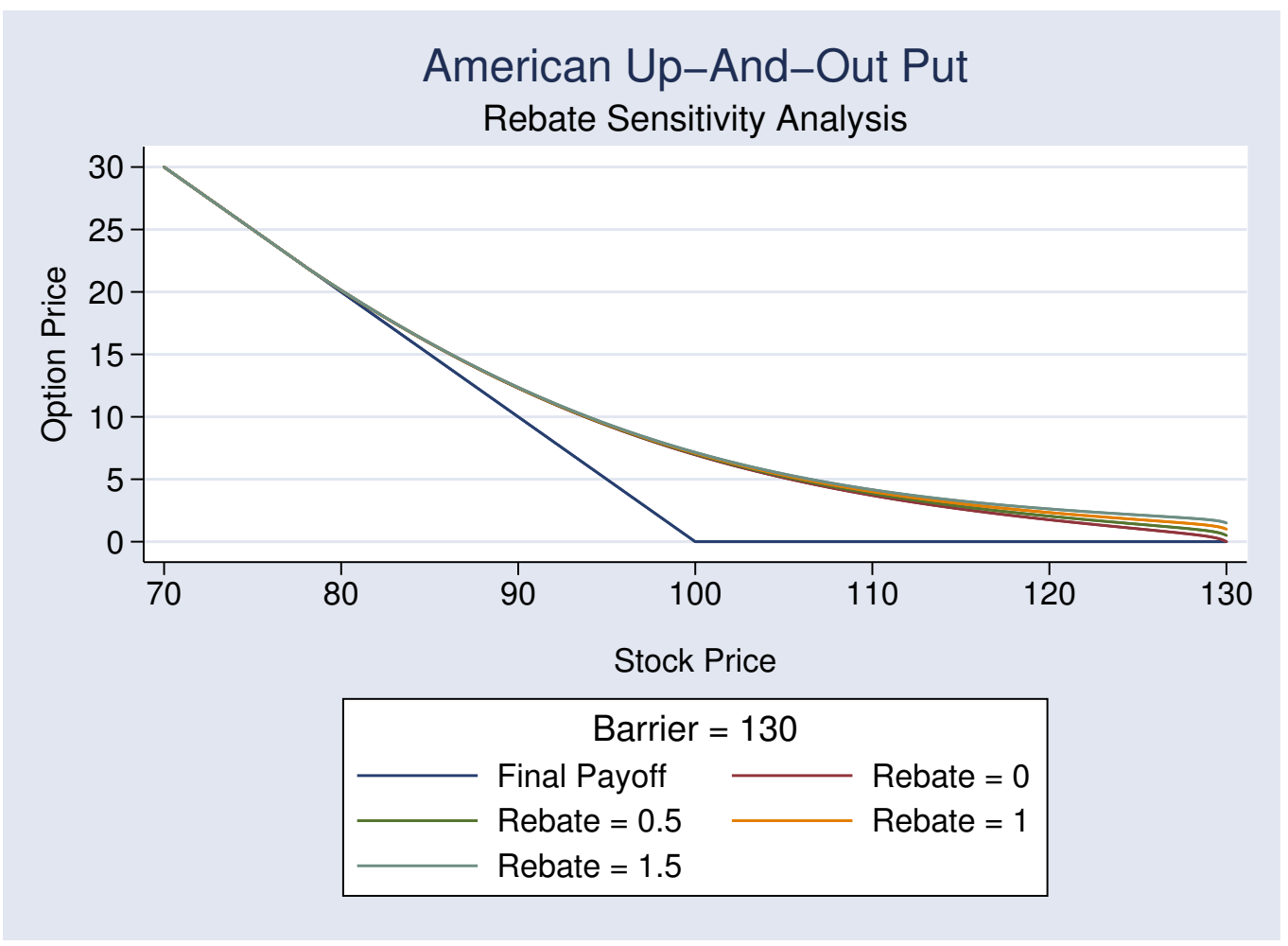

Figure 5.21: American Up-And-Out Put: Rebate Sensitivity Analysis 
This graph is a zoom of the previous one in the out of the money area. We can see more clearly that, as expected, the barrier value increases as the rebate increases.

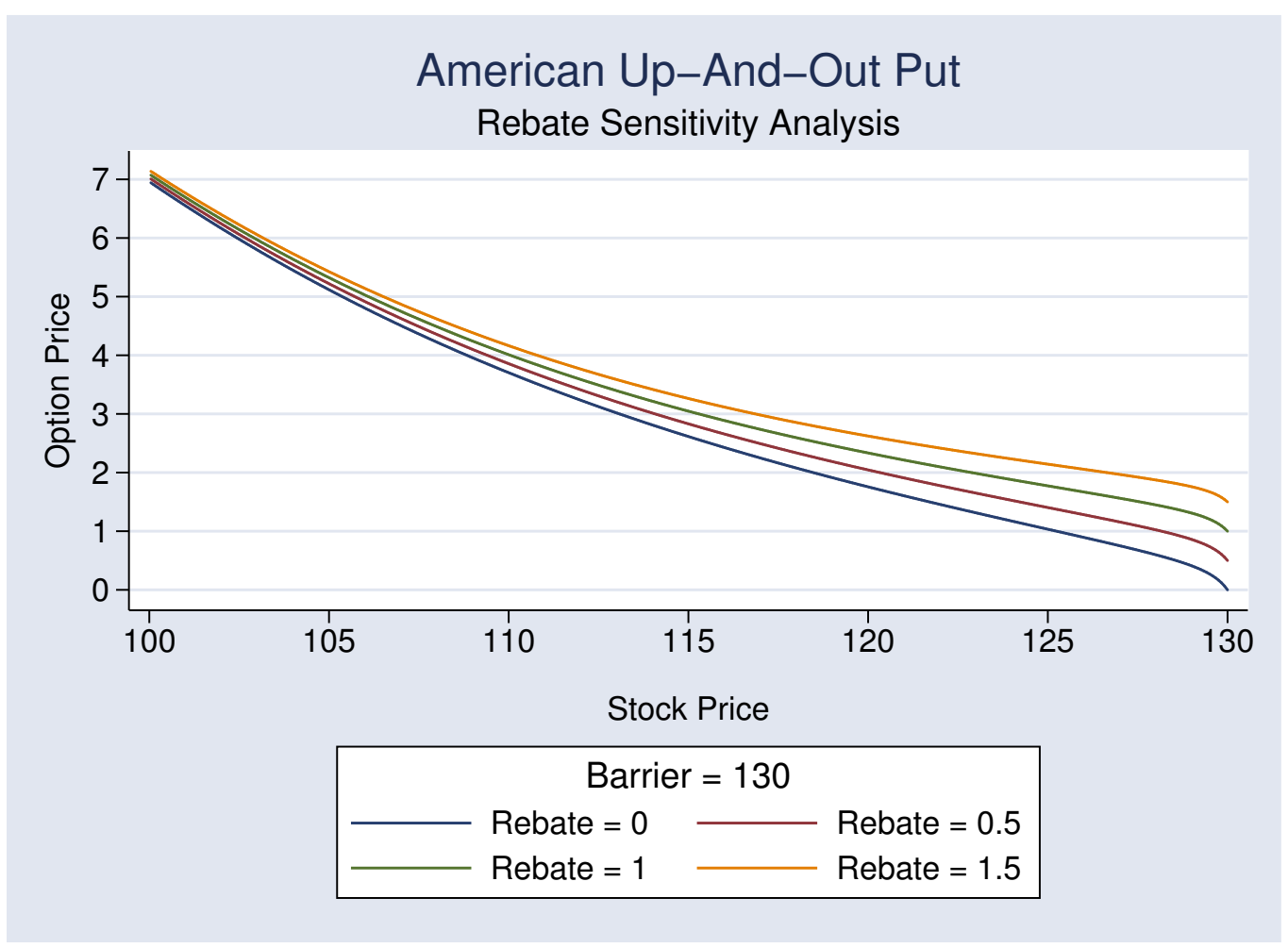

Figure 5.22: American Up-And-Out Put: Rebate Sensitivity Analysis, Detail 


\subsubsection{Down-And-Out Puts: Sensitivity Analysis}

We consider now some experiments on American down-and-out put options. In particular we want to study the sensitivity analysis with respect to $\nu$ and $\vartheta$. We remember that for the European down-and-out put case we realized sensitivity experiments with respect to $\nu$ and $\vartheta$ in different hypotheses of rebate. In the American case here, the difference in rebate is no more interesting, unless the rebate is higher than the intrinsic value of the option at the time the stock reaches the barrier, which is an unusual case in the market. In fact if the option is American and the barrier is reached when the option is in the money, we can always early exercise the barrier option to avoid the lost of the difference between the intrinsic value and the rebate.

The numerical experiments presented here can be obtained using the code in $\mathrm{C}$ language available in appendix B. The European down-and-out put case studied here is realized by choosing the following variable values:

- callput $=0$

- euroamerican $=1$

- barrier_switch $=1$

- up_and_out $=0$

in the $\mathrm{C}$ code.

\section{Sensitivity with Respect to $\nu$}

We consider now the sensitivity of an American up-and-out put option with respect to $\vartheta$. The rebate level used here is $\$ 40$ but any value less of $\$ 40$ would have produced the same result. The parameters used in this experiment are:

- $T=1 \quad$ Time to maturity in years;

- $K=\$ 100$ Strike price;

- barrier $=4.09434$, corresponding to $S=\$ 60$;

- rebate $=40$;

- $r=0.03 \quad$ Interest rate;

- $q=0.01$ Dividend yield; 
- $\sigma=0.2$

- $\vartheta=-0.1$;

- $N=5100$ Number of space intervals;

- $M=300 \quad$ Number of time intervals;

- $x_{\text {min }}=4.09434$ Min $x$ value considered, corresponding to $S=\$ 60$;

- $x_{\max }=5.85793$ Max $x$ value considered, corresponding to $S=\$ 350$;

- $\Delta x=0.000346$ Size of a space interval;

- $\Delta t=0.003333$ Size of a time interval;

- $\frac{\Delta t}{\Delta x}=9.639$

Using these parameters we study the impact of changing $\nu$ from 0.1 to 0.7 . Volatility, skewness and kurtosis are affected by the movement in $\nu$ in the following way:

\begin{tabular}{|c|c|c|c|}
\hline$\nu$ & 0.1 & 0.3 & 0.7 \\
\hline$\sqrt{E\left[(X(t)-E[X(t)])^{2}\right]}$ & 0.202 & 0.207 & 0.217 \\
\hline$\frac{E\left[(X(t)-E[X(t)])^{3}\right]}{\sqrt{\left\{E\left[(X(t)-E[X(t)])^{2}\right]\right\}^{3}}}$ & -0.147 & -0.424 & -0.921 \\
\hline$\frac{E\left[(X(t)-E[X(t)])^{4}\right]}{\left\{E\left[(X(t)-E[X(t)])^{2}\right]\right\}^{2}}$ & 3.314 & 4.021 & 5.679 \\
\hline
\end{tabular}

We can see from the table above that, as $\nu$ increases, we have higher kurtosis, volatility and skewness. 
Figure 5.23 shows the value of an up-and-out put for 3 levels of $\nu$ : $0.1,0.3$ and 0.7. Although in this scale the lines are not too far from each other, we should be able to individuate a central area, where the option is at the money or a bit in the money where the option with lowest $\nu$ is worth the most. As we move to the out of the money area, we can see that now it is the option with highest $\nu$ to be worth the most. Finally in the area deep in the money we have that all the options considered have the same value since they are all early exercised.

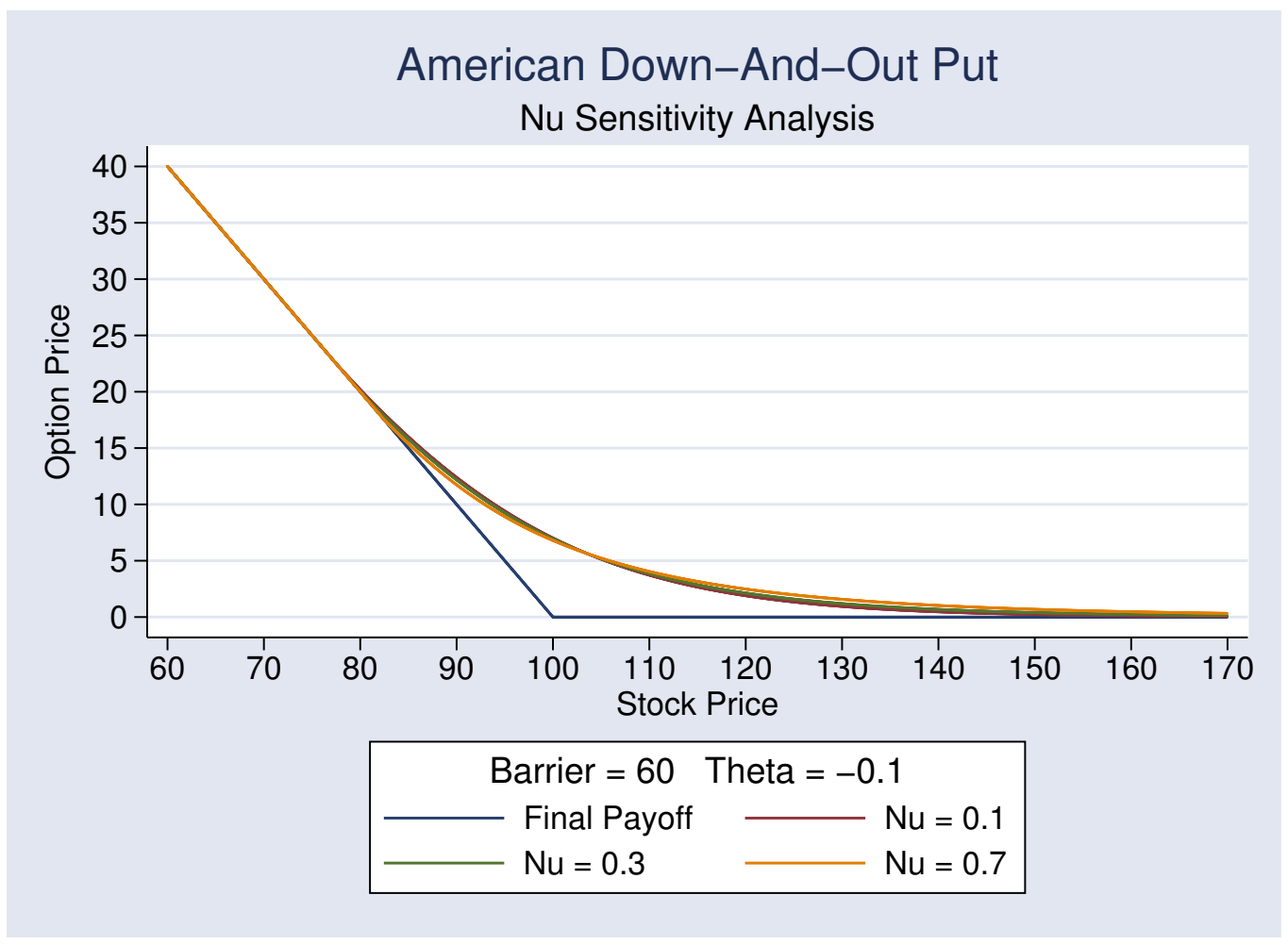

Figure 5.23: American Down-And-Out Put: $\nu$ Sensitivity Analysis 
In this graph we consider the same barrier option studied in the previous graph but we focus our attention on the stock range from $\$ 80$ to $\$ 110$. In this graph we can better note how the relative values of the options with different $\nu$ are switched in order depending on the stock level.

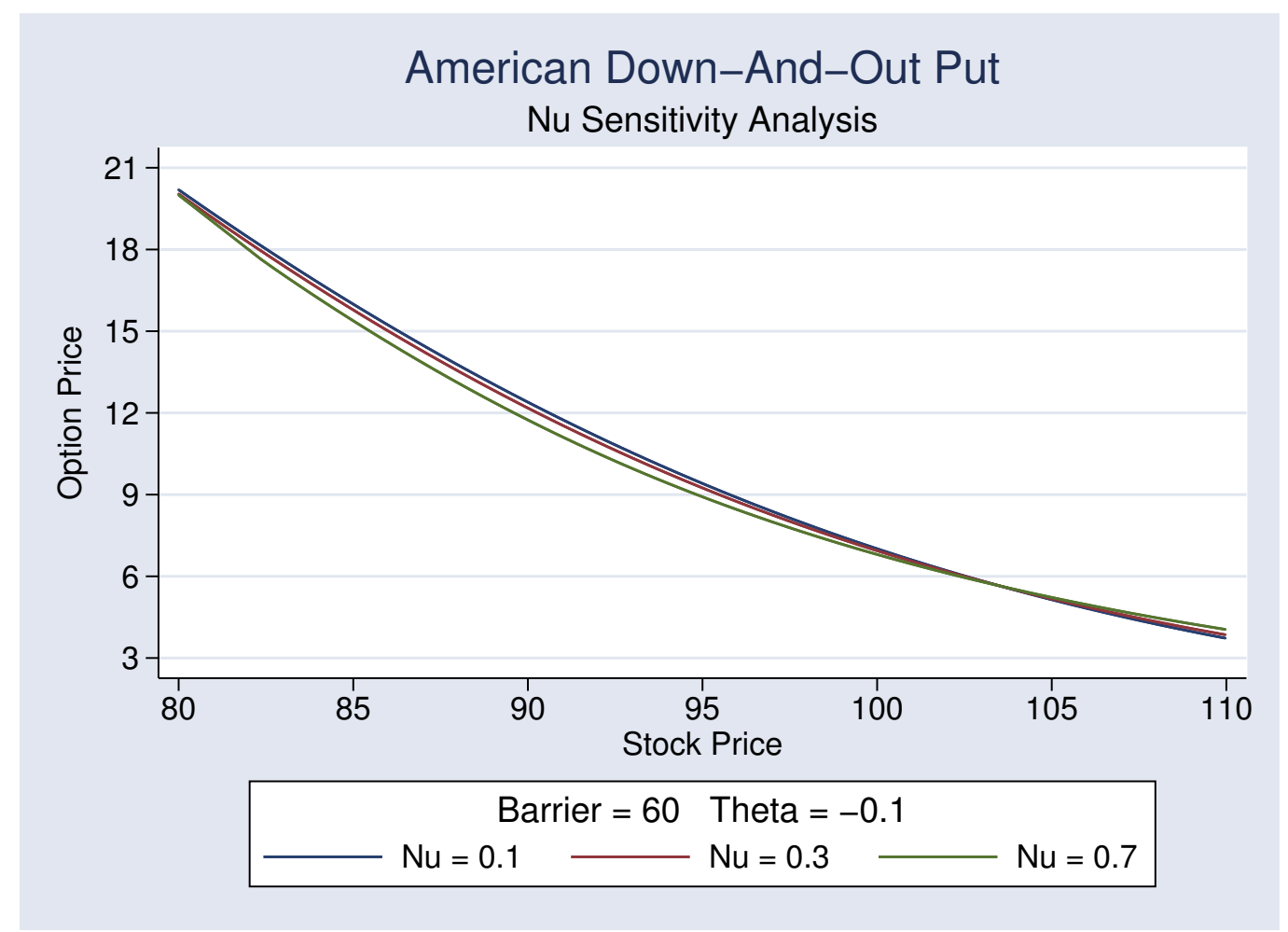

Figure 5.24: American Down-And-Out Put: $\nu$ Sensitivity Analysis, Detail 
This graph is a zoom of the out of the money area for the same options considered above. We can see that here having a larger $\nu$ and, as a consequence a larger kurtosis, volatility and skewness, increases the value of the option, because it is more likely that the stock has a large jump and moves in the money.

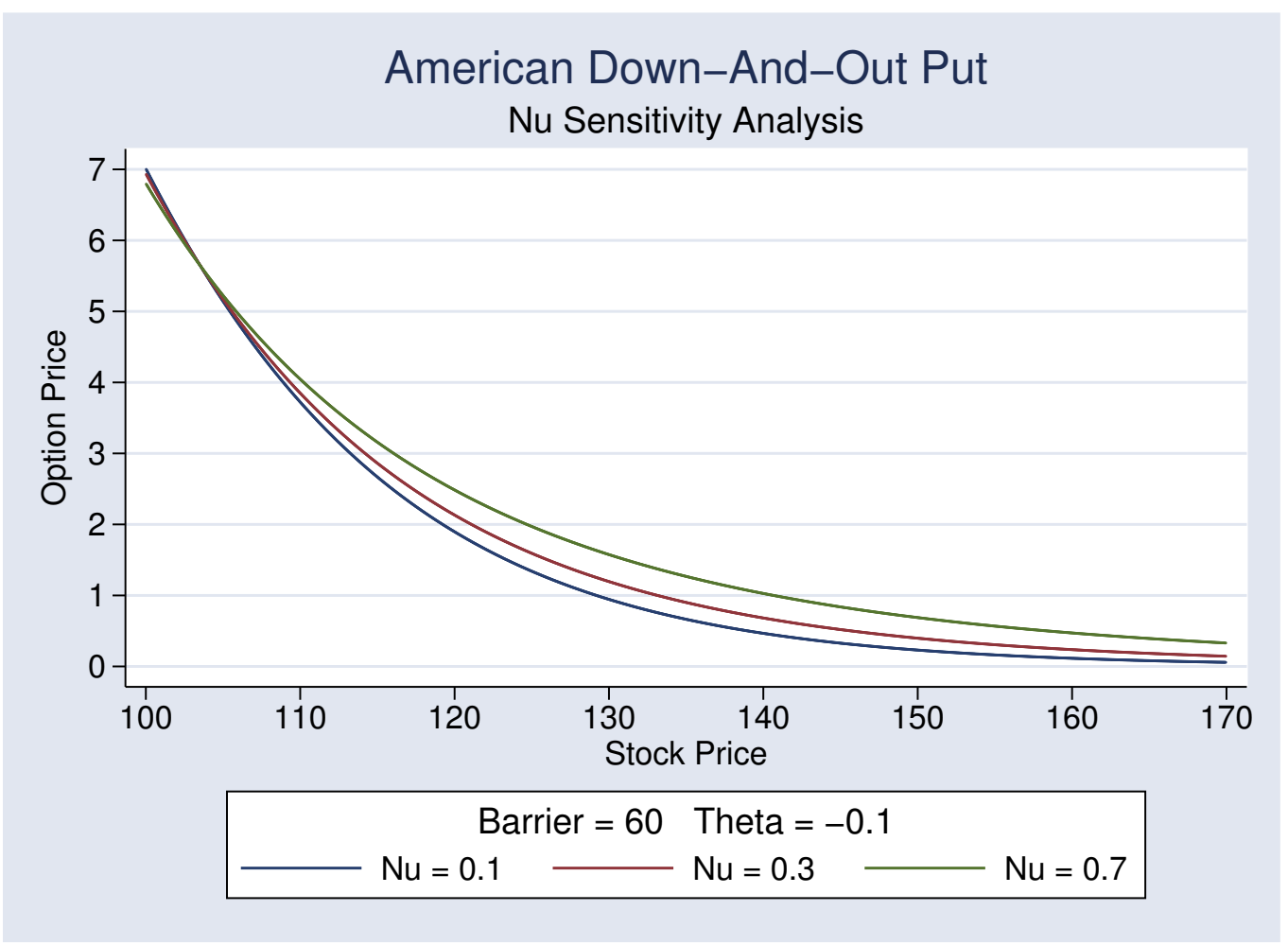

Figure 5.25: American Down-And-Out Put: $\nu$ Sensitivity Analysis, Detail 


\section{Sensitivity with Respect to $\vartheta$}

Let's study now the sensitivity of an American down-and-out put options with respect to $\vartheta$. We consider here a rebate of $\$ 40$, but, as discussed, any rebate lower than $\$ 40$ would produce the same results. The parameters used in this experiment are:

- $T=1 \quad$ Time to maturity in years;

- $K=\$ 100$ Strike price;

- barrier $=4.09434$, corresponding to $S=\$ 60$;

- rebate $=40$;

- $r=0.03$ Interest rate;

- $q=0.01 \quad$ Dividend yield;

- $\sigma=0.2$;

- $\nu=0.2$

- $N=5100$ Number of space intervals;

- $M=300$ Number of time intervals;

- $x_{\min }=4.09434$ Min $x$ value considered, corresponding to $S=\$ 60$;

- $x_{\max }=5.85793$ Max $x$ value considered, corresponding to $S=\$ 350$;

- $\Delta x=0.000346 \quad$ Size of a space interval;

- $\Delta t=0.003333$ Size of a time interval;

- $\frac{\Delta t}{\Delta x}=9.639$;

Keeping $\sigma, \nu$ and all the other parameters constant, we study the impact of moving $\vartheta$ from 0 to -0.5 . The impact of moving $\vartheta$ on volatility, skewness and kurtosis is presented in the following table: 


\begin{tabular}{|c|c|c|c|c|}
\hline$\vartheta$ & 0 & -0.1 & -0.3 & -0.5 \\
\hline$\sqrt{E\left[(X(t)-E[X(t)])^{2}\right]}$ & 0.200 & 0.205 & 0.241 & 0.300 \\
\hline$\frac{E\left[(X(t)-E[X(t)])^{3}\right]}{\sqrt{\left\{E\left[(X(t)-E[X(t)])^{2}\right]\right\}^{3}}}$ & 0.000 & -0.288 & -0.670 & -0.815 \\
\hline$\frac{E\left[(X(t)-E[X(t)])^{4}\right]}{\left\{E\left[(X(t)-E[X(t)])^{2}\right]\right\}^{2}}$ & 3.600 & 3.656 & 3.915 & 4.081 \\
\hline
\end{tabular}

We can see that as $\vartheta$ increases in absolute value, negative skewness volatility and kurtosis increase.

The graph shows the impact on the American barrier option price of moving $\vartheta$. We can see that the put value grows as $\vartheta$ becomes more negative.

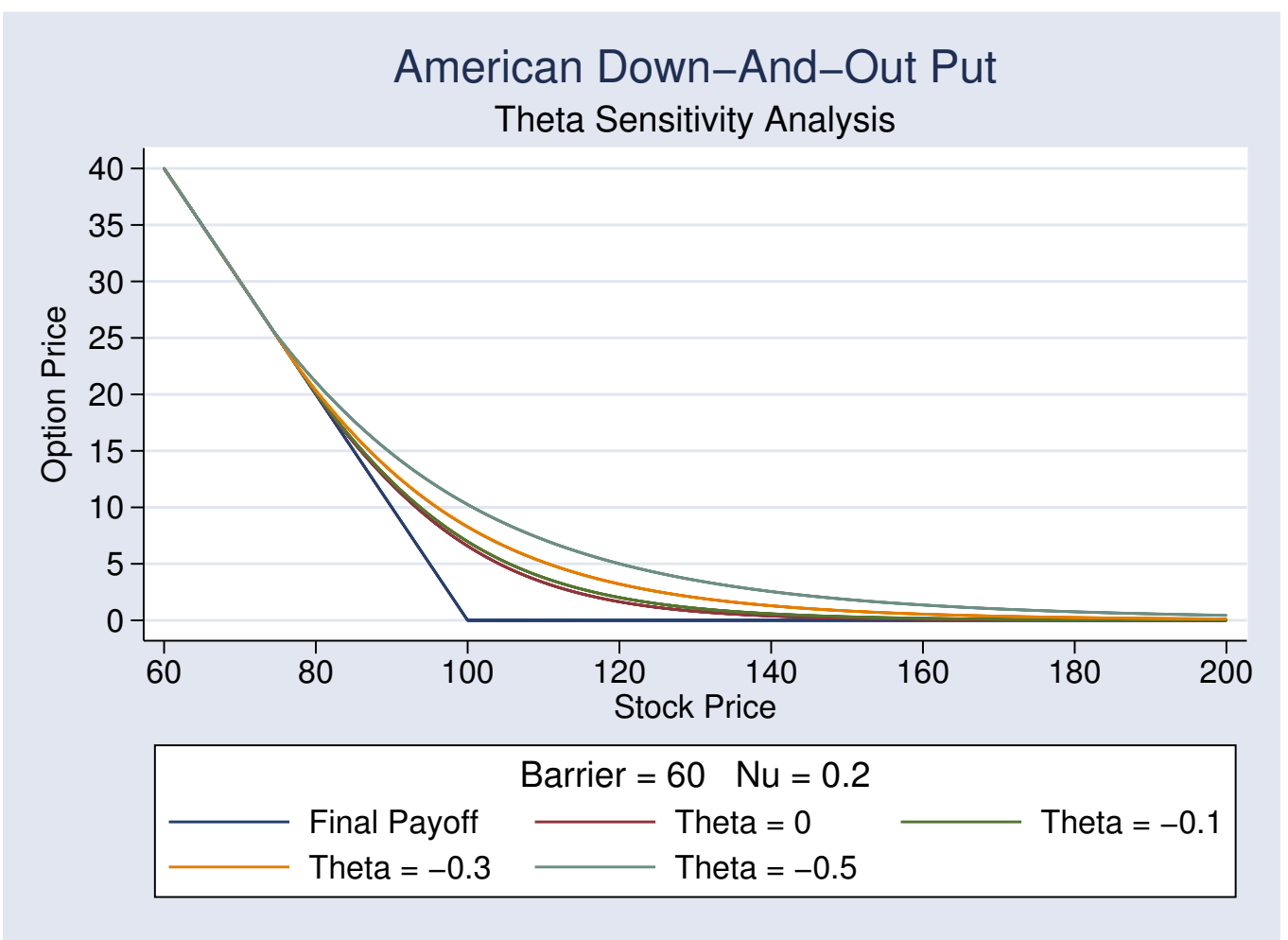

Figure 5.26: American Down-And-Out Put: $\vartheta$ Sensitivity Analysis 
This graph is a zoom of the previous one. We can see that as $\vartheta$ increases in absolute value, we have a larger volatility, kurtosis and negative skewness and hence a more expensive option.

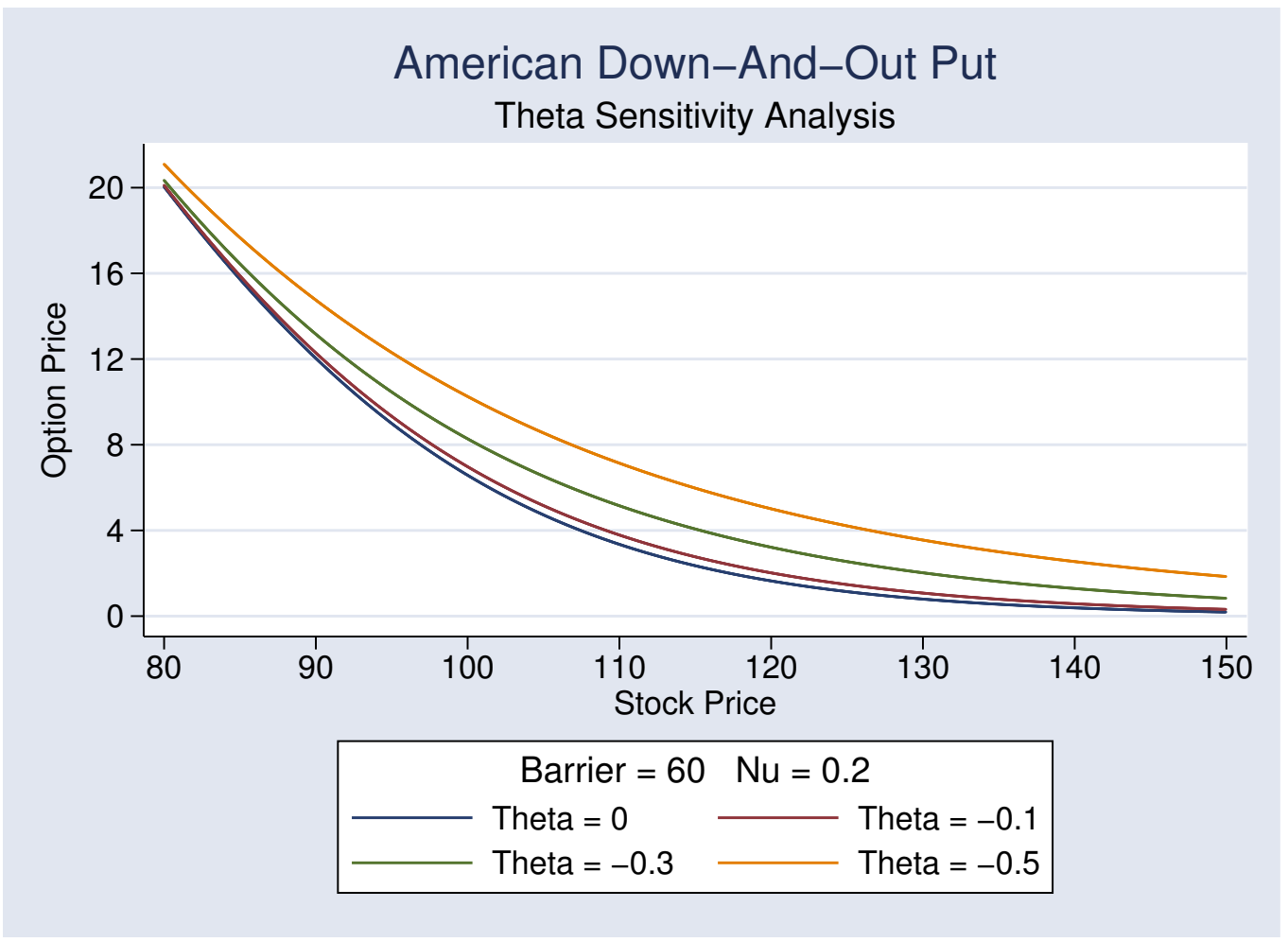

Figure 5.27: American Down-And-Out Put: $\vartheta$ Sensitivity Analysis, Detail 


\section{Chapter 6}

\section{Conclusions}

In this work we studied option pricing under the variance gamma process. The interest in this topic comes from the attempt of improving the theory of option pricing especially with respect to its benchmark represented by the Black, Scholes and Merton approach. The classic approach presents in fact several limits and a number of authors have proposed extensions and improvements to correct biases presented by the model. Our interest in particular is focused on two elements which are interconnected: the misspecification of the underlying price process with respect to the presence of skewness and higher than normal kurtosis and the inability of the Black and Scholes model to price consistently options having different moneyness. These limits come from the fact that the model assumes a geometric Brownian motion as process followed by the underlying returns. Although this process presents the advantage of being easily tractable, it is unable to capture different market premia for up moves versus down moves and for large moves versus small moves in the underlying process. This is reflected in the volatility smiles commonly exhibited by the market data.

Among the various approaches presented in literature to improve the pricing and hedging performances of the Black and Scholes model, the variance gamma belongs to the family of pure jump processes. This technique is a significant move from the classical approach because the familiar continuous process frame is replaced with a purely discontinuous approach, with the result that the dynamic hedging argument for option pricing cannot be used anymore. Complete market hypothesis has therefore to be abandoned and options become completing instruments necessary to hedge jump risks.

This new framework can however be reconnected to the traditional Brow- 
nian motion. In particular we can note that the variance gamma process is a semimartingale and we can therefore use a result by Monroe $^{1}$ who shows that every semimartingale can be written as a Brownian motion, possibly defined on some adequately extended probability space, evaluated at a random time. In the variance gamma case, the time change is realized by valuing the Brownian motion at a stochastic time defined by a gamma process. In this way the pure jump process can be considered as continuous process in the stochastic time. We note that the choice of a process of this kind is a reasonable one, because the fact that the process is a semimartingale with non zero martingale component is equivalent to require to be in a arbitrage free model in the "no free lunch with vanishing risk" meaning of Delbaen and Schachermayer ${ }^{2}$.

In a pure jump process, all the underlying price moves happen via jumps. Here high activity is accounted for by an infinite number of small jumps; while the activity of various jump size is realized by requiring that small jumps occurs at a higher rate than larger rates. This is a different situation compared with jump diffusion models where the process accounts for high activity small price moves using an infinite variation diffusion process and for low activity large moves using an orthogonal pure jump process, often in the form of a Poisson process. Being a process of finite variation, the variance gamma can be written as the difference of two increasing processes. In particular, we can define the variance gamma process, as the difference of two gamma processes. This definition is equivalent to the previous one which describes the variance gamma process as a Brownian motion valued at a random time given by a gamma process.

The variance gamma process has been known in financial literature for some time: the first complete representation of the process in its symmetric form is due to Madan and Seneta in $1990^{3}$. Madan and Milne in $1991^{4}$ studied then an equilibrium option pricing model for the variance gamma and finally Madan, Carr and Chang in $1998^{5}$ published a paper in which they price

\footnotetext{
${ }^{1}$ I. Monroe, "Processes That Can Be Embedded In A Brownian Motion", The Annals of Probability, Vol. 6, 1978, pages 42-56.

${ }^{2}$ Freddy Delbaen and Walter Schachermayer, "A General Version of the Fundamental Theorem of Asset Pricing", Mathematische Annalen, Vol. 300, 1994, pages 463-520.

${ }^{3}$ Dilib B. Madan and Eugene Seneta "The Variance Gamma (V.G.) Model for Share Market Return", The Journal of Business, vol 63. no.4, 1990, pages 511-524.

${ }^{4}$ Dibip B. Madan and Frank Milne, "Option Pricing with V.G. Martingale Components", Mathematical Finance, Vol. 1, No. 4, October 1991, pages 39-55.

${ }^{5}$ Dilip B Madan, Peter P. Carr and Eric C. Chang, "The Variance Gamma Process and
} 
European vanilla option under the non symmetric variance gamma process. In this last paper Madan, Carr and Chang show that the variance gamma provides an improvement with respect to the geometric Brownian motion. The variance gamma presents in fact two additional parameters compared with the geometric Brownian motion which allow the control of skewness and kurtosis. Using these parameters it is possible to fit the process so that it can consistently price options with different moneyness at a certain maturity. Madan, Carr and Chang show that the underlying return processes exhibit fat tails both statistically and risk neutrally and that they present often negative skew especially in the risk neutral case. Moreover they show that the option prices obtained on the basis of the variance gamma exhibit less biases than those obtained under the Black and Scholes model.

As it is the case also for the geometric Brownian motion, an analytical solution of the option pricing problem is not available for American options. Recently Hirsa and Madan ${ }^{6}$ presented a numerical solution of the problem in terms of a finite difference scheme which can be used to price options under Variance Gamma. In particular they provide an algorithm to price American vanilla options. When the underlying return follows a variance gamma process, the equation describing its dynamic can be written in the form of a partial integro differential equation having integral terms describing the impact of jumps in the underlying price. Its is important to properly discretize these integrals to solve the problem.

Our original contribution to the research consists first of all in a detailed study of the nature of the variance gamma process. We analyze the process from a theoretical point of view and we study how to implement numerical schemes to price options under variance gamma. In particular, we use the numerical scheme developed by Hirsa and Madan, for vanilla options, improving it when necessary, to study the price of European and American vanilla options. Numerous experiments are realized to show the sensitivity of both vanilla European and American option prices to several parameters including the parameters controlling the volatility, the skewness and the kurtosis as well as interest rates and dividends. Moreover the work presents results in terms of the implied volatility obtainable when option prices computed by using the variance gamma model are plugged in the traditional

Option Pricing",European Finance Review, 2, 1998, pages 79-105.

${ }^{6}$ Ali Hirsa and Dilip B. Madan, "Pricing American Options Under Variance Gamma", Journal of Computational Finance, Vol. 7, No. 2, Winter 2003/2004, pages 63-80. 
Black and Scholes model; this shows that it is possible to replicate volatility smiles exhibited by the market with a single set of parameters. Finally a comparison between option prices obtainable under geometric Brownian motion and under variance gamma is showed. In realizing this experiment we have been careful in selecting the appropriate parameters to be used. In particular the volatility of the two processes is different, as the parameters employed are the ones implied by the S\&P 500 for the corresponding model as reported by Madan, Carr and Chang.

The most significant innovative results are however in the chapter devoted to the pricing of European and American barrier options. The pricing of barrier options is obtained using a finite difference scheme. In this case the algorithm used for vanilla options has to be modified to account for the cases in which the underlying jumps across the barrier. The analysis is concentrated on pricing knock-outs since knock-ins can always be obtained as difference between vanilla and out barrier options. To solve the problem we make sure that the limit of the range of log prices considered for the numerical scheme is exactly positioned at the barrier level. This assures a smooth increase in the accuracy of the scheme as the number of space intervals increases. The solution of the scheme for the variance gamma case is different from the geometric Brownian case. In the traditional approach in fact, it is possible to ignore the behavior of the option after the stock reaches the out barrier because the underlying process is continuous and so the stock has to reach exactly the barrier before reaching values outside the barrier. In the variance gamma case, on the contrary, having a purely discontinuous process, it is necessary to consider also the option value for values of the underlying outside the barrier for cases in cases in which the underlying jumps across the barrier without having ever been exactly at the barrier level. For example in the cases of rebate payment, the option is worth the rebate not just at the barrier level, when the barrier is reached for the first time, but also for values outside the barrier if the stock was previously inside the barrier and has jumped outside it and it is a first passage of the knock-out level.

After having designed the procedure to be followed to discretize the jump integrals and price barrier options, we also implemented the finite difference scheme with $\mathrm{C}$ code. The program we realized covers all the cases of relative position of the barrier with respect to the underlying level for both European and American barrier option. We present also a large number of experiments for both European and American barrier options, showing sensitivity analysis 
for the option prices as the rebate received for first passage of the barrier and the parameters controlling volatility, skewness and kurtosis of the process are modified. All the experiments presented in this work for both vanilla and barrier options are realized with the $\mathrm{C}$ code reported in the appendix.

This work opens several perspective for future research which are of interest and which we would like to consider. First of all we would like to compare more in detail the results obtained under variance gamma with those ones obtainable under the geometric Brownian motion. In particular we would like to realize a more complete analysis of the differences for the barrier option case and see how the prices obtainable using the Rubinstein and Reiener ${ }^{7}$ closed form solutions for European barrier options are different from the ones obtained under variance gamma as the skewness and the kurtosis vary. Moreover, we would like to replicate the results obtained in this dissertation using Monte Carlo to provide more insights in terms of accuracy and convergence of the numerical procedure designed here. This would be more challenging for the American option case, since the Monte Carlo is not as efficient when early exercise is allowed. However the interest here would be in creating a benchmark to be used to compare the results obtained with the finite difference method and so we would be more focused on the convergence of the scheme rather then in the speed of the algorithm.

On a more advanced level, we would like to develop similar numerical approaches to be applied to the extensions of the variance gamma model. In particular it would be interesting to work with the CGMY and realize a finite difference scheme capable of pricing options under this model. For this process, if we try to solve the PIDE in a similar way we did for the variance gamma, one issue we will have to keep in mind is that, as we noted in the text, the Lévy measure tends to infinity as the jump size tends to zero. In the CGMY case, when the parameter $Y$ is larger than 1, the linear approximation we used for small jumps in the variance gamma case, needs to be properly modified to solve the divergence issue. Other extensions of the variance gamma we would like to work on are the stochastic volatility variance gamma model and the stochastic volatility CGMY model. We have seen in fact that, although the variance gamma provides material improvement in pricing options across moneyness, it still has problems in consistently pricing options with different maturities. Further extensions of the model considering

\footnotetext{
${ }^{7}$ Mark Rubinstein and Eric Reiner, "Breaking down the barriers", Risk, Vol. 4, No. 8, September 1991, pages. 28-35.
} 
stochastic volatility on top of the variance gamma and the CGMY model seem to be able to handle also this additional level of complexity. It would be interesting to study how to implement these schemes numerically to provide prices for American and exotic options. 


\section{Appendix A}

\section{A.1 Numerical Solution of the Jump Integral in the Variance Gamma PIDE for Vanilla European Options}

In order to define a routine to solve numerically the PIDE describing the dynamic of European option prices under variance gamma

$$
\begin{array}{r}
h W\left(x_{i-1}, t_{j}\right)+(1+r \Delta t) W\left(x_{i}, t_{j}\right)-h W\left(x_{i+1}, t_{j}\right)= \\
=W\left(x_{i}, t_{j+1}\right)+\Delta t \int_{-\infty}^{+\infty}\left[W\left(x_{i}+y, t_{j+1}\right)-W\left(x_{i}, t_{j+1}\right)\right] k(y) d y
\end{array}
$$

we need to evaluate numerically the integral

$$
\int_{-\infty}^{+\infty}\left[W\left(x_{i}+y, t_{j+1}\right)-W\left(x_{i}, t_{j+1}\right)\right] k(y) d y
$$

The best way to solve it is to split it in 6 parts and evaluate each part separately as suggested by Hirsa and Madan ${ }^{1}$. Therefore we can write

$$
\begin{aligned}
& \int_{-\infty}^{+\infty}\left[W\left(x_{i}+y, t_{j+1}\right)-W\left(x_{i}, t_{j+1}\right)\right] k(y) d y= \\
& =\int_{-\infty}^{x_{0}-x_{i}}\left[W\left(x_{i}+y, t_{j+1}\right)-W\left(x_{i}, t_{j+1}\right)\right] k(y) d y+
\end{aligned}
$$

\footnotetext{
${ }^{1}$ Ali Hirsa and Dilip B. Madan, "Pricing American Options Under Variance Gamma", Journal of Computational Finance, Vol. 7, No. 2, Winter 2003/2004, pages 63-80.
} 


$$
\begin{aligned}
& +\int_{x_{0}-x_{i}}^{-\Delta x}\left[W\left(x_{i}+y, t_{j+1}\right)-W\left(x_{i}, t_{j+1}\right)\right] k(y) d y+ \\
& +\int_{-\Delta x}^{0}\left[W\left(x_{i}+y, t_{j+1}\right)-W\left(x_{i}, t_{j+1}\right)\right] k(y) d y+ \\
& +\int_{0}^{\Delta x}\left[W\left(x_{i}+y, t_{j+1}\right)-W\left(x_{i}, t_{j+1}\right)\right] k(y) d y+ \\
& +\int_{\Delta x}^{(N-i) \Delta x}\left[W\left(x_{i}+y, t_{j+1}\right)-W\left(x_{i}, t_{j+1}\right)\right] k(y) d y+ \\
& +\int_{(N-i) \Delta x}^{+\infty}\left[W\left(x_{i}+y, t_{j+1}\right)-W\left(x_{i}, t_{j+1}\right)\right] k(y) d y
\end{aligned}
$$

The solution of the integrals A.3 and A.8 is different in the case of a put or a call option, let's start to consider the case of a put option.

\section{A.1.1 Solution of Integrals (A.6 and A.5)}

Let's start solving integral (A.6). Remember the value of $k(y)$ for $y>0$ from equation (2.23) that is

$$
\frac{\mu_{p}^{2} \exp \left(-\frac{\mu_{p}}{\nu_{p}} x\right)}{\nu_{p} x} d x
$$

By using use equation (2.17)

$$
\frac{\mu_{p}^{2}}{\nu_{p}}=\frac{\mu_{n}^{2}}{\nu_{n}}=\frac{1}{\nu}
$$

we can write integral (A.6) as

$$
\int_{0}^{\Delta x}\left[W\left(x_{i}+y, t_{j+1}\right)-W\left(x_{i}, t_{j+1}\right)\right] \frac{e^{-\frac{\mu_{p}}{\nu_{p}} y}}{\nu y} d y
$$

but for $\Delta x$ small enough.

$$
W\left(x_{i}+y, t_{j+1}\right)-W\left(x_{i}, t_{j+1}\right) \simeq \frac{W\left(x_{i+1}, t_{j+1}\right)-W\left(x_{i}, t_{j+1}\right)}{\Delta x} y
$$


So the integral becomes

$$
\begin{array}{r}
\int_{0}^{\Delta x} \frac{W\left(x_{i+1}, t_{j+1}\right)-W\left(x_{i}, t_{j+1}\right)}{\Delta x} \cdot y \cdot \frac{e^{-\frac{\mu_{p}}{\nu_{p}} y}}{\nu y} d y= \\
=\frac{\left[W\left(x_{i+1}, t_{j+1}\right)-W\left(x_{i}, t_{j+1}\right)\right]\left(1-e^{-\frac{\mu_{p}}{\nu_{p}} \Delta x}\right)}{\nu \Delta x \frac{\mu_{p}}{\nu_{p}}}
\end{array}
$$

In the implementation of the scheme, however, this jump will be treated implicitly, that is we are going to have

$$
\begin{array}{r}
\int_{0}^{\Delta x} \frac{W\left(x_{i+1}, t_{j}\right)-W\left(x_{i}, t_{j}\right)}{\Delta x} \cdot y \cdot \frac{e^{-\frac{\mu_{p}}{\nu_{p}} y}}{\nu y} d y= \\
=\frac{\left[W\left(x_{i+1}, t_{j}\right)-W\left(x_{i}, t_{j}\right)\right]\left(1-e^{-\frac{\mu_{p}}{\nu_{p}} \Delta x}\right)}{\nu \Delta x \frac{\mu_{p}}{\nu_{p}}}
\end{array}
$$

rather then the previous expression. This approach is used to improve the stability of the system.

We can solve in the same way the integral (A.5). The only difference is that we have to use the part of equation (2.23) corresponding to negative $y$ that is

$$
\frac{\mu_{n}^{2} \exp \left(-\frac{\mu_{n}}{\nu_{n}}|x|\right)}{\nu_{n}|x|} d x
$$

Again, for $\Delta x$ small enough we can write

$$
W\left(x_{i}+y, t_{j+1}\right)-W\left(x_{i}, t_{j+1}\right) \simeq \frac{W\left(x_{i-1}, t_{j+1}\right)-W\left(x_{i}, t_{j+1}\right)}{\Delta x}|y|
$$

and using the same change of variable as before,

$$
\frac{\mu_{p}^{2}}{\nu_{p}}=\frac{\mu_{n}^{2}}{\nu_{n}}=\frac{1}{\nu}
$$

we can write the integral as

$$
\int_{-\Delta x}^{0}\left[W\left(x_{i}+y, t_{j+1}\right)-W\left(x_{i}, t_{j+1}\right)\right] k(y) d y=
$$




$$
=\frac{\left[W\left(x_{i-1}, t_{j+1}\right)-W\left(x_{i}, t_{j+1}\right)\right]\left(1-e^{-\frac{\mu_{n}}{\nu_{n}} \Delta x}\right)}{\nu \Delta x \frac{\mu_{n}}{\nu_{n}}}
$$

Also this jump is going to be treated implicitly as the previous one, so in reality we are going to have

$$
\begin{gathered}
\int_{-\Delta x}^{0}\left[W\left(x_{i}+y, t_{j}\right)-W\left(x_{i}, t_{j}\right)\right] k(y) d y= \\
=\frac{\left[W\left(x_{i-1}, t_{j}\right)-W\left(x_{i}, t_{j}\right)\right]\left(1-e^{-\frac{\mu_{n}}{\nu_{n}} \Delta x}\right)}{\nu \Delta x \frac{\mu_{n}}{\nu_{n}}}
\end{gathered}
$$

rather than the previous one.

\section{A.1.2 Solution of Integrals (A.7 and A.4)}

Let's consider the integral (A.7). By substituting the value of $k(y)$, from equation (2.23), and using equation (2.17), we can write our integral as

$$
\begin{gathered}
\int_{\Delta x}^{(N-i) \Delta x}\left[W\left(x_{i}+y, t_{j+1}\right)-W\left(x_{i}, t_{j+1}\right)\right] k(y) d y= \\
\int_{\Delta x}^{(N-i) \Delta x}\left[W\left(x_{i}+y, t_{j+1}\right)-W\left(x_{i}, t_{j+1}\right)\right] \frac{e^{-\frac{\mu_{p}}{\nu_{p}} y}}{\nu y} d y
\end{gathered}
$$

and we can rewrite the integral as a summation of integrals in the following way

$$
=\sum_{k=1}^{N-i-1} \int_{k \Delta x}^{(k+1) \Delta x}\left[W\left(x_{i}+y, t_{j+1}\right)-W\left(x_{i}, t_{j+1}\right)\right] \frac{e^{-\frac{\mu_{p}}{\nu_{p}} y}}{\nu y} d y
$$

Now we can note that for $\Delta x$ enough small we can write the following approximation:

$$
\begin{array}{r}
W\left(x_{i}+y, t_{j+1}\right)-W\left(x_{i+k}, t_{j+1}\right) \simeq \\
\simeq \frac{W\left(x_{i+k+1}, t_{j+1}\right)-W\left(x_{i+k}, t_{j+1}\right)}{\Delta x}(y-k \Delta x)
\end{array}
$$


By using this we can rewrite the expression (A.11) in the following way:

$$
\begin{gathered}
\sum_{k=1}^{N-i-1} \int_{k \Delta x}^{(k+1) \Delta x}\left[W\left(x_{i}+y, t_{j+1}\right)-W\left(x_{i+k}, t_{j+1}\right)+\right. \\
\left.+W\left(x_{i+k}, t_{j+1}\right)-W\left(x_{i}, t_{j+1}\right)\right] \frac{e^{-\frac{\mu_{p}}{\nu_{p}} y}}{\nu y} d y= \\
\sum_{k=1}^{N-i-1} \int_{k \Delta x}^{(k+1) \Delta x}\left[\frac{W\left(x_{i+k+1}, t_{j+1}\right)-W\left(x_{i+k}, t_{j+1}\right)}{\Delta x} \cdot(y-k \Delta x)+\right. \\
\left.+W\left(x_{i+k}, t_{j+1}\right)-W\left(x_{i}, t_{j+1}\right)\right] \frac{e^{-\frac{\mu_{p}}{\nu_{p}} y}}{\nu y} d y
\end{gathered}
$$

And finally we get ${ }^{2}$

$$
\begin{aligned}
& \sum_{k=1}^{N-i-1} \frac{1}{\nu \Delta x}\left[W\left(x_{i+k+1}, t_{j+1}\right)-W\left(x_{i+k}, t_{j+1}\right)\right] \frac{\nu_{p}}{\mu_{p}}\left(e^{-\frac{\mu_{p}}{\nu_{p}} k \Delta x}-e^{-\frac{\mu_{p}}{\nu_{p}}(k+1) \Delta x}\right)+ \\
& +\sum_{k=1}^{N-i-1} \frac{1}{\nu}\left[W\left(x_{i+k}, t_{j+1}\right)-W\left(x_{i}, t_{j+1}\right)-k\left[W\left(x_{i+k+1}, t_{j+1}\right)-W\left(x_{i+k}, t_{j+1}\right)\right] .\right.
\end{aligned}
$$

${ }^{2}$ The exponential integral is defined as

$$
\operatorname{expint}_{n}(x)=\int_{1}^{\infty} \frac{e^{-x t}}{t^{n}} d t
$$

for $x>0$ and $n=0,1, \ldots$ In our case $\mathrm{n}=1$, so we omitted the indication in the name of the function. To obtain the two exponential integrals you can write

$$
\int_{k \Delta x}^{(k+1) \Delta x} \frac{e^{-\frac{\mu_{p}}{\nu_{p}} y}}{y} d y=\int_{k \Delta x}^{+\infty} \frac{e^{-\frac{\mu_{p}}{\nu_{p}} y}}{y} d y-\int_{(k+1) \Delta x}^{+\infty} \frac{e^{-\frac{\mu_{p}}{\nu_{p}} y}}{y} d y
$$

and do the change of variable $y=z k \Delta x$ in the first integral and $y=z(k+1) \Delta x$ in the second integral, to get

$$
\int_{1}^{+\infty} \frac{e^{-\frac{\mu_{p}}{\nu_{p}} z k \Delta x}}{z} d z-\int_{1}^{+\infty} \frac{e^{-\frac{\mu_{p}}{\nu_{p}} z(k+1) \Delta x}}{z} d z
$$




$$
\cdot\left[\operatorname{expint}\left(\frac{\mu_{p}}{\nu_{p}} k \Delta x\right)-\operatorname{expint}\left(\frac{\mu_{p}}{\nu_{p}}(k+1) \Delta x\right)\right]
$$

In a similar way we can solve the integral (A.4)

$$
\int_{x_{0}-x_{i}}^{-\Delta x}\left[W\left(x_{i}+y, t_{j+1}\right)-W\left(x_{i}, t_{j+1}\right)\right] \frac{e^{-\frac{\mu_{n}}{\nu_{n}}|y|}}{\nu|y|} d y
$$

by redefining $y \triangleq-y$ you have

$$
\int_{\Delta x}^{x_{i}-x_{0}}\left[W\left(x_{i}-y, t_{j+1}\right)-W\left(x_{i}, t_{j+1}\right)\right] \frac{e^{-\frac{\mu_{n}}{\nu_{n}} y}}{\nu y} d y
$$

Considering the approximation, for small $\Delta x$,

$$
\begin{array}{r}
W\left(x_{i}-y, t_{j+1}\right)-W\left(x_{i-k}, t_{j+1}\right) \simeq \\
\simeq \frac{W\left(x_{i-k-1}, t_{j+1}\right)-W\left(x_{i-k}, t_{j+1}\right)}{\Delta x}(y-k \Delta x)
\end{array}
$$

and working as we did to obtain the equation (A.12) we get

$$
\begin{gathered}
\sum_{k=1}^{i-1} \frac{1}{\nu \Delta x}\left[W\left(x_{i-k-1}, t_{j+1}\right)-W\left(x_{i-k}, t_{j+1}\right)\right] \frac{\nu_{n}}{\mu_{n}}\left(e^{-\frac{\mu_{n}}{\nu_{n}} k \Delta x}-e^{-\frac{\mu_{n}}{\nu_{n}}(k+1) \Delta x}\right)+ \\
+\sum_{k=1}^{i-1} \frac{1}{\nu}\left[W\left(x_{i-k}, t_{j+1}\right)-W\left(x_{i}, t_{j+1}\right)\right)-k\left[W\left(x_{i-k-1}, t_{j+1}\right)-W\left(x_{i-k}, t_{j+1}\right)\right] \cdot \\
\cdot\left[\operatorname{expint}\left(\frac{\mu_{n}}{\nu_{n}} k \Delta x\right)-\operatorname{expint}\left(\frac{\mu_{n}}{\nu_{n}}(k+1) \Delta x\right)\right]
\end{gathered}
$$

\section{A.1.3 Solution of Integral (A.8) in the Case of Vanilla Put Options}

Let's consider the integral (A.8)

$$
\int_{(N-i) \Delta x}^{+\infty}\left[W\left(x_{i}+y, t_{j+1}\right)-W\left(x_{i}, t_{j+1}\right)\right] \frac{e^{-\frac{\mu_{p}}{\nu_{p}} y}}{\nu y} d y
$$


We can note that here the jump expressed in the terms $W\left(x_{i}+y, t_{j+1}\right)$ is positive and huge, so that we reach points which are bigger than the biggest point we consider in our grid: $S_{\max }$, and we can approximate $V\left(S_{\max }, t\right) \simeq 0$, being the option a put. Because we defined $x \triangleq \ln (S)$, we have

$$
W\left(x_{i}+y, t_{j+1}\right) \simeq 0
$$

for $y \geq(N-i) \Delta x$ because $(N-i) \Delta x=x_{N}-x_{i}$ and $e^{x_{N}}=S_{\max }$. Therefore the first term in the integral cancel out and we can rewrite our integral as

$$
\int_{(N-i) \Delta x}^{+\infty}-W\left(x_{i}, t_{j+1}\right) \frac{e^{-\frac{\mu_{p}}{\nu_{p}} y}}{\nu y} d y
$$

and defining

$$
y \triangleq(N-i) \Delta x z
$$

and utilizing the exponential integral function we have

$$
-\frac{1}{\nu} W\left(x_{i}, t_{j+1}\right) \operatorname{expint}\left[(N-i) \frac{\mu_{p}}{\nu_{p}} \Delta x\right]
$$

\section{A.1.4 Solution of Integral (A.3) in the Case of Vanilla Put Options}

Finally we have to evaluate the integral

$$
\int_{-\infty}^{x_{0}-x_{i}}\left[W\left(x_{i}+y, t_{j+1}\right)-W\left(x_{i}, t_{j+1}\right)\right] \frac{e^{-\frac{\mu_{n}}{\nu_{n}}|y|}}{\nu|y|} d y
$$

Redefine $y \triangleq-y$, so you have

$$
\int_{x_{i}-x_{0}}^{+\infty}\left[W\left(x_{i}-y, t_{j+1}\right)-W\left(x_{i}, t_{j+1}\right)\right] \frac{e^{-\frac{\mu_{n}}{\nu_{n}} y}}{\nu y} d y
$$

In this case the jump expressed by the term $W\left(x_{i}-y, t_{j+1}\right)$ is huge but negative, so we end up in points which are smaller than the our smallest 
point in the grid: $S_{\min }$. Therefore, because we are considering a put option we can write

$$
W\left(x_{i}-y, t_{j+1}\right) \simeq K e^{-r\left(T-t_{j+1}\right)}-e^{x_{i}-y} e^{-q\left(T-t_{j+1}\right)}
$$

for $y>x_{i}-x_{0}$, where $T=M \Delta t$ is the expiration of the contingent claim. Hence the integral becomes

$$
\int_{x_{i}-x_{0}}^{+\infty}\left[K e^{-r\left(T-t_{j+1}\right)}-e^{x_{i}-y} e^{-q\left(T-t_{j+1}\right)}-W\left(x_{i}, t_{j+1}\right)\right] \frac{e^{-\frac{\mu_{n}}{\nu_{n}} y}}{\nu y} d y
$$

And again, by using the exponential integral notation, we get

$$
\begin{gathered}
\frac{1}{\nu}\left\{\left[K e^{-r\left(T-t_{j+1}\right)}-W\left(x_{i}, t_{j+1}\right)\right] \text { expint }\left[\left(x_{i}-x_{0}\right) \frac{\mu_{n}}{\nu_{n}}\right]+\right. \\
\left.-e^{-q\left(T-t_{j+1}\right)} e^{x_{i}} \operatorname{expint}\left[\left(x_{i}-x_{0}\right)\left(\frac{\mu_{n}}{\nu_{n}}+1\right)\right]\right\}
\end{gathered}
$$

\section{A.1.5 Numerical Solution of the Jump Integral for Eu- ropean Vanilla Put Options}

We can now put together the integrals (A.9), (A.10), (A.12), (A.13), (A.14) and (A.15) to get the numerical solution of the integral in the case of a put option

$$
\begin{gathered}
\int_{-\infty}^{+\infty}\left[W\left(x_{i}+y, t_{j+1}\right)-W\left(x_{i}, t_{j+1}\right)\right] k(y) d y= \\
=\frac{\left[W\left(x_{i+1}, t_{j+1}\right)-W\left(x_{i}, t_{j+1}\right)\right]\left(1-e^{-\frac{\mu_{p}}{\nu_{p}} \Delta x}\right)}{\nu \Delta x \frac{\mu_{p}}{\nu_{p}}}+ \\
+\frac{\left[W\left(x_{i-1}, t_{j+1}\right)-W\left(x_{i}, t_{j+1}\right)\right]\left(1-e^{-\frac{\mu_{n}}{\nu_{n}} \Delta x}\right)}{\nu \Delta x \frac{\mu_{n}}{\nu_{n}}}+ \\
+\sum_{k=1}^{N-i-1} \frac{1}{\nu \Delta x}\left[W\left(x_{i+k+1}, t_{j+1}\right)-W\left(x_{i+k}, t_{j+1}\right)\right] \cdot \frac{\nu_{p}}{\mu_{p}} \cdot\left[e^{-\frac{\mu_{p}}{\nu_{p}} k \Delta x}-e^{-\frac{\mu_{p}}{\nu_{p}}(k+1) \Delta x}\right]+
\end{gathered}
$$




$$
\begin{aligned}
& +\sum_{k=1}^{N-i-1} \frac{1}{\nu}\left\{W\left(x_{i+k}, t_{j+1}\right)-W\left(x_{i}, t_{j+1}\right)-k\left[W\left(x_{i+k+1}, t_{j+1}\right)-W\left(x_{i+k}, t_{j+1}\right)\right]\right\} . \\
& \cdot\left\{\operatorname{expint}\left(\frac{\mu_{p}}{\nu_{p}} k \Delta x\right)-\operatorname{expint}\left[\frac{\mu_{p}}{\nu_{p}}(k+1) \Delta x\right]\right\}+ \\
& +\sum_{k=1}^{i-1} \frac{1}{\nu \Delta x}\left[W\left(x_{i-k-1}, t_{j+1}\right)-W\left(x_{i-k}, t_{j+1}\right)\right] \frac{\nu_{n}}{\mu_{n}}\left[e^{-\frac{\mu_{n}}{\nu_{n}} k \Delta x}-e^{-\frac{\mu_{n}}{\nu_{n}}(k+1) \Delta x}\right]+ \\
& +\sum_{k=1}^{i-1} \frac{1}{\nu}\left\{W\left(x_{i-k}, t_{j+1}\right)-W\left(x_{i}, t_{j+1}\right)-k\left[W\left(x_{i-k-1}, t_{j+1}\right)-W\left(x_{i-k}, t_{j+1}\right)\right]\right\} \cdot \\
& \cdot\left\{\operatorname{expint}\left(\frac{\mu_{n}}{\nu_{n}} k \Delta x\right)-\operatorname{expint}\left[\frac{\mu_{n}}{\nu_{n}}(k+1) \Delta x\right]\right\}+ \\
& -\frac{1}{\nu} W\left(x_{i}, t_{j+1}\right) \text { expint }\left[(N-i) \frac{\mu_{p}}{\nu_{p}} \Delta x\right]+ \\
& +\frac{1}{\nu}\left\{\left[K e^{-r\left(T-t_{j+1}\right)}-W\left(x_{i}, t_{j+1}\right)\right] \operatorname{expint}\left[\left(x_{i}-x_{0}\right) \frac{\mu_{n}}{\nu_{n}}\right]+\right. \\
& \left.-e^{-q\left(T-t_{j+1}\right)} e^{x_{i}} \operatorname{expint}\left[\left(x_{i}-x_{0}\right)\left(\frac{\mu_{n}}{\nu_{n}}+1\right)\right]\right\}
\end{aligned}
$$

However as noted above, the small jumps will are implemented implicitly, so we are going to use expression

$$
\begin{aligned}
& \frac{\left[W\left(x_{i+1}, t_{j}\right)-W\left(x_{i}, t_{j}\right)\right]\left(1-e^{-\frac{\mu_{p}}{\nu_{p}} \Delta x}\right)}{\nu \Delta x \frac{\mu_{p}}{\nu_{p}}}+ \\
& +\frac{\left[W\left(x_{i-1}, t_{j}\right)-W\left(x_{i}, t_{j}\right)\right]\left(1-e^{-\frac{\mu_{n}}{\nu_{n}} \Delta x}\right)}{\nu \Delta x \frac{\mu_{n}}{\nu_{n}}}
\end{aligned}
$$

in substitution to the first two lines of the solutions (A.16) and we are going to move these terms to the left hand side of the PIDE (A.1). We note that this approach is slightly different from the one suggested by Hirsa and Madan ${ }^{3}$,

\footnotetext{
${ }^{3}$ Ali Hirsa and Dilip B. Madan, "Pricing American Options Under Variance Gamma", Journal of Computational Finance, Vol. 7, No. 2, Winter 2003/2004, pages 63-80.
} 
in fact they treat implicitly also the terms containing $W\left(x_{i}, t_{j+1}\right)$ for large jumps. This means that they use the following expression

$$
-\frac{1}{\nu} W\left(x_{i}, t_{j}\right) \text { expint }\left[(N-i) \frac{\mu_{p}}{\nu_{p}} \Delta x\right]-\frac{1}{\nu} W\left(x_{i}, t_{j}\right) \operatorname{expint}\left[\left(x_{i}-x_{0}\right) \frac{\mu_{n}}{\nu_{n}}\right]
$$

rather than the corresponding one with $W\left(x_{i}, t_{j+1}\right)$ in equation (A.16). This implicit term is then taken to the left hand side of the equation (A.1) to realize the implementation of the system. We decided not to treat implicitly this part because, based on our numerical experiments, it did not provide an improvement in terms of stability.

\section{A.1.6 Solution of Integral (A.8) in the Case of Vanilla Call Options}

Let's consider the integral A.8

$$
\int_{(N-i) \Delta x}^{+\infty}\left[W\left(x_{i}+y, t_{j+1}\right)-W\left(x_{i}, t_{j+1}\right)\right] \frac{e^{-\frac{\mu_{p}}{\nu_{p}} y}}{\nu y} d y
$$

As in the case of a put option we have a positive and huge jump expressed in the terms $W\left(x_{i}+y, t_{j+1}\right)$ is positive and huge, so that we reach points which are bigger than the biggest point we consider in our grid: $S_{\max }$. This time we are considering a call option, so we can approximates the value of $W$ as

$$
W\left(x_{i}+y, t_{j+1}\right) \simeq e^{x_{i}+y} e^{-q\left(T-t_{j+1}\right)}-K e^{-r\left(T-t_{j+1}\right)}
$$

for $y \geq(N-i) \Delta x$. Therefore we can rewrite our integral as

$$
\int_{(N-i) \Delta x}^{+\infty}\left[e^{x_{i}+y} e^{-q\left(T-t_{j+1}\right)}-K e^{-r\left(T-t_{j+1}\right)}-W\left(x_{i}, t_{j+1}\right)\right] \frac{e^{-\frac{\mu_{p}}{\nu_{p}} y}}{\nu y} d y
$$

and finally we get

$$
\begin{array}{r}
\frac{1}{\nu}\left\{e^{x_{i}} e^{-q\left(T-t_{j+1}\right)} \text { expint }\left[\left(\frac{\mu_{p}}{\nu_{p}}-1\right)(N-i) \Delta x\right]+\right. \\
\left.-\left[K e^{-r\left(T-t_{j+1}\right)}+W\left(x_{i}, t_{j+1}\right)\right] \operatorname{expint}\left[\frac{\mu_{p}}{\nu_{p}}(N-i) \Delta x\right]\right\}
\end{array}
$$




\section{A.1.7 Solution of Integral (A.3) in the Case of Vanilla Call Options}

Consider the integral

$$
\int_{-\infty}^{x_{0}-x_{i}}\left[W\left(x_{i}+y, t_{j+1}\right)-W\left(x_{i}, t_{j+1}\right)\right] \frac{e^{-\frac{\mu_{n}}{\nu_{n}}|y|}}{\nu|y|} d y
$$

As we did for the put option we can redefine $y \triangleq-y$, to have

$$
\int_{x_{i}-x_{0}}^{+\infty}\left[W\left(x_{i}-y, t_{j+1}\right)-W\left(x_{i}, t_{j+1}\right)\right] \frac{e^{-\frac{\mu_{n}}{\nu_{n}} y}}{\nu y} d y
$$

Here we have a huge negative jump, so we end up in points which are smaller than the our smallest point in the grid: $S_{m i n}$. Hence, because we are considering a call option, we can write

$$
W\left(x_{i}-y, t_{j+1}\right) \simeq 0
$$

for $y>x_{i}-x_{0}$, Therefore the integral becomes

$$
\int_{x_{i}-x_{0}}^{+\infty}\left[-W\left(x_{i}, t_{j+1}\right)\right] \frac{e^{-\frac{\mu_{n}}{\nu_{n}} y}}{\nu y} d y
$$

And again, by using the exponential integral notation, we get

$$
-\frac{1}{\nu} W\left(x_{i}, t_{j+1}\right) \operatorname{expint}\left[\left(x_{i}-x_{0}\right) \frac{\mu_{n}}{\nu_{n}}\right]
$$




\section{A.1.8 Numerical Solution of the Jump Integral for Eu- ropean Vanilla Call Options}

We can now put together the integrals (A.9), (A.10), (A.12), (A.13), (A.18) and (A.19) to get the following numerical solution of the integral in the case of a call option

$$
\begin{aligned}
& \int_{-\infty}^{+\infty}\left[W\left(x_{i}+y, t_{j+1}\right)-W\left(x_{i}, t_{j+1}\right)\right] k(y) d y= \\
& =\frac{\left[W\left(x_{i+1}, t_{j+1}\right)-W\left(x_{i}, t_{j+1}\right)\right]\left(1-e^{-\frac{\mu_{p}}{\nu_{p}} \Delta x}\right)}{\nu \Delta x \frac{\mu_{p}}{\nu_{p}}}+ \\
& +\frac{\left[W\left(x_{i-1}, t_{j+1}\right)-W\left(x_{i}, t_{j+1}\right)\right]\left(1-e^{-\frac{\mu_{n}}{\nu_{n}} \Delta x}\right)}{\nu \Delta x \frac{\mu_{n}}{\nu_{n}}}+ \\
& +\sum_{k=1}^{N-i-1} \frac{1}{\nu \Delta x}\left[W\left(x_{i+k+1}, t_{j+1}\right)-W\left(x_{i+k}, t_{j+1}\right)\right] \frac{\nu_{p}}{\mu_{p}}\left[e^{-\frac{\mu_{p}}{\nu_{p}} k \Delta x}-e^{-\frac{\mu_{p}}{\nu_{p}}(k+1) \Delta x}\right]+ \\
& +\sum_{k=1}^{N-i-1} \frac{1}{\nu}\left\{W\left(x_{i+k}, t_{j+1}\right)-W\left(x_{i}, t_{j+1}\right)-k\left[W\left(x_{i+k+1}, t_{j+1}\right)-W\left(x_{i+k}, t_{j+1}\right)\right]\right\} . \\
& \cdot\left\{\operatorname{expint}\left(\frac{\mu_{p}}{\nu_{p}} k \Delta x\right)-\operatorname{expint}\left[\frac{\mu_{p}}{\nu_{p}}(k+1) \Delta x\right]\right\}+ \\
& +\sum_{k=1}^{i-1} \frac{1}{\nu \Delta x}\left[W\left(x_{i-k-1}, t_{j+1}\right)-W\left(x_{i-k}, t_{j+1}\right)\right] \frac{\nu_{n}}{\mu_{n}}\left[e^{-\frac{\mu_{n}}{\nu_{n}} k \Delta x}-e^{-\frac{\mu_{n}}{\nu_{n}}(k+1) \Delta x}\right]+ \\
& +\sum_{k=1}^{i-1} \frac{1}{\nu}\left\{W\left(x_{i-k}, t_{j+1}\right)-W\left(x_{i}, t_{j+1}\right)-k\left[W\left(x_{i-k-1}, t_{j+1}\right)-W\left(x_{i-k}, t_{j+1}\right)\right]\right\} \cdot \\
& \cdot\left\{\operatorname{expint}\left(\frac{\mu_{n}}{\nu_{n}} k \Delta x\right)-\operatorname{expint}\left[\frac{\mu_{n}}{\nu_{n}}(k+1) \Delta x\right]\right\}+ \\
& +\frac{1}{\nu}\left\{e^{x_{i}} e^{-q\left(T-t_{j+1}\right)} e x p i n t\left[\left(\frac{\mu_{p}}{\nu_{p}}-1\right)(N-i) \Delta x\right]+\right.
\end{aligned}
$$




$$
\begin{gathered}
\left.-\left[K e^{-r\left(T-t_{j+1}\right)}+W\left(x_{i}, t_{j+1}\right)\right] \text { expint }\left[\frac{\mu_{p}}{\nu_{p}}(N-i) \Delta x\right]\right\}+ \\
-\frac{1}{\nu} W\left(x_{i}, t_{j+1}\right) \text { expint }\left[\left(x_{i}-x_{0}\right) \frac{\mu_{n}}{\nu_{n}}\right]
\end{gathered}
$$

However, as noted for the put case, the small jumps are treated implicitly in the implementation of the scheme to improve the stability of the system, so we are going to use expression

$$
\begin{gathered}
\frac{\left[W\left(x_{i+1}, t_{j}\right)-W\left(x_{i}, t_{j}\right)\right]\left(1-e^{-\frac{\mu_{p}}{\nu_{p}} \Delta x}\right)}{\nu \Delta x \frac{\mu_{p}}{\nu_{p}}}+ \\
+\frac{\left[W\left(x_{i-1}, t_{j}\right)-W\left(x_{i}, t_{j}\right)\right]\left(1-e^{-\frac{\mu_{n}}{\nu_{n}} \Delta x}\right)}{\nu \Delta x \frac{\mu_{n}}{\nu_{n}}}
\end{gathered}
$$

in substitution to the first two lines of the solutions (A.20). As we noted for the call option case, this algorithm is slightly different from the one suggested by Hirsa and Madan who treat implicitly also the terms containing $W\left(x_{i}, t_{j+1}\right)$ in the large jumps. The terms used by Hirsa and Madan are

$$
-\frac{1}{\nu} W\left(x_{i}, t_{j}\right) \text { expint }\left[(N-i) \frac{\mu_{p}}{\nu_{p}} \Delta x\right]-\frac{1}{\nu} W\left(x_{i}, t_{j}\right) \operatorname{expint}\left[\left(x_{i}-x_{0}\right) \frac{\mu_{n}}{\nu_{n}}\right]
$$

rather than the corresponding one with $W\left(x_{i}, t_{j+1}\right)$ in equation (A.20). We decided not to treat implicitly this part because, based on our numerical experiments, it did not provide an improvement in terms of stability. 


\section{A.2 Variance Gamma Difference Equation for European Vanilla Options}

\section{A.2.1 Difference Equation for Call Options}

We can write the difference equation to be used to price European call options by substituting equation (A.20) as modified by (A.21) in the initial PIDE (A.1). We obtain in this way:

$$
\begin{gathered}
W\left(x_{i-1}, t_{j}\right)\left\{h-\frac{\Delta t \cdot\left(1-e^{-\frac{\mu_{n}}{\nu_{n}} \Delta x}\right)}{\nu \Delta x \frac{\mu_{n}}{\nu_{n}}}\right\}+ \\
+W\left(x_{i}, t_{j}\right)\left\{(1+r \Delta t)+\frac{\Delta t \cdot\left(1-e^{-\frac{\mu_{p}}{\nu_{p}} \Delta x}\right)}{\nu \Delta x \frac{\mu_{p}}{\nu_{p}}}+\frac{\Delta t \cdot\left(1-e^{-\frac{\mu_{n}}{\nu_{n}} \Delta x}\right)}{\nu \Delta x \frac{\mu_{n}}{\nu_{n}}}\right\}+ \\
-W\left(x_{i+1}, t_{j}\right)\left\{h+\frac{\Delta t \cdot\left(1-e^{-\frac{\mu_{p}}{\nu_{p}} \Delta x}\right)}{\nu \Delta x \frac{\mu_{p}}{\nu_{p}}}\right\}=W\left(x_{i}, t_{j+1}\right)+ \\
+\Delta t \cdot\left\{\sum_{k=1}^{N-i-1} \frac{1}{\nu \Delta x}\left[W\left(x_{i+k+1}, t_{j+1}\right)-W\left(x_{i+k}, t_{j+1}\right)\right] \cdot\right. \\
+\sum_{k=1}^{N-i-1} \frac{1}{\nu}\left\{W\left(x_{i+k}, t_{j+1}\right)-W\left(x_{i}, t_{j+1}\right)-k\left[W\left(x_{i+k+1}, t_{j+1}\right)-W\left(x_{i+k}, t_{j+1}\right)\right]\right\} \\
\left.+\sum_{k=1}^{-\frac{\mu_{p}}{\nu_{p}} k \Delta x}-e^{-\frac{\mu_{p}}{\nu_{p}}(k+1) \Delta x}\right]+ \\
+\sum_{k=1}^{i-1} \frac{1}{\nu}\left\{W\left(x_{i-k}, t_{j+1}\right)-W\left(x_{i}, t_{j+1}\right)-k\left[W\left(x_{i-k-1}, t_{j+1}\right)-W\left(x_{i-k}, t_{j+1}\right)\right]\right\} \\
\left.\operatorname{expint}\left(\frac{\mu_{p}}{\nu_{p}} \cdot k \Delta x\right)-\operatorname{expint}\left[\frac{\mu_{p}}{\nu_{p}} \cdot(k+1) \Delta x\right]\right\}+ \\
\left.\left.+x_{i-k-1}, t_{j+1}\right)-W\left(x_{i-k}, t_{j+1}\right)\right] \frac{\nu_{n}}{\mu_{n}}\left[e^{-\frac{\mu_{n}}{\nu_{n}} k \Delta x}-e^{-\frac{\mu_{n}}{\nu_{n}}(k+1) \Delta x}\right]+
\end{gathered}
$$




$$
\begin{gathered}
\cdot\left\{\text { expint }\left(\frac{\mu_{n}}{\nu_{n}} k \Delta x\right)-\text { expint }\left[\frac{\mu_{n}}{\nu_{n}}(k+1) \Delta x\right]\right\}+ \\
+\frac{1}{\nu}\left\{e^{x_{i}} e^{-q\left(T-t_{j+1}\right)} \text { expint }\left[\left(\frac{\mu_{p}}{\nu_{p}}-1\right)(N-i) \Delta x\right]+\right. \\
\left.-\left[K e^{-r\left(T-t_{j+1}\right)}+W\left(x_{i}, t_{j+1}\right)\right] \text { expint }\left[\frac{\mu_{p}}{\nu_{p}}(N-i) \Delta x\right]\right\}+ \\
\left.-\frac{1}{\nu} W\left(x_{i}, t_{j+1}\right) \text { expint }\left[\left(x_{i}-x_{0}\right) \frac{\mu_{n}}{\nu_{n}}\right]\right\}
\end{gathered}
$$

We can see that the left hand side can be written as a tridiagonal matrix, hence it is possible to solve the system using the Gauss's elimination technique $^{4}$. The Gauss's elimination provides a much faster solution of the problem compared with the inversion of the matrix. Appendix B presents $\mathrm{C}$ code to solve this difference equation numerically.

\section{A.2.2 Difference Equation for Put Options}

The difference equation for European vanilla put options can be obtained substituting equation (A.16) as modified by equation (A.17) in the initial PIDE (A.1). By doing this, we obtain:

$$
\begin{gathered}
W\left(x_{i-1}, t_{j}\right)\left\{h-\frac{\Delta t \cdot\left(1-e^{-\frac{\mu_{n}}{\nu_{n}} \Delta x}\right)}{\nu \Delta x \frac{\mu_{n}}{\nu_{n}}}\right\}+ \\
+W\left(x_{i}, t_{j}\right)\left\{(1+r \Delta t)+\frac{\Delta t \cdot\left(1-e^{-\frac{\mu_{p}}{\nu_{p}} \Delta x}\right)}{\nu \Delta x \frac{\mu_{p}}{\nu_{p}}}+\frac{\Delta t \cdot\left(1-e^{-\frac{\mu_{n}}{\nu_{n}} \Delta x}\right)}{\nu \Delta x \frac{\mu_{n}}{\nu_{n}}}\right\}+ \\
-W\left(x_{i+1}, t_{j}\right)\left\{h+\frac{\Delta t \cdot\left(1-e^{-\frac{\mu_{p}}{\nu_{p}} \Delta x}\right)}{\nu \Delta x \frac{\mu_{p}}{\nu_{p}}}\right\}=W\left(x_{i}, t_{j+1}\right)+
\end{gathered}
$$

\footnotetext{
${ }^{4}$ John C. Strikwerda, Finite Difference Schemes and Partial Differential Equations, Wadsworth Inc., Belmont, California, 1989, pages 78-81.
} 


$$
\begin{aligned}
& +\Delta t \cdot\left\{\sum_{k=1}^{N-i-1} \frac{1}{\nu \Delta x}\left[W\left(x_{i+k+1}, t_{j+1}\right)-W\left(x_{i+k}, t_{j+1}\right)\right] .\right. \\
& \cdot \frac{\nu_{p}}{\mu_{p}} \cdot\left[e^{-\frac{\mu_{p}}{\nu_{p}} k \Delta x}-e^{-\frac{\mu_{p}}{\nu_{p}}(k+1) \Delta x}\right]+ \\
& +\sum_{k=1}^{N-i-1} \frac{1}{\nu}\left\{W\left(x_{i+k}, t_{j+1}\right)-W\left(x_{i}, t_{j+1}\right)-k\left[W\left(x_{i+k+1}, t_{j+1}\right)-W\left(x_{i+k}, t_{j+1}\right)\right]\right\} . \\
& \cdot\left\{\operatorname{expint}\left(\frac{\mu_{p}}{\nu_{p}} k \Delta x\right)-\operatorname{expint}\left[\frac{\mu_{p}}{\nu_{p}}(k+1) \Delta x\right]\right\}+ \\
& +\sum_{k=1}^{i-1} \frac{1}{\nu \Delta x}\left[W\left(x_{i-k-1}, t_{j+1}\right)-W\left(x_{i-k}, t_{j+1}\right)\right] \frac{\nu_{n}}{\mu_{n}}\left[e^{-\frac{\mu_{n}}{\nu_{n}} k \Delta x}-e^{-\frac{\mu_{n}}{\nu_{n}}(k+1) \Delta x}\right]+ \\
& +\sum_{k=1}^{i-1} \frac{1}{\nu}\left\{W\left(x_{i-k}, t_{j+1}\right)-W\left(x_{i}, t_{j+1}\right)-k\left[W\left(x_{i-k-1}, t_{j+1}\right)-W\left(x_{i-k}, t_{j+1}\right)\right]\right\} \cdot \\
& \cdot\left\{\operatorname{expint}\left(\frac{\mu_{n}}{\nu_{n}} k \Delta x\right)-\operatorname{expint}\left[\frac{\mu_{n}}{\nu_{n}}(k+1) \Delta x\right]\right\}+ \\
& -\frac{1}{\nu} W\left(x_{i}, t_{j+1}\right) \text { expint }\left[(N-i) \frac{\mu_{p}}{\nu_{p}} \Delta x\right]+ \\
& +\frac{1}{\nu}\left\{\left[K e^{-r\left(T-t_{j+1}\right)}-W\left(x_{i}, t_{j+1}\right)\right] \operatorname{expint}\left[\left(x_{i}-x_{0}\right) \frac{\mu_{n}}{\nu_{n}}\right]+\right. \\
& \left.\left.-e^{-q\left(T-t_{j+1}\right)} e^{x_{i}} \operatorname{expint}\left[\left(x_{i}-x_{0}\right)\left(\frac{\mu_{n}}{\nu_{n}}+1\right)\right]\right\}\right\}
\end{aligned}
$$

Again the left hand side of the equation can be written as a tridiagonal matrix and so we can solve the system using Gauss's elimination technique. Appendix B presents $\mathrm{C}$ code to solve this difference equation numerically. 


\section{A.3 Numerical Solution of the Jump Integral in the Variance Gamma PIDE for Amer- ican Vanilla Options}

In the same way as we did for the European case we write the integral as sums of integrals depending on the size of the jump

$$
\begin{aligned}
& \int_{-\infty}^{+\infty}\left[W\left(x_{i}+y, t_{j+1}\right)-W\left(x_{i}, t_{j+1}\right)\right] k(y) d y= \\
= & \int_{-\infty}^{x_{0}-x_{i}}\left[W\left(x_{i}+y, t_{j+1}\right)-W\left(x_{i}, t_{j+1}\right)\right] k(y) d y+ \\
+ & \int_{x_{0}-x_{i}}^{-\Delta x}\left[W\left(x_{i}+y, t_{j+1}\right)-W\left(x_{i}, t_{j+1}\right)\right] k(y) d y+ \\
& +\int_{-\Delta x}^{0}\left[W\left(x_{i}+y, t_{j+1}\right)-W\left(x_{i}, t_{j+1}\right)\right] k(y) d y+ \\
& +\int_{0}^{\Delta x}\left[W\left(x_{i}+y, t_{j+1}\right)-W\left(x_{i}, t_{j+1}\right)\right] k(y) d y+ \\
+ & \int_{\Delta x}^{x_{N}-x_{i}}\left[W\left(x_{i}+y, t_{j+1}\right)-W\left(x_{i}, t_{j+1}\right)\right] k(y) d y+ \\
& +\int_{x_{N}-x_{i}}^{+\infty}\left[W\left(x_{i}+y, t_{j+1}\right)-W\left(x_{i}, t_{j+1}\right)\right] k(y) d y
\end{aligned}
$$

Integrals from (A.24) to (A.27) do not need any modification with respect to the European case, so their solution can be found in sections A.1.1 and A.1.2.

To discuss about the other two integrals where the jump is so big that we finishe outside the range of values, we distinguish between calls and puts.

\section{A.3.1 Solution of Jump Integral for American Vanilla Call Options}

In the case of a call option, integral (A.23) is the same as the European case, while for integral (A.28), we have to keep in mind that it is possible that the option is exercised before maturity. Hence we can write that if

$$
y \in\left[x_{N}-x_{i},+\infty\right]
$$


the option value can be approximated by

$$
W\left(x_{i}+y, t_{j+1}\right) \simeq \max \left[e^{x_{i}+y} e^{-q\left(T-t_{j+1}\right)}-K e^{-r\left(T-t_{j+1}\right)}, e^{x_{i}+y}-K\right]
$$

If the option is indeed exercised and for every value of $y$ in the considered interval the payoff is higher than its discounted value, integral (A.28) can be written as

$$
\begin{array}{r}
\int_{(N-i) \Delta x}^{+\infty}\left[e^{x_{i}+y}-K-W\left(x_{i}, t_{j+1}\right)\right] \frac{e^{-\frac{\mu_{p}}{\nu_{p}} y}}{\nu y} d y= \\
=\frac{1}{\nu}\left\{e^{x_{i}} \text { expint }\left[\left(\frac{\mu_{p}}{\nu_{p}}-1\right)(N-i) \Delta x\right]+\right. \\
\left.-\left[K+W\left(x_{i}, t_{j+1}\right)\right] \text { expint }\left[\frac{\mu_{p}}{\nu_{p}}(N-i) \Delta x\right]\right\}
\end{array}
$$

And the whole jump integral (A.22) in the case of call options can be rewritten as

$$
\begin{aligned}
& \int_{-\infty}^{+\infty}\left[W\left(x_{i}+y, t_{j+1}\right)-W\left(x_{i}, t_{j+1}\right)\right] k(y) d y= \\
& =\frac{\left[W\left(x_{i+1}, t_{j+1}\right)-W\left(x_{i}, t_{j+1}\right)\right]\left(1-e^{-\frac{\mu_{p}}{\nu_{p}} \Delta x}\right)}{\nu \Delta x \frac{\mu_{p}}{\nu_{p}}}+ \\
& +\frac{\left[W\left(x_{i-1}, t_{j+1}\right)-W\left(x_{i}, t_{j+1}\right)\right]\left(1-e^{-\frac{\mu_{n}}{\nu_{n}} \Delta x}\right)}{\nu \Delta x \frac{\mu_{n}}{\nu_{n}}}+
\end{aligned}
$$

$+\sum_{k=1}^{N-i-1}\left\{\frac{1}{\nu \Delta x}\left[W\left(x_{i+k+1}, t_{j+1}\right)-W\left(x_{i+k}, t_{j+1}\right)\right] \frac{\nu_{p}}{\mu_{p}}\left[e^{-\frac{\mu_{p}}{\nu_{p}} k \Delta x}-e^{-\frac{\mu_{p}}{\nu_{p}}(k+1) \Delta x}\right]\right\}+$

$+\sum_{k=1}^{N-i-1} \frac{1}{\nu}\left\{W\left(x_{i+k}, t_{j+1}\right)-W\left(x_{i}, t_{j+1}\right)-k\left[W\left(x_{i+k+1}, t_{j+1}\right)-W\left(x_{i+k}, t_{j+1}\right)\right]\right\}$.

$$
\cdot\left\{\operatorname{expint}\left(\frac{\mu_{p}}{\nu_{p}} k \Delta x\right)-\operatorname{expint}\left[\frac{\mu_{p}}{\nu_{p}}(k+1) \Delta x\right]\right\}+
$$

$+\sum_{k=1}^{i-1}\left\{\frac{1}{\nu \Delta x}\left[W\left(x_{i-k-1}, t_{j+1}\right)-W\left(x_{i-k}, t_{j+1}\right)\right] \frac{\nu_{n}}{\mu_{n}}\left[e^{-\frac{\mu_{n}}{\nu_{n}} k \Delta x}-e^{-\frac{\mu_{n}}{\nu_{n}}(k+1) \Delta x}\right]\right\}+$ 


$$
\begin{gathered}
+\sum_{k=1}^{i-1} \frac{1}{\nu}\left\{W\left(x_{i-k}, t_{j+1}\right)-W\left(x_{i}, t_{j+1}\right)-k\left[W\left(x_{i-k-1}, t_{j+1}\right)-W\left(x_{i-k}, t_{j+1}\right)\right]\right\} \\
\cdot\left\{\text { expint }\left(\frac{\mu_{n}}{\nu_{n}} k \Delta x\right)-\operatorname{expint}\left[\frac{\mu_{n}}{\nu_{n}}(k+1) \Delta x\right]\right\}+ \\
+\frac{1}{\nu}\left\{e^{x_{i}} \operatorname{expint}\left[\left(\frac{\mu_{p}}{\nu_{p}}-1\right)(N-i) \Delta x\right]+\right. \\
\left.-\left[K+W\left(x_{i}, t_{j+1}\right)\right] \operatorname{expint}\left[\frac{\mu_{p}}{\nu_{p}}(N-i) \Delta x\right]\right\}+ \\
-\frac{1}{\nu} W\left(x_{i}, t_{j+1}\right) \operatorname{expint}\left(\frac{\mu_{n}}{\nu_{n}} i \Delta x\right)
\end{gathered}
$$

As for the European option case, small jumps are treated implicitly to improve the stability of the system and so, in the implementation of the solution, we will use

$$
\begin{array}{r}
\frac{\left[W\left(x_{i+1}, t_{j}\right)-W\left(x_{i}, t_{j}\right)\right]\left(1-e^{-\frac{\mu_{p}}{\nu_{p}} \Delta x}\right)}{\nu \Delta x \frac{\mu_{p}}{\nu_{p}}}+ \\
+\frac{\left[W\left(x_{i-1}, t_{j}\right)-W\left(x_{i}, t_{j}\right)\right]\left(1-e^{-\frac{\mu_{n}}{\nu_{n}} \Delta x}\right)}{\nu \Delta x \frac{\mu_{n}}{\nu_{n}}}
\end{array}
$$

instead of the first two lines of the right hand side of equation (A.29).

Note however that, if the option is not exercised for any value of $y$ in the considered range $\left[x_{N}-x_{i},+\infty\right]$, integral (A.28) is the same as in the European case and the whole jump integral (A.22) can be written down exactly as we did for the European call option, in equation (A.20), adjusted with equation $(\mathrm{A} .21)^{5}$. This is for example the case when $q=0$.

Note that if $r>q$ and $q>0$, there exists a point such that for values lower than this it is not profitable to exercise, while for values higher than this it is profitable exercise. This happens because for values just slightly in the money, $K$ and $e^{x_{i}}$ are not too different in size and the fact that $r$ is larger than $q$ puts more weight on the fact that the payoff is discounted, making

\footnotetext{
${ }^{5}$ The small jumps are not affected by this early exercise issue so the correction to make the small jumps implicit is not affect.
} 
the discounted value of the payoff larger than the non discounted payoff. However as the stock price increases, the fact that the stock is discounted at the dividend yield has a higher and higher impact until at some point it becomes profitable to early exercise. This point at which it starts to be profitable to early exercise may be outside the considered grid of values. This would give some problems because we would need to discount values up to that point and get the non discounted values after it. From a practical point of view, it would be necessary to check for each point in the time grid considered where the option starts to be exercised, if it is outside the grid, and make the discounted decisions as necessary. In the code, the approach used is to check whether the exercise boundary exists and it is outside the grid. If this is the case, a warning signal is given, so that it is possible to consider a larger interval inside which the exercise boundary is reached. If anyway, running the program, we still decide to leave the exercise point outside the grid, the following approximation is used.

$$
\begin{array}{r}
\int_{x_{N}-x_{i}}^{+\infty} W\left(x_{i}+y, t_{j+1}\right) k(y) d y= \\
=\max \left[\frac { 1 } { \nu } \left\{e^{x_{i}} \text { expint }\left[\left(\frac{\mu_{p}}{\nu_{p}}-1\right)(N-i) \Delta x\right]+\right.\right. \\
\left.-K \operatorname{expint}\left[\frac{\mu_{p}}{\nu_{p}}(N-i) \Delta x\right]\right\}, \\
\frac{1}{\nu}\left\{e^{x_{i}} e^{-q\left(T-t_{j+1}\right)} \operatorname{expint}\left[\left(\frac{\mu_{p}}{\nu_{p}}-1\right)(N-i) \Delta x\right]+\right. \\
\left.\left.-K e^{-r\left(T-t_{j+1}\right)} \operatorname{expint}\left[\frac{\mu_{p}}{\nu_{p}}(N-i) \Delta x\right]\right\}\right]
\end{array}
$$

This implies that at a certain time step either all or none of the options for stock prices higher than the upper limit in the grid, will be exercised. The decision is based on the fact that on average is convenient to exercise or not exercise. 


\section{A.3.2 Solution of Jump Integral for American Vanilla Put Options}

Let's consider now the put option case. If we remain inside the grid, we can use the results from the European case. Moreover if the jump is large and positive the option can be assumed to have no value, hence there is no early exercise issue. This takes care of integrals from A.24 to A.28 and we need only to consider integral (A.23). Here the jump takes the option so in the money that we can impose that it will be exercised for sure, the only unsure thing is about the timing of the early exercise depending on the proportion between dividend yield and risk free interest rate. We can write that if

$$
y \in\left[-\infty, x_{0}-x_{i}\right]
$$

the value of the put option can be written as

$$
W\left(x_{i}+y, t_{j+1}\right) \simeq \max \left[K e^{-r\left(T-t_{j+1}\right)}-e^{x_{i}+y} e^{-q\left(T-t_{j+1}\right)}, K-e^{x_{i}+y}\right]
$$

Assuming $r>q$, as it is generally the case, the put option would be instantaneously exercised. Changing the variable $y \triangleq-y$, we would have the following solution of integral (A.23):

$$
\begin{array}{r}
\int_{x_{i}-x_{0}}^{+\infty}\left[K-e^{x_{i}-y}-W\left(x_{i}, t_{j+1}\right)\right] \frac{e^{-\frac{\mu_{n}}{\nu_{n}} y}}{\nu y} d y= \\
=\frac{1}{\nu}\left\{\left[K-W\left(x_{i}, t_{j+1}\right)\right] \text { expint }\left(\frac{\mu_{n}}{\nu_{n}} i \Delta x\right)+\right. \\
\left.-e^{x_{i}} \operatorname{expint}\left[\left(\frac{\mu_{n}}{\nu_{n}}+1\right) i \Delta x\right]\right\}
\end{array}
$$

Integral (A.22) for put options in this case can be written as

$$
\begin{aligned}
& \int_{-\infty}^{+\infty}\left[W\left(x_{i}+y, t_{j+1}\right)-W\left(x_{i}, t_{j+1}\right)\right] k(y) d y= \\
= & \frac{\left[W\left(x_{i+1}, t_{j+1}\right)-W\left(x_{i}, t_{j+1}\right)\right]\left(1-e^{-\frac{\mu_{p}}{\nu_{p}} \Delta x}\right)}{\nu \Delta x \frac{\mu_{p}}{\nu_{p}}}+ \\
+ & \frac{\left[W\left(x_{i-1}, t_{j+1}\right)-W\left(x_{i}, t_{j+1}\right)\right]\left(1-e^{-\frac{\mu_{n}}{\nu_{n}} \Delta x}\right)}{\nu \Delta x \frac{\mu_{n}}{\nu_{n}}}+
\end{aligned}
$$




$$
\begin{gathered}
+\sum_{k=1}^{N-i-1} \frac{1}{\nu \Delta x}\left[W\left(x_{i+k+1}, t_{j+1}\right)-W\left(x_{i+k}, t_{j+1}\right)\right] \frac{\nu_{p}}{\mu_{p}}\left[e^{-\frac{\mu_{p}}{\nu_{p}} k \Delta x}-e^{-\frac{\mu_{p}}{\nu_{p}}(k+1) \Delta x}\right]+ \\
+\sum_{k=1}^{N-i-1} \frac{1}{\nu}\left\{W\left(x_{i+k}, t_{j+1}\right)-W\left(x_{i}, t_{j+1}\right)-k\left[W\left(x_{i+k+1}, t_{j+1}\right)-W\left(x_{i+k}, t_{j+1}\right)\right]\right\} \\
+\left\{\operatorname{expint}\left(\frac{\mu_{p}}{\nu_{p}} k \Delta x\right)-\operatorname{expint}\left[\frac{\mu_{p}}{\nu_{p}}(k+1) \Delta x\right]\right\}+ \\
+\sum_{k=1}^{i-1} \frac{1}{\nu \Delta x}\left[W\left(x_{i-k-1}, t_{j+1}\right)-W\left(x_{i-k}, t_{j+1}\right)\right] \frac{\nu_{n}}{\mu_{n}}\left[e^{-\frac{\mu_{n}}{\nu_{n}} k \Delta x}-e^{-\frac{\mu_{n}}{\nu_{n}}(k+1) \Delta x}\right]+ \\
\cdot\left\{\operatorname{expint}\left(\frac{\mu_{n}}{\nu_{n}} k \Delta x\right)-\operatorname{expint}\left[\frac{\mu_{n}}{\nu_{n}}(k+1) \Delta x\right]\right\}+ \\
-\frac{1}{\nu} W\left(x_{i}, t_{j+1}\right) \operatorname{expint}\left[(N-i) \frac{\mu_{p}}{\nu_{p}} \Delta x\right]+ \\
+\frac{1}{\nu}\left\{\left[K-W\left(x_{i-k}, t_{j+1}\right)-W\left(x_{j+1}\right)\right] \operatorname{expint}\left(\frac{\mu_{n}}{\nu_{n}} i \Delta x\right)+\right. \\
\left.\left.-e^{x_{i}} \operatorname{expint}\left[\left(\frac{\mu_{n}}{\nu_{n}}+1\right) i \Delta x\right)\right]\right\}
\end{gathered}
$$

Small jumps are as usual treated implicitly to improve the stability of the scheme, so we replace

$$
\begin{aligned}
& \frac{\left[W\left(x_{i+1}, t_{j}\right)-W\left(x_{i}, t_{j}\right)\right]\left(1-e^{-\frac{\mu_{p}}{\nu_{p}} \Delta x}\right)}{\nu \Delta x \frac{\mu_{p}}{\nu_{p}}}+ \\
& +\frac{\left[W\left(x_{i-1}, t_{j}\right)-W\left(x_{i}, t_{j}\right)\right]\left(1-e^{-\frac{\mu_{n}}{\nu_{n}} \Delta x}\right)}{\nu \Delta x \frac{\mu_{n}}{\nu_{n}}}
\end{aligned}
$$

in the first two lines of the right hand side of equation (A.31). 
Note however that, if the option is never exercised, in the unlikely case where risk free interest rate is zero and dividend yield is positive, integral (A.23) is the same as for the European case, as is the whole jump integral (A.22) which would be given by expression (A.16), as adjusted by expression (A.17) to make small jumps implicitly.

Also for put options it is possible to think about a case in which the exercise boundary is outside the considered range. However this is not a very interesting case from an economic point of view, because it would be given by a situation in which $r<q$ and $r>0$. The approach followed here is again to check if it happens that the exercise point exists and it is outside the considered grid. If this is the case a warning signal is given so that it is possible to consider a smaller lower limit. If anyway we still decide to leave the exercise point outside the grid, the following approximation, after having defined $y \triangleq-y$, is used:

$$
\begin{array}{r}
\int_{x_{i}-x_{0}}^{+\infty} W\left(x_{i}-y, t_{j+1}\right) \frac{e^{-\frac{\mu_{n}}{\nu_{n}} y}}{\nu y} d y= \\
=\max \left[\frac{1}{\nu}\left\{K \text { expint }\left(\frac{\mu_{n}}{\nu_{n}} i \Delta x\right)-e^{x_{i}} \operatorname{expint}\left[\left(\frac{\mu_{n}}{\nu_{n}}+1\right) i \Delta x\right]\right\},\right. \\
\frac{1}{\nu}\left\{K e^{-r\left(T-t_{j+1}\right)} \operatorname{expint}\left[\frac{\mu_{n}}{\nu_{n}} i \Delta x\right]+\right. \\
\left.\left.-e^{-q\left(T-t_{j+1}\right)} e^{x_{i}} \operatorname{expint}\left[\left(\frac{\mu_{n}}{\nu_{n}}+1\right) i \Delta x\right]\right\}\right]
\end{array}
$$

As for the call case, this is equivalent to decide that either all or none of the options outside the grid are exercised depending on which alternative on average is more profitable. 


\section{A.4 Numerical Solution of the Integral in the Heaviside Term of the PIDE for Ameri- can Vanilla Options}

The heaviside terms in the PIDE for American options differs depending if the option considered is a put or a call option, but the technique used of their solution is the same. Let's start considering the America call option case.

\section{A.4.1 Integral for American Vanilla Call Options}

Our goal is to rewrite the integral in the heaviside term in equations (4.24) so that it can used in a linear system to be solved with a finite difference numerical approach. The integral we are interested into is

$$
\int_{-\infty}^{x\left(\tau_{j+1}\right)-x_{i}}\left[W\left(x_{i}+y, t_{j+1}\right)+K-e^{x_{i}+y}\right] k(y) d y
$$

We know that the integral is multiplied by $1_{x_{i}>x\left(\tau_{j+1}\right)}$, therefore it is taken over a strictly negative interval and we therefore are not concern about divergent behavior of the Lévy measure for jump of size approaching to zero. Moreover, since we are working on a discrete space, $x\left(\tau_{j+1}\right)$ has to be on a node of the grid and $x_{i}-x\left(\tau_{j+1}\right)$ as to be at least as big as $\Delta x$, that is one space step in the grid. We define

$$
x\left(\tau_{j+1}\right) \triangleq l \Delta x=x_{l}
$$

for some integer $l$ between 0 and $N$ whose value determines the position of the exercise boundary in the grid. Note that in reality $l$ must be larger than zero, because at least it has to be such that $e^{x_{i}} \geq K$. Moreover if the option is not exercised for any point of the grid for a certain $t_{j+1}$, then $l$ is set equal to $N$. Because the integral is multiplied by the indicator function $1_{\left\{x_{i}>x\left(\tau_{j+1}\right)\right\}}$ presenting a strict inequality, positioning the exercise boundary at the limit of the grid, produces the result that the heaviside function does not impact that time step.

Following Hirsa and Madan ${ }^{6}$, let's split the integral in two pieces in the

\footnotetext{
${ }^{6}$ Ali Hirsa and Dilip B. Madan, "Pricing American Options Under Variance Gamma", Journal of Computational Finance, Vol. 7, No. 2, Winter 2003/2004, pages 63-80.
} 
following way:

$$
\begin{aligned}
& \int_{-\infty}^{x\left(\tau_{j+1}\right)-x_{i}}\left[W\left(x_{i}+y, t_{j+1}\right)+K-e^{x_{i}+y}\right] k(y) d y= \\
& =\int_{-\infty}^{x_{0}-x_{i}}\left[W\left(x_{i}+y, t_{j+1}\right)+K-e^{x_{i}+y}\right] k(y) d y+ \\
& \quad+\int_{x_{0}-x_{i}}^{(l-i) \Delta x}\left[W\left(x_{i}+y, t_{j+1}\right)+K-e^{x_{i}+y}\right] k(y) d y
\end{aligned}
$$

Remember that overall this integral describes the impact of a scenario in which the stock jump back to the continuation region after having reached the exercise region. Integral (A.34) describes the case in which the stock prices goes back to the continuation region because of a negative jump. In this case, however, the jump is not too big and stock reachs a value which is still inside the range considered. Integral (A.33), on the other side defines a situation where the negative jump is so large that not only the underlying goes back to the continuation region, but it even finishes outside the range considered in our grid. Let's see how to rewrite each of the two integrals.

\section{A.4.2 Solution of Integral (A.34)}

Remembering the definition of variance gamma Lévy density for negative jumps, we can write the integral as

$$
\int_{x_{0}-x_{i}}^{(l-i) \Delta x}\left[W\left(x_{i}+y, t_{j+1}\right)+K-e^{x_{i}+y}\right] \frac{e^{-\frac{\mu_{n}}{\nu_{n}}|y|}}{\nu|y|} d y
$$

Let's make a change of variable $y \triangleq-y$, the integral becomes

$$
\int_{(i-l) \Delta x}^{x_{i}-x_{0}}\left[W\left(x_{i}-y, t_{j+1}\right)+K-e^{x_{i}-y}\right] \frac{e^{-\frac{\mu_{n}}{\nu_{n}} y}}{\nu y} d y
$$

This integral can be written as sum of integrals in the following way

$$
\sum_{k=i-l}^{i-0-1}\left\{\int_{k \Delta x}^{(k+1) \Delta x}\left[W\left(x_{i}-y, t_{j+1}\right)+K-e^{x_{i}-y}\right] \frac{e^{-\frac{\mu_{n}}{\nu_{n}} y}}{\nu y} d y\right\}
$$


We have here a sum of jumps whose size is between $k \Delta x$ and $(k+1) \Delta x$. For every $k$ considered in the summation, we can approximate the value of the option via a linear interpolation in the following way:

$$
\begin{array}{r}
W\left(x_{i}-y, t_{j+1}\right)-W\left(x_{i-k}, t_{j+1}\right) \simeq \\
\simeq \frac{W\left(x_{i-k-1}, t_{j+1}\right)-W\left(x_{i-k}, t_{j+1}\right)}{\Delta x} \cdot(y-k \Delta x)
\end{array}
$$

Using this approximation, we can rewrite expression (A.35) as

$$
\begin{gathered}
\sum_{k=i-l}^{i-1}\left\{\int_{k \Delta x}^{(k+1) \Delta x}\left[\frac{W\left(x_{i-k-1}, t_{j+1}\right)-W\left(x_{i-k}, t_{j+1}\right)}{\Delta x}\right] \cdot y \frac{e^{-\frac{\mu_{n}}{\nu_{n}} y}}{\nu y} d y\right\}+ \\
-\sum_{k=i-l}^{i-1}\left\{\int_{k \Delta x}^{(k+1) \Delta x}\left[W\left(x_{i-k-1}, t_{j+1}\right)-W\left(x_{i-k}, t_{j+1}\right)\right] \cdot k \frac{e^{-\frac{\mu_{n}}{\nu_{n}} y}}{\nu y} d y\right\}+ \\
+\sum_{k=i-l}^{i-1}\left\{\int_{k \Delta x}^{(k+1) \Delta x}\left[W\left(x_{i-k}, t_{j+1}\right)+K\right] \cdot \frac{e^{-\frac{\mu_{n}}{\nu_{n}} y}}{\nu y} d y\right\}+ \\
-\sum_{k=i-l}^{i-1}\left\{\int_{k \Delta x}^{(k+1) \Delta x} e^{x_{i}-y} \cdot \frac{e^{-\frac{\mu_{n}}{\nu_{n}} y}}{\nu y} d y\right\}
\end{gathered}
$$

Integral in (A.36) can be solved to obtain

$$
\begin{aligned}
& \int_{k \Delta x}^{(k+1) \Delta x}\left[\frac{W\left(x_{i-k-1}, t_{j+1}\right)-W\left(x_{i-k}, t_{j+1}\right)}{\Delta x}\right] \cdot y \frac{e^{-\frac{\mu_{n}}{\nu_{n}} y}}{\nu y} d y= \\
= & \frac{W\left(x_{i-k-1}, t_{j+1}\right)-W\left(x_{i-k}, t_{j+1}\right)}{\nu \frac{\mu_{n}}{\nu_{n}} \Delta x} \cdot\left[e^{-\frac{\mu_{n}}{\nu_{n}} k \Delta x}-e^{-\frac{\mu_{n}}{\nu_{n}}(k+1) \Delta x}\right]
\end{aligned}
$$

We can use the expint $(\cdot)$ function

$$
\operatorname{expint}_{n}(x)=\int_{1}^{\infty} \frac{e^{-x t}}{t^{n}} d t
$$


for $x>0$ and $n=0,1, \ldots$ and in particular here with $n=1$. With this notation, the integral in (A.37) becomes

$$
\begin{array}{r}
\int_{k \Delta x}^{(k+1) \Delta x}\left[W\left(x_{i-k-1}, t_{j+1}\right)-W\left(x_{i-k}, t_{j+1}\right)\right] \cdot k \frac{e^{-\frac{\mu_{n}}{\nu_{n}} y}}{\nu y} d y= \\
=\left[W\left(x_{i-k-1}, t_{j+1}\right)-W\left(x_{i-k}, t_{j+1}\right)\right] \cdot \frac{k}{\nu} . \\
\cdot\left\{\text { expint }\left[\frac{\mu_{n}}{\nu_{n}} k \Delta x\right]-\text { expint }\left[\frac{\mu_{n}}{\nu_{n}}(k+1) \Delta x\right]\right\}
\end{array}
$$

Similarly the integral in (A.38) can be expressed as

$$
\begin{array}{r}
\int_{k \Delta x}^{(k+1) \Delta x}\left[W\left(x_{i-k}, t_{j+1}\right)+K\right] \cdot \frac{e^{-\frac{\mu_{n}}{\nu_{n}} y}}{\nu y} d y= \\
=\left[W\left(x_{i-k}, t_{j+1}\right)+K\right] \cdot \frac{1}{\nu} . \\
\cdot\left\{\text { expint }\left[\frac{\mu_{n}}{\nu_{n}} k \Delta x\right]-\text { expint }\left[\frac{\mu_{n}}{\nu_{n}}(k+1) \Delta x\right]\right\}
\end{array}
$$

Finally the integral in (A.39) can be rewritten as

$$
\begin{gathered}
\int_{k \Delta x}^{(k+1) \Delta x} e^{x_{i}-y} \cdot \frac{e^{-\frac{\mu_{n}}{\nu_{n}} y}}{\nu y} d y= \\
=\frac{e^{x_{i}}}{\nu} \cdot\left\{\text { expint }\left[\left(1+\frac{\mu_{n}}{\nu_{n}}\right) k \Delta x\right]-\text { expint }\left[\left(1+\frac{\mu_{n}}{\nu_{n}}\right)(k+1) \Delta x\right]\right\}
\end{gathered}
$$

Clearly it is true that

$$
\sum_{k=i-l}^{i-1}\{f[\beta k]-f[\beta(k+1)]\}=f[\beta(i-l)]-f[\beta i]
$$

Applying (A.44) to (A.43) and to part of (A.42) and substituting equations from (A.40) to (A.43) in integral (A.35) gives us the solution we were looking 
for

$$
\begin{gathered}
\int_{x_{0}-x_{i}}^{(l-i) \Delta x}\left[W\left(x_{i}+y, t_{j+1}\right)+K-e^{x_{i}+y}\right] k(y) d y= \\
=\sum_{k=i-l}^{i-1}\left\{\frac{W\left(x_{i-k-1}, t_{j+1}\right)-W\left(x_{i-k}, t_{j+1}\right)}{\nu \frac{\mu_{n}}{\nu_{n}} \Delta x} \cdot\left[e^{-\frac{\mu_{n}}{\nu_{n}} k \Delta x}-e^{-\frac{\mu_{n}}{\nu_{n}}(k+1) \Delta x}\right]+\right. \\
-\left[W\left(x_{i-k-1}, t_{j+1}\right)-W\left(x_{i-k}, t_{j+1}\right)\right] \cdot \frac{k}{\nu} \cdot \\
+\left[\operatorname{expint}\left(\frac{\mu_{n}}{\nu_{n}} k \Delta x\right)-\operatorname{expint}\left(\frac{\mu_{n}}{\nu_{n}}(k+1) \Delta x\right)\right]+ \\
\left.+W\left(x_{i-k}, t_{j+1}\right) \cdot \frac{1}{\nu} \cdot\left[\operatorname{expint}\left(\frac{\mu_{n}}{\nu_{n}} k \Delta x\right)-\operatorname{expint}\left(\frac{\mu_{n}}{\nu_{n}}(k+1) \Delta x\right)\right]\right\}+ \\
+\frac{K}{\nu} \cdot\left\{\operatorname{expint}\left[\frac{\mu_{n}}{\nu_{n}}(i-l) \Delta x\right]-\operatorname{expint}\left(\frac{\mu_{n}}{\nu_{n}} i \Delta x\right)\right\}+ \\
-\frac{e^{x_{i}}}{\nu} \cdot\left\{\operatorname{expint}\left[\left(1+\frac{\mu_{n}}{\nu_{n}}\right)(i-l) \Delta x\right]-\operatorname{expint}\left[\left(1+\frac{\mu_{n}}{\nu_{n}}\right) i \Delta x\right]\right\}
\end{gathered}
$$

which can be written also as:

$$
\begin{gathered}
\int_{x_{0}-x_{i}}^{(l-i) \Delta x}\left[W\left(x_{i}+y, t_{j+1}\right)+K-e^{x_{i}+y}\right] k(y) d y= \\
=\sum_{k=i-l}^{i-1}\left\{\frac{W\left(x_{i-k-1}, t_{j+1}\right)-W\left(x_{i-k}, t_{j+1}\right)}{\nu \frac{\mu_{n}}{\nu_{n}} \Delta x} \cdot\left[e^{-\frac{\mu_{n}}{\nu_{n}} k \Delta x}-e^{-\frac{\mu_{n}}{\nu_{n}}(k+1) \Delta x}\right]+\right. \\
+\frac{1}{\nu}\left[W\left(x_{i-k}, t_{j+1}\right)-k\left(W\left(x_{i-k-1}, t_{j+1}\right)-W\left(x_{i-k}, t_{j+1}\right)\right)\right] \\
\left.\cdot\left[\operatorname{expint}\left(\frac{\mu_{n}}{\nu_{n}} k \Delta x\right)-\text { expint }\left(\frac{\mu_{n}}{\nu_{n}}(k+1) \Delta x\right)\right]\right\}+ \\
+\frac{K}{\nu} \cdot\left\{\operatorname{expint}\left[\frac{\mu_{n}}{\nu_{n}}(i-l) \Delta x\right]-\text { expint }\left(\frac{\mu_{n}}{\nu_{n}} i \Delta x\right)\right\}+ \\
-\frac{e^{x_{i}}}{\nu} \cdot\left\{\operatorname{expint}\left[\left(1+\frac{\mu_{n}}{\nu_{n}}\right)(i-l) \Delta x\right]-\text { expint }\left[\left(1+\frac{\mu_{n}}{\nu_{n}}\right) i \Delta x\right]\right\}(A .4
\end{gathered}
$$




\section{A.4.3 Solution of Integral (A.33)}

Integral (A.33) is given by

$$
\int_{-\infty}^{x_{0}-x_{i}}\left[W\left(x_{i}+y, t_{j+1}\right)+K-e^{x_{i}+y}\right] k(y) d y
$$

Again we apply a change of variable $y \triangleq-y$ and the definition of variance gamma Lévy density to obtain

$$
\int_{x_{i}-x_{0}}^{+\infty}\left[W\left(x_{i}-y, t_{j+1}\right)+K-e^{x_{i}-y}\right] \frac{e^{-\frac{\mu_{n}}{\nu_{n}} y}}{\nu y} d y
$$

Here the negative jump is so big that the underlying finishing outside the range of values considered. Because we are considering a call option, we impose that values on the boundary $x_{0}$ or outside that boundary are zero. Hence we can write

$$
W\left(x_{i}-y, t_{j+1}\right) \simeq 0 \quad \text { for } \quad y \in\left[x_{i}-x_{0},+\infty\right]
$$

Hence the solution of integral (A.33) is just the solution of

$$
\int_{x_{i}-x_{0}}^{+\infty}\left(K-e^{x_{i}-y}\right) \frac{e^{-\frac{\mu_{n}}{\nu_{n}} y}}{\nu y} d y
$$

Using the definition of expint $(\cdot)$ we have

$$
\begin{array}{r}
\int_{x_{i}-x_{0}}^{+\infty}\left(K-e^{x_{i}-y}\right) \frac{e^{-\frac{\mu_{n}}{\nu_{n}} y}}{\nu y} d y= \\
\frac{K}{\nu} \cdot \operatorname{expint}\left(\frac{\mu_{n}}{\nu_{n}} i \Delta x\right)-\frac{e^{x_{i}}}{\nu} \cdot \operatorname{expint}\left[\left(1+\frac{\mu_{n}}{\nu_{n}}\right) i \Delta x\right]
\end{array}
$$




\section{A.4.4 Numerical Solution of the Integral in the Heav- iside Term of the American PIDE for Vanilla Call Options}

By combining equations (A.45) and (A.46) we have reached my goal of writing the integral in the heaviside term of the equation describing the dynamics of the American call option price in a proper way to allow the numerical solution of the equation. The result is

$$
\begin{gathered}
\int_{-\infty}^{x\left(\tau_{j+1}\right)-x_{i}}\left[W\left(x_{i}+y, t_{j+1}\right)+K-e^{x_{i}+y}\right] k(y) d y= \\
=\sum_{k=i-l}^{i-1}\left\{\frac{W\left(x_{i-k-1}, t_{j+1}\right)-W\left(x_{i-k}, t_{j+1}\right)}{\nu \frac{\mu_{n}}{\nu_{n}} \Delta x} \cdot\left[e^{-\frac{\mu_{n}}{\nu_{n}} k \Delta x}-e^{-\frac{\mu_{n}}{\nu_{n}}(k+1) \Delta x}\right]+\right. \\
+\frac{1}{\nu}\left[W\left(x_{i-k}, t_{j+1}\right)-k\left(W\left(x_{i-k-1}, t_{j+1}\right)-W\left(x_{i-k}, t_{j+1}\right)\right)\right] \\
\left.\cdot\left[\operatorname{expint}\left(\frac{\mu_{n}}{\nu_{n}} k \Delta x\right)-\operatorname{expint}\left(\frac{\mu_{n}}{\nu_{n}}(k+1) \Delta x\right)\right]\right\}+ \\
+\frac{K}{\nu} \cdot \operatorname{expint}\left[\frac{\mu_{n}}{\nu_{n}}(i-l) \Delta x\right]-\frac{e^{x_{i}}}{\nu} \cdot \operatorname{expint}\left[\left(1+\frac{\mu_{n}}{\nu_{n}}\right)(i-l) \Delta x\right]
\end{gathered}
$$

\section{A.4.5 Integral for American Vanilla Put Options}

Let's consider the integral which appear in the Heaviside term in equation $(4.25)$

$$
\int_{x\left(\tau_{j+1}\right)-x_{i}}^{+\infty}\left[W\left(x_{i}+y, t_{j+1}\right)-K+e^{x_{i}+y}\right] k(y) d y
$$

To evaluate numerically this integral we can, first of all, note that, because this integral is multiplied by the indicator function $1_{\left\{x_{i}<x\left(\tau_{j+1}\right)\right\}}$, the integral is taken over a strictly positive range, hence we do not have to deal with the issue of a Lévy measure which tends to infinity for jumps which tend to zero. Moreover, because we are working in discrete space, we know that 
$x\left(\tau_{j+1}\right)-x_{i}$ is at least equal to $\Delta x$, that is one space in our grid. Following Hirsa and Madan, we can split this integral in two parts in the following way:

$$
\begin{array}{r}
\int_{(l-i) \Delta x}^{(N-i) \Delta x}\left[W\left(x_{i}+y, t_{j+1}\right)-K+e^{x_{i}+y}\right] k(y) d y+ \\
\int_{(N-i) \Delta x}^{+\infty}\left[W\left(x_{i}+y, t_{j+1}\right)-K+e^{x_{i}+y}\right] k(y) d y
\end{array}
$$

where we defined

$$
x\left(\tau_{j+1}\right) \triangleq l \Delta x=x_{l}
$$

for some integer $l$ between 0 and $N$ whose value determines the position of the exercise boundary on the grid. In reality $l$ has to be lower than $N$, because it has to be at least such that $e^{x_{i}} \leq K$. Moreover if the option is not exercised for any grid point at a certain time step $t_{j+1}, l$ is set at 0 . Given that the integral is multiplied by the indicator function $1_{\left\{x_{i}<x\left(\tau_{j+1}\right)\right\}}$ presenting a strict inequality, if we position the exercise boundary at the extreme left of the grid, the heaviside function will not affect the time step because the indicator would always be equal to zero.

Integral (A.48) describe the case of a positive jump which takes the stock back to the continuation region, after it had reached the exercise region. The size of the jump $y$ here is however not too large and the stock value remains inside the range considered. On the contrary in the case of integral (A.49) the jump is so large that the underlying value is outside the considered range.

\section{A.4.6 Solution of Integral (A.48)}

We can write this integral as sum of integrals in the following way:

$$
\sum_{k=l-i}^{N-i-1}\left\{\int_{k \Delta x}^{(k+1) \Delta x}\left[W\left(x_{i}+y, t_{j+1}\right)-K+e^{x_{i}+y}\right] \frac{e^{-\frac{\mu_{p}}{\nu_{p}} y}}{\nu y} d y\right\}
$$

where we replaced $k(y) d y$ with the variance gamma Lévy measure for positive jumps. For each of the jumps of size $[k \Delta x,(k+1) \Delta x]$, it is possible to approximate the difference in the value of the option after the jump and the value of the option at the last point in the grid passed with the jump (that 
is $x_{i+k}$ ), with a linear interpolation of the option value at the two nodes $x_{i+k}$ and $x_{i+k+1}$. In a mathematical notation, this can be written as

$$
\begin{array}{r}
W\left(x_{i}+y, t_{j+1}\right)-W\left(x_{i+k}, t_{j+1}\right) \simeq \\
\simeq \frac{W\left(x_{i+k+1}, t_{j+1}\right)-W\left(x_{i+k}, t_{j+1}\right)}{\Delta x} \cdot(y-k \Delta x)
\end{array}
$$

Using this linear interpolation, we can rewrite expression (A.50) as

$$
\begin{gathered}
\sum_{k=l-i}^{N-i-1}\left\{\int_{k \Delta x}^{(k+1) \Delta x}\left[\frac{W\left(x_{i+k+1}, t_{j+1}\right)-W\left(x_{i+k}, t_{j+1}\right)}{\Delta x} \cdot(y-k \Delta x)\right] \frac{e^{-\frac{\mu_{p}}{\nu_{p}} y}}{\nu y} d y+\right. \\
\left.+\int_{k \Delta x}^{(k+1) \Delta x}\left[W\left(x_{i+k}, t_{j+1}\right)-K+e^{x_{i}+y}\right] \frac{e^{-\frac{\mu_{p}}{\nu_{p}} y}}{\nu y} d y\right\}
\end{gathered}
$$

Working in a similar way we did for integrals (A.36) to (A.39) we can rewrite integral (A.51) with the following approximation

$$
\begin{gathered}
\sum_{k=l-i}^{N-i-1}\left\{\frac{W\left(x_{i+k+1}, t_{j+1}\right)-W\left(x_{i+k}, t_{j+1}\right)}{\nu \frac{\mu_{p}}{\nu_{p}} \Delta x} \cdot\left[e^{-\frac{\mu_{p}}{\nu_{p}} k \Delta x}-e^{-\frac{\mu_{p}}{\nu_{p}}(k+1) \Delta x}\right]+\right. \\
+\frac{1}{\nu}\left[W\left(x_{i+k}, t_{j+1}\right)-k\left(W\left(x_{i+k+1}, t_{j+1}\right)-W\left(x_{i+k}, t_{j+1}\right)\right)\right] \cdot \\
\left.\cdot\left[\operatorname{expint}\left(\frac{\mu_{p}}{\nu_{p}} k \Delta x\right)-\operatorname{expint}\left(\frac{\mu_{p}}{\nu_{p}}(k+1) \Delta x\right)\right]\right\}+ \\
-\frac{K}{\nu} \cdot\left\{\operatorname{expint}\left[\frac{\mu_{p}}{\nu_{p}}(l-i) \Delta x\right]-\operatorname{expint}\left(\frac{\mu_{p}}{\nu_{p}}(N-i) \Delta x\right)\right\}+ \\
+\frac{e^{x_{i}}}{\nu} \cdot\left\{\operatorname{expint}\left[\left(\frac{\mu_{p}}{\nu_{p}}-1\right)(l-i) \Delta x\right]-\right. \\
\left.\operatorname{expint}\left[\left(\frac{\mu_{p}}{\nu_{p}}-1\right)(N-i) \Delta x\right]\right\}
\end{gathered}
$$




\section{A.4.7 Solution of Integral (A.49)}

The integral we are interested into refers to large positive jumps such that the underlying finishes outside the considered range. Because we are studying here put options, the value of the option for large values of the stock at the level or outside the boundary $x_{N}$ is equal zero. Therefore we can write

$$
W\left(x_{i}+y, t_{j+1}\right) \simeq 0 \quad \text { for } \quad y \in\left[x_{N}-x_{i},+\infty\right]
$$

Hence integral (A.49) is equal to

$$
\int_{(N-i) \Delta x}^{+\infty}\left(e^{x_{i}+y}-K\right) \frac{e^{-\frac{\mu_{p}}{\nu_{p}} y}}{\nu y} d y
$$

The solution of this integral is

$$
\frac{e^{x_{i}}}{\nu} \text { expint }\left[\left(\frac{\mu_{p}}{\nu_{p}}-1\right)(N-i) \Delta x\right]-\frac{K}{\nu} \text { expint }\left[\frac{\mu_{p}}{\nu_{p}}(N-i) \Delta x\right]
$$

\section{A.4.8 Numerical Solution of the Integral in the Heavi- side Term of the American PIDE for Vanilla Put Options}

Let's combine equations (A.52) and (A.53), this gives

$$
\begin{gathered}
\int_{x\left(\tau_{j+1}\right)-x_{i}}^{+\infty}\left[W\left(x_{i}+y, t_{j+1}\right)-K+e^{x_{i}+y}\right] k(y) d y= \\
=\sum_{k=l-i}^{N-i-1}\left\{\frac{W\left(x_{i+k+1}, t_{j+1}\right)-W\left(x_{i+k}, t_{j+1}\right)}{\nu \frac{\mu_{p}}{\nu_{p}} \Delta x} \cdot\left[e^{-\frac{\mu_{p}}{\nu_{p}} k \Delta x}-e^{-\frac{\mu_{p}}{\nu_{p}}(k+1) \Delta x}\right]+\right. \\
+\frac{1}{\nu}\left[W\left(x_{i+k}, t_{j+1}\right)-k\left(W\left(x_{i+k+1}, t_{j+1}\right)-W\left(x_{i+k}, t_{j+1}\right)\right)\right] \\
\left.\cdot\left[\operatorname{expint}\left(\frac{\mu_{p}}{\nu_{p}} k \Delta x\right)-\operatorname{expint}\left(\frac{\mu_{p}}{\nu_{p}}(k+1) \Delta x\right)\right]\right\}+ \\
-\frac{K}{\nu} \cdot \text { expint }\left[\frac{\mu_{p}}{\nu_{p}}(l-i) \Delta x\right]+\frac{e^{x_{i}}}{\nu} \cdot \operatorname{expint}\left[\left(\frac{\mu_{p}}{\nu_{p}}-1\right)(l-i) \Delta x\right]
\end{gathered}
$$




\section{A.5 Variance Gamma Difference Equation for American Vanilla Options}

\section{A.5.1 Difference Equation for Call Options}

Let's consider the PIDE for American vanilla call options:

$$
\begin{aligned}
& h W\left(x_{i-1}, t_{j}\right)+(1+r \Delta t) W\left(x_{i}, t_{j}\right)-h W\left(x_{i+1}, t_{j}\right)=W\left(x_{i}, t_{j+1}\right)+ \\
& +\Delta t \int_{-\infty}^{+\infty}\left[W\left(x_{i}+y, t_{j+1}\right)-W\left(x_{i}, t_{j+1}\right)\right] k(y) d y-1_{x_{i}>x\left(\tau_{j+1}\right)} \cdot \Delta t . \\
& \cdot\left\{r K-q e^{x_{i}}+\int_{-\infty}^{x\left(\tau_{j+1}\right)-x_{i}}\left[W\left(x_{i}+y, t_{j+1}\right)+K-e^{x_{i}+y}\right] k(y) d y\right\}
\end{aligned}
$$

Let's replace in this equation the discretization of the integral in the heaviside term from equation (A.47). Moreover if we assume that the option is exercised for all the cases in which the stock has a positive jump which takes it outside the considered range, we can use equation (A.29) as adjusted by expression (A.30) to discretize the jump integral. We can therefore write the difference equation in the following way:

$$
\begin{gathered}
W\left(x_{i-1}, t_{j}\right)\left\{h-\frac{\Delta t \cdot\left(1-e^{-\frac{\mu_{n}}{\nu_{n}} \Delta x}\right)}{\nu \Delta x \frac{\mu_{n}}{\nu_{n}}}\right\}+ \\
+W\left(x_{i}, t_{j}\right)\left\{(1+r \Delta t)+\frac{\Delta t \cdot\left(1-e^{-\frac{\mu_{p}}{\nu_{p}} \Delta x}\right)}{\nu \Delta x \frac{\mu_{p}}{\nu_{p}}}+\frac{\Delta t \cdot\left(1-e^{-\frac{\mu_{n}}{\nu_{n}} \Delta x}\right)}{\nu \Delta x \frac{\mu_{n}}{\nu_{n}}}\right\}+ \\
-W\left(x_{i+1}, t_{j}\right)\left\{h+\frac{\Delta t \cdot\left(1-e^{-\frac{\mu_{p}}{\nu_{p}} \Delta x}\right)}{\nu \Delta x^{\frac{\mu_{p}}{\nu_{p}}}}\right\}=W\left(x_{i}, t_{j+1}\right)+ \\
+\Delta t \cdot\left\{\sum_{k=1}^{N-i-1} \frac{1}{\nu \Delta x}\left[W\left(x_{i+k+1}, t_{j+1}\right)-W\left(x_{i+k}, t_{j+1}\right)\right] \cdot\right. \\
\cdot \frac{\nu_{p}}{\mu_{p}}\left[e^{-\frac{\mu_{p}}{\nu_{p}} k \Delta x}-e^{-\frac{\mu_{p}}{\nu_{p}}(k+1) \Delta x}\right]+
\end{gathered}
$$




$$
\begin{aligned}
& +\sum_{k=1}^{N-i-1} \frac{1}{\nu}\left\{W\left(x_{i+k}, t_{j+1}\right)-W\left(x_{i}, t_{j+1}\right)-k\left[W\left(x_{i+k+1}, t_{j+1}\right)-W\left(x_{i+k}, t_{j+1}\right)\right]\right\} . \\
& \cdot\left\{\operatorname{expint}\left(\frac{\mu_{p}}{\nu_{p}} k \Delta x\right)-\operatorname{expint}\left[\frac{\mu_{p}}{\nu_{p}}(k+1) \Delta x\right]\right\}+ \\
& +\sum_{k=1}^{i-1}\left\{\frac{1}{\nu \Delta x}\left[W\left(x_{i-k-1}, t_{j+1}\right)-W\left(x_{i-k}, t_{j+1}\right)\right] \frac{\nu_{n}}{\mu_{n}}\left[e^{-\frac{\mu_{n}}{\nu_{n}} k \Delta x}-e^{-\frac{\mu_{n}}{\nu_{n}}(k+1) \Delta x}\right]\right\}+ \\
& +\sum_{k=1}^{i-1} \frac{1}{\nu}\left\{W\left(x_{i-k}, t_{j+1}\right)-W\left(x_{i}, t_{j+1}\right)-k\left[W\left(x_{i-k-1}, t_{j+1}\right)-W\left(x_{i-k}, t_{j+1}\right)\right]\right\} . \\
& \cdot\left\{\operatorname{expint}\left(\frac{\mu_{n}}{\nu_{n}} k \Delta x\right)-\operatorname{expint}\left[\frac{\mu_{n}}{\nu_{n}}(k+1) \Delta x\right]\right\}+ \\
& +\frac{1}{\nu}\left\{e^{x_{i}} \operatorname{expint}\left[\left(\frac{\mu_{p}}{\nu_{p}}-1\right)(N-i) \Delta x\right]+\right. \\
& \left.-\left[K+W\left(x_{i}, t_{j+1}\right)\right] \operatorname{expint}\left[\frac{\mu_{p}}{\nu_{p}}(N-i) \Delta x\right]\right\}+ \\
& \left.-\frac{1}{\nu} W\left(x_{i}, t_{j+1}\right) \operatorname{expint}\left(\frac{\mu_{n}}{\nu_{n}} i \Delta x\right)\right\}+ \\
& -1_{x_{i}>x\left(\tau_{j+1}\right)} \cdot \Delta t \cdot\left\{r K-q e^{x_{i}}+\right. \\
& \sum_{k=i-l}^{i-1}\left\{\frac{W\left(x_{i-k-1}, t_{j+1}\right)-W\left(x_{i-k}, t_{j+1}\right)}{\nu \frac{\mu_{n}}{\nu_{n}} \Delta x} \cdot\left[e^{-\frac{\mu_{n}}{\nu_{n}} k \Delta x}-e^{-\frac{\mu_{n}}{\nu_{n}}(k+1) \Delta x}\right]+\right. \\
& +\frac{1}{\nu}\left[W\left(x_{i-k}, t_{j+1}\right)-k\left(W\left(x_{i-k-1}, t_{j+1}\right)-W\left(x_{i-k}, t_{j+1}\right)\right)\right] . \\
& \left.\cdot\left[\operatorname{expint}\left(\frac{\mu_{n}}{\nu_{n}} k \Delta x\right)-\operatorname{expint}\left(\frac{\mu_{n}}{\nu_{n}}(k+1) \Delta x\right)\right]\right\}+ \\
& \left.+\frac{K}{\nu} \cdot \operatorname{expint}\left[\frac{\mu_{n}}{\nu_{n}}(i-l) \Delta x\right]-\frac{e^{x_{i}}}{\nu} \cdot \operatorname{expint}\left[\left(1+\frac{\mu_{n}}{\nu_{n}}\right)(i-l) \Delta x\right]\right\}
\end{aligned}
$$


The left hand side can be written in tridiagonal matrix form and hence we can use Gauss's elimination method to solve the system. Appendix B provides $\mathrm{C}$ code to solve this difference equation numerically.

\section{A.5.2 Difference Equation for Put Options}

Consider the PIDE for American put options:

$$
\begin{aligned}
& h W\left(x_{i-1}, t_{j}\right)+(1+r \Delta t) W\left(x_{i}, t_{j}\right)-h W\left(x_{i+1}, t_{j}\right)=W\left(x_{i}, t_{j+1}\right)+ \\
& +\Delta t \int_{-\infty}^{+\infty}\left[W\left(x_{i}+y, t_{j+1}\right)-W\left(x_{i}, t_{j+1}\right)\right] k(y) d y-1_{x_{i}<x\left(\tau_{j+1}\right)} \cdot \Delta t . \\
& \quad \cdot\left\{q e^{x_{i}}-r K+\int_{x\left(\tau_{j+1}\right)-x_{i}}^{+\infty}\left[W\left(x_{i}+y, t_{j+1}\right)-K+e^{x_{i}+y}\right] k(y) d y\right\}
\end{aligned}
$$

We can rewrite this PIDE by replacing the integrals with the corresponding discretized forms. In particular the integral in the heaviside term can be replaced using equation (A.54). Moreover if we assume that the option is always early exercised every time that the there is a large negative jump which takes the stock outside the considered range, we can approximate the jump integral by using equation (A.31) as adjusted by expression (A.32). Using these approximation we can write the following difference equation for American vanilla put options:

$$
\begin{gathered}
W\left(x_{i-1}, t_{j}\right)\left\{h-\frac{\Delta t \cdot\left(1-e^{-\frac{\mu_{n}}{\nu_{n}} \Delta x}\right)}{\nu \Delta x \frac{\mu_{n}}{\nu_{n}}}\right\}+ \\
+W\left(x_{i}, t_{j}\right)\left\{(1+r \Delta t)+\frac{\Delta t \cdot\left(1-e^{-\frac{\mu_{p}}{\nu_{p}} \Delta x}\right)}{\nu \Delta x \frac{\mu_{p}}{\nu_{p}}}+\frac{\Delta t \cdot\left(1-e^{-\frac{\mu_{n}}{\nu_{n}} \Delta x}\right)}{\nu \Delta x \frac{\mu_{n}}{\nu_{n}}}\right\}+ \\
-W\left(x_{i+1}, t_{j}\right)\left\{h+\frac{\Delta t \cdot\left(1-e^{-\frac{\mu_{p}}{\nu_{p}} \Delta x}\right)}{\nu \Delta x \frac{\mu_{p}}{\nu_{p}}}\right\}=W\left(x_{i}, t_{j+1}\right)+
\end{gathered}
$$




$$
\begin{aligned}
& +\Delta t \cdot\left\{\sum_{k=1}^{N-i-1} \frac{1}{\nu \Delta x}\left[W\left(x_{i+k+1}, t_{j+1}\right)-W\left(x_{i+k}, t_{j+1}\right)\right] .\right. \\
& \cdot \frac{\nu_{p}}{\mu_{p}}\left[e^{-\frac{\mu_{p}}{\nu_{p}} k \Delta x}-e^{-\frac{\mu_{p}}{\nu_{p}}(k+1) \Delta x}\right]+ \\
& +\sum_{k=1}^{N-i-1} \frac{1}{\nu}\left\{W\left(x_{i+k}, t_{j+1}\right)-W\left(x_{i}, t_{j+1}\right)-k\left[W\left(x_{i+k+1}, t_{j+1}\right)-W\left(x_{i+k}, t_{j+1}\right)\right]\right\} . \\
& \cdot\left\{\operatorname{expint}\left(\frac{\mu_{p}}{\nu_{p}} k \Delta x\right)-\operatorname{expint}\left[\frac{\mu_{p}}{\nu_{p}}(k+1) \Delta x\right]\right\}+ \\
& +\sum_{k=1}^{i-1} \frac{1}{\nu \Delta x}\left[W\left(x_{i-k-1}, t_{j+1}\right)-W\left(x_{i-k}, t_{j+1}\right)\right] \frac{\nu_{n}}{\mu_{n}}\left[e^{-\frac{\mu_{n}}{\nu_{n}} k \Delta x}-e^{-\frac{\mu_{n}}{\nu_{n}}(k+1) \Delta x}\right]+ \\
& +\sum_{k=1}^{i-1} \frac{1}{\nu}\left\{W\left(x_{i-k}, t_{j+1}\right)-W\left(x_{i}, t_{j+1}\right)-k\left[W\left(x_{i-k-1}, t_{j+1}\right)-W\left(x_{i-k}, t_{j+1}\right)\right]\right\} \cdot \\
& \cdot\left\{\operatorname{expint}\left(\frac{\mu_{n}}{\nu_{n}} k \Delta x\right)-\operatorname{expint}\left[\frac{\mu_{n}}{\nu_{n}}(k+1) \Delta x\right]\right\}+ \\
& -\frac{1}{\nu} W\left(x_{i}, t_{j+1}\right) \operatorname{expint}\left[(N-i) \frac{\mu_{p}}{\nu_{p}} \Delta x\right]+ \\
& +\frac{1}{\nu}\left\{\left[K-W\left(x_{i}, t_{j+1}\right)\right] \operatorname{expint}\left(\frac{\mu_{n}}{\nu_{n}} i \Delta x\right)+\right. \\
& \left.-e^{x_{i}} \operatorname{expint}\left[\left(\frac{\mu_{n}}{\nu_{n}}+1\right) i \Delta x\right]\right\}+ \\
& -1_{x_{i}<x\left(\tau_{j+1}\right)} \cdot \Delta t \cdot\left\{q e^{x_{i}}-r K+\right. \\
& +\sum_{k=l-i}^{N-i-1}\left\{\frac{W\left(x_{i+k+1}, t_{j+1}\right)-W\left(x_{i+k}, t_{j+1}\right)}{\nu \frac{\mu_{p}}{\nu_{p}} \Delta x} \cdot\left[e^{-\frac{\mu_{p}}{\nu_{p}} k \Delta x}-e^{-\frac{\mu_{p}}{\nu_{p}}(k+1) \Delta x}\right]+\right.
\end{aligned}
$$




$$
\begin{array}{r}
+\frac{1}{\nu}\left[W\left(x_{i+k}, t_{j+1}\right)-k\left(W\left(x_{i+k+1}, t_{j+1}\right)-W\left(x_{i+k}, t_{j+1}\right)\right)\right] \cdot \\
\left.\cdot\left[\operatorname{expint}\left(\frac{\mu_{p}}{\nu_{p}} k \Delta x\right)-\operatorname{expint}\left(\frac{\mu_{p}}{\nu_{p}}(k+1) \Delta x\right)\right]\right\}+ \\
\left.-\frac{K}{\nu} \cdot \operatorname{expint}\left[\frac{\mu_{p}}{\nu_{p}}(l-i) \Delta x\right]+\frac{e^{x_{i}}}{\nu} \cdot \operatorname{expint}\left[\left(\frac{\mu_{p}}{\nu_{p}}-1\right)(l-i) \Delta x\right]\right\}
\end{array}
$$

We can see that the left hand side of the equation can be expressed in tridiagonal matrix form. We can therefore apply Gauss's elimination technique to solve it. Appendix B presents C code to solve numerically the difference equation.

\section{A.6 Numerical Solution of the Jump Integral in the Variance Gamma PIDE for Euro- pean Barrier Options}

European barrier options follow the same PIDE which describes the dynamics of the values for plain vanilla European options, that is

$$
\begin{array}{r}
h W\left(x_{i-1}, t_{j}\right)+(1+r \Delta t) W\left(x_{i}, t_{j}\right)-h W\left(x_{i+1}, t_{j}\right)= \\
=W\left(x_{i}, t_{j+1}\right)+\Delta t \int_{-\infty}^{+\infty}\left[W\left(x_{i}+y, t_{j+1}\right)-W\left(x_{i}, t_{j+1}\right)\right] k(y) d y
\end{array}
$$

We want here to evaluate numerically the integral in this PIDE so that it is possible to write the differential equation as a linear system of equations which can be solved to obtain barrier options prices. We will solve the knockout valuation explicitly, as it is easier than solving knock-in numerically. The correspondent knock-in case can be obtained as difference between plain vanilla and knock-out. Let's start writing the integral as sum of integrals so 
that we can separate different cases of jump size in the following way:

$$
\begin{aligned}
& \int_{-\infty}^{+\infty}\left[W\left(x_{i}+y, t_{j+1}\right)-W\left(x_{i}, t_{j+1}\right)\right] k(y) d y= \\
= & \int_{-\infty}^{x_{0}-x_{i}}\left[W\left(x_{i}+y, t_{j+1}\right)-W\left(x_{i}, t_{j+1}\right)\right] k(y) d y+ \\
+ & \int_{x_{0}-x_{i}}^{-\Delta x}\left[W\left(x_{i}+y, t_{j+1}\right)-W\left(x_{i}, t_{j+1}\right)\right] k(y) d y+ \\
& +\int_{-\Delta x}^{0}\left[W\left(x_{i}+y, t_{j+1}\right)-W\left(x_{i}, t_{j+1}\right)\right] k(y) d y+ \\
& +\int_{0}^{\Delta x}\left[W\left(x_{i}+y, t_{j+1}\right)-W\left(x_{i}, t_{j+1}\right)\right] k(y) d y+ \\
+ & \int_{\Delta x}^{x_{N}-x_{i}}\left[W\left(x_{i}+y, t_{j+1}\right)-W\left(x_{i}, t_{j+1}\right)\right] k(y) d y+ \\
& +\int_{x_{N}-x_{i}}^{+\infty}\left[W\left(x_{i}+y, t_{j+1}\right)-W\left(x_{i}, t_{j+1}\right)\right] k(y) d y
\end{aligned}
$$

As long as the jumps $y$ are not too big and the stock remains inside the range of values considered, the integrals can be valued in the exact same way we did for regular options. In fact, though the values on the boundary are different, being given by the rebate, where the boundary is given by the barrier, inside the boundary the dynamic is unchanged. Hence we do not need to spend more time for integrals from (A.58) to (A.61), their solution is already available in sections A.1.1 and A.1.2. Note that for these just mentioned cases it is not necessary to distinguish between call and put options, however for the remain two integrals, (A.57) and (A.62), it is necessary to distinguish the cases of put and call options as well as the fact that the barrier is below or above the current level of the stock.

\section{A.6.1 Solution of Integral for Up-And-Out Call Op- tions}

We solve as first case an up-and-out call option. In this case the boundary $x_{N}$ is given by $\ln (B)$, where $B$ is the out barrier. Consider integral (A.62), which describes large positive jumps which take the stock price outside the 
barrier. Once the barrier is passed, the option disappears and so its value is zero, however at the time of the first passage the rebate, if it exists, is paid. Hence for $y \in\left[x_{N}-x_{i},+\infty\right]$ we can write

$$
W\left(x_{i}+y, t_{j+1}\right)=R
$$

Hence integral (A.62) becomes

$$
\begin{array}{r}
\int_{x_{N}-x_{i}}^{+\infty}\left[W\left(x_{i}+y, t_{j+1}\right)-W\left(x_{i}, t_{j+1}\right)\right] k(y) d y= \\
=\int_{x_{N}-x_{i}}^{+\infty}\left[R-W\left(x_{i}, t_{j+1}\right)\right] \cdot \frac{e^{-\frac{\mu_{p}}{\nu p} y}}{\nu y} d y
\end{array}
$$

where we also used the definition of variance gamma Lévy measure for positive jumps. It is easy to rewrite this integral using the definition of exponential integral in the following way

$$
\frac{1}{\nu} \cdot\left[R-W\left(x_{i}, t_{j+1}\right)\right] \cdot \operatorname{expint}\left[\frac{\mu_{p}}{\nu_{p}}(N-i) \Delta x\right]
$$

We note that imposing that the option value for jumps larger than $\left(x_{N}-x_{i}\right)$ is always equal to the rebate implies that the rebate is paid both if the stock jumps exactly at the barrier and if the stock jump further. This is necessary because the variance gamma is not a continuous process and therefore it can reach a value larger than $\ln (B)$ without touching $\ln (B)$.

The rebate, if any, has to be paid only once meaning that after the stock has passed the barrier, the options is knocked out and if the stock later on passes the barrier again this is irrelevant and nothing is paid. In the case we are considering here, the double payment of the rebate cannot happen because we intentionally cut the grid at the barrier level and so once the barrier is reached the process is absorbed by the barrier and cannot go back. In particular we can see that when we write the PIDE as a system of equations, the three option values we need to obtain in each equation, $W\left(x_{i-1}, t_{j}\right), W\left(x_{i}, t_{j}\right)$ and $W\left(x_{i+1}, t_{j}\right)$ are a function of options at the previous time $j+1$ which correspond to stock values inside the grid. These stock can jump outside the grid and pass the barrier and this is the case described by equation A.62. However after the barrier is passed the options value is no more considered and only the rebate, if any, is paid. So the three unknown option values we try to solve in each equation do not depend on options corresponding to 
stock already outside the barrier and jumping from a position outside the barrier.

Let's look at integral (A.57) now. Making a change of variable $y \triangleq-y$, this becomes

$$
\int_{x_{i}-x_{0}}^{+\infty}\left[W\left(x_{i}-y, t_{j+1}\right)-W\left(x_{i}, t_{j+1}\right)\right] \frac{e^{-\frac{\mu_{n}}{\nu_{n}} y}}{\nu y} d y
$$

The negative jump takes the stock outside the range on which the grid is defined. Because we are talking about call options, we can impose that the value at the level of the boundary $x_{0}$ and outside it is equal to zero. Formally we can write that for $y \in\left[x_{i}-x_{0},+\infty\right]$ we can approximate

$$
W\left(x_{i}-y, t_{j+1}\right) \simeq 0
$$

It follows that integral (A.64) becomes

$$
\int_{x_{i}-x_{0}}^{+\infty}-W\left(x_{i}, t_{j+1}\right) \frac{e^{-\frac{\mu_{n}}{\nu_{n}} y}}{\nu y} d y
$$

which can be expressed as

$$
-\frac{1}{\nu} W\left(x_{i}, t_{j+1}\right) \cdot \operatorname{expint}\left(\frac{\mu_{n}}{\nu_{n}} i \Delta x\right)
$$

If we combine (A.63) and (A.65) with the results we found for vanilla European options for the other four integrals, that is (A.9), (A.10), (A.12) and (A.13), we can rewrite the integral (A.56) in a proper way to be used in the linear system as

$$
\begin{gathered}
\int_{-\infty}^{+\infty}\left[W\left(x_{i}+y, t_{j+1}\right)-W\left(x_{i}, t_{j+1}\right)\right] k(y) d y= \\
=\frac{\left[W\left(x_{i+1}, t_{j+1}\right)-W\left(x_{i}, t_{j+1}\right)\right]\left(1-e^{-\frac{\mu_{p}}{\nu_{p}} \Delta x}\right)}{\nu \Delta x \frac{\mu_{p}}{\nu_{p}}}+ \\
+\frac{\left[W\left(x_{i-1}, t_{j+1}\right)-W\left(x_{i}, t_{j+1}\right)\right]\left(1-e^{-\frac{\mu_{n}}{\nu_{n}} \Delta x}\right)}{\nu \Delta x \frac{\mu_{n}}{\nu_{n}}}+
\end{gathered}
$$




$$
\begin{aligned}
& +\sum_{k=1}^{N-i-1} \frac{1}{\nu \Delta x}\left[W\left(x_{i+k+1}, t_{j+1}\right)-W\left(x_{i+k}, t_{j+1}\right)\right] \frac{\nu_{p}}{\mu_{p}}\left[e^{-\frac{\mu_{p}}{\nu_{p}} k \Delta x}-e^{-\frac{\mu_{p}}{\nu_{p}}(k+1) \Delta x}\right]+ \\
& +\sum_{k=1}^{N-i-1} \frac{1}{\nu}\left[W\left(x_{i+k}, t_{j+1}\right)-W\left(x_{i}, t_{j+1}\right)-k\left[W\left(x_{i+k+1}, t_{j+1}\right)-W\left(x_{i+k}, t_{j+1}\right)\right]\right] \cdot \\
& \cdot\left\{\operatorname{expint}\left(\frac{\mu_{p}}{\nu_{p}} k \Delta x\right)-\operatorname{expint}\left[\frac{\mu_{p}}{\nu_{p}}(k+1) \Delta x\right]\right\}+ \\
& +\sum_{k=1}^{i-1} \frac{1}{\nu \Delta x}\left[W\left(x_{i-k-1}, t_{j+1}\right)-W\left(x_{i-k}, t_{j+1}\right)\right] \frac{\nu_{n}}{\mu_{n}}\left[e^{-\frac{\mu_{n}}{\nu_{n}} k \Delta x}-e^{-\frac{\mu_{n}}{\nu_{n}}(k+1) \Delta x}\right]+ \\
& +\sum_{k=1}^{i-1} \frac{1}{\nu}\left\{W\left(x_{i-k}, t_{j+1}\right)-W\left(x_{i}, t_{j+1}\right)-k\left[W\left(x_{i-k-1}, t_{j+1}\right)-W\left(x_{i-k}, t_{j+1}\right)\right]\right\} . \\
& \cdot\left\{\operatorname{expint}\left(\frac{\mu_{n}}{\nu_{n}} k \Delta x\right)-\operatorname{expint}\left[\frac{\mu_{n}}{\nu_{n}}(k+1) \Delta x\right]\right\}+ \\
& +\frac{1}{\nu} \cdot\left[R-W\left(x_{i}, t_{j+1}\right)\right] \cdot \operatorname{expint}\left[\frac{\mu_{p}}{\nu_{p}}(N-i) \Delta x\right]+ \\
& -\frac{1}{\nu} W\left(x_{i}, t_{j+1}\right) \cdot \operatorname{expint}\left(\frac{\mu_{n}}{\nu_{n}} i \Delta x\right)
\end{aligned}
$$

To improve the stability of the system, we treat small jumps implicitly by replacing the first two lines of the right hand side of equation (A.66) with

$$
\begin{aligned}
& \frac{\left[W\left(x_{i+1}, t_{j}\right)-W\left(x_{i}, t_{j}\right)\right]\left(1-e^{-\frac{\mu_{p}}{\nu_{p}} \Delta x}\right)}{\nu \Delta x \frac{\mu_{p}}{\nu_{p}}}+ \\
& +\frac{\left[W\left(x_{i-1}, t_{j}\right)-W\left(x_{i}, t_{j}\right)\right]\left(1-e^{-\frac{\mu_{n}}{\nu_{n}} \Delta x}\right)}{\nu \Delta x \frac{\mu_{n}}{\nu_{n}}}
\end{aligned}
$$




\section{A.6.2 Solution of Integral for Down-And-Out Call Op- tions}

When the barrier option is a down-and-out options, the out-barrier can be positioned at the level $x_{0}$. Let's solve first the integral (A.62). In this case the jump takes the stock to a value which is so high that we can assume that the option is exercised for sure; at this level we can also assume that the stock will not reach the barrier anymore. Therefore we can approximate the option value as the discounted value of the payoff; formally we can write for $j>x_{N}-x_{i}$

$$
W\left(x_{i}+y, t_{j+1}\right) \simeq e^{x_{i}+y} e^{-q\left(T-t_{j+1}\right)}-K e^{-r\left(T-t_{j+1}\right)}
$$

therefore we can write

$$
\begin{array}{r}
\int_{(N-i) \Delta x}^{+\infty}\left[W\left(x_{i}+y, t_{j+1}\right)-W\left(x_{i}, t_{j+1}\right)\right] k(y) d y= \\
\int_{(N-i) \Delta x}^{+\infty}\left[e^{x_{i}+y} e^{-q\left(T-t_{j+1}\right)}-K e^{-r\left(T-t_{j+1}\right)}-W\left(x_{i}, t_{j+1}\right)\right] \frac{e^{-\frac{\mu_{p}}{\nu_{p}} y}}{\nu y} d y
\end{array}
$$

which can be solved to obtain

$$
\begin{array}{r}
\frac{1}{\nu}\left\{e^{x_{i}} e^{-q\left(T-t_{j+1}\right)} \text { expint }\left[\left(\frac{\mu_{p}}{\nu_{p}}-1\right)(N-i) \Delta x\right]+\right. \\
\left.-\left[K e^{-r\left(T-t_{j+1}\right)}+W\left(x_{i}, t_{j+1}\right)\right] \text { expint }\left[\frac{\mu_{p}}{\nu_{p}}(N-i) \Delta x\right]\right\}
\end{array}
$$

Note that this is the same result we found for plain vanilla options in equation (A.18). The reason is that when we are at the higher boundary we are very far from the barrier and we can impose that the stock will never reach the barrier and it will be exercised for sure.

Let's now solve equation (A.57). Here the negative jump is so big that we pass the barrier level $x_{0} \triangleq \ln (B)$ and hence the rebate is paid and the option value goes to zero. Using the usual change of variable $y \triangleq-y$ and 
the definition of exponential integral, we can write

$$
\begin{array}{r}
\int_{-\infty}^{x_{0}-x_{i}}\left[W\left(x_{i}+y, t_{j+1}\right)-W\left(x_{i}, t_{j+1}\right)\right] k(y) d y= \\
=\int_{x_{i}-x_{0}}^{+\infty}\left[R-W\left(x_{i}, t_{j+1}\right)\right] \frac{e^{-\frac{\mu_{n}}{\nu_{n}} y}}{\nu y} d y= \\
=\frac{1}{\nu}\left[R-W\left(x_{i}, t_{j+1}\right)\right] \cdot \text { expint }\left(\frac{\mu_{n}}{\nu_{n}} i \Delta x\right)
\end{array}
$$

Similarly to the up-and-out call case, we note that here we do not impose that the option value is zero passed the barrier, but we impose that it is equal to the rebate. This is because, although it is true that the option in itself disappears once the barrier is passed, the cash flow associated to the rebate is received for any jump which passes the barrier for the first time.

Now combining (A.68) and (A.69) with integrals (A.9), (A.10), (A.12) and (A.13) we can express integral (A.56) as

$$
\begin{aligned}
& \int_{-\infty}^{+\infty}\left[W\left(x_{i}+y, t_{j+1}\right)-W\left(x_{i}, t_{j+1}\right)\right] k(y) d y= \\
& =\frac{\left[W\left(x_{i+1}, t_{j+1}\right)-W\left(x_{i}, t_{j+1}\right)\right]\left(1-e^{-\frac{\mu_{p}}{\nu_{p}} \Delta x}\right)}{\nu \Delta x \frac{\mu_{p}}{\nu_{p}}}+ \\
& +\frac{\left[W\left(x_{i-1}, t_{j+1}\right)-W\left(x_{i}, t_{j+1}\right)\right]\left(1-e^{-\frac{\mu_{n}}{\nu_{n}} \Delta x}\right)}{\nu \Delta x \frac{\mu_{n}}{\nu_{n}}}+
\end{aligned}
$$

$$
\begin{gathered}
+\sum_{k=1}^{N-i-1} \frac{1}{\nu \Delta x}\left[W\left(x_{i+k+1}, t_{j+1}\right)-W\left(x_{i+k}, t_{j+1}\right)\right] \frac{\nu_{p}}{\mu_{p}}\left[e^{-\frac{\mu_{p}}{\nu_{p}} k \Delta x}-e^{-\frac{\mu_{p}}{\nu_{p}}(k+1) \Delta x}\right]+ \\
+\sum_{k=1}^{N-i-1} \frac{1}{\nu}\left\{W\left(x_{i+k}, t_{j+1}\right)-W\left(x_{i}, t_{j+1}\right)-k\left[W\left(x_{i+k+1}, t_{j+1}\right)-W\left(x_{i+k}, t_{j+1}\right)\right]\right\} . \\
\cdot\left\{\operatorname{expint}\left(\frac{\mu_{p}}{\nu_{p}} k \Delta x\right)-\operatorname{expint}\left[\frac{\mu_{p}}{\nu_{p}}(k+1) \Delta x\right]\right\}+ \\
+\sum_{k=1}^{i-1} \frac{1}{\nu \Delta x}\left[W\left(x_{i-k-1}, t_{j+1}\right)-W\left(x_{i-k}, t_{j+1}\right)\right] \frac{\nu_{n}}{\mu_{n}}\left[e^{-\frac{\mu_{n}}{\nu_{n}} k \Delta x}-e^{-\frac{\mu_{n}}{\nu_{n}}(k+1) \Delta x}\right]+
\end{gathered}
$$




$$
\begin{gathered}
+\sum_{k=1}^{i-1} \frac{1}{\nu}\left\{W\left(x_{i-k}, t_{j+1}\right)-W\left(x_{i}, t_{j+1}\right)-k\left[W\left(x_{i-k-1}, t_{j+1}\right)-W\left(x_{i-k}, t_{j+1}\right)\right]\right\} \cdot \\
\cdot\left\{\text { expint }\left(\frac{\mu_{n}}{\nu_{n}} k \Delta x\right)-\text { expint }\left[\frac{\mu_{n}}{\nu_{n}}(k+1) \Delta x\right]\right\}+ \\
+\frac{1}{\nu}\left\{e^{x_{i}} e^{-q\left(T-t_{j+1}\right)} \operatorname{expint}\left[\left(\frac{\mu_{p}}{\nu_{p}}-1\right)(N-i) \Delta x\right]+\right. \\
-\left[K e^{-r\left(T-t_{j+1}\right)}+W\left(x_{i}, t_{j+1}\right)\right] \operatorname{expint}\left[\frac{\mu_{p}}{\nu_{p}}(N-i) \Delta x\right]+ \\
\left.+\left[R-W\left(x_{i}, t_{j+1}\right)\right] \cdot \operatorname{expint}\left(\frac{\mu_{n}}{\nu_{n}} i \Delta x\right)\right\}
\end{gathered}
$$

To improve the stability of the system, we treat small jumps implicitly by replacing the first two lines of the right hand side of equation (A.70) with

$$
\begin{aligned}
& \frac{\left[W\left(x_{i+1}, t_{j}\right)-W\left(x_{i}, t_{j}\right)\right]\left(1-e^{-\frac{\mu_{p}}{\nu_{p}} \Delta x}\right)}{\nu \Delta x \frac{\mu_{p}}{\nu_{p}}}+ \\
& +\frac{\left[W\left(x_{i-1}, t_{j}\right)-W\left(x_{i}, t_{j}\right)\right]\left(1-e^{-\frac{\mu_{n}}{\nu_{n}} \Delta x}\right)}{\nu \Delta x \frac{\mu_{n}}{\nu_{n}}}
\end{aligned}
$$

\section{A.6.3 Solution of Integral for Up-And-Out Put Op- tions}

When we look at the upper boundary $x_{N}$, this is set at the value of the barrier expressed as $\ln (B)$. As in the up-and-out call case, the option value is given by the rebate, as the option in itself is knocked out, hence we get

$$
\begin{array}{r}
\int_{x_{N}-x_{i}}^{+\infty}\left[W\left(x_{i}+y, t_{j+1}\right)-W\left(x_{i}, t_{j+1}\right)\right] k(y) d y= \\
\frac{1}{\nu}\left[R-W\left(x_{i}, t_{j+1}\right)\right] \cdot \text { expint }\left[\frac{\mu_{p}}{\nu_{p}}(N-i) \Delta x\right]
\end{array}
$$


In the case of large negative jumps, on the other side, we can impose that the put will be exercised and we can approximate its value with the discounted payoff. For $y>\left(x_{i}-x_{0}\right)$ we write

$$
W\left(x_{i}-y, t_{j+1}\right) \simeq K e^{-r\left(T-t_{j+1}\right)}-e^{x_{i}-y} e^{-q\left(T-t_{j+1}\right)}
$$

and hence we solve

$$
\begin{gathered}
\int_{-\infty}^{x_{0}-x_{i}}\left[W\left(x_{i}+y, t_{j+1}\right)-W\left(x_{i}, t_{j+1}\right)\right] \frac{e^{-\frac{\mu_{n}}{\nu_{n}}|y|}}{\nu|y|} d y= \\
=\int_{x_{i}-x_{0}}^{+\infty}\left[K e^{-r\left(T-t_{j+1}\right)}-e^{x_{i}-y} e^{-q\left(T-t_{j+1}\right)}-W\left(x_{i}, t_{j+1}\right)\right] \frac{e^{-\frac{\mu_{n}}{\nu_{n}} y}}{\nu y} d y= \\
=\frac{1}{\nu}\left\{\left[K e^{-r\left(T-t_{j+1}\right)}-W\left(x_{i}, t_{j+1}\right)\right] \operatorname{expint}\left(\frac{\mu_{n}}{\nu_{n}} i \Delta x\right)+\right. \\
\left.-e^{x_{i}} e^{-q\left(T-t_{j+1}\right)} \operatorname{expint}\left[\left(\frac{\mu_{n}}{\nu_{n}}+1\right) i \Delta x\right]\right\}
\end{gathered}
$$

So combining expressions (A.72) and (A.73) with (A.9), (A.10), (A.12) and (A.13) we can write

$$
\begin{aligned}
& \int_{-\infty}^{+\infty}\left[W\left(x_{i}+y, t_{j+1}\right)-W\left(x_{i}, t_{j+1}\right)\right] k(y) d y= \\
= & \frac{\left[W\left(x_{i+1}, t_{j+1}\right)-W\left(x_{i}, t_{j+1}\right)\right]\left(1-e^{-\frac{\mu_{p}}{\nu_{p}} \Delta x}\right)}{\nu \Delta x \frac{\mu_{p}}{\nu_{p}}}+ \\
+ & \frac{\left[W\left(x_{i-1}, t_{j+1}\right)-W\left(x_{i}, t_{j+1}\right)\right]\left(1-e^{-\frac{\mu_{n}}{\nu_{n}} \Delta x}\right)}{\nu \Delta x x_{n}}+
\end{aligned}
$$

$$
\begin{gathered}
+\sum_{k=1}^{N-i-1} \frac{1}{\nu \Delta x}\left[W\left(x_{i+k+1}, t_{j+1}\right)-W\left(x_{i+k}, t_{j+1}\right)\right] \cdot \frac{\nu_{p}}{\mu_{p}}\left[e^{-\frac{\mu_{p}}{\nu_{p}} k \Delta x}-e^{-\frac{\mu_{p}}{\nu_{p}}(k+1) \Delta x}\right]+ \\
+\sum_{k=1}^{N-i-1} \frac{1}{\nu}\left\{W\left(x_{i+k}, t_{j+1}\right)-W\left(x_{i}, t_{j+1}\right)-k\left[W\left(x_{i+k+1}, t_{j+1}\right)-W\left(x_{i+k}, t_{j+1}\right)\right]\right\} \cdot \\
\cdot\left\{\text { expint }\left(\frac{\mu_{p}}{\nu_{p}} k \Delta x\right)-\text { expint }\left[\frac{\mu_{p}}{\nu_{p}}(k+1) \Delta x\right]\right\}+
\end{gathered}
$$




$$
\begin{gathered}
+\sum_{k=1}^{i-1} \frac{1}{\nu \Delta x}\left[W\left(x_{i-k-1}, t_{j+1}\right)-W\left(x_{i-k}, t_{j+1}\right)\right] \cdot \frac{\nu_{n}}{\mu_{n}}\left[e^{-\frac{\mu_{n}}{\nu_{n}} k \Delta x}-e^{-\frac{\mu_{n}}{\nu_{n}}(k+1) \Delta x}\right]+ \\
+\sum_{k=1}^{i-1} \frac{1}{\nu}\left\{W\left(x_{i-k}, t_{j+1}\right)-W\left(x_{i}, t_{j+1}\right)-k\left[W\left(x_{i-k-1}, t_{j+1}\right)-W\left(x_{i-k}, t_{j+1}\right)\right]\right\} \\
\cdot\left\{\operatorname{expint}\left(\frac{\mu_{n}}{\nu_{n}} k \Delta x\right)-\operatorname{expint}\left[\frac{\mu_{n}}{\nu_{n}}(k+1) \Delta x\right]\right\}+ \\
+\frac{1}{\nu}\left[R-W\left(x_{i}, t_{j+1}\right)\right] \cdot \operatorname{expint}\left[\frac{\mu_{p}}{\nu_{p}}(N-i) \Delta x\right] \\
+\frac{1}{\nu}\left\{\left[K e^{-r\left(T-t_{j+1}\right)}-W\left(x_{i}, t_{j+1}\right)\right] \operatorname{expint}\left(\frac{\mu_{n}}{\nu_{n}} i \Delta x\right)+\right. \\
\left.-e^{x_{i}} e^{-q\left(T-t_{j+1}\right)} \operatorname{expint}^{-}\left[\left(\frac{\mu_{n}}{\nu_{n}}+1\right) i \Delta x\right]\right\}
\end{gathered}
$$

To improve the stability of the system, we treat small jumps implicitly by replacing the first two lines of the right hand side of equation (A.74) with

$$
\begin{aligned}
& \frac{\left[W\left(x_{i+1}, t_{j}\right)-W\left(x_{i}, t_{j}\right)\right]\left(1-e^{-\frac{\mu_{p}}{\nu_{p}} \Delta x}\right)}{\nu \Delta x \frac{\mu_{p}}{\nu_{p}}}+ \\
& +\frac{\left[W\left(x_{i-1}, t_{j}\right)-W\left(x_{i}, t_{j}\right)\right]\left(1-e^{-\frac{\mu_{n}}{\nu_{n}} \Delta x}\right)}{\nu \Delta x \frac{\mu_{n}}{\nu_{n}}}
\end{aligned}
$$

\section{A.6.4 Solution of Integral for Down-And-Out Put Op- tions}

Finally, for the down-and-out put option, we can impose that the option is worthless if the stock has a large an positive jump because it is very deep 
out of the money. Hence we can impose

$$
\begin{array}{r}
\int_{x_{N}-x_{i}}^{+\infty}\left[W\left(x_{i}+y, t_{j+1}\right)-W\left(x_{i}, t_{j+1}\right)\right] k(y) d y= \\
-\frac{1}{\nu} W\left(x_{i}, t_{j+1}\right) \cdot \text { expint }\left[\frac{\mu_{p}}{\nu_{p}}(N-i) \Delta x\right]
\end{array}
$$

If the jump is large and negative, on the other side, the stock will pass the barrier $x_{0} \triangleq \ln (B)$ and the rebate is going to be paid, as the option disappears. We can write

$$
\begin{array}{r}
\int_{-\infty}^{x_{0}-x_{i}}\left[W\left(x_{i}+y, t_{j+1}\right)-W\left(x_{i}, t_{j+1}\right)\right] k(y) d y= \\
=\frac{1}{\nu}\left[R-W\left(x_{i}, t_{j+1}\right)\right] \cdot \operatorname{expint}\left(\frac{\mu_{n}}{\nu_{n}} i \Delta x\right)
\end{array}
$$

The combination of (A.76) and (A.77) with (A.9), (A.10), (A.12) and (A.13) gives in the case of down-and-out put options

$$
\begin{aligned}
& \int_{-\infty}^{+\infty}\left[W\left(x_{i}+y, t_{j+1}\right)-W\left(x_{i}, t_{j+1}\right)\right] k(y) d y= \\
& =\frac{\left[W\left(x_{i+1}, t_{j+1}\right)-W\left(x_{i}, t_{j+1}\right)\right]\left(1-e^{-\frac{\mu_{p}}{\nu_{p}} \Delta x}\right)}{\nu \Delta x \frac{\mu_{p}}{\nu_{p}}}+ \\
& +\frac{\left[W\left(x_{i-1}, t_{j+1}\right)-W\left(x_{i}, t_{j+1}\right)\right]\left(1-e^{-\frac{\mu_{n}}{\nu_{n}} \Delta x}\right)}{\nu \Delta x \frac{\mu_{n}}{\nu_{n}}}+
\end{aligned}
$$

$+\sum_{k=1}^{N-i-1} \frac{1}{\nu \Delta x}\left[W\left(x_{i+k+1}, t_{j+1}\right)-W\left(x_{i+k}, t_{j+1}\right)\right] \cdot \frac{\nu_{p}}{\mu_{p}}\left[e^{-\frac{\mu_{p}}{\nu_{p}} k \Delta x}-e^{-\frac{\mu_{p}}{\nu_{p}}(k+1) \Delta x}\right]+$ $+\sum_{k=1}^{N-i-1} \frac{1}{\nu}\left[W\left(x_{i+k}, t_{j+1}\right)-W\left(x_{i}, t_{j+1}\right)-k\left[W\left(x_{i+k+1}, t_{j+1}\right)-W\left(x_{i+k}, t_{j+1}\right)\right]\right]$.

$$
\cdot\left\{\operatorname{expint}\left(\frac{\mu_{p}}{\nu_{p}} k \Delta x\right)-\operatorname{expint}\left[\frac{\mu_{p}}{\nu_{p}}(k+1) \Delta x\right]\right\}+
$$




$$
\begin{gathered}
+\sum_{k=1}^{i-1} \frac{1}{\nu \Delta x}\left[W\left(x_{i-k-1}, t_{j+1}\right)-W\left(x_{i-k}, t_{j+1}\right)\right] \cdot \frac{\nu_{n}}{\mu_{n}}\left[e^{-\frac{\mu_{n}}{\nu_{n}} k \Delta x}-e^{-\frac{\mu_{n}}{\nu_{n}}(k+1) \Delta x}\right]+ \\
+\sum_{k=1}^{i-1} \frac{1}{\nu}\left\{W\left(x_{i-k}, t_{j+1}\right)-W\left(x_{i}, t_{j+1}\right)-k\left[W\left(x_{i-k-1}, t_{j+1}\right)-W\left(x_{i-k}, t_{j+1}\right)\right]\right\} \\
\cdot\left\{\operatorname{expint}\left(\frac{\mu_{n}}{\nu_{n}} k \Delta x\right)-\operatorname{expint}\left[\frac{\mu_{n}}{\nu_{n}}(k+1) \Delta x\right]\right\}+ \\
-\frac{1}{\nu} W\left(x_{i}, t_{j+1}\right) \cdot \operatorname{expint}\left[\frac{\mu_{p}}{\nu_{p}}(N-i) \Delta x\right]+ \\
+\frac{1}{\nu}\left[R-W\left(x_{i}, t_{j+1}\right)\right] \operatorname{expint}\left(\frac{\mu_{n}}{\nu_{n}} i \Delta x\right)
\end{gathered}
$$

To improve the stability of the system, we treat small jumps implicitly by replacing the first two lines of the right hand side of equation (A.78) with

$$
\begin{aligned}
& \frac{\left[W\left(x_{i+1}, t_{j}\right)-W\left(x_{i}, t_{j}\right)\right]\left(1-e^{-\frac{\mu_{p}}{\nu_{p}} \Delta x}\right)}{\nu \Delta x \frac{\mu_{p}}{\nu_{p}}}+ \\
& +\frac{\left[W\left(x_{i-1}, t_{j}\right)-W\left(x_{i}, t_{j}\right)\right]\left(1-e^{-\frac{\mu_{n}}{\nu_{n}} \Delta x}\right)}{\nu \Delta x_{\mu_{n}}^{\mu_{n}}}
\end{aligned}
$$

\section{A.7 Variance Gamma Difference Equation for European Barrier Options}

\section{A.7.1 Difference Equation for European Up-And-Out Calls}

To obtain the difference equation to price European up-and-out calls under variance gamma, we have to replace the integral in equation (A.55) with its 
discrete approximation given by equation (A.66) as adjusted by expression (A.67)

$$
\begin{aligned}
& W\left(x_{i-1}, t_{j}\right)\left\{h-\frac{\Delta t \cdot\left(1-e^{-\frac{\mu_{n}}{\nu_{n}} \Delta x}\right)}{\nu \Delta x \frac{\mu_{n}}{\nu_{n}}}\right\}+ \\
& +W\left(x_{i}, t_{j}\right)\left\{(1+r \Delta t)+\frac{\Delta t \cdot\left(1-e^{-\frac{\mu_{p}}{\nu_{p}} \Delta x}\right)}{\nu \Delta x \frac{\mu_{p}}{\nu_{p}}}+\frac{\Delta t \cdot\left(1-e^{-\frac{\mu_{n}}{\nu_{n}} \Delta x}\right)}{\nu \Delta x \frac{\mu_{n}}{\nu_{n}}}\right\}+ \\
& -W\left(x_{i+1}, t_{j}\right)\left\{h+\frac{\Delta t \cdot\left(1-e^{-\frac{\mu_{p}}{\nu_{p}} \Delta x}\right)}{\nu \Delta x \frac{\mu_{p}}{\nu_{p}}}\right\}=W\left(x_{i}, t_{j+1}\right)+ \\
& +\Delta t \cdot\left\{\sum_{k=1}^{N-i-1} \frac{1}{\nu \Delta x}\left[W\left(x_{i+k+1}, t_{j+1}\right)-W\left(x_{i+k}, t_{j+1}\right)\right] \cdot\right. \\
& \cdot \frac{\nu_{p}}{\mu_{p}}\left[e^{-\frac{\mu_{p}}{\nu_{p}} k \Delta x}-e^{-\frac{\mu_{p}}{\nu_{p}}(k+1) \Delta x}\right]+ \\
& +\sum_{k=1}^{N-i-1} \frac{1}{\nu}\left\{W\left(x_{i+k}, t_{j+1}\right)-W\left(x_{i}, t_{j+1}\right)-k\left[W\left(x_{i+k+1}, t_{j+1}\right)-W\left(x_{i+k}, t_{j+1}\right)\right]\right\} . \\
& \cdot\left\{\operatorname{expint}\left(\frac{\mu_{p}}{\nu_{p}} k \Delta x\right)-\operatorname{expint}\left[\frac{\mu_{p}}{\nu_{p}}(k+1) \Delta x\right]\right\}+ \\
& +\sum_{k=1}^{i-1} \frac{1}{\nu \Delta x}\left[W\left(x_{i-k-1}, t_{j+1}\right)-W\left(x_{i-k}, t_{j+1}\right)\right] \frac{\nu_{n}}{\mu_{n}}\left[e^{-\frac{\mu_{n}}{\nu_{n}} k \Delta x}-e^{-\frac{\mu_{n}}{\nu_{n}}(k+1) \Delta x}\right]+ \\
& +\sum_{k=1}^{i-1} \frac{1}{\nu}\left\{W\left(x_{i-k}, t_{j+1}\right)-W\left(x_{i}, t_{j+1}\right)-k\left[W\left(x_{i-k-1}, t_{j+1}\right)-W\left(x_{i-k}, t_{j+1}\right)\right]\right\} . \\
& \cdot\left\{\operatorname{expint}\left(\frac{\mu_{n}}{\nu_{n}} k \Delta x\right)-\operatorname{expint}\left[\frac{\mu_{n}}{\nu_{n}}(k+1) \Delta x\right]\right\}+ \\
& +\frac{1}{\nu}\left[R-W\left(x_{i}, t_{j+1}\right)\right] \cdot \operatorname{expint}\left[\frac{\mu_{p}}{\nu_{p}}(N-i) \Delta x\right]+ \\
& \left.-\frac{1}{\nu} W\left(x_{i}, t_{j+1}\right) \cdot \operatorname{expint}\left(\frac{\mu_{n}}{\nu_{n}} i \Delta x\right)\right\}
\end{aligned}
$$


The left hand side of this difference equation can be written in tridiagonal matrix form, hence the system can be solved using the Gauss's elimination technique. Appendix B reports $\mathrm{C}$ code which can be used to solve numerically this difference equation.

\section{A.7.2 Difference Equation for European Down-And- Out Calls}

To obtain the difference equation to price European down-and-out calls under variance gamma, we have to replace the integral in equation (A.55) with its discrete approximation given by equation (A.70) as adjusted by expression (A.71)

$$
\begin{gathered}
W\left(x_{i-1}, t_{j}\right)\left\{h-\frac{\Delta t \cdot\left(1-e^{-\frac{\mu_{n}}{\nu_{n}} \Delta x}\right)}{\nu \Delta x \frac{\mu_{n}}{\nu_{n}}}\right\}+ \\
+W\left(x_{i}, t_{j}\right)\left\{(1+r \Delta t)+\frac{\Delta t \cdot\left(1-e^{-\frac{\mu_{p}}{\nu_{p}} \Delta x}\right)}{\nu \Delta x \frac{\mu_{p}}{\nu_{p}}}+\frac{\Delta t \cdot\left(1-e^{-\frac{\mu_{n}}{\nu_{n}} \Delta x}\right)}{\nu \Delta x \frac{\mu_{n}}{\nu_{n}}}\right\}+ \\
-W\left(x_{i+1}, t_{j}\right)\left\{h+\frac{\Delta t \cdot\left(1-e^{-\frac{\mu_{p}}{\nu_{p}} \Delta x}\right)}{\nu \Delta x \frac{\mu_{p}}{\nu_{p}}}\right\}=W\left(x_{i}, t_{j+1}\right)+ \\
+\Delta t \cdot\left\{\sum_{k=1}^{N-i-1} \frac{1}{\nu \Delta x}\left[W\left(x_{i+k+1}, t_{j+1}\right)-W\left(x_{i+k}, t_{j+1}\right)\right] \cdot\right. \\
+\sum_{k=1}^{N-i-1} \frac{1}{\nu}\left\{W\left(x_{i+k}, t_{j+1}\right)-W\left(x_{i}, t_{j+1}\right)-k\left[W\left(x_{i+k+1}, t_{j+1}\right)-W\left(x_{i+k}, t_{j+1}\right)\right]\right\} \\
+\left\{e^{-\frac{\mu_{p}}{\nu_{p}} k \Delta x}-e^{-\frac{\mu_{p}}{\nu_{p}}(k+1) \Delta x}\right]+ \\
+\sum_{k=1}^{\mu_{p}} \frac{1}{\nu \Delta x}\left[W\left(x_{i-k-1}, t_{j+1}\right)-W\left(x_{i-k}, t_{j+1}\right)\right] \frac{\nu_{n}}{\mu_{n}}\left[e^{-\frac{\mu_{n}}{\nu_{n}} k \Delta x}-e^{-\frac{\mu_{n}}{\nu_{n}}(k+1) \Delta x}\right]+ \\
\left.\mu_{p} k \Delta x\right)-e x p i n t\left[\frac{\mu_{p}}{\nu_{p}}(k+1) \Delta x\right]+
\end{gathered}
$$




$$
\begin{gathered}
+\sum_{k=1}^{i-1} \frac{1}{\nu}\left\{W\left(x_{i-k}, t_{j+1}\right)-W\left(x_{i}, t_{j+1}\right)-k\left[W\left(x_{i-k-1}, t_{j+1}\right)-W\left(x_{i-k}, t_{j+1}\right)\right]\right\} \\
\cdot\left\{\operatorname{expint}\left(\frac{\mu_{n}}{\nu_{n}} k \Delta x\right)-\operatorname{expint}\left[\frac{\mu_{n}}{\nu_{n}}(k+1) \Delta x\right]\right\}+ \\
+\frac{1}{\nu}\left\{e^{x_{i}} e^{-q\left(T-t_{j+1}\right)} \operatorname{expint}\left[\left(\frac{\mu_{p}}{\nu_{p}}-1\right)(N-i) \Delta x\right]+\right. \\
-\left[K e^{-r\left(T-t_{j+1}\right)}+W\left(x_{i}, t_{j+1}\right)\right] \operatorname{expint}\left[\frac{\mu_{p}}{\nu_{p}}(N-i) \Delta x\right]+ \\
\left.\left.+\left[R-W\left(x_{i}, t_{j+1}\right)\right] \cdot \operatorname{expint}\left(\frac{\mu_{n}}{\nu_{n}} i \Delta x\right)\right\}\right\}
\end{gathered}
$$

The left hand side of this difference equation can be written in tridiagonal matrix form, hence the system can be solved using the Gauss's elimination technique. Appendix B reports $\mathrm{C}$ code which can be used to solve numerically this difference equation.

\section{A.7.3 Difference Equation for European Up-And-Out Puts}

To obtain the difference equation to price European up-and-out puts under variance gamma, we have to replace the integral in equation (A.55) with its discrete approximation given by equation (A.74) as adjusted by expression (A.75)

$$
\begin{gathered}
W\left(x_{i-1}, t_{j}\right)\left\{h-\frac{\Delta t \cdot\left(1-e^{-\frac{\mu_{n}}{\nu_{n}} \Delta x}\right)}{\nu \Delta x \frac{\mu_{n}}{\nu_{n}}}\right\}+ \\
+W\left(x_{i}, t_{j}\right)\left\{(1+r \Delta t)+\frac{\Delta t \cdot\left(1-e^{-\frac{\mu_{p}}{\nu_{p}} \Delta x}\right)}{\nu \Delta x \frac{\mu_{p}}{\nu_{p}}}+\frac{\Delta t \cdot\left(1-e^{-\frac{\mu_{n}}{\nu_{n}} \Delta x}\right)}{\nu \Delta x \frac{\mu_{n}}{\nu_{n}}}\right\}+ \\
-W\left(x_{i+1}, t_{j}\right)\left\{h+\frac{\Delta t \cdot\left(1-e^{-\frac{\mu_{p}}{\nu_{p}} \Delta x}\right)}{\nu \Delta x \frac{\mu_{p}}{\nu_{p}}}\right\}=W\left(x_{i}, t_{j+1}\right)+
\end{gathered}
$$




$$
\begin{aligned}
& +\Delta t \cdot\left\{\sum_{k=1}^{N-i-1} \frac{1}{\nu \Delta x}\left[W\left(x_{i+k+1}, t_{j+1}\right)-W\left(x_{i+k}, t_{j+1}\right)\right] \cdot\right. \\
& \cdot \frac{\nu_{p}}{\mu_{p}}\left[e^{-\frac{\mu_{p}}{\nu_{p}} k \Delta x}-e^{-\frac{\mu_{p}}{\nu_{p}}(k+1) \Delta x}\right]+ \\
& +\sum_{k=1}^{N-i-1} \frac{1}{\nu}\left\{W\left(x_{i+k}, t_{j+1}\right)-W\left(x_{i}, t_{j+1}\right)-k\left[W\left(x_{i+k+1}, t_{j+1}\right)-W\left(x_{i+k}, t_{j+1}\right)\right]\right\} . \\
& \cdot\left\{\operatorname{expint}\left(\frac{\mu_{p}}{\nu_{p}} k \Delta x\right)-\operatorname{expint}\left[\frac{\mu_{p}}{\nu_{p}}(k+1) \Delta x\right]\right\}+ \\
& +\sum_{k=1}^{i-1} \frac{1}{\nu \Delta x}\left[W\left(x_{i-k-1}, t_{j+1}\right)-W\left(x_{i-k}, t_{j+1}\right)\right] \cdot \frac{\nu_{n}}{\mu_{n}}\left[e^{-\frac{\mu_{n}}{\nu_{n}} k \Delta x}-e^{-\frac{\mu_{n}}{\nu_{n}}(k+1) \Delta x}\right]+ \\
& +\sum_{k=1}^{i-1} \frac{1}{\nu}\left\{W\left(x_{i-k}, t_{j+1}\right)-W\left(x_{i}, t_{j+1}\right)-k\left[W\left(x_{i-k-1}, t_{j+1}\right)-W\left(x_{i-k}, t_{j+1}\right)\right]\right\} . \\
& \cdot\left\{\operatorname{expint}\left(\frac{\mu_{n}}{\nu_{n}} k \Delta x\right)-\operatorname{expint}\left[\frac{\mu_{n}}{\nu_{n}}(k+1) \Delta x\right]\right\}+ \\
& +\frac{1}{\nu}\left[R-W\left(x_{i}, t_{j+1}\right)\right] \cdot \operatorname{expint}\left[\frac{\mu_{p}}{\nu_{p}}(N-i) \Delta x\right] \\
& +\frac{1}{\nu}\left\{\left[K e^{-r\left(T-t_{j+1}\right)}-W\left(x_{i}, t_{j+1}\right)\right] \operatorname{expint}\left(\frac{\mu_{n}}{\nu_{n}} i \Delta x\right)+\right. \\
& \left.\left.-e^{x_{i}} e^{-q\left(T-t_{j+1}\right)} \operatorname{expint}\left[\left(\frac{\mu_{n}}{\nu_{n}}+1\right) i \Delta x\right]\right\}\right\}
\end{aligned}
$$

The left hand side of this difference equation can be written in tridiagonal matrix form, hence the system can be solved using the Gauss's elimination technique. Appendix B reports $\mathrm{C}$ code which can be used to solve numerically this difference equation. 


\section{A.7.4 Difference Equation for European Down-And- Out Puts}

To obtain the difference equation to price European down-and-out puts under variance gamma, we have to replace the integral in equation (A.55) with its discrete approximation given by equation (A.78) as adjusted by expression (A.79)

$$
\begin{aligned}
& W\left(x_{i-1}, t_{j}\right)\left\{h-\frac{\Delta t \cdot\left(1-e^{-\frac{\mu_{n}}{\nu_{n}} \Delta x}\right)}{\nu \Delta x \frac{\mu_{n}}{\nu_{n}}}\right\}+ \\
& +W\left(x_{i}, t_{j}\right)\left\{(1+r \Delta t)+\frac{\Delta t \cdot\left(1-e^{-\frac{\mu_{p}}{\nu_{p}} \Delta x}\right)}{\nu \Delta x \frac{\mu_{p}}{\nu_{p}}}+\frac{\Delta t \cdot\left(1-e^{-\frac{\mu_{n}}{\nu_{n}} \Delta x}\right)}{\nu \Delta x \frac{\mu_{n}}{\nu_{n}}}\right\}+ \\
& -W\left(x_{i+1}, t_{j}\right)\left\{h+\frac{\Delta t \cdot\left(1-e^{-\frac{\mu_{p}}{\nu_{p}} \Delta x}\right)}{\nu \Delta x \frac{\mu_{p}}{\nu_{p}}}\right\}=W\left(x_{i}, t_{j+1}\right)+ \\
& +\Delta t \cdot\left\{\sum_{k=1}^{N-i-1} \frac{1}{\nu \Delta x}\left[W\left(x_{i+k+1}, t_{j+1}\right)-W\left(x_{i+k}, t_{j+1}\right)\right] \cdot\right. \\
& \cdot \frac{\nu_{p}}{\mu_{p}}\left[e^{-\frac{\mu_{p}}{\nu_{p}} k \Delta x}-e^{-\frac{\mu_{p}}{\nu_{p}}(k+1) \Delta x}\right]+ \\
& +\sum_{k=1}^{N-i-1} \frac{1}{\nu}\left[W\left(x_{i+k}, t_{j+1}\right)-W\left(x_{i}, t_{j+1}\right)-k\left[W\left(x_{i+k+1}, t_{j+1}\right)-W\left(x_{i+k}, t_{j+1}\right)\right]\right] . \\
& \cdot\left\{\operatorname{expint}\left(\frac{\mu_{p}}{\nu_{p}} k \Delta x\right)-\operatorname{expint}\left[\frac{\mu_{p}}{\nu_{p}}(k+1) \Delta x\right]\right\}+ \\
& +\sum_{k=1}^{i-1} \frac{1}{\nu \Delta x}\left[W\left(x_{i-k-1}, t_{j+1}\right)-W\left(x_{i-k}, t_{j+1}\right)\right] \cdot \frac{\nu_{n}}{\mu_{n}}\left[e^{-\frac{\mu_{n}}{\nu_{n}} k \Delta x}-e^{-\frac{\mu_{n}}{\nu_{n}}(k+1) \Delta x}\right]+ \\
& +\sum_{k=1}^{i-1} \frac{1}{\nu}\left\{W\left(x_{i-k}, t_{j+1}\right)-W\left(x_{i}, t_{j+1}\right)-k\left[W\left(x_{i-k-1}, t_{j+1}\right)-W\left(x_{i-k}, t_{j+1}\right)\right]\right\} . \\
& \cdot\left\{\operatorname{expint}\left(\frac{\mu_{n}}{\nu_{n}} k \Delta x\right)-\operatorname{expint}\left[\frac{\mu_{n}}{\nu_{n}}(k+1) \Delta x\right]\right\}+
\end{aligned}
$$




$$
\begin{aligned}
& -\frac{1}{\nu} W\left(x_{i}, t_{j+1}\right) \cdot \operatorname{expint}\left[\frac{\mu_{p}}{\nu_{p}}(N-i) \Delta x\right]+ \\
& \left.+\frac{1}{\nu}\left[R-W\left(x_{i}, t_{j+1}\right)\right] \operatorname{expint}\left(\frac{\mu_{n}}{\nu_{n}} i \Delta x\right)\right\}
\end{aligned}
$$

The left hand side of this difference equation can be written in tridiagonal matrix form, hence the system can be solved using the Gauss's elimination technique. Appendix B reports $\mathrm{C}$ code which can be used to solve numerically this difference equation.

\section{A.8 Numerical Solution of Jump Integral in the Variance Gamma PIDE for Ameri- can Barrier Options}

Consider the American barrier option PIDE, given by

$$
\begin{aligned}
& h W\left(x_{i-1}, t_{j}\right)+(1+r \Delta t) W\left(x_{i}, t_{j}\right)-h W\left(x_{i+1}, t_{j}\right)=W\left(x_{i}, t_{j+1}\right)+ \\
& +\Delta t \int_{-\infty}^{+\infty}\left[W\left(x_{i}+y, t_{j+1}\right)-W\left(x_{i}, t_{j+1}\right)\right] k(y) d y-1_{x_{i}>x\left(\tau_{j+1}\right)} \cdot \Delta t . \\
& \cdot\left\{r K-q e^{x_{i}}+\int_{-\infty}^{x\left(\tau_{j+1}\right)-x_{i}}\left[W\left(x_{i}+y, t_{j+1}\right)+K-e^{x_{i}+y}\right] k(y) d y\right\}
\end{aligned}
$$

for call options and by

$$
\begin{aligned}
& h W\left(x_{i-1}, t_{j}\right)+(1+r \Delta t) W\left(x_{i}, t_{j}\right)-h W\left(x_{i+1}, t_{j}\right)=W\left(x_{i}, t_{j+1}\right)+ \\
& +\Delta t \int_{-\infty}^{+\infty}\left[W\left(x_{i}+y, t_{j+1}\right)-W\left(x_{i}, t_{j+1}\right)\right] k(y) d y-1_{x_{i}<x\left(\tau_{j+1}\right)} \cdot \Delta t . \\
& \cdot\left\{q e^{x_{i}}-r K+\int_{x\left(\tau_{j+1}\right)-x_{i}}^{+\infty}\left[W\left(x_{i}+y, t_{j+1}\right)-K+e^{x_{i}+y}\right] k(y) d y\right\}
\end{aligned}
$$


for put options. We define the first integral in those equations as a the "jump integral" and the second integral as "integral in the heaviside term". We are interested here in rewriting the jump integral in a proper way to be solved numerically. As usual we approach it by splitting it in several integrals depending on the size of the jump.

$$
\begin{aligned}
& \int_{-\infty}^{+\infty}\left[W\left(x_{i}+y, t_{j+1}\right)-W\left(x_{i}, t_{j+1}\right)\right] k(y) d y= \\
= & \int_{-\infty}^{x_{0}-x_{i}}\left[W\left(x_{i}+y, t_{j+1}\right)-W\left(x_{i}, t_{j+1}\right)\right] k(y) d y+ \\
+ & \int_{x_{0}-x_{i}}^{-\Delta x}\left[W\left(x_{i}+y, t_{j+1}\right)-W\left(x_{i}, t_{j+1}\right)\right] k(y) d y+ \\
& +\int_{-\Delta x}^{0}\left[W\left(x_{i}+y, t_{j+1}\right)-W\left(x_{i}, t_{j+1}\right)\right] k(y) d y+ \\
& +\int_{0}^{\Delta x}\left[W\left(x_{i}+y, t_{j+1}\right)-W\left(x_{i}, t_{j+1}\right)\right] k(y) d y+ \\
+ & \int_{\Delta x}^{x_{N}-x_{i}}\left[W\left(x_{i}+y, t_{j+1}\right)-W\left(x_{i}, t_{j+1}\right)\right] k(y) d y+ \\
& +\int_{x_{N}-x_{i}}^{+\infty}\left[W\left(x_{i}+y, t_{j+1}\right)-W\left(x_{i}, t_{j+1}\right)\right] k(y) d y
\end{aligned}
$$

The only difference with the European barrier case is when the option is so in the money and far from the barrier that we can assume that it will be exercised for sure. The European option is, in this case, approximated with the discounted value of the payoff. On the other side when the option is American, it is possible to exercise it immediately as it becomes profitable to do so. The proportion between dividend yield and risk interest rate will drive the decision of early exercise. Getting into the detail of the solution, if the jump is relatively small and stock remains inside the considered range, then the integrals can be rewritten in the same way we did for the European barrier case, which in itself was the same as the European vanilla case. Hence for the solution of integrals from (A.84) to (A.87) we can just refers to A.1.1 and A.1.2. For the solution of integrals (A.83) and (A.88) we need to distinguish between call and put options and on the basis of the barrier position with respect to the stock price. 


\section{A.8.1 Solution of the Jump Integral for American Up- And-Out Call Options}

If the option is an up-and-out, we impose that the boundary is at the barrier level, that is $x_{N}=\ln (B)$. If the jump is large and positive the stock reaches the barrier level, the option is knocked out and the rebate is paid. Hence we can write that the option is worth the rebate that is paid for it

$$
W\left(x_{i}+y, t_{j+1}\right)=R \quad \text { for } \quad y \in\left[\ln (B)-x_{i},+\infty\right]
$$

And integral (A.88) becomes

$$
\frac{1}{\nu}\left[R-W\left(x_{i}, t_{j+1}\right)\right] \cdot \operatorname{expint}\left[\frac{\mu_{p}}{\nu_{p}}(N-i) \Delta x\right]
$$

If the jump is large and negative, $y<x_{0}-x_{i}$, then the call options can be imposed to have a value close to zero and equation (A.83) becomes

$$
-\frac{1}{\nu} W\left(x_{i}, t_{j+1}\right) \cdot \operatorname{expint}\left(\frac{\mu_{n}}{\nu_{n}} i \Delta x\right)
$$

We can note that the values of the integrals (A.88) and (A.83) are the same as those we found for European up-and-out call options. So the whole integral (A.82) for up-and-out American call options can be written in the same way as we did for the European counterpart and is given by

$$
\begin{gathered}
\int_{-\infty}^{+\infty}\left[W\left(x_{i}+y, t_{j+1}\right)-W\left(x_{i}, t_{j+1}\right)\right] k(y) d y= \\
=\frac{\left[W\left(x_{i+1}, t_{j+1}\right)-W\left(x_{i}, t_{j+1}\right)\right]\left(1-e^{-\frac{\mu_{p}}{\nu_{p}} \Delta x}\right)}{\nu \Delta x \frac{\mu_{p}}{\nu_{p}}}+ \\
+\frac{\left[W\left(x_{i-1}, t_{j+1}\right)-W\left(x_{i}, t_{j+1}\right)\right]\left(1-e^{-\frac{\mu_{n}}{\nu_{n}} \Delta x}\right)}{\nu \Delta x \frac{\mu_{n}}{\nu_{n}}}+ \\
+\sum_{k=1}^{N-i-1} \frac{1}{\nu \Delta x}\left[W\left(x_{i+k+1}, t_{j+1}\right)-W\left(x_{i+k}, t_{j+1}\right)\right] \frac{\nu_{p}}{\mu_{p}}\left[e^{-\frac{\mu_{p}}{\nu_{p}} k \Delta x}-e^{-\frac{\mu_{p}}{\nu_{p}}(k+1) \Delta x}\right]+ \\
+\sum_{k=1}^{N-i-1} \frac{1}{\nu}\left[W\left(x_{i+k}, t_{j+1}\right)-W\left(x_{i}, t_{j+1}\right)-k\left[W\left(x_{i+k+1}, t_{j+1}\right)-W\left(x_{i+k}, t_{j+1}\right)\right]\right] \\
\cdot\left\{\operatorname{expint}\left(\frac{\mu_{p}}{\nu_{p}} k \Delta x\right)-\operatorname{expint}\left[\frac{\mu_{p}}{\nu_{p}}(k+1) \Delta x\right]\right\}+
\end{gathered}
$$




$$
\begin{gathered}
+\sum_{k=1}^{i-1} \frac{1}{\nu \Delta x}\left[W\left(x_{i-k-1}, t_{j+1}\right)-W\left(x_{i-k}, t_{j+1}\right)\right] \frac{\nu_{n}}{\mu_{n}}\left[e^{-\frac{\mu_{n}}{\nu_{n}} k \Delta x}-e^{-\frac{\mu_{n}}{\nu_{n}}(k+1) \Delta x}\right]+ \\
+\sum_{k=1}^{i-1} \frac{1}{\nu}\left\{W\left(x_{i-k}, t_{j+1}\right)-W\left(x_{i}, t_{j+1}\right)-k\left[W\left(x_{i-k-1}, t_{j+1}\right)-W\left(x_{i-k}, t_{j+1}\right)\right]\right\} \\
\cdot\left\{\operatorname{expint}\left(\frac{\mu_{n}}{\nu_{n}} k \Delta x\right)-\operatorname{expint}\left[\frac{\mu_{n}}{\nu_{n}}(k+1) \Delta x\right]\right\}+ \\
+\frac{1}{\nu}\left[R-W\left(x_{i}, t_{j+1}\right)\right] \cdot \operatorname{expint}\left[\frac{\mu_{p}}{\nu_{p}}(N-i) \Delta x\right]+ \\
-\frac{1}{\nu} W\left(x_{i}, t_{j+1}\right) \cdot \operatorname{expint}\left(\frac{\mu_{n}}{\nu_{n}} i \Delta x\right)
\end{gathered}
$$

As usual to improve the stability of the scheme, we treat the small jumps implicitly. In particular we replace the first two lines of the right hand side of equation (A.91) with the following expression:

$$
\begin{aligned}
& \frac{\left[W\left(x_{i+1}, t_{j}\right)-W\left(x_{i}, t_{j}\right)\right]\left(1-e^{-\frac{\mu_{p}}{\nu_{p}} \Delta x}\right)}{\nu \Delta x \frac{\mu_{p}}{\nu_{p}}}+ \\
& +\frac{\left[W\left(x_{i-1}, t_{j}\right)-W\left(x_{i}, t_{j}\right)\right]\left(1-e^{-\frac{\mu_{n}}{\nu_{n}} \Delta x}\right)}{\nu \Delta x \frac{\mu_{n}}{\nu_{n}}}
\end{aligned}
$$

\section{A.8.2 Solution of the Jump Integral for American Down- And-Out Call Options}

In the case of a down and out option call, a large positive jump

$$
y \in\left[x_{N}-x_{i},+\infty\right]
$$

takes the stock outside the considered range. The stock value is here so high and it is so far from the down barrier that we can impose that the option is going to be exercised for sure. The decision if the exercise is immediate is going to depend on the proportion between risk free interest rate and dividend yield. We can therefore approximate

$$
W\left(x_{i}+y, t_{j+1}\right) \simeq \max \left[e^{x_{i}+y} e^{-q\left(T-t_{j+1}\right)}-K e^{-r\left(T-t_{j+1}\right)}, e^{x_{i}+y}-K\right]
$$


If it is profitable to early exercise for every stock value higher than the upper limit in the grid, can rewrite equation (A.88) as

$$
\begin{array}{r}
\int_{(N-i) \Delta x}^{+\infty}\left[W\left(x_{i}+y, t_{j+1}\right)-W\left(x_{i}, t_{j+1}\right)\right] k(y) d y= \\
\int_{(N-i) \Delta x}^{+\infty}\left[e^{x_{i}+y}-K-W\left(x_{i}, t_{j+1}\right)\right] \frac{e^{-\frac{\mu_{p}}{\nu_{p}} y}}{\nu y} d y
\end{array}
$$

The solution of this integral is given by

$$
\begin{array}{r}
\frac{1}{\nu}\left\{e^{x_{i}} \operatorname{expint}\left[\left(\frac{\mu_{p}}{\nu_{p}}-1\right)(N-i) \Delta x\right]+\right. \\
\left.-\left[K+W\left(x_{i}, t_{j+1}\right)\right] \operatorname{expint}\left[\frac{\mu_{p}}{\nu_{p}}(N-i) \Delta x\right]\right\}
\end{array}
$$

In the case of large negative jumps, the stock reaches the barrier $x_{0} \triangleq \ln (B)$, the options is deactivated and the rebate, if any, is paid. So we can say that the option for large negative jumps is worth the rebate paid. Hence we write, using the usual change of variable $y \triangleq-y$

$$
\begin{gathered}
\int_{-\infty}^{x_{0}-x_{i}}\left[W\left(x_{i}+y, t_{j+1}\right)-W\left(x_{i}, t_{j+1}\right)\right] k(y) d y= \\
=\int_{x_{i}-x_{0}}^{+\infty}\left[R-W\left(x_{i}, t_{j+1}\right)\right] \frac{e^{-\frac{\mu_{n}}{\nu_{n}} y}}{\nu y} d y= \\
=\frac{1}{\nu}\left[R-W\left(x_{i}, t_{j+1}\right)\right] \cdot \operatorname{expint}\left(\frac{\mu_{n}}{\nu_{n}} i \Delta x\right)
\end{gathered}
$$

Now combining equations (A.94) and (A.95) with the results we found for the vanilla European case, integrals (A.9), (A.10), (A.12) and (A.13), we can express integral (A.82) as

$$
\begin{aligned}
& \int_{-\infty}^{+\infty}\left[W\left(x_{i}+y, t_{j+1}\right)-W\left(x_{i}, t_{j+1}\right)\right] k(y) d y= \\
= & \frac{\left[W\left(x_{i+1}, t_{j+1}\right)-W\left(x_{i}, t_{j+1}\right)\right]\left(1-e^{-\frac{\mu_{p}}{\nu_{p}} \Delta x}\right)}{\nu \Delta x \frac{\mu_{p}}{\nu_{p}}}+ \\
+ & \frac{\left[W\left(x_{i-1}, t_{j+1}\right)-W\left(x_{i}, t_{j+1}\right)\right]\left(1-e^{-\frac{\mu_{n}}{\nu_{n}} \Delta x}\right)}{\nu \Delta x \frac{\mu_{n}}{\nu_{n}}}+
\end{aligned}
$$




$$
\begin{aligned}
& +\sum_{k=1}^{N-i-1} \frac{1}{\nu \Delta x}\left[W\left(x_{i+k+1}, t_{j+1}\right)-W\left(x_{i+k}, t_{j+1}\right)\right] \frac{\nu_{p}}{\mu_{p}}\left[e^{-\frac{\mu_{p}}{\nu_{p}} k \Delta x}-e^{-\frac{\mu_{p}}{\nu_{p}}(k+1) \Delta x}\right]+ \\
& +\sum_{k=1}^{N-i-1} \frac{1}{\nu}\left\{W\left(x_{i+k}, t_{j+1}\right)-W\left(x_{i}, t_{j+1}\right)-k\left[W\left(x_{i+k+1}, t_{j+1}\right)-W\left(x_{i+k}, t_{j+1}\right)\right]\right\} . \\
& \cdot\left\{\operatorname{expint}\left(\frac{\mu_{p}}{\nu_{p}} k \Delta x\right)-\operatorname{expint}\left[\frac{\mu_{p}}{\nu_{p}}(k+1) \Delta x\right]\right\}+ \\
& +\sum_{k=1}^{i-1} \frac{1}{\nu \Delta x}\left[W\left(x_{i-k-1}, t_{j+1}\right)-W\left(x_{i-k}, t_{j+1}\right)\right] \frac{\nu_{n}}{\mu_{n}}\left[e^{-\frac{\mu_{n}}{\nu_{n}} k \Delta x}-e^{-\frac{\mu_{n}}{\nu_{n}}(k+1) \Delta x}\right]+ \\
& +\sum_{k=1}^{i-1} \frac{1}{\nu}\left\{W\left(x_{i-k}, t_{j+1}\right)-W\left(x_{i}, t_{j+1}\right)-k\left[W\left(x_{i-k-1}, t_{j+1}\right)-W\left(x_{i-k}, t_{j+1}\right)\right]\right\} . \\
& \cdot\left\{\operatorname{expint}\left(\frac{\mu_{n}}{\nu_{n}} k \Delta x\right)-\operatorname{expint}\left[\frac{\mu_{n}}{\nu_{n}}(k+1) \Delta x\right]\right\}+ \\
& +\frac{1}{\nu}\left\{e^{x_{i}} \operatorname{expint}\left[\left(\frac{\mu_{p}}{\nu_{p}}-1\right)(N-i) \Delta x\right]+\right. \\
& -\left[K+W\left(x_{i}, t_{j+1}\right)\right] \operatorname{expint}\left[\frac{\mu_{p}}{\nu_{p}}(N-i) \Delta x\right]+ \\
& \left.+\left[R-W\left(x_{i}, t_{j+1}\right)\right] \cdot \operatorname{expint}\left(\frac{\mu_{n}}{\nu_{n}} i \Delta x\right)\right\}
\end{aligned}
$$

As usual to improve the stability of the scheme, we treat the small jumps implicitly. In particular we replace the first two lines of the right hand side of equation (A.96) with the following expression:

$$
\begin{aligned}
& \frac{\left[W\left(x_{i+1}, t_{j}\right)-W\left(x_{i}, t_{j}\right)\right]\left(1-e^{-\frac{\mu_{p}}{\nu_{p}} \Delta x}\right)}{\nu \Delta x \frac{\mu_{p}}{\nu_{p}}}+ \\
& +\frac{\left[W\left(x_{i-1}, t_{j}\right)-W\left(x_{i}, t_{j}\right)\right]\left(1-e^{-\frac{\mu_{n}}{\nu_{n}} \Delta x}\right)}{\nu \Delta x \frac{\mu_{n}}{\nu_{n}}}
\end{aligned}
$$


Note anyway that if $q$ is equal to zero, it is never convenient to early exercise the call option and hence integral (A.88) would be the same as the one for the European down-and-out call case and it would be given by expression (A.68). In this case, the whole integral (A.82) would be given by (A.70).

In general if $r>q$ and $q>0$, there is going to be a point such that for values lower than this point, it is not profitable to early exercise, while it is profitable to early exercise for values larger than this point. It is possible that this point is outside the considered range of values. In this case, if the jump takes the stock relatively close to $x_{N}$, the maximum in equation (A.93) is given by the discounted payoff, while for larger jumps the maximum is given by the non discounted payoff. In the code, the approach used to solve the issue is to check if the exercise boundary exists and is outside the grid. If this is the case a warning signal is given, so that it is possible to increase the size of the grid. If anyway we still decide to leave the exercise point outside the grid the following approximation is used

$$
\begin{gathered}
\int_{x_{N}-x_{i}}^{+\infty} W\left(x_{i}+y, t_{j+1}\right) k(y) d y= \\
=\max \left[\frac { 1 } { \nu } \left\{e^{x_{i}} \text { expint }\left[\left(\frac{\mu_{p}}{\nu_{p}}-1\right)(N-i) \Delta x\right]+\right.\right. \\
\left.- \text { Kexpint }\left[\frac{\mu_{p}}{\nu_{p}}(N-i) \Delta x\right]\right\}, \\
\frac{1}{\nu}\left\{e^{x_{i}} e^{-q\left(T-t_{j+1}\right)} \text { expint }\left[\left(\frac{\mu_{p}}{\nu_{p}}-1\right)(N-i) \Delta x\right]+\right. \\
\left.\left.-K e^{-r\left(T-t_{j+1}\right)} \text { expint }\left[\frac{\mu_{p}}{\nu_{p}}(N-i) \Delta x\right]\right\}\right]
\end{gathered}
$$

This implies that at each time step, either all or none of the options corresponding to stock prices larger than the upper boundary are exercised. The decision is based on the fact that on average it is convenient or not to early exercise.

\section{A.8.3 Solution of the Jump Integral for American Up- And-Out Put Options}

For up-and-out options, we set the up boundary at the barrier value $x_{N}=$ $\ln (B)$. For jumps $y>\ln (B)-x_{i}$ the option is knocked out and the rebate 
is paid. Hence we can impose that the option is worth the rebate and write integral (A.88) as

$$
\begin{array}{r}
\int_{x_{N}-x_{i}}^{+\infty}\left[W\left(x_{i}+y, t_{j+1}\right)-W\left(x_{i}, t_{j+1}\right)\right] k(y) d y= \\
\frac{1}{\nu}\left[R-W\left(x_{i}, t_{j+1}\right)\right] \cdot \text { expint }\left[\frac{\mu_{p}}{\nu_{p}}(N-i) \Delta x\right]
\end{array}
$$

In the case of negative jumps which are large enough to finish outside the considered range, we can impose that the option will be exercised for sure. The proportion between $r$ and $q$ will determine the timing of the exercise. If risk free interest rate is higher than dividend yield, as it is usually the case, a put option which is deep in the money and far from the barrier will be exercised right away. After the usual change of variable $y \triangleq-y$, we can write that for $y>\left(x_{i}-x_{0}\right)$ we have

$$
W\left(x_{i}-y, t_{j+1}\right) \simeq \max \left[K e^{-r\left(T-t_{j+1}\right)}-e^{x_{i}-y} e^{-q\left(T-t_{j+1}\right)}, K-e^{x_{i}-y}\right]
$$

If for every stock value on the left of the lower grid limit, it is profitable to early exercise, we can solve integral (A.83) as

$$
\begin{gathered}
\int_{-\infty}^{x_{0}-x_{i}}\left[W\left(x_{i}+y, t_{j+1}\right)-W\left(x_{i}, t_{j+1}\right)\right] \frac{e^{-\frac{\mu_{n}}{\nu_{n}}|y|}}{\nu|y|} d y= \\
=\int_{x_{i}-x_{0}}^{+\infty}\left[K-e^{x_{i}-y}-W\left(x_{i}, t_{j+1}\right)\right] \frac{e^{-\frac{\mu_{n}}{\nu_{n}} y}}{\nu y} d y= \\
=\frac{1}{\nu}\left\{\left[K-W\left(x_{i}, t_{j+1}\right)\right] \operatorname{expint}\left(\frac{\mu_{n}}{\nu_{n}} i \Delta x\right)+\right. \\
\left.-e^{x_{i}} \text { expint }\left[\left(\frac{\mu_{n}}{\nu_{n}}+1\right) i \Delta x\right]\right\}
\end{gathered}
$$

Combining (A.98) and (A.99) with (A.9), (A.10), (A.12) and (A.13) we can write

$$
\begin{aligned}
& \int_{-\infty}^{+\infty}\left[W\left(x_{i}+y, t_{j+1}\right)-W\left(x_{i}, t_{j+1}\right)\right] k(y) d y= \\
= & \frac{\left[W\left(x_{i+1}, t_{j+1}\right)-W\left(x_{i}, t_{j+1}\right)\right]\left(1-e^{-\frac{\mu_{p}}{\nu_{p}} \Delta x}\right)}{\nu \Delta x \frac{\mu_{p}}{\nu_{p}}}+
\end{aligned}
$$




$$
+\frac{\left[W\left(x_{i-1}, t_{j+1}\right)-W\left(x_{i}, t_{j+1}\right)\right]\left(1-e^{-\frac{\mu_{n}}{\nu_{n}} \Delta x}\right)}{\nu \Delta x \frac{\mu_{n}}{\nu_{n}}}+
$$

$$
\begin{gathered}
+\sum_{k=1}^{N-i-1} \frac{1}{\nu \Delta x}\left[W\left(x_{i+k+1}, t_{j+1}\right)-W\left(x_{i+k}, t_{j+1}\right)\right] \cdot \frac{\nu_{p}}{\mu_{p}}\left[e^{-\frac{\mu_{p}}{\nu_{p}} k \Delta x}-e^{-\frac{\mu_{p}}{\nu_{p}}(k+1) \Delta x}\right]+ \\
+\sum_{k=1}^{N-i-1} \frac{1}{\nu}\left[W\left(x_{i+k}, t_{j+1}\right)-W\left(x_{i}, t_{j+1}\right)-k\left[W\left(x_{i+k+1}, t_{j+1}\right)-W\left(x_{i+k}, t_{j+1}\right)\right]\right] \cdot \\
\cdot\left\{\operatorname{expint}\left(\frac{\mu_{p}}{\nu_{p}} k \Delta x\right)-\operatorname{expint}\left[\frac{\mu_{p}}{\nu_{p}}(k+1) \Delta x\right]\right\}+
\end{gathered}
$$$$
+\sum_{k=1}^{i-1} \frac{1}{\nu \Delta x}\left[W\left(x_{i-k-1}, t_{j+1}\right)-W\left(x_{i-k}, t_{j+1}\right)\right] \cdot \frac{\nu_{n}}{\mu_{n}}\left[e^{-\frac{\mu_{n}}{\nu_{n}} k \Delta x}-e^{-\frac{\mu_{n}}{\nu_{n}}(k+1) \Delta x}\right]+
$$$$
+\sum_{k=1}^{i-1} \frac{1}{\nu}\left\{W\left(x_{i-k}, t_{j+1}\right)-W\left(x_{i}, t_{j+1}\right)-k\left[W\left(x_{i-k-1}, t_{j+1}\right)-W\left(x_{i-k}, t_{j+1}\right)\right]\right\} .
$$$$
\cdot\left\{\operatorname{expint}\left(\frac{\mu_{n}}{\nu_{n}} k \Delta x\right)-\operatorname{expint}\left[\frac{\mu_{n}}{\nu_{n}}(k+1) \Delta x\right]\right\}+
$$$$
+\frac{1}{\nu}\left[R-W\left(x_{i}, t_{j+1}\right)\right] \cdot \operatorname{expint}\left[\frac{\mu_{p}}{\nu_{p}}(N-i) \Delta x\right]+
$$$$
+\frac{1}{\nu}\left\{\left[K-W\left(x_{i}, t_{j+1}\right)\right] \operatorname{expint}\left(\frac{\mu_{n}}{\nu_{n}} i \Delta x\right)+\right.
$$$$
\left.-e^{x_{i}} \text { expint }\left[\left(\frac{\mu_{n}}{\nu_{n}}+1\right) i \Delta x\right]\right\}
$$

As usual to improve the stability of the scheme, we treat the small jumps implicitly. In particular we replace the first two lines of the right hand side of equation (A.100) with the following expression: 


$$
\begin{aligned}
& \frac{\left[W\left(x_{i+1}, t_{j}\right)-W\left(x_{i}, t_{j}\right)\right]\left(1-e^{-\frac{\mu_{p}}{\nu_{p}} \Delta x}\right)}{\nu \Delta x \frac{\mu_{p}}{\nu_{p}}}+ \\
& +\frac{\left[W\left(x_{i-1}, t_{j}\right)-W\left(x_{i}, t_{j}\right)\right]\left(1-e^{-\frac{\mu_{n}}{\nu_{n}} \Delta x}\right)}{\nu \Delta x \frac{\mu_{n}}{\nu_{n}}}
\end{aligned}
$$

Equation (A.100) is valid for the cases in which it is profitable to early exercise the option for all the stock prices smaller than the lower boundary. On the contrary, in the unlikely case in which it is never convenient to early exercise, for example if the risk free interest rate is zero, integral (A.83) will be valued in the same way we did for the European up-and-out put option and will be given by (A.73). In this case the whole integral (A.82) will be the same as the European case and will be equal to (A.74).

In general if $q>r$ and $r>0$ as the stock price moves more and more in the money, there is going to exist a stock price such that for values larger than this price, it is not convenient to exercise the option, while for values lower than this price, it is convenient to early exercise. This exercise level can be also outside the considered grid on the left of the lower limit. Clearly a case in which dividend yield is higher than risk free interest rate has a limited economic meaning, however the approach used in the code to solve the issue is to print a warning in the case the exercise boundary exists and it is outside the grid. In this way it is possible to reduce the size of the lower limit to include also the exercise point. If anyway we still like to run the program with the exercise boundary outside the grid, the following approximation is used:

$$
\begin{gathered}
\int_{x_{i}-x_{0}}^{+\infty} W\left(x_{i}-y, t_{j+1}\right) \frac{e^{-\frac{\mu_{n}}{\nu_{n}} y}}{\nu y} d y= \\
=\max \left[\frac{1}{\nu}\left\{K \operatorname{expint}\left(\frac{\mu_{n}}{\nu_{n}} i \Delta x\right)-e^{x_{i}} \operatorname{expint}\left[\left(\frac{\mu_{n}}{\nu_{n}}+1\right) i \Delta x\right]\right\},\right. \\
\frac{1}{\nu}\left\{K e^{-r\left(T-t_{j+1}\right)} \operatorname{expint}\left[\frac{\mu_{n}}{\nu_{n}} i \Delta x\right]+\right. \\
\left.\left.-e^{-q\left(T-t_{j+1}\right)} e^{x_{i}} \operatorname{expint}\left[\left(\frac{\mu_{n}}{\nu_{n}}+1\right) i \Delta x\right]\right\}\right]
\end{gathered}
$$


This approximation corresponds to requiring that either all or none of the options with stock prices smaller than the lower limit are exercised, depending on what is profitable on average.

\section{A.8.4 Solution of the Jump Integral for American Down- And-Out Put Options}

The American down-and-out put options case is the same as the European one. In fact if the stock moves outside the range on a positive jump the option is worth about zero because it is a put options, on the other side if the jump is negative and the stock passes the barrier $x_{0} \triangleq \ln (B)$, the option is worth the rebate which is paid upon passage of the barrier. Hence integral (A.88) can valued as

$$
-\frac{1}{\nu} W\left(x_{i}, t_{j+1}\right) \cdot \operatorname{expint}\left[\frac{\mu_{p}}{\nu_{p}}(N-i) \Delta x\right]
$$

and integral (A.83) can be written as

$$
\frac{1}{\nu}\left[R-W\left(x_{i}, t_{j+1}\right)\right] \cdot \operatorname{expint}\left(\frac{\mu_{n}}{\nu_{n}} i \Delta x\right)
$$

Putting expressions (A.102) and (A.103) together with (A.9), (A.10), (A.12) and (A.13), we can write the jump integral for down-and-out American put options in the same we wrote the down-and-out European put option integral, that is

$$
\begin{aligned}
& \int_{-\infty}^{+\infty}\left[W\left(x_{i}+y, t_{j+1}\right)-W\left(x_{i}, t_{j+1}\right)\right] k(y) d y= \\
& =\frac{\left[W\left(x_{i+1}, t_{j+1}\right)-W\left(x_{i}, t_{j+1}\right)\right]\left(1-e^{-\frac{\mu_{p}}{\nu_{p}} \Delta x}\right)}{\nu \Delta x \frac{\mu_{p}}{\nu_{p}}}+ \\
& +\frac{\left[W\left(x_{i-1}, t_{j+1}\right)-W\left(x_{i}, t_{j+1}\right)\right]\left(1-e^{-\frac{\mu_{n}}{\nu_{n}} \Delta x}\right)}{\nu \Delta x \frac{\mu_{n}}{\nu_{n}}}+
\end{aligned}
$$




$$
\begin{aligned}
& +\sum_{k=1}^{N-i-1} \frac{1}{\nu \Delta x}\left[W\left(x_{i+k+1}, t_{j+1}\right)-W\left(x_{i+k}, t_{j+1}\right)\right] \cdot \frac{\nu_{p}}{\mu_{p}}\left[e^{-\frac{\mu_{p}}{\nu_{p}} k \Delta x}-e^{-\frac{\mu_{p}}{\nu_{p}}(k+1) \Delta x}\right]+ \\
& +\sum_{k=1}^{N-i-1} \frac{1}{\nu}\left[W\left(x_{i+k}, t_{j+1}\right)-W\left(x_{i}, t_{j+1}\right)-k\left[W\left(x_{i+k+1}, t_{j+1}\right)-W\left(x_{i+k}, t_{j+1}\right)\right]\right] . \\
& \cdot\left\{\operatorname{expint}\left(\frac{\mu_{p}}{\nu_{p}} k \Delta x\right)-\operatorname{expint}\left[\frac{\mu_{p}}{\nu_{p}}(k+1) \Delta x\right]\right\}+ \\
& +\sum_{k=1}^{i-1} \frac{1}{\nu \Delta x}\left[W\left(x_{i-k-1}, t_{j+1}\right)-W\left(x_{i-k}, t_{j+1}\right)\right] \cdot \frac{\nu_{n}}{\mu_{n}}\left(e^{-\frac{\mu_{n}}{\nu_{n}} k \Delta x}-e^{-\frac{\mu_{n}}{\nu_{n}}(k+1) \Delta x}\right)+ \\
& +\sum_{k=1}^{i-1} \frac{1}{\nu}\left\{W\left(x_{i-k}, t_{j+1}\right)-W\left(x_{i}, t_{j+1}\right)-k\left[W\left(x_{i-k-1}, t_{j+1}\right)-W\left(x_{i-k}, t_{j+1}\right)\right]\right\} . \\
& \cdot\left\{\operatorname{expint}\left(\frac{\mu_{n}}{\nu_{n}} k \Delta x\right)-\operatorname{expint}\left[\frac{\mu_{n}}{\nu_{n}}(k+1) \Delta x\right]\right\}+ \\
& -\frac{1}{\nu} W\left(x_{i}, t_{j+1}\right) \cdot \operatorname{expint}\left[\frac{\mu_{p}}{\nu_{p}}(N-i) \Delta x\right]+ \\
& +\frac{1}{\nu}\left[R-W\left(x_{i}, t_{j+1}\right)\right] \cdot \operatorname{expint}\left(\frac{\mu_{n}}{\nu_{n}} i \Delta x\right)
\end{aligned}
$$

As usual to improve the stability of the scheme, we treat the small jumps implicitly. In particular we replace the first two lines of the right hand side of equation (A.104) with the following expression:

$$
\begin{aligned}
& \frac{\left[W\left(x_{i+1}, t_{j}\right)-W\left(x_{i}, t_{j}\right)\right]\left(1-e^{-\frac{\mu_{p}}{\nu_{p}} \Delta x}\right)}{\nu \Delta x \frac{\mu_{p}}{\nu_{p}}}+ \\
& +\frac{\left[W\left(x_{i-1}, t_{j}\right)-W\left(x_{i}, t_{j}\right)\right]\left(1-e^{-\frac{\mu_{n}}{\nu_{n}} \Delta x}\right)}{\nu \Delta x \frac{\mu_{n}}{\nu_{n}}}
\end{aligned}
$$




\section{A.9 Numerical Solution of the Integral in the Heaviside Term of the Variance Gamma PIDE for American Barrier Options}

The PIDE describing the dynamics of the American barrier option value when the underlying follows a variance gamma model is different depending on the option being a call or a put; we examine each case separately.

\section{A.9.1 Numerical Solution of the Integral for American Barrier Call Options}

We want to rewrite the following integral in such a way that the PIDE can be solved using a finite difference technique:

$$
\int_{-\infty}^{x\left(\tau_{j+1}\right)-x_{i}}\left[W\left(x_{i}+y, t_{j+1}\right)+K-e^{x_{i}+y}\right] k(y) d y
$$

We note that this integral is taken over a strictly negative interval because the integral is multiplied by the indicator function $1_{x_{i}>x\left(\tau_{j+1}\right)}$. This means that we do not have to consider the case of jumps whose size tends to zero and hence we do not have to be worried about Lévy measures diverging for small jumps. Let's define the position of the exercise boundary in the following way:

$$
x\left(\tau_{j+1}\right) \triangleq l \Delta x=x_{l}
$$

for some integer $l$ between 0 and $N$. In reality $l$ has be to larger than 0 because a necessary condition to early exercise the call option is that it is in the money, that is $e^{x_{i}} \geq K$. Moreover note that if the option is not exercised for any point in the grid at a certain time step $t_{j+1}$, then $l$ is equal to $N$. Because the integral is multiplied by the indicator function $1_{\left\{x_{i}>x\left(\tau_{j+1}\right)\right\}}$, if we position the exercise boundary at the $x_{N}$ level, the heaviside term does not impact the PIDE, since the indicator function would be equal to zero. With this notation we can rewrite integral (A.106) as 


$$
\begin{aligned}
& \int_{-\infty}^{x_{0}-x_{i}}\left[W\left(x_{i}+y, t_{j+1}\right)+K-e^{x_{i}+y}\right] k(y) d y+ \\
& +\int_{x_{0}-x_{i}}^{(l-i) \Delta x}\left[W\left(x_{i}+y, t_{j+1}\right)+K-e^{x_{i}+y}\right] k(y) d y
\end{aligned}
$$

Integral (A.108) defines the case of a jump which is negative and not too big so that the stock value remains inside the considered range. Being inside the range, the option dynamics is the same as that one of American vanilla calls. Therefore we do not have to worry about this integral and we can get its solution from equation (A.45) in section A.4.2. The only thing we have to be careful about is that if the option is a down-and-out call, at $x_{0} \triangleq \ln (B)$, the option is worth the rebate.

\section{American Up-And-Out Call}

Let's now move to integral (A.107) and let's try to solve it in the hypothesis of an up-and-out call option. Given that the option is a call option, its value for very small values of the underlying can be approximated to zero. This is the case when the jump is large and negative and the stock finishes outside the considered range. We can therefore write

$$
W\left(x_{i}-y, t_{j+1}\right) \simeq 0 \quad \text { for } \quad y \in\left[x_{i}-x_{0},+\infty\right]
$$

where, as usual, we had the change of variable $y \triangleq-y$. So we can evaluate the integral in the following way.

$$
\begin{array}{r}
\int_{-\infty}^{x_{0}-x_{i}}\left[W\left(x_{i}+y, t_{j+1}\right)+K-e^{x_{i}+y}\right] k(y) d y= \\
\int_{x_{i}-x_{0}}^{+\infty}\left[K-e^{x_{i}-y}\right] \frac{e^{-\frac{\mu_{n}}{\nu_{n}} y}}{\nu y} d y= \\
\frac{K}{\nu} \operatorname{expint}\left(\frac{\mu_{n}}{\nu_{n}} i \Delta x\right)-\frac{e^{x_{i}}}{\nu} \operatorname{expint}\left[\left(\frac{\mu_{n}}{\nu_{n}}+1\right) i \Delta x\right]
\end{array}
$$

We note that this is the same result we found for the up-and-out call option. Combining equation (A.109) with the solution of integral (A.108), given by 
equation (A.45), we can write integral (A.106) for up-and-out calls and in the following way:

$$
\begin{gathered}
\int_{-\infty}^{x\left(\tau_{j+1}\right)-x_{i}}\left[W\left(x_{i}+y, t_{j+1}\right)+K-e^{x_{i}+y}\right] k(y) d y= \\
=\sum_{k=i-l}^{i-1}\left\{\frac{W\left(x_{i-k-1}, t_{j+1}\right)-W\left(x_{i-k}, t_{j+1}\right)}{\nu \frac{\mu_{n}}{\nu_{n}} \Delta x} \cdot\left[e^{-\frac{\mu_{n}}{\nu_{n}} k \Delta x}-e^{-\frac{\mu_{n}}{\nu_{n}}(k+1) \Delta x}\right]+\right. \\
+\frac{1}{\nu}\left\{W\left(x_{i-k}, t_{j+1}\right)-k\left[W\left(x_{i-k-1}, t_{j+1}\right)-W\left(x_{i-k}, t_{j+1}\right)\right]\right\} \\
\left.\cdot\left[\operatorname{expint}\left(\frac{\mu_{n}}{\nu_{n}} k \Delta x\right)-\operatorname{expint}\left(\frac{\mu_{n}}{\nu_{n}}(k+1) \Delta x\right)\right]\right\}+ \\
+\frac{K}{\nu} \cdot \operatorname{expint}\left[\frac{\mu_{n}}{\nu_{n}}(i-l) \Delta x\right]+ \\
-\frac{e^{x_{i}}}{\nu} \cdot \operatorname{expint}\left[\left(1+\frac{\mu_{n}}{\nu_{n}}\right)(i-l) \Delta x\right]
\end{gathered}
$$

\section{American Down-And-Out Call}

We consider now integral (A.107) in the case of a down-and-out call. Here the negative jump is such that the stock passes the barrier. This determines the payment of the rebate and the deactivation of the option. As in the jump integral case, we note that here the rebate has to be paid not only when the stock jumps exactly at the barrier, but also when it jumps further left with respect to the barrier. Moreover we can say that, because we imposed that the lower boundary of the grid is exactly at the barrier level, we are sure that we are not considering paths of the stock which have already passed the barrier and have come back to cross it again. So we can write

$$
W\left(x_{i}-y, t_{j+1}\right)=R \quad \text { for } \quad y \in\left[x_{i}-\ln (B),+\infty\right]
$$


where we imposed $x_{0}=\ln (B)$. Hence we can solve integral (A.107) as

$$
\begin{array}{r}
\int_{-\infty}^{x_{0}-x_{i}}\left[W\left(x_{i}+y, t_{j+1}\right)+K-e^{x_{i}+y}\right] k(y) d y= \\
\int_{x_{i}-x_{0}}^{+\infty}\left[R+K-e^{x_{i}-y}\right] \frac{e^{-\frac{\mu_{n}}{\nu_{n}} y}}{\nu y} d y= \\
\frac{R+K}{\nu} \operatorname{expint}\left(\frac{\mu_{n}}{\nu_{n}} i \Delta x\right)-\frac{e^{x_{i}}}{\nu} \operatorname{expint}\left[\left(\frac{\mu_{n}}{\nu_{n}}+1\right) i \Delta x\right]
\end{array}
$$

Combing (A.111) with the solution of integral (A.108) given by equation (A.45) we can write integral (A.106) for down-and-out calls in the following way:

$$
\begin{gathered}
\int_{-\infty}^{x\left(\tau_{j+1}\right)-x_{i}}\left[W\left(x_{i}+y, t_{j+1}\right)+K-e^{x_{i}+y}\right] k(y) d y= \\
=\sum_{k=i-l}^{i-1}\left\{\frac{W\left(x_{i-k-1}, t_{j+1}\right)-W\left(x_{i-k}, t_{j+1}\right)}{\nu \frac{\mu_{n}}{\nu_{n}} \Delta x} \cdot\left[e^{-\frac{\mu_{n}}{\nu_{n}} k \Delta x}-e^{-\frac{\mu_{n}}{\nu_{n}}(k+1) \Delta x}\right]+\right. \\
+\frac{1}{\nu}\left\{W\left(x_{i-k}, t_{j+1}\right)-k\left[W\left(x_{i-k-1}, t_{j+1}\right)-W\left(x_{i-k}, t_{j+1}\right)\right]\right\} \\
+\frac{K}{\nu} \cdot \operatorname{expint}\left[\frac{\mu_{n}}{\nu_{n}}(i-l) \Delta x\right]-\frac{e^{x_{i}}}{\nu} \cdot \operatorname{expint}\left[\left(1+\frac{\mu_{n}}{\nu_{n}}\right)(i-l) \Delta x\right]+ \\
+\frac{R}{\nu} \operatorname{expint}\left(\frac{\mu_{n}}{\nu_{n}} i \Delta x\right)
\end{gathered}
$$




\section{A.9.2 Numerical Solution of the Integral for American Barrier Put Options}

The integral in the heaviside term in the case of put options is given by

$$
\int_{x\left(\tau_{j+1}\right)-x_{i}}^{+\infty}\left[W\left(x_{i}+y, t_{j+1}\right)-K+e^{x_{i}+y}\right] k(y) d y
$$

Being multiplied by an indicator function which reflects stock prices inside the exercise region, the integral is taken over a strictly positive interval. Let's define $x\left(\tau_{j+1}\right) \triangleq l \Delta x=x_{l}$ for some integer $l$ between 0 and $N$. In reality, $l$ has to be lower than $N$ because a necessary condition for early exercise is that the option is in the money which, in the case of a put option, means $e^{x_{i}} \leq K$. Moreover note that, if the option is not exercised for any grid point at a certain time step $t_{j+1}$, then we have $l=0$. We see that the integral is multiplied by $1_{\left\{x_{i}<x\left(\tau_{j+1}\right)\right\}}$, so if we position $l$ at the lower end of the grid, the indicator function is going to be always equal to zero and hence the heaviside function is not going to impact the option PIDE.

We can now divide the integral in the familiar way:

$$
\begin{array}{r}
\int_{(l-i) \Delta x}^{(N-i) \Delta x}\left[W\left(x_{i}+y, t_{j+1}\right)-K+e^{x_{i}+y}\right] k(y) d y+ \\
\int_{(N-i) \Delta x}^{+\infty}\left[W\left(x_{i}+y, t_{j+1}\right)-K+e^{x_{i}+y}\right] k(y) d y
\end{array}
$$

As long as the jump is small and the stock price remains inside the range of values in which our grid for the numerical solution is defined, we can use the results from the American vanilla case. Hence the solution of integral (A.114) is given by equation (A.52) in section A.4.6. The only thing we need to remember is that, if the option is an up-and-out put, when the stock is at the node $x_{N} \triangleq \ln (B)$, the option is worth the rebate. 


\section{American Up-And-Out Put}

We consider now integral (A.115) for up-and-out put options. We set the limit of the grid at the barrier level $x_{N}=\ln (B)$. For large positive jumps the stock passes the barrier and so the option becomes worthless and the rebate is paid. Hence we can write

$$
W\left(x_{i}+y, t_{j+1}\right)=R \quad \text { for } \quad y \in\left[\ln (B)-x_{i},+\infty\right]
$$

Integral (A.115) can be rewritten in the following way

$$
\begin{array}{r}
\int_{(N-i) \Delta x}^{+\infty}\left[W\left(x_{i}+y, t_{j+1}\right)-K+e^{x_{i}+y}\right] k(y) d y= \\
=\frac{e^{x_{i}}}{\nu} \text { expint }\left[\left(\frac{\mu_{p}}{\nu_{p}}-1\right)(N-i) \Delta x\right]+ \\
+\frac{R-K}{\nu} \text { expint }\left[\frac{\mu_{p}}{\nu_{p}}(N-i) \Delta x\right]
\end{array}
$$

Combining equation (A.116) with the solution of equation (A.114) provided by equation (A.52), we can rewrite integral (A.113) for up-and-out put as

$$
\begin{gathered}
\int_{x\left(\tau_{j+1}\right)-x_{i}}^{+\infty}\left[W\left(x_{i}+y, t_{j+1}\right)-K+e^{x_{i}+y}\right] k(y) d y= \\
=\sum_{k=l-i}^{N-i-1}\left\{\frac{W\left(x_{i+k+1}, t_{j+1}\right)-W\left(x_{i+k}, t_{j+1}\right)}{\nu \frac{\mu_{p}}{\nu_{p}} \Delta x} \cdot\left[e^{-\frac{\mu_{p}}{\nu_{p}} k \Delta x}-e^{-\frac{\mu_{p}}{\nu_{p}}(k+1) \Delta x}\right]+\right. \\
+\frac{1}{\nu}\left\{W\left(x_{i+k}, t_{j+1}\right)-k\left[W\left(x_{i+k+1}, t_{j+1}\right)-W\left(x_{i+k}, t_{j+1}\right)\right]\right\} . \\
\left.\cdot\left[\operatorname{expint}\left(\frac{\mu_{p}}{\nu_{p}} k \Delta x\right)-\operatorname{expint}\left(\frac{\mu_{p}}{\nu_{p}}(k+1) \Delta x\right)\right]\right\}+ \\
\cdot \text { expint }\left[\frac{\mu_{p}}{\nu_{p}}(l-i) \Delta x\right]+\frac{e^{x_{i}}}{\nu} \cdot \operatorname{expint}\left[\left(\frac{\mu_{p}}{\nu_{p}}-1\right)(l-i) \Delta x\right] \\
\text { expint }\left[\frac{\mu_{p}}{\nu_{p}}(N-i) \Delta x\right]
\end{gathered}
$$




\section{American Down-And-Out Put}

In the case of Down-And-Out Put options, if the stock has a positive large jump such that the stock passes the grid limit, we can say that it is so out of the money that its value can be approximated by zero. Formally we write

$$
W\left(x_{i}+y, t_{j+1}\right) \simeq 0 \quad \text { for } \quad y \in\left[\ln (B)-x_{i},+\infty\right]
$$

Integral (A.115) can then be rewritten in the following way

$$
\frac{e^{x_{i}}}{\nu} \text { expint }\left[\left(\frac{\mu_{p}}{\nu_{p}}-1\right)(N-i) \Delta x\right]-\frac{K}{\nu} \text { expint }\left[\frac{\mu_{p}}{\nu_{p}}(N-i) \Delta x\right](
$$

Combing equation (A.118) with the solution of equation (A.114) provided by equation (A.52), we write integral (A.113) for down-and-out put options in the following way:

$$
\begin{gathered}
\int_{x\left(\tau_{j+1}\right)-x_{i}}^{+\infty}\left[W\left(x_{i}+y, t_{j+1}\right)-K+e^{x_{i}+y}\right] k(y) d y= \\
=\sum_{k=l-i}^{N-i-1}\left\{\frac{W\left(x_{i+k+1}, t_{j+1}\right)-W\left(x_{i+k}, t_{j+1}\right)}{\nu \frac{\mu_{p}}{\nu_{p}} \Delta x} \cdot\left[e^{-\frac{\mu_{p}}{\nu_{p}} k \Delta x}-e^{-\frac{\mu_{p}}{\nu_{p}}(k+1) \Delta x}\right]+\right. \\
+\frac{1}{\nu}\left\{W\left(x_{i+k}, t_{j+1}\right)-k\left[W\left(x_{i+k+1}, t_{j+1}\right)-W\left(x_{i+k}, t_{j+1}\right)\right]\right\} \\
\left.\cdot\left[\operatorname{expint}\left(\frac{\mu_{p}}{\nu_{p}} k \Delta x\right)-\operatorname{expint}\left(\frac{\mu_{p}}{\nu_{p}}(k+1) \Delta x\right)\right]\right\}+ \\
-\frac{K}{\nu} \cdot \operatorname{expint}\left[\frac{\mu_{p}}{\nu_{p}}(l-i) \Delta x\right]+ \\
+\frac{e^{x_{i}}}{\nu} \cdot \operatorname{expint}\left[\left(\frac{\mu_{p}}{\nu_{p}}-1\right)(l-i) \Delta x\right]
\end{gathered}
$$




\section{A.10 Variance Gamma Difference Equation for American Barrier Options}

\section{A.10.1 Difference Equation for American Up-And-Out Calls}

To obtain the difference equation under variance gamma of an American up-and-out call option, we replace the integral in the heaviside term in the PIDE (A.80) with the discretization in equation (A.110) and the jump integral in the PIDE with the approximation in equation (A.91) as modified by expression (A.92). We obtain in this way the following difference equation.

$$
\begin{gathered}
W\left(x_{i-1}, t_{j}\right)\left\{h-\frac{\Delta t \cdot\left(1-e^{-\frac{\mu_{n}}{\nu_{n}} \Delta x}\right)}{\left.\nu \Delta x^{\frac{\mu_{n}}{\nu_{n}}}\right\}}\right\}+ \\
+W\left(x_{i}, t_{j}\right)\left\{(1+r \Delta t)+\frac{\Delta t \cdot\left(1-e^{-\frac{\mu_{p}}{\nu_{p}} \Delta x}\right)}{\nu \Delta x \frac{\mu_{p}}{\nu_{p}}}+\frac{\Delta t \cdot\left(1-e^{-\frac{\mu_{n}}{\nu_{n}} \Delta x}\right)}{\nu \Delta x_{\frac{\mu_{n}}{\nu_{n}}}}\right\}+ \\
-W\left(x_{i+1}, t_{j}\right)\left\{h+\frac{\Delta t \cdot\left(1-e^{-\frac{\mu_{p}}{\nu_{p}} \Delta x}\right)}{\nu \Delta x \frac{\mu_{p}}{\nu_{p}}}\right\}=W\left(x_{i}, t_{j+1}\right)+ \\
+\Delta t \cdot\left\{\sum_{k=1}^{N-i-1} \frac{1}{\nu \Delta x}\left[W\left(x_{i+k+1}, t_{j+1}\right)-W\left(x_{i+k}, t_{j+1}\right)\right] \cdot\right. \\
+\sum_{k=1}^{N-i-1} \frac{\nu_{p}}{\mu_{p}}\left[e^{-\frac{\mu_{p}}{\nu_{p}} k \Delta x}-e^{-\frac{\mu_{p}}{\nu_{p}}(k+1) \Delta x}\right]+ \\
\left.\cdot\left\{x_{i+k}, t_{j+1}\right)-W\left(x_{i}, t_{j+1}\right)-k\left[W\left(x_{i+k+1}, t_{j+1}\right)-W\left(x_{i+k}, t_{j+1}\right)\right]\right] \\
+\sum_{k=1}^{i-1} \frac{1}{\nu \Delta x}\left[W\left(x_{i-k-1}, t_{j+1}\right)-W\left(x_{i-k}, t_{j+1}\right)\right] \frac{\nu_{n}}{\mu_{n}}\left[e^{-\frac{\mu_{n}}{\nu_{n}} k \Delta x}-e^{-\frac{\mu_{n}}{\nu_{n}}(k+1) \Delta x}\right]+
\end{gathered}
$$




$$
\begin{aligned}
& +\sum_{k=1}^{i-1} \frac{1}{\nu}\left\{W\left(x_{i-k}, t_{j+1}\right)-W\left(x_{i}, t_{j+1}\right)-k\left[W\left(x_{i-k-1}, t_{j+1}\right)-W\left(x_{i-k}, t_{j+1}\right)\right]\right\} . \\
& \cdot\left\{\operatorname{expint}\left(\frac{\mu_{n}}{\nu_{n}} k \Delta x\right)-\operatorname{expint}\left[\frac{\mu_{n}}{\nu_{n}}(k+1) \Delta x\right]\right\}+ \\
& +\frac{1}{\nu}\left[R-W\left(x_{i}, t_{j+1}\right)\right] \cdot \operatorname{expint}\left[\frac{\mu_{p}}{\nu_{p}}(N-i) \Delta x\right]+ \\
& \left.-\frac{1}{\nu} W\left(x_{i}, t_{j+1}\right) \cdot \operatorname{expint}\left(\frac{\mu_{n}}{\nu_{n}} i \Delta x\right)\right\}+ \\
& -1_{x_{i}>x\left(\tau_{j+1}\right)} \cdot \Delta t \cdot\left\{r K-q e^{x_{i}}+\right. \\
& +\sum_{k=i-l}^{i-1}\left\{\frac{W\left(x_{i-k-1}, t_{j+1}\right)-W\left(x_{i-k}, t_{j+1}\right)}{\nu \frac{\mu_{n}}{\nu_{n}} \Delta x} \cdot\left[e^{-\frac{\mu_{n}}{\nu_{n}} k \Delta x}-e^{-\frac{\mu_{n}}{\nu_{n}}(k+1) \Delta x}\right]+\right. \\
& +\frac{1}{\nu}\left\{W\left(x_{i-k}, t_{j+1}\right)-k\left[W\left(x_{i-k-1}, t_{j+1}\right)-W\left(x_{i-k}, t_{j+1}\right)\right]\right\} . \\
& \left.\cdot\left[\operatorname{expint}\left(\frac{\mu_{n}}{\nu_{n}} k \Delta x\right)-\operatorname{expint}\left(\frac{\mu_{n}}{\nu_{n}}(k+1) \Delta x\right)\right]\right\}+ \\
& +\frac{K}{\nu} \cdot \operatorname{expint}\left[\frac{\mu_{n}}{\nu_{n}}(i-l) \Delta x\right]+ \\
& \left.-\frac{e^{x_{i}}}{\nu} \cdot \operatorname{expint}\left[\left(1+\frac{\mu_{n}}{\nu_{n}}\right)(i-l) \Delta x\right]\right\}
\end{aligned}
$$

The left hand side of the difference equation can be written in the form of a tridiagonal matrix. Hence we can easily solve the system using the Gauss's elimination method. In Appendix B we present a code in $\mathrm{C}$ language which can be used to solve numerically the difference equation. 


\section{A.10.2 Difference Equation for American Down-And- Out Calls}

We can write the difference equation to price American down-and-out call options under variance gamma, by substituting equation (A.112) and equation (A.96) as modified by expression (A.97) in the initial PIDE (A.80). This corresponds to assuming that the option is always early exercised in the case that there is a large positive jump which takes the stock outside the considered range. We obtain in this way:

$$
\begin{gathered}
W\left(x_{i-1}, t_{j}\right)\left\{h-\frac{\Delta t \cdot\left(1-e^{-\frac{\mu_{n}}{\nu_{n}} \Delta x}\right)}{\nu \Delta x \frac{\mu_{n}}{\nu_{n}}}\right\}+ \\
+W\left(x_{i}, t_{j}\right)\left\{(1+r \Delta t)+\frac{\Delta t \cdot\left(1-e^{-\frac{\mu_{p}}{\nu_{p}} \Delta x}\right)}{\nu \Delta x \frac{\mu_{p}}{\nu_{p}}}+\frac{\Delta t \cdot\left(1-e^{-\frac{\mu_{n}}{\nu_{n}} \Delta x}\right)}{\nu \Delta x \frac{\mu_{n}}{\nu_{n}}}\right\}+ \\
-W\left(x_{i+1}, t_{j}\right)\left\{h+\frac{\Delta t \cdot\left(1-e^{-\frac{\mu_{p}}{\nu_{p}} \Delta x}\right)}{\nu \Delta x \frac{\mu_{p}}{\nu_{p}}}\right\}=W\left(x_{i}, t_{j+1}\right)+ \\
+\Delta t \cdot\left\{\sum_{k=1}^{N-i-1} \frac{1}{\nu \Delta x}\left[W\left(x_{i+k+1}, t_{j+1}\right)-W\left(x_{i+k}, t_{j+1}\right)\right] \cdot\right. \\
+\sum_{k=1}^{N-i-1} \frac{1}{\nu}\left\{W\left(x_{i+k}, t_{j+1}\right)-W\left(x_{i}, t_{j+1}\right)-k\left[W\left(x_{i+k+1}, t_{j+1}\right)-W\left(x_{i+k}, t_{j+1}\right)\right]\right\} \\
\left.+\sum_{k=1}^{-\frac{\mu_{p}}{\nu_{p}} k \Delta x}-e^{-\frac{\mu_{p}}{\nu_{p}}(k+1) \Delta x}\right]+ \\
+\sum_{k=1}^{i-1} \frac{1}{\nu}\left\{W\left(x_{i-k}, t_{j+1}\right)-W\left(x_{i}, t_{j+1}\right)-k\left[W\left(x_{i-k-1}, t_{j+1}\right)-W\left(x_{i-k}, t_{j+1}\right)\right]\right\} \\
\left.\operatorname{expint}\left(\frac{\mu_{p}}{\nu_{p}} k \Delta x\right)-\operatorname{expint}\left[\frac{\mu_{p}}{\nu_{p}}(k+1) \Delta x\right]\right\}+ \\
\left.\left.+x_{i-k-1}, t_{j+1}\right)-W\left(x_{i-k}, t_{j+1}\right)\right] \frac{\nu_{n}}{\mu_{n}}\left[e^{-\frac{\mu_{n}}{\nu_{n}} k \Delta x}-e^{-\frac{\mu_{n}}{\nu_{n}}(k+1) \Delta x}\right]+
\end{gathered}
$$




$$
\begin{aligned}
& \cdot\left\{\operatorname{expint}\left(\frac{\mu_{n}}{\nu_{n}} k \Delta x\right)-\operatorname{expint}\left[\frac{\mu_{n}}{\nu_{n}}(k+1) \Delta x\right]\right\}+ \\
& +\frac{1}{\nu}\left\{e^{x_{i}} \operatorname{expint}\left[\left(\frac{\mu_{p}}{\nu_{p}}-1\right)(N-i) \Delta x\right]+\right. \\
& -\left[K+W\left(x_{i}, t_{j+1}\right)\right] \operatorname{expint}\left[\frac{\mu_{p}}{\nu_{p}}(N-i) \Delta x\right]+ \\
& \left.\left.+\left[R-W\left(x_{i}, t_{j+1}\right)\right] \cdot \operatorname{expint}\left(\frac{\mu_{n}}{\nu_{n}} i \Delta x\right)\right\}\right\}+ \\
& -1_{x_{i}>x\left(\tau_{j+1}\right)} \cdot \Delta t \cdot\left\{r K-q e^{x_{i}}+\right. \\
& +\sum_{k=i-l}^{i-1}\left\{\frac{W\left(x_{i-k-1}, t_{j+1}\right)-W\left(x_{i-k}, t_{j+1}\right)}{\nu \frac{\mu_{n}}{\nu_{n}} \Delta x} \cdot\left[e^{-\frac{\mu_{n}}{\nu_{n}} k \Delta x}-e^{-\frac{\mu_{n}}{\nu_{n}}(k+1) \Delta x}\right]+\right. \\
& +\frac{1}{\nu}\left\{W\left(x_{i-k}, t_{j+1}\right)-k\left[W\left(x_{i-k-1}, t_{j+1}\right)-W\left(x_{i-k}, t_{j+1}\right)\right]\right\} \cdot \\
& \left.\cdot\left[\operatorname{expint}\left(\frac{\mu_{n}}{\nu_{n}} k \Delta x\right)-\operatorname{expint}\left(\frac{\mu_{n}}{\nu_{n}}(k+1) \Delta x\right)\right]\right\}+ \\
& +\frac{K}{\nu} \cdot \operatorname{expint}\left[\frac{\mu_{n}}{\nu_{n}}(i-l) \Delta x\right]-\frac{e^{x_{i}}}{\nu} \cdot \operatorname{expint}\left[\left(1+\frac{\mu_{n}}{\nu_{n}}\right)(i-l) \Delta x\right]+ \\
& \left.+\frac{R}{\nu} \operatorname{expint}\left(\frac{\mu_{n}}{\nu_{n}} i \Delta x\right)\right\}
\end{aligned}
$$

The left hand side of the difference equation can be written in the form of a tridiagonal matrix. Hence we can easily solve the system using the Gauss's elimination method. In Appendix B we present a code in C language which can be used to solve numerically the difference equation. 


\section{A.10.3 Difference Equation for American Up-And-Out Puts}

We can write the difference equation to price American up-and-out put options under variance gamma, by substituting equation (A.117) and equation (A.100) as modified by expression (A.101) in the initial PIDE (A.81). This corresponds to assuming that the option is always early exercised in the case that there is a large negative jump which takes the stock outside the considered range. We obtain in this way:

$$
\begin{gathered}
W\left(x_{i-1}, t_{j}\right)\left\{h-\frac{\Delta t \cdot\left(1-e^{-\frac{\mu_{n}}{\nu_{n}} \Delta x}\right)}{\nu \Delta x \frac{\mu_{n}}{\nu_{n}}}\right\}+ \\
+W\left(x_{i}, t_{j}\right)\left\{(1+r \Delta t)+\frac{\Delta t \cdot\left(1-e^{-\frac{\mu_{p}}{\nu_{p}} \Delta x}\right)}{\nu \Delta x \frac{\mu_{p}}{\nu_{p}}}+\frac{\Delta t \cdot\left(1-e^{-\frac{\mu_{n}}{\nu_{n}} \Delta x}\right)}{\nu \Delta x \frac{\mu_{n}}{\nu_{n}}}\right\}+ \\
-W\left(x_{i+1}, t_{j}\right)\left\{h+\frac{\Delta t \cdot\left(1-e^{-\frac{\mu_{p}}{\nu_{p}} \Delta x}\right)}{\nu \Delta x \frac{\mu_{p}}{\nu_{p}}}\right\}=W\left(x_{i}, t_{j+1}\right)+ \\
+\Delta t \cdot\left\{\sum_{k=1}^{N-i-1} \frac{1}{\nu \Delta x}\left[W\left(x_{i+k+1}, t_{j+1}\right)-W\left(x_{i+k}, t_{j+1}\right)\right] \cdot\right. \\
+\sum_{k=1}^{N-i-1} \frac{1}{\nu}\left[W\left(x_{i+k}, t_{j+1}\right)-W\left(x_{i}, t_{j+1}\right)-k\left[W\left(x_{i+k+1}, t_{j+1}\right)-W\left(x_{i+k}, t_{j+1}\right)\right]\right] \\
+\sum_{k=1} \frac{\nu_{p}}{\mu_{p}}\left[e^{-\frac{\mu_{p}}{\nu_{p}} k \Delta x}-e^{-\frac{\mu_{p}}{\nu_{p}}(k+1) \Delta x}\right]+ \\
+\sum_{k=1}^{i-1} \frac{1}{\nu \Delta x}\left\{W\left(x_{i-k}, t_{j+1}\right)-W\left(x_{i}, t_{j+1}\right)-k\left[W\left(x_{i-k-1}, t_{j+1}\right)-W\left(x_{i-k}, t_{j+1}\right)\right]\right\} \\
\left.\operatorname{expint}^{i-1}\left(\frac{\mu_{p}}{\nu_{p}} k \Delta x\right)-e x p i n t\left[\frac{\mu_{p}}{\nu_{p}}(k+1) \Delta x\right]\right\}+ \\
\left.\left.\sum_{i-k-1}, t_{j+1}\right)-W\left(x_{i-k}, t_{j+1}\right)\right] \cdot \frac{\nu_{n}}{\mu_{n}}\left[e^{-\frac{\mu_{n}}{\nu_{n}} k \Delta x}-e^{-\frac{\mu_{n}}{\nu_{n}}(k+1) \Delta x}\right]+
\end{gathered}
$$




$$
\begin{aligned}
& \cdot\left\{\operatorname{expint}\left(\frac{\mu_{n}}{\nu_{n}} k \Delta x\right)-\operatorname{expint}\left[\frac{\mu_{n}}{\nu_{n}}(k+1) \Delta x\right]\right\}+ \\
& +\frac{1}{\nu}\left[R-W\left(x_{i}, t_{j+1}\right)\right] \cdot \operatorname{expint}\left[\frac{\mu_{p}}{\nu_{p}}(N-i) \Delta x\right]+ \\
& +\frac{1}{\nu}\left\{\left[K-W\left(x_{i}, t_{j+1}\right)\right] \operatorname{expint}\left(\frac{\mu_{n}}{\nu_{n}} i \Delta x\right)+\right. \\
& \left.\left.-e^{x_{i}} \operatorname{expint}\left[\left(\frac{\mu_{n}}{\nu_{n}}+1\right) i \Delta x\right]\right\}\right\}+ \\
& -1_{x_{i}<x\left(\tau_{j+1}\right)} \cdot \Delta t \cdot\left\{q e^{x_{i}}-r K+\right. \\
& +\sum_{k=l-i}^{N-i-1}\left\{\frac{W\left(x_{i+k+1}, t_{j+1}\right)-W\left(x_{i+k}, t_{j+1}\right)}{\nu \frac{\mu_{p}}{\nu_{p}} \Delta x} \cdot\left[e^{-\frac{\mu_{p}}{\nu_{p}} k \Delta x}-e^{-\frac{\mu_{p}}{\nu_{p}}(k+1) \Delta x}\right]+\right. \\
& +\frac{1}{\nu}\left\{W\left(x_{i+k}, t_{j+1}\right)-k\left[W\left(x_{i+k+1}, t_{j+1}\right)-W\left(x_{i+k}, t_{j+1}\right)\right]\right\} . \\
& \left.\cdot\left[\operatorname{expint}\left(\frac{\mu_{p}}{\nu_{p}} k \Delta x\right)-\operatorname{expint}\left(\frac{\mu_{p}}{\nu_{p}}(k+1) \Delta x\right)\right]\right\}+ \\
& -\frac{K}{\nu} \cdot \operatorname{expint}\left[\frac{\mu_{p}}{\nu_{p}}(l-i) \Delta x\right]+\frac{e^{x_{i}}}{\nu} \cdot \operatorname{expint}\left[\left(\frac{\mu_{p}}{\nu_{p}}-1\right)(l-i) \Delta x\right] \\
& \left.+\frac{R}{\nu} \text { expint }\left[\frac{\mu_{p}}{\nu_{p}}(N-i) \Delta x\right]\right\}
\end{aligned}
$$

The left hand side of the difference equation can be written in the form of a tridiagonal matrix. Hence we can easily solve the system using the Gauss's elimination method. In Appendix B we present a code in $\mathrm{C}$ language which can be used to solve numerically the difference equation. 


\section{A.10.4 Difference Equation for American Down-And- Out Puts}

We can write the difference equation to price American down-and-out put options under variance gamma, by substituting equation (A.119) and equation (A.104) as modified by expression (A.105) in the initial PIDE (A.81). We obtain in this way:

$$
\begin{gathered}
W\left(x_{i-1}, t_{j}\right)\left\{h-\frac{\Delta t \cdot\left(1-e^{-\frac{\mu_{n}}{\nu_{n}} \Delta x}\right)}{\nu \Delta x \frac{\mu_{n}}{\nu_{n}}}\right\}+ \\
+W\left(x_{i}, t_{j}\right)\left\{(1+r \Delta t)+\frac{\Delta t \cdot\left(1-e^{-\frac{\mu_{p}}{\nu_{p}} \Delta x}\right)}{\nu \Delta x \frac{\mu_{p}}{\nu_{p}}}+\frac{\Delta t \cdot\left(1-e^{-\frac{\mu_{n}}{\nu_{n}} \Delta x}\right)}{\nu \Delta x \frac{\mu_{n}}{\nu_{n}}}\right\}+ \\
-W\left(x_{i+1}, t_{j}\right)\left\{h+\frac{\Delta t \cdot\left(1-e^{-\frac{\mu_{p}}{\nu_{p}} \Delta x}\right)}{\nu \Delta x \frac{\mu_{p}}{\nu_{p}}}\right\}=W\left(x_{i}, t_{j+1}\right)+ \\
+\Delta t \cdot\left\{\sum_{k=1}^{N-i-1} \frac{1}{\nu \Delta x}\left[W\left(x_{i+k+1}, t_{j+1}\right)-W\left(x_{i+k}, t_{j+1}\right)\right] \cdot\right. \\
+\sum_{k=1}^{N-i-1} \frac{1}{\nu}\left[W\left(x_{i+k}, t_{j+1}\right)-W\left(x_{i}, t_{j+1}\right)-k\left[W\left(x_{i+k+1}, t_{j+1}\right)-W\left(x_{i+k}, t_{j+1}\right)\right]\right] \\
\left.+\sum_{k=1}^{-\frac{\mu_{p}}{\nu_{p}} k \Delta x}-e^{-\frac{\mu_{p}}{\nu_{p}}(k+1) \Delta x}\right]+ \\
+\sum_{k=1}^{i-1} \frac{1}{\nu}\left\{W\left(x_{i-k}, t_{j+1}\right)-W\left(x_{i}, t_{j+1}\right)-k\left[W\left(x_{i-k-1}, t_{j+1}\right)-W\left(x_{i-k}, t_{j+1}\right)\right]\right\} . \\
\left.\operatorname{expint}\left(\frac{\mu_{p}}{\nu_{p}} k \Delta x\right)-e x p i n t\left[\frac{\mu_{p}}{\nu_{p}}(k+1) \Delta x\right]\right\}+ \\
\sum_{i-1}\left[W\left(x_{i-k-1}, t_{j+1}\right)-W\left(x_{i-k}, t_{j+1}\right)\right] \cdot \frac{\nu_{n}}{\mu_{n}}\left(e^{-\frac{\mu_{n}}{\nu_{n}} k \Delta x}-e^{-\frac{\mu_{n}}{\nu_{n}}(k+1) \Delta x}\right)+
\end{gathered}
$$




$$
\begin{aligned}
& \cdot\left\{\operatorname{expint}\left(\frac{\mu_{n}}{\nu_{n}} k \Delta x\right)-\operatorname{expint}\left[\frac{\mu_{n}}{\nu_{n}}(k+1) \Delta x\right]\right\}+ \\
& -\frac{1}{\nu} W\left(x_{i}, t_{j+1}\right) \cdot \operatorname{expint}\left[\frac{\mu_{p}}{\nu_{p}}(N-i) \Delta x\right]+ \\
& \left.+\frac{1}{\nu}\left[R-W\left(x_{i}, t_{j+1}\right)\right] \cdot \operatorname{expint}\left(\frac{\mu_{n}}{\nu_{n}} i \Delta x\right)\right\}+ \\
& -1_{x_{i}<x\left(\tau_{j+1}\right)} \cdot \Delta t \cdot\left\{q e^{x_{i}}-r K+\right. \\
& +\sum_{k=l-i}^{N-i-1}\left\{\frac{W\left(x_{i+k+1}, t_{j+1}\right)-W\left(x_{i+k}, t_{j+1}\right)}{\nu \frac{\mu_{p}}{\nu_{p}} \Delta x} \cdot\left[e^{-\frac{\mu_{p}}{\nu_{p}} k \Delta x}-e^{-\frac{\mu_{p}}{\nu_{p}}(k+1) \Delta x}\right]+\right. \\
& +\frac{1}{\nu}\left\{W\left(x_{i+k}, t_{j+1}\right)-k\left[W\left(x_{i+k+1}, t_{j+1}\right)-W\left(x_{i+k}, t_{j+1}\right)\right]\right\} . \\
& \left.\cdot\left[\operatorname{expint}\left(\frac{\mu_{p}}{\nu_{p}} k \Delta x\right)-\operatorname{expint}\left(\frac{\mu_{p}}{\nu_{p}}(k+1) \Delta x\right)\right]\right\}+ \\
& -\frac{K}{\nu} \cdot \operatorname{expint}\left[\frac{\mu_{p}}{\nu_{p}}(l-i) \Delta x\right]+ \\
& \left.+\frac{e^{x_{i}}}{\nu} \cdot \operatorname{expint}\left[\left(\frac{\mu_{p}}{\nu_{p}}-1\right)(l-i) \Delta x\right]\right\}
\end{aligned}
$$

The left hand side of the difference equation can be written in the form of a tridiagonal matrix. Hence we can easily solve the system using the Gauss's elimination method. In Appendix B we present a code in C language which can be used to solve numerically the difference equation. 


\section{Appendix B}

\section{Option Pricing under the Variance Gamma Process C Code}

// Option Pricing Under the Variance Gamma Process.c

// Author: Filippo Fiorani

// This code allows to price options under the variance gamma // process using a finite difference scheme

// Pricing of vanilla European and American options as well as

// European and American barrier option is possible

// The desired option and the correspondent parameters can be // selected at the beginnning of the main function

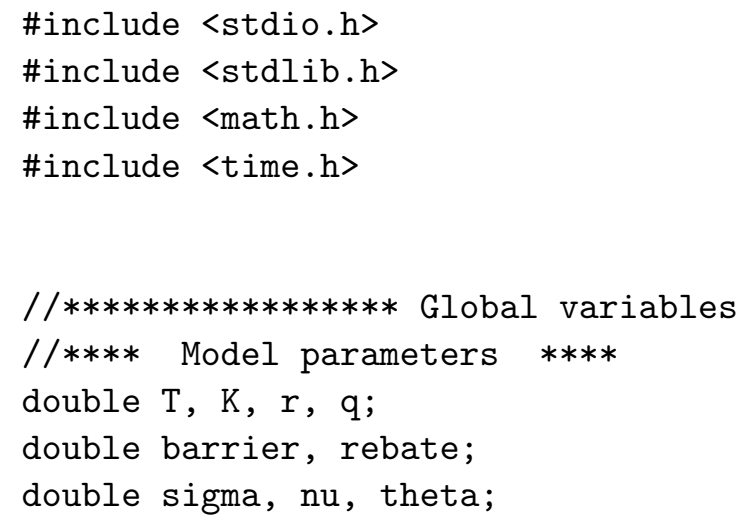




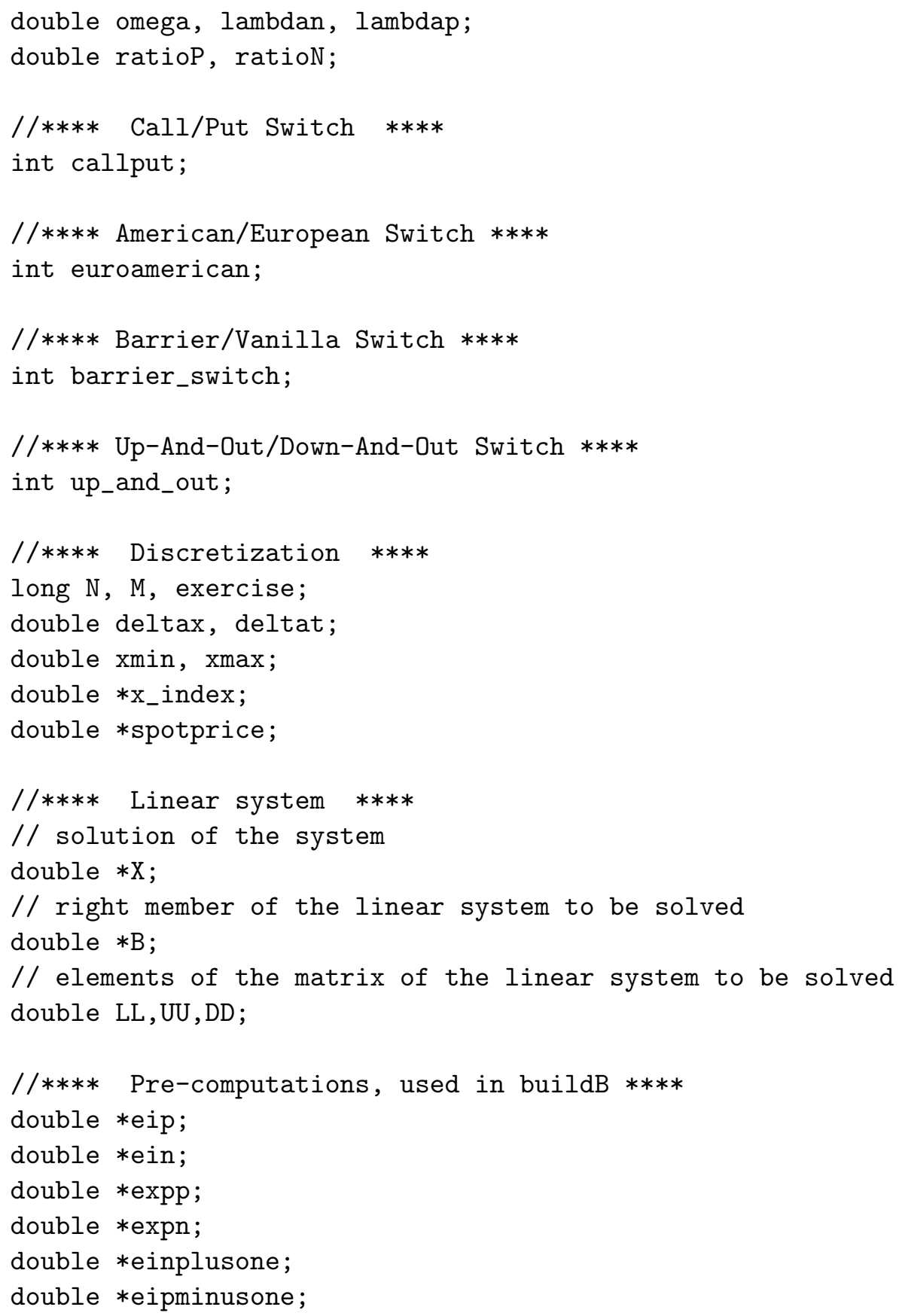




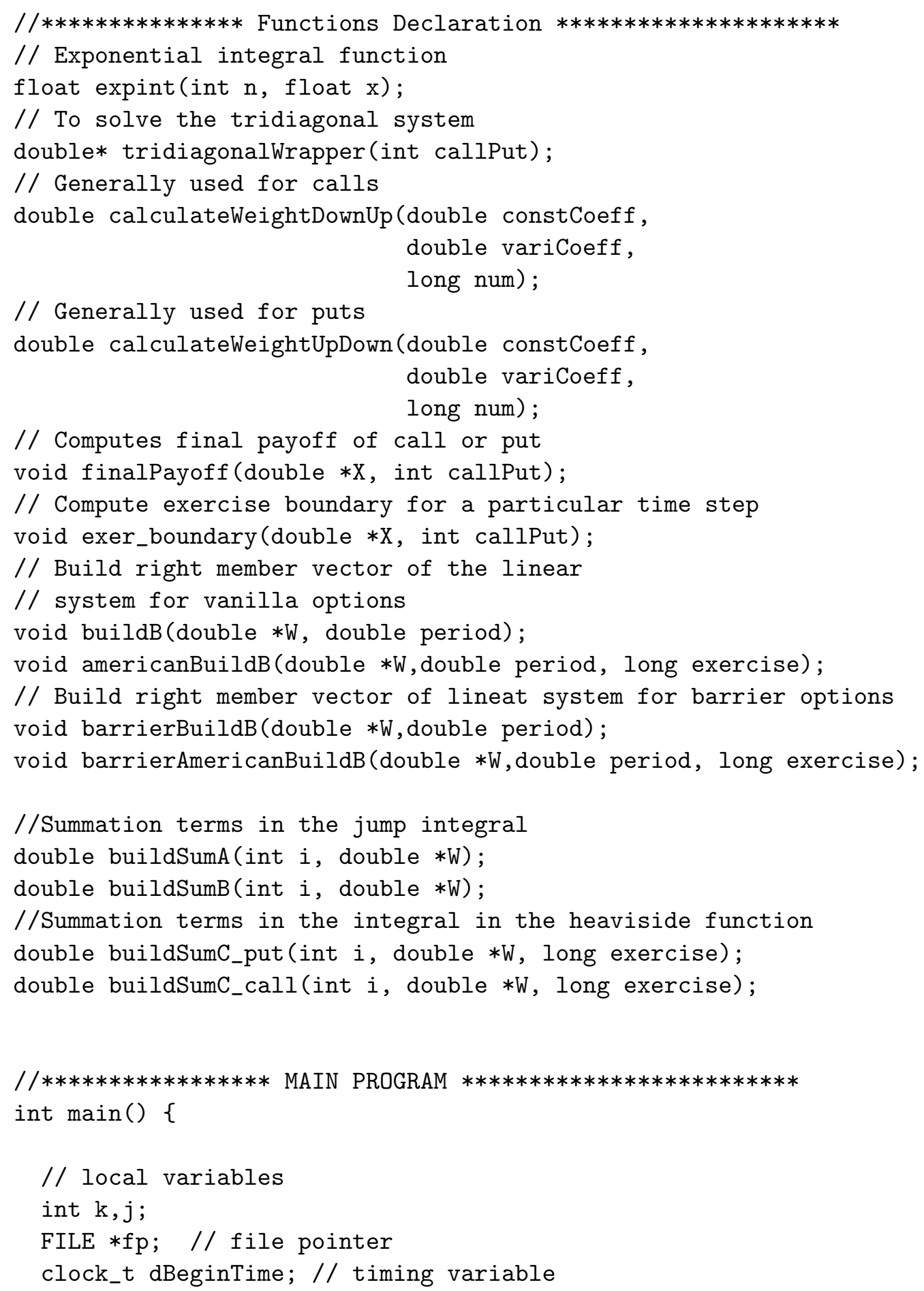




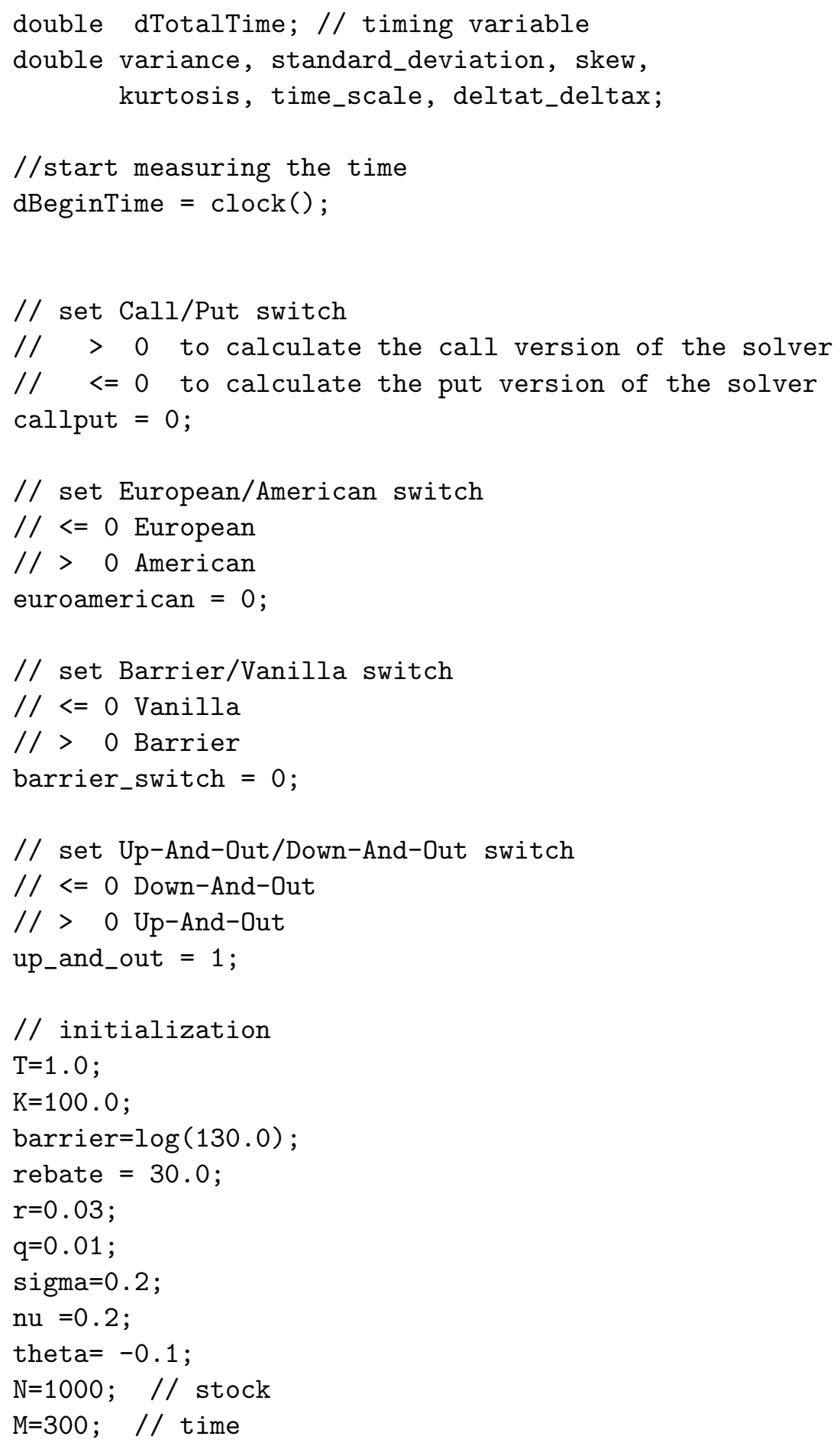




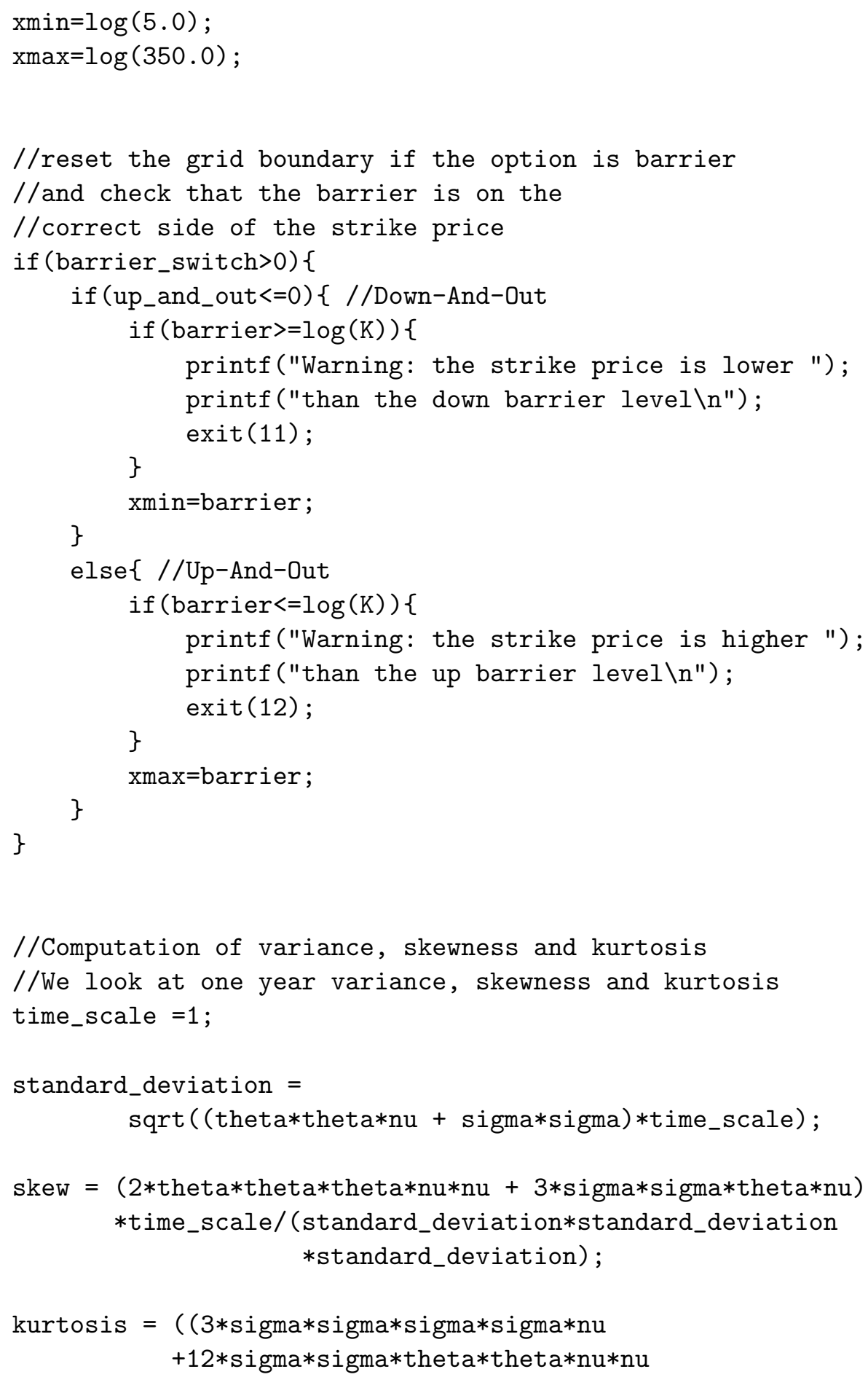




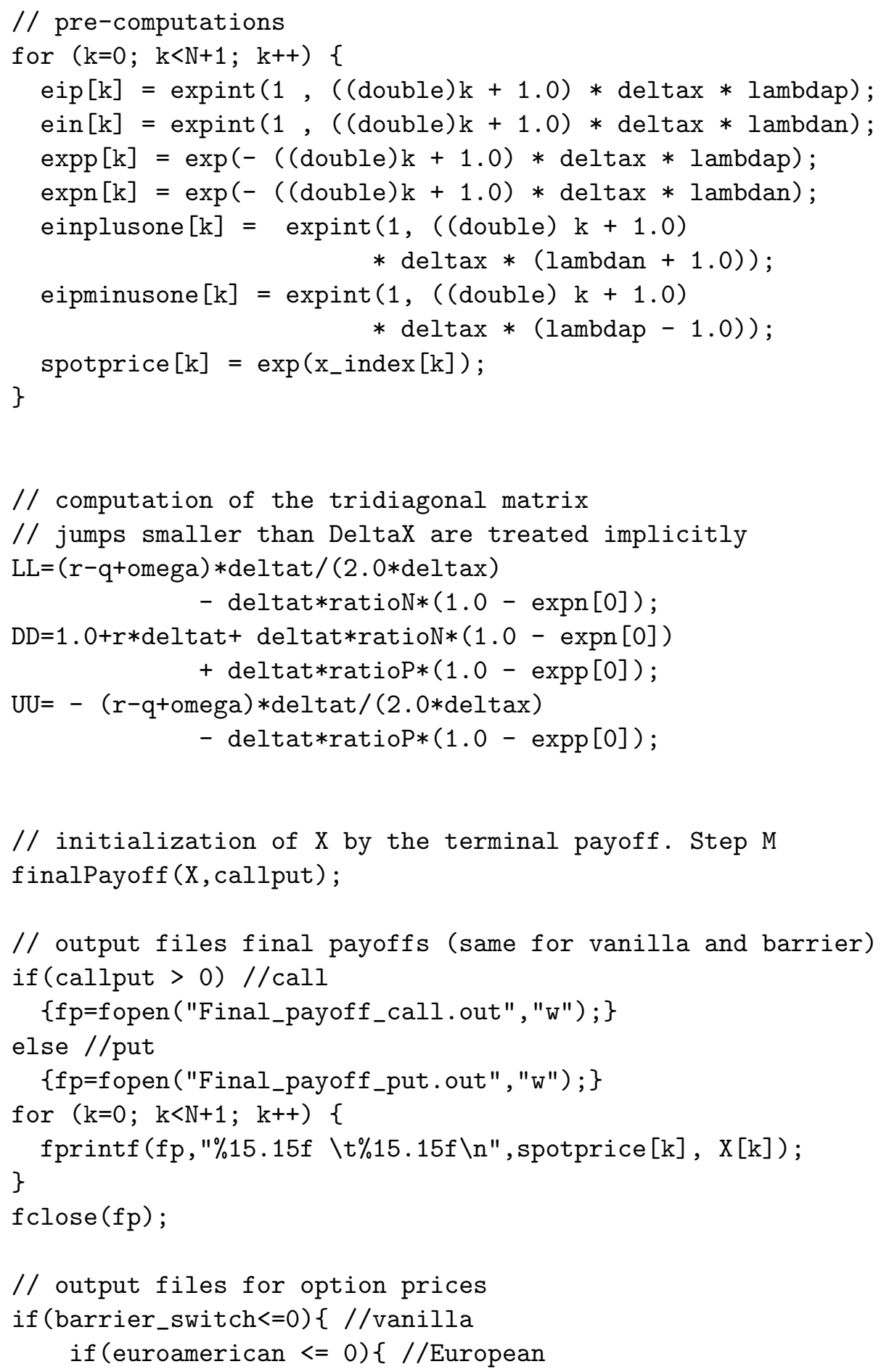




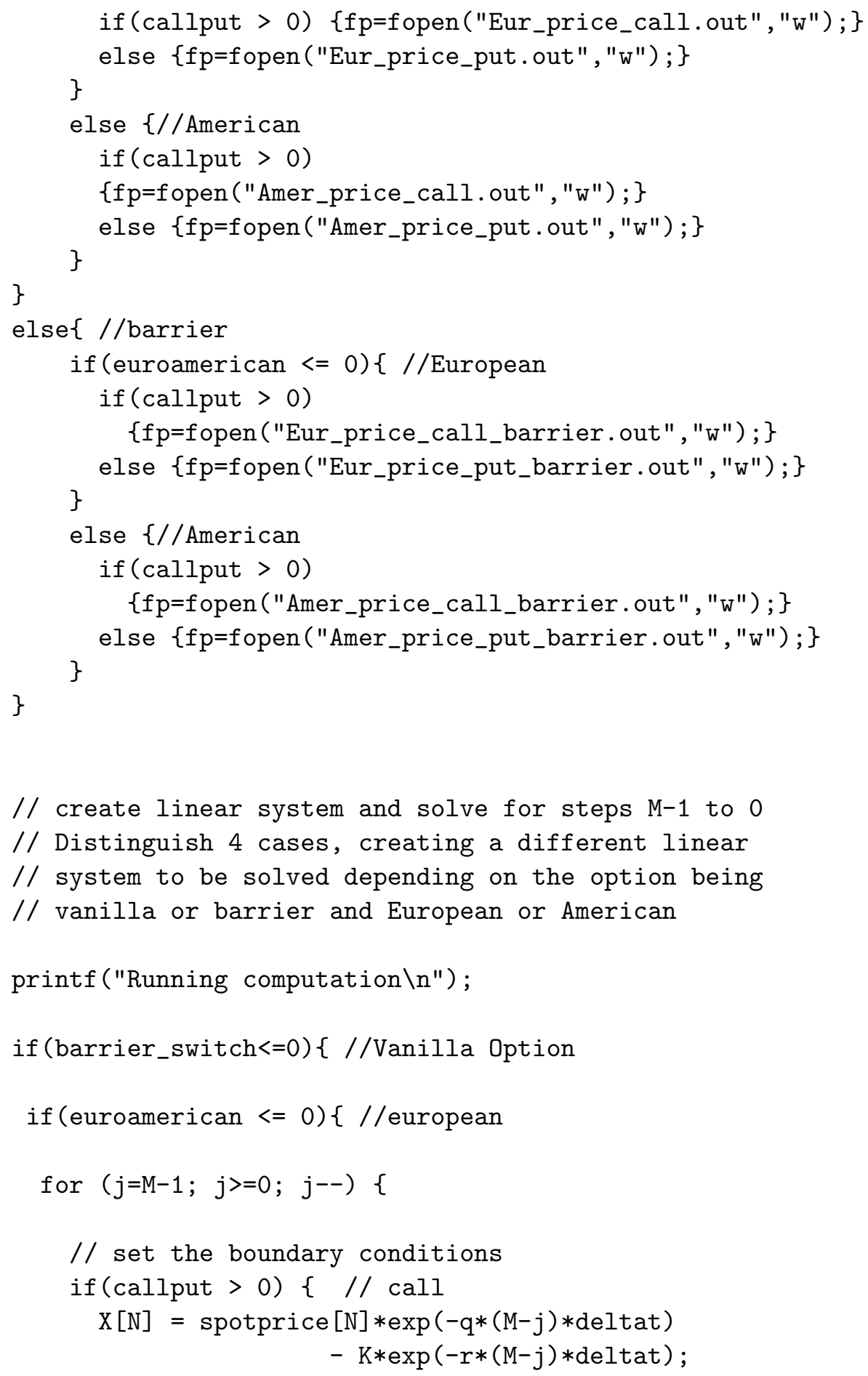




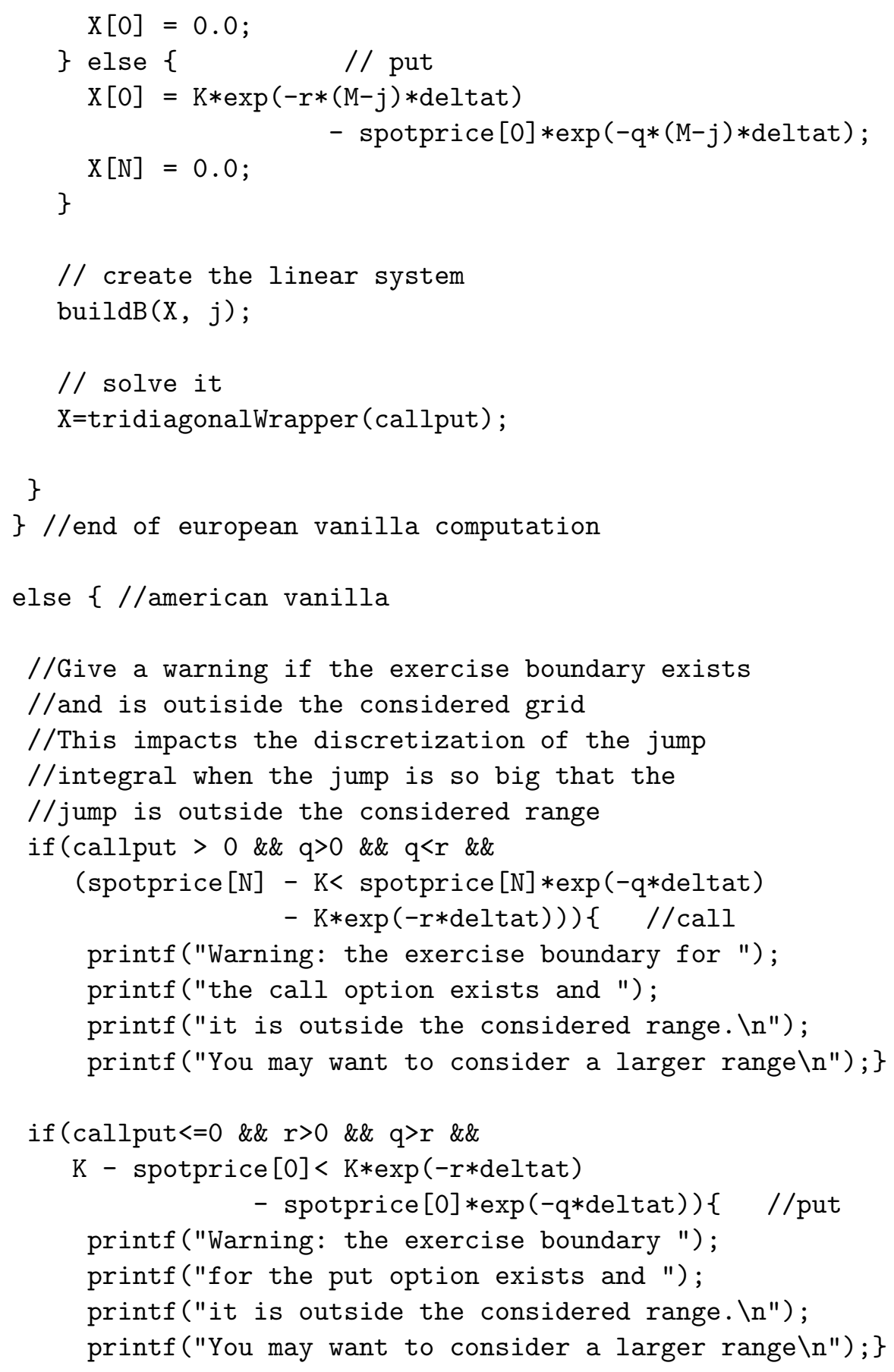




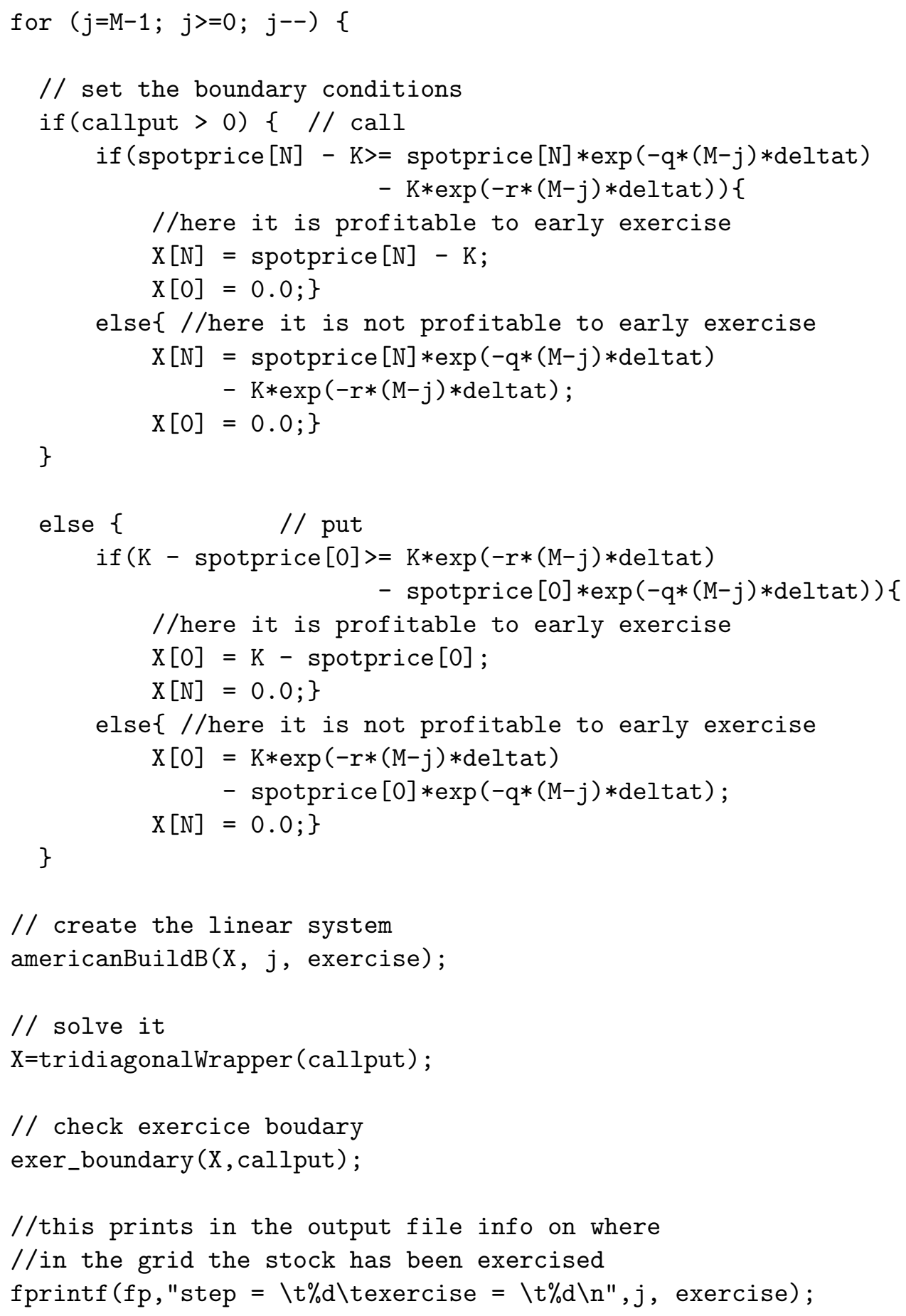




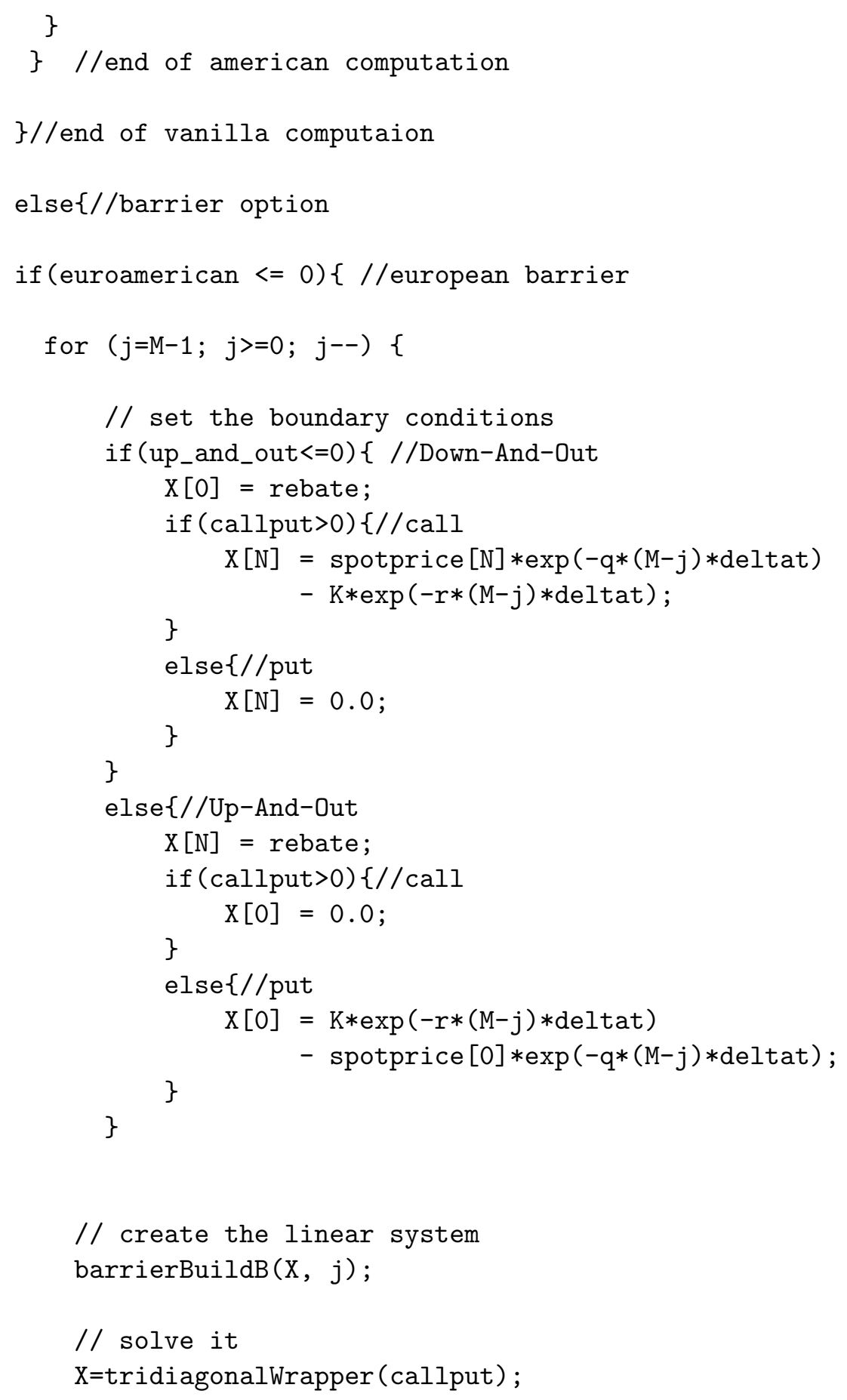




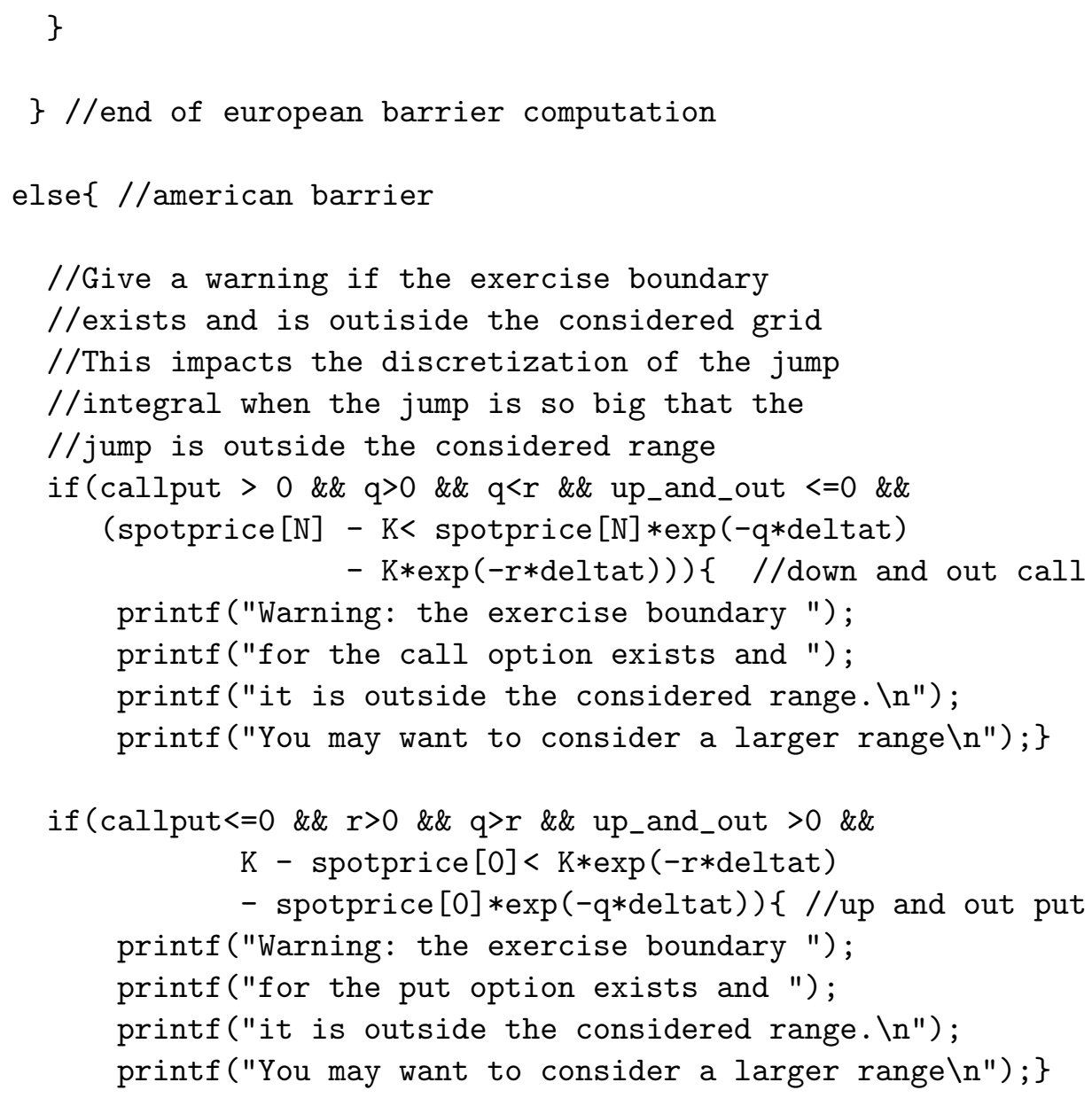




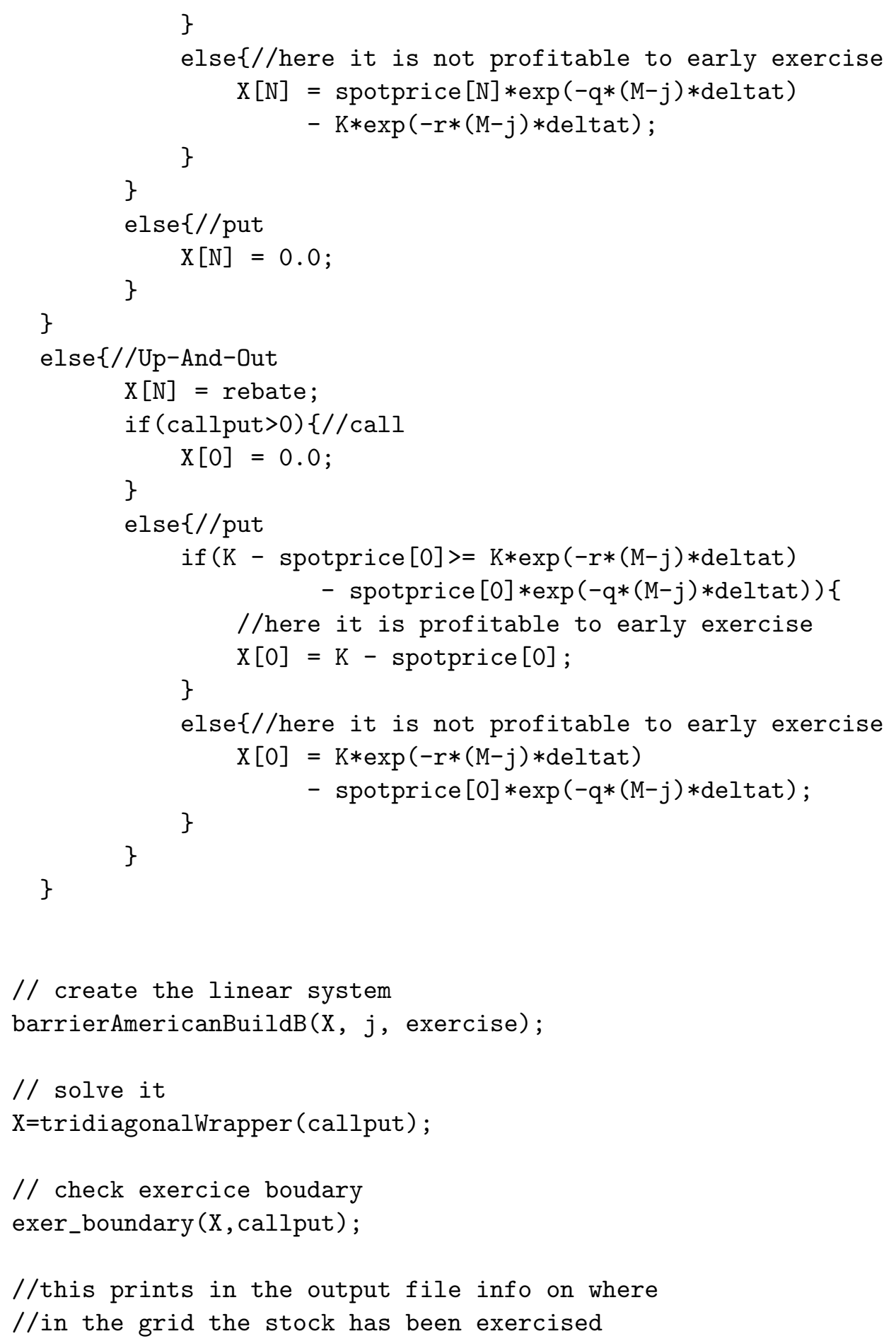




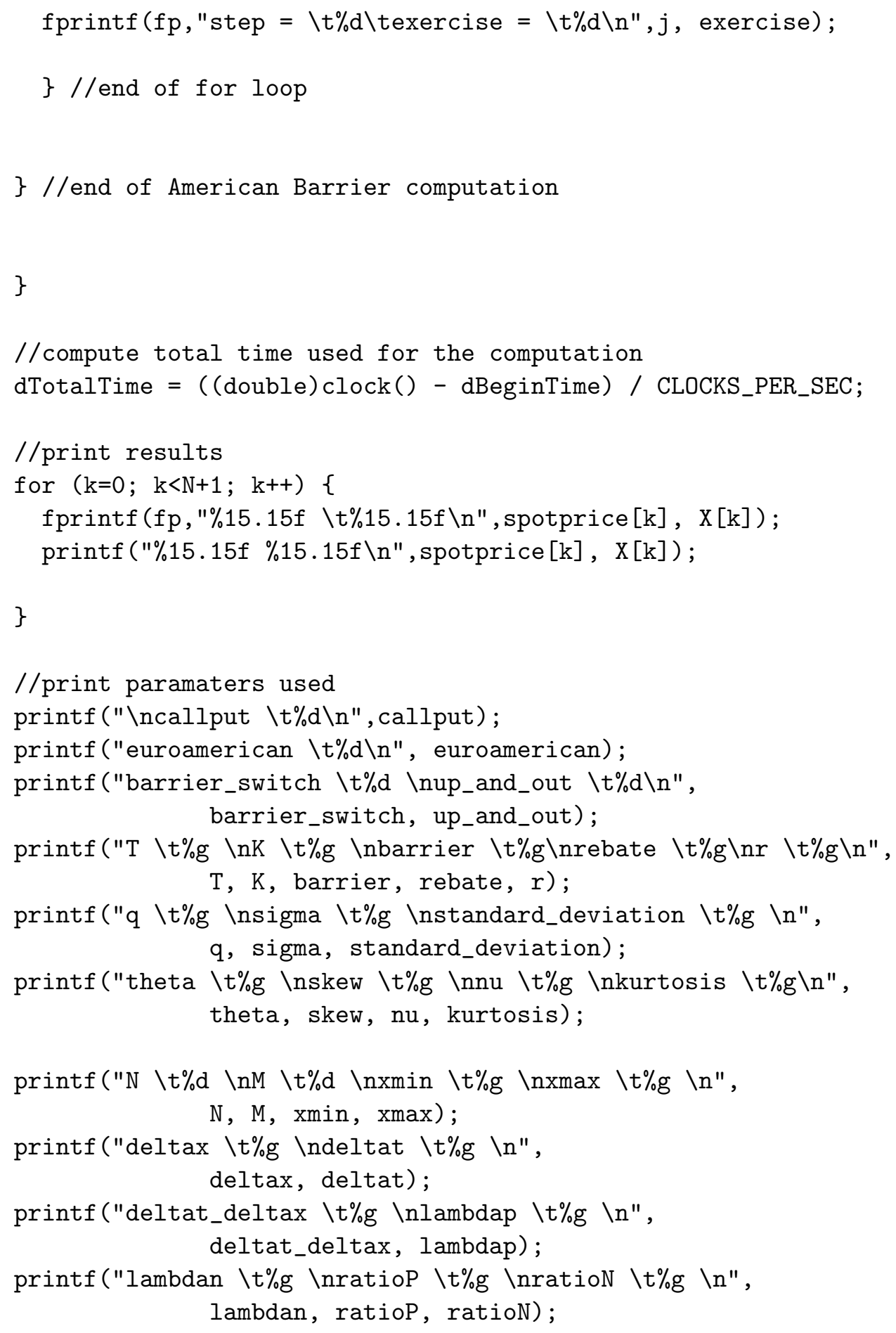




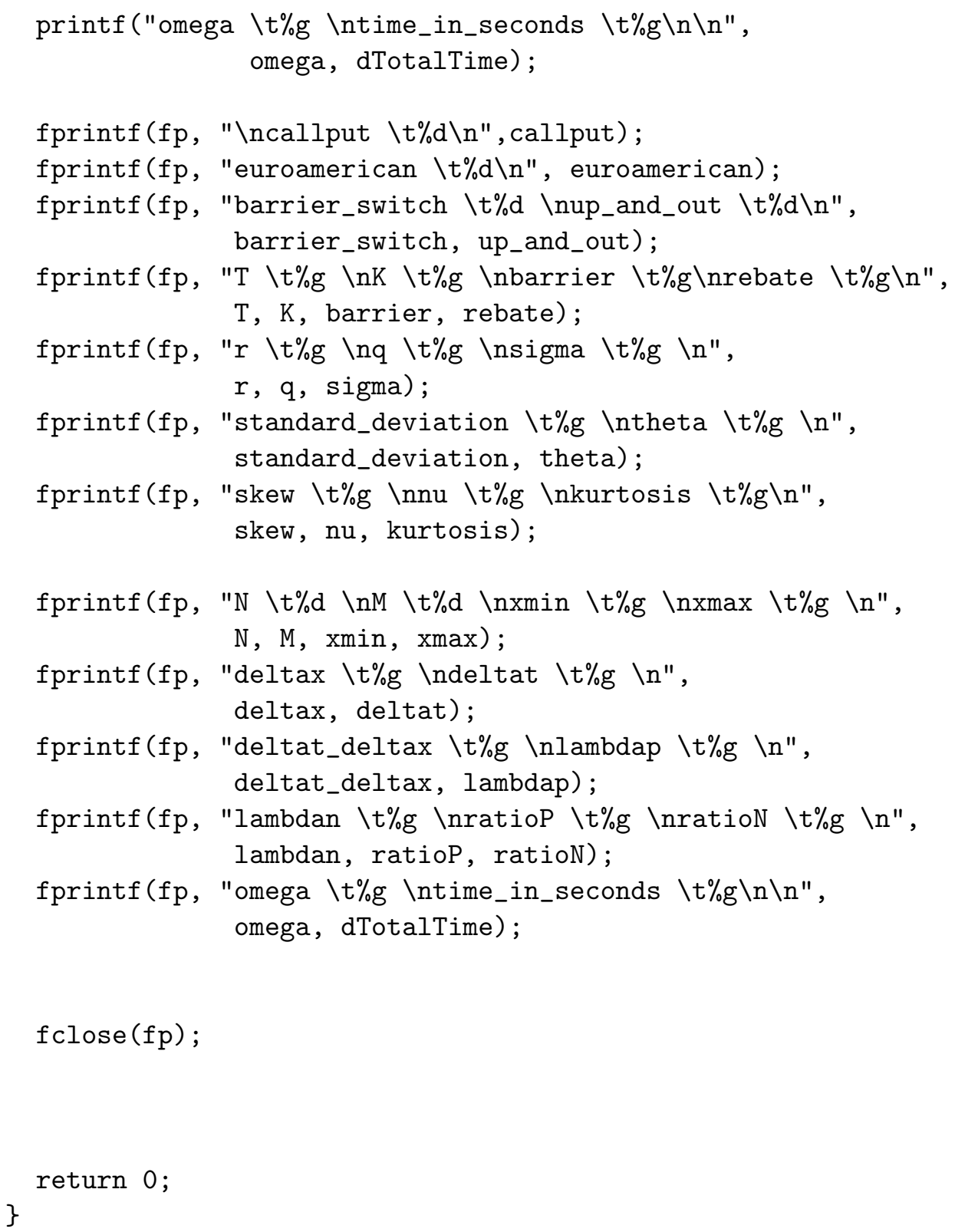


$/ / * * * * * * * * * * * * * * * * * * * * * * * * * * * * * * * * * * * * * * * * * * * * * * * * * * * * * * * * * * * * *)$

$/ / * * * * * * * * * * * * * * * * *$ SUB ROUTINES

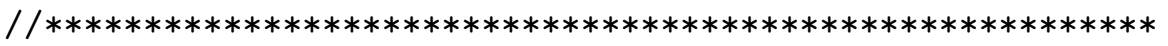

// Use this funciton to solve the tridiagonal system.

// The "callPut" argument should be > 0 to calculate

// the call version of the solver (calculates the $\mathrm{X}$

// values from 0 to $\mathrm{N}$ ), "callPut" should be $<=0$

// to call the put version of the solver.

//

// Assumptions when using this is that the X array memory

// has already been allocated, and that the B array values

// have already been calculated. X and B should be global

// arrays as should be the constant coefficients

// that make up the three diagonals.

double* tridiagonalWrapper (int callPut) \{

// use a recursive algorithm to calculate the values in the

// X array, or solve the tridiagonal linear system.

if (callPut > 0) $\{/ /$ call

calculateWeightDownUp $(0, / /$ const coeff

$0, / /$ variable coeff

1 // step number

);

\} else $\{/ /$ put

calculateWeightUpDown $(0, / /$ const coeff

$0, / /$ variable coeff

$\mathrm{N}-1 / /$ step number

) ;

\}

return $\mathrm{X}$;

\}

// Generally used for calls. Easiest to call through the

// tridiagonalWrapper.

double calculateWeightDownUp(double constCoeff,

double variCoeff,

long num) \{ 


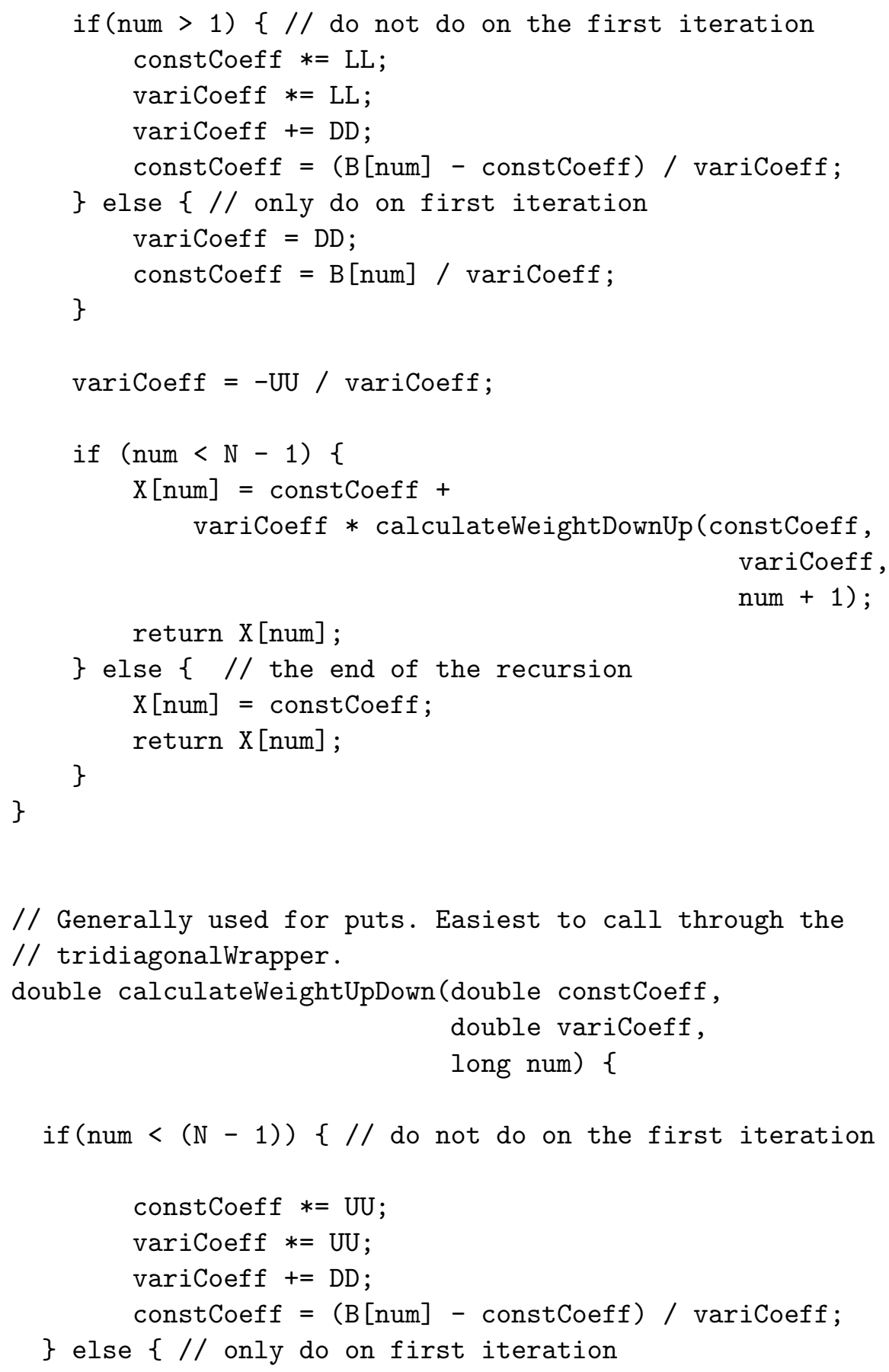




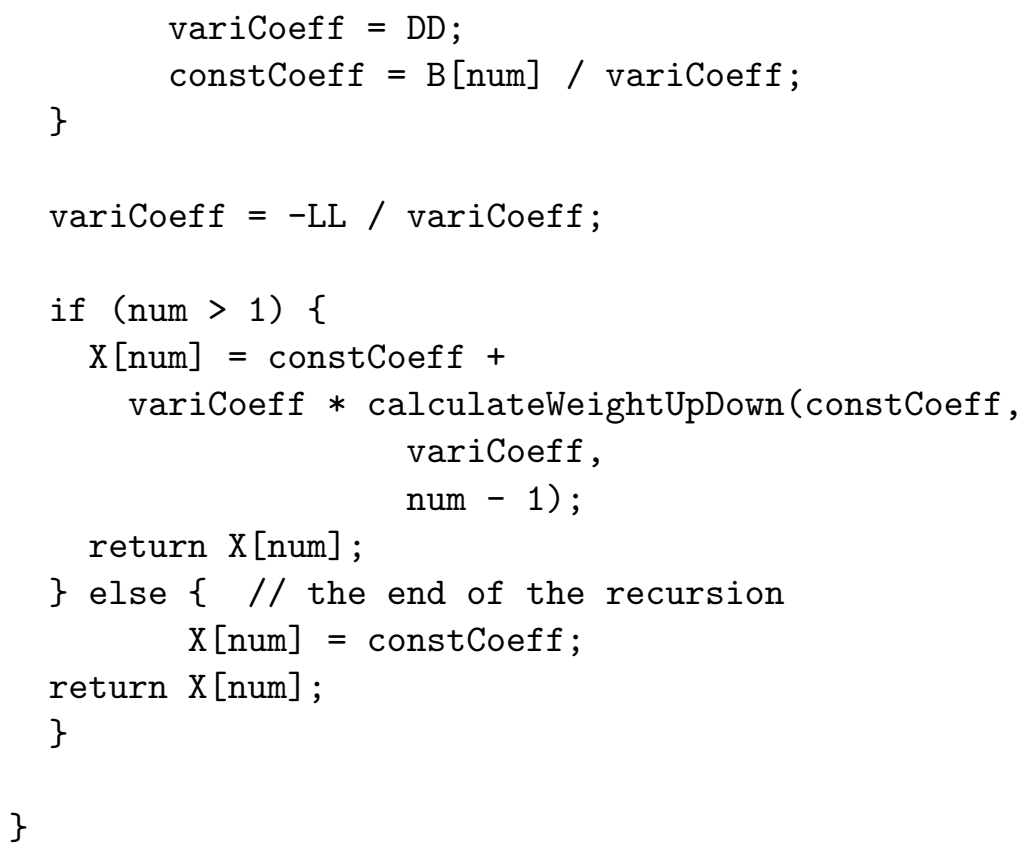




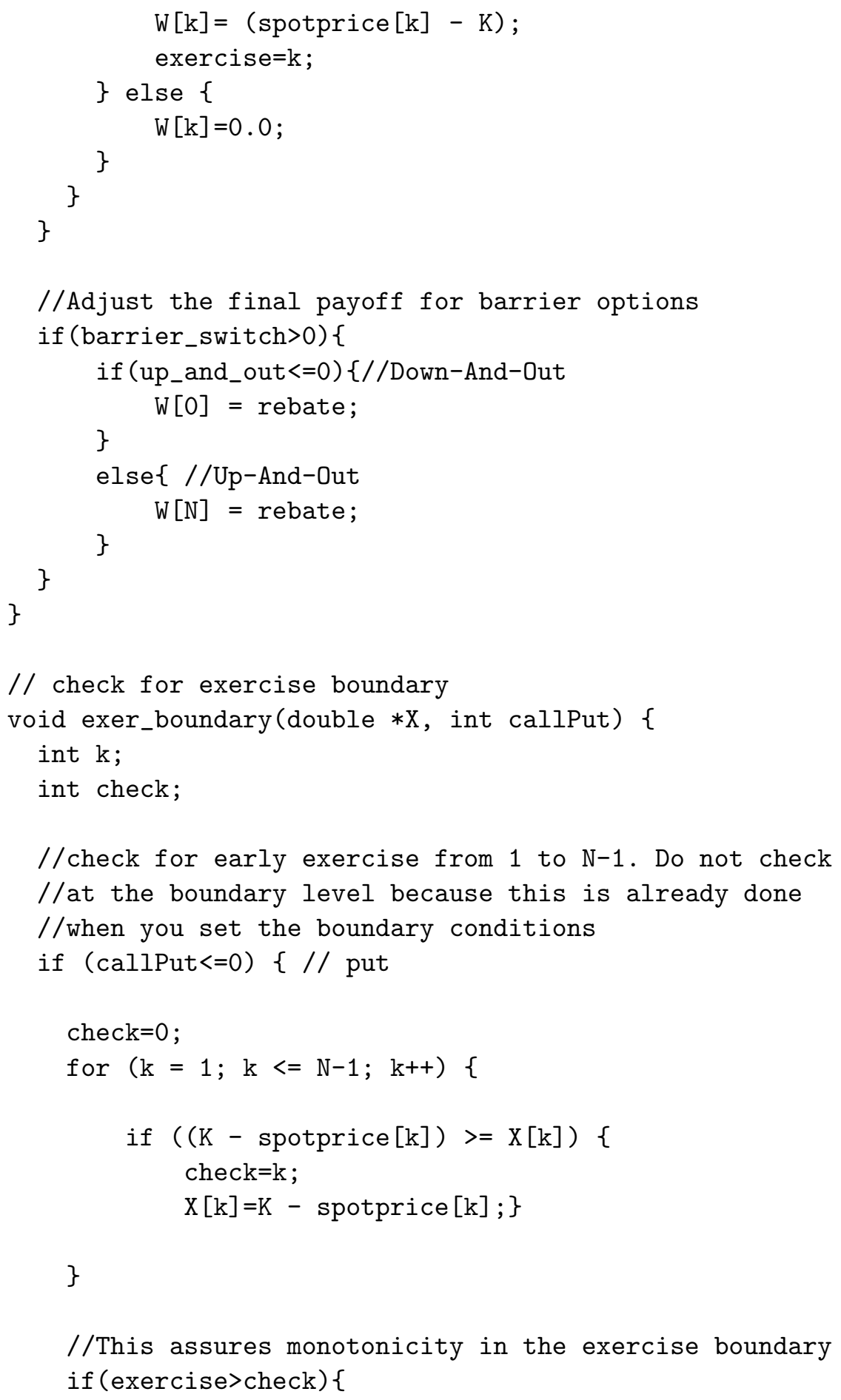




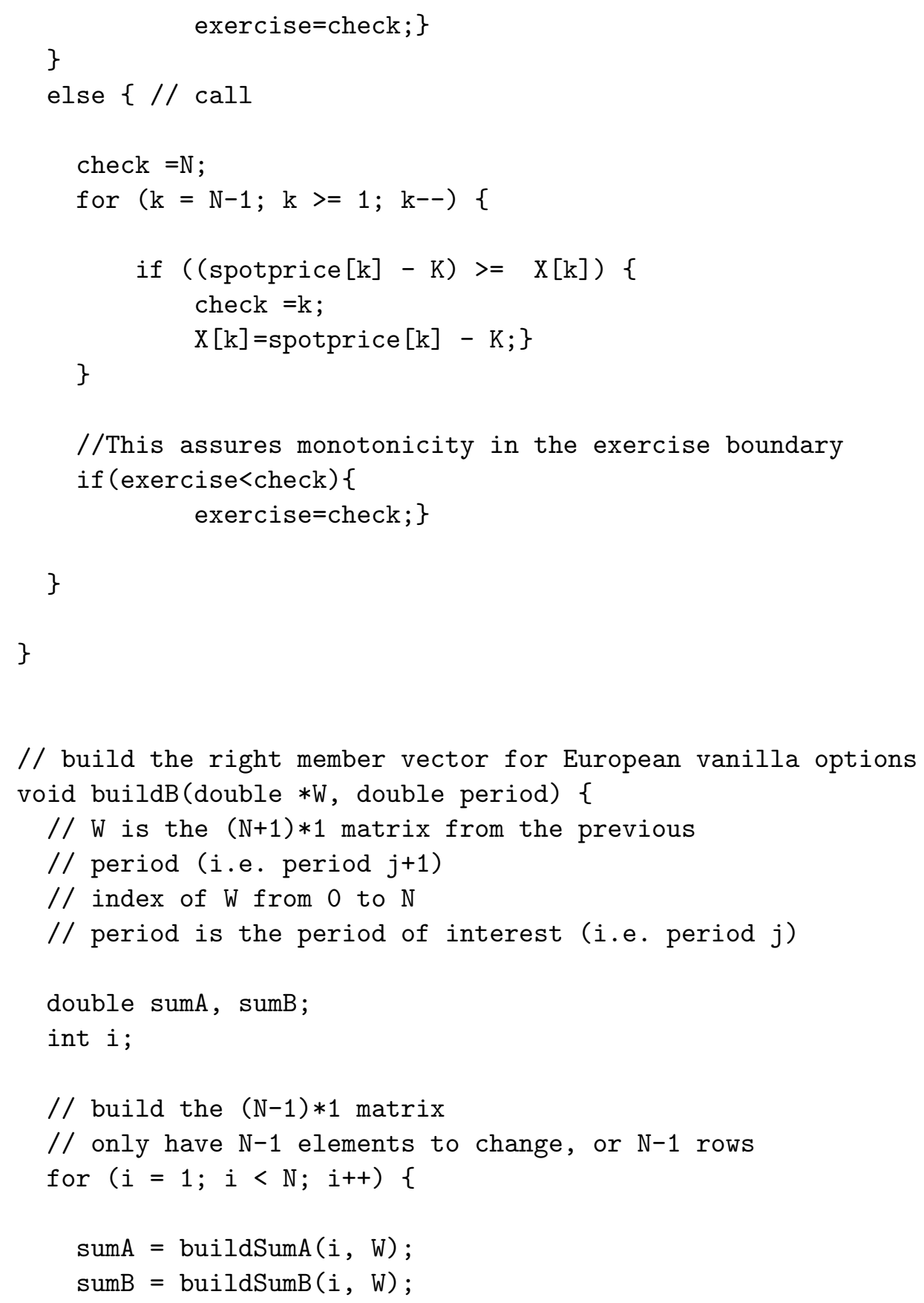




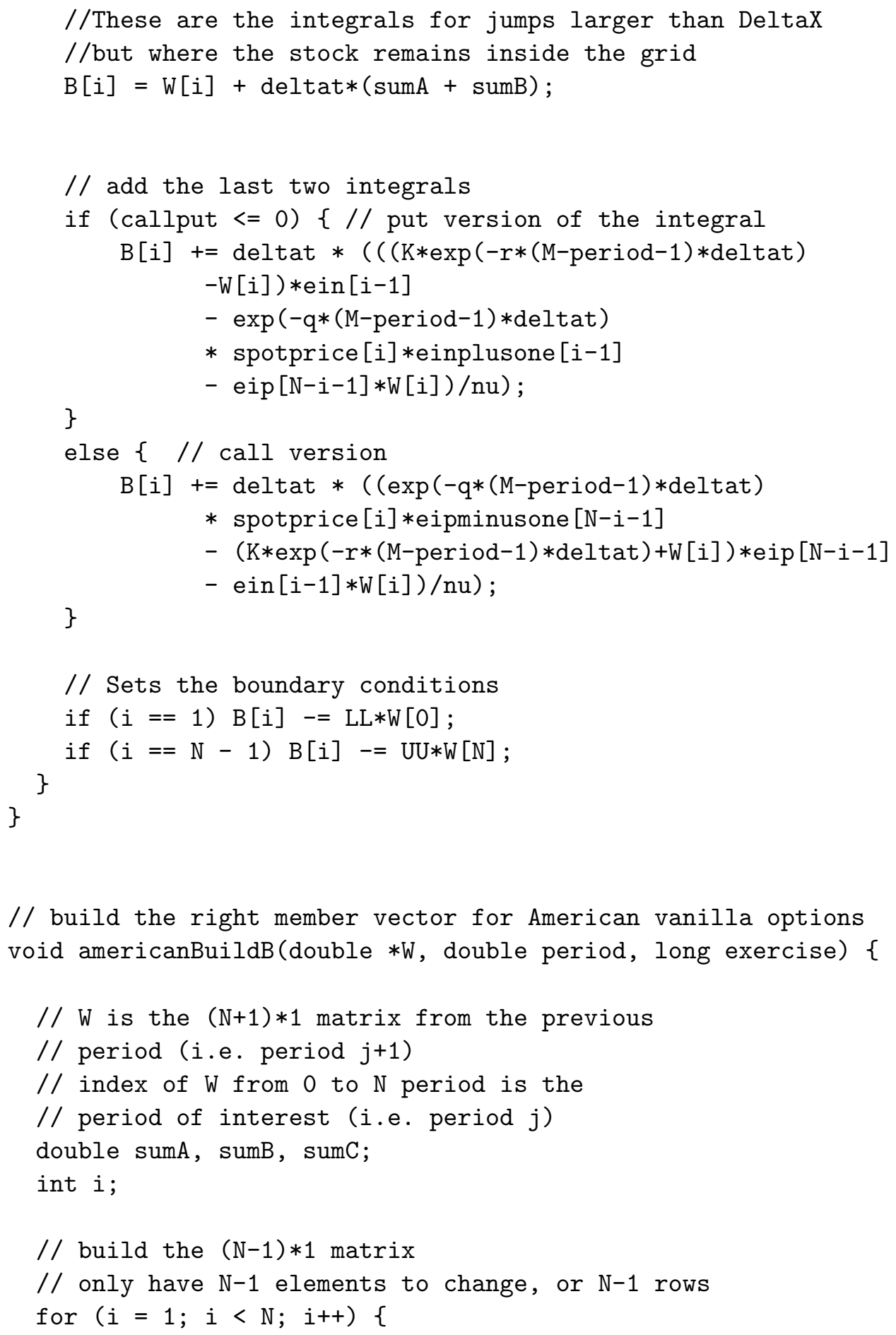




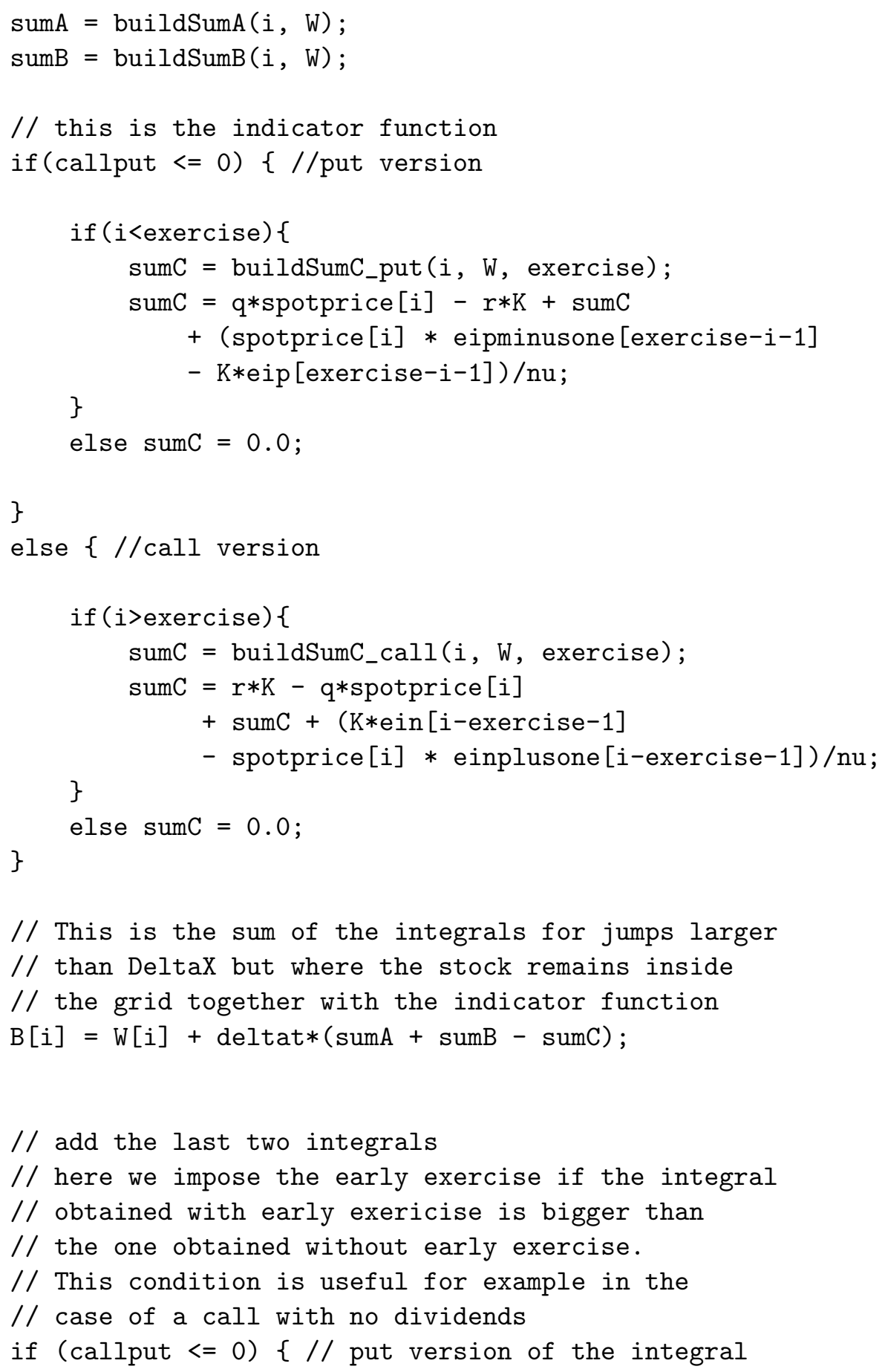




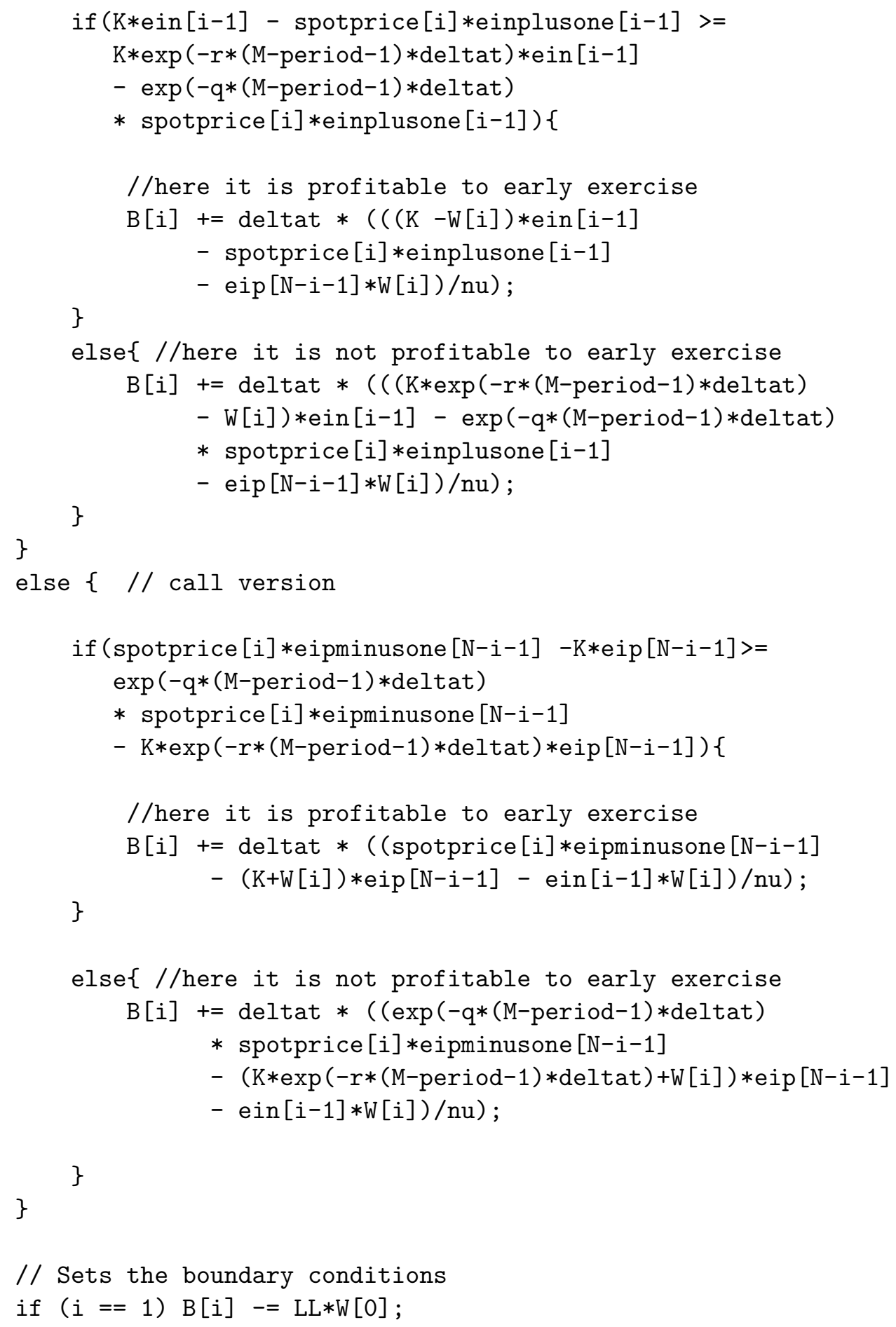




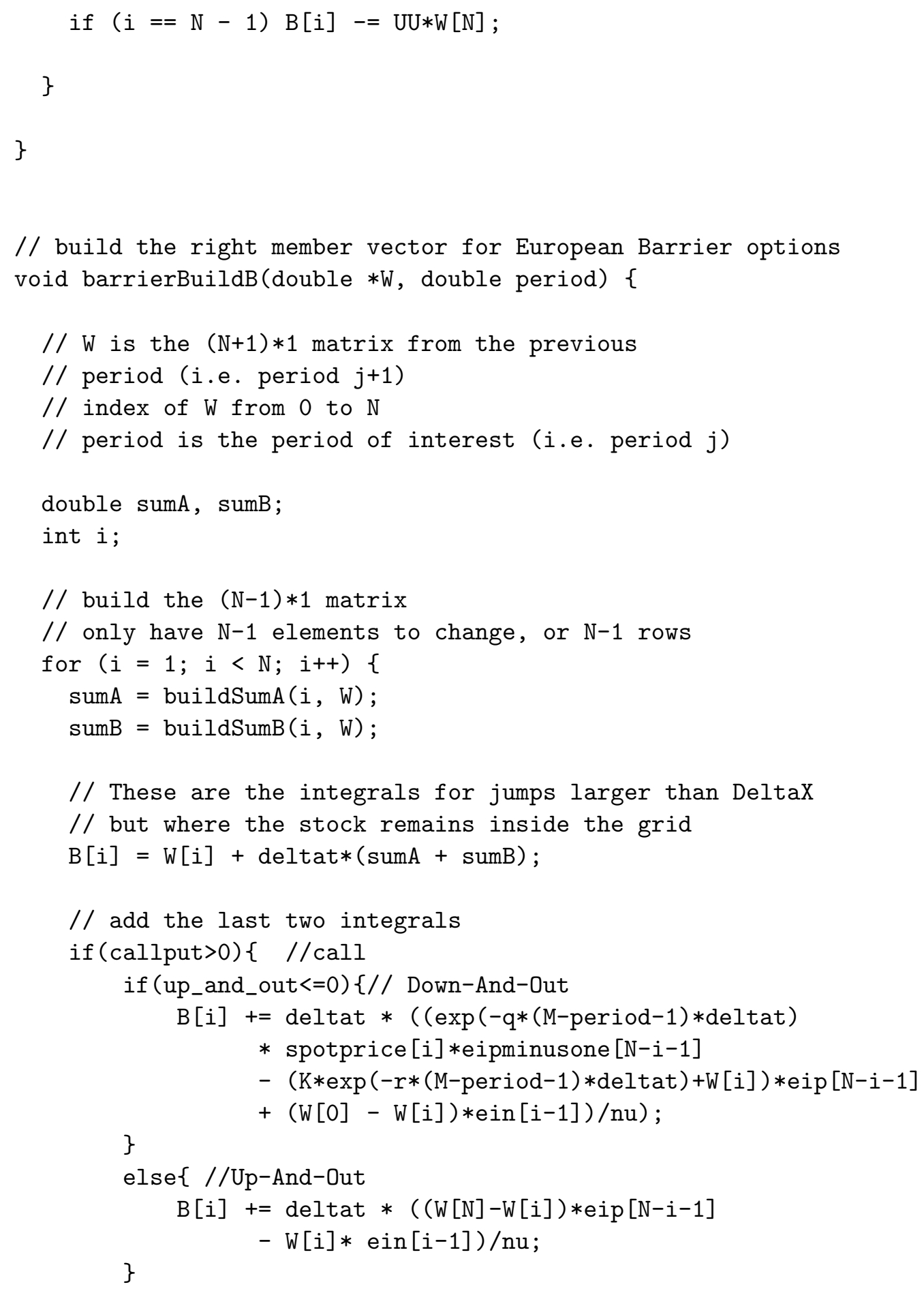




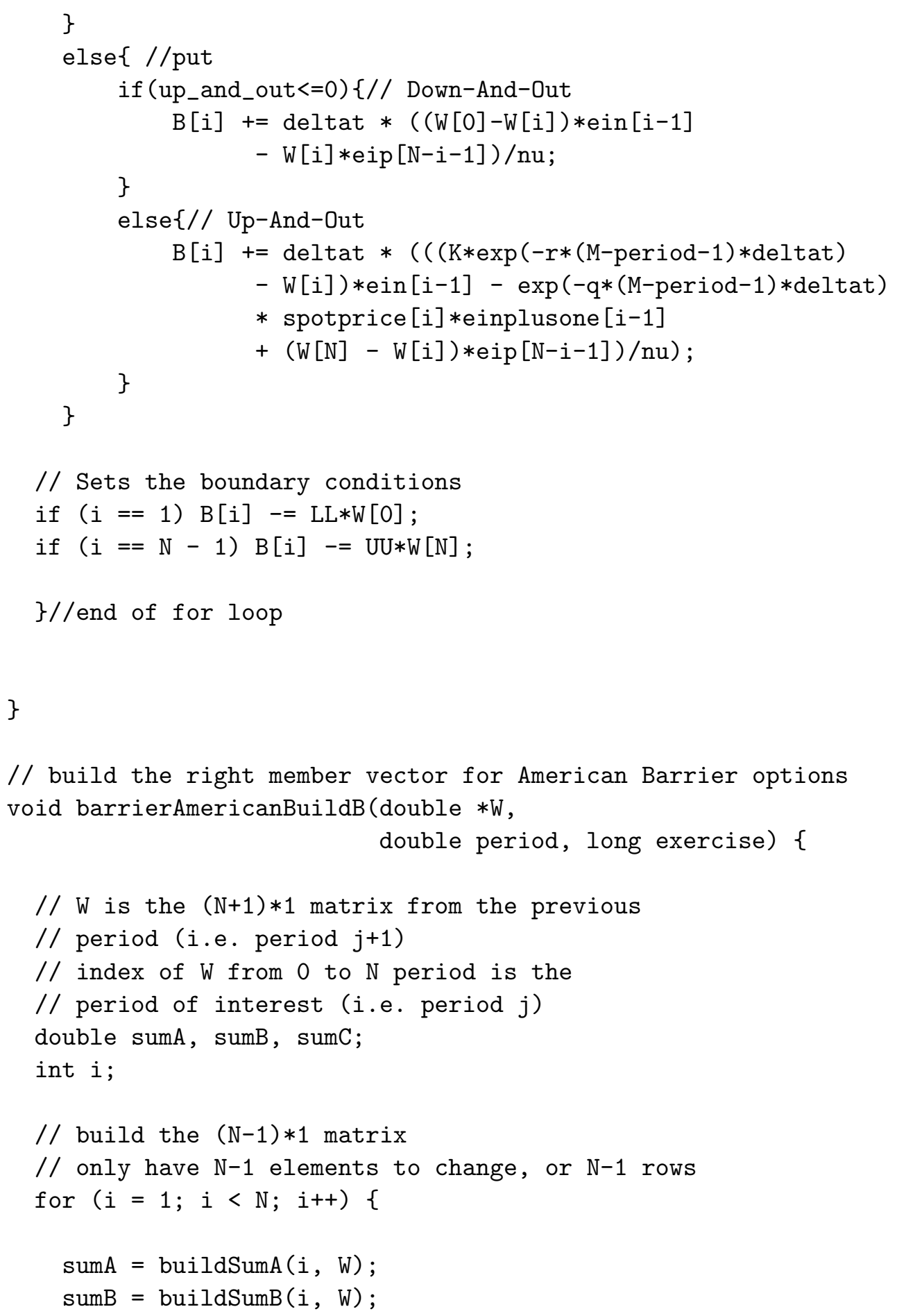




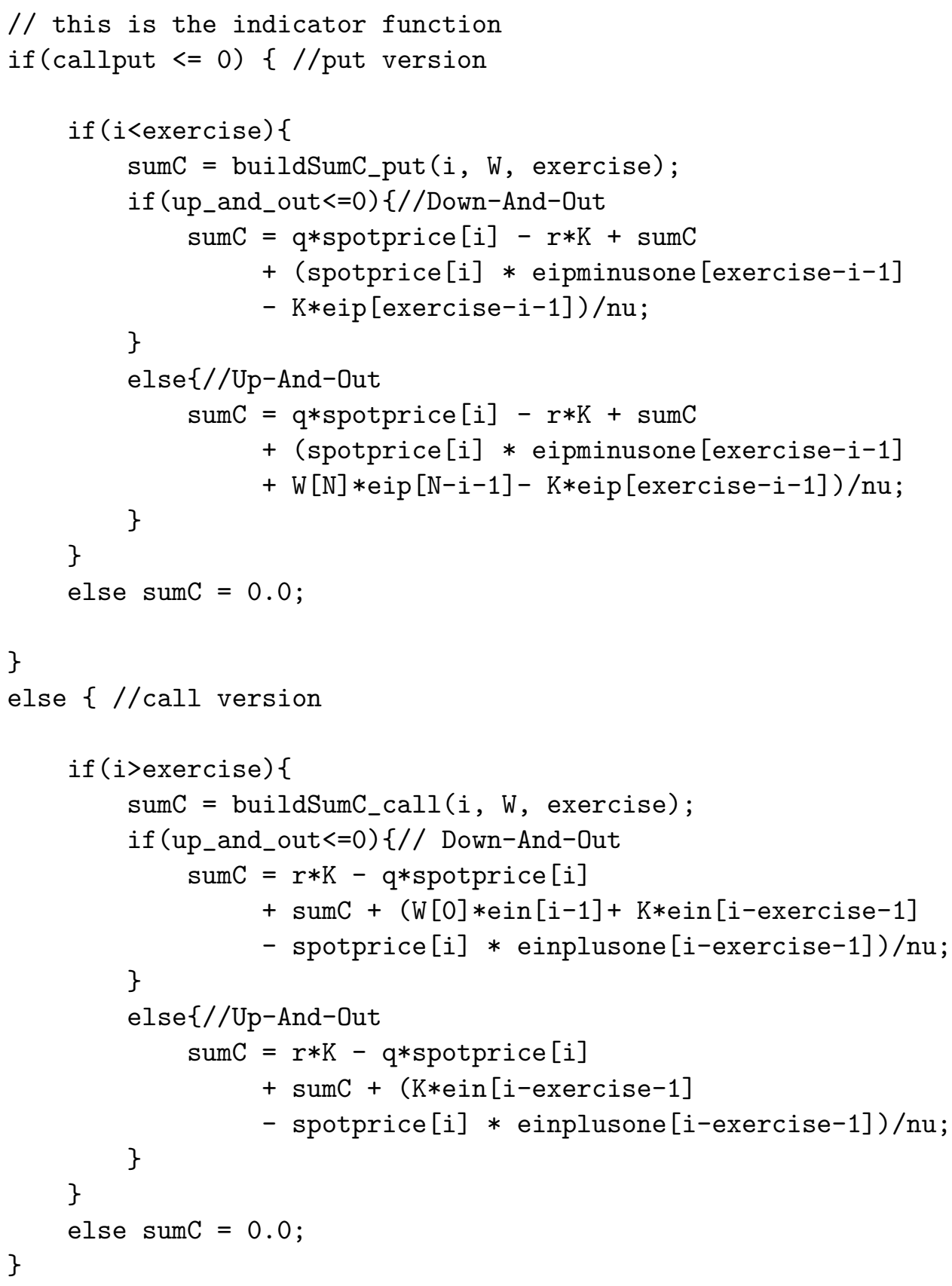




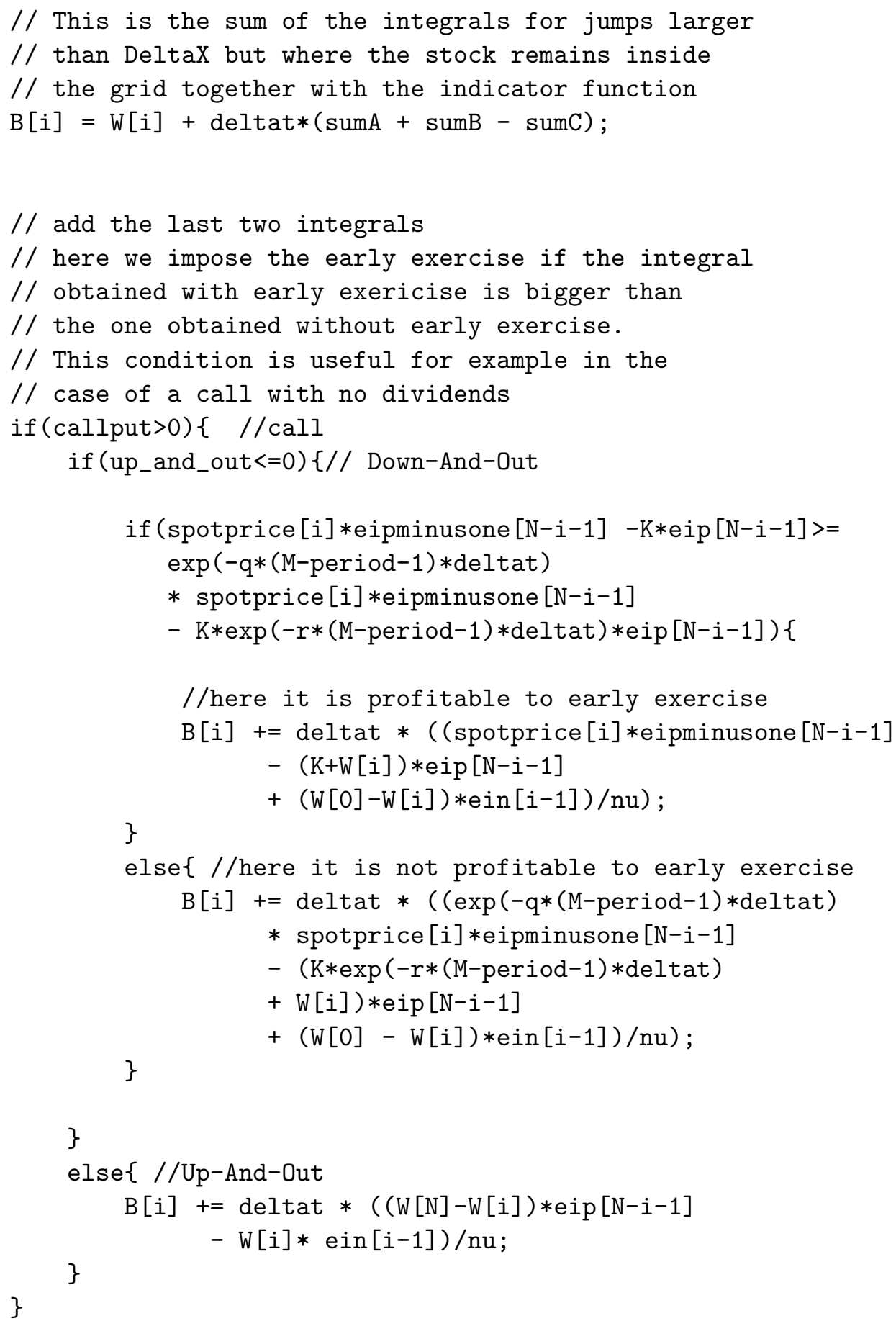




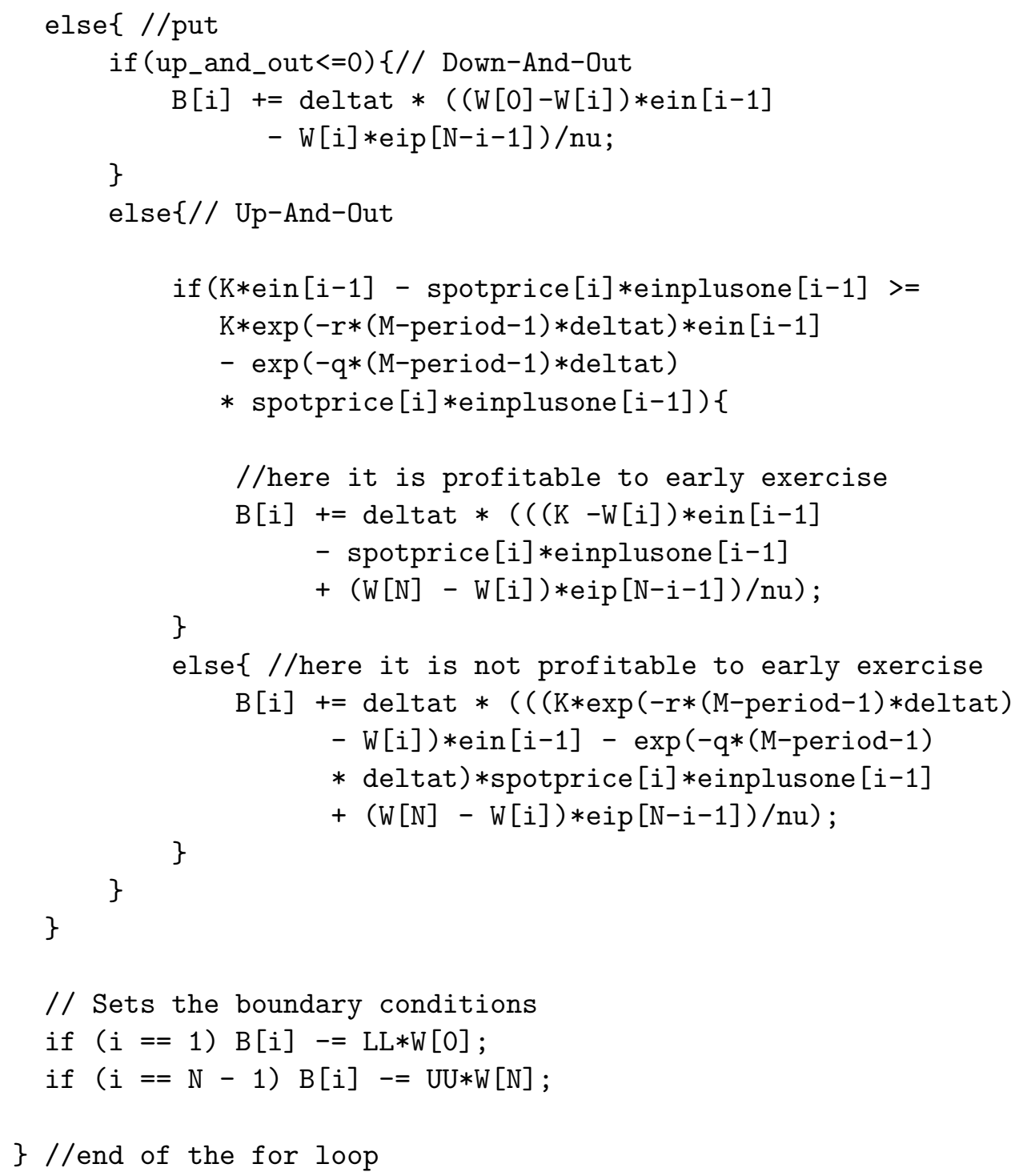




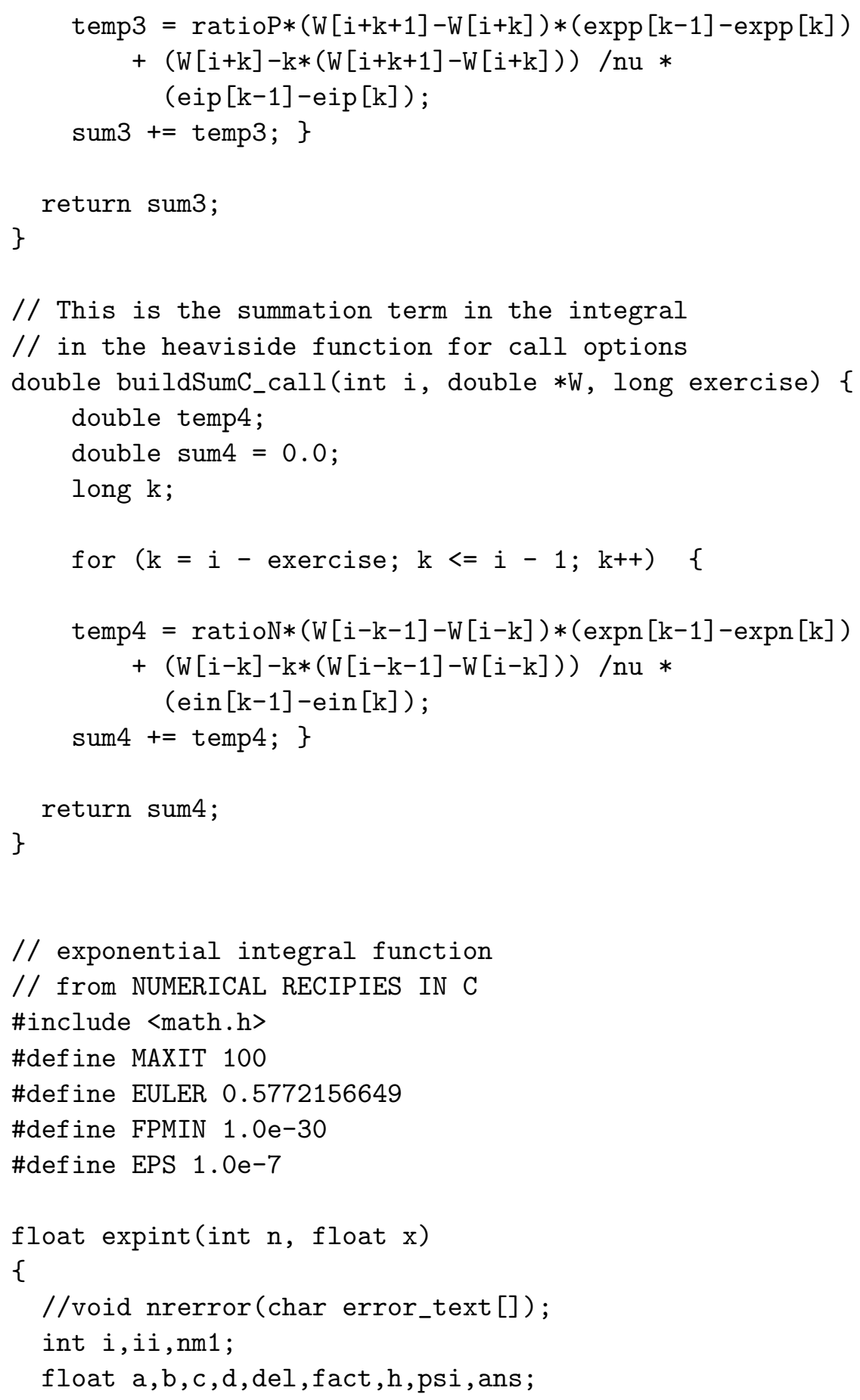




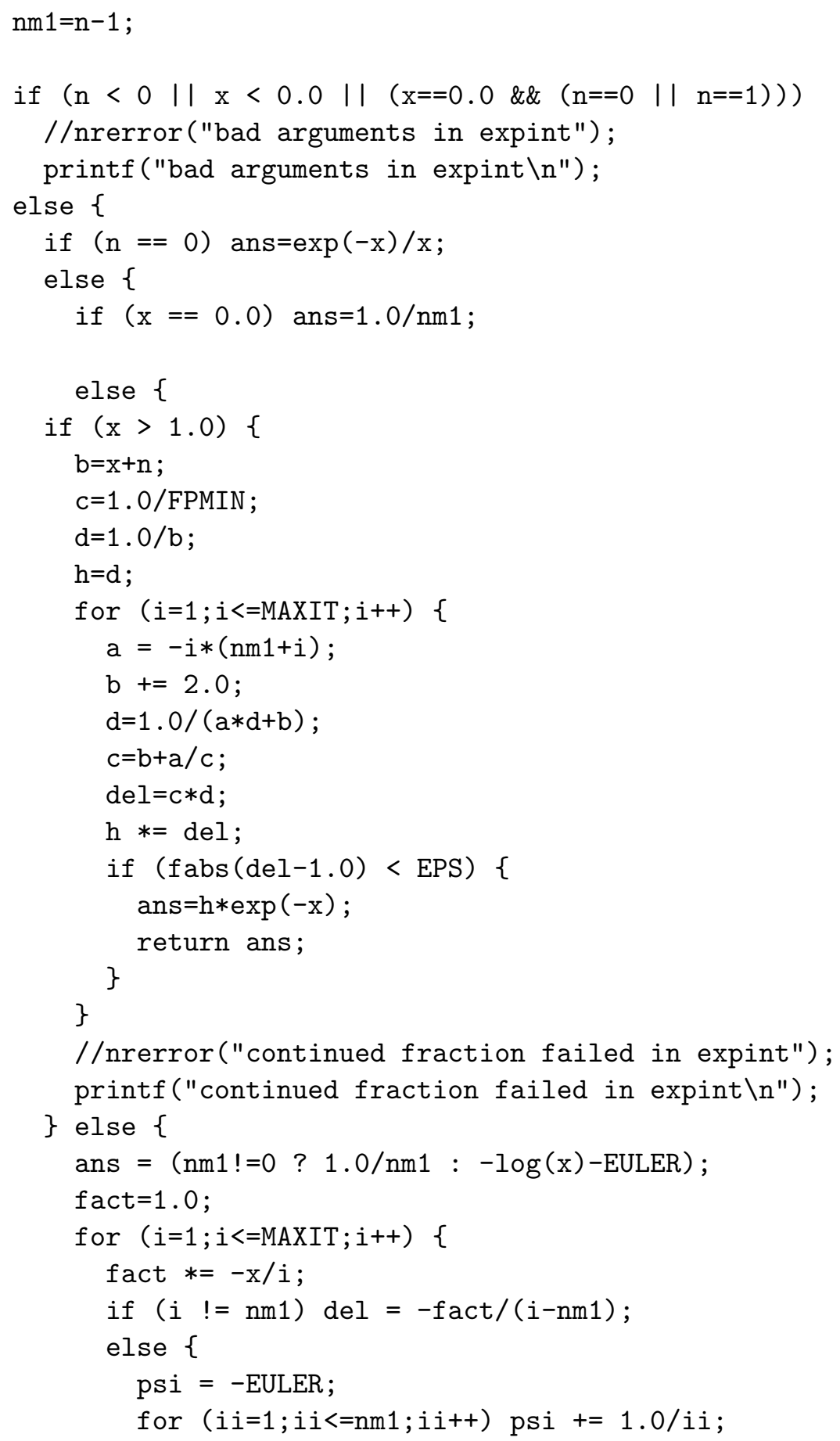




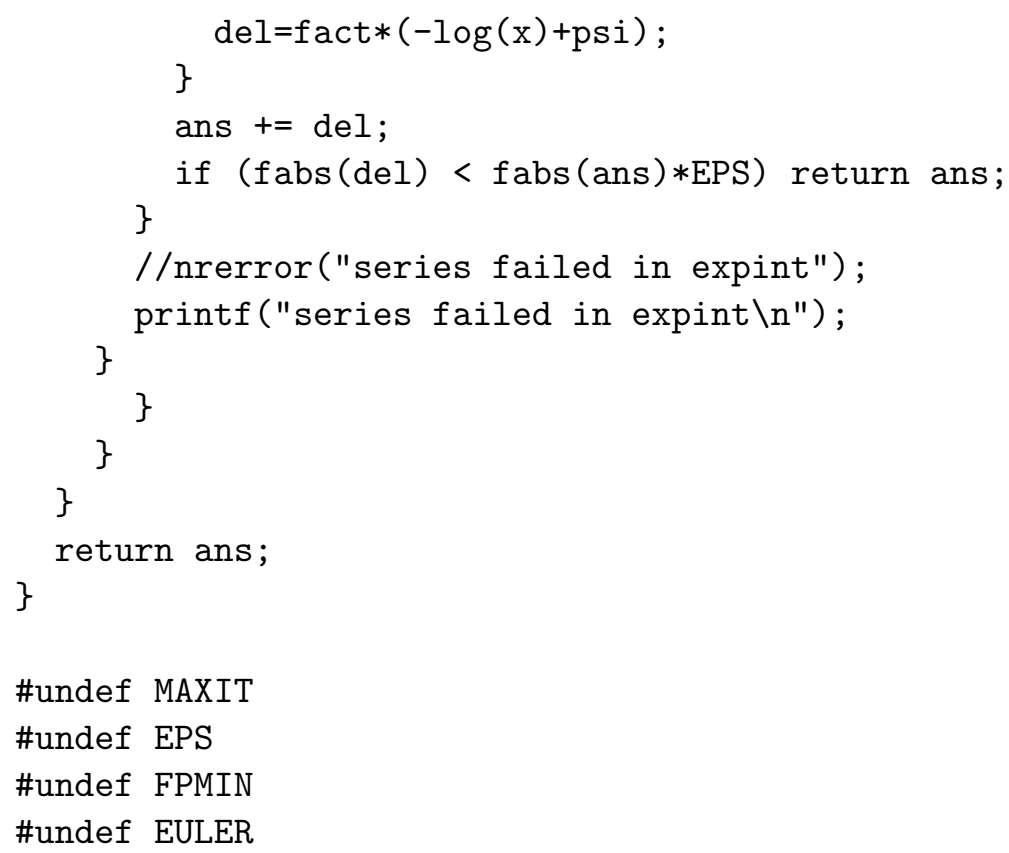

//End of Option Pricing Under the Variance Gamma Process.c 


\section{Appendix C}

\section{Bibliography}

Aase Knut K., "A Jump Diffusion Consumption Based Capital Asset Pricing Model and the Equity Premium Puzzle", Mathematical Finance, Vol. 3, 1993, pages 65-84.

Ahn Chang M. and Thompson Howard E., "Jump-Diffusion Processes and the Term Structure of Interest Rates", The Journal of Finance, Vol. 43, No. 1, 1988, pages 155-174.

Albanese Claudio, Jaimungal Sebastian and Rubisov Dimitri H., "A TwoState Jump Model", Quantitative Finance, Vol. 3, 2003, pages 145-154.

Albanese Claudio, Jaimungal Sebastian and Rubisov Dimitri H., "Jumping in Line", Risk, February 2001, pages 65-68.

Amin Kaushik I., "Jump Diffusion Option Valuation in Discrete Time", The Journal of Finance, Vol. 48, No. 5, December 1993, pages 1833-1863.

Amin Kaushik I. and Jarrow Robert A., "Pricing Options on Risky Assets in a Stochastic Interest Rate Economy", Mathematical Finance, Vol. 2, 1992, pages 217-237.

Amin Kaushik I. and Ng Victor K., "Option Valuation with Systematic Stochastic Volatility", The Journal of Finance, Vol. 48, No. 3, 1993, pages 881-910. 
Andersen Torben G., "Stochastic Autoregressive Volatility: A Framework for Volatility Modelling", Mathematical Finance, Vol. 4, 1994, pages 75-102.

Andersen Torben G., "Return Volatility and Trading Volume. An Information Flow Interpretation of Stochastic Volatility", The Journal of Finance, Vol. 51, No.1, March 1996, pages 169-204.

Ané Thierry and Geman Hélyette, "Order Flow, Transaction Clock and Normality of Asset Returns", The Journal of Finance, Vol. 55, 2000, pages 2259-2284.

Ané Thierry and Labidi Chiraz, "Implied Volatility Surfaces and Market Activity over Time", Journal of Economics and Finance, Vol. 25, Nr. 3, Fall 2001, pages 259-275.

Arnaiz Óscar G., "Revisiting the Variance Gamma Model", working paper.

Bailey Warren and Stulz René, "The Pricing of Stock Index Options in a General Equilibrium Model", Journal of Financial and Quantitative Analysis, Vol. 24, 1989, pages 1-12.

Bakshi Gurdip, Cao Charles and Chen Zhiwu, "Empirical Performance of Alternative Option Pricing Models", The Journal of Finance, Vol. 52, No. 5, December 1997, pages 2003-2049.

Bakshi Gurdip and Chen Zhiwu, "An Alternative Valuation Model for Contingent Claims", Journal of Financial Economics, Vol. 44, 1997, pages 123165.

Bakshi Gurdip and Chen Zhiwu, "Equilibrium Valuation of Foreign Exchange Claims", The Journal of Finance, Vol. 52, 1997, pages 799-826.

Bakshi Gurdip and Madan Dilip B., "What is the Probability of a Stock Market Crash?", working paper, 1998.

Bakshi Gurdip and Madan Dilip B., "Spanning and Derivative-Security Valuation", Journal of Financial Economics, 55, 2000, pages 205-238. 
Bates David S., "Post-'87 Crash Fears in S\&P 500 Future Options", Journal of Econometrics, 94, 2000, pages 181-238.

Bates David S., "Jumps and Stochastic Volatility : Exchange Rate Processes Implicit in Deutsche Mark Options", The Review of Financial Studies, Vol. 9, No. 1, 1996, pages 69-107.

Bates David S., "The Crash of '87: Was It Expected? The Evidence from Options Markets", The Journal of Finance, Vol. 46, No. 3, July 1991, pages 1009-1044.

Benhamou Eric, "Option Pricing with Lévy Process", working paper, 2000.

Berger Eric, "Barrier Options", in Israel Nelken, edit., The Handbook of Exotic Options: Instruments, Analysis and Applications, Richard D. Irwin, New York, 1996, page 215.

Bertoin J., Lévy Processes, Cambridge University Press, Cambridge, 1996.

Black Fisher and Scholes Myron, "The Pricing of Options and Corporate Liabilities", Journal of Political Economy, Vol. 81, No. 3, May/June 1973, pages 637-654.

Bouchaud M., Cont R., Potters M., "Financial Markets as Adaptive Systems", Europhysics Letters, Vol. 41, No. 3, 1998.

Boyle Phelim P. and Lau Sok Hoon, "Bumping Up Against the Barrier with the Binomial Method", The Journal of Derivatives, Summer 1994, pages 6-14.

Brennan Michael J. and Schwartz Eduardo S., "Finite Difference Methods and Jump Processes Arising in the Pricing of Contingent Claims: A Synthesis", The Journal of Financial and Quantitative Analysis, Vol. 13, No. 3, Sept. 1978.

Broadie Mark, Chernov Mikhail and Johannes Michael, "Model Specification and Risk Premiums: The Evidence from the Future Options", working paper, 2003. 
Carr Peter, Geman Hélyette, Madan Dilip B. and Yor Marc, "Stochastic Volatility for Lévy Processes", Mathematical Finance, Vol. 13, No. 3, July 2003, pages 345-382.

Carr Peter, Geman Hélyette, Madan Dilip B. and Yor Marc, "Pricing Options on Realized Variance", working paper, 2003.

Carr Peter, Geman Hélyette, Madan Dilip B. and Yor Marc, "The Fine Structure of Asset Returns: An Empirical Investigation", The Journal of Business, Vol. 75, No. 2, 2002, pages 305-332.

Carr Peter, Jarrow R. A. and Myneni R., "Alternative Characterization of American Put Options", Mathematical Finance, 2, 1992, pages 87-106.

Carr Peter, Jin Xing and Madan Dilib B., "Optimal Investment in Derivative Securities", Finance and Stochastics, Vol. 5, 2001, pages 33-59.

Carr Peter and Madan Dilib B., "Option Valuation Using the Fast Fourier Transform", Journal of Computational Finance, 2(4), 1999, pages 61-73.

Carr Peter and Madan Dilib B., "Optimal Positioning in Derivative Securities", Quantitative Finance, Vol. 1, 2001, pages 19-37.

Carr Peter and Wu Liuren, "Time-Changed Lévy Processes and Option Pricing", forthcoming in Journal of Financial Economics.

Chan T., "Pricing Contingent Claims on Stock Driven by Lévy Processes", The Annals of Applied Probability, Vol. 9, No. 2, pages 504-528, 1999.

Clark P. K., "A Subordinated Stochastic Process Model with Finite Variance for Speculative Prices", Econometrica, Vol. 41, 1973, pages 135-156.

Cont Rama and Voltchkova Ekaterina, "A Finite Difference Scheme for Option Pricing in Jump Diffusion and Exponential Lévy Models", working paper, 2003.

Cox J. C., Ingersoll J. and Ross S., "A Theory of the Term Structure of Interest Rates", Econometrica, 41, 1985, pages 135-156. 
Cox J. C., Ross S. A., "The Valuation of Options for Alternative Stochastic Processes", Journal of Financial Economics, 3, 1976, pages 145-166.

Delbaen Freddy and Schachermayer Walter, "A General Version of the Fundamental Theorem of Asset Pricing", Mathematische Annalen, Vol. 300, 1994, pages 463-520.

Derman Emanuel and Kani Iraj, "Stochastic Implied Trees: Arbitrage Pricing with Stochastic Term and Strike Structure of Volatility", International Journal of Theoretical and Applied Finance, Vol. 3, 1998, pages 7-22.

Derman Emanuel and Kani Iraj, "Riding on the Smile", Risk, 7, 1994, pages 32-39.

Duan Jin-Chuan, "The GARCH Option Pricing Model", Mathematical Finance, Vol. 5, No. 1, January 1995, pages 13-32.

Duffie D., Pan J. and Singleton K., "Transform Analysis and Asset Pricing for Affine Jump-Diffusions", Econometrica, Vol. 68, 2000, pages 1343-1376.

Dumas Bernard, Fleming Jeff and Whaley Robert E., "Implied Volatility Functions: Empirical Tests", The Journal of Finance, Vol. 53, No. 6, 1998, pages 2059-2106.

Dupire Bruno, "Pricing with a Smile", Risk, 7, 1994, pages 18-20.

Engle R. F., "Autoregressive Conditional Heteroskedasticity with Estimates of the Variance of United Kingdom Inflation", Econometrica, Vol. 61, 1982, pages 929-952.

Fama Eugene F., "The Behavior of Stock Market Prices", The Journal of Business, 38, 1965, pages 34-105.

Feller W. E., An Introduction to Probability Theory and its Applications, 2nd Edition, Wiley, New York, 1971.

Fiorani Filippo, Le Barrier Option nel Mercato Finanziario: Replica Statica 
e Dinamica, Tesi discussa alla Facoltà di Economia e Commercio, Università degli Studi di Torino, academic year 1998-1999.

Fristedt B., Sample Properties of Stochastic Processes with Stationary, Independent Increments. In P. Ney and S. C. Port (editors), Advances in Probability and Related Topics, Vol. 3, New York, Marcel Dekker, 1974.

Gallant A. R., Rossi P. E. and Tauchen G., "Stock Prices and Volume", Review of Financial Studies, Vol. 5, 1992, pages 199-242.

Geman Hélyette, "Pure Jump Lévy Processes for Asset Price Modelling", Journal of Banking and Finance, Vol. 26, 2002, pages 1297-1316.

Geman Hélyette and Ané Thierry, "Stochastic Subordination", Risk, September 1996.

Geman Hélyette, Madan Dilip B. and Yor Marc, "Asset Prices are Brownian Motion: Only in Business Time", working paper, 1998.

Geman Hélyette, Madan Dilip B. and Yor Marc, "Time Changes Hidden in Brownian Subordination", working paper, 2000.

Geman Hélyette, Madan Dilip B. and Yor Marc, "Time Changes for Lévy Processes", Mathematical Finance, Vol. 11, No. 1, January 2001, pages 7996.

Geman Hélyette, Madan Dilip B. and Yor Marc, "Stochastic Volatility, Jumps and Hidden Time Changes", forthcoming in Finance and Stochastic, 2001.

Gradshetyn I. S. and Ryzhik I. M. , Tables of Integrals, Series and Products, Academic Press, New York, 1980.

Gukhal C. R., "Analytical Valuation of American Options on Jump-Diffusion Processes", Mathematical Finance, 11, 2001, pages 97-115.

Harrison J. Michael and Kreps David M., "Martingales and Arbitrage in Multiperiod Securities Markets", Journal of Economic Theory, Vol. 20, 1979, pages 381-408. 
Harrison J. Michael and Pliska Stanley R., "Martingales and Stochastic Integrals in the Theory of Continuous Trading", Stochastic Processes and their Applications, Vol. 11, 1981, pages 215-260.

Heston Steven L. , "Invisible Parameters in Option Pricing", The Journal of Finance, Vol. 48, No. 3, 1993, pages 933-947.

Heston Steven L., "A Closed Form Solution for Options with Stochastic Volatility with Applications to Bond and Currency Options", Review of Financial Studies, 6, 1993, pages 327-343.

Hirsa Ali and Madan Dilip B., "Pricing American Options Under Variance Gamma", Journal of Computational Finance, Vol. 7, No. 2, Winter 2003/2004, pages 63-80.

Hull John C. and White A., "The Pricing of Options on Assets with Stochastic Volatility", The Journal of Finance, 42, 1987, pages 281-300.

Humbert Pierre, "The confluent hypergeometric function of two variables", Proceedings of the Royal Society of Edinburgh, 1920, pages 73-85.

Jarrow Robert A. and Rudd Andrew, "Approximate Option Valuation for Arbitrary Stochastic Processes", Journal of Financial Economics, Vol. 10, No. 3, November 1982, pages 349-369.

Jarrow Robert A. and Rosenfeld Eric R., "Jump Risks and the Intertemporal Capital Asset Pricing Model", The Journal of Business, Vol. 57, No. 3, 1984, pages 337-351.

Johnson H. and Shanno D., "Option Pricing when the Variance is Changing", Journal of Financial and Quantitative Analysis, Vol. 22, 1987, pages 143-151.

Jorion P., "On Jump Processes in the Foreign Exchange and Stock Market", Review of Financial Studies, Vol. 1, No. 4, 1988.

Karatzas Ioannis and Shreve Steven E., Methods of Mathematical Finance, Application of Mathematics, Vol. 39, Springer, New York and Heidelberg, 
1998.

Karatzas Ioannis and Shreve Steven E., Brownian Motion and Stochastic Calculus, Springer Verlag, second edition, 1991.

Karpoff Jonathan M., "The Relationship Between Price Changes and Trading Volume: A Survey", The Journal of Financial and Quantitative Analysis, Vol. 22, 1987, pages 109-126.

Këllezi Evis and Webber Nick, "Numerical Methods for Lévy Processes: Lattice Methods and the Density, the Subordinator and the Time Copula", working paper, 2003.

Kon T. S., "Models of Stock Returns: A Comparison", The Journal of Finance, Vol. 39, No. 1, 1984, pages 147-165.

Konikov Mikhail and Madan Dilip B. , "Stochastic Volatility via Markov Chain", working paper, Robert H. Smith School of Business, University of Mariland, 2001.

Konikov Mikhail and Madan Dilip B., "Option Pricing Using Variance Gamma Markov Chains", Review of Derivatives Research, Vol. 5, 2002, pages 81-115.

Kou S. G., "A Jump Diffusion Model for Option Pricing with Three Properties: Leptokurtic Feature, Volatility Smile, and Analytical Tractability", working paper, 1999.

Lam K., Chang E. and Lee M. C., "An Empirical Test of the Variance Gamma Option Pricing Model", Pacific-Basin Finance Journal, Vol. 10, No. 3, 2002, pages 267-285.

Lucas R. E., "Asset Prices in an Exchange Economy", Econometrica, 46, 1978, pages 1429-1445.

Madan Dilib B., "Purely Discontinuous Asset Price Processes", working paper, 1999.

Madan Dilib B., Carr Peter P. and Chang Eric C., "The Variance Gamma 
Process and Option Pricing", European Finance Review 2, 1998, pages 79105.

Madan Dibip B. and Milne Frank, "Option Pricing with V.G. Martingale Components", Mathematical Finance, Vol. 1, No. 4, October 1991, pages $39-55$.

Madan Dilip B. and Seneta Eugene, "Simulation of Estimates Using the Empirical Characteristic Function", International Statistical Review, 55, 1987, pages 153-161.

Madan Dilip B. and Seneta Eugene, "Chebyshev Polynomial Approximation and Characteristic Function Estimation", Journal of the Royal Statistical Society, ser. B, 49, 1987, pages 163-169.

Madan Dilib B. and Seneta Eugene, "The Variance Gamma (V.G.) Model for Share Market Returns", The Journal of Business, 63(4), 1990, pages 511-524.

Mandelbrot Benoit, "New Methods in Statistical Economics", The Journal of Political Economy, Vol. 71, No. 5, October 1963, pages 421-440.

Mandelbrot Benoit, "The Variation of Certain Speculative Prices", The Journal of Business, 36, 1963.

Melino Angelo and Turnbull Stuart M., "Pricing Foreign Currency Options with Stochastic Volatility", Journal of Econometrics, Vol. 45, No. 1-2, JulyAug. 1990, pages 239-265.

Melino Angelo and Turnbull Stuart M., "Misspecification and the Pricing and Hedging of Long-Term Foreign Currency Options", Journal of International Money and Finance, Vol. 4, 1995, pages 373-393.

Merton Robert C., "Theory of Rational Option Pricing", Bell Journal of Economics and Management Science, Vol. 4, 1973, pages 141-183.

Merton Robert C., "Option Pricing when Underlying Stock Returns are Discontinuous", Journal of Financial Economics, 3, 1976, pages 125-144. 
Merton Robert C., "The Impact on Option Pricing of Specification Error in the Underlying Stock Price Returns", The Journal of Finance, Vol. 31, No. 2, May 1976, pages 333-350.

Monroe I., "Processes That Can Be Embedded In A Brownian Motion", The Annals of Probability, Vol. 6, 1978, pages 42-56.

Naik V. and Lee M., "General Equilibrium Pricing of Options on the Market Portfolio with Discontinuous Returns", Review of Financial Studies, Vol. 3, 1990, pages 493-521.

Nandi Saikat, "Pricing and Hedging Index Options under Stochastic Volatility: An Empirical Examination", working paper 96-9, Federal Reserve Bank of Atlanta, August 1996.

Nelson D., "Conditional Heteroskedasticity in Asset Returns: A New Approach", Econometrica, Vol. 59, 1991, pages 347-370.

Poon Ser-Huang and Granger Clive W. J., "Forecasting Volatility in Financial Markets: A Review", Journal of Economic Literature, Vol. 41, No. 2, June 2003, pages 478-539.

Press S. J., "A Compound Events Model for Security Prices", The Journal of Business, 40, July 1967, pages 317-335.

Press William H., Teukolsky Saul A., Vetterling William T. and Flannery Brian P. Numerical Recipes in C, Cambridge University Press, 1992.

Revuz Daniel and Yor Marc, Continuous Martingales and Brownian Motion, Springer-Verlag, Berlin, 1991.

Rogers L. C. and Williams D., Diffusions, Markov Processes and Martingales, Vol. 2, John Wiley \& Sons, New York, 1987.

Rubinstein Mark, "Implied Binomial Trees", The Journal of Finance, Vol. 49, 1994, pages 771-818.

Rubinstein Mark and Reiner Eric, "Breaking down the barriers", Risk, Vol. 
4, No. 8, September 1991, pages. 28-35.

Sato K., Lévy Processes and Infinitely Divisible Distributions, Cambridge University Press, 1999.

Scott Louis O., "Option Pricing when the Variance Changes Randomly: Theory, Estimation and an Application", Journal of Financial and Quantitative Analysis, Vol. 22, 1987, pages 419-438.

Scott Luis O., "Pricing Stock Options in a Jump-Diffusion Model with Stochastic Volatility and Interest Rates: Applications of Fourier Inversion Methods", Mathematical Finance, Vol. 7, No. 4, October 1997, pages 413-426.

Smith G. D., "Numerical Solution of Partial Differential Equations: Finite Difference Methods", $3^{\text {rd }}$ ed., Oxford University Press, 1998.

Stein Elias and Stein Jeremy, "Stock Price Distribution with Stochastic Volatility: An Analysis Approach", Review of Financial Studies, Vol. 4, 1991, pages 727-752.

Strikwerda John C., Finite Difference Schemes and Partial Differential Equations, Wadsworth Inc., Belmont, California, 1989.

Tauchen G. and Pitts M., "The Price-Volume Relationship on Speculative Markets", Econometrica, Vol. 51, 1983, pages 485-505.

Trippi Robert R. and Don M. Chance, "Quick Valuation of the 'Bermuda' Capped Option", Journal of Portfolio Management, Vol. 20, N. 1, 1993, pages 93-99.

Webber Nick and Ribeiro Claudia, "Valuing Path-Dependent Options in the Variance-Gamma Model by Monte Carlo with a Gamma Bridge", Journal of Computational Finance, Vol. 7, No. 2, Winter 2003/2004, pages 81-100.

Wiggins James, "Option Values under Stochastic Volatility", Journal of Financial Economics, Vol. 19, 1987, pages 351-372. 